\title{
Theoretical Studies on Synthetic and Biosynthetic Oxidopyrylium-Alkene Cycloadditions: Pericyclic Pathways to Intricarene
}

\author{
Selina C. Wang and Dean J. Tantillo* \\ Department of Chemistry \\ University of California, Davis \\ One Shields Avenue \\ Davis, CA 95616
}

tantillo@chem.ucdavis.edu

\section{Table of Contents}

\section{S2 Full Gaussian citation.}

S3-S28 Geometries and energies for structures mentioned in Methods section (Table 1).

S29-S48 Geometries and energies for structures mentioned in Figure 1 (B3LYP/6-31G(d) and B3LYP/6-311+G(d,p)) and in solvent (water).

S49-S232 Geometries and energies for structures mentioned in Figures 2 (B3LYP/6-31G(d) in solvents (water, DMSO, $\mathrm{CH}_{3} \mathrm{CN}$ ), B3LYP/6$311+G(d, p), M P 2 / 6-31 G(d)), 3$ and 5, Tables 2-5 and Chart 3.

S233-S240 Investigations on interconversion of 2 to its endo/exo atropisomer.

S241

$\mathrm{S} 242$

S243-S245 Summary of energies, dipole moments, BSSE (for some) of the transition state structures.

S246-S249 Overall cationic structures. 


\section{$\underline{\text { Gaussian citation }}$}

Frisch, M. J., et al., Gaussian 03, revision B.04; Gaussian, Inc.: Pittsburgh, PA, 2004. Frisch, M. J.; Trucks, G. W.; Schlegel, H. B.; Scuseria, G. E.; Robb, M. A.; Cheeseman, J. R.; Montgomery, J. A., Jr.; Vreven, T.; Kudin, K. N.; Burant, J. C.; Millam, J. M.; lyengar, S. S.; Tomasi, J.; Barone, V.; Mennucci, B.; Cossi, M.; Scalmani, G.; Rega, N.; Petersson, G. A.; Nakatsuji, H.; Hada, M.; Ehara, M.; Toyota, K.; Fukuda, R.; Hasegawa, J.; Ishida, M.; Nakajima, T.; Honda, Y.; Kitao, O.; Nakai, H.; Klene, M.; Li, X.; Knox, J. E.; Hratchian, H. P.; Cross, J. B.; Bakken, V.; Adamo, C.; Jaramillo, J.; Gomperts, R.; Stratmann, R. E.; Yazyev, O.; Austin, A. J.; Cammi, R.; Pomelli, C.; Ochterski, J. W.; Ayala, P. Y.; Morokuma, K.; Voth, G. A.; Salvador, P.; Dannenberg, J. J.; Zakrzewski, V. G.; Dapprich, S.; Daniels, A. D.; Strain, M. C.; Farkas, O.; Malick, D. K.; Rabuck, A. D.; Raghavachari, K.; Foresman, J. B.; Ortiz, J. V.; Cui, Q.; Baboul, A. G.; Clifford, S.; Cioslowski, J.; Stefanov, B. B.; Liu, G.; Liashenko, A.; Piskorz, P.; Komaromi, I.; Martin, R. L.; Fox, D. J.; Keith, T.; Al-Laham, M. A.; Peng, C. Y.; Nanayakkara, A.; Challacombe, M.; Gill, P. M. W.; Johnson, B.; Chen, W.; Wong, M. W.; Gonzalez, C.; Pople, J. A. Gaussian 03, revision B.04; Gaussian, Inc.: Pittsburgh, PA, 2004.

The default integration grid was used for all calculations. 


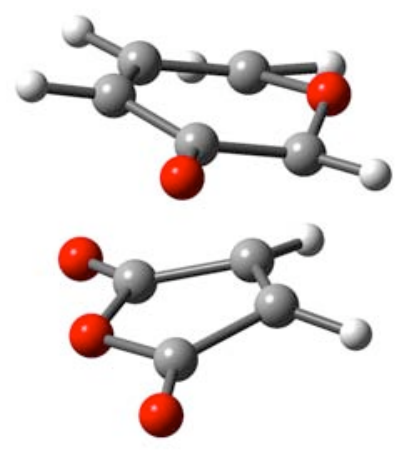

$\mathrm{HF}=-722.5911936$ hartrees $(-453433.199895936 \mathrm{kcal} / \mathrm{mol})$ Imaginary Frequencies: $1(-349.43951 / \mathrm{cm})$

Zero-point correction $=0.137478$ (Hartree/Particle)

Thermal correction to Energy= 0.148309

Thermal correction to Enthalpy=

0.149253

Thermal correction to Gibbs Free Energy=

Sum of electronic and zero-point Energies=

0.100159

Sum of electronic and thermal Energies=

$-722.453715$

Sum of electronic and thermal Enthalpies=

$-722.442885$

Sum of electronic and thermal Free Energies $=\quad-722.491034$

Coordinates (from last standard orientation):

\begin{tabular}{llrrr} 
Center & Atomic & \multicolumn{3}{c}{ Coordinates (Angstroms) } \\
Number & Number & X & $Y$ & $Z$ \\
- \hdashline 1 & 8 & 1.267470 & -1.433464 & 1.158413 \\
2 & 8 & 2.532534 & 1.321100 & -0.691176 \\
3 & 6 & 1.658460 & -0.174195 & 0.915712 \\
4 & 8 & -1.639843 & 0.984602 & -0.654608 \\
5 & 6 & 1.875924 & 0.309578 & -0.460771 \\
6 & 6 & 1.210176 & -0.523180 & -1.463884 \\
7 & 6 & 0.493651 & -1.621507 & -1.083207 \\
8 & 1 & -0.063854 & -2.216592 & -1.799843 \\
9 & 6 & 0.429850 & -2.002605 & 0.282824 \\
10 & 6 & -1.256332 & -0.523364 & 1.070163 \\
11 & 1 & -1.572637 & -1.283346 & 1.771584 \\
12 & 6 & -0.475440 & 0.592196 & 1.327833 \\
13 & 6 & -0.773599 & 1.591898 & 0.266152 \\
14 & 1 & 2.250972 & 0.241220 & 1.723587 \\
15 & 1 & 0.077317 & -2.971479 & 0.612516 \\
16 & 1 & 1.308460 & -0.235341 & -2.505708 \\
17 & 1 & -0.112402 & 0.923875 & 2.291415 \\
18 & 6 & -2.026088 & -0.263350 & -0.169010 \\
19 & 8 & -2.841645 & -0.943198 & -0.731683 \\
20 & 8 & -0.406949 & 2.724563 & 0.143001
\end{tabular}




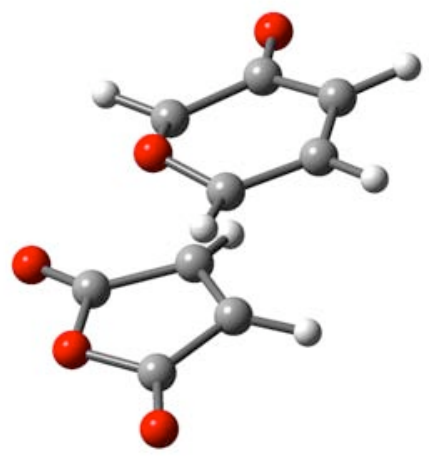

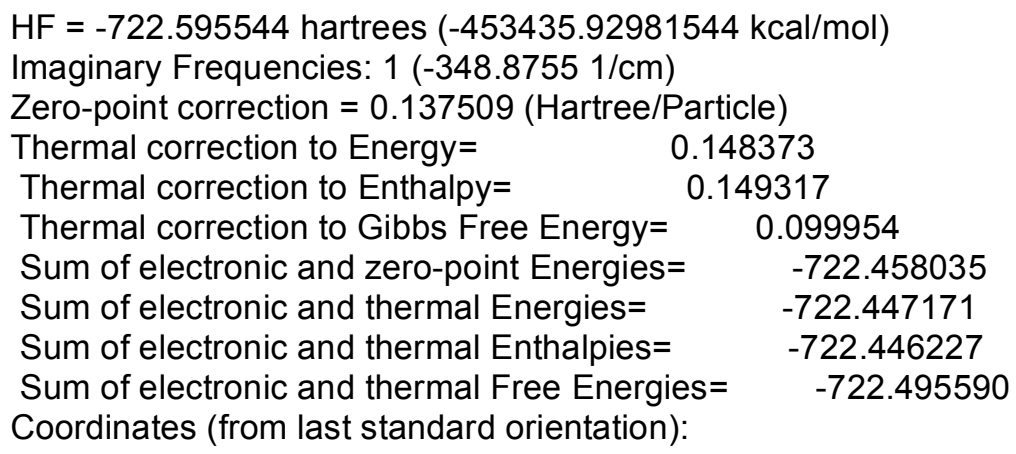

\begin{tabular}{|c|c|c|c|c|}
\hline \multirow{2}{*}{$\begin{array}{l}\text { Center } \\
\text { Number }\end{array}$} & \multirow{2}{*}{$\begin{array}{l}\text { Atomic } \\
\text { Number }\end{array}$} & \multicolumn{3}{|c|}{ Coordinates (Angstroms) } \\
\hline & & & $X \quad Y$ & Z \\
\hline 1 & 8 & 0.511323 & -0.339702 & 1.380279 \\
\hline 2 & 8 & 2.940682 & 1.508086 & -0.461931 \\
\hline 3 & 6 & 1.086987 & 0.704005 & 0.764002 \\
\hline 4 & 6 & 2.292324 & 0.537386 & -0.072219 \\
\hline 5 & 6 & 2.567625 & -0.856133 & -0.407772 \\
\hline 6 & 6 & 1.691947 & -1.836947 & -0.033214 \\
\hline 7 & 1 & 1.822001 & -2.869657 & -0.344061 \\
\hline 8 & 6 & 0.575004 & -1.531506 & 0.782517 \\
\hline 9 & 6 & -0.792121 & -0.597088 & -1.050690 \\
\hline 10 & 6 & -0.423502 & $2 \quad 0.727551$ & -0.891661 \\
\hline 11 & 1 & 0.911954 & 1.643570 & 1.276559 \\
\hline 12 & 1 & -0.051016 & -2.275236 & 1.258484 \\
\hline 13 & 1 & 3.461372 & $2-1.072008$ & -0.984807 \\
\hline 14 & 6 & -2.057229 & $9-0.816749$ & -0.319586 \\
\hline 15 & 6 & -1.499147 & $\begin{array}{ll}7 & 1.379722\end{array}$ & -0.082255 \\
\hline 16 & 8 & -2.399877 & 0.390849 & 0.303209 \\
\hline 17 & 8 & -1.635092 & 2.528017 & 0.235794 \\
\hline 18 & 1 & -0.439370 & $0 \quad-1.303562$ & -1.787649 \\
\hline 19 & 1 & 0.207654 & $4 \quad 1.324045$ & -1.538158 \\
\hline 20 & 8 & -2.737527 & $\begin{array}{ll}7 & -1.800822\end{array}$ & -0.209238 \\
\hline
\end{tabular}




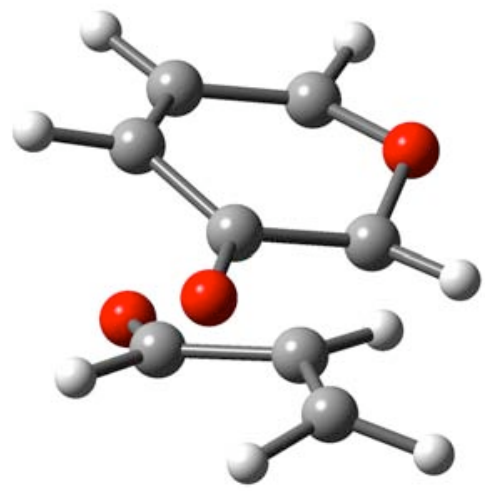

$\mathrm{HF}=-535.2155024$ hartrees $(-335853.079911024 \mathrm{kcal} / \mathrm{mol})$

Imaginary Frequencies: 1 (-360.9456 1/cm)

Zero-point correction $=0.143731$ (Hartree/Particle)

Thermal correction to Energy=

0.153432

Thermal correction to Enthalpy=

0.154376

Thermal correction to Gibbs Free Energy=

Sum of electronic and zero-point Energies=

0.108228

$-535.071771$

Sum of electronic and thermal Energies $=\quad-535.062070$

Sum of electronic and thermal Enthalpies $=\quad-535.061126$

Sum of electronic and thermal Free Energies $=\quad-535.107274$

Coordinates (from last standard orientation):

\begin{tabular}{|c|c|c|c|c|}
\hline \multirow{2}{*}{$\begin{array}{l}\text { Center } \\
\text { Number }\end{array}$} & \multirow{2}{*}{$\begin{array}{l}\text { Atomic } \\
\text { Number }\end{array}$} & \multicolumn{3}{|c|}{ Coordinates (Angstroms) } \\
\hline & & & $X \quad Y$ & Z \\
\hline 1 & 8 & -0.680572 & 0.154525 & 1.665529 \\
\hline 2 & 8 & -2.646955 & -0.604551 & -1.213668 \\
\hline 3 & 6 & -1.359924 & -0.591034 & 0.770921 \\
\hline 4 & 6 & -1.810230 & -0.027361 & -0.515602 \\
\hline 5 & 6 & -1.120856 & 1.208232 & -0.859999 \\
\hline 6 & 6 & -0.129506 & 1.695193 & -0.047155 \\
\hline 7 & 1 & 0.461028 & 2.562905 & -0.326105 \\
\hline 8 & 6 & 0.143987 & 1.088320 & 1.195689 \\
\hline 9 & 6 & 1.484757 & -0.948431 & 0.318375 \\
\hline 10 & 1 & 1.992698 & -0.985565 & 1.278637 \\
\hline 11 & 6 & 0.367609 & -1.730353 & 0.073344 \\
\hline 12 & 1 & -1.975446 & -1.341905 & 1.254213 \\
\hline 13 & 1 & 0.780912 & 1.517957 & 1.957508 \\
\hline 14 & 1 & -1.397866 & 1.705211 & -1.784540 \\
\hline 15 & 1 & 0.111500 & -2.523912 & 0.768956 \\
\hline 16 & 6 & 2.196534 & -0.254774 & -0.759662 \\
\hline 17 & 8 & 3.290911 & 0.270329 & -0.642003 \\
\hline 18 & 1 & 0.024452 & -1.886731 & -0.946584 \\
\hline 19 & 1 & 1.661425 & -0.249140 & -1.736423 \\
\hline
\end{tabular}




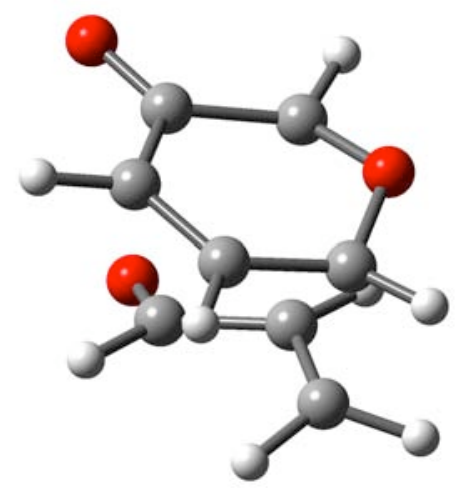

$\mathrm{HF}=-535.2105672$ hartrees $(-335849.983023672 \mathrm{kcal} / \mathrm{mol})$

Imaginary Frequencies: $1(-368.17441 / \mathrm{cm})$

Zero-point correction $=0.143605$ (Hartree/Particle)

Thermal correction to Energy=

0.153297

Thermal correction to Enthalpy=

0.154241

Thermal correction to Gibbs Free Energy=

Sum of electronic and zero-point Energies=

0.108184

Sum of electronic and thermal Energies=

$-535.066962$

Sum of electronic and thermal Enthalpies=

$-535.057270$

$-535.056326$

Sum of electronic and thermal Free Energies=

$-535.102383$

Coordinates (from last standard orientation):

\begin{tabular}{|c|c|c|c|c|}
\hline \multirow{2}{*}{$\begin{array}{l}\text { Center } \\
\text { Number }\end{array}$} & \multirow{2}{*}{$\begin{array}{l}\text { Atomic } \\
\text { Number }\end{array}$} & \multicolumn{3}{|c|}{ Coordinates (Angstroms) } \\
\hline & & & $\begin{array}{l}X \\
Y\end{array}$ & Z \\
\hline 1 & 8 & -1.322761 & -0.476479 & 1.253661 \\
\hline 2 & 6 & -1.789223 & -0.965641 & 0.087730 \\
\hline 3 & 6 & -1.863123 & -0.085297 & -1.026835 \\
\hline 4 & 6 & -1.185291 & 1.098730 & -1.021143 \\
\hline 5 & 6 & -0.331208 & 1.454744 & 0.111528 \\
\hline 6 & 6 & -0.389199 & 0.471001 & 1.200476 \\
\hline 7 & 6 & 1.097033 & -1.235084 & 0.251525 \\
\hline 8 & 6 & 0.152455 & -2.032235 & -0.372081 \\
\hline 9 & 1 & -2.498733 & -1.768981 & 0.242527 \\
\hline 10 & 1 & 0.038283 & 0.706346 & 2.168840 \\
\hline 11 & 1 & -1.246700 & 1.801463 & -1.846424 \\
\hline 12 & 1 & 0.068157 & -2.017062 & -1.455482 \\
\hline 13 & 1 & -2.451798 & -0.402271 & -1.883610 \\
\hline 14 & 8 & 0.375608 & 2.460824 & 0.186030 \\
\hline 15 & 1 & 1.404217 & -1.442986 & 1.273138 \\
\hline 16 & 6 & 2.018879 & -0.387439 & -0.527178 \\
\hline 17 & 1 & 1.698447 & -0.196879 & -1.576973 \\
\hline 18 & 8 & 3.063910 & 0.058358 & -0.097094 \\
\hline 19 & 1 & -0.207865 & -2.933926 & 0.113075 \\
\hline
\end{tabular}




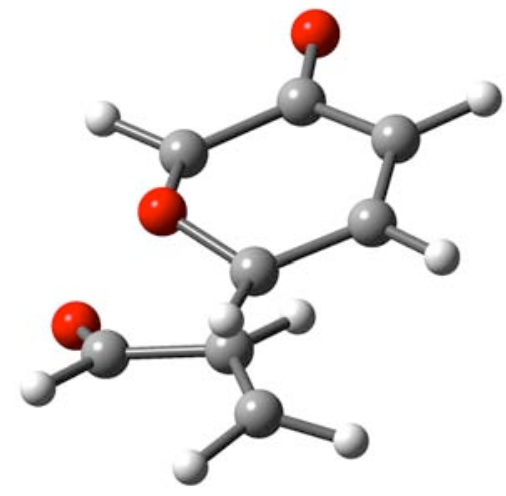

$\mathrm{HF}=-535.2122008$ hartrees $(-335851.008124008 \mathrm{kcal} / \mathrm{mol})$ Imaginary Frequencies: $1(-361.99651 / \mathrm{cm})$

Zero-point correction $=0.143720($ Hartree/Particle $)$

Thermal correction to Energy=

0.153372

Thermal correction to Enthalpy=

0.154317

Thermal correction to Gibbs Free Energy=

Sum of electronic and zero-point Energies=

0.108361

Sum of electronic and thermal Energies=

$-535.068480$

$-535.058828$

$\begin{array}{lc}\text { Sum of electronic and thermal Enthalpies }= & -535.057884 \\ \text { Sum of electronic and thermal Free Energies }= & -535.103840\end{array}$

Coordinates (from last standard orientation):

\begin{tabular}{|c|c|c|c|c|}
\hline \multirow{2}{*}{$\begin{array}{l}\text { Center } \\
\text { Number }\end{array}$} & \multirow{2}{*}{$\begin{array}{l}\text { Atomic } \\
\text { Number }\end{array}$} & \multicolumn{3}{|c|}{ Coordinates (Angstroms) } \\
\hline & & & $\begin{array}{ll}X \quad Y\end{array}$ & Z \\
\hline 1 & 8 & 0.457703 & -0.658216 & 1.372626 \\
\hline 2 & 8 & 0.926077 & 2.488921 & -0.228188 \\
\hline 3 & 6 & 0.197597 & 0.578084 & 0.954268 \\
\hline 4 & 6 & 1.062013 & 1.285893 & 0.000494 \\
\hline 5 & 6 & 2.067859 & 0.416716 & -0.608644 \\
\hline 6 & 6 & 2.054670 & -0.925149 & -0.365252 \\
\hline 7 & 1 & 2.744214 & -1.603019 & -0.861322 \\
\hline 8 & 6 & 1.10 & -1.487123 & 0.529054 \\
\hline 9 & 6 & -0.680566 & -1.654657 & -0.862338 \\
\hline 10 & 6 & -1.243400 & -0.391984 & -0.798490 \\
\hline 11 & 1 & -0.438630 & 1.135773 & 1.632434 \\
\hline 12 & 1 & 1.206147 & -2.482574 & 0.942468 \\
\hline 13 & 1 & 2.799573 & 0.876189 & -1.266013 \\
\hline 14 & 6 & -2.419199 & -0.150432 & 0.057936 \\
\hline 15 & 8 & -3.170557 & 0.800989 & -0.037792 \\
\hline 16 & 1 & -0.105433 & -1.959538 & -1.729285 \\
\hline 17 & 1 & -1.038420 & 0.361710 & -1.552829 \\
\hline 18 & 1 & -1.117738 & -2.463591 & -0.280556 \\
\hline 19 & 1 & -2.595733 & -0.946598 & 0.819763 \\
\hline
\end{tabular}




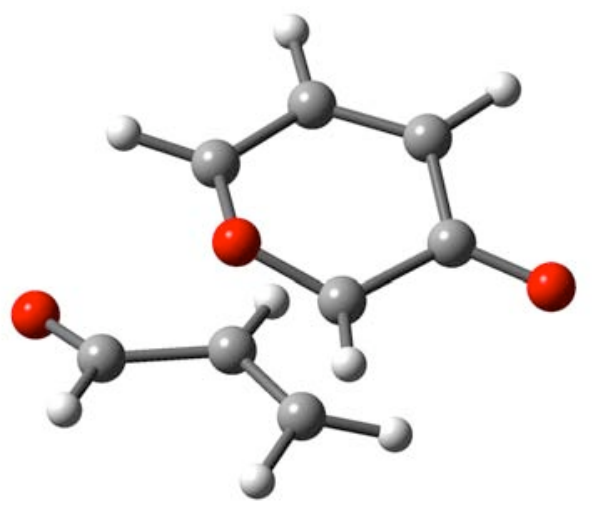

$\mathrm{HF}=-535.2172583$ hartrees $(-335854.181755833 \mathrm{kcal} / \mathrm{mol})$ Imaginary Frequencies: $1(-346.06001 / \mathrm{cm})$

Zero-point correction $=0.143803$ (Hartree/Particle)

Thermal correction to Energy=

0.153498

Thermal correction to Enthalpy=

0.154442

Thermal correction to Gibbs Free Energy=

Sum of electronic and zero-point Energies=

0.108292

Sum of electronic and thermal Energies=

$-535.073455$

Sum of electronic and thermal Enthalpies= $-535.063760$

Sum of electronic and thermal Free Energies= $-535.062816$ Coordinates (from last standard orientation):

\begin{tabular}{|c|c|c|c|c|}
\hline \multirow{2}{*}{$\begin{array}{l}\text { Center } \\
\text { Number }\end{array}$} & \multirow{2}{*}{$\begin{array}{l}\text { Atomic } \\
\text { Number }\end{array}$} & \multicolumn{3}{|c|}{ Coordinates (Angstroms) } \\
\hline & & & $\begin{array}{ll}X & Y\end{array}$ & Z \\
\hline 1 & 8 & 0.212780 & 0.322848 & 1.468021 \\
\hline 2 & 6 & -0.252912 & 1.291279 & 0.682545 \\
\hline 3 & 6 & 0.515602 & 1.733459 & -0.411186 \\
\hline 4 & 6 & 1.660056 & 1.070018 & -0.776299 \\
\hline 5 & 6 & 2.014132 & -0.186073 & -0.132665 \\
\hline 6 & 6 & 1.069394 & -0.568265 & 0.933366 \\
\hline 7 & 6 & -0.412577 & -1.619418 & -0.274469 \\
\hline 8 & 6 & -1.324853 & -0.674433 & -0.709347 \\
\hline 9 & 1 & -1.064110 & 1.850318 & 1.129391 \\
\hline 10 & 1 & 1.366137 & -1.327470 & 1.648853 \\
\hline 11 & 1 & 2.308312 & 1.438880 & -1.565051 \\
\hline 12 & 6 & -2.583881 & -0.441436 & 0.002612 \\
\hline 13 & 8 & -3.503601 & 0.247848 & -0.407974 \\
\hline 14 & 1 & 0.329350 & -2.035122 & -0.948965 \\
\hline 15 & 1 & -1.217941 & -0.189101 & -1.674819 \\
\hline 16 & 1 & 0.181641 & 2.624413 & -0.934957 \\
\hline 17 & 8 & 2.957559 & -0.916487 & -0.445918 \\
\hline 18 & 1 & -2.657563 & -0.966357 & 0.984089 \\
\hline 19 & 1 & -0.689495 & -2.260020 & 0.561084 \\
\hline
\end{tabular}




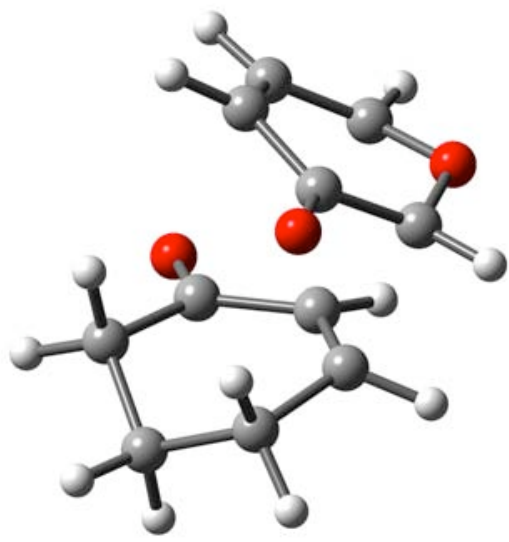

$\mathrm{HF}=-651.9621877$ hartrees $(-409112.792403627 \mathrm{kcal} / \mathrm{mol})$ Imaginary Frequencies: $1(-405.22231 / \mathrm{cm})$

Zero-point correction $=0.210197$ (Hartree/Particle)

Thermal correction to Energy $=\quad 0.221703$

Thermal correction to Enthalpy= $\quad 0.222648$

Thermal correction to Gibbs Free Energy $=\quad 0.172571$

Sum of electronic and zero-point Energies $=\quad-651.751991$

Sum of electronic and thermal Energies $=\quad-651.740484$

Sum of electronic and thermal Enthalpies $=\quad-651.739540$

Sum of electronic and thermal Free Energies $=\quad-651.789617$

Coordinates (from last standard orientation):

\begin{tabular}{|c|c|c|c|c|}
\hline \multirow{2}{*}{$\begin{array}{l}\text { Center } \\
\text { Number }\end{array}$} & \multirow{2}{*}{$\begin{array}{l}\text { Atomic } \\
\text { Number }\end{array}$} & \multicolumn{3}{|c|}{ Coordinates (Angstroms) } \\
\hline & & & $X \quad Y$ & Z \\
\hline 1 & 8 & -1.661143 & -1.182498 & -1.127776 \\
\hline 2 & 8 & -2.300703 & 1.799174 & 0.721546 \\
\hline 3 & 6 & -1.805688 & 0.139540 & -0.883989 \\
\hline 4 & 6 & -1.935114 & 0.640211 & 0.488640 \\
\hline 5 & 6 & -1.505017 & -0.320349 & 1.497027 \\
\hline 6 & 6 & -0.939980 & -1.504054 & 1.110935 \\
\hline 7 & 1 & -0.502938 & -2.193766 & 1.826981 \\
\hline 8 & 6 & -0.897795 & -1.860537 & -0.260561 \\
\hline 9 & 6 & 0.947446 & -0.596435 & -1.021112 \\
\hline 10 & 1 & 1.016219 & -1.378437 & -1.771485 \\
\hline 11 & 6 & 0.316729 & 0.619564 & -1.330527 \\
\hline 12 & 1 & -2.354605 & 0.642810 & -1.672528 \\
\hline 13 & 1 & -0.660751 & - -2.861092 & -0.598984 \\
\hline 14 & 1 & -1.606982 & -0.042487 & 2.541686 \\
\hline 15 & 1 & -0.020794 & 0.728897 & -2.358539 \\
\hline 16 & 6 & 1.918773 & -0.719381 & 0.090129 \\
\hline 17 & 8 & 2.549240 & -1.751306 & 0.281009 \\
\hline 18 & 6 & 0.685393 & 1.915411 & -0.619950 \\
\hline 19 & 6 & 2.027764 & 1.804120 & 0.124418 \\
\hline 20 & 6 & 2.094455 & 0.517863 & 0.962091 \\
\hline 21 & 1 & 0.737582 & 2.717031 & -1.366376 \\
\hline 22 & 1 & -0.100632 & 2.222132 & 0.081962 \\
\hline 23 & 1 & 2.173067 & 2.682306 & 0.764000 \\
\hline 24 & 1 & 2.851979 & 1.799976 & -0.601464 \\
\hline 25 & 1 & 3.040170 & 0.418813 & 1.503410 \\
\hline 26 & 1 & 1.286753 & 0.525135 & 1.710508 \\
\hline
\end{tabular}




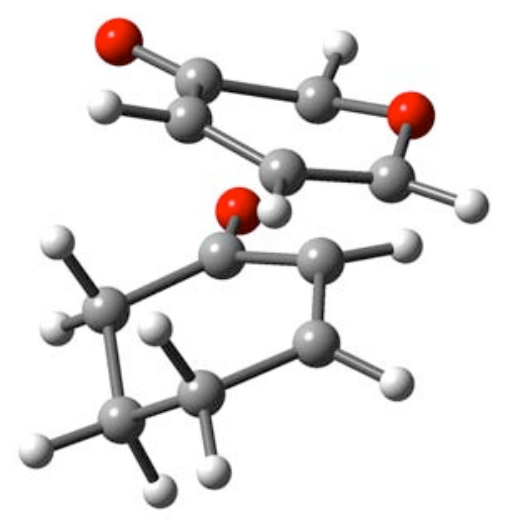

$\mathrm{HF}=-651.9584258$ hartrees $(-409110.431773758 \mathrm{kcal} / \mathrm{mol})$

Imaginary Frequencies: $1(-383.10331 / \mathrm{cm})$

Zero-point correction $=0.209489$ (Hartree/Particle)

Thermal correction to Energy $=\quad 0.221059$

Thermal correction to Enthalpy $=\quad 0.222003$

Thermal correction to Gibbs Free Energy $=\quad 0.172030$

Sum of electronic and zero-point Energies $=\quad-651.748937$

Sum of electronic and thermal Energies $=\quad-651.737367$

Sum of electronic and thermal Enthalpies $=\quad-651.736423$

Sum of electronic and thermal Free Energies $=\quad-651.786396$

Coordinates (from last standard orientation):

\begin{tabular}{llrrr} 
Center & Atomic & \multicolumn{3}{c}{ Coordinates (Angstroms) } \\
Number & Number & X & $Y$ & $Z$ \\
-1 & 8 & -1.916744 & -0.544308 & -1.281471 \\
2 & 6 & -2.321783 & 0.666060 & -0.846254 \\
3 & 6 & -2.369532 & 0.924657 & 0.524843 \\
4 & 6 & -1.779643 & 0.064474 & 1.430081 \\
5 & 6 & -0.986416 & -1.045242 & 0.945971 \\
6 & 6 & -0.962146 & -1.156838 & -0.540680 \\
7 & 6 & 0.587965 & -0.053914 & -1.203153 \\
8 & 1 & 0.484862 & -0.437545 & -2.216881 \\
9 & 6 & 0.279813 & 1.299907 & -0.996326 \\
10 & 1 & -2.894002 & 1.205516 & -1.590021 \\
11 & 1 & -0.690877 & -2.125011 & -0.951547 \\
12 & 1 & -1.876943 & 0.200472 & 2.502394 \\
13 & 1 & -0.112200 & 1.888490 & -1.819082 \\
14 & 6 & 1.768567 & -0.676477 & -0.491237 \\
15 & 8 & 2.273990 & -1.690408 & -0.934008 \\
16 & 1 & -2.872360 & 1.830262 & 0.853823 \\
17 & 8 & -0.320453 & -1.821586 & 1.641954 \\
18 & 6 & 0.742710 & 1.990953 & 0.248545 \\
19 & 1 & 0.665544 & 3.078610 & 0.142921 \\
20 & 1 & 0.085411 & 1.702767 & 1.089965 \\
21 & 6 & 2.179503 & 1.569054 & 0.624686 \\
22 & 6 & 2.288596 & 0.041037 & 0.743661
\end{tabular}




$\begin{array}{rrrrr}23 & 1 & 3.317965 & -0.289439 & 0.912092 \\ 24 & 1 & 1.686642 & -0.319379 & 1.590616 \\ 25 & 1 & 2.466114 & 2.040846 & 1.571782 \\ 26 & 1 & 2.879692 & 1.932793 & -0.138687\end{array}$

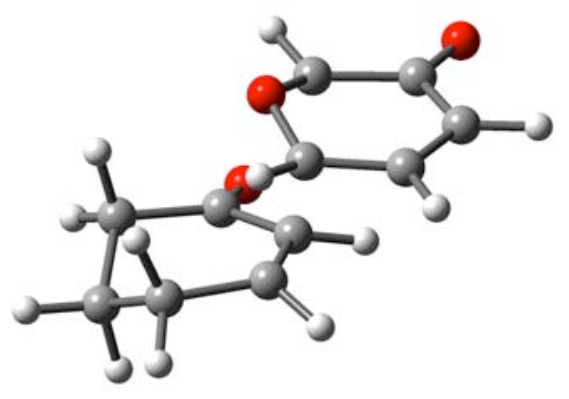

$\mathrm{HF}=-651.9579404$ hartrees $(-409110.127180404 \mathrm{kcal} / \mathrm{mol})$

Imaginary Frequencies: $1(-396.41811 / \mathrm{cm})$

Zero-point correction $=0.209633$ (Hartree/Particle)

Thermal correction to Energy $=\quad 0.221372$

Thermal correction to Enthalpy $=\quad 0.222317$

Thermal correction to Gibbs Free Energy $=\quad 0.171410$

Sum of electronic and zero-point Energies $=\quad-651.748307$

Sum of electronic and thermal Energies $=\quad-651.736568$

Sum of electronic and thermal Enthalpies $=\quad-651.735624$

Sum of electronic and thermal Free Energies $=\quad-651.786531$

Coordinates (from last standard orientation):

\begin{tabular}{|c|c|c|c|c|}
\hline \multirow{2}{*}{$\begin{array}{l}\text { Center } \\
\text { Number }\end{array}$} & \multirow{2}{*}{$\begin{array}{l}\text { Atomic } \\
\text { Numbe }\end{array}$} & \multicolumn{3}{|c|}{ Coordinates (Angstroms) } \\
\hline & & & $\begin{array}{ll}X \quad Y\end{array}$ & Z \\
\hline 1 & 8 & 0.750703 & -0.723396 & 1.337028 \\
\hline 2 & 8 & 2.643438 & 1.948405 & -0.054910 \\
\hline 3 & 6 & 1.039841 & 0.518614 & 0.936056 \\
\hline 4 & 6 & 2.209747 & 0.804662 & 0.086483 \\
\hline 5 & 6 & 2.754731 & -0.391320 & -0.551903 \\
\hline 6 & 6 & 2.139013 & -1.597521 & -0.383091 \\
\hline 7 & 1 & 2.478858 & -2.488589 & -0.904597 \\
\hline 8 & 6 & 1.006449 & -1.720363 & 0.464963 \\
\hline 9 & 6 & -0.704229 & -1.010587 & -0.905291 \\
\hline 10 & 6 & -0.491324 & 0.367701 & -0.845071 \\
\hline 11 & 1 & 0.679585 & 1.281091 & 1.618073 \\
\hline 12 & 1 & 0.654467 & -2.678193 & 0.827780 \\
\hline 13 & 1 & 3.640458 & -0.276213 & -1.169057 \\
\hline 14 & 6 & -1.471406 & 1.286613 & -0.202727 \\
\hline 15 & 8 & -1.417537 & 2.495446 & -0.363118 \\
\hline 16 & 1 & -0.276581 & I -1.548142 & -1.746099 \\
\hline 17 & 1 & 0.155498 & 0.853558 & -1.568460 \\
\hline 18 & 6 & -1.899565 & $5-1.651153$ & -0.210718 \\
\hline 19 & 1 & -2.215220 & -2.538481 & -0.771889 \\
\hline 20 & 1 & -1.626357 & -2.003637 & 0.793552 \\
\hline
\end{tabular}




$\begin{array}{rrrrr}21 & 6 & -3.067701 & -0.663883 & -0.053554 \\ 22 & 6 & -2.579185 & 0.628790 & 0.616251 \\ 23 & 1 & -3.379386 & 1.362941 & 0.746673 \\ 24 & 1 & -2.182319 & 0.392745 & 1.616054 \\ 25 & 1 & -3.489957 & -0.427678 & -1.039127 \\ 26 & 1 & -3.870119 & -1.122366 & 0.536695\end{array}$

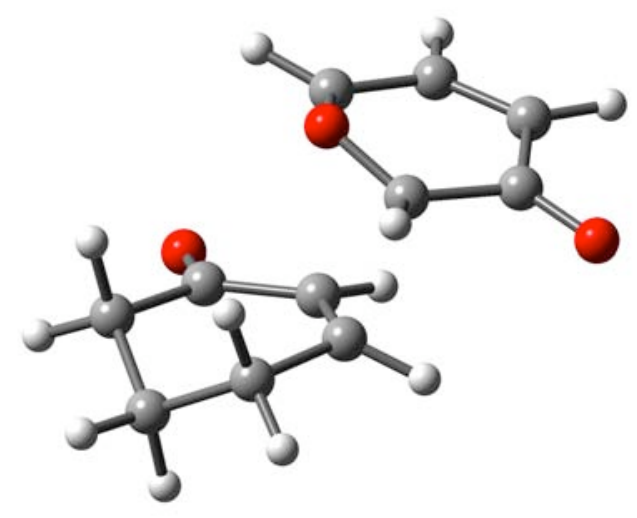

$\mathrm{HF}=-651.9623427$ hartrees $(-409112.889667677 \mathrm{kcal} / \mathrm{mol})$ Imaginary Frequencies: $1(-394.23831 / \mathrm{cm})$

Zero-point correction $=0.209808$ (Hartree/Particle)

Thermal correction to Energy $=\quad 0.221534$

Thermal correction to Enthalpy $=\quad 0.222478$

Thermal correction to Gibbs Free Energy $=\quad 0.171515$

Sum of electronic and zero-point Energies $=\quad-651.752534$

Sum of electronic and thermal Energies $=\quad-651.740809$

Sum of electronic and thermal Enthalpies $=\quad-651.739865$

Sum of electronic and thermal Free Energies $=\quad-651.790827$

Coordinates (from last standard orientation):

\begin{tabular}{ccccc}
$\begin{array}{c}\text { Center } \\
\text { Number }\end{array}$ & Atomic & \multicolumn{3}{c}{ Coordinates (Angstroms) } \\
- & Number & X & $Y$ & $Z$ \\
-1 & 8 & 0.780210 & 0.080500 & 1.499541 \\
2 & 6 & 0.706604 & 1.322956 & 1.016058 \\
3 & 6 & 1.714218 & 1.787301 & 0.143987 \\
4 & 6 & 2.628831 & 0.918864 & -0.394303 \\
5 & 6 & 2.463096 & -0.512408 & -0.199903 \\
6 & 6 & 1.342286 & -0.851368 & 0.698780 \\
7 & 6 & -0.309192 & -0.991903 & -0.669858 \\
8 & 6 & -0.663243 & 0.339359 & -0.902276 \\
9 & 1 & 0.068205 & 1.971644 & 1.601272 \\
10 & 1 & 1.332752 & -1.836576 & 1.152438 \\
11 & 1 & 3.451170 & 1.260854 & -1.014784 \\
12 & 6 & -1.921732 & 0.940805 & -0.419484 \\
13 & 8 & -2.247573 & 2.083894 & -0.719018 \\
14 & 1 & 0.347197 & -1.472494 & -1.392739 \\
15 & 1 & -0.134007 & 0.925231 & -1.646200 \\
16 & 6 & -1.266678 & -1.906869 & 0.095733
\end{tabular}




$\begin{array}{rrrrr}17 & 1 & -1.172056 & -2.929268 & -0.287131 \\ 18 & 1 & -0.996442 & -1.949630 & 1.159255 \\ 19 & 6 & -2.721562 & -1.421156 & 0.002821 \\ 20 & 6 & -2.817090 & 0.048036 & 0.436837 \\ 21 & 1 & -3.836795 & 0.439867 & 0.375728 \\ 22 & 1 & -2.497289 & 0.140623 & 1.486793 \\ 23 & 1 & -3.081468 & -1.521777 & -1.029540 \\ 24 & 1 & -3.366568 & -2.049425 & 0.628697 \\ 25 & 8 & 3.119395 & -1.398240 & -0.756076 \\ 26 & 1 & 1.735825 & 2.850018 & -0.079716\end{array}$

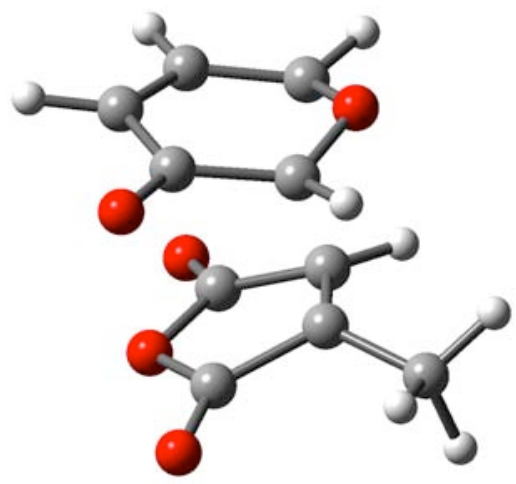

$\mathrm{HF}=-761.9089728$ hartrees $(-478105.499521728 \mathrm{kcal} / \mathrm{mol})$ Imaginary Frequencies: $1(-379.13341 / \mathrm{cm})$

Zero-point correction $=0.165502$ (Hartree/Particle)

Thermal correction to Energy $=$

0.177939

Thermal correction to Enthalpy= 0.178884

Thermal correction to Gibbs Free Energy=

Sum of electronic and zero-point Energies=

0.126667

Sum of electronic and thermal Energies=

$-761.743471$

Sum of electronic and thermal Enthalpies=

$-761.731033$

Sum of electronic and thermal Free Energies=

$-761.730089$

Coordinates (from last standard orientation):

\begin{tabular}{ccrrr}
\hline & Center & Atomic & \multicolumn{3}{c}{ Coordinates (Angstroms) } \\
Number & Number & X & $Y$ & $Z$ \\
- \hdashline & 8 & 1.422379 & -0.503940 & 1.573676 \\
2 & 8 & 2.236760 & 1.171800 & -1.451662 \\
3 & 6 & 1.538683 & 0.518566 & 0.708527 \\
4 & 8 & -1.560310 & 0.026314 & -1.247272 \\
5 & 6 & 1.817631 & 0.278595 & -0.719397 \\
6 & 6 & 1.510779 & -1.090143 & -1.138919 \\
7 & 6 & 1.008429 & -1.986373 & -0.241968 \\
8 & 1 & 0.698121 & -2.982806 & -0.540255 \\
9 & 6 & 0.833267 & -1.618283 & 1.121722 \\
10 & 6 & -1.190889 & -0.452453 & 0.996198 \\
11 & 1 & -1.497569 & -0.885415 & 1.939867 \\
12 & 6 & -0.743423 & 0.853048 & 0.790709 \\
13 & 6 & -1.054277 & 1.177276 & -0.635672 \\
14 & 1 & 1.927015 & 1.415996 & 1.176454 \\
15 & 1 & 0.662583 & -2.344907 & 1.906234
\end{tabular}




$\begin{array}{rrrrr}16 & 1 & 1.689944 & -1.353539 & -2.176431 \\ 17 & 6 & -1.754349 & -0.956094 & -0.274753 \\ 18 & 8 & -2.287735 & -2.002589 & -0.528924 \\ 19 & 8 & -0.945583 & 2.217044 & -1.220817 \\ 20 & 6 & -0.688586 & 1.963709 & 1.808438 \\ 21 & 1 & -0.182052 & 1.643873 & 2.724039 \\ 22 & 1 & -0.178998 & 2.840180 & 1.400468 \\ 23 & 1 & -1.706719 & 2.270482 & 2.080315\end{array}$

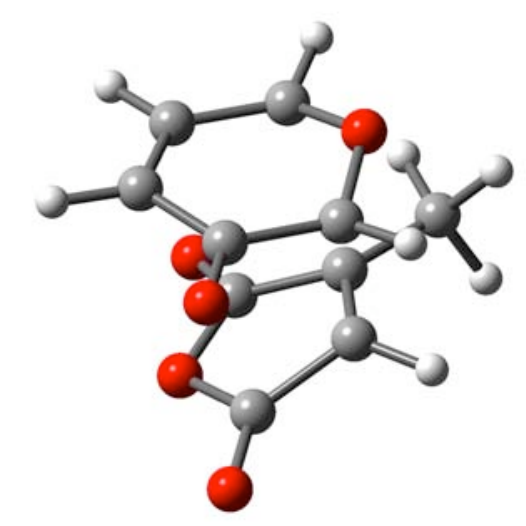

$\mathrm{HF}=-761.9104835$ hartrees $(-478106.447501085 \mathrm{kcal} / \mathrm{mol})$ Imaginary Frequencies: 1 (-372.4060 1/cm)

Zero-point correction $=0.165456$ (Hartree/Particle)

Thermal correction to Energy $=\quad 0.177931$

Thermal correction to Enthalpy= $\quad 0.178875$

Thermal correction to Gibbs Free Energy $=0.126593$

Sum of electronic and zero-point Energies $=\quad-761.745027$

Sum of electronic and thermal Energies $=\quad-761.732552$

Sum of electronic and thermal Enthalpies $=\quad-761.731608$

Sum of electronic and thermal Free Energies $=\quad-761.783890$

Coordinates (from last standard orientation):

\begin{tabular}{llrrr} 
Center & Atomic & \multicolumn{3}{c}{ Coordinates (Angstroms) } \\
Number & Number & $X$ & $Y$ & $Z$ \\
\hline 1 & 8 & -0.623877 & -1.925059 & -0.691527 \\
2 & 8 & -3.153914 & 0.483158 & -0.083176 \\
3 & 6 & -1.422529 & -0.875326 & -0.947952 \\
4 & 8 & 0.876162 & 1.677588 & 0.705383 \\
5 & 6 & -2.160155 & -0.201081 & 0.141896 \\
6 & 6 & -1.549642 & -0.419158 & 1.452754 \\
7 & 6 & -0.441562 & -1.210699 & 1.570670 \\
8 & 1 & 0.071501 & -1.334912 & 2.519455 \\
9 & 6 & 0.093423 & -1.878661 & 0.441211 \\
10 & 6 & 1.395867 & -0.159148 & -0.639371 \\
11 & 6 & 0.326005 & 0.507043 & -1.237329 \\
12 & 6 & 0.054049 & 1.726206 & -0.431387 \\
13 & 1 & -1.884715 & -0.937385 & -1.927508 \\
14 & 1 & 0.812332 & -2.684400 & 0.512903 \\
15 & 1 & -2.003793 & 0.064849 & 2.311392 \\
16 & 6 & 1.756869 & 0.608382 & 0.581848 \\
17 & 8 & 2.639873 & 0.410125 & 1.374986
\end{tabular}




$\begin{array}{rrrrr}18 & 8 & -0.690288 & 2.641738 & -0.634055 \\ 19 & 6 & 2.379815 & -1.091143 & -1.285306 \\ 20 & 1 & 1.874223 & -1.860897 & -1.876712 \\ 21 & 1 & 3.039727 & -0.535063 & -1.964584 \\ 22 & 1 & 3.012776 & -1.569514 & -0.533017 \\ 23 & 1 & 0.101452 & 0.518428 & -2.297018\end{array}$

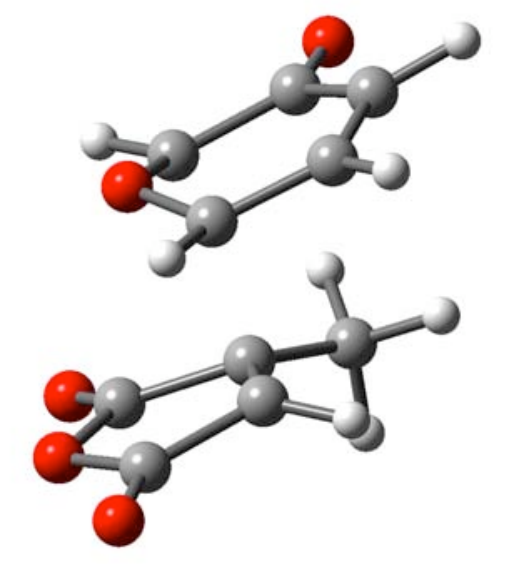

$\mathrm{HF}=-761.9143727$ hartrees $(-478108.888012977 \mathrm{kcal} / \mathrm{mol})$

Imaginary Frequencies: $1(-382.85211 / \mathrm{cm})$

Zero-point correction $=0.165588$ (Hartree/Particle)

Thermal correction to Energy $=\quad 0.178030$

Thermal correction to Enthalpy $=\quad 0.178974$

Thermal correction to Gibbs Free Energy $=\quad 0.126658$

Sum of electronic and zero-point Energies $=\quad-761.748785$

Sum of electronic and thermal Energies $=\quad-761.736343$

Sum of electronic and thermal Enthalpies $=\quad-761.735398$

Sum of electronic and thermal Free Energies $=\quad-761.787715$

Coordinates (from last standard orientation):

\begin{tabular}{ccccc} 
Center & Atomic & \multicolumn{3}{c}{ Coordinates (Angstroms) } \\
Number & Number & X & $Y$ & $Z$ \\
- \hdashline 1 & 8 & -0.364288 & -0.860498 & -1.353843 \\
2 & 8 & -2.972667 & 1.288751 & -0.238996 \\
3 & 6 & -1.009892 & 0.281474 & -1.076794 \\
4 & 6 & -2.261234 & 0.284854 & -0.307145 \\
5 & 6 & -2.518308 & -0.978134 & 0.384789 \\
6 & 6 & -1.583359 & -1.969596 & 0.360190 \\
7 & 1 & -1.700475 & -2.884960 & 0.933516 \\
8 & 6 & -0.399438 & -1.822264 & -0.418922 \\
9 & 6 & 0.841148 & -0.528829 & 1.016495 \\
10 & 6 & 0.418472 & 0.766130 & 0.708269 \\
11 & 1 & -0.824705 & 1.060339 & -1.808386 \\
12 & 1 & 0.260904 & -2.649129 & -0.649539 \\
13 & 1 & -3.451282 & -1.075569 & 0.931333 \\
14 & 6 & 2.099329 & -0.796137 & 0.283768 \\
15 & 6 & 1.435666 & 1.313946 & -0.244835 \\
16 & 8 & 2.357670 & 0.307902 & -0.532391 \\
17 & 8 & 1.527978 & 2.414964 & -0.714112 \\
18 & 1 & 0.602420 & -1.084097 & 1.913717
\end{tabular}




$\begin{array}{rrrrr}19 & 8 & 2.824548 & -1.754259 & 0.302005 \\ 20 & 6 & -0.379903 & 1.711761 & 1.562225 \\ 21 & 1 & -0.976493 & 2.402975 & 0.962062 \\ 22 & 1 & -1.053788 & 1.166948 & 2.228520 \\ 23 & 1 & 0.302616 & 2.309372 & 2.179234\end{array}$

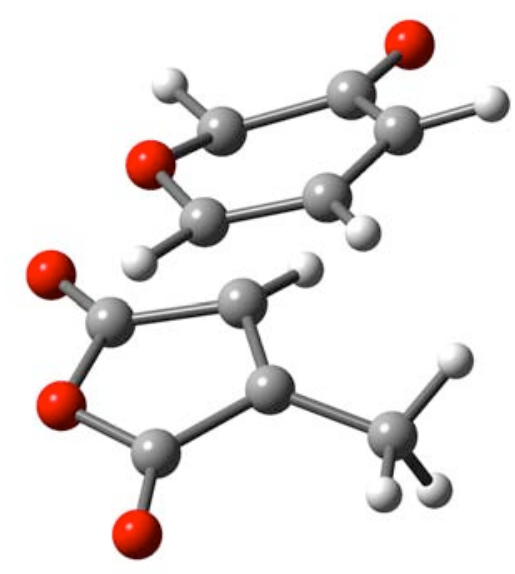

$\mathrm{HF}=-761.9155031$ hartrees $(-478109.597350281 \mathrm{kcal} / \mathrm{mol})$ Imaginary Frequencies: $1(-366.97871 / \mathrm{cm})$

Zero-point correction $=0.165427$ (Hartree/Particle)

Thermal correction to Energy=

0.177941

Thermal correction to Enthalpy=

0.178886

Thermal correction to Gibbs Free Energy=

Sum of electronic and zero-point Energies=

0.126144

Sum of electronic and thermal Energies=

$-761.750076$

Sum of electronic and thermal Enthalpies=

$-761.737562$

$-761.736617$

Sum of electronic and thermal Free Energies=

$-761.789359$

Coordinates (from last standard orientation):

\begin{tabular}{ccccc} 
Center & Atomic & \multicolumn{3}{c}{ Coordinates (Angstroms) } \\
Number & Number & X & $Y$ & $Z$ \\
- \hdashline 1 & 8 & -0.548586 & 0.384666 & -1.546671 \\
2 & 8 & -2.907480 & 1.274830 & 0.980555 \\
3 & 6 & -1.088747 & 1.068778 & -0.515442 \\
4 & 6 & -2.282807 & 0.553508 & 0.201847 \\
5 & 6 & -2.551683 & -0.850400 & -0.066072 \\
6 & 6 & -1.685268 & -1.574874 & -0.851067 \\
7 & 1 & -1.804248 & -2.645722 & -0.987199 \\
8 & 6 & -0.617525 & -0.948115 & -1.510873 \\
9 & 6 & 0.765708 & -0.636561 & 0.857886 \\
10 & 6 & 0.412909 & 0.712077 & 0.876286 \\
11 & 1 & -0.983126 & 2.141572 & -0.640935 \\
12 & 1 & 0.019411 & -1.423891 & -2.244974 \\
13 & 1 & -3.431090 & -1.298872 & 0.385259 \\
14 & 6 & 1.981473 & -0.770322 & 0.031920 \\
15 & 6 & 1.503503 & 1.449832 & 0.154943 \\
16 & 8 & 2.351044 & 0.513242 & -0.412387 \\
17 & 8 & 1.665455 & 2.631268 & 0.022624 \\
18 & 8 & 2.633694 & -1.742231 & -0.246125
\end{tabular}




$\begin{array}{rrrrr}19 & 6 & 0.279808 & -1.739327 & 1.736891 \\ 20 & 1 & 0.630547 & -1.590694 & 2.767017 \\ 21 & 1 & -0.815856 & -1.768704 & 1.774861 \\ 22 & 1 & 0.657075 & -2.704368 & 1.388496 \\ 23 & 1 & -0.129961 & 1.208899 & 1.675585\end{array}$

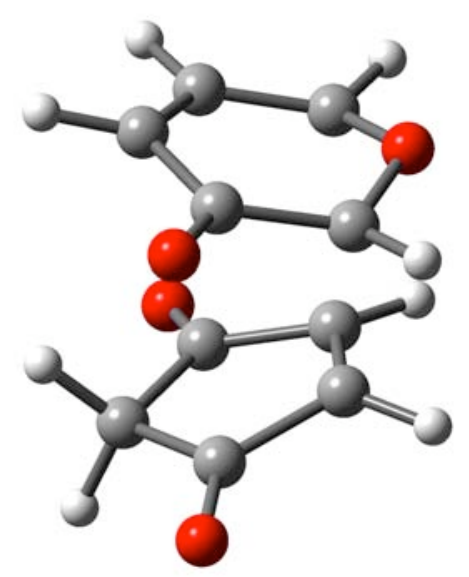

$\mathrm{HF}=-686.657475$ hartrees $(-430884.43213725 \mathrm{kcal} / \mathrm{mol})$ Imaginary Frequencies: $1(-370.50921 / \mathrm{cm})$

Zero-point correction $=0.160550($ Hartree/Particle $)$

Thermal correction to Energy $=\quad 0.171908$

Thermal correction to Enthalpy= $\quad 0.172852$

Thermal correction to Gibbs Free Energy $=\quad 0.122468$

Sum of electronic and zero-point Energies $=\quad-686.496925$

Sum of electronic and thermal Energies $=\quad-686.485567$

Sum of electronic and thermal Enthalpies $=\quad-686.484623$

Sum of electronic and thermal Free Energies $=\quad-686.535007$

Coordinates (from last standard orientation):

\begin{tabular}{llrrr} 
Center & Atomic & \multicolumn{3}{c}{ Coordinates (Angstroms) } \\
Number & Number & $X$ & $Y$ & $Z$ \\
-1 & 8 & 1.332401 & -1.289225 & 1.248938 \\
2 & 8 & 2.433868 & 1.381002 & -0.821474 \\
3 & 6 & 1.635093 & -0.026217 & 0.905205 \\
4 & 6 & 1.855838 & 0.343060 & -0.507726 \\
5 & 6 & 1.298005 & -0.634224 & -1.442947 \\
6 & 6 & 0.623149 & -1.725975 & -0.976645 \\
7 & 1 & 0.130490 & -2.424722 & -1.645951 \\
8 & 6 & 0.535817 & -1.975736 & 0.418743 \\
9 & 6 & -1.228692 & -0.544027 & 1.044875 \\
10 & 1 & -1.497883 & -1.286235 & 1.787903 \\
11 & 6 & -0.498787 & 0.622510 & 1.290114 \\
12 & 6 & -0.827689 & 1.649274 & 0.250005 \\
13 & 1 & 2.197733 & 0.490859 & 1.674745 \\
14 & 1 & 0.231390 & -2.930764 & 0.827277 \\
15 & 1 & 1.435405 & -0.453156 & -2.504689 \\
16 & 1 & -0.162789 & 0.945711 & 2.269451 \\
17 & 6 & -2.032182 & -0.398701 & -0.202218 \\
18 & 8 & -2.819130 & -1.214022 & -0.644376
\end{tabular}




$\begin{array}{lllll}19 & 8 & -0.503278 & 2.816595 & 0.277751 \\ 20 & 6 & -1.707347 & 0.969912 & -0.803282 \\ 21 & 1 & -1.186465 & 0.874793 & -1.763829 \\ 22 & 1 & -2.618011 & 1.549465 & -0.988369\end{array}$

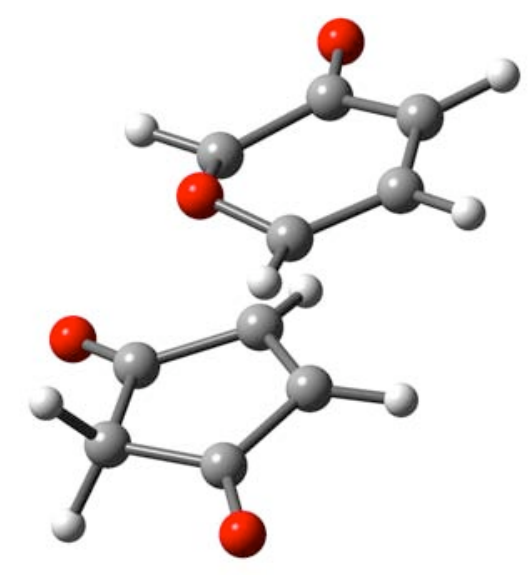

$\mathrm{HF}=-686.6622097$ hartrees $(-430887.403208847 \mathrm{kcal} / \mathrm{mol})$ Imaginary Frequencies: $1(-362.84071 / \mathrm{cm})$

Zero-point correction $=0.160635$ (Hartree/Particle)

Thermal correction to Energy=

0.171996

Thermal correction to Enthalpy=

0.172941

Thermal correction to Gibbs Free Energy=

Sum of electronic and zero-point Energies=

0.122517

Sum of electronic and thermal Energies=

$-686.501575$

$-686.490213$

Sum of electronic and thermal Enthalpies=

$-686.489269$

Sum of electronic and thermal Free Energies=

$-686.539693$

Coordinates (from last standard orientation):

\begin{tabular}{|c|c|c|c|c|}
\hline \multirow{2}{*}{$\begin{array}{l}\text { Center } \\
\text { Number }\end{array}$} & \multirow{2}{*}{$\begin{array}{l}\text { Atomic } \\
\text { Number }\end{array}$} & \multicolumn{3}{|c|}{ Coordinates (Angstroms) } \\
\hline & & & $X \quad Y$ & Z \\
\hline 1 & 8 & 0.528418 & -0.345244 & 1.396836 \\
\hline 2 & 8 & 2.964156 & 1.481105 & -0.456174 \\
\hline 3 & 6 & 1.092505 & 0.695517 & 0.758173 \\
\hline 4 & 6 & 2.300998 & 0.517322 & -0.073541 \\
\hline 5 & 6 & 2.559706 & -0.877657 & -0.413208 \\
\hline 6 & 6 & 1.675660 & -1.850275 & -0.035491 \\
\hline 7 & 1 & 1.790858 & -2.882783 & -0.352841 \\
\hline 8 & 6 & 0.569235 & -1.536135 & 0.790344 \\
\hline 9 & 6 & -0.794538 & -0.599945 & -1.022469 \\
\hline 10 & 6 & -0.408983 & $\begin{array}{ll}3 & 0.732508\end{array}$ & -0.866110 \\
\hline 11 & 1 & 0.936283 & 1.637450 & 1.272535 \\
\hline 12 & 1 & -0.052736 & $6 \quad-2.278354$ & 1.273648 \\
\hline 13 & 1 & 3.447499 & $9-1.101825$ & -0.996233 \\
\hline 14 & 6 & -2.074899 & $9-0.868186$ & -0.319123 \\
\hline 15 & 6 & -1.471016 & $6 \quad 1.460680$ & -0.087496 \\
\hline 16 & 8 & -1.524272 & 2.658844 & 0.094285 \\
\hline 17 & 1 & -0.413346 & $6-1.288602$ & -1.766125 \\
\hline 18 & 1 & 0.243100 & 1.286372 & -1.533291 \\
\hline 19 & 8 & -2.700707 & $7-1.912688$ & -0.331998 \\
\hline 20 & 6 & -2.476729 & 0.419532 & 0.405035 \\
\hline
\end{tabular}




$\begin{array}{lllll}21 & 1 & -3.504896 & 0.711754 & 0.167682 \\ 22 & 1 & -2.419148 & 0.299684 & 1.494358 \\ -\end{array}$

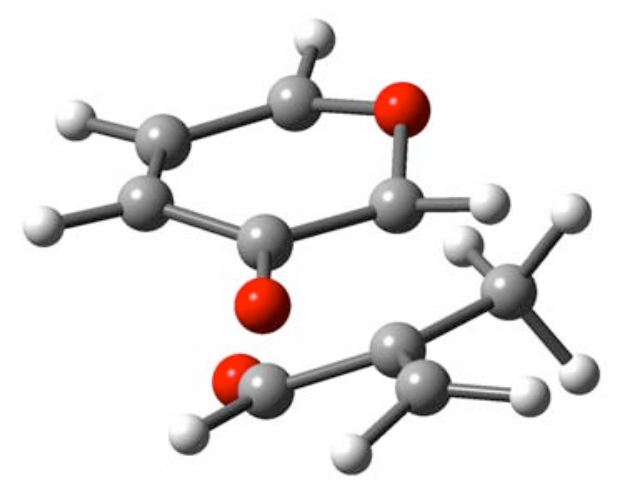

$\mathrm{HF}=-574.5345066$ hartrees $(-360526.148236566 \mathrm{kcal} / \mathrm{mol})$ Imaginary Frequencies: 1 (-365.3179 1/cm)

Zero-point correction $=0.171732$ (Hartree/Particle)

Thermal correction to Energy $=\quad 0.183064$

Thermal correction to Enthalpy= $\quad 0.184008$

Thermal correction to Gibbs Free Energy $=0.134213$

Sum of electronic and zero-point Energies $=\quad-574.362775$

Sum of electronic and thermal Energies $=\quad-574.351442$

Sum of electronic and thermal Enthalpies $=\quad-574.350498$

Sum of electronic and thermal Free Energies $=\quad-574.400294$

Coordinates (from last standard orientation):

\begin{tabular}{|c|c|c|c|c|}
\hline \multirow{2}{*}{$\begin{array}{l}\text { Center } \\
\text { Number }\end{array}$} & \multirow{2}{*}{$\begin{array}{l}\text { Atomic } \\
\text { Number }\end{array}$} & \multicolumn{3}{|c|}{ Coordinates (Angstroms) } \\
\hline & & & $X \quad Y$ & Z \\
\hline 1 & 8 & -0.609177 & -0.713135 & 1.452264 \\
\hline 2 & 8 & -2.991184 & -0.319409 & -1.172432 \\
\hline 3 & 6 & -1.320986 & -1.023459 & 0.348504 \\
\hline 4 & 6 & -2.121666 & -0.000550 & -0.357988 \\
\hline 5 & 6 & -1.711820 & 1.355294 & -0.032494 \\
\hline 6 & 6 & -0.660223 & 1.570797 & 0.825947 \\
\hline 7 & 1 & -0.278570 & 2.569568 & 1.016513 \\
\hline 8 & 6 & -0.058999 & 0.501160 & 1.510628 \\
\hline 9 & 6 & 1.421066 & -0.566272 & -0.453265 \\
\hline 10 & 6 & 0.318735 & -1.180853 & -1.037395 \\
\hline 11 & 1 & -1.706140 & -2.036538 & 0.392191 \\
\hline 12 & 1 & 0.648242 & 0.609766 & 2.321757 \\
\hline 13 & 1 & -2.240269 & 2.176822 & -0.505984 \\
\hline 14 & 1 & 0.245820 & -2.265427 & -1.008308 \\
\hline 15 & 6 & 1.831147 & 0.764933 & -0.927976 \\
\hline 16 & 8 & 2.882339 & 1.313045 & -0.635482 \\
\hline 17 & 1 & -0.167932 & -0.720436 & -1.893148 \\
\hline 18 & 1 & 1.106024 & 1.253107 & -1.615160 \\
\hline 19 & 6 & 2.355890 & -1.307407 & 0.466900 \\
\hline 20 & 1 & 3.008643 & -1.978845 & -0.107888 \\
\hline 21 & 1 & 3.003129 & -0.608704 & 1.002490 \\
\hline 22 & 1 & 1.806365 & -1.925183 & 1.185566 \\
\hline
\end{tabular}




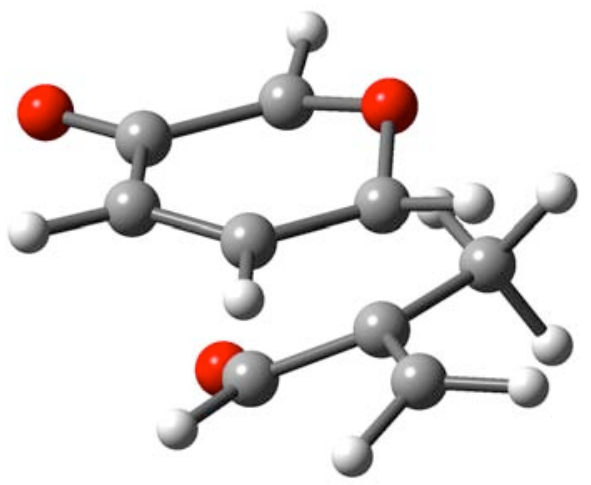

$\mathrm{HF}=-574.527926$ hartrees $(-360522.01884426 \mathrm{kcal} / \mathrm{mol})$ Imaginary Frequencies: $1(-386.98731 / \mathrm{cm})$

Zero-point correction $=0.171820($ Hartree/Particle $)$

Thermal correction to Energy=

0.183003

Thermal correction to Enthalpy=

0.183947

Thermal correction to Gibbs Free Energy=

Sum of electronic and zero-point Energies=

0.134814

Sum of electronic and thermal Energies=

$-574.356106$

Sum of electronic and thermal Enthalpies=

$-574.344923$

$-574.343979$

Sum of electronic and thermal Free Energies $=\quad-574.393112$

Coordinates (from last standard orientation):

\begin{tabular}{|c|c|c|c|c|}
\hline \multirow{2}{*}{$\begin{array}{l}\text { Center } \\
\text { Number }\end{array}$} & \multirow{2}{*}{$\begin{array}{l}\text { Atomic } \\
\text { Number }\end{array}$} & \multicolumn{3}{|c|}{ Coordinates (Angstroms) } \\
\hline & & & $\begin{array}{ll}X & Y\end{array}$ & Z \\
\hline 1 & 8 & -0.929027 & -1.051780 & 1.169425 \\
\hline 2 & 6 & -1.392670 & -1.535151 & -0.001359 \\
\hline 3 & 6 & -2.105632 & -0.641765 & -0.853668 \\
\hline 4 & 6 & -2.004115 & 0.706329 & -0.680891 \\
\hline 5 & 6 & -1.121865 & 1.252346 & 0.351486 \\
\hline 6 & 6 & -0.511820 & 0.214930 & 1.189346 \\
\hline 7 & 6 & 1.343466 & -0.602881 & -0.271467 \\
\hline 8 & 6 & 0.597111 & -1.523846 & -0.998884 \\
\hline 9 & 1 & -1.650157 & -2.584773 & 0.072791 \\
\hline 10 & 1 & -0.010356 & 0.485583 & 2.111498 \\
\hline 11 & 1 & -2.552794 & 1.410134 & -1.299296 \\
\hline 12 & 1 & 0.250880 & -1.262544 & -1.994904 \\
\hline 13 & 1 & -2.696219 & -1.072739 & -1.657830 \\
\hline 14 & 8 & -0.892164 & 2.449872 & 0.532366 \\
\hline 15 & 6 & 1.634115 & 0.710562 & -0.887296 \\
\hline 16 & 1 & 0.986943 & 0.972453 & -1.753250 \\
\hline 17 & 8 & 2.523403 & 1.454325 & -0.519709 \\
\hline 18 & 1 & 0.736267 & -2.586994 & -0.820447 \\
\hline 19 & 6 & 2.219271 & -1.035592 & 0.882306 \\
\hline 20 & 1 & 3.129901 & -1.519294 & 0.504740 \\
\hline 21 & 1 & 2.535903 & -0.174868 & 1.475927 \\
\hline 22 & 1 & 1.704764 & -1.755889 & 1.526680 \\
\hline
\end{tabular}




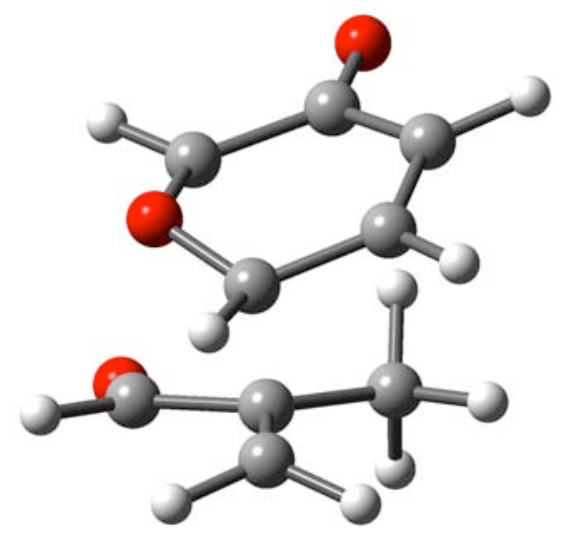

$\mathrm{HF}=-574.5317615$ hartrees $(-360524.425658865 \mathrm{kcal} / \mathrm{mol})$ Imaginary Frequencies: $1(-379.01771 / \mathrm{cm})$

Zero-point correction $=0.172065$ (Hartree/Particle)

Thermal correction to Energy=

0.183118

Thermal correction to Enthalpy=

0.184062

Thermal correction to Gibbs Free Energy=

Sum of electronic and zero-point Energies=

0.135355

Sum of electronic and thermal Energies=

$-574.359696$

Sum of electronic and thermal Enthalpies=

$-574.348644$

$-574.347699$

Sum of electronic and thermal Free Energies $=\quad-574.396406$

Coordinates (from last standard orientation):

\begin{tabular}{|c|c|c|c|c|}
\hline \multirow{2}{*}{$\begin{array}{l}\text { Center } \\
\text { Number }\end{array}$} & \multirow{2}{*}{$\begin{array}{l}\text { Atomic } \\
\text { Number }\end{array}$} & \multicolumn{3}{|c|}{ Coordinates (Angstroms) } \\
\hline & & & $X \quad Y$ & Z \\
\hline 1 & 8 & -0.843751 & -0.863528 & -1.389046 \\
\hline 2 & 8 & -0.774162 & 2.492785 & -0.192707 \\
\hline 3 & 6 & -0.457311 & 0.400365 & -1.228833 \\
\hline 4 & 6 & -1.069642 & 1.294390 & -0.254021 \\
\hline 5 & 6 & -2.035389 & 0.622368 & 0.617170 \\
\hline 6 & 6 & -2.148009 & -0.733975 & 0.594743 \\
\hline 7 & 1 & -2.796754 & -1.269060 & 1.283076 \\
\hline 8 & 6 & -1.364104 & -1.502953 & -0.318881 \\
\hline 9 & 6 & 0.533673 & -1.719904 & 0.709901 \\
\hline 10 & 6 & 1.203088 & $8-0.505978$ & 0.579891 \\
\hline 11 & 1 & 0.092787 & $7 \quad 0.794480$ & -2.075633 \\
\hline 12 & 1 & -1.616606 & $6-2.529769$ & -0.555983 \\
\hline 13 & 1 & -2.622018 & 81.241203 & 1.289316 \\
\hline 14 & 6 & 2.177433 & $3-0.369526$ & -0.523210 \\
\hline 15 & 8 & 3.028812 & $2 \quad 0.499783$ & -0.586805 \\
\hline 16 & 1 & 0.134549 & $9-2.010730$ & 1.677243 \\
\hline 17 & 1 & 0.817807 & $7 \quad-2.546246$ & 0.061816 \\
\hline 18 & 1 & 2.109113 & $3-1.162238$ & -1.303627 \\
\hline 19 & 6 & 1.271851 & 10.526416 & 1.673911 \\
\hline 20 & 1 & 2.280911 & 0.532503 & 2.104881 \\
\hline 21 & 1 & 1.089056 & 1.535999 & 1.291446 \\
\hline 22 & 1 & 0.554426 & 0.314320 & 2.471895 \\
\hline
\end{tabular}




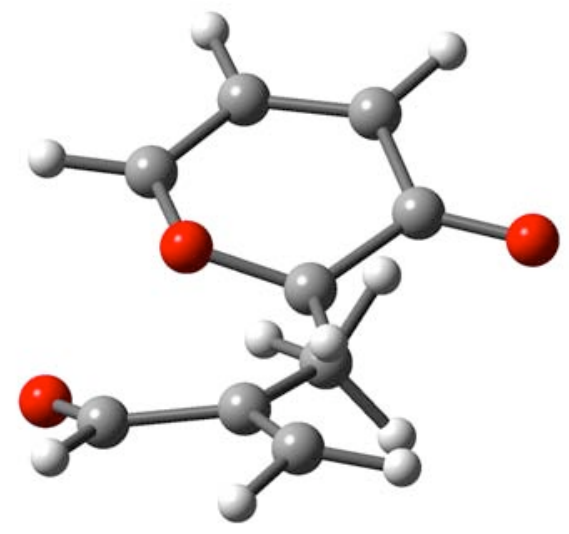

$\mathrm{HF}=-574.5377353$ hartrees $(-360528.174278103 \mathrm{kcal} / \mathrm{mol})$

Imaginary Frequencies: $1(-336.86631 / \mathrm{cm})$

Zero-point correction $=0.171833$ (Hartree/Particle)

Thermal correction to Energy $=\quad 0.183039$

Thermal correction to Enthalpy= $\quad 0.183983$

Thermal correction to Gibbs Free Energy $=\quad 0.134418$

Sum of electronic and zero-point Energies $=\quad-574.365902$

Sum of electronic and thermal Energies $=\quad-574.354696$

Sum of electronic and thermal Enthalpies $=\quad-574.353752$

Sum of electronic and thermal Free Energies $=\quad-574.403317$

Coordinates (from last standard orientation):

\begin{tabular}{ccccc}
\hline Center & Atomic & \multicolumn{3}{c}{ Coordinates (Angstroms) } \\
Number & Number & X & $Y$ & $Z$ \\
\hline 1 & 8 & -0.604530 & 1.321864 & -1.138208 \\
2 & 6 & -0.110947 & 1.788727 & 0.010998 \\
3 & 6 & -0.655007 & 1.380339 & 1.227202 \\
4 & 6 & -1.622952 & 0.395144 & 1.276940 \\
5 & 6 & -1.992281 & -0.324691 & 0.072655 \\
6 & 6 & -1.264451 & 0.145721 & -1.126502 \\
7 & 6 & 0.385461 & -1.171081 & -1.178606 \\
8 & 6 & 1.281977 & -0.849045 & -0.170223 \\
9 & 1 & 0.559126 & 2.626646 & -0.124928 \\
10 & 1 & -1.677964 & -0.090728 & -2.101267 \\
11 & 1 & -2.127033 & 0.136917 & 2.203019 \\
12 & 6 & 2.397854 & 0.058772 & -0.481972 \\
13 & 8 & 3.313054 & 0.334482 & 0.279363 \\
14 & 1 & -0.252848 & -2.047098 & -1.080022 \\
15 & 1 & -0.305277 & 1.873764 & 2.129091 \\
16 & 8 & -2.781308 & -1.271880 & 0.000027 \\
17 & 1 & 2.375118 & 0.473604 & -1.515176 \\
18 & 1 & 0.635374 & -0.886125 & -2.199142 \\
19 & 6 & 1.214884 & -1.468379 & 1.191052 \\
20 & 1 & 1.281827 & -2.561547 & 1.133481 \\
21 & 1 & 2.030939 & -1.095254 & 1.814723 \\
22 & 1 & 0.255773 & -1.238937 & 1.681492 \\
$------------------1 .-19$
\end{tabular}




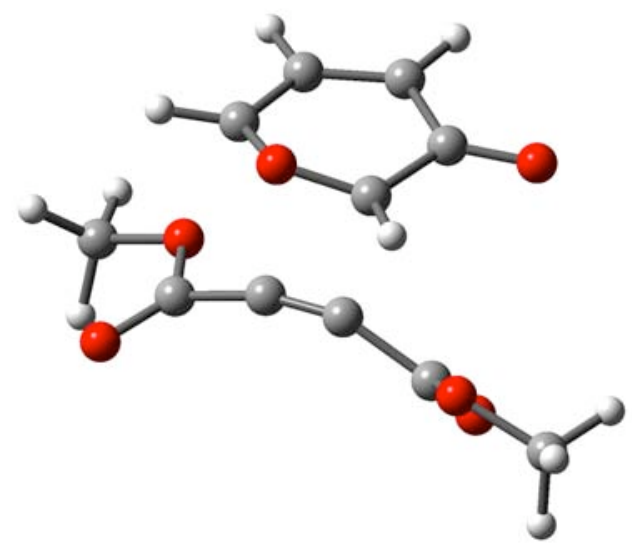

$\mathrm{HF}=-876.3776393$ hartrees $(-549935.732437143 \mathrm{kcal} / \mathrm{mol})$

Imaginary Frequencies: 1 (-307.1004 1/cm)

Zero-point correction $=0.196328$ (Hartree/Particle)

Thermal correction to Energy $=\quad 0.213361$

Thermal correction to Enthalpy= $\quad 0.214305$

Thermal correction to Gibbs Free Energy $=\quad 0.149054$

Sum of electronic and zero-point Energies $=\quad-876.181311$

Sum of electronic and thermal Energies $=\quad-876.164278$

Sum of electronic and thermal Enthalpies $=\quad-876.163334$

Sum of electronic and thermal Free Energies $=\quad-876.228585$

Coordinates (from last standard orientation):

\begin{tabular}{|c|c|c|c|c|}
\hline \multirow{2}{*}{$\begin{array}{l}\text { Center } \\
\text { Number }\end{array}$} & \multirow{2}{*}{$\begin{array}{l}\text { Atomic } \\
\text { Number }\end{array}$} & \multicolumn{3}{|c|}{ Coordinates (Angstroms) } \\
\hline & & & $\mathrm{K} \quad \mathrm{Y}$ & Z \\
\hline 1 & 8 & 0.005415 & 1.536185 & 1.601380 \\
\hline 2 & 6 & -1.112918 & 1.762587 & 0.915903 \\
\hline 3 & 6 & -1.035145 & 2.264932 & -0.398894 \\
\hline 4 & 6 & 0.173177 & 2.353243 & -1.039715 \\
\hline 5 & 6 & 1.373711 & 1.804982 & -0.421460 \\
\hline 6 & 6 & 1.122307 & 1.255868 & 0.921154 \\
\hline 7 & 6 & 0.388445 & -0.714424 & 0.179888 \\
\hline 8 & 6 & -0.850761 & -0.698699 & 0.226422 \\
\hline 9 & 1 & 38725 & 6743 & 1.548226 \\
\hline 10 & 1 & 949210 & 2361 & 1.567564 \\
\hline 11 & 1 & 0.266281 & 2.791985 & -2.028179 \\
\hline 12 & 1 & -1.957756 & 2.578041 & -0.877409 \\
\hline 13 & 8 & 2.489798 & 1.740481 & -0.944244 \\
\hline 14 & 6 & 1.624638 & -1.382795 & -0.259931 \\
\hline 15 & 6 & -2.254701 & -1.028150 & 0.292870 \\
\hline 16 & 8 & 1.706579 & -2.004508 & -1.294687 \\
\hline 17 & 8 & -2.770038 & -1.642307 & 1.205991 \\
\hline 18 & 8 & -2.923703 & -0.566533 & -0.789600 \\
\hline 19 & 8 & 2.628258 & -1.218026 & 0.619036 \\
\hline 20 & 6 & -4.321250 & -0.906234 & -0.824063 \\
\hline 21 & 1 & -4.845689 & -0.475595 & 0.033521 \\
\hline 22 & 1 & -4.696954 & -0.484670 & -1.756617 \\
\hline 23 & 1 & -4.452827 & -1.991101 & -0.812179 \\
\hline 24 & 6 & 3.895205 & -1.765872 & 0.205932 \\
\hline 25 & 1 & 4.574529 & -1.577829 & 1.037187 \\
\hline
\end{tabular}




$\begin{array}{rrrrr}26 & 1 & 3.804871 & -2.837429 & 0.013588 \\ 27 & 1 & 4.240343 & -1.257490 & -0.697354\end{array}$

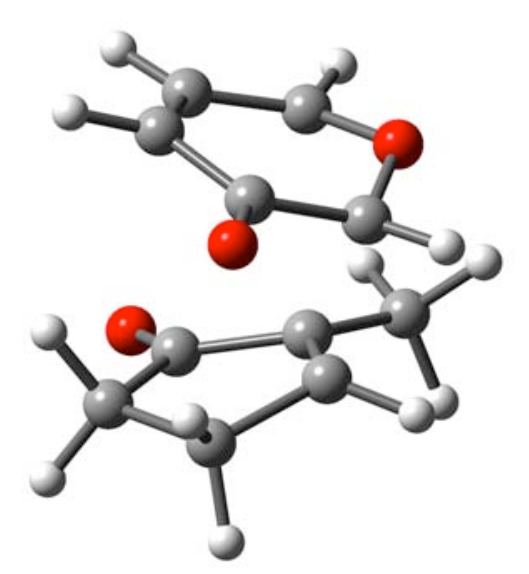

$\mathrm{HF}=-651.9658373$ hartrees $(-409115.082564123 \mathrm{kcal} / \mathrm{mol})$ Imaginary Frequencies: 1 (-391.3348 1/cm)

Zero-point correction $=0.208568$ (Hartree/Particle)

Thermal correction to Energy $=\quad 0.220647$

Thermal correction to Enthalpy $=\quad 0.221591$

Thermal correction to Gibbs Free Energy $=0.170606$

Sum of electronic and zero-point Energies $=\quad-651.757269$

Sum of electronic and thermal Energies $=\quad-651.745191$

Sum of electronic and thermal Enthalpies $=\quad-651.744247$

Sum of electronic and thermal Free Energies $=\quad-651.795231$

Coordinates (from last standard orientation):

\begin{tabular}{|c|c|c|c|c|}
\hline \multirow{2}{*}{$\begin{array}{l}\text { Center } \\
\text { Number }\end{array}$} & \multirow{2}{*}{$\begin{array}{l}\text { Atomic } \\
\text { Number }\end{array}$} & \multicolumn{3}{|c|}{ Coordinates (Angstroms) } \\
\hline & & & $X \quad Y$ & Z \\
\hline 1 & 8 & -0.863977 & -1.813422 & -0.445630 \\
\hline 2 & 8 & -2.967360 & 1.056266 & -0.388339 \\
\hline 3 & 6 & -1.447606 & -0.681768 & -0.909407 \\
\hline 4 & 6 & -2.152005 & 0.223346 & 0.020824 \\
\hline 5 & 6 & -1.714934 & 0.072165 & 1.400071 \\
\hline 6 & 6 & -0.732041 & -0.835010 & 1.710605 \\
\hline 7 & 1 & -0.316543 & -0.904713 & 2.711595 \\
\hline 8 & 6 & -0.236740 & -1.717090 & 0.732526 \\
\hline 9 & 6 & 1.347120 & -0.242859 & -0.600371 \\
\hline 10 & 6 & 0.347197 & 0.397084 & -1.349717 \\
\hline 11 & 6 & 0.205954 & 1.857864 & -0.925266 \\
\hline 12 & 1 & -1.890968 & -0.836076 & -1.887733 \\
\hline 13 & 1 & 0.384387 & -2.574740 & 0.953867 \\
\hline 14 & 1 & -2.173198 & 0.704273 & 2.154497 \\
\hline 15 & 1 & 0.202491 & 0.144109 & -2.398005 \\
\hline 16 & 1 & 0.682749 & 2.484457 & -1.690360 \\
\hline 17 & 6 & 1.824588 & 0.670694 & 0.466239 \\
\hline 18 & 8 & 2.752254 & 0.456158 & 1.230536 \\
\hline 19 & 1 & -0.833733 & 2.188122 & -0.851203 \\
\hline 20 & 6 & 2.164611 & -1.426534 & -1.031519 \\
\hline 21 & 1 & 2.873625 & -1.131672 & -1.818336 \\
\hline 22 & 1 & 2.753545 & -1.809556 & -0.192928 \\
\hline 23 & 1 & 1.544521 & -2.230160 & -1.442761 \\
\hline
\end{tabular}




\begin{tabular}{lllll}
24 & 6 & 0.975817 & 1.946111 & 0.407212 \\
25 & 1 & 1.615899 & 2.829443 & 0.491432 \\
26 & 1 & 0.298120 & 1.960489 & 1.270210 \\
\hline
\end{tabular}

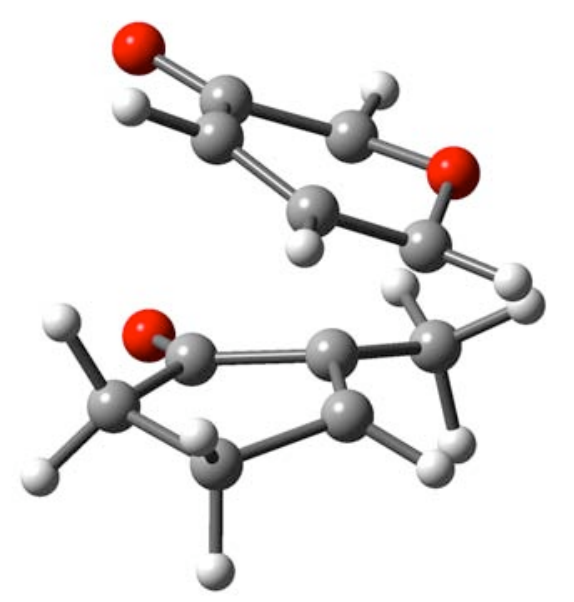

$\mathrm{HF}=-651.9577406$ hartrees $(-409110.001803906 \mathrm{kcal} / \mathrm{mol})$ Imaginary Frequencies: $1(-418.39681 / \mathrm{cm})$

Zero-point correction $=0.208356($ Hartree/Particle $)$

Thermal correction to Energy $=\quad 0.220449$

Thermal correction to Enthalpy= $\quad 0.221394$

Thermal correction to Gibbs Free Energy $=\quad 0.170409$

Sum of electronic and zero-point Energies $=\quad-651.749384$

Sum of electronic and thermal Energies $=\quad-651.737291$

Sum of electronic and thermal Enthalpies $=\quad-651.736347$

Sum of electronic and thermal Free Energies $=\quad-651.787332$

Coordinates (from last standard orientation):

\begin{tabular}{|c|c|c|c|c|}
\hline \multirow{2}{*}{$\begin{array}{l}\text { Center } \\
\text { Number }\end{array}$} & \multirow{2}{*}{$\begin{array}{l}\text { Atomic } \\
\text { Number }\end{array}$} & \multicolumn{3}{|c|}{ Coordinates (Angstroms) } \\
\hline & & & $\begin{array}{ll}X \quad Y\end{array}$ & Z \\
\hline 1 & 8 & 1.328725 & -1.056419 & -1.221324 \\
\hline 2 & 6 & 1.629881 & -1.434991 & 0.041859 \\
\hline 3 & 6 & 2.189655 & -0.450617 & 0.904712 \\
\hline 4 & 6 & 2.034434 & 0.875333 & 0.625203 \\
\hline 5 & 6 & 1.216145 & 1.288145 & -0.514283 \\
\hline 6 & 6 & 0.785896 & 0.161504 & -1.357101 \\
\hline 7 & 6 & -1.130768 & -0.692296 & -0.340306 \\
\hline 8 & 6 & -0.545199 & -1.407457 & 0.713456 \\
\hline 9 & 6 & -0.709775 & -0.662177 & 2.036778 \\
\hline 10 & 1 & 1.943638 & $3-2.471455$ & 0.083845 \\
\hline 11 & 1 & 0.432065 & $5 \quad 0.359954$ & -2.362507 \\
\hline 12 & 1 & 2.472660 & 1.654146 & 1.241965 \\
\hline 13 & 1 & -0.512867 & $7-2.493592$ & 0.711530 \\
\hline 14 & 1 & -1.425798 & $8-1.214015$ & 2.660414 \\
\hline 15 & 6 & -1.737304 & $4 \quad 0.561045$ & 0.199543 \\
\hline 16 & 8 & -2.511289 & 1.283149 & -0.397809 \\
\hline 17 & 1 & 0.215769 & $9-0.590904$ & 2.615940 \\
\hline 18 & 1 & 2.710677 & -0.788872 & 1.796397 \\
\hline 19 & 8 & 0.884934 & 2.449855 & -0.762681 \\
\hline 20 & 6 & -1.675638 & $\begin{array}{ll}8 & -1.300258\end{array}$ & -1.607189 \\
\hline 21 & 1 & -2.006033 & $3-0.515859$ & -2.293849 \\
\hline
\end{tabular}




$\begin{array}{rrrrr}22 & 1 & -0.938657 & -1.936865 & -2.106715 \\ 23 & 1 & -2.550098 & -1.924113 & -1.379087 \\ 24 & 6 & -1.277098 & 0.720476 & 1.650515 \\ 25 & 1 & -2.116934 & 1.040278 & 2.273893 \\ 26 & 1 & -0.524751 & 1.516383 & 1.693562\end{array}$

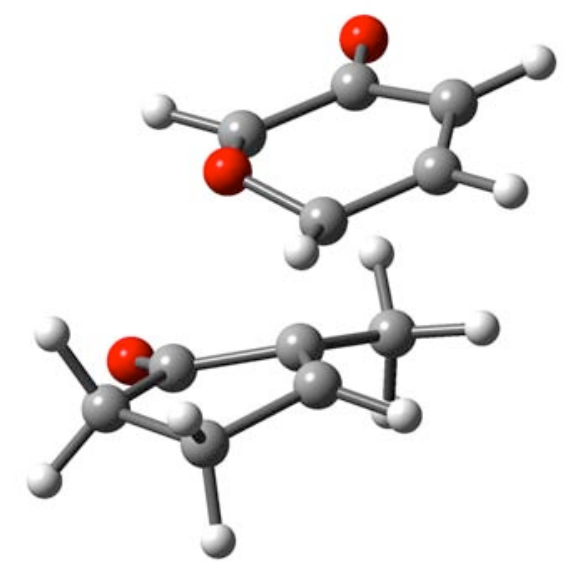

$\mathrm{HF}=-651.9623718$ hartrees $(-409112.907928218 \mathrm{kcal} / \mathrm{mol})$ Imaginary Frequencies: 1 (-409.0897 1/cm)

Zero-point correction $=0.208242($ Hartree/Particle $)$

Thermal correction to Energy $=\quad 0.220460$

Thermal correction to Enthalpy $=\quad 0.221405$

Thermal correction to Gibbs Free Energy $=\quad 0.170027$

Sum of electronic and zero-point Energies $=\quad-651.754130$

Sum of electronic and thermal Energies $=\quad-651.741911$

Sum of electronic and thermal Enthalpies $=\quad-651.740967$

Sum of electronic and thermal Free Energies $=\quad-651.792345$

Coordinates (from last standard orientation):

\begin{tabular}{|c|c|c|c|c|}
\hline \multirow{2}{*}{$\begin{array}{l}\text { Center } \\
\text { Number }\end{array}$} & \multirow{2}{*}{$\begin{array}{l}\text { Atomic } \\
\text { Number }\end{array}$} & \multicolumn{3}{|c|}{ Coordinates (Angstroms) } \\
\hline & & & $\begin{array}{ll}X \quad Y\end{array}$ & Z \\
\hline 1 & 8 & -0.489864 & -1.071311 & -1.274592 \\
\hline 2 & 8 & -2.333208 & 1.884155 & -0.534285 \\
\hline 3 & 6 & -0.806762 & 0.223490 & -1.217399 \\
\hline 4 & 6 & -1.948963 & 0.712388 & -0.452893 \\
\hline 5 & 6 & -2.545098 & -0.307159 & 0.411341 \\
\hline 6 & 6 & -1.932942 & -1.513503 & 0.562655 \\
\hline 7 & 1 & -2.300462 & -2.259745 & 1.262245 \\
\hline 8 & 6 & -0.743933 & -1.813516 & -0.170153 \\
\hline 9 & 6 & 0.854815 & -0.939013 & 1.004564 \\
\hline 10 & 6 & 0.756623 & $3 \quad 0.439546$ & 0.776444 \\
\hline 11 & 1 & -0.409391 & 0.803078 & -2.043054 \\
\hline 12 & 1 & -0.405811 & -2.835730 & -0.295380 \\
\hline 13 & 1 & -3.456353 & $3-0.047801$ & 0.941733 \\
\hline 14 & 6 & 2.087278 & $3-1.518081$ & 0.304139 \\
\hline 15 & 6 & 1.826154 & 0.850426 & -0.175671 \\
\hline 16 & 8 & 2.140795 & 1.995728 & -0.447452 \\
\hline 17 & 1 & 1.908421 & $1-2.484457$ & -0.179453 \\
\hline 18 & 1 & 0.516145 & $5-1.378633$ & 1.938974 \\
\hline 19 & 1 & 2.867075 & -1.688462 & 1.058769 \\
\hline 20 & 6 & 0.108753 & 1.466948 & 1.659943 \\
\hline
\end{tabular}




$\begin{array}{rrrrr}21 & 1 & 0.886238 & 1.998450 & 2.224460 \\ 22 & 1 & -0.435961 & 2.221161 & 1.083838 \\ 23 & 1 & -0.580473 & 1.008549 & 2.375168 \\ 24 & 6 & 2.523519 & -0.419535 & -0.683798 \\ 25 & 1 & 3.604921 & -0.262898 & -0.726558 \\ 26 & 1 & 2.187202 & -0.634042 & -1.705148\end{array}$

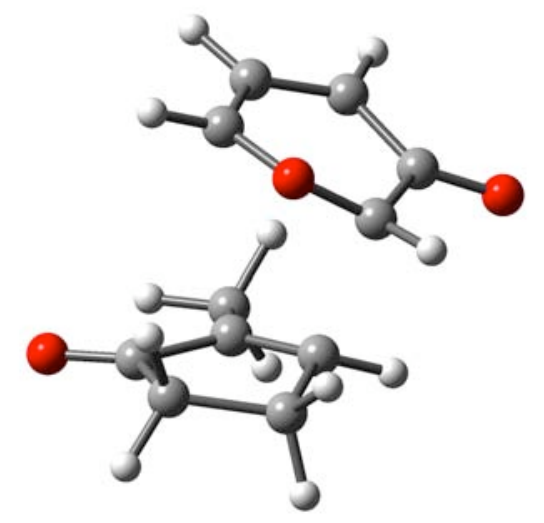

$\mathrm{HF}=-651.9690969$ hartrees $(-409117.127995719 \mathrm{kcal} / \mathrm{mol})$

Imaginary Frequencies: 1 (-370.4492 1/cm)

Zero-point correction $=0.208173$ (Hartree/Particle)

Thermal correction to Energy $=\quad 0.220402$

Thermal correction to Enthalpy $=\quad 0.221346$

Thermal correction to Gibbs Free Energy $=0.169586$

Sum of electronic and zero-point Energies $=\quad-651.760924$

Sum of electronic and thermal Energies $=\quad-651.748695$

Sum of electronic and thermal Enthalpies $=\quad-651.747750$

Sum of electronic and thermal Free Energies $=\quad-651.799511$

Coordinates (from last standard orientation):

\begin{tabular}{|c|c|c|c|c|}
\hline \multirow{2}{*}{$\begin{array}{l}\text { Center } \\
\text { Number }\end{array}$} & \multirow{2}{*}{$\begin{array}{l}\text { Atomic } \\
\text { Number }\end{array}$} & \multicolumn{3}{|c|}{ Coordinates (Angstroms) } \\
\hline & & & $\begin{array}{ll}X & Y\end{array}$ & Z \\
\hline 1 & 8 & 0.599298 & 0.554079 & -1.593712 \\
\hline 2 & 6 & 0.564715 & -0.779126 & -1.698771 \\
\hline 3 & 6 & 1.475024 & -1.564424 & -1.000128 \\
\hline 4 & 6 & 2.333277 & -1.004243 & -0.068884 \\
\hline 5 & 6 & 2.194565 & 0.391599 & 0.289902 \\
\hline 6 & 6 & 1.123032 & 1.089836 & -0.462921 \\
\hline 7 & 6 & -0.458093 & 1.034638 & 0.802991 \\
\hline 8 & 6 & -0.913306 & -0.284151 & 0.906190 \\
\hline 9 & 1 & -0.065014 & -1.124094 & -2.507739 \\
\hline 10 & 1 & 1.205679 & 2.168824 & -0.547657 \\
\hline 11 & 1 & 116492 & -1.584149 & 0.409116 \\
\hline 12 & 6 & -1.516943 & 1.892595 & 0.098085 \\
\hline 13 & 6 & -2.137521 & -0.464922 & 0.098335 \\
\hline 14 & 8 & -2.808345 & -1.483961 & 0.020218 \\
\hline 15 & 1 & -1.091257 & 2.613959 & -0.606568 \\
\hline 16 & 1 & 0.155950 & 1.473264 & 1.591011 \\
\hline 17 & 1 & -2.052057 & 2.474942 & 0.858842 \\
\hline 18 & 6 & -0.393892 & -1.342758 & 1.810498 \\
\hline
\end{tabular}




$\begin{array}{rrrrr}19 & 1 & -0.455463 & -1.033387 & 2.861975 \\ 20 & 1 & -0.969263 & -2.263600 & 1.677288 \\ 21 & 1 & 0.670660 & -1.552050 & 1.616377 \\ 22 & 6 & -2.458208 & 0.876573 & -0.576830 \\ 23 & 1 & -3.520310 & 1.111011 & -0.457421 \\ 24 & 1 & -2.268195 & 0.795928 & -1.653696 \\ 25 & 8 & 2.821671 & 0.989144 & 1.171492 \\ 26 & 1 & 1.495893 & -2.628439 & -1.216306\end{array}$




\section{Intricarene Formation (see Figure 1 in text)}

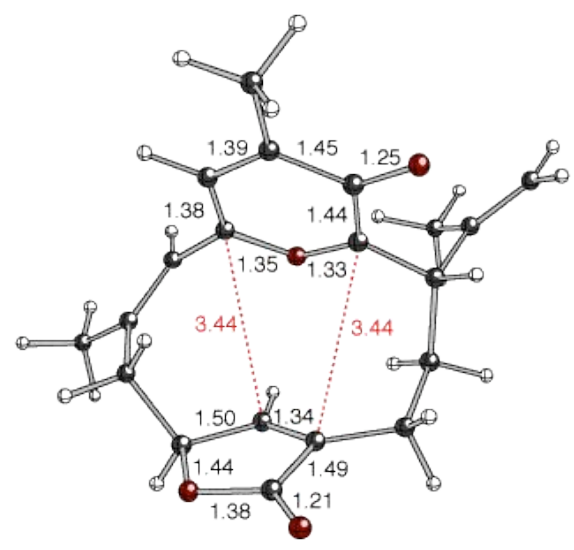

(2)

B3LYP/6-31G(d)

$\mathrm{HF}=-1076.195295$ hartrees $(-675323.30956545 \mathrm{kcal} / \mathrm{mol})$ Imaginary Frequencies: none found

Zero-point correction $=0.380148$ (Hartree/Particle)

Thermal correction to Energy $=\quad 0.402686$

Thermal correction to Enthalpy= $\quad 0.403630$

Thermal correction to Gibbs Free Energy $=\quad 0.328673$

Sum of electronic and zero-point Energies $=\quad-1075.815147$

Sum of electronic and thermal Energies $=\quad-1075.792609$

Sum of electronic and thermal Enthalpies $=\quad-1075.791665$

Sum of electronic and thermal Free Energies $=\quad-1075.866623$

Coordinates (from last standard orientation):

\begin{tabular}{|c|c|c|c|c|}
\hline \multirow{2}{*}{$\begin{array}{l}\text { Center } \\
\text { Number }\end{array}$} & \multirow{2}{*}{$\begin{array}{l}\text { Atomic } \\
\text { Number }\end{array}$} & \multicolumn{3}{|c|}{ Coordinates (Angstroms) } \\
\hline & & & $\begin{array}{ll}X & Y\end{array}$ & Z \\
\hline 1 & 8 & 0.473751 & 0.625765 & -0.704386 \\
\hline 2 & 6 & 2.091086 & -1.136631 & -0.317071 \\
\hline 3 & 1 & 2.557664 & -1.475820 & 0.613577 \\
\hline 4 & 8 & 2.887022 & 0.513045 & 1.918249 \\
\hline 5 & 6 & 1.456430 & 0.168577 & 0.072576 \\
\hline 6 & 8 & -3.196851 & -1.273031 & 1.170822 \\
\hline 7 & 6 & 1.948926 & 0.924716 & 1.202497 \\
\hline 8 & 8 & -1.944822 & -2.654308 & 2.464066 \\
\hline 9 & 6 & 1.257327 & 2.183247 & 1.422021 \\
\hline 10 & 6 & 0.216660 & 67111 & 0.580902 \\
\hline 11 & 1 & -0.3 & 3.500753 & 0.751479 \\
\hline 12 & 6 & -0.189968 & 1.779455 & -0.482947 \\
\hline 13 & 6 & -1.318180 & 2.030203 & -1.407544 \\
\hline 14 & 1 & -1.094890 & 2.563701 & -2.333039 \\
\hline 15 & 6 & -2.585806 & 1.642616 & -1.172763 \\
\hline 16 & 6 & -2.976752 & 0.876696 & 0.075879 \\
\hline 17 & 1 & -3.949411 & 1.222892 & 0.446194 \\
\hline 18 & 1 & -2.244577 & 1.045436 & 0.870245 \\
\hline 19 & 6 & -3.059692 & -0.660521 & -0.122132 \\
\hline 20 & 1 & -3.947563 & -0.924749 & -0.710943 \\
\hline 21 & 6 & -1.801908 & -1.252416 & -0.685761 \\
\hline
\end{tabular}




$\begin{array}{rrrrr}22 & 1 & -1.439662 & -1.000233 & -1.673474 \\ 23 & 6 & -1.200605 & -2.017979 & 0.230613 \\ 24 & 6 & 0.102838 & -2.762945 & 0.196245 \\ 25 & 1 & 0.522349 & -2.767884 & 1.210240 \\ 26 & 1 & -0.118495 & -3.817572 & -0.021846 \\ 27 & 6 & 1.144898 & -2.252536 & -0.822889 \\ 28 & 1 & 0.645132 & -1.947736 & -1.749154 \\ 29 & 1 & 1.792947 & -3.093432 & -1.096861 \\ 30 & 6 & 3.236776 & -0.849366 & -1.302714 \\ 31 & 6 & 4.497092 & -0.789957 & -0.860560 \\ 32 & 1 & 5.322196 & -0.567702 & -1.533758 \\ 33 & 1 & 4.739616 & -0.932871 & 0.187955 \\ 34 & 6 & 2.878210 & -0.597730 & -2.747793 \\ 35 & 1 & 2.121784 & 0.192274 & -2.844173 \\ 36 & 1 & 2.460878 & -1.492295 & -3.228267 \\ 37 & 1 & 3.759147 & -0.293090 & -3.320884 \\ 38 & 6 & 1.718583 & 3.011125 & 2.577672 \\ 39 & 1 & 1.135623 & 3.929612 & 2.691819 \\ 40 & 1 & 2.779242 & 3.265395 & 2.456957 \\ 41 & 1 & 1.664336 & 2.423579 & 3.502615 \\ 42 & 6 & -3.687632 & 1.937305 & -2.158616 \\ 43 & 1 & -3.314929 & 2.462467 & -3.042948 \\ 44 & 1 & -4.468280 & 2.554785 & -1.694862 \\ 45 & 1 & -4.180540 & 1.014480 & -2.493370 \\ 46 & 6 & -2.092093 & -2.059927 & 1.426132 \\ ----------------------\end{array}$

\section{B3LYP/6-31+G(d,p)}

$\mathrm{HF}=-1076.2631438$ hartrees $(-675365.885365938 \mathrm{kcal} / \mathrm{mol})$ Imaginary Frequencies: none found

Zero-point correction $=0.377792($ Hartree/Particle $)$

Thermal correction to Energy= $\quad 0.400436$

Thermal correction to Enthalpy $=\quad 0.401380$

Thermal correction to Gibbs Free Energy $=\quad 0.326213$

Sum of electronic and zero-point Energies $=\quad-1075.885352$

Sum of electronic and thermal Energies $=\quad-1075.862708$

Sum of electronic and thermal Enthalpies $=\quad-1075.861763$

Sum of electronic and thermal Free Energies $=\quad-1075.936931$

Coordinates (from last standard orientation):

\begin{tabular}{|c|c|c|c|c|}
\hline Center & Atomic & & ordinates (A & ngstroms) \\
\hline Number & Number & $x$ & $Y$ & Z \\
\hline 1 & 8 & 0.496630 & 0.655082 & -0.708018 \\
\hline 2 & 6 & 2.143365 & -1.077238 & -0.328652 \\
\hline 3 & 1 & 2.632085 & -1.412653 & 0.591599 \\
\hline 4 & 8 & 2.829410 & 0.533596 & 1.990037 \\
\hline 5 & 6 & 1.474407 & 0.204290 & 0.083609 \\
\hline 6 & 8 & -3.172279 & -1.334321 & 1.129766 \\
\hline 7 & 6 & 1.899108 & 0.938509 & 1.252222 \\
\hline 8 & 8 & -1.921442 & -2.700340 & 2.439977 \\
\hline 9 & 6 & 1.160202 & 2.166297 & 1.491537 \\
\hline 10 & 6 & 0.127550 & 2.539230 & 0.632255 \\
\hline 11 & 1 & -0.441306 & 3.445841 & 0.817294 \\
\hline 12 & 6 & -0.217843 & 1.772482 & -0.467386 \\
\hline 13 & 6 & -1.331682 & 2.008282 & -1.414244 \\
\hline 14 & 1 & -1.095280 & 2.539528 & -2.337163 \\
\hline
\end{tabular}




\begin{tabular}{rrrrr}
15 & 6 & -2.599459 & 1.602644 & -1.202017 \\
16 & 6 & -3.002724 & 0.824954 & 0.036620 \\
17 & 1 & -3.997300 & 1.138526 & 0.373544 \\
18 & 1 & -2.303943 & 1.018948 & 0.854403 \\
19 & 6 & -3.028735 & -0.714381 & -0.162933 \\
20 & 1 & -3.895234 & -1.011135 & -0.767001 \\
21 & 6 & -1.743210 & -1.269773 & -0.698576 \\
22 & 1 & -1.367296 & -1.007282 & -1.677966 \\
23 & 6 & -1.140311 & -2.027061 & 0.226100 \\
24 & 6 & 0.184698 & -2.734138 & 0.208139 \\
25 & 1 & 0.604918 & -2.704842 & 1.220852 \\
26 & 1 & -0.007882 & -3.798229 & 0.014171 \\
27 & 6 & 1.211667 & -2.214426 & -0.822392 \\
28 & 1 & 0.703223 & -1.925927 & -1.748370 \\
29 & 1 & 1.874342 & -3.044342 & -1.091695 \\
30 & 6 & 3.268976 & -0.770342 & -1.331093 \\
31 & 6 & 4.545295 & -0.780279 & -0.924542 \\
32 & 1 & 5.356885 & -0.555554 & -1.612155 \\
33 & 1 & 4.812236 & -0.990642 & 0.106418 \\
34 & 6 & 2.883987 & -0.441210 & -2.754374 \\
35 & 1 & 2.171094 & 0.391115 & -2.798301 \\
36 & 1 & 2.406624 & -1.293295 & -3.253725 \\
37 & 1 & 3.764950 & -0.163105 & -3.339198 \\
38 & 6 & 1.552689 & 2.979031 & 2.682036 \\
39 & 1 & 0.933960 & 3.873177 & 2.793350 \\
40 & 1 & 2.607372 & 3.269952 & 2.605277 \\
41 & 1 & 1.486228 & 2.367986 & 3.590129 \\
42 & 6 & -3.689310 & 1.885721 & -2.204611 \\
43 & 1 & -3.307782 & 2.407556 & -3.086152 \\
44 & 1 & -4.476842 & 2.501981 & -1.752839 \\
45 & 1 & -4.172277 & 0.958333 & -2.538262 \\
46 & 6 & -2.056555 & -2.098936 & 1.401919 \\
-------------------------- & \\
\hline-------19 &
\end{tabular}

in $\mathrm{H}_{2} \mathrm{O}$ B3LYP/6-31G(d)

$\mathrm{HF}=-1076.2274727$ hartrees $(-675343.501393977 \mathrm{kcal} / \mathrm{mol})$ Imaginary Frequencies: none found Zero-point correction $=0.376491$ (Hartree/Particle)

Thermal correction to Energy $=\quad 0.398969$ Thermal correction to Enthalpy $=0.399913$ Thermal correction to Gibbs Free Energy $=0.325332$

Sum of electronic and zero-point Energies= $\quad-1075.850981$

Sum of electronic and thermal Energies $=\quad-1075.828503$

Sum of electronic and thermal Enthalpies $=\quad-1075.827559$

Sum of electronic and thermal Free Energies $=\quad-1075.902141$

Coordinates (from last standard orientation):

\begin{tabular}{llrrr} 
Center & Atomic & \multicolumn{3}{c}{ Coordinates (Angstroms) } \\
Number & Number & \multicolumn{1}{c}{ X } & \multicolumn{1}{c}{$Y$} & \multicolumn{1}{c}{ Z } \\
\hline 1 & 8 & 0.469373 & 0.616432 & -0.699893 \\
2 & 6 & 2.103771 & -1.118516 & -0.314433 \\
3 & 1 & 2.565467 & -1.496942 & 0.610884 \\
4 & 8 & 2.839997 & 0.552874 & 1.971576 \\
5 & 6 & 1.455625 & 0.178789 & 0.088778 \\
6 & 8 & -3.129596 & -1.275877 & 1.233630 \\
7 & 6 & 1.907883 & 0.947052 & 1.217189 \\
8 & 8 & -1.826451 & -2.600633 & 2.516405
\end{tabular}




\begin{tabular}{|c|c|c|c|c|}
\hline 9 & 6 & 1.206560 & 2.199655 & 1.409663 \\
\hline 10 & 6 & 0.168477 & 2.566567 & 0.554905 \\
\hline 11 & 1 & -0.373057 & 3.497493 & 0.711036 \\
\hline 12 & 6 & -0.215509 & 1.758989 & -0.499516 \\
\hline 13 & 6 & -1.336574 & 1.978759 & -1.439261 \\
\hline 14 & 1 & -1.103357 & 2.486568 & -2.377811 \\
\hline 15 & 6 & -2.600036 & 1.580767 & -1.200462 \\
\hline 16 & 6 & -2.989326 & 0.848523 & 0.069609 \\
\hline 17 & 1 & -3.969402 & 1.198600 & 0.421217 \\
\hline 18 & 1 & -2.262310 & 1.049139 & 0.863858 \\
\hline 19 & 6 & -3.058591 & -0.692633 & -0.094073 \\
\hline 20 & 1 & -3.994329 & -1.000112 & -0.654686 \\
\hline 21 & 6 & -1.817507 & -1.287119 & -0.677842 \\
\hline 22 & 1 & -1.500432 & -1.074251 & -1.692005 \\
\hline 23 & 6 & -1.172823 & -2.027635 & 0.233968 \\
\hline 24 & 6 & 0.129743 & -2.773459 & 0.151815 \\
\hline 25 & 1 & 0.569081 & -2.821888 & 1.157480 \\
\hline 26 & 1 & -0.093442 & -3.817306 & -0.115333 \\
\hline 27 & 6 & 1.159605 & -2.221552 & -0.858776 \\
\hline 28 & 1 & 0.652069 & -1.891745 & -1.773197 \\
\hline 29 & 1 & 1.813170 & -3.052917 & -1.160783 \\
\hline 30 & 6 & 3.248133 & -0.813710 & -1.296712 \\
\hline 31 & 6 & 4.519448 & -0.855673 & -0.883144 \\
\hline 32 & 1 & 5.343748 & -0.636792 & -1.558940 \\
\hline 33 & 1 & 4.776840 & -1.098647 & 0.144217 \\
\hline 34 & 6 & 2.883303 & -0.452991 & -2.717156 \\
\hline 35 & 1 & 2.185031 & 0.392976 & -2.753987 \\
\hline 36 & 1 & 2.390168 & -1.287460 & -3.232426 \\
\hline 37 & 1 & 3.774867 & -0.182339 & -3.291876 \\
\hline 38 & 6 & 1.635368 & 3.058960 & 2.556658 \\
\hline 39 & 1 & 1.033223 & 3.968407 & 2.636972 \\
\hline 40 & 1 & 2.692718 & 3.335716 & 2.448263 \\
\hline 41 & 1 & 1.569731 & 2.495006 & 3.496405 \\
\hline 42 & 6 & -3.697998 & 1.821474 & -2.203611 \\
\hline 43 & 1 & -3.327426 & 2.330661 & -3.098633 \\
\hline 44 & 1 & -4.497620 & 2.430553 & -1.760382 \\
\hline 45 & 1 & -4.161858 & 0.874289 & -2.511754 \\
\hline 46 & 6 & -2.014462 & -2.036477 & 1.455355 \\
\hline
\end{tabular}

transition state structure $2 \rightarrow 1$ 


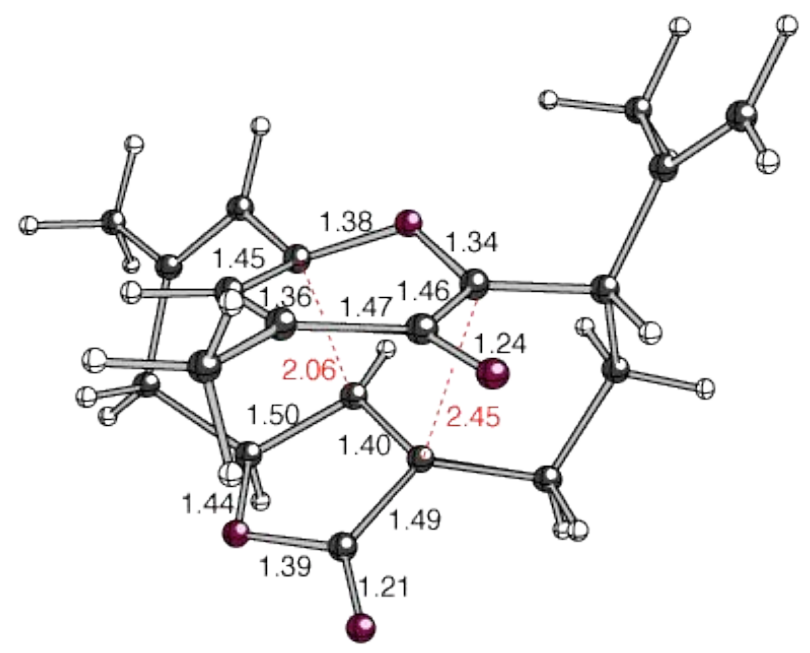

B3LYP/6-31G(d)

$\mathrm{HF}=-1076.16339$ hartrees $(-675303.2888589 \mathrm{kcal} / \mathrm{mol})$

Imaginary Frequencies: $1(-410.92081 / \mathrm{cm})$

Zero-point correction $=0.379871$ (Hartree/Particle)

Thermal correction to Energy=

0.401196

Thermal correction to Enthalpy=

0.402140

Thermal correction to Gibbs Free Energy $=\quad 0.331294$

Sum of electronic and zero-point Energies=

$-1075.783519$

Sum of electronic and thermal Energies $=\quad-1075.762194$

Sum of electronic and thermal Enthalpies $=\quad-1075.761250$

Sum of electronic and thermal Free Energies $=\quad-1075.832096$

Coordinates (from last standard orientation):

\begin{tabular}{llrrr} 
Center & Atomic & \multicolumn{3}{c}{ Coordinates (Angstroms) } \\
Number & Number & X & $Y$ & $Z$ \\
-1 & 8 & 0.532995 & 1.070027 & 0.599494 \\
2 & 6 & 2.573057 & 0.258952 & -0.426426 \\
3 & 1 & 3.130189 & -0.678294 & -0.340666 \\
4 & 8 & 2.007607 & -2.131845 & 1.110435 \\
5 & 6 & 1.332400 & 0.012223 & 0.376825 \\
6 & 8 & -2.246073 & -1.575698 & -1.014455 \\
7 & 6 & 1.145558 & -1.243864 & 1.105068 \\
8 & 8 & -0.567245 & -3.073914 & -1.304209 \\
9 & 6 & -0.144074 & -1.360115 & 1.795363 \\
10 & 6 & -1.070891 & -0.386692 & 1.586655 \\
11 & 1 & -2.058253 & -0.467923 & 2.028463 \\
12 & 6 & -0.800563 & 0.785837 & 0.785819 \\
13 & 6 & -1.732488 & 1.923678 & 0.863387 \\
14 & 1 & -1.381255 & 2.872083 & 1.265248 \\
15 & 6 & -2.992785 & 1.766968 & 0.415840 \\
16 & 6 & -3.444547 & 0.462010 & -0.230062 \\
17 & 1 & -4.395077 & 0.632003 & -0.747114 \\
18 & 1 & -3.658383 & -0.299805 & 0.528587 \\
19 & 6 & -2.429509 & -0.161771 & -1.236592 \\
20 & 1 & -2.844812 & -0.047469 & -2.247901 \\
21 & 6 & -1.023989 & 0.371614 & -1.220627 \\
22 & 1 & -0.844244 & 1.389223 & -1.550428 \\
23 & 6 & -0.130434 & -0.676573 & -1.466159 \\
24 & 6 & 1.142272 & -0.693554 & -2.280712
\end{tabular}




$\begin{array}{rrrrr}25 & 1 & 1.610276 & -1.675996 & -2.147790 \\ 26 & 1 & 0.892973 & -0.612916 & -3.349895 \\ 27 & 6 & 2.132184 & 0.418531 & -1.909454 \\ 28 & 1 & 1.663243 & 1.399557 & -2.056547 \\ 29 & 1 & 3.012661 & 0.385766 & -2.562282 \\ 30 & 6 & 3.468174 & 1.381765 & 0.100634 \\ 31 & 6 & 4.587212 & 1.070491 & 0.763198 \\ 32 & 1 & 5.245318 & 1.840904 & 1.158048 \\ 33 & 1 & 4.881511 & 0.039563 & 0.941003 \\ 34 & 6 & 3.066742 & 2.816816 & -0.143739 \\ 35 & 1 & 2.060114 & 3.023731 & 0.236616 \\ 36 & 1 & 3.057121 & 3.056641 & -1.215010 \\ 37 & 1 & 3.766123 & 3.503007 & 0.343472 \\ 38 & 6 & -0.374184 & -2.586055 & 2.621241 \\ 39 & 1 & -1.365636 & -2.586433 & 3.083006 \\ 40 & 1 & 0.389047 & -2.675150 & 3.402947 \\ 41 & 1 & -0.269011 & -3.473706 & 1.986090 \\ 42 & 6 & -4.018022 & 2.864628 & 0.516748 \\ 43 & 1 & -3.604628 & 3.764928 & 0.981659 \\ 44 & 1 & -4.883232 & 2.543667 & 1.113125 \\ 45 & 1 & -4.406961 & 3.134954 & -0.474513 \\ 46 & 6 & -0.922004 & -1.921037 & -1.268047 \\ ---------------------------------------------------\end{array}$

\section{B3LYP/6-31+G(d,p)}

$\mathrm{HF}=-1076.2299903$ hartrees $(-675345.081213153 \mathrm{kcal} / \mathrm{mol})$ Imaginary Frequencies: $1(-413.98181 / \mathrm{cm})$

Zero-point correction $=0.377568$ (Hartree/Particle)

Thermal correction to Energy $=\quad 0.398952$

Thermal correction to Enthalpy $=\quad 0.399896$

Thermal correction to Gibbs Free Energy $=\quad 0.329028$

Sum of electronic and zero-point Energies $=\quad-1075.852422$

Sum of electronic and thermal Energies $=\quad-1075.831038$

Sum of electronic and thermal Enthalpies $=\quad-1075.830094$

Sum of electronic and thermal Free Energies $=\quad-1075.900962$

Coordinates (from last standard orientation):

\begin{tabular}{ccccc} 
Center & Atomic & \multicolumn{3}{c}{ Coordinates (Angstroms) } \\
Number & Number & X & $Y$ & $Z$ \\
- \hdashline 1 & 8 & 0.533846 & 1.071759 & 0.581024 \\
2 & 6 & 2.568637 & 0.251787 & -0.444612 \\
3 & 1 & 3.121717 & -0.688332 & -0.369415 \\
4 & 8 & 2.036282 & -2.100653 & 1.174337 \\
5 & 6 & 1.340395 & 0.014632 & 0.379299 \\
6 & 8 & -2.265345 & -1.587269 & -1.019863 \\
7 & 6 & 1.161152 & -1.221108 & 1.141045 \\
8 & 8 & -0.606238 & -3.095740 & -1.368539 \\
9 & 6 & -0.125584 & -1.331600 & 1.837721 \\
10 & 6 & -1.061194 & -0.370805 & 1.602019 \\
11 & 1 & -2.047712 & -0.451498 & 2.045054 \\
12 & 6 & -0.800357 & 0.785777 & 0.774291 \\
13 & 6 & -1.733068 & 1.925927 & 0.851213 \\
14 & 1 & -1.379818 & 2.874491 & 1.249890 \\
15 & 6 & -2.998217 & 1.765720 & 0.413986 \\
16 & 6 & -3.451685 & 0.454442 & -0.218722
\end{tabular}




\begin{tabular}{rrrrr}
17 & 1 & -4.407421 & 0.615778 & -0.726974 \\
18 & 1 & -3.653409 & -0.300851 & 0.549310 \\
19 & 6 & -2.442840 & -0.167306 & -1.228546 \\
20 & 1 & -2.855878 & -0.044848 & -2.239566 \\
21 & 6 & -1.033565 & 0.356471 & -1.209676 \\
22 & 1 & -0.849415 & 1.370424 & -1.547750 \\
23 & 6 & -0.146555 & -0.697249 & -1.470971 \\
24 & 6 & 1.122192 & -0.700008 & -2.291531 \\
25 & 1 & 1.599785 & -1.679041 & -2.173178 \\
26 & 1 & 0.861486 & -0.611876 & -3.357143 \\
27 & 6 & 2.107198 & 0.417383 & -1.921599 \\
28 & 1 & 1.633267 & 1.396741 & -2.058600 \\
29 & 1 & 2.981550 & 0.391125 & -2.581804 \\
30 & 6 & 3.485759 & 1.365252 & 0.067750 \\
31 & 6 & 4.628818 & 1.039130 & 0.686559 \\
32 & 1 & 5.302820 & 1.803155 & 1.065240 \\
33 & 1 & 4.921014 & 0.004311 & 0.840078 \\
34 & 6 & 3.084106 & 2.805656 & -0.145653 \\
35 & 1 & 2.096029 & 3.014850 & 0.277392 \\
36 & 1 & 3.034010 & 3.054512 & -1.212802 \\
37 & 1 & 3.808056 & 3.480114 & 0.319723 \\
38 & 6 & -0.346379 & -2.523613 & 2.714336 \\
39 & 1 & -1.343670 & -2.515880 & 3.161420 \\
40 & 1 & 0.406571 & -2.560097 & 3.509355 \\
41 & 1 & -0.216783 & -3.440143 & 2.127917 \\
42 & 6 & -4.026533 & 2.860603 & 0.517351 \\
43 & 1 & -3.613175 & 3.762637 & 0.977017 \\
44 & 1 & -4.885322 & 2.536262 & 1.119623 \\
45 & 1 & -4.420687 & 3.124779 & -0.472591 \\
46 & 6 & -0.945842 & -1.937988 & -1.293903 \\
------------------------------------------ \\
& & & & \\
\hline
\end{tabular}

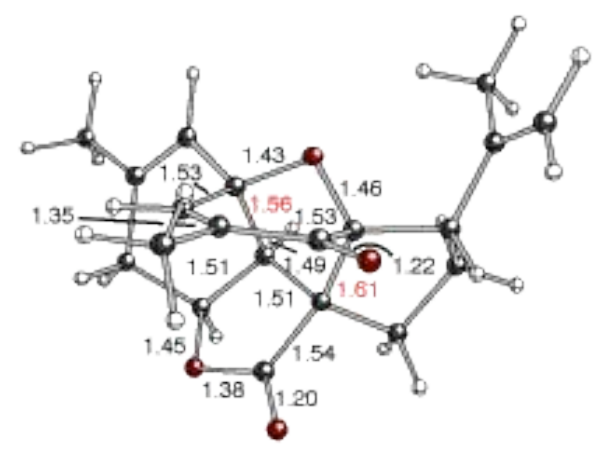

1 (computed structure B3LYP/6-31G(d))

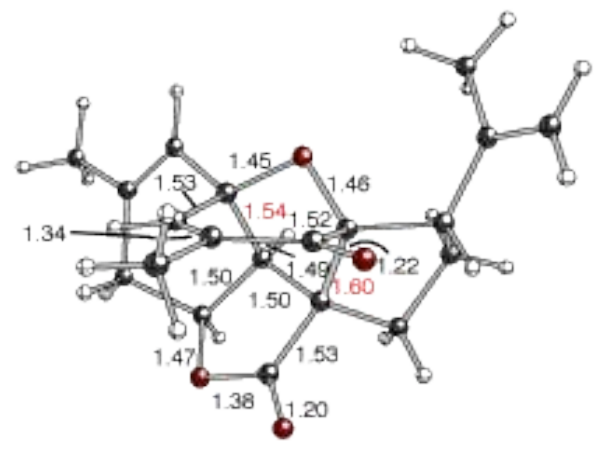

1 (X-ray structure $)^{\text {ret. } 1}$

\section{B3LYP/6-31G(d)}

$\mathrm{HF}=-1076.2144616$ hartrees $(-675335.336798616 \mathrm{kcal} / \mathrm{mol})$ Imaginary Frequencies: none found

Zero-point correction $=0.382982$ (Hartree/Particle)

Thermal correction to Energy $=\quad 0.403788$

Thermal correction to Enthalpy= $\quad 0.404732$

Thermal correction to Gibbs Free Energy $=\quad 0.335599$

Sum of electronic and zero-point Energies $=\quad-1075.831480$

Sum of electronic and thermal Energies $=\quad-1075.810674$

Sum of electronic and thermal Enthalpies $=\quad-1075.809729$

Sum of electronic and thermal Free Energies $=\quad-1075.878862$ 
Coordinates (from last standard orientation):

\begin{tabular}{|c|c|c|c|c|}
\hline \multirow{2}{*}{$\begin{array}{l}\text { Center } \\
\text { Number }\end{array}$} & \multirow{2}{*}{$\begin{array}{l}\text { Atomic } \\
\text { Number }\end{array}$} & \multicolumn{3}{|c|}{ Coordinates (Angstroms) } \\
\hline & & & $\begin{array}{l}K \\
Y \quad Y\end{array}$ & Z \\
\hline 1 & 8 & 0.454832 & -0.977838 & -0.790417 \\
\hline 2 & 6 & 2.467931 & -0.448729 & 0.575392 \\
\hline 3 & 1 & 3.030365 & 0.472075 & 0.770700 \\
\hline 4 & 8 & 2.211948 & 1.970535 & -1.012196 \\
\hline 5 & 6 & 1.128687 & 0.045116 & -0.001128 \\
\hline 6 & 8 & -1.924084 & 1.455377 & 1.426034 \\
\hline 7 & 6 & 1.215702 & 1.277418 & -0.904529 \\
\hline 8 & 8 & -0.056564 & 2.709847 & 1.592764 \\
\hline 9 & 6 & -0.072461 & 1.583172 & -1.585128 \\
\hline 10 & 6 & -1.099047 & 0.740722 & -1.365092 \\
\hline 11 & 1 & -2.086479 & 0.971999 & -1.754891 \\
\hline 12 & 6 & -0.924024 & -0.608575 & -0.660148 \\
\hline 13 & 6 & -1.993856 & -1.552591 & -1.131586 \\
\hline 14 & 1 & -1.785078 & -2.306895 & -1.885332 \\
\hline 15 & 6 & -3.216731 & -1.363536 & -0.604162 \\
\hline 16 & 6 & -3.473011 & -0.253708 & 0.426314 \\
\hline 17 & 1 & -4.358377 & -0.511775 & 1.018010 \\
\hline 18 & 1 & -3.727262 & 0.681157 & -0.091419 \\
\hline 19 & 6 & -2.277246 & 0.046177 & 1.377802 \\
\hline 20 & 1 & -2.531617 & -0.250236 & 2.401022 \\
\hline 21 & 6 & -1.011254 & -0.611652 & 0.894657 \\
\hline 22 & 1 & -0.919669 & -1.651776 & 1.212339 \\
\hline 23 & 6 & 0.156926 & 0.273202 & 1.265627 \\
\hline 24 & 6 & 1.021401 & -0.102856 & 2.487995 \\
\hline 25 & 1 & 1.496648 & 0.808053 & 2.870553 \\
\hline 26 & 1 & 0.424200 & -0.532022 & 3.300934 \\
\hline 27 & 6 & 2.082441 & -1.066101 & 1.936516 \\
\hline 28 & 1 & 1.650483 & -2.064103 & 1.797206 \\
\hline 29 & 1 & 2.944096 & -1.171567 & 2.604010 \\
\hline 30 & 6 & 3.308229 & -1.310655 & -0.357452 \\
\hline 31 & 6 & 4.303468 & -0.757430 & -1.058849 \\
\hline 32 & 1 & 4.914911 & -1.348049 & -1.737087 \\
\hline 33 & 1 & 4.525808 & 0.303802 & -0.995240 \\
\hline 34 & 6 & 2.994607 & -2.784679 & -0.458373 \\
\hline 35 & 1 & 1.947374 & -2.945108 & -0.736187 \\
\hline 36 & 1 & 3.158101 & -3.299865 & 0.497990 \\
\hline 37 & 1 & 3.631147 & -3.266726 & -1.206805 \\
\hline 38 & 6 & -0.156586 & 2.854470 & -2.378050 \\
\hline 39 & 1 & -1.149814 & 2.986397 & -2.817049 \\
\hline 40 & 1 & 0.588684 & 2.856417 & -3.181614 \\
\hline 41 & 1 & 0.068475 & 3.716438 & -1.739660 \\
\hline 42 & 6 & -4.415035 & -2.178037 & -1.013375 \\
\hline 43 & 1 & -4.155710 & -2.922525 & -1.772169 \\
\hline 44 & 1 & -5.209180 & -1.539009 & -1.423562 \\
\hline 45 & 1 & -4.850093 & -2.702330 & -0.151574 \\
\hline 46 & 6 & -0.556151 & 1.626984 & 1.435292 \\
\hline
\end{tabular}

\section{B3LYP/6-31+G(d,p)}

$\mathrm{HF}=-1076.2793856$ hartrees $(-675376.077257856 \mathrm{kcal} / \mathrm{mol})$ Imaginary Frequencies: none found 


$\begin{array}{lc}\text { Zero-point correction }=0.380689 & \text { (Hartree/Particle) } \\ \text { Thermal correction to Energy= } & 0.401565 \\ \text { Thermal correction to Enthalpy= } & 0.402509 \\ \text { Thermal correction to Gibbs Free Energy= } & 0.333262 \\ \text { Sum of electronic and zero-point Energies= } & -1075.898697 \\ \text { Sum of electronic and thermal Energies= } & -1075.877821 \\ \text { Sum of electronic and thermal Enthalpies }= & -1075.876877 \\ \text { Sum of electronic and thermal Free Energies }= & -1075.946123 \\ \text { Coordinates (from last standard orientation): } & \end{array}$

\begin{tabular}{|c|c|c|c|c|}
\hline \multirow{2}{*}{$\begin{array}{l}\text { Center } \\
\text { Number }\end{array}$} & \multirow{2}{*}{$\begin{array}{l}\text { Atomic } \\
\text { Number }\end{array}$} & \multicolumn{3}{|c|}{ Coordinates (Angstroms) } \\
\hline & & & $X \quad Y$ & Z \\
\hline 1 & 8 & 0.450551 & -0.978440 & -0.792006 \\
\hline 2 & 6 & 2.467366 & -0.445893 & 0.572201 \\
\hline 3 & 1 & 3.026332 & 0.475420 & 0.773614 \\
\hline 4 & 8 & 2.207262 & 1.973065 & -1.022511 \\
\hline 5 & 6 & 1.128572 & 0.048305 & -0.006757 \\
\hline 6 & 8 & -1.927095 & 1.453236 & 1.441201 \\
\hline 7 & 6 & 1.211032 & 1.277288 & -0.917068 \\
\hline 8 & 8 & -0.064635 & 2.713048 & 1.616262 \\
\hline 9 & 6 & -0.076559 & 1.579983 & -1.600992 \\
\hline 10 & 6 & -1.103585 & 0.737805 & -1.374125 \\
\hline 11 & 1 & -2.091712 & 0.970804 & -1.760391 \\
\hline 12 & 6 & -0.929795 & $5-0.607778$ & -0.662703 \\
\hline 13 & 6 & -1.999534 & $4-1.553718$ & -1.131249 \\
\hline 14 & 1 & -1.791976 & $6-2.305809$ & -1.887245 \\
\hline 15 & 6 & -3.222834 & $4-1.365714$ & -0.599267 \\
\hline 16 & 6 & -3.475607 & $7-0.254892$ & 0.431349 \\
\hline 17 & 1 & -4.361756 & $6-0.509079$ & 1.022015 \\
\hline 18 & 1 & -3.726138 & $8 \quad 0.679127$ & -0.089258 \\
\hline 19 & 6 & -2.279640 & 0.040192 & 1.381425 \\
\hline 20 & 1 & -2.530730 & -0.261195 & 2.403617 \\
\hline 21 & 6 & -1.012489 & -0.609113 & 0.891725 \\
\hline 22 & 1 & -0.914521 & $1-1.648772$ & 1.207963 \\
\hline 23 & 6 & 0.156106 & 0.276770 & 1.263311 \\
\hline 24 & 6 & 1.018704 & $4-0.107280$ & 2.485725 \\
\hline 25 & 1 & 1.495326 & 0.798172 & 2.877625 \\
\hline 26 & 1 & 0.416815 & $5-0.541760$ & 3.291750 \\
\hline 27 & 6 & 2.078900 & -1.069280 & 1.930438 \\
\hline 28 & 1 & 1.649308 & -2.067357 & 1.788201 \\
\hline 29 & 1 & 2.940995 & $5-1.174520$ & 2.596428 \\
\hline 30 & 6 & 3.325820 & J -1.303199 & -0.349643 \\
\hline 31 & 6 & 4.362880 & -0.753682 & -0.996822 \\
\hline 32 & 1 & 4.990923 & $\begin{array}{l}3 \\
-1.344629\end{array}$ & -1.658475 \\
\hline 33 & 1 & 4.601455 & 0.301144 & -0.900083 \\
\hline 34 & 6 & 2.998052 & $2-2.771609$ & -0.492174 \\
\hline 35 & 1 & 1.963256 & -2.915441 & -0.818158 \\
\hline 36 & 1 & 3.115222 & $2-3.303387$ & 0.460787 \\
\hline 37 & 1 & 3.662877 & $7-3.245598$ & -1.219729 \\
\hline 38 & 6 & -0.165457 & $\begin{array}{l}7 \\
2.841198\end{array}$ & -2.409478 \\
\hline 39 & 1 & -1.164056 & 2.967452 & -2.835967 \\
\hline 40 & 1 & 0.567743 & 2.826791 & -3.223074 \\
\hline 41 & 1 & 0.071698 & 3.711707 & -1.788627 \\
\hline 42 & 6 & -4.423916 & -2.178075 & -1.005739 \\
\hline 43 & 1 & -4.167957 & $7 \quad-2.920213$ & -1.766854 \\
\hline
\end{tabular}




$\begin{array}{llrrr}44 & 1 & -5.216746 & -1.535513 & -1.410850 \\ 45 & 1 & -4.854364 & -2.702676 & -0.142913 \\ 46 & 6 & -0.559791 & 1.628368 & 1.445854\end{array}$

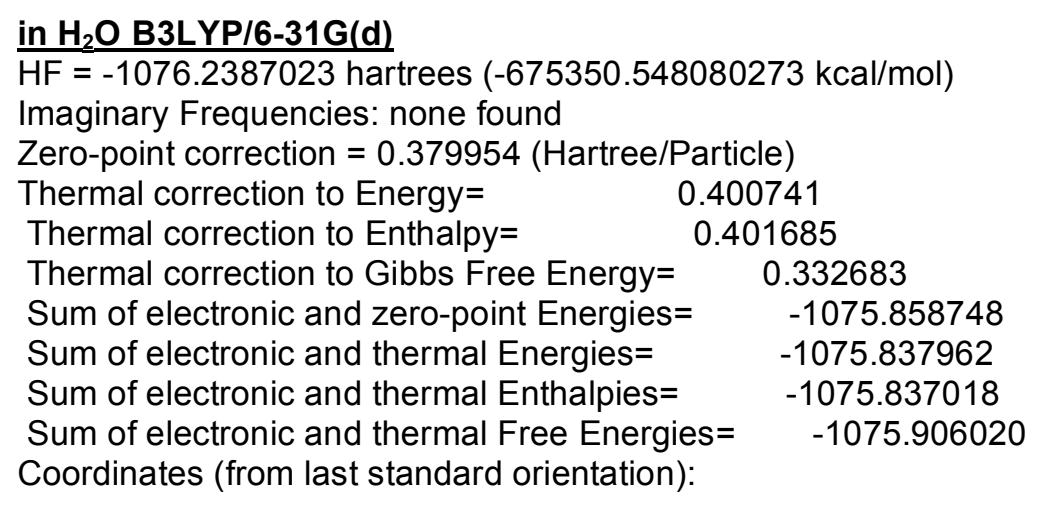

\begin{tabular}{llrrr} 
Center & Atomic & \multicolumn{3}{c}{ Coordinates (Angstroms) } \\
Number & Number & X & $Y$ & $Z$ \\
- \hdashline 1 & 8 & 0.456443 & -0.973937 & -0.800334 \\
2 & 6 & 2.471375 & -0.448646 & 0.565952 \\
3 & 1 & 3.029162 & 0.473153 & 0.788331 \\
4 & 8 & 2.179332 & 2.014600 & -0.968248 \\
5 & 6 & 1.128614 & 0.043523 & -0.005615 \\
6 & 8 & -1.899408 & 1.453513 & 1.437657 \\
7 & 6 & 1.197047 & 1.287657 & -0.893421 \\
8 & 8 & -0.035972 & 2.693847 & 1.620844 \\
9 & 6 & -0.082684 & 1.579852 & -1.584196 \\
10 & 6 & -1.108765 & 0.735902 & -1.355279 \\
11 & 1 & -2.100033 & 0.971588 & -1.736134 \\
12 & 6 & -0.929358 & -0.614573 & -0.656505 \\
13 & 6 & -2.002191 & -1.552082 & -1.133707 \\
14 & 1 & -1.803518 & -2.296916 & -1.901643 \\
15 & 6 & -3.222665 & -1.353255 & -0.603004 \\
16 & 6 & -3.471880 & -0.255401 & 0.442120 \\
17 & 1 & -4.346835 & -0.529247 & 1.044582 \\
18 & 1 & -3.743897 & 0.681468 & -0.069393 \\
19 & 6 & -2.270567 & 0.031037 & 1.386029 \\
20 & 1 & -2.517220 & -0.254393 & 2.416660 \\
21 & 6 & -1.009054 & -0.626024 & 0.897152 \\
22 & 1 & -0.919607 & -1.689688 & 1.228209 \\
23 & 6 & 0.162430 & 0.255882 & 1.269744 \\
24 & 6 & 1.026812 & -0.142994 & 2.486335 \\
25 & 1 & 1.505130 & 0.756082 & 2.895578 \\
26 & 1 & 0.422700 & -0.594885 & 3.281452 \\
27 & 6 & 2.085722 & -1.095388 & 1.913528 \\
28 & 1 & 1.648663 & -2.088383 & 1.755639 \\
29 & 1 & 2.949166 & -1.213833 & 2.577721 \\
30 & 6 & 3.319215 & -1.290043 & -0.379179 \\
31 & 6 & 4.309240 & -0.722877 & -1.078748 \\
32 & 1 & 4.925745 & -1.301958 & -1.763139 \\
33 & 1 & 4.528130 & 0.338620 & -0.999370 \\
34 & 6 & 3.023585 & -2.767180 & -0.491243 \\
35 & 1 & 1.971546 & -2.938795 & -0.743588
\end{tabular}




$\begin{array}{rrrrr}36 & 1 & 3.212538 & -3.287049 & 0.457898 \\ 37 & 1 & 3.650066 & -3.233872 & -1.258535 \\ 38 & 6 & -0.180895 & 2.844497 & -2.389342 \\ 39 & 1 & -1.186636 & 2.973749 & -2.800245 \\ 40 & 1 & 0.536615 & 2.833122 & -3.219220 \\ 41 & 1 & 0.064356 & 3.717649 & -1.772145 \\ 42 & 6 & -4.430152 & -2.145958 & -1.026430 \\ 43 & 1 & -4.179867 & -2.876674 & -1.802385 \\ 44 & 1 & -5.216735 & -1.485850 & -1.418512 \\ 45 & 1 & -4.867923 & -2.680889 & -0.172167 \\ 46 & 6 & -0.543279 & 1.606540 & 1.450985 \\ -\end{array}$

\section{isomeric structure 2}

\section{B3LYP/6-31G(d)}

$\mathrm{HF}=-1076.1886099$ hartrees $(-675319.114598349 \mathrm{kcal} / \mathrm{mol})$

Imaginary Frequencies: none found

Zero-point correction $=0.380630$ (Hartree/Particle)

Thermal correction to Energy $=\quad 0.403120$

Thermal correction to Enthalpy= $\quad 0.404064$

Thermal correction to Gibbs Free Energy $=\quad 0.329879$

Sum of electronic and zero-point Energies $=\quad-1075.807980$

Sum of electronic and thermal Energies $=\quad-1075.785490$

Sum of electronic and thermal Enthalpies $=\quad-1075.784545$

Sum of electronic and thermal Free Energies $=\quad-1075.858731$

Coordinates (from last standard orientation):

\begin{tabular}{|c|c|c|c|c|}
\hline \multirow{2}{*}{$\begin{array}{l}\text { Center } \\
\text { Number }\end{array}$} & \multirow{2}{*}{$\begin{array}{l}\text { Atomic } \\
\text { Number }\end{array}$} & \multicolumn{3}{|c|}{ Coordinates (Angstroms) } \\
\hline & & & $X \quad Y$ & Z \\
\hline 1 & 8 & 0.080052 & 0.329424 & 1.062059 \\
\hline 2 & 6 & 1.891407 & -1.011745 & 0.421399 \\
\hline 3 & 1 & 1.176080 & -1.638245 & 0.961865 \\
\hline 4 & 6 & 1.233041 & 0.345164 & 0.389338 \\
\hline 5 & 8 & -2.511660 & -1.732223 & -0.534615 \\
\hline 6 & 8 & -0.787011 & -3.162565 & -0.192060 \\
\hline 7 & 6 & -0.830800 & 1.313890 & 1.061005 \\
\hline 8 & 6 & -2.074903 & 0.898238 & 1.730659 \\
\hline 9 & 1 & -2.020681 & 0.758819 & 2.810474 \\
\hline 10 & 6 & -3.195240 & 0.524195 & 1.090528 \\
\hline 11 & 6 & -3.393193 & 0.605634 & -0.415889 \\
\hline 12 & 1 & -3.026933 & 1.562791 & -0.801826 \\
\hline 13 & 1 & -4.469960 & 0.586038 & -0.612834 \\
\hline 14 & 6 & -2.731468 & -0.520545 & -1.274918 \\
\hline 15 & 1 & -3.432554 & -0.764005 & -2.085897 \\
\hline 16 & 6 & -1.386448 & -0.203421 & -1.860905 \\
\hline 17 & 1 & -1.211099 & 0.678767 & -2.467987 \\
\hline 18 & 6 & -0.497231 & -1.159875 & -1.561955 \\
\hline 19 & 6 & 0.930455 & -1.281058 & -2.029276 \\
\hline 20 & 1 & 0.967958 & -2.047000 & -2.816628 \\
\hline 21 & 1 & 1.200362 & -0.333380 & -2.506440 \\
\hline 22 & 6 & 2.032023 & -1.637855 & -0.996974 \\
\hline 23 & 1 & 2.978128 & -1.329958 & -1.447344 \\
\hline 24 & 1 & 2.069806 & -2.721955 & -0.851687 \\
\hline 25 & 6 & 3.191035 & -1.033872 & 1.237746 \\
\hline 26 & 6 & 3.163427 & -1.526404 & 2.481607 \\
\hline
\end{tabular}




$\begin{array}{rrrrr}27 & 1 & 4.056024 & -1.548958 & 3.102354 \\ 28 & 1 & 2.254793 & -1.926488 & 2.925113 \\ 29 & 6 & 4.460523 & -0.497934 & 0.621932 \\ 30 & 1 & 4.292602 & 0.475557 & 0.151376 \\ 31 & 1 & 4.827035 & -1.165609 & -0.169591 \\ 32 & 1 & 5.250527 & -0.416922 & 1.375271 \\ 33 & 6 & -4.361775 & -0.044467 & 1.856221 \\ 34 & 1 & -4.166245 & -0.083821 & 2.931603 \\ 35 & 1 & -4.571389 & -1.063922 & 1.506847 \\ 36 & 1 & -5.273209 & 0.545751 & 1.690869 \\ 37 & 6 & -1.205329 & -2.151593 & -0.700581 \\ 38 & 6 & -0.502466 & 2.493919 & 0.414492 \\ 39 & 6 & 1.632717 & 1.508063 & -0.357083 \\ 40 & 6 & 0.708952 & 2.627982 & -0.260534 \\ 41 & 8 & 2.673662 & 1.539111 & -1.058370 \\ 42 & 6 & 1.100396 & 3.877953 & -0.982755 \\ 43 & 1 & 2.062465 & 4.245600 & -0.604304 \\ 44 & 1 & 1.272813 & 3.659364 & -2.044034 \\ 45 & 1 & 0.348329 & 4.666785 & -0.888138 \\ 46 & 1 & -1.215931 & 3.313182 & 0.440482 \\ -------------------------------------------\end{array}$

\section{B3LYP/6-31+G(d,p)}

$\mathrm{HF}=-1076.2552023$ hartrees $(-675360.901995273 \mathrm{kcal} / \mathrm{mol})$

Imaginary Frequencies: none found

Zero-point correction $=0.378006$ (Hartree/Particle)

Thermal correction to Energy $=\quad 0.400686$

Thermal correction to Enthalpy $=\quad 0.401630$

Thermal correction to Gibbs Free Energy $=\quad 0.326626$

Sum of electronic and zero-point Energies $=\quad-1075.877196$

Sum of electronic and thermal Energies $=\quad-1075.854516$

Sum of electronic and thermal Enthalpies $=\quad-1075.853572$

Sum of electronic and thermal Free Energies $=\quad-1075.928577$

Coordinates (from last standard orientation):

\begin{tabular}{|c|c|c|c|c|}
\hline \multirow{2}{*}{$\begin{array}{l}\text { Center } \\
\text { Number }\end{array}$} & \multirow{2}{*}{$\begin{array}{l}\text { Atomic } \\
\text { Number }\end{array}$} & \multicolumn{3}{|c|}{ Coordinates (Angstroms) } \\
\hline & & & $X \quad Y$ & Z \\
\hline 1 & 8 & 0.086375 & 0.374982 & 1.033043 \\
\hline 2 & 6 & 1.885680 & -1.003893 & 0.445273 \\
\hline 3 & 1 & 1.161010 & -1.599228 & 1.007568 \\
\hline 4 & 6 & 1.246469 & 0.360966 & 0.369016 \\
\hline 5 & 8 & -2.550927 & -1.752214 & -0.511339 \\
\hline 6 & 8 & -0.875256 & -3.246153 & -0.190795 \\
\hline 7 & 6 & 4042 & 1.367649 & 1.001433 \\
\hline 8 & 6 & -2.058794 & 0.992838 & 1.695725 \\
\hline 9 & 1 & -1.998685 & 0.918325 & 2.781475 \\
\hline 10 & 6 & -3.190817 & 0.595359 & 1.087766 \\
\hline 11 & 6 & -3.415930 & 0.593835 & -0.418762 \\
\hline 12 & 1 & -3.097202 & 1.546307 & -0.856550 \\
\hline 13 & 1 & -4.494276 & 0.524408 & -0.588993 \\
\hline 14 & 6 & -2.737536 & -0.533516 & -1.255411 \\
\hline 15 & 1 & -3.422278 & -0.769990 & -2.082642 \\
\hline 16 & 6 & -1.377665 & -0.243462 & -1.819689 \\
\hline 17 & 1 & -1.172567 & 0.635828 & -2.420985 \\
\hline 18 & 6 & -0.515711 & -1.227474 & -1.522930 \\
\hline
\end{tabular}




\begin{tabular}{rrrrr}
19 & 6 & 0.908709 & -1.382698 & -1.988697 \\
20 & 1 & 0.934736 & -2.194018 & -2.728445 \\
21 & 1 & 1.172508 & -0.466058 & -2.525576 \\
22 & 6 & 2.020984 & -1.684048 & -0.948213 \\
23 & 1 & 2.961105 & -1.386773 & -1.417130 \\
24 & 1 & 2.069786 & -2.760552 & -0.758030 \\
25 & 6 & 3.181922 & -1.027556 & 1.268983 \\
26 & 6 & 3.135577 & -1.483074 & 2.529705 \\
27 & 1 & 4.025328 & -1.508176 & 3.153369 \\
28 & 1 & 2.214303 & -1.847006 & 2.977080 \\
29 & 6 & 4.468958 & -0.546598 & 0.644394 \\
30 & 1 & 4.337377 & 0.424245 & 0.157945 \\
31 & 1 & 4.810558 & -1.240906 & -0.133889 \\
32 & 1 & 5.258803 & -0.480798 & 1.398130 \\
33 & 6 & -4.357955 & 0.096763 & 1.901509 \\
34 & 1 & -4.145108 & 0.111923 & 2.973373 \\
35 & 1 & -4.601778 & -0.932827 & 1.611536 \\
36 & 1 & -5.253995 & 0.703427 & 1.717492 \\
37 & 6 & -1.258127 & -2.209814 & -0.680067 \\
38 & 6 & -0.467422 & 2.529441 & 0.332460 \\
39 & 6 & 1.667370 & 1.506264 & -0.391178 \\
40 & 6 & 0.754478 & 2.637489 & -0.333243 \\
41 & 8 & 2.727258 & 1.514424 & -1.071193 \\
42 & 6 & 1.162481 & 3.875467 & -1.066043 \\
43 & 1 & 2.122481 & 4.240209 & -0.681388 \\
44 & 1 & 1.344858 & 3.645716 & -2.122803 \\
45 & 1 & 0.413346 & 4.667523 & -0.985500 \\
46 & 1 & -1.171680 & 3.356477 & 0.334050 \\
---------------------------------------------------- \\
\hline
\end{tabular}

\section{in $\mathrm{H}_{2} \mathrm{O}$ B3LYP/6-31G(d)}

$\mathrm{HF}=-1076.2166409$ hartrees $(-675336.704331159 \mathrm{kcal} / \mathrm{mol})$ Imaginary Frequencies: none found

Zero-point correction $=0.376980($ Hartree/Particle $)$

Thermal correction to Energy $=\quad 0.399469$

Thermal correction to Enthalpy $=\quad 0.400413$

Thermal correction to Gibbs Free Energy $=\quad 0.326181$

Sum of electronic and zero-point Energies $=\quad-1075.839661$

Sum of electronic and thermal Energies $=\quad-1075.817172$

Sum of electronic and thermal Enthalpies $=\quad-1075.816228$

Sum of electronic and thermal Free Energies $=\quad-1075.890460$

Coordinates (from last standard orientation):

\begin{tabular}{|c|c|c|c|c|}
\hline \multirow{2}{*}{$\begin{array}{l}\text { Center } \\
\text { Number }\end{array}$} & \multirow{2}{*}{$\begin{array}{l}\text { Atomic } \\
\text { Number }\end{array}$} & \multicolumn{3}{|c|}{ Coordinates (Angstroms) } \\
\hline & & & $X \quad Y$ & Z \\
\hline 1 & 8 & 0.088388 & 0.338796 & 1.048930 \\
\hline 2 & 6 & 1.902340 & -0.992523 & 0.408232 \\
\hline 3 & 1 & 1.172813 & -1.616025 & 0.933410 \\
\hline 4 & 6 & 1.251577 & 0.370934 & 0.387013 \\
\hline 5 & 8 & -2.494068 & -1.764546 & -0.540648 \\
\hline 6 & 8 & -0.779531 & -3.200503 & -0.237488 \\
\hline 7 & 6 & -0.836587 & 1.307956 & 1.035450 \\
\hline 8 & 6 & -2.071669 & 0.894715 & 1.721476 \\
\hline 9 & 1 & -2.004230 & 0.786925 & 2.805992 \\
\hline 10 & 6 & -3.201047 & 0.513470 & 1.100285 \\
\hline
\end{tabular}




\begin{tabular}{rrrrr}
11 & 6 & -3.431769 & 0.556126 & -0.404331 \\
12 & 1 & -3.133646 & 1.528934 & -0.817047 \\
13 & 1 & -4.509738 & 0.468645 & -0.578720 \\
14 & 6 & -2.730754 & -0.533586 & -1.269076 \\
15 & 1 & -3.434782 & -0.786750 & -2.123156 \\
16 & 6 & -1.389048 & -0.201286 & -1.837861 \\
17 & 1 & -1.220966 & 0.689961 & -2.436571 \\
18 & 6 & -0.493892 & -1.162784 & -1.559818 \\
19 & 6 & 0.932026 & -1.270077 & -2.038078 \\
20 & 1 & 0.977177 & -2.037073 & -2.825177 \\
21 & 1 & 1.178137 & -0.319400 & -2.524085 \\
22 & 6 & 2.049227 & -1.606589 & -1.014217 \\
23 & 1 & 2.990443 & -1.282475 & -1.471382 \\
24 & 1 & 2.113713 & -2.694152 & -0.874388 \\
25 & 6 & 3.189381 & -1.051491 & 1.241417 \\
26 & 6 & 3.144546 & -1.578828 & 2.471099 \\
27 & 1 & 4.030617 & -1.628012 & 3.100564 \\
28 & 1 & 2.225431 & -1.977479 & 2.895251 \\
29 & 6 & 4.473140 & -0.514416 & 0.657480 \\
30 & 1 & 4.318252 & 0.479466 & 0.226334 \\
31 & 1 & 4.832302 & -1.154385 & -0.160256 \\
32 & 1 & 5.259647 & -0.474121 & 1.418313 \\
33 & 6 & -4.364204 & -0.011193 & 1.903034 \\
34 & 1 & -4.146496 & -0.036244 & 2.975254 \\
35 & 1 & -4.618271 & -1.028053 & 1.573711 \\
36 & 1 & -5.259867 & 0.604542 & 1.742193 \\
37 & 6 & -1.198223 & -2.166454 & -0.724452 \\
38 & 6 & -0.524666 & 2.487296 & 0.382644 \\
39 & 6 & 1.649906 & 1.542999 & -0.332131 \\
40 & 6 & 0.697118 & 2.636913 & -0.272613 \\
41 & 8 & 2.725856 & 1.608995 & -0.995159 \\
42 & 6 & 1.058789 & 3.893914 & -0.999228 \\
43 & 1 & 2.002432 & 4.299000 & -0.610293 \\
44 & 1 & 1.243151 & 3.676179 & -2.059563 \\
45 & 1 & 0.278158 & 4.655446 & -0.917077 \\
46 & 1 & -1.256595 & 3.292539 & 0.383665 \\
------------------------------------------------ \\
\hline
\end{tabular}

isomeric transition state structure $2 \rightarrow 1$

\section{B3LYP/6-31G(d)}

$\mathrm{HF}=-1076.1555441$ hartrees $(-675298.365478191 \mathrm{kcal} / \mathrm{mol})$

Imaginary Frequencies: $1(-438.34271 / \mathrm{cm})$

Zero-point correction $=0.379837($ Hartree/Particle $)$

Thermal correction to Energy $=\quad 0.401122$

Thermal correction to Enthalpy= $\quad 0.402066$

Thermal correction to Gibbs Free Energy $=\quad 0.331585$

Sum of electronic and zero-point Energies $=\quad-1075.775707$

Sum of electronic and thermal Energies $=\quad-1075.754422$

Sum of electronic and thermal Enthalpies $=\quad-1075.753478$

Sum of electronic and thermal Free Energies $=\quad-1075.823959$

Coordinates (from last standard orientation):

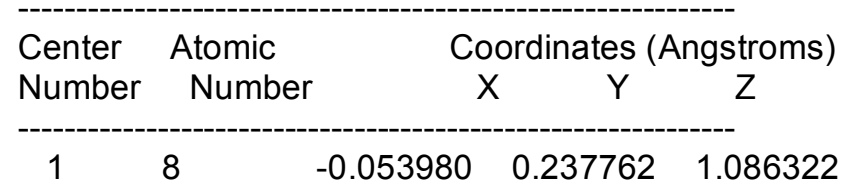




$\begin{array}{ccccc}2 & 6 & 1.965705 & -1.011940 & 0.468451 \\ 3 & 1 & 1.387699 & -1.776730 & 0.991624 \\ 4 & 6 & 0.991779 & 0.118670 & 0.224066 \\ 5 & 8 & -2.136726 & -1.912430 & -0.491633 \\ 6 & 8 & -0.263171 & -3.088447 & -0.087260 \\ 7 & 6 & -1.074481 & 1.026015 & 0.650835 \\ 8 & 6 & -2.320017 & 0.815331 & 1.409703 \\ 9 & 1 & -2.256445 & 0.984607 & 2.485482 \\ 10 & 6 & -3.477515 & 0.392405 & 0.882220 \\ 11 & 6 & -3.657757 & 0.011603 & -0.572397 \\ 12 & 1 & -3.862596 & 0.899760 & -1.188529 \\ 13 & 1 & -4.551687 & -0.617568 & -0.657880 \\ 14 & 6 & -2.498938 & -0.741426 & -1.244982 \\ 15 & 1 & -2.881004 & -1.091254 & -2.218300 \\ 16 & 6 & -1.194230 & -0.030935 & -1.457633 \\ 17 & 1 & -1.145132 & 0.821737 & -2.125372 \\ 18 & 6 & -0.120127 & -0.899849 & -1.201844 \\ 19 & 6 & 1.169088 & -1.216344 & -1.950311 \\ 20 & 1 & 0.993357 & -2.041984 & -2.650507 \\ 21 & 1 & 1.466180 & -0.345346 & -2.539927 \\ 22 & 6 & 2.301347 & -1.584312 & -0.946296 \\ 23 & 1 & 3.244016 & -1.184698 & -1.320874 \\ 24 & 1 & 2.401126 & -2.669454 & -0.860445 \\ 25 & 6 & 3.146163 & -0.646423 & 1.382401 \\ 26 & 6 & 2.941006 & -0.612881 & 2.704338 \\ 27 & 1 & 3.737001 & -0.349876 & 3.396541 \\ 28 & 1 & 1.972690 & -0.842634 & 3.143085 \\ 29 & 6 & 4.501294 & -0.350708 & 0.790238 \\ 30 & 1 & 4.438035 & 0.452357 & 0.050167 \\ 31 & 1 & 4.912094 & -1.231616 & 0.278640 \\ 32 & 1 & 5.209073 & -0.062632 & 1.574141 \\ 33 & 6 & -4.702335 & 0.225550 & 1.744697 \\ 34 & 1 & -4.519426 & 0.537761 & 2.777047 \\ 35 & 1 & -5.027416 & -0.823358 & 1.752924 \\ 36 & 1 & -5.544363 & 0.813475 & 1.354188 \\ 37 & 6 & -0.777247 & -2.085903 & -0.521571 \\ 38 & 6 & -0.716396 & 2.264774 & 0.044120 \\ 39 & 6 & 1.470866 & 1.365120 & -0.438403 \\ 40 & 6 & 0.553217 & 2.494533 & -0.413596 \\ 41 & 8 & 2.567461 & 1.408782 & -1.018903 \\ 42 & 6 & 1.020716 & 3.794310 & -0.995961 \\ 43 & 1 & 1.841428 & 4.213013 & -0.400572 \\ 44 & 1 & 1.427689 & 3.636553 & -2.001613 \\ 45 & 1 & 0.213086 & 4.531449 & -1.040392 \\ 46 & 1 & -1.496895 & 3.015563 & -0.056102 \\ --------------------------------------------- \\ & & & \end{array}$

\section{B3LYP/6-31+G(d,p)}

$\mathrm{HF}=-1076.2202891$ hartrees $(-675338.993613141 \mathrm{kcal} / \mathrm{mol})$ Imaginary Frequencies: 1 (-444.0485 1/cm)

Zero-point correction $=0.377365$ (Hartree/Particle)

Thermal correction to Energy $=\quad 0.398737$

Thermal correction to Enthalpy= $\quad 0.399681$

Thermal correction to Gibbs Free Energy $=\quad 0.329025$

Sum of electronic and zero-point Energies $=\quad-1075.842924$

Sum of electronic and thermal Energies $=\quad-1075.821552$ 
Sum of electronic and thermal Enthalpies $=\quad-1075.820608$

Sum of electronic and thermal Free Energies $=\quad-1075.891264$ Coordinates (from last standard orientation):

\begin{tabular}{|c|c|c|c|c|}
\hline \multirow{2}{*}{$\begin{array}{l}\text { Center } \\
\text { Number }\end{array}$} & \multirow{2}{*}{$\begin{array}{l}\text { Atomic } \\
\text { Number }\end{array}$} & \multicolumn{3}{|c|}{ Coordinates (Angstroms) } \\
\hline & & & $\begin{array}{ll}X & Y\end{array}$ & Z \\
\hline 1 & 8 & -0.054300 & 0.243989 & 1.085652 \\
\hline 2 & 6 & 1.969835 & -1.005614 & 0.463914 \\
\hline 3 & 1 & 1.395290 & -1.772079 & 0.988012 \\
\hline 4 & 6 & 0.991289 & 0.122180 & 0.219883 \\
\hline 5 & 8 & -2.135859 & -1.920813 & -0.494592 \\
\hline 6 & 8 & -0.262374 & -3.098815 & -0.094148 \\
\hline 7 & 6 & -1.077766 & 1.028152 & 0.646830 \\
\hline 8 & 6 & -2.322724 & 0.818592 & 1.408523 \\
\hline 9 & 1 & -2.256772 & 0.993796 & 2.482989 \\
\hline 10 & 6 & -3.481863 & 0.390862 & 0.884120 \\
\hline 11 & 6 & -3.662052 & 0.002250 & -0.568805 \\
\hline 12 & 1 & -3.872231 & 0.888066 & -1.185766 \\
\hline 13 & 1 & -4.551933 & -0.631967 & -0.650251 \\
\hline 14 & 6 & -2.501361 & -0.743704 & -1.243444 \\
\hline 15 & 1 & -2.880303 & -1.092999 & -2.217651 \\
\hline 16 & 6 & -1.196756 & -0.031773 & -1.451175 \\
\hline 17 & 1 & -1.147073 & 0.819581 & -2.120231 \\
\hline 18 & 6 & -0.118881 & -0.900932 & -1.195986 \\
\hline 19 & 6 & 1.166334 & -1.219123 & -1.952239 \\
\hline 20 & 1 & 0.986679 & -2.051703 & -2.642293 \\
\hline 21 & 1 & 1.453793 & -0.352441 & -2.552845 \\
\hline 22 & 6 & 2.306897 & -1.574578 & -0.952305 \\
\hline 23 & 1 & 3.242477 & -1.161823 & -1.329609 \\
\hline 24 & 1 & 2.420480 & -2.658190 & -0.866330 \\
\hline 25 & 6 & 3.153889 & -0.649142 & 1.378153 \\
\hline 26 & 6 & 2.957368 & -0.643437 & 2.704846 \\
\hline 27 & 1 & 3.758571 & -0.390055 & 3.393774 \\
\hline 28 & 1 & 1.993916 & -0.889789 & 3.143909 \\
\hline 29 & 6 & 4.507197 & -0.344221 & 0.784777 \\
\hline 30 & 1 & 4.443126 & 0.459857 & 0.046581 \\
\hline 31 & 1 & 4.919152 & -1.223912 & 0.273752 \\
\hline 32 & 1 & 5.212118 & -0.056356 & 1.570184 \\
\hline 33 & 6 & -4.705395 & 0.222460 & 1.748459 \\
\hline 34 & 1 & -4.523209 & 0.542726 & 2.777642 \\
\hline 35 & 1 & -5.022441 & -0.828043 & 1.762397 \\
\hline 36 & 1 & -5.549062 & 0.803271 & 1.353282 \\
\hline 37 & 6 & -0.777099 & -2.092053 & -0.521948 \\
\hline 38 & 6 & -0.720260 & 2.269417 & 0.043712 \\
\hline 39 & 6 & 1.467080 & 1.373298 & -0.438306 \\
\hline 40 & 6 & 0.550577 & 2.503353 & -0.412187 \\
\hline 41 & 8 & 2.567615 & 1.417745 & -1.015739 \\
\hline 42 & 6 & 1.012102 & 3.809874 & -0.984113 \\
\hline 43 & 1 & 1.844675 & 4.214952 & -0.397509 \\
\hline 44 & 1 & 1.398833 & 3.669128 & -1.999792 \\
\hline 45 & 1 & 0.204713 & 4.547347 & -0.999592 \\
\hline 46 & 1 & -1.501926 & 3.018622 & -0.056288 \\
\hline
\end{tabular}




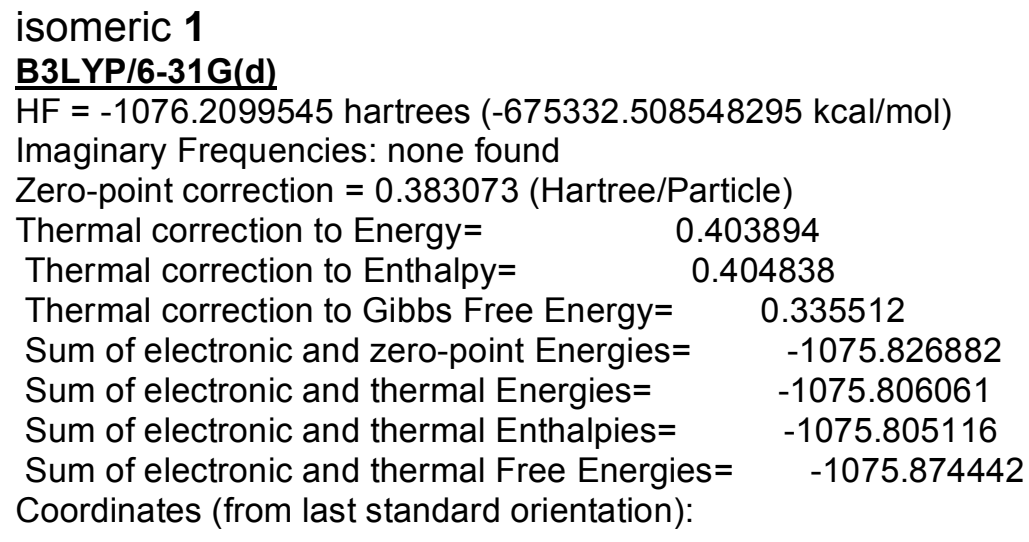

\begin{tabular}{|c|c|c|c|c|}
\hline Center & Atomic & & ordinates ( & gstroms) \\
\hline Number & Numbe & $\gamma$ & $\begin{array}{l}K \quad Y \\
K \quad\end{array}$ & Z \\
\hline 1 & 8 & -0.113067 & 0.374354 & 1.080207 \\
\hline 2 & 6 & 2.020986 & -0.939663 & 0.483891 \\
\hline 3 & 1 & 1.596183 & -1.727244 & 1.114414 \\
\hline 4 & 6 & 0.860693 & -0.007598 & 0.113739 \\
\hline 5 & 8 & -1.978455 & -2.078441 & -0.280203 \\
\hline 6 & 8 & -0.071719 & -3.023251 & 0.393659 \\
\hline 7 & 6 & -1.190774 & 0.920234 & 0.250878 \\
\hline 8 & 6 & -2.443943 & 0.934608 & 1.071275 \\
\hline 9 & 1 & -2.368728 & 1.483717 & 2.008303 \\
\hline 10 & 6 & -3.595415 & 0.348219 & 0.727436 \\
\hline 11 & 6 & -3.732363 & -0.434823 & -0.558473 \\
\hline 12 & 1 & -4.199150 & 0.192263 & -1.333422 \\
\hline 13 & 1 & -4.412666 & -1.283130 & -0.411736 \\
\hline 14 & 6 & -2.411197 & -0.968506 & -1.096594 \\
\hline 15 & 1 & -2.559812 & -1.354050 & -2.113615 \\
\hline 16 & 6 & -1.237212 & 0.006278 & -1.069682 \\
\hline 17 & 1 & -1.220172 & 0.631246 & -1.964177 \\
\hline 18 & 6 & 0.009405 & -0.869421 & -0.856166 \\
\hline 19 & 6 & 1.077567 & -1.382849 & -1.841453 \\
\hline 20 & 1 & 0.777847 & -2.300284 & -2.355770 \\
\hline 21 & 1 & 1.294610 & -0.626964 & -2.600442 \\
\hline 22 & 6 & 2.328449 & -1.605660 & -0.914011 \\
\hline 23 & 1 & 3.204975 & -1.152119 & -1.377894 \\
\hline 24 & 1 & 2.520335 & -2.672539 & -0.777031 \\
\hline 25 & 6 & 3.190540 & -0.347852 & 1.252122 \\
\hline 26 & 6 & 3.052054 & 0.719252 & 2.045692 \\
\hline 27 & 1 & 3.883032 & 1.091502 & 2.639727 \\
\hline 28 & 1 & 2.108119 & 1.247731 & 2.147239 \\
\hline 29 & 6 & 4.495134 & -1.103112 & 1.172147 \\
\hline
\end{tabular}




$\begin{array}{rrrrr}30 & 1 & 4.916797 & -1.076241 & 0.159670 \\ 31 & 1 & 4.356500 & -2.162795 & 1.427853 \\ 32 & 1 & 5.237377 & -0.683477 & 1.857895 \\ 33 & 6 & -4.827332 & 0.434097 & 1.587937 \\ 34 & 1 & -4.661771 & 1.054343 & 2.473786 \\ 35 & 1 & -5.138764 & -0.565145 & 1.920608 \\ 36 & 1 & -5.672930 & 0.856340 & 1.027313 \\ 37 & 6 & -0.626061 & -2.098858 & -0.145360 \\ 38 & 6 & -0.740721 & 2.311443 & -0.151898 \\ 39 & 6 & 1.389707 & 1.281309 & -0.607032 \\ 40 & 6 & 0.537288 & 2.500096 & -0.519352 \\ 41 & 8 & 2.425232 & 1.288217 & -1.254283 \\ 42 & 6 & 1.140559 & 3.806469 & -0.947974 \\ 43 & 1 & 1.966157 & 4.089718 & -0.284127 \\ 44 & 1 & 1.566978 & 3.721122 & -1.953265 \\ 45 & 1 & 0.396591 & 4.608643 & -0.938997 \\ 46 & 1 & -1.471612 & 3.118340 & -0.164095\end{array}$

\section{B3LYP/6-31+(d,p)}

$\mathrm{HF}=-1076.2733506$ hartrees $(-675372.290235006 \mathrm{kcal} / \mathrm{mol})$ Imaginary Frequencies: none found

Zero-point correction $=0.380577($ Hartree/Particle $)$

Thermal correction to Energy $=\quad 0.401506$

Thermal correction to Enthalpy= $\quad 0.402451$

Thermal correction to Gibbs Free Energy $=\quad 0.332877$

Sum of electronic and zero-point Energies $=\quad-1075.892774$

Sum of electronic and thermal Energies $=\quad-1075.871844$

Sum of electronic and thermal Enthalpies $=\quad-1075.870900$

Sum of electronic and thermal Free Energies $=\quad-1075.940474$

Coordinates (from last standard orientation):

\begin{tabular}{|c|c|c|c|c|}
\hline \multirow{2}{*}{$\begin{array}{l}\text { Center } \\
\text { Number }\end{array}$} & \multirow{2}{*}{$\begin{array}{l}\text { Atomic } \\
\text { Number }\end{array}$} & \multicolumn{3}{|c|}{ Coordinates (Angstroms) } \\
\hline & & & $X \quad Y$ & Z \\
\hline 1 & 8 & -0.110952 & 0.378172 & 1.083349 \\
\hline 2 & 6 & 2.029109 & -0.928422 & 0.474600 \\
\hline 3 & 1 & 1.608934 & -1.719797 & 1.103571 \\
\hline 4 & 6 & 0.863761 & 0.001487 & 0.113624 \\
\hline 5 & 8 & -1.970457 & -2.090409 & -0.299133 \\
\hline 6 & 8 & -0.062784 & -3.025412 & 0.387875 \\
\hline 7 & 6 & -1.196613 & 0.921342 & 0.257220 \\
\hline 8 & 6 & -2.448533 & 0.926465 & 1.079887 \\
\hline 9 & 1 & -2.376379 & 1.472684 & 2.018523 \\
\hline 10 & 6 & -3.597958 & 0.332476 & 0.734762 \\
\hline 11 & 6 & -3.731741 & -0.446945 & -0.553922 \\
\hline 12 & 1 & -4.205653 & 0.181650 & -1.322565 \\
\hline 13 & 1 & -4.405096 & -1.300150 & -0.408211 \\
\hline 14 & 6 & -2.410197 & -0.967699 & -1.101253 \\
\hline 15 & 1 & -2.558611 & -1.343862 & -2.121171 \\
\hline 16 & 6 & -1.238575 & 0.009013 & -1.064426 \\
\hline 17 & 1 & -1.220188 & 0.636190 & -1.957196 \\
\hline 18 & 6 & 0.011450 & -0.863384 & -0.855069 \\
\hline 19 & 6 & 1.076536 & -1.370115 & -1.848279 \\
\hline 20 & 1 & 0.778313 & -2.288992 & -2.359925 \\
\hline 21 & 1 & 1.283564 & -0.612782 & -2.608340 \\
\hline
\end{tabular}




$\begin{array}{rrrrr}22 & 6 & 2.334589 & -1.587121 & -0.928478 \\ 23 & 1 & 3.204348 & -1.122791 & -1.394165 \\ 24 & 1 & 2.535996 & -2.652583 & -0.797122 \\ 25 & 6 & 3.205601 & -0.354214 & 1.245227 \\ 26 & 6 & 3.095261 & 0.726412 & 2.028787 \\ 27 & 1 & 3.935319 & 1.075444 & 2.623092 \\ 28 & 1 & 2.168975 & 1.286072 & 2.118592 \\ 29 & 6 & 4.487153 & -1.150977 & 1.182078 \\ 30 & 1 & 4.914867 & -1.146473 & 0.172672 \\ 31 & 1 & 4.311016 & -2.201811 & 1.447424 \\ 32 & 1 & 5.236108 & -0.747560 & 1.868896 \\ 33 & 6 & -4.829580 & 0.403996 & 1.597399 \\ 34 & 1 & -4.668884 & 1.024285 & 2.483157 \\ 35 & 1 & -5.127402 & -0.599364 & 1.927538 \\ 36 & 1 & -5.678665 & 0.817820 & 1.037484 \\ 37 & 6 & -0.620038 & -2.101423 & -0.151887 \\ 38 & 6 & -0.752827 & 2.316406 & -0.138434 \\ 39 & 6 & 1.379580 & 1.298168 & -0.607775 \\ 40 & 6 & 0.524278 & 2.514467 & -0.509464 \\ 41 & 8 & 2.409713 & 1.308859 & -1.265632 \\ 42 & 6 & 1.116112 & 3.829662 & -0.927269 \\ 43 & 1 & 1.954746 & 4.101760 & -0.276624 \\ 44 & 1 & 1.518874 & 3.766622 & -1.943355 \\ 45 & 1 & 0.368605 & 4.626473 & -0.884867 \\ 46 & 1 & -1.487158 & 3.119918 & -0.143057 \\ ----------------------------------------------------\end{array}$

\section{in $\mathrm{H}_{2} \mathrm{O}$ B3LYP/6-31G(d)}

$\mathrm{HF}=-1076.2339694$ hartrees $(-675347.578138194 \mathrm{kcal} / \mathrm{mol})$ Imaginary Frequencies: none found

Zero-point correction $=0.379995$ (Hartree/Particle)

Thermal correction to Energy $=\quad 0.400762$

Thermal correction to Enthalpy= $\quad 0.401707$

Thermal correction to Gibbs Free Energy $=\quad 0.332811$

Sum of electronic and zero-point Energies $=\quad-1075.853975$

Sum of electronic and thermal Energies $=\quad-1075.833207$

Sum of electronic and thermal Enthalpies $=\quad-1075.832263$

Sum of electronic and thermal Free Energies $=\quad-1075.901158$

Coordinates (from last standard orientation):

\begin{tabular}{|c|c|c|c|c|}
\hline \multirow{2}{*}{$\begin{array}{l}\text { Center } \\
\text { Number }\end{array}$} & \multirow{2}{*}{$\begin{array}{l}\text { Atomic } \\
\text { Number }\end{array}$} & \multicolumn{3}{|c|}{ Coordinates (Angstroms) } \\
\hline & & & $\begin{array}{l}X \\
X\end{array}$ & Z \\
\hline 1 & 8 & -0.110019 & 0.366609 & 1.081600 \\
\hline 2 & 6 & 2.032147 & -0.926761 & 0.466998 \\
\hline 3 & 1 & 1.618653 & -1.717612 & 1.103259 \\
\hline 4 & 6 & 0.862367 & -0.000302 & 0.106674 \\
\hline 5 & 8 & -1.956148 & -2.086894 & -0.263764 \\
\hline 6 & 8 & -0.053175 & -3.016653 & 0.405856 \\
\hline 7 & 6 & -1.203076 & 0.910995 & 0.248959 \\
\hline 8 & 6 & -2.451592 & 0.918666 & 1.075863 \\
\hline 9 & 1 & -2.385461 & 1.485080 & 2.005099 \\
\hline 10 & 6 & -3.600774 & 0.325080 & 0.731695 \\
\hline 11 & 6 & -3.733070 & -0.457649 & -0.554031 \\
\hline 12 & 1 & -4.199284 & 0.173172 & -1.328469 \\
\hline 13 & 1 & -4.413795 & -1.309368 & -0.412904 \\
\hline
\end{tabular}




\begin{tabular}{rrrrr}
14 & 6 & -2.410890 & -0.975246 & -1.096344 \\
15 & 1 & -2.553514 & -1.381572 & -2.107989 \\
16 & 6 & -1.243795 & 0.004182 & -1.075115 \\
17 & 1 & -1.241152 & 0.632784 & -1.999450 \\
18 & 6 & 0.010107 & -0.864403 & -0.862207 \\
19 & 6 & 1.075960 & -1.373443 & -1.852078 \\
20 & 1 & 0.776339 & -2.290790 & -2.368195 \\
21 & 1 & 1.277758 & -0.613690 & -2.612332 \\
22 & 6 & 2.334027 & -1.590537 & -0.933727 \\
23 & 1 & 3.209393 & -1.135418 & -1.400513 \\
24 & 1 & 2.536254 & -2.656854 & -0.798942 \\
25 & 6 & 3.207489 & -0.344916 & 1.233241 \\
26 & 6 & 3.096309 & 0.744004 & 2.002300 \\
27 & 1 & 3.932918 & 1.102325 & 2.597910 \\
28 & 1 & 2.171065 & 1.309466 & 2.077187 \\
29 & 6 & 4.488183 & -1.144431 & 1.187117 \\
30 & 1 & 4.914531 & -1.167507 & 0.175916 \\
31 & 1 & 4.311037 & -2.190521 & 1.475056 \\
32 & 1 & 5.241140 & -0.727591 & 1.863759 \\
33 & 6 & -4.837066 & 0.419085 & 1.584890 \\
34 & 1 & -4.672802 & 1.037852 & 2.472849 \\
35 & 1 & -5.161979 & -0.579069 & 1.909987 \\
36 & 1 & -5.672518 & 0.848785 & 1.014306 \\
37 & 6 & -0.614580 & -2.091901 & -0.144574 \\
38 & 6 & -0.756868 & 2.303775 & -0.141964 \\
39 & 6 & 1.378963 & 1.299424 & -0.602042 \\
40 & 6 & 0.522178 & 2.506363 & -0.503322 \\
41 & 8 & 2.424330 & 1.317683 & -1.240789 \\
42 & 6 & 1.105333 & 3.826621 & -0.919696 \\
43 & 1 & 1.938917 & 4.111101 & -0.264917 \\
44 & 1 & 1.509509 & 3.768991 & -1.937126 \\
45 & 1 & 0.349989 & 4.617402 & -0.882066 \\
46 & 1 & -1.495013 & 3.105429 & -0.161462 \\
----------------------------------------------------- \\
\hline
\end{tabular}




\section{Tables 2-5)}

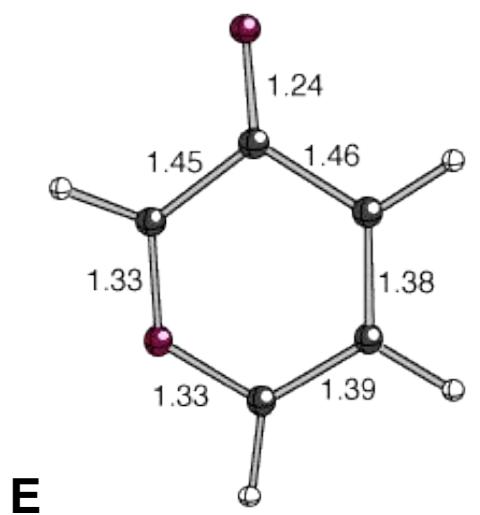

B3LYP/6-31G(d)

$\mathrm{HF}=-343.3138655$ hartrees $(-215432.883739905 \mathrm{kcal} / \mathrm{mol})$

Imaginary Frequencies: none found

Zero-point correction $=0.079960$ (Hartree/Particle $)$

Thermal correction to Energy $=\quad 0.085234$

Thermal correction to Enthalpy $=\quad 0.086178$

Thermal correction to Gibbs Free Energy $=0.050954$

Sum of electronic and zero-point Energies $=\quad-343.233906$

Sum of electronic and thermal Energies $=\quad-343.228632$

Sum of electronic and thermal Enthalpies $=\quad-343.227688$

Sum of electronic and thermal Free Energies $=\quad-343.262911$

Coordinates (from last standard orientation):

\begin{tabular}{|c|c|c|c|c|}
\hline \multirow{2}{*}{$\begin{array}{l}\text { Center } \\
\text { Number }\end{array}$} & \multirow{2}{*}{$\begin{array}{l}\text { Atomic } \\
\text { Number }\end{array}$} & \multicolumn{3}{|c|}{ Coordinates (Angstroms) } \\
\hline & & & $X \quad Y$ & Z \\
\hline 1 & 8 & 1.033576 & -1.169952 & 0.000012 \\
\hline 2 & 8 & -2.332575 & 0.009847 & -0.000201 \\
\hline 3 & 6 & -0.291833 & -1.164950 & 0.000178 \\
\hline 4 & 6 & -1.093209 & 0.039104 & -0.000025 \\
\hline 5 & 6 & -0.266718 & 1.238287 & 0.000126 \\
\hline 6 & 6 & 1.116420 & 1.174840 & 0.000012 \\
\hline 7 & 1 & 1.722383 & 2.076020 & 0.000340 \\
\hline 8 & 6 & 1.759464 & -0.052287 & -0.000115 \\
\hline 9 & 1 & 2.823291 & -0.240305 & -0.000804 \\
\hline 10 & 1 & -0.779666 & $6 \quad 2.195797$ & 0.000324 \\
\hline 11 & 1 & -0.718755 & $5-2.160636$ & 0.000598 \\
\hline
\end{tabular}

\section{B3LYP/6-31+(d,p)}

$\mathrm{HF}=-343.3375721$ hartrees $(-215447.759868471 \mathrm{kcal} / \mathrm{mol})$

Imaginary Frequencies: none found

Zero-point correction $=0.079585$ (Hartree/Particle)

Thermal correction to Energy $=\quad 0.084883$

Thermal correction to Enthalpy $=\quad 0.085828$

Thermal correction to Gibbs Free Energy $=0.050534$

Sum of electronic and zero-point Energies $=\quad-343.257987$

Sum of electronic and thermal Energies $=\quad-343.252689$ 
Sum of electronic and thermal Enthalpies=

$-343.251744$

Sum of electronic and thermal Free Energies=

$-343.287038$

Coordinates (from last standard orientation):

\begin{tabular}{|c|c|c|c|c|}
\hline \multirow{2}{*}{$\begin{array}{l}\text { Center } \\
\text { Number }\end{array}$} & \multirow{2}{*}{$\begin{array}{l}\text { Atomic } \\
\text { Number }\end{array}$} & \multicolumn{3}{|c|}{ Coordinates (Angstroms) } \\
\hline & & & $X \quad Y$ & Z \\
\hline 1 & 8 & 1.032769 & -1.170354 & 0.000002 \\
\hline 2 & 8 & -2.333451 & 0.010820 & -0.000051 \\
\hline 3 & 6 & -0.294033 & -1.166957 & 0.000054 \\
\hline 4 & 6 & -1.087920 & 0.039391 & -0.000016 \\
\hline 5 & 6 & -0.268182 & 1.239330 & 0.000056 \\
\hline 6 & 6 & 1.117035 & 1.174946 & -0.000003 \\
\hline 7 & 1 & 1.722215 & 2.076055 & -0.000021 \\
\hline 8 & 6 & 1.760359 & -0.052485 & -0.000044 \\
\hline 9 & 1 & 2.823921 & -0.239620 & -0.000078 \\
\hline 10 & 1 & -0.781723 & $3 \quad 2.196031$ & 0.000111 \\
\hline 11 & 1 & -0.722522 & $2-2.161550$ & 0.000103 \\
\hline
\end{tabular}

\section{MP2/6-31G(d)}

$\mathrm{HF}=-341.2961699$ hartrees $(-214166.759573949 \mathrm{kcal} / \mathrm{mol})$

Imaginary Frequencies: none found

Zero-point correction $=0.080558$ (Hartree/Particle)

Thermal correction to Energy $=\quad 0.085956$

Thermal correction to Enthalpy $=\quad 0.086900$

Thermal correction to Gibbs Free Energy $=0.051409$

Sum of electronic and zero-point Energies $=\quad-342.221346$

Sum of electronic and thermal Energies $=\quad-342.215948$

Sum of electronic and thermal Enthalpies $=\quad-342.215004$

Sum of electronic and thermal Free Energies $=\quad-342.250495$

Coordinates (from last standard orientation):

\begin{tabular}{|c|c|c|c|c|}
\hline \multirow{2}{*}{$\begin{array}{l}\text { Center } \\
\text { Number }\end{array}$} & \multirow{2}{*}{$\begin{array}{l}\text { Atomic } \\
\text { Number }\end{array}$} & \multicolumn{3}{|c|}{ Coordinates (Angstroms) } \\
\hline & & & $\begin{array}{ll}X \quad Y\end{array}$ & Z \\
\hline 1 & 8 & 1.039512 & -1.175566 & 0.000001 \\
\hline 2 & 8 & -2.334235 & 0.006125 & -0.000099 \\
\hline 3 & 6 & -0.293511 & -1.171286 & 0.000088 \\
\hline 4 & 6 & -1.092923 & 0.040093 & 0.000004 \\
\hline 5 & 6 & -0.268728 & 1.244946 & 0.000094 \\
\hline 6 & 6 & 1.110916 & 1.179570 & -0.000020 \\
\hline 7 & 1 & 1.716208 & 2.081799 & -0.000001 \\
\hline 8 & 6 & 1.763627 & -0.047136 & -0.000053 \\
\hline 9 & 1 & 2.828208 & -0.235587 & -0.000213 \\
\hline 10 & 1 & -0.785298 & 2.201720 & 0.000158 \\
\hline 11 & 1 & -0.717616 & -2.169519 & 0.000155 \\
\hline
\end{tabular}

in $\mathrm{H}_{2} \mathrm{O}$ B3LYP/6-31G(d)

$\mathrm{HF}=-343.3327666$ hartrees $(-215444.744369166 \mathrm{kcal} / \mathrm{mol})$ Imaginary Frequencies: none found

Zero-point correction $=0.079181$ (Hartree/Particle $)$

Thermal correction to Energy $=\quad 0.084386$

Thermal correction to Enthalpy $=\quad 0.085330$

Thermal correction to Gibbs Free Energy $=\quad 0.050264$ 
Sum of electronic and zero-point Energies= Sum of electronic and thermal Energies= Sum of electronic and thermal Enthalpies= Sum of electronic and thermal Free Energies= Coordinates (from last standard orientation):
$-343.253586$

$-343.248380$

$-343.247436$

$-343.282502$

\begin{tabular}{ccrrr} 
Center & Atomic & \multicolumn{3}{c}{ Coordinates (Angstroms) } \\
Number & Number & \multicolumn{2}{c}{ X } & \multicolumn{1}{c}{$Y$} \\
\hdashline 1 & 8 & 1.029208 & -1.169120 & 0.000003 \\
2 & 8 & -2.339013 & 0.018475 & 0.000029 \\
3 & 6 & -0.298361 & -1.164101 & -0.000045 \\
4 & 6 & -1.081022 & 0.038792 & -0.000002 \\
5 & 6 & -0.264753 & 1.232106 & -0.000020 \\
6 & 6 & 1.122241 & 1.172338 & -0.000002 \\
7 & 1 & 1.728469 & 2.075637 & -0.000004 \\
8 & 6 & 1.758999 & -0.054273 & 0.000014 \\
9 & 1 & 2.826117 & -0.244791 & 0.000091 \\
10 & 1 & -0.772606 & 2.195431 & -0.000027 \\
11 & 1 & -0.726169 & -2.170298 & 0.000014
\end{tabular}

in $\mathrm{H}_{2} \mathrm{O} M P 2 / 6-31 \mathrm{G}(\mathrm{d})$

$\mathrm{HF}=-341.3226755$ hartrees $(-214183.392103005 \mathrm{kcal} / \mathrm{mol})$

Imaginary Frequencies: none found

Zero-point correction $=0.079646($ Hartree/Particle $)$

Thermal correction to Energy $=\quad 0.084994$

Thermal correction to Enthalpy $=\quad 0.085938$

Thermal correction to Gibbs Free Energy $=0.050532$

Sum of electronic and zero-point Energies= $\quad-342.240308$

Sum of electronic and thermal Energies $=\quad-342.234960$

Sum of electronic and thermal Enthalpies $=\quad-342.234016$

Sum of electronic and thermal Free Energies $=\quad-342.269422$

Coordinates (from last standard orientation):

\begin{tabular}{|c|c|c|c|c|}
\hline \multirow{2}{*}{$\begin{array}{l}\text { Center } \\
\text { Number }\end{array}$} & \multirow{2}{*}{$\begin{array}{l}\text { Atomic } \\
\text { Number }\end{array}$} & \multicolumn{3}{|c|}{ Coordinates (Angstroms) } \\
\hline & & & $Y$ & Z \\
\hline 1 & 8 & 1.033616 & -1.173475 & 0.000001 \\
\hline 2 & 8 & -2.336886 & 0.011886 & -0.000064 \\
\hline 3 & 6 & -0.296963 & -1.170641 & 0.000022 \\
\hline 4 & 6 & -1.081450 & 0.042367 & 0.000026 \\
\hline 5 & 6 & -0.269500 & 1.238716 & 0.000058 \\
\hline 6 & 6 & 1.114596 & 1.175956 & -0.000019 \\
\hline 7 & 1 & 1.720812 & 2.080704 & -0.000008 \\
\hline 8 & 6 & 1.763189 & -0.048057 & -0.000034 \\
\hline 9 & 1 & 2.831185 & -0.239426 & -0.000070 \\
\hline 10 & 1 & -0.781001 & 2.201518 & 0.000098 \\
\hline 11 & 1 & -0.724065 & -2.180125 & 0.000164 \\
\hline
\end{tabular}

in $\mathrm{CH}_{3} \mathrm{CN}$ B3LYP/6-31G(d)

$\mathrm{HF}=-343.323777$ hartrees $(-215439.10330527 \mathrm{kcal} / \mathrm{mol})$

Imaginary Frequencies: none found

Zero-point correction $=0.080019($ Hartree/Particle $)$

Thermal correction to Energy $=\quad 0.085237$

Thermal correction to Enthalpy $=0.086181$

Thermal correction to Gibbs Free Energy $=0.051082$ 
Sum of electronic and zero-point Energies= Sum of electronic and thermal Energies= Sum of electronic and thermal Enthalpies= Sum of electronic and thermal Free Energies= Coordinates (from last standard orientation):
$-343.243758$

$-343.238540$

$-343.237596$

$-343.272695$

\begin{tabular}{ccrrr} 
Center & Atomic & \multicolumn{3}{c}{ Coordinates (Angstroms) } \\
Number & Number & \multicolumn{1}{c}{ X } & \multicolumn{1}{c}{$Y$} & \multicolumn{1}{c}{ Y } \\
\hdashline 1 & 8 & 1.032306 & -1.168448 & 0.000000 \\
2 & 8 & -2.337135 & 0.014220 & -0.000021 \\
3 & 6 & -0.294721 & -1.163512 & 0.000024 \\
4 & 6 & -1.086849 & 0.037490 & -0.000018 \\
5 & 6 & -0.265915 & 1.233301 & 0.000016 \\
6 & 6 & 1.119169 & 1.173703 & 0.000000 \\
7 & 1 & 1.724028 & 2.075579 & 0.000065 \\
8 & 6 & 1.758962 & -0.053180 & -0.000017 \\
9 & 1 & 2.823908 & -0.240959 & -0.000091 \\
10 & 1 & -0.774999 & 2.193702 & 0.000057 \\
11 & 1 & -0.718174 & -2.161318 & 0.000109
\end{tabular}

\section{in DMSO B3LYP/6-31G(d)}

$\mathrm{HF}=-343.3237914$ hartrees $(-215439.112341414 \mathrm{kcal} / \mathrm{mol})$

Imaginary Frequencies: none found

Zero-point correction $=0.080020($ Hartree $/$ Particle $)$

Thermal correction to Energy $=\quad 0.085239$

Thermal correction to Enthalpy= $\quad 0.086183$

Thermal correction to Gibbs Free Energy $=\quad 0.051083$

Sum of electronic and zero-point Energies= $\quad-343.243771$

Sum of electronic and thermal Energies $=\quad-343.238553$

Sum of electronic and thermal Enthalpies $=\quad-343.237609$

Sum of electronic and thermal Free Energies= $\quad-343.272709$

Coordinates (from last standard orientation):

\begin{tabular}{ccrrr}
\hline $\begin{array}{c}\text { Center } \\
\text { Number }\end{array}$ & Atomic & \multicolumn{3}{c}{ Coordinates (Angstroms) } \\
\cline { 1 - 2 } & Numb & \multicolumn{1}{c}{ Y } & \multicolumn{1}{c}{$Z$} \\
\hline 1 & 8 & 1.032318 & -1.168434 & 0.000017 \\
2 & 8 & -2.337176 & 0.014146 & -0.000380 \\
3 & 6 & -0.294633 & -1.163365 & 0.000322 \\
4 & 6 & -1.087010 & 0.037504 & 0.000000 \\
5 & 6 & -0.265916 & 1.233277 & 0.000353 \\
6 & 6 & 1.119146 & 1.173593 & -0.000058 \\
7 & 1 & 1.724097 & 2.075437 & -0.000141 \\
8 & 6 & 1.759023 & -0.053196 & -0.000278 \\
9 & 1 & 2.824039 & -0.240862 & -0.000501 \\
10 & 1 & -0.774679 & 2.193896 & 0.000825 \\
11 & 1 & -0.718254 & -2.161045 & 0.000683
\end{tabular}

\section{Ethene}

\section{B3LYP/6-31G(d)}

$\mathrm{HF}=-78.5874582$ hartrees $(-49314.415895082 \mathrm{kcal} / \mathrm{mol})$

Imaginary Frequencies: none found

Zero-point correction $=0.051231$ (Hartree/Particle)

Thermal correction to Energy=

0.054273

Thermal correction to Enthalpy=

0.055217 
Thermal correction to Gibbs Free Energy=

Sum of electronic and zero-point Energies=

Sum of electronic and thermal Energies=

Sum of electronic and thermal Enthalpies=

Sum of electronic and thermal Free Energies=

Coordinates (from last standard orientation):
0.029047

$-78.536227$

$-78.533185$

$-78.532241$

$-78.558411$

\begin{tabular}{|c|c|c|c|c|}
\hline \multirow{2}{*}{$\begin{array}{l}\text { Center } \\
\text { Number }\end{array}$} & \multirow{2}{*}{$\begin{array}{l}\text { Atomic } \\
\text { Number }\end{array}$} & \multicolumn{3}{|c|}{ Coordinates (Angstroms) } \\
\hline & & & $\begin{array}{ll}X & Y\end{array}$ & Z \\
\hline 1 & 6 & -0.665445 & -0.000008 & -0.000014 \\
\hline 2 & 1 & -1.239225 & 0.923719 & 0.000044 \\
\hline 3 & 6 & 0.665443 & -0.000008 & -0.000013 \\
\hline 4 & 1 & 1.239229 & 0.923717 & 0.000039 \\
\hline 5 & 1 & -1.239317 & -0.923674 & 0.000040 \\
\hline 6 & 1 & 1.239326 & -0.923667 & 0.000042 \\
\hline
\end{tabular}

\section{in $\mathrm{H}_{2} \mathrm{O}$ B3LYP/6-31G(d)}

$\mathrm{HF}=-78.5884795$ hartrees $(-49315.056771045 \mathrm{kcal} / \mathrm{mol})$ Imaginary Frequencies: none found

Zero-point correction $=0.051075$ (Hartree/Particle)

Thermal correction to Energy $=\quad 0.054119$

Thermal correction to Enthalpy= $\quad 0.055063$

Thermal correction to Gibbs Free Energy $=\quad 0.028889$

Sum of electronic and zero-point Energies $=\quad-78.537404$

Sum of electronic and thermal Energies $=\quad-78.534361$

Sum of electronic and thermal Enthalpies $=\quad-78.533417$

Sum of electronic and thermal Free Energies $=\quad-78.559591$

Coordinates (from last standard orientation):

\begin{tabular}{|c|c|c|c|c|}
\hline \multirow{2}{*}{$\begin{array}{l}\text { Center } \\
\text { Number }\end{array}$} & \multirow{2}{*}{$\begin{array}{l}\text { Atomic } \\
\text { Number }\end{array}$} & & \multicolumn{2}{|c|}{ Coordinates (Angstroms) } \\
\hline & & & $\begin{array}{ll}X \quad Y\end{array}$ & Z \\
\hline 1 & 6 & 0.665993 & -0.000022 & -0.000028 \\
\hline 2 & 1 & 1.239656 & 0.924559 & 0.000098 \\
\hline 3 & 6 & -0.665987 & -0.000026 & -0.000021 \\
\hline 4 & 1 & -1.239676 & 0.924551 & 0.000049 \\
\hline 5 & 1 & 1.239945 & -0.924422 & 0.000059 \\
\hline 6 & 1 & -1.239962 & -0.924404 & 0.000087 \\
\hline
\end{tabular}

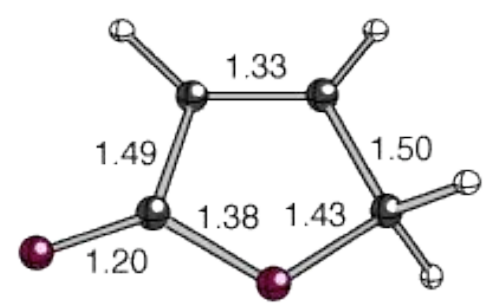

IV

B3LYP/6-31G(d)

$\mathrm{HF}=-305.2641846$ hartrees $(-191556.328478346 \mathrm{kcal} / \mathrm{mol})$

Imaginary Frequencies: none found

Zero-point correction $=0.074913($ Hartree/Particle $)$ 


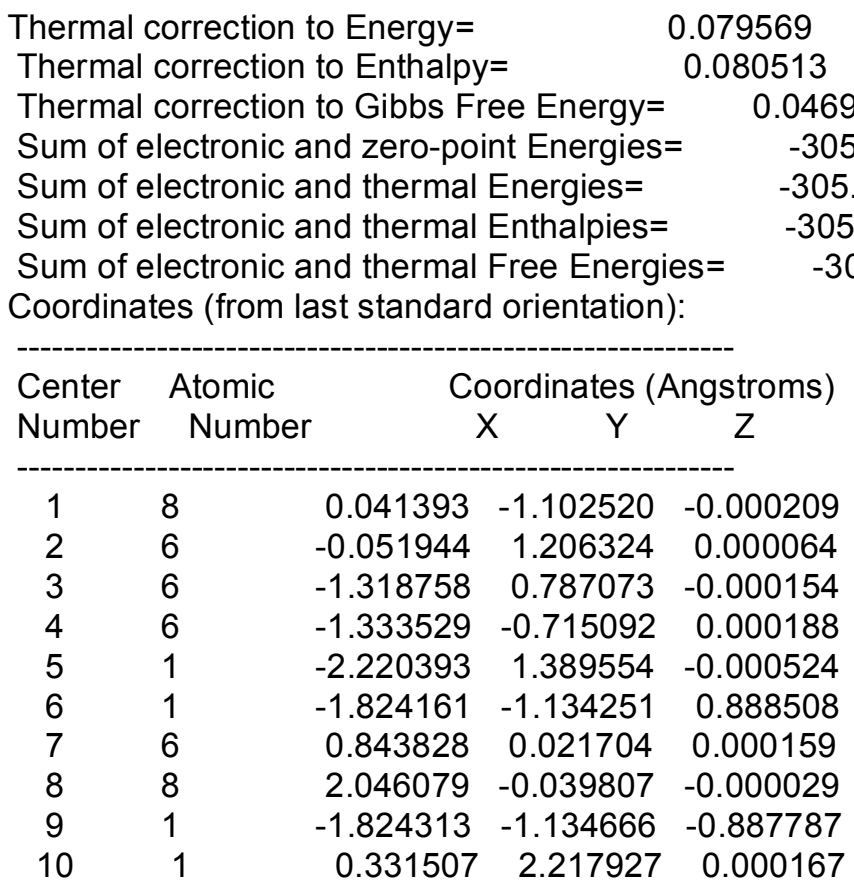

\section{B3LYP/6-31+G(d,p)}

$\mathrm{HF}=-305.2846075$ hartrees $(-191569.144052325 \mathrm{kcal} / \mathrm{mol})$

Imaginary Frequencies: none found

Zero-point correction $=0.074507$ (Hartree/Particle)

Thermal correction to Energy $=\quad 0.079178$

Thermal correction to Enthalpy= $\quad 0.080123$

Thermal correction to Gibbs Free Energy $=\quad 0.046530$

Sum of electronic and zero-point Energies $=\quad-305.210101$

Sum of electronic and thermal Energies $=\quad-305.205429$

Sum of electronic and thermal Enthalpies $=\quad-305.204485$

Sum of electronic and thermal Free Energies $=\quad-305.238078$

Coordinates (from last standard orientation):

\begin{tabular}{|c|c|c|c|c|}
\hline \multirow{2}{*}{$\begin{array}{l}\text { Center } \\
\text { Number }\end{array}$} & \multirow{2}{*}{$\begin{array}{l}\text { Atomic } \\
\text { Number }\end{array}$} & & \multicolumn{2}{|c|}{ Coordinates (Angstroms) } \\
\hline & & & $\begin{array}{ll}X & Y\end{array}$ & Z \\
\hline 1 & 8 & -0.041541 & -1.102529 & -0.000015 \\
\hline 2 & 6 & 0.050230 & 1.206543 & -0.000017 \\
\hline 3 & 6 & 1.319628 & 0.787103 & 0.000029 \\
\hline 4 & 6 & 1.338006 & -0.714324 & 0.000016 \\
\hline 5 & 1 & 2.218980 & 1.392163 & 0.000015 \\
\hline 6 & 1 & 1.823190 & -1.133931 & -0.889673 \\
\hline 7 & 6 & -0.843170 & 0.021577 & -0.000097 \\
\hline 8 & 8 & -2.048333 & -0.040810 & 0.000059 \\
\hline 9 & 1 & 1.823166 & -1.133910 & 0.889727 \\
\hline 10 & 1 & -0.334502 & 2.216997 & -0.000008 \\
\hline
\end{tabular}

\section{MP2/6-31G(d)}

$\mathrm{HF}=-303.5147341$ hartrees $(-190458.530795091 \mathrm{kcal} / \mathrm{mol})$

Imaginary Frequencies: none found

Zero-point correction $=0.075702($ Hartree/Particle $)$ 


\begin{tabular}{|c|c|c|c|c|c|}
\hline \multicolumn{4}{|c|}{ Thermal correction to Energy= } & \multicolumn{2}{|c|}{0.080393} \\
\hline \multicolumn{6}{|c|}{ Thermal correction to Enthalpy $=\quad 0.081338$} \\
\hline \multirow{5}{*}{\multicolumn{5}{|c|}{$\begin{array}{l}\text { Thermal correction to Gibbs Free Energy= } \\
\text { Sum of electronic and zero-point Energies= } \\
\text { Sum of electronic and thermal Energies= } \\
\text { Sum of electronic and thermal Enthalpies= } \\
\text { Sum of electronic and thermal Free Energies= } \\
\text { Coordinates (from last standard orientation): }\end{array}$}} & \\
\hline & & & & & \\
\hline & & & & & \\
\hline & & & & & \\
\hline & & & & & \\
\hline \multirow{2}{*}{$\begin{array}{l}\text { Center } \\
\text { Number }\end{array}$} & \multirow{2}{*}{$\begin{array}{l}\text { Atomic } \\
\text { Number }\end{array}$} & & \multicolumn{3}{|c|}{ Coordinates (Angstroms) } \\
\hline & & & $X \quad Y$ & & \\
\hline 1 & & & & & \\
\hline 2 & & 0.049660 & 4529 & & 007 \\
\hline 3 & & 1.320938 & 36984 & -0.6 & \\
\hline 4 & & 1.339408 & -0.711722 & 0.0 & 0008 \\
\hline 5 & 1 & 2.219033 & 4926 & -0.0 & 0016 \\
\hline 6 & & 1.822778 & -1.1 & & \\
\hline 7 & 6 & -0.842135 & 0.020403 & & \\
\hline 8 & 8 & -2.052467 & -0.038664 & & 00001 \\
\hline 9 & 1 & 1.822772 & -1.129965 & & \\
\hline & & & & & \\
\hline
\end{tabular}

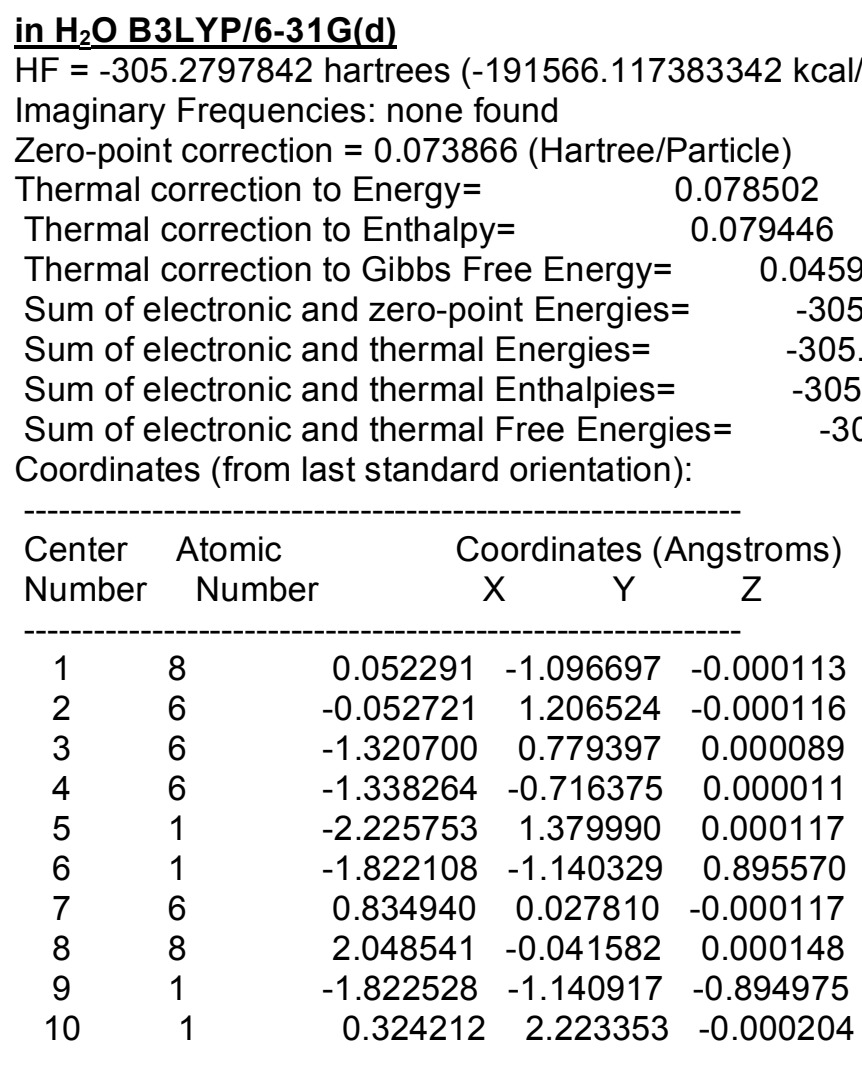

in $\mathrm{H}_{2} \mathrm{O} \mathrm{MP} 2 / 6-31 \mathrm{G}(\mathrm{d})$

$\mathrm{HF}=-303.5339554$ hartrees $(-190470.592353054 \mathrm{kcal} / \mathrm{mol})$ Imaginary Frequencies: none found

Zero-point correction $=0.074762($ Hartree/Particle $)$ 
Thermal correction to Energy= $\quad 0.079415$

Thermal correction to Enthalpy=

0.080359

Thermal correction to Gibbs Free Energy=

Sum of electronic and zero-point Energies=

0.046803

Sum of electronic and thermal Energies=

$-304.308759$

$-304.304106$

$-304.303162$

Sum of electronic and thermal Free Energies=

$-304.336717$

Coordinates (from last standard orientation):

\begin{tabular}{ccrrr}
\hline $\begin{array}{c}\text { Center } \\
\text { Number }\end{array}$ & Atomic & \multicolumn{3}{c}{ Coordinates (Angstroms) } \\
\hline 1 & 8 & $X$ & $Y$ & $Z$ \\
2 & 6 & -0.051031 & -1.099380 & -0.000152 \\
3 & 6 & -1.321622 & 0.780138 & 0.000052 \\
4 & 6 & -1.344253 & -0.713282 & -0.000017 \\
5 & 1 & -2.223466 & 1.386336 & -0.000060 \\
6 & 1 & -1.820743 & -1.136582 & 0.897412 \\
7 & 6 & 0.834076 & 0.025181 & 0.000343 \\
8 & 8 & 2.053099 & -0.040254 & -0.000059 \\
9 & 1 & -1.821134 & -1.137014 & -0.896967 \\
10 & 1 & 0.326131 & 2.222401 & -0.000350 \\
- \hdashline------- &
\end{tabular}

\section{in $\mathrm{CH}_{3} \mathrm{CN}$ B3LYP/6-31G(d)}

$\mathrm{HF}=-305.2729137$ hartrees $(-191561.806075887 \mathrm{kcal} / \mathrm{mol})$

Imaginary Frequencies: none found

Zero-point correction $=0.074752($ Hartree/Particle $)$

Thermal correction to Energy $=\quad 0.079385$

Thermal correction to Enthalpy $=\quad 0.080330$

Thermal correction to Gibbs Free Energy $=\quad 0.046818$

Sum of electronic and zero-point Energies $=\quad-305.198162$

Sum of electronic and thermal Energies $=\quad-305.193528$

Sum of electronic and thermal Enthalpies $=\quad-305.192584$

Sum of electronic and thermal Free Energies $=\quad-305.226096$

Coordinates (from last standard orientation):

\begin{tabular}{|c|c|c|c|c|}
\hline \multirow{2}{*}{$\begin{array}{l}\text { Center } \\
\text { Number }\end{array}$} & \multirow{2}{*}{$\begin{array}{l}\text { Atomic } \\
\text { Number }\end{array}$} & & \multicolumn{2}{|c|}{ Coordinates (Angstroms) } \\
\hline & & & $\begin{array}{ll}X & Y\end{array}$ & Z \\
\hline 1 & 8 & 0.049552 & -1.098511 & 0.000016 \\
\hline 2 & 6 & -0.054000 & 1.206607 & 0.000029 \\
\hline 3 & 6 & -1.320880 & 0.780974 & -0.000060 \\
\hline 4 & 6 & -1.335119 & -0.717223 & 0.000044 \\
\hline 5 & 1 & -2.224887 & 1.380159 & 0.000061 \\
\hline 6 & 1 & -1.820611 & -1.138815 & 0.889886 \\
\hline 7 & 6 & 0.837891 & 0.027409 & -0.000176 \\
\hline 8 & 8 & 2.047461 & -0.040296 & 0.000063 \\
\hline 9 & 1 & -1.820577 & -1.138835 & -0.889798 \\
\hline 10 & 1 & 0.322615 & $5 \quad 2.221336$ & 0.000202 \\
\hline
\end{tabular}

in DMSO B3LYP/6-31G(d)

$\mathrm{HF}=-305.2729752$ hartrees $(-191561.844667752 \mathrm{kcal} / \mathrm{mol})$

Imaginary Frequencies: none found

Zero-point correction $=0.074752$ (Hartree/Particle)

Thermal correction to Energy=

0.079385 


\begin{tabular}{|c|c|c|c|c|c|}
\hline \multicolumn{6}{|l|}{ Ther } \\
\hline \multirow{6}{*}{\multicolumn{5}{|c|}{$\begin{array}{l}\text { Thermal correction to Gibbs Free Energy= } \\
\text { Sum of electronic and zero-point Energies= } \\
\text { Sum of electronic and thermal Energies= } \\
\text { Sum of electronic and thermal Enthalpies= } \\
\text { Sum of electronic and thermal Free Energies= } \\
\text { Coordinates (from last standard orientation): }\end{array}$}} & 0.0468 \\
\hline & & & & & \\
\hline & & & & & \\
\hline & & & & & \\
\hline & & & & & \\
\hline & & & & & \\
\hline \multirow{2}{*}{$\begin{array}{l}\text { Center } \\
\text { Number }\end{array}$} & \multirow{2}{*}{$\begin{array}{l}\text { Atomic } \\
\text { Numbel }\end{array}$} & & \multicolumn{3}{|c|}{ Coordinates (Angstroms) } \\
\hline & & & $\mathrm{Y}$ & 7 & \\
\hline 1 & 8 & -0.049688 & -1.098418 & & \\
\hline 2 & 6 & 0.054081 & 1.206652 & -0.0 & 0060 \\
\hline 3 & 6 & 1.320929 & 0.780856 & & 0049 \\
\hline 4 & 6 & 1.335109 & -0.717312 & & 0000 \\
\hline 5 & 1 & 2.225020 & 1.379921 & -0.0 & 0047 \\
\hline 6 & 1 & 1.820550 & -1.139019 & -0.8 & 9817 \\
\hline 7 & 6 & -0.837855 & 0.027548 & 0.0 & 0207 \\
\hline 8 & 8 & -2.047458 & -0.040315 & & 0038 \\
\hline 9 & 1 & 1.820343 & -1.138960 & & 9947 \\
\hline 10 & 1 & -0.322331 & 2.221461 & & 0219 \\
\hline
\end{tabular}

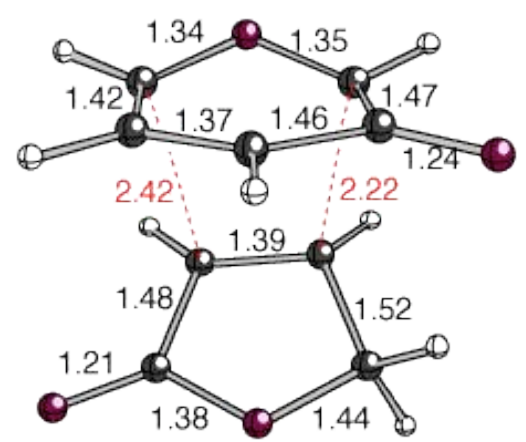

\section{B3LYP/6-31G(d)}

$\mathrm{HF}=-648.5648665$ hartrees $(-406980.939377415 \mathrm{kcal} / \mathrm{mol})$

Imaginary Frequencies: $1(-392.08081 / \mathrm{cm})$

Zero-point correction $=0.157139($ Hartree/Particle $)$

Thermal correction to Energy $=\quad 0.167185$

Thermal correction to Enthalpy $=\quad 0.168129$

Thermal correction to Gibbs Free Energy $=\quad 0.121212$

Sum of electronic and zero-point Energies $=\quad-648.407727$

Sum of electronic and thermal Energies $=\quad-648.397682$

Sum of electronic and thermal Enthalpies $=\quad-648.396738$

Sum of electronic and thermal Free Energies $=\quad-648.443655$

Coordinates (from last standard orientation):

\begin{tabular}{|c|c|c|c|c|}
\hline \multirow{2}{*}{$\begin{array}{l}\text { Center } \\
\text { Number }\end{array}$} & \multirow{2}{*}{$\begin{array}{l}\text { Atomic } \\
\text { Number }\end{array}$} & & \multicolumn{2}{|c|}{ Coordinates (Angstroms) } \\
\hline & & & $\begin{array}{ll}X \quad Y\end{array}$ & Z \\
\hline 1 & 8 & 1.290601 & -1.317247 & 1.120750 \\
\hline 2 & 8 & 2.295899 & 1.585773 & -0.688271 \\
\hline 3 & 6 & 1.615231 & -0.028154 & 0.892823 \\
\hline 4 & 8 & -1.629325 & 1.221011 & -0.584768 \\
\hline 5 & 6 & 1.771244 & 0.486949 & -0.473102 \\
\hline 6 & 6 & 1.158727 & -0.367701 & -1.482024 \\
\hline
\end{tabular}




$\begin{array}{ccccc}7 & 6 & 0.476016 & -1.491367 & -1.104394 \\ 8 & 1 & -0.081590 & -2.088418 & -1.819055 \\ 9 & 6 & 0.446789 & -1.887117 & 0.253936 \\ 10 & 6 & -1.227746 & -0.345133 & 1.061299 \\ 11 & 1 & -1.529255 & -1.136882 & 1.732642 \\ 12 & 6 & -0.410931 & 0.738678 & 1.356776 \\ 13 & 6 & -0.767522 & 1.841582 & 0.382378 \\ 14 & 1 & 2.238781 & 0.381445 & 1.680313 \\ 15 & 1 & 0.100757 & -2.858900 & 0.582338 \\ 16 & 1 & 1.232508 & -0.062235 & -2.520807 \\ 17 & 1 & -0.066999 & 0.982069 & 2.355890 \\ 18 & 1 & -1.325462 & 2.638631 & 0.891376 \\ 19 & 6 & -2.010849 & -0.029645 & -0.153504 \\ 20 & 8 & -2.826665 & -0.696165 & -0.743403 \\ 21 & 1 & 0.081442 & 2.288776 & -0.142285 \\ -----------------------------------------------------\end{array}$

\section{B3LYP/6-31+G(d,p)}

$\mathrm{HF}=-648.6050427$ hartrees $(-407006.150344677 \mathrm{kcal} / \mathrm{mol})$ Imaginary Frequencies: $1(-399.72871 / \mathrm{cm})$

Zero-point correction $=0.156406$ (Hartree/Particle)

Thermal correction to Energy $=\quad 0.166486$

Thermal correction to Enthalpy $=\quad 0.167430$

Thermal correction to Gibbs Free Energy $=\quad 0.120417$

Sum of electronic and zero-point Energies $=\quad-648.448636$

Sum of electronic and thermal Energies $=\quad-648.438556$

Sum of electronic and thermal Enthalpies $=\quad-648.437612$

Sum of electronic and thermal Free Energies $=\quad-648.484626$

Coordinates (from last standard orientation):

\begin{tabular}{|c|c|c|c|c|}
\hline \multirow{2}{*}{$\begin{array}{l}\text { Center } \\
\text { Number }\end{array}$} & \multirow{2}{*}{$\begin{array}{l}\text { Atomic } \\
\text { Number }\end{array}$} & \multicolumn{3}{|c|}{ Coordinates (Angstroms) } \\
\hline & & & $X \quad Y$ & Z \\
\hline 1 & 8 & 1.293609 & -1.306661 & 1.129537 \\
\hline 2 & 8 & 2.299773 & 1.595954 & -0.679973 \\
\hline 3 & 6 & 1.606213 & -0.010843 & 0.902461 \\
\hline 4 & 8 & -1.660323 & 1.214381 & -0.587533 \\
\hline 5 & 6 & 1.779735 & 0.490488 & -0.465167 \\
\hline 6 & 6 & 1.193927 & -0.372039 & -1.480486 \\
\hline 7 & 6 & 0.505389 & -1.494493 & -1.103790 \\
\hline 8 & 1 & -0.039349 & -2.097448 & -1.823031 \\
\hline 9 & 6 & 0.462548 & -1.886188 & 0.255756 \\
\hline 10 & 6 & -1.224427 & $7 \quad-0.352608$ & 1.047955 \\
\hline 11 & 1 & -1.520723 & $3-1.142768$ & 1.722939 \\
\hline 12 & 6 & -0.410016 & $6 \quad 0.739479$ & 1.338683 \\
\hline 13 & 6 & -0.783631 & 1.841704 & 0.369233 \\
\hline 14 & 1 & 2.219858 & 0.406461 & 1.693333 \\
\hline 15 & 1 & 0.112094 & -2.856171 & 0.583852 \\
\hline 16 & 1 & 1.282885 & -0.073427 & -2.519713 \\
\hline 17 & 1 & -0.076215 & $5 \quad 0.989140$ & 2.339538 \\
\hline 18 & 1 & -1.341764 & 2.633522 & 0.883806 \\
\hline 19 & 6 & -2.024354 & -0.038545 & -0.154290 \\
\hline 20 & 8 & -2.848291 & -0.710425 & -0.733489 \\
\hline 21 & 1 & 0.052757 & 2.292977 & -0.171190 \\
\hline
\end{tabular}




\begin{tabular}{|c|c|c|c|c|}
\hline \multicolumn{5}{|c|}{ MP2/6-31G(d) } \\
\hline \multicolumn{5}{|c|}{$\begin{array}{l}\mathrm{HF}=-644.7804479 \text { hartrees }(-404606.178861729 \mathrm{kc} \\
\text { Imaginary Frequencies: } 1(-326.64051 / \mathrm{cm}) \\
\text { Zero-point correction }=0.158793(\text { Hartree/Particle })\end{array}$} \\
\hline \multicolumn{4}{|c|}{ Thermal correction to Energy $=$} & 0.168809 \\
\hline \multicolumn{4}{|c|}{ Thermal correction to Enthalpy= } & 0.169753 \\
\hline \multicolumn{4}{|c|}{ Thermal correction to Gibbs Free Energy= } & 0.122 \\
\hline \multicolumn{4}{|c|}{$\begin{array}{l}\text { Thermal correction to Gibbs Free Energy= } \\
\text { Sum of electronic and zero-point Energies= }\end{array}$} & \\
\hline \multicolumn{5}{|c|}{ Sum of electronic and thermal Energies $=$} \\
\hline \multicolumn{5}{|c|}{ Sum of electronic and thermal Enthalpies= } \\
\hline \multirow{2}{*}{\multicolumn{5}{|c|}{$\begin{array}{l}\text { Sum of electronic and thermal Free Energies= } \\
\text { Coordinates (from last standard orientation): }\end{array}$}} \\
\hline & & & & \\
\hline \multirow{2}{*}{$\begin{array}{l}\text { Center } \\
\text { Number }\end{array}$} & \multirow{2}{*}{$\begin{array}{l}\text { Atomic } \\
\text { Number }\end{array}$} & \multicolumn{3}{|c|}{ Coordinates (Angstroms) } \\
\hline & & & $X \quad Y$ & Z \\
\hline 1 & 8 & 1.255008 & -1.403201 & 1.056166 \\
\hline 2 & 8 & 2.311084 & 16093 & -0.702734 \\
\hline 3 & 6 & 1.652931 & 7589 & 3881 \\
\hline 4 & 8 & -1.522627 & 1.281099 & 41229 \\
\hline 5 & 6 & 1.733564 & 0.431224 & -0.504890 \\
\hline 6 & 6 & 1.002120 & -0.333047 & -1.498419 \\
\hline 7 & 6 & 0.305607 & -1.454656 & -1.124244 \\
\hline 8 & 1 & -0.324996 & -1.989867 & -1.829047 \\
\hline 9 & 6 & 0.319301 & -1.896297 & 0.217063 \\
\hline 10 & 6 & -1.189893 & -0.313676 & 1.095931 \\
\hline 11 & 1 & -1.529215 & -1.094898 & $1.7647 \xi$ \\
\hline 12 & 6 & -0.333553 & 0.727072 & 1.39626 \\
\hline 13 & 6 & -0.643908 & 1.856342 & 0.445941 \\
\hline 14 & 1 & 2.340859 & 0.212534 & 1.609769 \\
\hline 15 & 1 & -0.032317 & -2.873342 & 0.530377 \\
\hline 16 & 1 & 1.027721 & 0.007223 & -2.530142 \\
\hline 17 & 1 & 0.055647 & 0.928242 & 2.390180 \\
\hline 18 & 1 & -1.183100 & 2.656776 & 0.966914 \\
\hline 19 & 6 & -1.951844 & 0.041053 & -0.12155 \\
\hline 20 & 8 & -2.785764 & -0.600738 & \\
\hline 21 & 1 & 0.217844 & 2.284757 & -0.07358 \\
\hline
\end{tabular}

in $\mathrm{H}_{2} \mathrm{O}$ B3LYP/6-31G(d)

$\mathrm{HF}=-648.5929389 \mathrm{hartrees}(-406998.555089139 \mathrm{kcal} / \mathrm{mol})$ Imaginary Frequencies: $1(-412.45181 / \mathrm{cm})$

Zero-point correction $=0.155083$ (Hartree/Particle)

Thermal correction to Energy $=\quad 0.165154$

Thermal correction to Enthalpy= $\quad 0.166099$

Thermal correction to Gibbs Free Energy $=0.119065$

Sum of electronic and zero-point Energies $=\quad-648.437856$

Sum of electronic and thermal Energies $=\quad-648.427785$

Sum of electronic and thermal Enthalpies $=\quad-648.426840$

Sum of electronic and thermal Free Energies $=\quad-648.473874$

Coordinates (from last standard orientation):

\begin{tabular}{lllll} 
Center & Atomic & & \multicolumn{2}{c}{ Coordinates (Angstroms) } \\
Number & Number & $X$ & $Y$ & $Z$ \\
\hline 1 & 8 & 1.329878 & -1.253151 & 1.146993
\end{tabular}




$\begin{array}{ccrrr}2 & 8 & 2.236840 & 1.645231 & -0.721218 \\ 3 & 6 & 1.566273 & 0.058757 & 0.899401 \\ 4 & 8 & -1.666087 & 1.187009 & -0.587060 \\ 5 & 6 & 1.739819 & 0.527615 & -0.478567 \\ 6 & 6 & 1.194815 & -0.372006 & -1.475623 \\ 7 & 6 & 0.563016 & -1.523906 & -1.082283 \\ 8 & 1 & 0.061002 & -2.174996 & -1.795277 \\ 9 & 6 & 0.548780 & -1.898892 & 0.278912 \\ 10 & 6 & -1.220980 & -0.390441 & 1.031515 \\ 11 & 1 & -1.492219 & -1.205735 & 1.691111 \\ 12 & 6 & -0.417947 & 0.710108 & 1.339936 \\ 13 & 6 & -0.824099 & 1.824129 & 0.400978 \\ 14 & 1 & 2.158146 & 0.521406 & 1.694554 \\ 15 & 1 & 0.239534 & -2.879139 & 0.628953 \\ 16 & 1 & 1.277436 & -0.096669 & -2.525028 \\ 17 & 1 & -0.102757 & 0.952185 & 2.355515 \\ 18 & 1 & -1.423834 & 2.578256 & 0.935334 \\ 19 & 6 & -2.012540 & -0.071149 & -0.161893 \\ 20 & 8 & -2.842460 & -0.730167 & -0.761082 \\ 21 & 1 & -0.005494 & 2.328037 & -0.120482 \\ ----------------------------------------------\end{array}$

\section{in $\mathrm{H}_{2} \mathrm{O}$ MP2/6-31G(d)}

$\mathrm{HF}=-644.8143284$ hartrees $(-404627.439214284 \mathrm{kcal} / \mathrm{mol})$ Imaginary Frequencies: $1(-337.08371 / \mathrm{cm})$

Zero-point correction $=0.157077$ (Hartree/Particle)

Thermal correction to Energy $=\quad 0.167023$

Thermal correction to Enthalpy $=\quad 0.167967$

Thermal correction to Gibbs Free Energy $=\quad 0.121372$

Sum of electronic and zero-point Energies $=\quad-646.545359$

Sum of electronic and thermal Energies $=\quad-646.535413$

Sum of electronic and thermal Enthalpies $=\quad-646.534469$

Sum of electronic and thermal Free Energies $=\quad-646.581064$

Coordinates (from last standard orientation):

\begin{tabular}{ccrrr} 
Center & Atomic & \multicolumn{3}{c}{ Coordinates (Angstroms) } \\
Number & Number & $X$ & $Y$ & $Z$ \\
- \hdashline 1 & 8 & -1.306372 & 1.335174 & 1.083884 \\
2 & 8 & -2.235237 & -1.579468 & -0.750867 \\
3 & 6 & -1.616473 & 0.044709 & 0.848667 \\
4 & 8 & 1.549778 & -1.251185 & -0.538637 \\
5 & 6 & -1.695382 & -0.472258 & -0.519390 \\
6 & 6 & -1.025138 & 0.349877 & -1.497237 \\
7 & 6 & -0.377626 & 1.496287 & -1.095967 \\
8 & 1 & 0.206771 & 2.092783 & -1.796402 \\
9 & 6 & -0.415231 & 1.908312 & 0.249281 \\
10 & 6 & 1.179842 & 0.363038 & 1.067834 \\
11 & 1 & 1.500016 & 1.165491 & 1.725461 \\
12 & 6 & 0.344488 & -0.696941 & 1.387653 \\
13 & 6 & 0.699354 & -1.842214 & 0.476165 \\
14 & 1 & -2.278183 & -0.369496 & 1.621141 \\
15 & 1 & -0.101230 & 2.892103 & 0.591785 \\
16 & 1 & -1.050947 & 0.048209 & -2.544060 \\
17 & 1 & -0.017125 & -0.891790 & 2.395696 \\
18 & 1 & 1.285767 & -2.599348 & 1.020018
\end{tabular}




$\begin{array}{rrrrr}19 & 6 & 1.945821 & -0.000940 & -0.133815 \\ 20 & 8 & 2.786277 & 0.631906 & -0.754594 \\ 21 & 1 & -0.138571 & -2.328582 & -0.031076\end{array}$

\begin{tabular}{|c|c|c|c|c|}
\hline \multicolumn{5}{|c|}{$\begin{array}{l}\mathrm{HF}=-648.579368 \text { hartrees }(-406990.03921368 \mathrm{kcal} / \mathrm{m} \\
\text { Imaginary Frequencies: } 1(-405.26761 / \mathrm{cm}) \\
\text { Zero-point correction }=0.156764(\text { Hartree } / \text { Particle })\end{array}$} \\
\hline \multicolumn{4}{|c|}{ Thermal correction to Energy $=$} & 0.166811 \\
\hline \multicolumn{4}{|c|}{ Thermal correction to Enthalpy= } & 0.167755 \\
\hline \multicolumn{4}{|c|}{$\begin{array}{l}\text { Thermal correction to Enthalpy= } \\
\text { Thermal correction to Gibbs Free Energy= }\end{array}$} & \\
\hline \multicolumn{5}{|c|}{$\begin{array}{l}\text { Thermal correction to Gibbs Free Energy= } \\
\text { Sum of electronic and zero-point Energies= }\end{array}$} \\
\hline \multicolumn{5}{|c|}{ Sum of electronic and thermal Energies= } \\
\hline \multicolumn{5}{|c|}{ Sum of electronic and thermal Enthalpies= } \\
\hline \multirow{2}{*}{\multicolumn{5}{|c|}{$\begin{array}{l}\text { Sum of electronic and thermal Free Energies= } \\
\text { Coordinates (from last standard orientation): }\end{array}$}} \\
\hline & & & & \\
\hline \multirow{2}{*}{$\begin{array}{l}\text { Center } \\
\text { Number }\end{array}$} & \multirow{2}{*}{$\begin{array}{l}\text { Atomic } \\
\text { Number }\end{array}$} & \multicolumn{3}{|c|}{ Coordinates (Angstroms) } \\
\hline & & & $X \quad Y$ & \\
\hline 1 & 8 & 31272 & -1.258879 & \\
\hline 2 & 8 & 24524 & & 580 \\
\hline 3 & 6 & 78750 & & 530 \\
\hline 4 & 8 & 7904 & & 569 \\
\hline 5 & 6 & 88 & & \\
\hline 6 & & & & \\
\hline 7 & & & & \\
\hline 8 & & & & \\
\hline 9 & 6 & 0.539665 & 6343 & 8354 \\
\hline 10 & 6 & -1.223383 & 87212 & 1.038786 \\
\hline 11 & 1 & -1.501818 & & 1.698775 \\
\hline 12 & 6 & 424673 & & 1.350245 \\
\hline 13 & 6 & & & \\
\hline 14 & 1 & & & \\
\hline 15 & 1 & & & \\
\hline 16 & 1 & 3799 & & -2 \\
\hline 17 & 1 & -0.107251 & & 2.360455 \\
\hline 18 & 1 & -1.405155 & 2.587657 & 0.926984 \\
\hline 19 & 6 & -2.010026 & -0.069276 & -0.163632 \\
\hline 20 & 8 & -2.829630 & -0.731611 & \\
\hline 21 & 1 & 0.014448 & 2.310628 & -0.114273 \\
\hline
\end{tabular}

in DMSO B3LYP/6-31G(d)

$\mathrm{HF}=-648.5793834$ hartrees $(-406990.048877334 \mathrm{kcal} / \mathrm{mol})$ Imaginary Frequencies: $1(-405.25481 / \mathrm{cm})$

Zero-point correction $=0.156761$ (Hartree/Particle)

Thermal correction to Energy $=\quad 0.166810$

Thermal correction to Enthalpy $=\quad 0.167754$

Thermal correction to Gibbs Free Energy $=\quad 0.120768$

Sum of electronic and zero-point Energies= $\quad-648.422622$

Sum of electronic and thermal Energies $=\quad-648.412573$

Sum of electronic and thermal Enthalpies= $\quad-648.411629$

Sum of electronic and thermal Free Energies $=\quad-648.458615$

Coordinates (from last standard orientation): 


\begin{tabular}{|c|c|c|c|c|}
\hline \multirow{2}{*}{$\begin{array}{l}\text { Center } \\
\text { Number }\end{array}$} & \multirow{2}{*}{$\begin{array}{l}\text { Atomic } \\
\text { Number }\end{array}$} & \multicolumn{3}{|c|}{ Coordinates (Angstroms) } \\
\hline & & & $Y$ & $Z$ \\
\hline 1 & 8 & 1.330636 & -1.260619 & 1.142019 \\
\hline 2 & 8 & 2.227097 & 1.646550 & -0.718492 \\
\hline 3 & 6 & 1.579421 & 0.045904 & 0.895815 \\
\hline 4 & 8 & -1.658214 & 1.192652 & -0.583255 \\
\hline 5 & 6 & 1.740044 & 0.528356 & -0.480775 \\
\hline 6 & 6 & 1.186780 & -0.370764 & -1.477961 \\
\hline 7 & 6 & 0.553595 & -1.520493 & -1.085404 \\
\hline 8 & 1 & 0.046254 & -2.165762 & -1.796523 \\
\hline 9 & 6 & 0.538608 & -1.896486 & 0.276557 \\
\hline 10 & 6 & -1.223416 & -0.387453 & 1.038183 \\
\hline 11 & 1 & -1.501885 & -1.197550 & 1.698052 \\
\hline 12 & 6 & -0.424243 & 0.711040 & 1.349853 \\
\hline 13 & 6 & -0.816011 & 1.823605 & 0.402155 \\
\hline 14 & 1 & 2.175109 & 0.499108 & 1.683205 \\
\hline 15 & 1 & 0.230753 & -2.875604 & 0.623483 \\
\hline 16 & 1 & 1.264709 & -0.090835 & -2.524352 \\
\hline 17 & 1 & -0.106685 & 0.949990 & 2.360095 \\
\hline 18 & 1 & -1.403311 & 2.587889 & 0.927330 \\
\hline 19 & 6 & -2.011069 & -0.068492 & -0.163351 \\
\hline 20 & 8 & -2.832348 & -0.729618 & -0.763647 \\
\hline 21 & 1 & 0.015448 & 2.309745 & -0.114720 \\
\hline
\end{tabular}

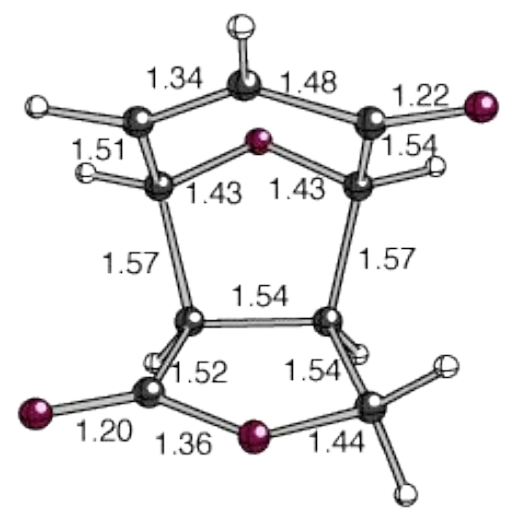

B3LYP/6-31G(d)

$\mathrm{HF}=-648.6323456$ hartrees $(-407023.283187456 \mathrm{kcal} / \mathrm{mol})$ Imaginary Frequencies: none found

Zero-point correction $=0.162112($ Hartree/Particle $)$

Thermal correction to Energy $=\quad 0.171443$

Thermal correction to Enthalpy= $\quad 0.172387$

Thermal correction to Gibbs Free Energy $=\quad 0.126884$

Sum of electronic and zero-point Energies $=\quad-648.470233$

Sum of electronic and thermal Energies $=\quad-648.460903$

Sum of electronic and thermal Enthalpies $=\quad-648.459958$

Sum of electronic and thermal Free Energies $=\quad-648.505462$

Coordinates (from last standard orientation):

\begin{tabular}{llll} 
Center & Atomic & \multicolumn{3}{c}{ Coordinates (Angstroms) } \\
Number Number & $X$ & $Y$
\end{tabular}




$\begin{array}{ccrrr}1 & 8 & 1.160140 & -1.189322 & 1.290429 \\ 2 & 8 & 2.427018 & 1.228754 & -0.936698 \\ 3 & 6 & 1.329622 & 0.185064 & 0.937311 \\ 4 & 8 & -1.575030 & 1.326424 & -0.585726 \\ 5 & 6 & 1.744317 & 0.298359 & -0.537359 \\ 6 & 6 & 1.179689 & -0.746616 & -1.416926 \\ 7 & 6 & 0.297518 & -1.621343 & -0.904188 \\ 8 & 1 & -0.227359 & -2.341596 & -1.525904 \\ 9 & 6 & -0.017593 & -1.568433 & 0.575907 \\ 10 & 6 & -1.021077 & -0.406048 & 0.923334 \\ 11 & 1 & -1.653101 & -0.697689 & 1.765526 \\ 12 & 6 & -0.089459 & 0.783956 & 1.221922 \\ 13 & 6 & -0.619335 & 1.917718 & 0.320389 \\ 14 & 1 & 2.111699 & 0.616480 & 1.564258 \\ 15 & 1 & -0.348052 & -2.536552 & 0.955024 \\ 16 & 1 & 1.460253 & -0.727960 & -2.465895 \\ 17 & 1 & -0.123001 & 1.073874 & 2.274166 \\ 18 & 1 & -1.140415 & 2.684345 & 0.901504 \\ 19 & 6 & -1.892131 & 0.050410 & -0.241548 \\ 20 & 8 & -2.725111 & -0.594256 & -0.825407 \\ 21 & 1 & 0.154528 & 2.397910 & -0.282513 \\ -----------------------------------------------\end{array}$

\section{B3LYP/6-31+G(d,p)}

$\mathrm{HF}=-648.6705294$ hartrees $(-407047.243903794 \mathrm{kcal} / \mathrm{mol})$ Imaginary Frequencies: none found Zero-point correction $=0.161339($ Hartree/Particle $)$ Thermal correction to Energy $=\quad 0.170701$ Thermal correction to Enthalpy $=\quad 0.171645$ Thermal correction to Gibbs Free Energy $=0.126085$ Sum of electronic and zero-point Energies $=\quad-648.509191$ Sum of electronic and thermal Energies $=\quad-648.499828$ Sum of electronic and thermal Enthalpies $=\quad-648.498884$ Sum of electronic and thermal Free Energies $=\quad-648.544444$ Coordinates (from last standard orientation):

\begin{tabular}{|c|c|c|c|c|}
\hline \multirow{2}{*}{$\begin{array}{l}\text { Center } \\
\text { Number }\end{array}$} & \multirow{2}{*}{$\begin{array}{l}\text { Atomic } \\
\text { Number }\end{array}$} & \multicolumn{3}{|c|}{ Coordinates (Angstroms) } \\
\hline & & & $\begin{array}{ll}X & Y\end{array}$ & Z \\
\hline 1 & 8 & 1.148988 & -1.185578 & 1.296445 \\
\hline 2 & 8 & 2.481036 & 1.214369 & -0.904544 \\
\hline 3 & 6 & 1.328251 & 0.191275 & 0.945553 \\
\hline 4 & 8 & -1.614876 & 1.323284 & -0.587308 \\
\hline 5 & 6 & 1.772501 & 0.294234 & -0.519676 \\
\hline 6 & 6 & 1.214007 & -0.742188 & -1.411452 \\
\hline 7 & 6 & 0.315705 & -1.610703 & -0.912085 \\
\hline 8 & 1 & -0.201809 & -2.327605 & -1.543368 \\
\hline 9 & 6 & -0.020214 & -1.565440 & 0.563164 \\
\hline 10 & 6 & -1.025111 & -0.405236 & 0.910262 \\
\hline 11 & 1 & -1.647899 & -0.699224 & 1.758143 \\
\hline 12 & 6 & -0.096497 & 0.790964 & 1.201518 \\
\hline 13 & 6 & -0.623211 & 1.917122 & 0.287212 \\
\hline 14 & 1 & 2.099478 & 0.620640 & 1.586359 \\
\hline 15 & 1 & -0.354343 & -2.535387 & 0.932569 \\
\hline 16 & 1 & 1.511516 & -0.726665 & -2.455328 \\
\hline 17 & 1 & -0.141694 & 1.092166 & 2.249764 \\
\hline
\end{tabular}




$\begin{array}{rrrrr}18 & 1 & -1.121999 & 2.703349 & 0.859613 \\ 19 & 6 & -1.916032 & 0.046703 & -0.238841 \\ 20 & 8 & -2.762779 & -0.601864 & -0.803968 \\ 21 & 1 & 0.141403 & 2.370640 & -0.346683 \\ - & 1 & -\end{array}$

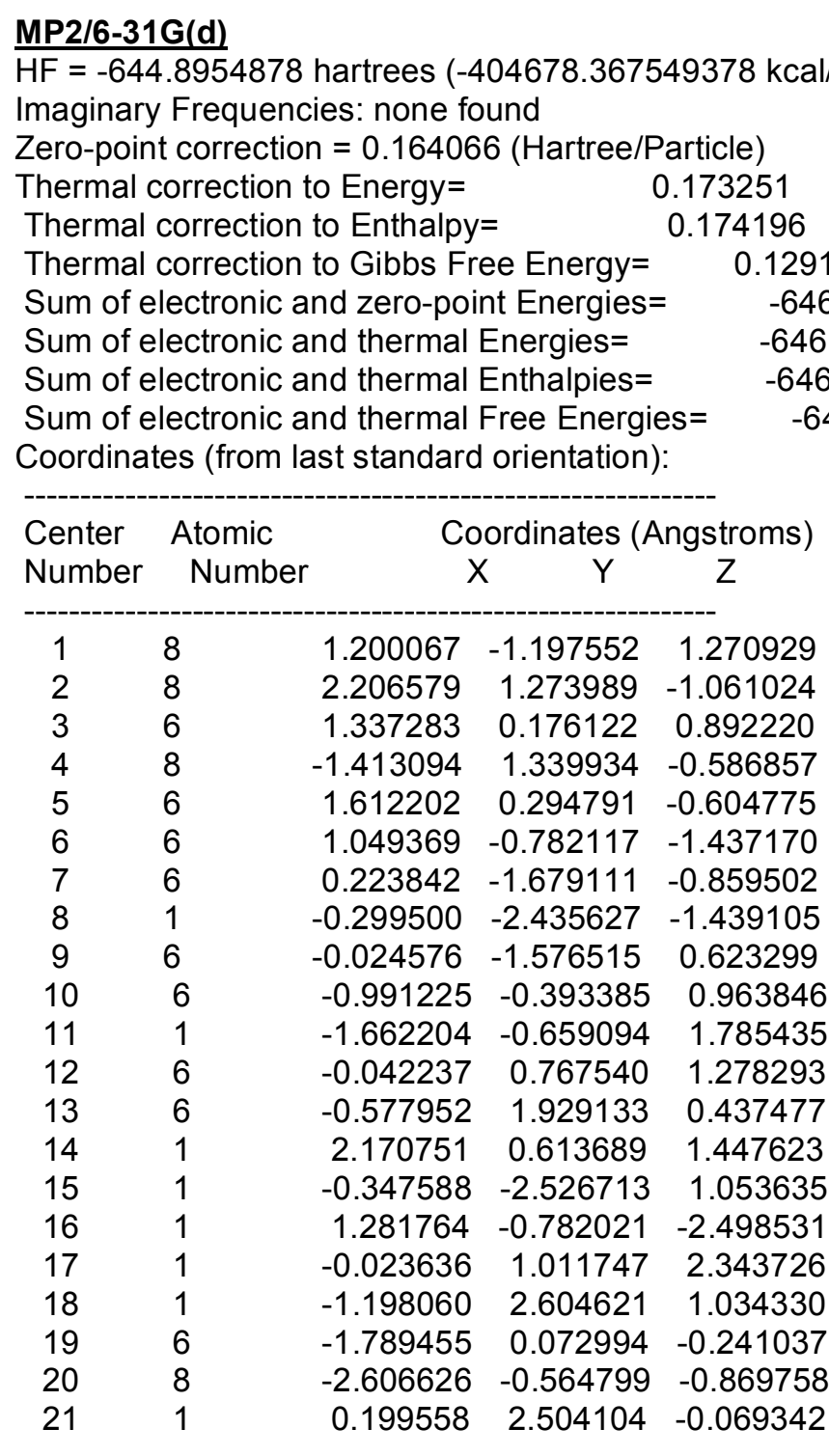

in $\mathrm{H}_{2} \mathrm{O} \mathrm{B} 3 \mathrm{LYP} / 6-31 \mathrm{G}(\mathrm{d})$

$\mathrm{HF}=-648.6633368$ hartrees $(-407042.730475368 \mathrm{kcal} / \mathrm{mol})$ Imaginary Frequencies: none found Zero-point correction $=0.156799($ Hartree/Particle $)$

Thermal correction to Energy= $\quad 0.166210$ Thermal correction to Enthalpy $=\quad 0.167154$ Thermal correction to Gibbs Free Energy $=\quad 0.121413$ Sum of electronic and zero-point Energies= $\quad-648.506538$ Sum of electronic and thermal Energies $=\quad-648.497127$ Sum of electronic and thermal Enthalpies= $\quad-648.496182$ Sum of electronic and thermal Free Energies $=\quad-648.541924$ 
Coordinates (from last standard orientation):

\begin{tabular}{|c|c|c|c|c|}
\hline Center & Atomic & & ordinates ( & ngstroms) \\
\hline Number & Number & & $\mathrm{Y}$ & Z \\
\hline 1 & 8 & 1.160114 & -1.179000 & 1.293748 \\
\hline 2 & 8 & 2.446898 & 1.221476 & -0.938266 \\
\hline 3 & 6 & 1.327396 & 0.199645 & 0.939466 \\
\hline 4 & 8 & -1.583970 & 1.314075 & -0.597185 \\
\hline 5 & 6 & 1.749344 & 0.296959 & -0.530112 \\
\hline 6 & 6 & 1.194280 & -0.748449 & -1.409408 \\
\hline 7 & 6 & 0.306788 & -1.621625 & -0.897312 \\
\hline 8 & 1 & -0.203424 & -2.352448 & -1.522715 \\
\hline 9 & 6 & -0.019531 & -1.571961 & 0.578682 \\
\hline 10 & 6 & -1.023699 & -0.406698 & 0.919351 \\
\hline 11 & 1 & -1.659334 & -0.696527 & 1.766892 \\
\hline 12 & 6 & -0.096041 & 0.789453 & 1.215144 \\
\hline 13 & 6 & -0.625541 & 1.918188 & 0.314758 \\
\hline 14 & 1 & 2.109239 & 0.643289 & 1.578230 \\
\hline 15 & 1 & -0.357577 & -2.566018 & 0.966975 \\
\hline 16 & 1 & 1.486820 & -0.742938 & -2.458031 \\
\hline 17 & 1 & -0.134402 & 1.088118 & 2.292451 \\
\hline 18 & 1 & -1.159133 & 2.679715 & 0.890974 \\
\hline 19 & 6 & -1.893647 & 0.045963 & -0.238625 \\
\hline 20 & 8 & -2.740122 & -0.590037 & -0.829597 \\
\hline 21 & 1 & 0.138357 & 2.405842 & -0.296046 \\
\hline
\end{tabular}

\section{in $\mathrm{H}_{2} \mathrm{O} M P 2 / 6-31 \mathrm{G}(\mathrm{d})$}

$\mathrm{HF}=-644.9314353$ hartrees $(-404700.924965103 \mathrm{kcal} / \mathrm{mol})$ Imaginary Frequencies: none found

Zero-point correction $=0.158746($ Hartree/Particle $)$

Thermal correction to Energy $=\quad 0.167799$

Thermal correction to Enthalpy= $\quad 0.168743$

Thermal correction to Gibbs Free Energy $=\quad 0.124447$

Sum of electronic and zero-point Energies= $\quad-646.621669$

Sum of electronic and thermal Energies $=\quad-646.612615$

Sum of electronic and thermal Enthalpies $=\quad-646.611671$

Sum of electronic and thermal Free Energies $=\quad-646.655967$

Coordinates (from last standard orientation):

\begin{tabular}{|c|c|c|c|c|}
\hline Center & Atomic & & ordinates (A & ngstroms) \\
\hline Number & Number & & $\quad \mathrm{Y}$ & Z \\
\hline 1 & 8 & 1.233752 & -1.140155 & 1.288639 \\
\hline 2 & 8 & 2.146691 & 1.305481 & -1.108065 \\
\hline 3 & 6 & 1.329792 & 0.233721 & 0.884125 \\
\hline 4 & 8 & -1.434510 & 1.304402 & -0.603428 \\
\hline 5 & 6 & 1.588295 & 0.318688 & -0.614628 \\
\hline 6 & 6 & 1.070130 & -0.798633 & -1.420203 \\
\hline 7 & 6 & 0.271916 & -1.706833 & -0.819586 \\
\hline 8 & 1 & -0.211222 & -2.505745 & -1.381317 \\
\hline 9 & 6 & 0.011166 & -1.575342 & 0.657636 \\
\hline 10 & 6 & -0.982657 & -0.404142 & 0.964681 \\
\hline 11 & 1 & -1.665930 & -0.669903 & 1.784653 \\
\hline 12 & 6 & -0.063770 & 0.783950 & 1.275081 \\
\hline 13 & 6 & -0.632210 & 1.928158 & 0.442325 \\
\hline 14 & 1 & 2.161060 & 0.715251 & 1.432061 \\
\hline
\end{tabular}




$\begin{array}{rrrrr}15 & 1 & -0.295677 & -2.547868 & 1.128508 \\ 16 & 1 & 1.314523 & -0.828136 & -2.481572 \\ 17 & 1 & -0.044045 & 1.032442 & 2.370557 \\ 18 & 1 & -1.296126 & 2.567330 & 1.032180 \\ 19 & 6 & -1.770497 & 0.033350 & -0.249856 \\ 20 & 8 & -2.572865 & -0.617847 & -0.895449 \\ 21 & 1 & 0.119881 & 2.544095 & -0.056097\end{array}$

\section{in $\mathrm{CH}_{3} \mathrm{CN}$ B3LYP/6-31G(d)}

$\mathrm{HF}=-648.6453177 \mathrm{hartrees}(-407031.423309927 \mathrm{kcal} / \mathrm{mol})$ Imaginary Frequencies: none found

Zero-point correction $=0.161393$ (Hartree/Particle)

Thermal correction to Energy $=\quad 0.170741$

Thermal correction to Enthalpy= $\quad 0.171685$

Thermal correction to Gibbs Free Energy $=\quad 0.126104$

Sum of electronic and zero-point Energies $=\quad-648.483925$

Sum of electronic and thermal Energies $=\quad-648.474577$

Sum of electronic and thermal Enthalpies $=\quad-648.473633$

Sum of electronic and thermal Free Energies $=\quad-648.519214$

Coordinates (from last standard orientation):

\begin{tabular}{|c|c|c|c|c|}
\hline \multirow{2}{*}{$\begin{array}{l}\text { Center } \\
\text { Number }\end{array}$} & \multirow{2}{*}{$\begin{array}{l}\text { Atomic } \\
\text { Number }\end{array}$} & \multicolumn{3}{|c|}{ Coordinates (Angstroms) } \\
\hline & & & $X \quad Y$ & Z \\
\hline 1 & 8 & 1.159400 & -1.186045 & 1.289621 \\
\hline 2 & 8 & 2.444467 & 1.226613 & -0.930041 \\
\hline 3 & 6 & 1.327327 & 0.191259 & 0.941031 \\
\hline 4 & 8 & -1.584201 & 1.319323 & -0.590123 \\
\hline 5 & 6 & 1.750605 & 0.300297 & -0.529980 \\
\hline 6 & 6 & 1.192503 & -0.742953 & -1.413016 \\
\hline 7 & 6 & 0.306524 & -1.618309 & -0.905164 \\
\hline 8 & 1 & -0.203476 & -2.346884 & -1.530485 \\
\hline 9 & 6 & -0.017527 & -1.571499 & 0.572681 \\
\hline 10 & 6 & -1.023231 & -0.409599 & 0.919196 \\
\hline 11 & 1 & -1.652751 & -0.701978 & 1.764117 \\
\hline 12 & 6 & -0.095315 & 0.784210 & 1.217617 \\
\hline 13 & 6 & -0.622850 & 1.915271 & 0.315330 \\
\hline 14 & 1 & 2.102082 & 0.626876 & 1.576892 \\
\hline 15 & 1 & -0.349472 & -2.543190 & 0.950038 \\
\hline 16 & 1 & 1.484428 & -0.731836 & -2.459769 \\
\hline 17 & 1 & -0.133396 & 1.077149 & 2.271293 \\
\hline 18 & 1 & -1.143912 & 2.682623 & 0.894081 \\
\hline 19 & 6 & -1.897037 & 0.047015 & -0.239172 \\
\hline 20 & 8 & -2.741717 & -0.588721 & -0.824856 \\
\hline 21 & 1 & 0.146927 & 2.393722 & -0.294119 \\
\hline
\end{tabular}

\section{in DMSO B3LYP/6-31G(d)}

$\mathrm{HF}=-648.6453922$ hartrees $(-407031.470059422 \mathrm{kcal} / \mathrm{mol})$

Imaginary Frequencies: none found

Zero-point correction $=0.161388$ (Hartree/Particle)

Thermal correction to Energy $=\quad 0.170736$

Thermal correction to Enthalpy $=\quad 0.171680$

Thermal correction to Gibbs Free Energy $=\quad 0.126102$

Sum of electronic and zero-point Energies $=\quad-648.484004$ 
Sum of electronic and thermal Energies=

$-648.474656$

Sum of electronic and thermal Enthalpies=

$-648.473712$

Sum of electronic and thermal Free Energies=

$-648.519290$

Coordinates (from last standard orientation):

\begin{tabular}{llrrr} 
Center & Atomic & \multicolumn{3}{c}{ Coordinates (Angstroms) } \\
Number & Number & X & $Y$ & $Z$ \\
-1 & 8 & 1.160201 & -1.184978 & 1.290098 \\
2 & 8 & 2.442626 & 1.227373 & -0.931597 \\
3 & 6 & 1.327310 & 0.192213 & 0.940672 \\
4 & 8 & -1.584395 & 1.318980 & -0.590090 \\
5 & 6 & 1.749796 & 0.300635 & -0.530608 \\
6 & 6 & 1.192131 & -0.743528 & -1.412818 \\
7 & 6 & 0.307043 & -1.619298 & -0.904097 \\
8 & 1 & -0.202488 & -2.348776 & -1.528753 \\
9 & 6 & -0.016786 & -1.571476 & 0.573738 \\
10 & 6 & -1.022938 & -0.409666 & 0.919374 \\
11 & 1 & -1.652764 & -0.701858 & 1.764146 \\
12 & 6 & -0.095483 & 0.784506 & 1.217730 \\
13 & 6 & -0.623644 & 1.915447 & 0.315759 \\
14 & 1 & 2.102127 & 0.628624 & 1.575939 \\
15 & 1 & -0.348298 & -2.542996 & 0.951991 \\
16 & 1 & 1.483751 & -0.732932 & -2.459666 \\
17 & 1 & -0.133409 & 1.077192 & 2.271511 \\
18 & 1 & -1.145358 & 2.682282 & 0.894595 \\
19 & 6 & -1.896378 & 0.046389 & -0.239447 \\
20 & 8 & -2.740397 & -0.589803 & -0.825672 \\
21 & 1 & 0.145874 & 2.394551 & -0.293495
\end{tabular}

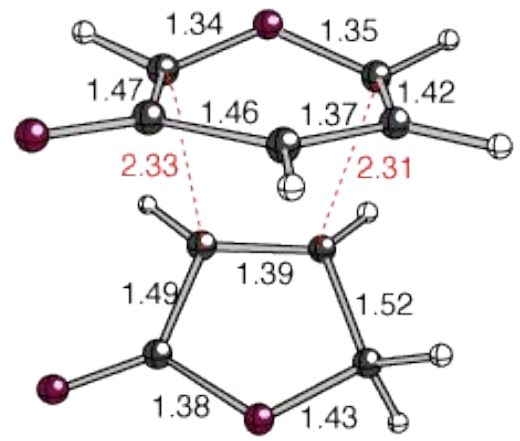

\section{B3LYP/6-31G(d)}

$\mathrm{HF}=-648.555403$ hartrees $(-406975.00093653 \mathrm{kcal} / \mathrm{mol})$ Imaginary Frequencies: $1(-403.73681 / \mathrm{cm})$

Zero-point correction $=0.156597$ (Hartree/Particle)

Thermal correction to Energy=

0.166858

Thermal correction to Enthalpy=

0.167802

Thermal correction to Gibbs Free Energy=

Sum of electronic and zero-point Energies=

0.119903

Sum of electronic and thermal Energies=

$-648.398806$

$-648.388545$

Sum of electronic and thermal Enthalpies=

$-648.387601$

Sum of electronic and thermal Free Energies=

$-648.435500$

Coordinates (from last standard orientation): 


\begin{tabular}{|c|c|c|c|c|}
\hline \multirow{2}{*}{$\begin{array}{l}\text { Center } \\
\text { Number }\end{array}$} & \multirow{2}{*}{$\begin{array}{l}\text { Atomic } \\
\text { Numbe }\end{array}$} & \multicolumn{3}{|c|}{ Coordinates (Angstroms) } \\
\hline & & & $\begin{array}{ll}X & Y\end{array}$ & Z \\
\hline 1 & 8 & 1.655748 & -0.716031 & -1.204770 \\
\hline 2 & 6 & 1.397557 & -1.571491 & -0.196474 \\
\hline 3 & 8 & -2.010740 & -0.113229 & 0.907642 \\
\hline 4 & 6 & 1.433579 & -1.073076 & 1.134253 \\
\hline 5 & 6 & 1.389050 & 0.269087 & 1.376810 \\
\hline 6 & 6 & 1.276032 & 1.214106 & 0.265389 \\
\hline 7 & 6 & 1.254328 & 0.548952 & -1.049040 \\
\hline 8 & 6 & -0.952794 & -0.183684 & -1.150356 \\
\hline 9 & 1 & -0.896361 & 0.092281 & -2.194420 \\
\hline 10 & 6 & -0.870776 & -1.467558 & -0.635067 \\
\hline 11 & 6 & -1.644818 & -1.475954 & 0.667955 \\
\hline 12 & 1 & 1.685705 & -2.586038 & -0.443839 \\
\hline 13 & 1 & 1.382536 & 1.146076 & -1.945494 \\
\hline 14 & 1 & 1.444147 & 0.675886 & 2.381582 \\
\hline 15 & 1 & -0.772750 & -2.366159 & -1.232532 \\
\hline 16 & 1 & -2.558787 & -2.078546 & 0.573858 \\
\hline 17 & 6 & -1.731255 & 0.657206 & -0.204455 \\
\hline 18 & 8 & -2.097569 & 1.797704 & -0.308717 \\
\hline 19 & 1 & -1.087762 & -1.836010 & 1.537496 \\
\hline 20 & 1 & 1.475790 & -1.794571 & 1.945796 \\
\hline 21 & 8 & 1.205319 & 2.436751 & 0.371278 \\
\hline
\end{tabular}

\section{B3LYP/6-31+G(d,p)}

$\mathrm{HF}=-648.5948254$ hartrees $(-406999.738886754 \mathrm{kcal} / \mathrm{mol})$ Imaginary Frequencies: $1(-413.61531 / \mathrm{cm})$

Zero-point correction $=0.155858$ (Hartree/Particle)

Thermal correction to Energy $=\quad 0.166154$

Thermal correction to Enthalpy= $\quad 0.167098$

Thermal correction to Gibbs Free Energy $=0.119096$

Sum of electronic and zero-point Energies $=\quad-648.438967$

Sum of electronic and thermal Energies $=\quad-648.428672$

Sum of electronic and thermal Enthalpies $=\quad-648.427727$

Sum of electronic and thermal Free Energies $=\quad-648.475730$

Coordinates (from last standard orientation):

\begin{tabular}{|c|c|c|c|c|}
\hline \multirow{2}{*}{$\begin{array}{l}\text { Center } \\
\text { Number }\end{array}$} & \multirow{2}{*}{$\begin{array}{l}\text { Atomic } \\
\text { Number }\end{array}$} & \multicolumn{3}{|c|}{ Coordinates (Angstroms) } \\
\hline & & & $X \quad Y$ & Z \\
\hline 1 & 8 & 1.631605 & -0.727592 & -1.224344 \\
\hline 2 & 6 & 1.371565 & -1.587495 & -0.218251 \\
\hline 3 & 8 & -2.037159 & -0.094622 & 0.915564 \\
\hline 4 & 6 & 1.439364 & -1.099886 & 1.116005 \\
\hline 5 & 6 & 1.436347 & 0.242132 & 1.370208 \\
\hline 6 & 6 & 1.325136 & 1.193215 & 0.266128 \\
\hline 7 & 6 & 1.248942 & 0.544400 & -1.053592 \\
\hline 8 & 6 & -0.958085 & -0.155370 & -1.132949 \\
\hline 9 & 1 & -0.908672 & 0.121709 & -2.176886 \\
\hline 10 & 6 & -0.886491 & $1-1.446842$ & -0.623784 \\
\hline 11 & 6 & -1.665317 & $7-1.460712$ & 0.676506 \\
\hline 12 & 1 & 1.634040 & -2.606015 & -0.476283 \\
\hline 13 & 1 & 1.369196 & 1.147269 & -1.947035 \\
\hline 14 & 1 & 1.519228 & 0.640389 & 2.376146 \\
\hline
\end{tabular}




\begin{tabular}{|c|c|c|c|c|}
\hline 15 & 1 & -0.805457 & -2.340088 & -1.231239 \\
\hline 16 & 1 & -2.579276 & -2.060488 & 0.577124 \\
\hline 17 & 6 & -1.751310 & 0.677296 & -0.192964 \\
\hline 18 & 8 & -2.132768 & 1.814555 & -0.304742 \\
\hline 19 & 1 & -1.111968 & -1.816252 & 1.549451 \\
\hline 20 & 1 & 1.474635 & -1.827915 & 1.921464 \\
\hline 21 & 8 & 1.294243 & 2.420279 & 0.383949 \\
\hline
\end{tabular}

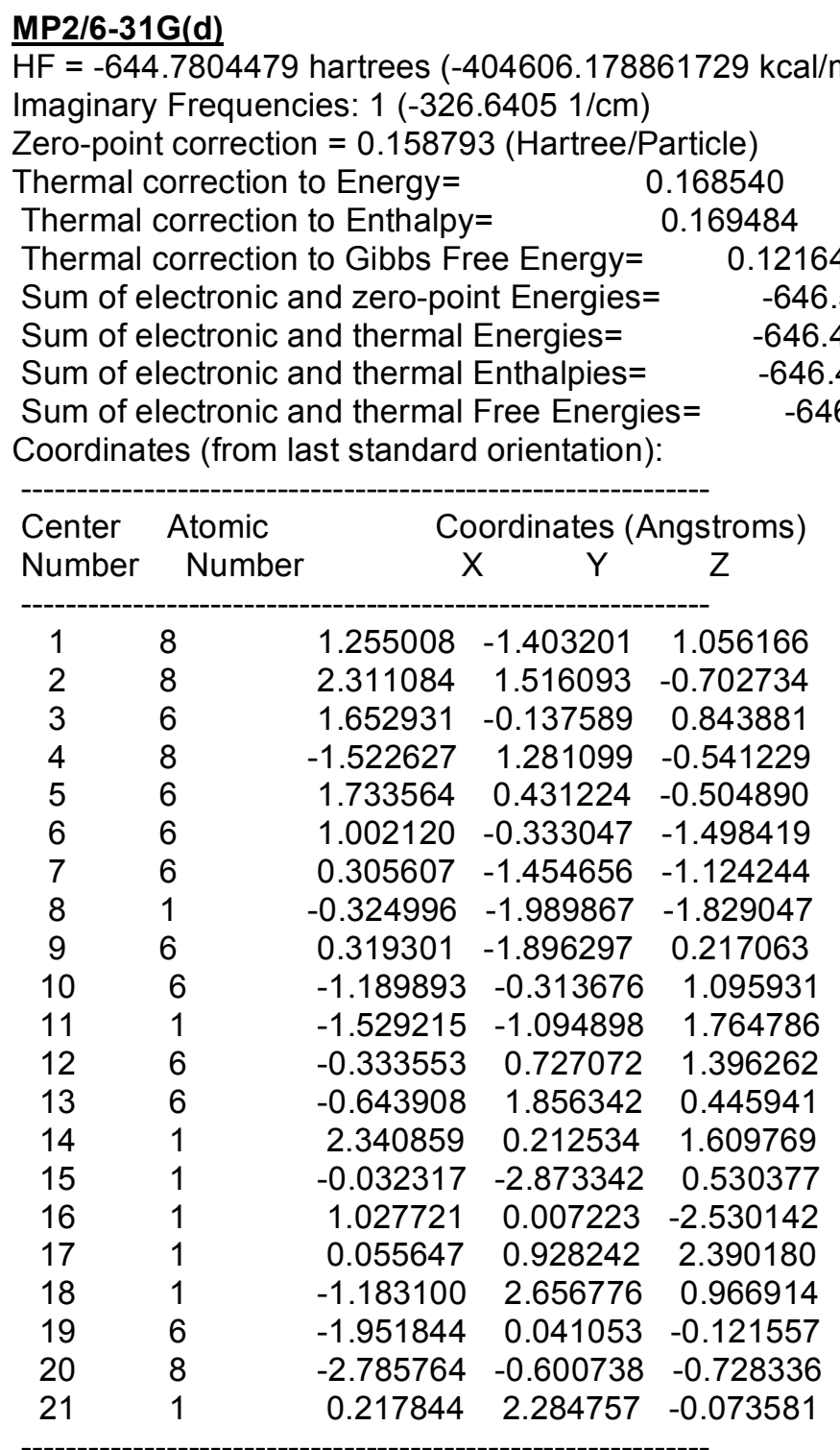

\section{in $\mathrm{H}_{2} \mathrm{O}$ B3LYP/6-31G(d)}

$\mathrm{HF}=-648.5912626$ hartrees $(-406997.503194126 \mathrm{kcal} / \mathrm{mol})$ Imaginary Frequencies: $1(-437.02891 / \mathrm{cm})$

Zero-point correction $=0.154628$ (Hartree/Particle)

Thermal correction to Energy $=\quad 0.164719$

Thermal correction to Enthalpy= $\quad 0.165663$

Thermal correction to Gibbs Free Energy $=0.118516$

Sum of electronic and zero-point Energies $=\quad-648.436635$ 


\begin{tabular}{|c|c|c|c|c|}
\hline & & & & \\
\hline Number & Number & & Y & Z \\
\hline 1 & 8 & 1.629391 & -0.855119 & -1.149020 \\
\hline 2 & 6 & 1.212035 & -1.657914 & -0.140615 \\
\hline 3 & 8 & -2.036664 & 0.002056 & 0.859553 \\
\hline 4 & 6 & 1.267376 & -1.132549 & 1.181261 \\
\hline 5 & 6 & 1.368512 & 0.213454 & 1.390666 \\
\hline 6 & 6 & 1.365277 & 1.126601 & 0.258833 \\
\hline 7 & 6 & 1.364297 & 0.447989 & -1.036023 \\
\hline 8 & 6 & -0.917876 & -0.104531 & -1.157070 \\
\hline 9 & 1 & -0.804776 & 0.184024 & -2.195368 \\
\hline 10 & 6 & -0.911528 & -1.404284 & -0.651128 \\
\hline 11 & 6 & -1.766183 & -1.394982 & 0.598666 \\
\hline 12 & 1 & 1.397534 & -2.713315 & -0.353038 \\
\hline 13 & 1 & 1.572942 & 1.009024 & -1.952325 \\
\hline 14 & 1 & 1.435147 & 0.633113 & 2.392486 \\
\hline 15 & 1 & -0.864534 & -2.297940 & -1.272514 \\
\hline 16 & 1 & -2.726305 & -1.902104 & 0.410030 \\
\hline 17 & 6 & -1.655245 & 0.757415 & -0.217969 \\
\hline 18 & 8 & -1.929930 & 1.939673 & -0.288884 \\
\hline 19 & 1 & -1.315526 & -1.826576 & 1.498689 \\
\hline 20 & 1 & 1.195310 & -1.833619 & 2.010856 \\
\hline 21 & 8 & 1.355981 & 2.368414 & 0.341033 \\
\hline
\end{tabular}

\section{in $\mathrm{H}_{2} \mathrm{O}$ MP2/6-31G(d)}

$\mathrm{HF}=-644.8126926$ hartrees $(-404626.412733426 \mathrm{kcal} / \mathrm{mol})$ Imaginary Frequencies: $1(-338.07451 / \mathrm{cm})$

Zero-point correction $=0.156576$ (Hartree/Particle)

Thermal correction to Energy $=\quad 0.166546$

Thermal correction to Enthalpy= $\quad 0.167490$

Thermal correction to Gibbs Free Energy $=\quad 0.120762$

Sum of electronic and zero-point Energies $=\quad-646.545106$

Sum of electronic and thermal Energies $=\quad-646.535136$

Sum of electronic and thermal Enthalpies $=\quad-646.534192$

Sum of electronic and thermal Free Energies $=\quad-646.580920$

Coordinates (from last standard orientation):

\begin{tabular}{rrrrr}
\multicolumn{3}{c}{$\begin{array}{c}\text { Center } \\
\text { Number }\end{array}$} & Atomic & \multicolumn{3}{c}{ Coordinates (Angstroms) } \\
\hline 1 & 8 & 1.617478 & -1.032860 & -1.030439 \\
2 & 6 & 1.041723 & -1.745407 & -0.033399 \\
3 & 8 & -1.991946 & 0.127206 & 0.800533 \\
4 & 6 & 1.019868 & -1.159898 & 1.265113 \\
5 & 6 & 1.192510 & 0.186133 & 1.419962 \\
6 & 6 & 1.382999 & 1.036763 & 0.253452 \\
7 & 6 & 1.474982 & 0.302311 & -0.994580 \\
8 & 6 & -0.845311 & -0.042969 & -1.197527 \\
9 & 1 & -0.660172 & 0.259730 & -2.223722
\end{tabular}




$\begin{array}{rrrrr}10 & 6 & -0.971865 & -1.338658 & -0.720405 \\ 11 & 6 & -1.874702 & -1.283101 & 0.488804 \\ 12 & 1 & 1.159648 & -2.824716 & -0.184184 \\ 13 & 1 & 1.830540 & 0.788023 & -1.912505 \\ 14 & 1 & 1.204462 & 0.651009 & 2.405763 \\ 15 & 1 & -0.959533 & -2.220365 & -1.357795 \\ 16 & 1 & -2.877235 & -1.661981 & 0.234874 \\ 17 & 6 & -1.503543 & 0.867463 & -0.249876 \\ 18 & 8 & -1.638905 & 2.080517 & -0.290150 \\ 19 & 1 & -1.517227 & -1.797103 & 1.387249 \\ 20 & 1 & 0.825060 & -1.811017 & 2.117100 \\ 21 & 8 & 1.450185 & 2.285213 & 0.288051 \\ --------------------------------------------\end{array}$

\section{in $\mathrm{CH}_{3} \mathrm{CN}$ B3LYP/6-31G(d)}

$\mathrm{HF}=-648.5748698 \mathrm{hartrees}(-406987.216548198 \mathrm{kcal} / \mathrm{mol})$ Imaginary Frequencies: $1(-425.19951 / \mathrm{cm})$

Zero-point correction $=0.156574$ (Hartree/Particle)

Thermal correction to Energy $=\quad 0.166705$

Thermal correction to Enthalpy $=\quad 0.167649$

Thermal correction to Gibbs Free Energy $=\quad 0.120293$

Sum of electronic and zero-point Energies $=\quad-648.418296$

Sum of electronic and thermal Energies $=\quad-648.408165$

Sum of electronic and thermal Enthalpies $=\quad-648.407221$

Sum of electronic and thermal Free Energies $=\quad-648.454577$

Coordinates (from last standard orientation):

\begin{tabular}{|c|c|c|c|c|}
\hline \multirow{2}{*}{$\begin{array}{l}\text { Center } \\
\text { Number }\end{array}$} & \multirow{2}{*}{$\begin{array}{l}\text { Atomic } \\
\text { Number }\end{array}$} & \multicolumn{3}{|c|}{ Coordinates (Angstroms) } \\
\hline & & & $\begin{array}{l}X \\
Y\end{array}$ & Z \\
\hline 1 & 8 & 1.653764 & -0.764458 & -1.177693 \\
\hline 2 & 6 & 1.320453 & -1.601597 & -0.171472 \\
\hline 3 & 8 & -2.020722 & -0.077072 & 0.881550 \\
\hline 4 & 6 & 1.362125 & -1.092168 & 1.156300 \\
\hline 5 & 6 & 1.377412 & 0.254015 & 1.383154 \\
\hline 6 & 6 & 1.303354 & 1.184915 & 0.263818 \\
\hline 7 & 6 & 1.306865 & 0.517197 & -1.041648 \\
\hline 8 & 6 & -0.930174 & -0.153456 & -1.154182 \\
\hline 9 & 1 & -0.849775 & 0.126692 & -2.195986 \\
\hline 10 & 6 & -0.866789 & -1.445000 & -0.640916 \\
\hline 11 & 6 & -1.683245 & -1.456036 & 0.635073 \\
\hline 12 & 1 & 1.565104 & -2.633094 & -0.402080 \\
\hline 13 & 1 & 1.469396 & 1.097666 & -1.945570 \\
\hline 14 & 1 & 1.436479 & 0.663904 & 2.387267 \\
\hline 15 & 1 & -0.789252 & -2.338288 & -1.249880 \\
\hline 16 & 1 & -2.613207 & -2.022579 & 0.494257 \\
\hline 17 & 6 & -1.699224 & 0.687340 & -0.213517 \\
\hline 18 & 8 & -2.036804 & 1.846842 & -0.301129 \\
\hline 19 & 1 & -1.175080 & -1.843368 & 1.521128 \\
\hline 20 & 1 & 1.356018 & -1.806534 & 1.975215 \\
\hline 21 & 8 & 1.235718 & 2.417729 & 0.361771 \\
\hline
\end{tabular}

\section{in DMSO B3LYP/6-31G(d)}

$\mathrm{HF}=-648.5749403$ hartrees $(-406987.260787653 \mathrm{kcal} / \mathrm{mol})$

Imaginary Frequencies: $1(-425.41101 / \mathrm{cm})$ 


\begin{tabular}{|c|c|c|c|c|}
\hline \multicolumn{5}{|c|}{ Zero-point correction = $0.156560($ Hartree/Particle $)$} \\
\hline \multicolumn{4}{|c|}{ Thermal correction to Energy $=\quad 0$} & 0.166696 \\
\hline \multirow{2}{*}{\multicolumn{5}{|c|}{$\begin{array}{lc}\text { Thermal correction to Enthalpy }= & 0.167640 \\
\text { Thermal correction to Gibbs Free Energy }= & 0.1202\end{array}$}} \\
\hline & & & & 0.1202 \\
\hline \multicolumn{5}{|c|}{$\begin{array}{l}\text { Thermal correction to Gibbs Free Energy= } \\
\text { Sum of electronic and zero-point Energies= }\end{array}$} \\
\hline \multicolumn{5}{|c|}{ Sum of electronic and thermal Energies $=$} \\
\hline \multicolumn{5}{|c|}{ Sum of electronic and thermal Enthalpies $=\quad-648$} \\
\hline \multirow{2}{*}{\multicolumn{5}{|c|}{ Sum of electronic and thermal Free Energies $=\quad-6$}} \\
\hline & & & & \\
\hline \multirow{2}{*}{$\begin{array}{l}\text { Center } \\
\text { Number }\end{array}$} & \multirow{2}{*}{$\begin{array}{l}\text { Atomic } \\
\text { Numbe }\end{array}$} & & \multicolumn{2}{|c|}{ Coordinates (Angstroms) } \\
\hline & & & $X \quad Y$ & Z \\
\hline 1 & 8 & 1.655293 & -0.757178 & -1.179954 \\
\hline 2 & 6 & 1.327458 & -1.596983 & -0.174224 \\
\hline 3 & 8 & -2.019310 & -0.082443 & 0.882856 \\
\hline 4 & 6 & 1.368869 & -1.089003 & 1.154018 \\
\hline 5 & 6 & 1.378589 & 0.256995 & 1.382487 \\
\hline 6 & 6 & 1.298282 & 8861 & 64465 \\
\hline 7 & 6 & 1.302354 & 0.522714 & -1.041874 \\
\hline 8 & 6 & -0.930304 & -0.157519 & -1.153720 \\
\hline 9 & 1 & -0.851914 & 0.121541 & -2.195986 \\
\hline 10 & 6 & -0.862162 & -1.448343 & -0.639153 \\
\hline 11 & 6 & -1.676698 & -1.460534 & 0.638038 \\
\hline 12 & 1 & 1.575511 & -2.627276 & -0.406649 \\
\hline 13 & 1 & 1.461085 & 1.105029 & -1.945296 \\
\hline 14 & 1 & 1.437551 & 0.665882 & 2.387023 \\
\hline 15 & 1 & -0.782307 & -2.342145 & -1.247052 \\
\hline 16 & 1 & -2.604648 & -2.030937 & 0.499533 \\
\hline 17 & 6 & -1.701895 & 0.681599 & -0.213651 \\
\hline 18 & 8 & -2.044122 & 1.839619 & -0.302785 \\
\hline 19 & 1 & -1.165751 & -1.844420 & 1.524030 \\
\hline 20 & 1 & 1.367145 & -1.804397 & 1.972063 \\
\hline 21 & 8 & 1.225185 & 2.421252 & 0.364135 \\
\hline
\end{tabular}

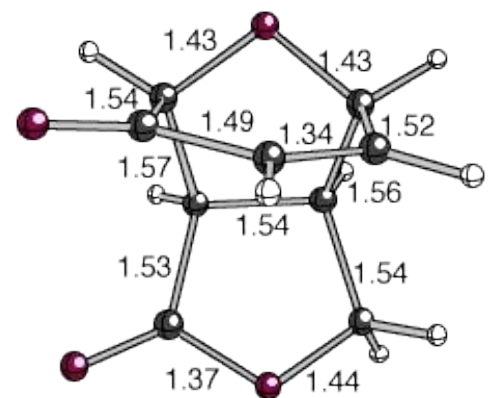

B3LYP/6-31G(d)

$\mathrm{HF}=-648.6279457$ hartrees $(-407020.522206207 \mathrm{kcal} / \mathrm{mol})$ Imaginary Frequencies: none found Zero-point correction $=0.161859$ (Hartree/Particle)

Thermal correction to Energy= 0.171255

Thermal correction to Enthalpy= 0.172199

Thermal correction to Gibbs Free Energy $=0.126425$

Sum of electronic and zero-point Energies= $-648.466086$

Sum of electronic and thermal Energies= $-648.456691$

Sum of electronic and thermal Enthalpies= $-648.455746$ 
Sum of electronic and thermal Free Energies=

$-648.501521$

Coordinates (from last standard orientation):

\begin{tabular}{ccrcc} 
Center & Atomic & \multicolumn{3}{c}{ Coordinates (Angstroms) } \\
Number & Number & X & $Y$ & $Z$ \\
- \hdashline 1 & 8 & 1.177853 & -1.341326 & -1.103193 \\
2 & 6 & 0.253543 & -1.815324 & -0.120680 \\
3 & 8 & -2.001772 & 0.614377 & 0.757063 \\
4 & 6 & 0.697036 & -1.360501 & 1.254508 \\
5 & 6 & 1.383365 & -0.214547 & 1.390791 \\
6 & 6 & 1.644086 & 0.624304 & 0.192886 \\
7 & 6 & 0.983938 & 0.078396 & -1.083927 \\
8 & 6 & -0.581476 & 0.219962 & -1.095994 \\
9 & 1 & -0.913131 & 0.455724 & -2.110367 \\
10 & 6 & -1.079940 & -1.146692 & -0.587470 \\
11 & 6 & -2.139617 & -0.788238 & 0.472883 \\
12 & 1 & 0.227070 & -2.905720 & -0.189187 \\
13 & 1 & 1.463260 & 0.513555 & -1.960821 \\
14 & 1 & 1.761139 & 0.142695 & 2.344395 \\
15 & 1 & -1.508880 & -1.755895 & -1.386185 \\
16 & 1 & -3.154956 & -0.960793 & 0.101510 \\
17 & 6 & -1.185360 & 1.244232 & -0.137231 \\
18 & 8 & -1.005789 & 2.432157 & -0.120527 \\
19 & 1 & -2.021052 & -1.328012 & 1.416522 \\
20 & 1 & 0.449595 & -1.979363 & 2.114536 \\
21 & 8 & 2.310147 & 1.640824 & 0.211034 \\
-----------------
\end{tabular}

\section{B3LYP/6-31+G(d,p)}

$\mathrm{HF}=-648.6653864$ hartrees $(-407044.016619864 \mathrm{kcal} / \mathrm{mol})$ Imaginary Frequencies: none found

Zero-point correction $=0.161116($ Hartree/Particle $)$

Thermal correction to Energy $=\quad 0.170511$

Thermal correction to Enthalpy $=\quad 0.171455$

Thermal correction to Gibbs Free Energy $=\quad 0.125759$

Sum of electronic and zero-point Energies $=\quad-648.504270$

Sum of electronic and thermal Energies $=\quad-648.494875$

Sum of electronic and thermal Enthalpies $=\quad-648.493931$

Sum of electronic and thermal Free Energies $=\quad-648.539627$

Coordinates (from last standard orientation):

\begin{tabular}{llrrr} 
Center & Atomic & \multicolumn{3}{c}{ Coordinates (Angstroms) } \\
Number & Number & X & $Y$ & $Z$ \\
- \hdashline 1 & 8 & 1.212913 & -1.287292 & -1.125306 \\
2 & 6 & 0.312749 & -1.806537 & -0.139003 \\
3 & 8 & -2.033902 & 0.548211 & 0.765023 \\
4 & 6 & 0.758211 & -1.357860 & 1.237069 \\
5 & 6 & 1.415155 & -0.194556 & 1.385209 \\
6 & 6 & 1.636569 & 0.666992 & 0.196787 \\
7 & 6 & 0.977404 & 0.128167 & -1.083035 \\
8 & 6 & -0.592557 & 0.216905 & -1.083816 \\
9 & 1 & -0.933695 & 0.449613 & -2.095509 \\
10 & 6 & -1.043668 & -1.172113 & -0.586539 \\
11 & 6 & -2.111407 & -0.863752 & 0.481255
\end{tabular}




$\begin{array}{rrrrr}12 & 1 & 0.320465 & -2.895602 & -0.223584 \\ 13 & 1 & 1.435731 & 0.590836 & -1.956419 \\ 14 & 1 & 1.793418 & 0.158166 & 2.339780 \\ 15 & 1 & -1.456121 & -1.785113 & -1.390516 \\ 16 & 1 & -3.120888 & -1.075175 & 0.116683 \\ 17 & 6 & -1.240907 & 1.212316 & -0.124913 \\ 18 & 8 & -1.119103 & 2.409542 & -0.111485 \\ 19 & 1 & -1.962962 & -1.394852 & 1.424953 \\ 20 & 1 & 0.539836 & -1.996157 & 2.090198 \\ 21 & 8 & 2.279457 & 1.700905 & 0.221310 \\ -\end{array}$

\begin{tabular}{|c|c|c|c|c|}
\hline \multirow{2}{*}{\multicolumn{5}{|c|}{ MP2/6-31G(d) }} \\
\hline & & & & \\
\hline \multirow{2}{*}{\multicolumn{5}{|c|}{$\begin{array}{l}\mathrm{HF}=-644.8907265 \text { hartrees }(-404675.379786015 \mathrm{kcal} / \mathrm{r} \\
\text { Imaginary Frequencies: none found } \\
\text { Zero-point correction }=0.163765 \text { (Hartree/Particle) }\end{array}$}} \\
\hline & & & & \\
\hline Thermal & corre & to Energy= & & Particle) \\
\hline \multicolumn{4}{|c|}{ Thermal correction to Enthalpy= } & 0.173956 \\
\hline \multicolumn{4}{|c|}{ Thermal correction to Gibbs Free Energy= } & \\
\hline \multicolumn{4}{|c|}{ Sum of electronic and zero-point Energies= } & \\
\hline \multicolumn{4}{|c|}{$\begin{array}{l}\text { Sum of electronic and zero-point Energies= } \\
\text { Sum of electronic and thermal Energies }=\end{array}$} & \\
\hline \multirow{2}{*}{\multicolumn{5}{|c|}{ Sum of electronic and thermal Enthalpies= }} \\
\hline \multirow{2}{*}{\multicolumn{5}{|c|}{$\begin{array}{l}\text { Sum of electronic and thermal Free Energies= } \\
\text { Coordinates (from last standard orientation): }\end{array}$}} \\
\hline & & & & \\
\hline \multirow{2}{*}{$\begin{array}{l}\text { Center } \\
\text { Number }\end{array}$} & \multirow{2}{*}{\multicolumn{2}{|c|}{$\begin{array}{l}\text { Atomic } \\
\text { Number }\end{array}$}} & \multicolumn{2}{|c|}{ Coordinates (Angstroms) } \\
\hline & & & $X \quad Y$ & Z \\
\hline 1 & 8 & 0.827374 & -1.715655 & -0.844059 \\
\hline 2 & 6 & -0.238010 & -1.763663 & 0.115039 \\
\hline 3 & 8 & -1.835731 & 4923 & 12388 \\
\hline 4 & 6 & 0.218541 & -1.1 & 7214 \\
\hline 5 & 6 & 1.199345 & -0.229242 & 3487 \\
\hline 6 & 6 & 1.758245 & 0.240551 & 33902 \\
\hline 7 & 6 & 0.988749 & -0.301086 & -1.065593 \\
\hline 8 & 6 & -0.474814 & 0.234137 & -1.135857 \\
\hline 9 & 1 & -0.730622 & 0.527998 & -2.158671 \\
\hline 10 & 6 & -1.322188 & -0.918390 & -0.591449 \\
\hline 11 & 6 & -2.368685 & -0.223947 & 0.279920 \\
\hline 12 & 1 & -0.520529 & -2.813462 & 0.239213 \\
\hline 13 & 1 & 1.558924 & -0.153886 & -1.984744 \\
\hline 14 & 1 & 1.593271 & 0.205060 & 2.338579 \\
\hline 15 & 1 & -1.777616 & -1.522433 & -1.381043 \\
\hline 16 & 1 & -3.309537 & -0.080576 & -0.260920 \\
\hline 17 & 6 & -0.781568 & 1.395665 & -0.200134 \\
\hline 18 & 8 & -0.206449 & 2.459422 & -0.130915 \\
\hline 19 & 1 & -2.580041 & -0.740088 & 1.220283 \\
\hline 20 & 1 & -0.250853 & -1.483601 & 2.343193 \\
\hline 21 & 8 & 2.732221 & 0.980064 & 0.035703 \\
\hline
\end{tabular}

in $\mathrm{H}_{2} \mathrm{O}$ B3LYP/6-31G(d)

$\mathrm{HF}=-648.6628023$ hartrees $(-407042.395071273 \mathrm{kcal} / \mathrm{mol})$ Imaginary Frequencies: none found

Zero-point correction $=0.156756$ (Hartree/Particle)

Thermal correction to Energy=

0.166166 


\begin{tabular}{|c|c|c|c|c|}
\hline \multicolumn{4}{|c|}{ Thermal correction to Enthalpy= } & \\
\hline \multirow{6}{*}{\multicolumn{4}{|c|}{$\begin{array}{l}\text { Thermal correction to Gibbs Free Energy= } \\
\text { Sum of electronic and zero-point Energies= } \\
\text { Sum of electronic and thermal Energies= } \\
\text { Sum of electronic and thermal Enthalpies= } \\
\text { Sum of electronic and thermal Free Energies= } \\
\text { Coordinates (from last standard orientation): }\end{array}$}} & ( \\
\hline & & & & $=$ \\
\hline & & & & \\
\hline & & & & \\
\hline & & & & $e s=$ \\
\hline & & & & \\
\hline \multirow{2}{*}{$\begin{array}{l}\text { Center } \\
\text { Number }\end{array}$} & \multirow{2}{*}{$\begin{array}{l}\text { Atomic } \\
\text { Numbe }\end{array}$} & \multicolumn{3}{|c|}{ Coordinates (Angstroms) } \\
\hline & & & $X \quad Y$ & Z \\
\hline 1 & 8 & 1.086937 & -1.453549 & -1.053204 \\
\hline 2 & 6 & 0.113165 & -1.829239 & -0.064738 \\
\hline 3 & 8 & -1.961201 & 0.763013 & $0.72220 \varsigma$ \\
\hline 4 & 6 & 0.577045 & -1.348521 & 1.291767 \\
\hline 5 & 6 & 1.362867 & -0.260523 & 1.393537 \\
\hline 6 & 6 & 1.682820 & 0.511402 & 0.173729 \\
\hline 7 & 6 & 50 & 909 & 89948 \\
\hline 8 & 6 & -0.554540 & 0.236073 & -1.101490 \\
\hline 9 & 1 & -0.875081 & 0.496705 & -2.120116 \\
\hline 10 & 6 & -1.157317 & -1.084636 & -0.582126 \\
\hline 11 & 6 & -2.218434 & -0.639569 & 0.435344 \\
\hline 12 & 1 & 0.005767 & -2.926945 & -0.086360 \\
\hline 13 & 1 & 1.518649 & 0.356588 & -2.005578 \\
\hline 14 & 1 & 1.756342 & 0.098299 & 2.343240 \\
\hline 15 & 1 & -1.613601 & -1.695516 & -1.400886 \\
\hline 16 & 1 & -3.229412 & -0.706921 & 0.022271 \\
\hline 17 & 6 & -1.074651 & 1.299477 & -0.148021 \\
\hline 18 & 8 & -0.781968 & 2.475382 & -0.108490 \\
\hline 19 & 1 & -2.187946 & -1.174863 & 1.388778 \\
\hline 20 & 1 & 0.259854 & -1.901619 & 2.175248 \\
\hline 21 & 8 & 2.405531 & 1.500022 & 0.168871 \\
\hline
\end{tabular}

\section{in $\mathrm{H}_{2} \mathrm{O}$ MP2/6-31G(d)}

$\mathrm{HF}=-644.9315699$ hartrees $(-404701.009427949 \mathrm{kcal} / \mathrm{mol})$ Imaginary Frequencies: none found Zero-point correction $=0.158704$ (Hartree/Particle)

Thermal correction to Energy $=\quad 0.167769$ Thermal correction to Enthalpy= $\quad 0.168713$ Thermal correction to Gibbs Free Energy $=0.124384$ Sum of electronic and zero-point Energies $=\quad-646.621999$ Sum of electronic and thermal Energies $=\quad-646.612934$ Sum of electronic and thermal Enthalpies $=\quad-646.611990$ Sum of electronic and thermal Free Energies $=\quad-646.656319$ Coordinates (from last standard orientation):

\begin{tabular}{llrrr} 
Center & Atomic & \multicolumn{3}{c}{ Coordinates (Angstroms) } \\
Number & Number & \multicolumn{1}{c}{ X } & $Y$ & \multicolumn{1}{c}{ Z } \\
\hdashline 1 & 8 & 0.819823 & -1.715046 & -0.864490 \\
2 & 6 & -0.253264 & -1.774889 & 0.097291 \\
3 & 8 & -1.805785 & 1.094134 & 0.625235 \\
4 & 6 & 0.217961 & -1.191030 & 1.402886 \\
5 & 6 & 1.206915 & -0.271659 & 1.420839 \\
6 & 6 & 1.745470 & 0.225008 & 0.138162
\end{tabular}




\begin{tabular}{|c|c|c|c|c|}
\hline 7 & 6 & 0.988019 & -0.297648 & -1.072790 \\
\hline 8 & 6 & -0.473972 & 0.244876 & -1.132995 \\
\hline 9 & 1 & -0.732958 & 0.547301 & -2.159850 \\
\hline 10 & 6 & -1.328147 & -0.905791 & -0.591612 \\
\hline 11 & 6 & -2.357942 & -0.214231 & 0.297035 \\
\hline 12 & 1 & -0.552141 & -2.833460 & 0.209665 \\
\hline 13 & 1 & 1.572478 & -0.128016 & -2.019196 \\
\hline 14 & 1 & 1.609837 & 0.140285 & 2.345868 \\
\hline 15 & 1 & -1.818704 & -1.506479 & -1.404927 \\
\hline 16 & 1 & -3.302049 & -0.049611 & -0.231232 \\
\hline 17 & 6 & -0.768418 & 1.398129 & -0.196312 \\
\hline 18 & 8 & -0.190996 & 2.470062 & -0.126482 \\
\hline 19 & 1 & -2.557899 & -0.724451 & 1.243825 \\
\hline 20 & 1 & -0.251626 & -1.529917 & 2.327126 \\
\hline 21 & 8 & 2.698625 & 1.001819 & 0.05494 \\
\hline
\end{tabular}

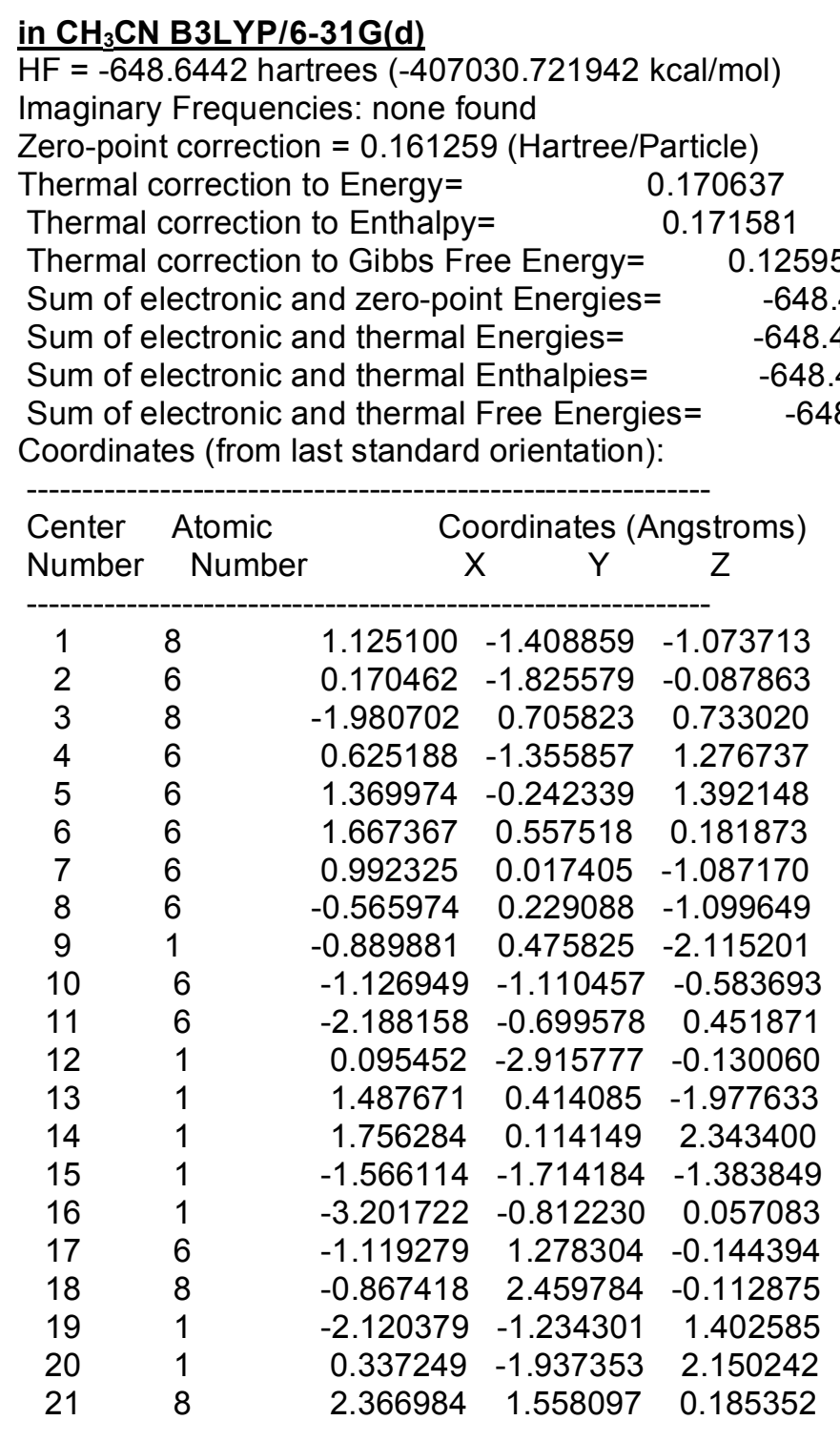


in DMSO B3LYP/6-31G(d)

$\mathrm{HF}=-648.6443384$ hartrees $(-407030.808789384 \mathrm{kcal} / \mathrm{mol})$

Imaginary Frequencies: none found

Zero-point correction $=0.161271$ (Hartree/Particle)

Thermal correction to Energy=

0.170645

Thermal correction to Enthalpy=

0.171589

Thermal correction to Gibbs Free Energy=

Sum of electronic and zero-point Energies=

0.125968

Sum of electronic and thermal Energies=

$-648.483068$

Sum of electronic and thermal Enthalpies= $-648.473693$

$-648.472749$

Sum of electronic and thermal Free Energies=

$-648.518371$

Coordinates (from last standard orientation):

\begin{tabular}{|c|c|c|c|c|}
\hline \multirow{2}{*}{$\begin{array}{l}\text { Center } \\
\text { Number }\end{array}$} & \multirow{2}{*}{$\begin{array}{l}\text { Atomic } \\
\text { Number }\end{array}$} & \multicolumn{3}{|c|}{ Coordinates (Angstroms) } \\
\hline & & & $\begin{array}{ll}X & Y\end{array}$ & Z \\
\hline 1 & 8 & 1.146149 & -1.384959 & -1.084392 \\
\hline 2 & 6 & 0.201332 & -1.824618 & -0.098992 \\
\hline 3 & 8 & -1.987966 & 0.675735 & 0.739086 \\
\hline 4 & 6 & 0.653552 & -1.359306 & 1.267872 \\
\hline 5 & 6 & 1.378248 & -0.233276 & 1.389933 \\
\hline 6 & 6 & 1.656120 & 0.581977 & 0.185565 \\
\hline 7 & 6 & 0.990113 & 0.038747 & -1.086982 \\
\hline 8 & 6 & -0.571552 & 0.224017 & -1.098254 \\
\hline 9 & 1 & -0.899088 & 0.465011 & -2.113976 \\
\hline 10 & 6 & -1.109291 & -1.125493 & -0.583242 \\
\hline 11 & 6 & -2.168792 & -0.734268 & 0.462147 \\
\hline 12 & 1 & 0.142913 & -2.915409 & -0.150324 \\
\hline 13 & 1 & 1.478546 & 0.450396 & -1.974519 \\
\hline 14 & 1 & 1.761848 & 0.122187 & 2.342669 \\
\hline 15 & 1 & -1.546181 & -1.731771 & -1.382711 \\
\hline 16 & 1 & -3.183794 & -0.868541 & 0.078054 \\
\hline 17 & 6 & -1.142220 & 1.263753 & -0.143120 \\
\hline 18 & 8 & -0.914640 & 2.450284 & -0.115400 \\
\hline 19 & 1 & -2.079992 & -1.265502 & 1.413097 \\
\hline 20 & 1 & 0.379598 & -1.953421 & 2.137338 \\
\hline 21 & 8 & 2.334092 & 1.597421 & 0.195807 \\
\hline
\end{tabular}

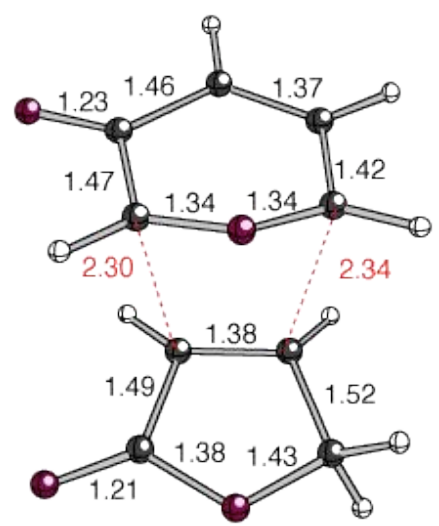

B3LYP/6-31G(d)

$\mathrm{HF}=-648.5599837$ hartrees $(-406977.875371587 \mathrm{kcal} / \mathrm{mol})$

Imaginary Frequencies: 1 (-404.1706 1/cm)

Zero-point correction $=0.156691$ (Hartree/Particle) 


\begin{tabular}{|c|c|c|c|c|}
\hline \multicolumn{4}{|c|}{ Thermal correction to Energy= } & 0.166941 \\
\hline \multicolumn{4}{|c|}{ Thermal correction to Enthalpy= } & 0.167885 \\
\hline Thermal & \multicolumn{4}{|c|}{ Thermal correction to Gibbs Free Energy $=\quad 0.1201$} \\
\hline \multicolumn{4}{|c|}{ Sum of electronic and zero-point Energies= } & \\
\hline \multirow{2}{*}{\multicolumn{5}{|c|}{$\begin{array}{ll}\text { Sum of electronic and thermal Energies }= & -648 . \\
\text { Sum of electronic and thermal Enthalpies }= & -648\end{array}$}} \\
\hline & & & & \\
\hline \multirow{2}{*}{\multicolumn{5}{|c|}{ Sum of electronic and thermal Free Energies $=-64$}} \\
\hline & & & & \\
\hline \multirow{2}{*}{$\begin{array}{l}\text { Center } \\
\text { Number }\end{array}$} & \multirow{2}{*}{$\begin{array}{l}\text { Atomic } \\
\text { Number }\end{array}$} & \multicolumn{3}{|c|}{ Coordinates (Angstroms) } \\
\hline & & & $X \quad Y$ & Z \\
\hline 1 & 8 & 0.483185 & -0.502855 & 1.365388 \\
\hline 2 & 8 & 2.357346 & 1113 & -0.324751 \\
\hline 3 & 6 & 0.779915 & 0.688433 & 0.838457 \\
\hline 4 & 6 & 1.962378 & 0.884804 & -0.019487 \\
\hline 5 & 6 & 2.551674 & -0.370294 & -0.475920 \\
\hline 6 & 6 & 1.941937 & -1.556939 & -0.184762 \\
\hline 7 & 1 & 2.308663 & 9213 & -0.583463 \\
\hline 8 & 6 & 1464 & 0420 & 0.631627 \\
\hline 9 & 6 & -0.817831 & -0.992057 & -0.970862 \\
\hline 10 & 6 & -0.717961 & 0.386431 & -0.883841 \\
\hline 11 & 1 & 0.381489 & 1.519688 & 1.409780 \\
\hline 12 & 1 & 0.409210 & -2.501769 & 1.083637 \\
\hline 13 & 1 & 3.457400 & -0.317558 & -1.072089 \\
\hline 14 & 6 & -2.109917 & -1.398511 & -0.292488 \\
\hline 15 & 6 & -1.894400 & 0.890463 & -0.121387 \\
\hline 16 & 8 & -2.628185 & -0.199964 & 0.293299 \\
\hline 17 & 8 & -2.215840 & 2.019616 & 0.147248 \\
\hline 18 & 1 & -2.008339 & -2.146630 & 0.500164 \\
\hline 19 & 1 & -0.372854 & -1.600857 & -1.747102 \\
\hline 20 & 1 & -0.175776 & 1.050245 & -1.543047 \\
\hline 21 & 1 & -2.835401 & -1.778643 & -1.025367 \\
\hline
\end{tabular}

\section{B3LYP/6-31+G(d,p)}

$\mathrm{HF}=-648.6000975$ hartrees $(-407003.047182225 \mathrm{kcal} / \mathrm{mol})$

Imaginary Frequencies: $1(-412.57571 / \mathrm{cm})$

Zero-point correction $=0.156007$ (Hartree/Particle)

Thermal correction to Energy $=\quad 0.166263$

Thermal correction to Enthalpy $=\quad 0.167207$

Thermal correction to Gibbs Free Energy $=\quad 0.119521$

Sum of electronic and zero-point Energies $=\quad-648.444091$

Sum of electronic and thermal Energies $=\quad-648.433834$

Sum of electronic and thermal Enthalpies $=\quad-648.432890$

Sum of electronic and thermal Free Energies $=\quad-648.480577$

Coordinates (from last standard orientation):

\begin{tabular}{|c|c|c|c|c|}
\hline \multirow{2}{*}{$\begin{array}{l}\text { Center } \\
\text { Number }\end{array}$} & \multirow{2}{*}{$\begin{array}{l}\text { Atomic } \\
\text { Number }\end{array}$} & & \multicolumn{2}{|c|}{ Coordinates (Angstroms) } \\
\hline & & & $X \quad Y$ & Z \\
\hline 1 & 8 & 0.496110 & $0-0.505494$ & 1.367889 \\
\hline 2 & 8 & 2.360650 & $0 \quad 2.015998$ & -0.320201 \\
\hline 3 & 6 & 0.779419 & 0.690602 & 0.836284 \\
\hline 4 & 6 & 1.964740 & $0 \quad 0.885037$ & -0.016800 \\
\hline 5 & 6 & 2.556911 & $1-0.363345$ & -0.481897 \\
\hline
\end{tabular}




$\begin{array}{ccrrr}6 & 6 & 1.946664 & -1.552421 & -0.194629 \\ 7 & 1 & 2.311387 & -2.491863 & -0.600854 \\ 8 & 6 & 0.789912 & -1.590958 & 0.627873 \\ 9 & 6 & -0.814508 & -0.993037 & -0.960331 \\ 10 & 6 & -0.715246 & 0.389070 & -0.866277 \\ 11 & 1 & 0.387043 & 1.520431 & 1.414014 \\ 12 & 1 & 0.420163 & -2.505525 & 1.074890 \\ 13 & 1 & 3.460260 & -0.306432 & -1.080489 \\ 14 & 6 & -2.108647 & -1.406835 & -0.291065 \\ 15 & 6 & -1.904280 & 0.887083 & -0.119981 \\ 16 & 8 & -2.637463 & -0.204628 & 0.289327 \\ 17 & 8 & -2.238602 & 2.017653 & 0.136923 \\ 18 & 1 & -2.011102 & -2.149024 & 0.506495 \\ 19 & 1 & -0.370344 & -1.594132 & -1.742707 \\ 20 & 1 & -0.183712 & 1.053029 & -1.533849 \\ 21 & 1 & -2.829057 & -1.785891 & -1.028062 \\ --------------------------------------------------\end{array}$

\begin{tabular}{|c|c|c|c|c|}
\hline \multicolumn{5}{|c|}{$\begin{array}{l}\mathrm{HF}=-644.7768067 \text { hartrees }(-404603.893972317 \mathrm{kcal} / \mathrm{r} \\
\text { Imaginary Frequencies: } 1(-340.65661 / \mathrm{cm}) \\
\text { Zero-point correction }=0.158406(\text { Hartree } / \text { Particle })\end{array}$} \\
\hline \multicolumn{4}{|c|}{ Thermal correction to Energy $=$} & 0.168640 \\
\hline \multicolumn{4}{|c|}{ Thermal correction to Enthalpy= } & 0.169584 \\
\hline \multicolumn{4}{|c|}{ Thermal correction to Gibbs Free Eneray= } & \\
\hline \multicolumn{5}{|c|}{ Sum of electronic and zero-point Energies= } \\
\hline \multicolumn{5}{|c|}{ Sum of electronic and thermal Energies= } \\
\hline \multicolumn{5}{|c|}{ Sum of electronic and thermal Enthalpies= } \\
\hline \multirow{2}{*}{\multicolumn{5}{|c|}{$\begin{array}{l}\text { Sum of electronic and thermal Free Energies= } \\
\text { Coordinates (from last standard orientation): }\end{array}$}} \\
\hline & & & & \\
\hline \multirow{2}{*}{$\begin{array}{l}\text { Center } \\
\text { Number }\end{array}$} & \multirow{2}{*}{$\begin{array}{l}\text { Atomic } \\
\text { Number }\end{array}$} & \multicolumn{3}{|c|}{ Coordinates (Angstroms) } \\
\hline & & & $X \quad Y$ & Z \\
\hline 1 & 8 & 0.426890 & -0.559395 & 1.349543 \\
\hline 2 & 8 & 2.296943 & 2.030636 & -0.259114 \\
\hline 3 & 6 & 0.736778 & 0.659905 & 73975 \\
\hline 4 & 6 & 5264 & 7274 & 721 \\
\hline 5 & 6 & 3356 & -0. & 068 \\
\hline 6 & 6 & 1.912102 & -1.551035 & 1173 \\
\hline 7 & 1 & 2.294195 & -2.477250 & -0.644718 \\
\hline 8 & 6 & 0.740976 & -1.619492 & 0.581052 \\
\hline 9 & 6 & -0.810626 & -1.019503 & -0.974547 \\
\hline 10 & 6 & -0.683059 & 0.351649 & -0.932068 \\
\hline 11 & 1 & 0.331914 & 1.462643 & 1.484717 \\
\hline 12 & 1 & 31196 & -2.547862 & 1.013312 \\
\hline 13 & 1 & 3.417025 & -0.267401 & -1.062600 \\
\hline 14 & 6 & -2.083578 & -1.380011 & -0.242857 \\
\hline 15 & 6 & -1.817417 & 0.908219 & -0.152131 \\
\hline 16 & 8 & -2.549485 & -0.154418 & 0.342470 \\
\hline 17 & 8 & -2.114652 & 2.060731 & 0.076390 \\
\hline 18 & 1 & -1.969959 & -2.108701 & 0.564870 \\
\hline 19 & 1 & -0.396531 & -1.657247 & -1.748530 \\
\hline 20 & 1 & -0.110987 & 0.987201 & -1.597428 \\
\hline 21 & 1 & -2.847205 & -1.748546 & -0.939355 \\
\hline
\end{tabular}


in $\mathrm{H}_{2} \mathrm{O}$ B3LYP/6-31G(d)

$\mathrm{HF}=-648.5926936$ hartrees $(-406998.401160936 \mathrm{kcal} / \mathrm{mol})$

Imaginary Frequencies: $1(-428.78131 / \mathrm{cm})$

Zero-point correction $=0.154629$ (Hartree/Particle)

Thermal correction to Energy $=\quad 0.164802$

Thermal correction to Enthalpy= $\quad 0.165746$

Thermal correction to Gibbs Free Energy $=0.118356$

Sum of electronic and zero-point Energies $=\quad-648.438064$

Sum of electronic and thermal Energies $=\quad-648.427892$

Sum of electronic and thermal Enthalpies $=\quad-648.426948$

Sum of electronic and thermal Free Energies $=\quad-648.474337$

Coordinates (from last standard orientation):

\begin{tabular}{|c|c|c|c|c|}
\hline \multirow{2}{*}{$\begin{array}{l}\text { Center } \\
\text { Number }\end{array}$} & \multirow{2}{*}{$\begin{array}{l}\text { Atomic } \\
\text { Number }\end{array}$} & \multicolumn{3}{|c|}{ Coordinates (Angstroms) } \\
\hline & & & $X \quad Y$ & Z \\
\hline 1 & 8 & 0.490499 & -0.589153 & 1.347938 \\
\hline 2 & 8 & 2.333409 & 2.043392 & -0.192700 \\
\hline 3 & 6 & 0.791968 & 0.635813 & 0.913983 \\
\hline 4 & 6 & 1.937026 & 0.886087 & 0.040411 \\
\hline 5 & 6 & 2.517593 & -0.323692 & -0.518682 \\
\hline 6 & 6 & 1.907955 & -1.527921 & -0.312126 \\
\hline 7 & 1 & 2.260822 & -2.438722 & -0.793312 \\
\hline 8 & 6 & 0.753615 & -1.621827 & 0.515562 \\
\hline 9 & 6 & -0.830572 & -1.050655 & -0.903847 \\
\hline 10 & 6 & -0.713991 & 0.335973 & -0.921913 \\
\hline 11 & 1 & 0.400135 & 1.429412 & 1.558173 \\
\hline 12 & 1 & 0.397672 & -2.575634 & 0.913822 \\
\hline 13 & 1 & 3.414377 & -0.232585 & -1.128360 \\
\hline 14 & 6 & -2.100654 & -1.382693 & -0.148035 \\
\hline 15 & 6 & -1.851327 & 0.910184 & -0.180787 \\
\hline 16 & 8 & -2.580452 & -0.115304 & 0.357796 \\
\hline 17 & 8 & -2.177087 & 2.070732 & -0.015474 \\
\hline 18 & 1 & -1.980266 & -2.062224 & 0.706114 \\
\hline 19 & 1 & -0.473439 & -1.705939 & -1.690507 \\
\hline 20 & 1 & -0.155155 & 0.937498 & -1.628002 \\
\hline 21 & 1 & -2.864770 & -1.796766 & -0.825800 \\
\hline
\end{tabular}

\section{in $\mathrm{H}_{2} \mathrm{O}$ MP2/6-31G(d)}

$\mathrm{HF}=-644.8177014 \mathrm{hartrees}(-404629.555805514 \mathrm{kcal} / \mathrm{mol})$

Imaginary Frequencies: 1 (-337.7160 1/cm)

Zero-point correction $=0.156467$ (Hartree/Particle)

Thermal correction to Energy $=\quad 0.166461$

Thermal correction to Enthalpy $=\quad 0.167406$

Thermal correction to Gibbs Free Energy $=\quad 0.120782$

Sum of electronic and zero-point Energies $=\quad-646.547009$

Sum of electronic and thermal Energies $=\quad-646.537015$

Sum of electronic and thermal Enthalpies $=\quad-646.536070$

Sum of electronic and thermal Free Energies $=\quad-646.582694$

Coordinates (from last standard orientation):

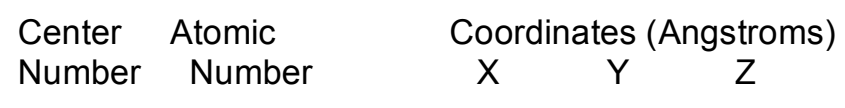




$\begin{array}{rrrrr}1 & 8 & 0.428628 & -0.634351 & 1.334922 \\ 2 & 8 & 2.246233 & 2.062508 & -0.151863 \\ 3 & 6 & 0.730944 & 0.611409 & 0.932930 \\ 4 & 6 & 1.867605 & 0.890832 & 0.071765 \\ 5 & 6 & 2.473269 & -0.298834 & -0.505804 \\ 6 & 6 & 1.889165 & -1.519625 & -0.319900 \\ 7 & 1 & 2.267127 & -2.417161 & -0.810098 \\ 8 & 6 & 0.722659 & -1.645874 & 0.485856 \\ 9 & 6 & -0.815190 & -1.068145 & -0.922553 \\ 10 & 6 & -0.671544 & 0.307796 & -0.962756 \\ 11 & 1 & 0.324923 & 1.380844 & 1.602866 \\ 12 & 1 & 0.372689 & -2.612984 & 0.865997 \\ 13 & 1 & 3.381185 & -0.182808 & -1.097406 \\ 14 & 6 & -2.066471 & -1.366870 & -0.130457 \\ 15 & 6 & -1.770443 & 0.919000 & -0.199766 \\ 16 & 8 & -2.496614 & -0.083913 & 0.391196 \\ 17 & 8 & -2.071591 & 2.093989 & -0.052475 \\ 18 & 1 & -1.934717 & -2.043890 & 0.723729 \\ 19 & 1 & -0.472464 & -1.744168 & -1.702185 \\ 20 & 1 & -0.088910 & 0.891654 & -1.668940 \\ 21 & 1 & -2.863051 & -1.755484 & -0.784096 \\ ------------------------------------------------------\end{array}$

\section{in $\mathrm{CH}_{3} \mathrm{CN}$ B3LYP/6-31G(d)}

$\mathrm{HF}=-648.5768927$ hartrees $(-406988.485938177 \mathrm{kcal} / \mathrm{mol})$ Imaginary Frequencies: $1(-419.73821 / \mathrm{cm})$

Zero-point correction $=0.156559($ Hartree/Particle $)$

Thermal correction to Energy $=\quad 0.166741$

Thermal correction to Enthalpy $=\quad 0.167685$

Thermal correction to Gibbs Free Energy $=\quad 0.120230$

Sum of electronic and zero-point Energies $=\quad-648.420334$

Sum of electronic and thermal Energies $=\quad-648.410152$

Sum of electronic and thermal Enthalpies $=\quad-648.409207$

Sum of electronic and thermal Free Energies $=\quad-648.456663$

Coordinates (from last standard orientation):

\begin{tabular}{|c|c|c|c|c|}
\hline \multirow{2}{*}{$\begin{array}{l}\text { Center } \\
\text { Number }\end{array}$} & \multirow{2}{*}{$\begin{array}{l}\text { Atomic } \\
\text { Number }\end{array}$} & \multicolumn{3}{|c|}{ Coordinates (Angstroms) } \\
\hline & & & $X \quad Y$ & Z \\
\hline 1 & 8 & 0.490345 & -0.559272 & 1.355013 \\
\hline 2 & 8 & 2.340420 & 2.035770 & -0.238020 \\
\hline 3 & 6 & 0.787955 & 0.654137 & 0.885914 \\
\hline 4 & 6 & 1.947233 & 0.888374 & 0.018857 \\
\hline 5 & 6 & 2.530867 & -0.338072 & -0.505923 \\
\hline 6 & 6 & 1.923890 & -1.537756 & -0.268328 \\
\hline 7 & 1 & 2.282000 & -2.459171 & -0.720132 \\
\hline 8 & 6 & 0.769031 & -1.610561 & 0.557763 \\
\hline 9 & 6 & -0.825258 & -1.030107 & -0.927140 \\
\hline 10 & 6 & -0.715544 & $4 \quad 0.354142$ & -0.904691 \\
\hline 11 & 1 & 0.395781 & 1.457396 & 1.503095 \\
\hline 12 & 1 & 0.409943 & $3-2.545507$ & 0.972547 \\
\hline 13 & 1 & 3.428487 & $7 \quad-0.261334$ & -1.112675 \\
\hline 14 & 6 & -2.104041 & $1-1.391272$ & -0.199659 \\
\hline 15 & 6 & -1.870632 & 0.902196 & -0.158892 \\
\hline 16 & 8 & -2.600831 & $1-0.148682$ & 0.334221 \\
\hline 17 & 8 & -2.196988 & $8 \quad 2.052227$ & 0.041123 \\
\hline
\end{tabular}




\begin{tabular}{rrrrr}
18 & 1 & -1.992216 & -2.093949 & 0.631464 \\
19 & 1 & -0.434943 & -1.667316 & -1.710635 \\
20 & 1 & -0.163274 & 0.978364 & -1.593316 \\
21 & 1 & -2.850356 & -1.795326 & -0.896447 \\
\hline
\end{tabular}

\section{in DMSO B3LYP/6-31G(d)}

$\mathrm{HF}=-648.5769078$ hartrees $(-406988.495413578 \mathrm{kcal} / \mathrm{mol})$ Imaginary Frequencies: $1(-419.29421 / \mathrm{cm})$

Zero-point correction $=0.156556$ (Hartree/Particle)

Thermal correction to Energy= $\quad 0.166738$

Thermal correction to Enthalpy= $\quad 0.167683$

Thermal correction to Gibbs Free Energy $=\quad 0.120238$

Sum of electronic and zero-point Energies $=\quad-648.420351$

Sum of electronic and thermal Energies $=\quad-648.410169$

Sum of electronic and thermal Enthalpies $=\quad-648.409225$

Sum of electronic and thermal Free Energies $=\quad-648.456670$

Coordinates (from last standard orientation):

\begin{tabular}{|c|c|c|c|c|}
\hline \multirow{2}{*}{$\begin{array}{l}\text { Center } \\
\text { Number }\end{array}$} & \multirow{2}{*}{$\begin{array}{l}\text { Atomic } \\
\text { Number }\end{array}$} & \multicolumn{3}{|c|}{ Coordinates (Angstroms) } \\
\hline & & & $X \quad Y$ & Z \\
\hline 1 & 8 & 0.490150 & -0.563379 & 1.352175 \\
\hline 2 & 8 & 2.343686 & 2.036007 & -0.228876 \\
\hline 3 & 6 & 0.787456 & 0.651377 & 0.886697 \\
\hline 4 & 6 & 1.947553 & 0.888373 & 0.021580 \\
\hline 5 & 6 & 2.530125 & -0.336623 & -0.508099 \\
\hline 6 & 6 & 1.923396 & -1.537099 & -0.274314 \\
\hline 7 & 1 & 2.281825 & -2.456938 & -0.729040 \\
\hline 8 & 6 & 0.768907 & -1.612774 & 0.552283 \\
\hline 9 & 6 & -0.830361 & -1.034084 & -0.924748 \\
\hline 10 & 6 & -0.717030 & 0.349921 & -0.908242 \\
\hline 11 & 1 & 0.393790 & 1.452657 & 1.505492 \\
\hline 12 & 1 & 0.411823 & $\begin{array}{ll}3 & -2.549017\end{array}$ & 0.965847 \\
\hline 13 & 1 & 3.428025 & $5-0.257690$ & -1.114157 \\
\hline 14 & 6 & -2.106834 & $4-1.388793$ & -0.189827 \\
\hline 15 & 6 & -1.868505 & 0.904493 & -0.162229 \\
\hline 16 & 8 & -2.599473 & $3-0.142312$ & 0.338819 \\
\hline 17 & 8 & -2.192517 & $\begin{array}{l}7 \quad 2.056094 \\
\end{array}$ & 0.032493 \\
\hline 18 & 1 & -1.992489 & $\begin{array}{ll}9 & -2.086823\end{array}$ & 0.644942 \\
\hline 19 & 1 & -0.445356 & $6 \quad-1.675640$ & -1.707370 \\
\hline 20 & 1 & -0.163745 & $5 \quad 0.969227$ & -1.600363 \\
\hline 21 & 1 & -2.856874 & $4-1.795799$ & -0.880842 \\
\hline
\end{tabular}




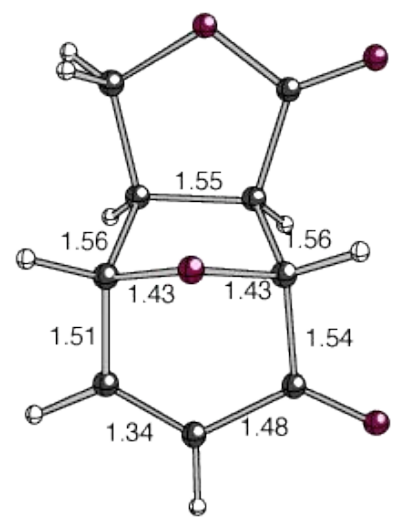

B3LYP/6-31G(d)

$\mathrm{HF}=-648.6353577$ hartrees $(-407025.173310327 \mathrm{kcal} / \mathrm{mol})$ Imaginary Frequencies: none found

Zero-point correction $=0.161911$ (Hartree/Particle)

Thermal correction to Energy $=\quad 0.171403$

Thermal correction to Enthalpy= $\quad 0.172347$

Thermal correction to Gibbs Free Energy $=0.126192$

Sum of electronic and zero-point Energies $=\quad-648.473446$

Sum of electronic and thermal Energies $=\quad-648.463955$

Sum of electronic and thermal Enthalpies $=\quad-648.463011$

Sum of electronic and thermal Free Energies $=\quad-648.509166$

Coordinates (from last standard orientation):

\begin{tabular}{ccrrr} 
Center & Atomic & \multicolumn{3}{c}{ Coordinates (Angstroms) } \\
Number & Number & X & $Y$ & $Z$ \\
- \hdashline 1 & 8 & 0.477221 & -0.471652 & 1.368915 \\
2 & 8 & 2.443847 & 1.978673 & -0.198917 \\
3 & 6 & 0.532669 & 0.719322 & 0.578565 \\
4 & 6 & 1.943059 & 0.884728 & -0.009610 \\
5 & 6 & 2.588038 & -0.392089 & -0.397187 \\
6 & 6 & 1.906940 & -1.539218 & -0.245610 \\
7 & 1 & 2.304672 & -2.491566 & -0.590810 \\
8 & 6 & 0.536205 & -1.516008 & 0.393443 \\
9 & 6 & -0.559146 & -1.097089 & -0.631801 \\
10 & 6 & -0.500031 & 0.444650 & -0.559997 \\
11 & 1 & 0.276194 & 1.580278 & 1.197640 \\
12 & 1 & 0.314654 & -2.458486 & 0.902107 \\
13 & 1 & 3.582412 & -0.340520 & -0.831518 \\
14 & 6 & -1.996450 & -1.435682 & -0.162413 \\
15 & 6 & -1.905222 & 0.883750 & -0.149567 \\
16 & 8 & -2.706694 & -0.200462 & 0.017358 \\
17 & 8 & -2.290660 & 2.009847 & 0.018572 \\
18 & 1 & -2.002696 & -1.963818 & 0.796504 \\
19 & 1 & -0.363819 & -1.493135 & -1.630554 \\
20 & 1 & -0.230795 & 0.952269 & -1.489289 \\
21 & 1 & -2.546719 & -2.030466 & -0.896441 \\
-------------------------------------------
\end{tabular}

\section{B3LYP/6-31+G(d,p)}

$\mathrm{HF}=-648.6734208$ hartrees $(-407049.058286208 \mathrm{kcal} / \mathrm{mol})$

Imaginary Frequencies: none found

Zero-point correction $=0.161122($ Hartree/Particle $)$ 


\begin{tabular}{|c|c|c|c|c|}
\hline \multirow{2}{*}{\multicolumn{5}{|c|}{ Thermal correction to Energy= }} \\
\hline & & & & \\
\hline \multicolumn{5}{|c|}{ Thermal correction to Enthalpy= } \\
\hline \multicolumn{5}{|c|}{ Thermal correction to Gibbs Free Energy $=\quad 0.1254$} \\
\hline \multicolumn{3}{|c|}{$\begin{array}{lr}\text { Thermal correction to Gibbs Free Energy }= & 0.1254 \\
\text { Sum of electronic and zero-point Energies }= & -648\end{array}$} & int Energies= & -648 \\
\hline \\
\hline \multirow{2}{*}{\multicolumn{5}{|c|}{$\begin{array}{l}\text { Sum of electronic and thermal Enthalpies }= \\
\text { Sum of electronic and thermal Free Energies }=\end{array}$}} \\
\hline & & & & \\
\hline \multicolumn{5}{|c|}{ Coordinates (from last standard orientation): } \\
\hline \multirow{2}{*}{$\begin{array}{l}\text { Center } \\
\text { Number }\end{array}$} & \multirow{2}{*}{$\begin{array}{l}\text { Atomic } \\
\text { Numbe }\end{array}$} & \multicolumn{3}{|c|}{ Coordinates (Angstroms) } \\
\hline & & & $X \quad Y$ & Z \\
\hline 1 & 8 & 0.486930 & -0.471856 & 1.371250 \\
\hline 2 & 8 & 2.442743 & 1.981686 & -0.202680 \\
\hline 3 & 6 & 0.535265 & 0.723595 & 0.583285 \\
\hline 4 & 6 & 1.942781 & 0.884998 & -0.011845 \\
\hline 5 & 6 & 2.590509 & -0.389050 & -0.401405 \\
\hline 6 & 6 & 1.908866 & -1.537890 & -0.249125 \\
\hline 7 & 1 & 2.308799 & -2.489200 & -0.593687 \\
\hline 8 & 6 & 0.5 & 7660 & 93438 \\
\hline 9 & 6 & -0.557996 & -1.095345 & -0.627817 \\
\hline 10 & 6 & -0.502637 & 0.448039 & -0.550574 \\
\hline 11 & 1 & 0.286480 & 1.582887 & 1.207172 \\
\hline 12 & 1 & 0.321142 & -2.460452 & 0.901623 \\
\hline 13 & 1 & 3.584103 & -0.336865 & -0.836153 \\
\hline 14 & 6 & -1.995178 & -1.443368 & -0.167613 \\
\hline 15 & 6 & -1.910305 & 0.881516 & -0.145823 \\
\hline 16 & 8 & -2.708283 & -0.204899 & 0.018808 \\
\hline 17 & 8 & -2.304097 & 2.008127 & 0.014434 \\
\hline 18 & 1 & -2.006600 & -1.974616 & 0.788729 \\
\hline 19 & 1 & -0.358614 & -1.484966 & -1.628015 \\
\hline 20 & 1 & -0.237448 & 0.957449 & -1.479756 \\
\hline 21 & 1 & -2.544501 & -2.027711 & -0.909543 \\
\hline
\end{tabular}

\section{MP2/6-31G(d)}

$\mathrm{HF}=-644.9008248$ hartrees $(-404681.716570248 \mathrm{kcal} / \mathrm{mol})$ Imaginary Frequencies: none found Zero-point correction $=0.163898$ (Hartree/Particle)

Thermal correction to Energy $=\quad 0.173353$ Thermal correction to Enthalpy $=\quad 0.174297$ Thermal correction to Gibbs Free Energy $=\quad 0.128129$ Sum of electronic and zero-point Energies $=\quad-646.586748$ Sum of electronic and thermal Energies $=\quad-646.577293$ Sum of electronic and thermal Enthalpies $=\quad-646.576349$ Sum of electronic and thermal Free Energies $=\quad-646.622518$ Coordinates (from last standard orientation):

\begin{tabular}{|c|c|c|c|c|}
\hline \multirow{2}{*}{$\begin{array}{l}\text { Center } \\
\text { Number }\end{array}$} & \multirow{2}{*}{$\begin{array}{l}\text { Atomic } \\
\text { Number }\end{array}$} & & \multicolumn{2}{|c|}{ Coordinates (Angstroms) } \\
\hline & & & $X \quad Y$ & Z \\
\hline 1 & 8 & 0.478923 & $3-0.526945$ & 1.361304 \\
\hline 2 & 8 & 2.414556 & $6 \quad 2.003520$ & -0.107965 \\
\hline 3 & 6 & 0.515495 & 0.686203 & 0.595220 \\
\hline 4 & 6 & 1.914389 & 0.888001 & 0.025108 \\
\hline 5 & 6 & 2.560866 & $6-0.357712$ & -0.436988 \\
\hline
\end{tabular}




$\begin{array}{ccrrr}6 & 6 & 1.893564 & -1.522451 & -0.315623 \\ 7 & 1 & 2.296298 & -2.453357 & -0.711567 \\ 8 & 6 & 0.544033 & -1.541476 & 0.348024 \\ 9 & 6 & -0.568476 & -1.108139 & -0.629271 \\ 10 & 6 & -0.485454 & 0.423214 & -0.561056 \\ 11 & 1 & 0.236692 & 1.526271 & 1.236444 \\ 12 & 1 & 0.346961 & -2.500959 & 0.837094 \\ 13 & 1 & 3.546241 & -0.282213 & -0.889322 \\ 14 & 6 & -1.972555 & -1.422373 & -0.075534 \\ 15 & 6 & -1.880795 & 0.889393 & -0.178128 \\ 16 & 8 & -2.699233 & -0.182555 & 0.024274 \\ 17 & 8 & -2.253384 & 2.034330 & -0.049965 \\ 18 & 1 & -1.919941 & -1.857448 & 0.927147 \\ 19 & 1 & -0.427649 & -1.506127 & -1.637909 \\ 20 & 1 & -0.185636 & 0.924268 & -1.486322 \\ 21 & 1 & -2.546249 & -2.085190 & -0.727270 \\ --------------------------------------------------\end{array}$

\begin{tabular}{|c|c|c|c|c|}
\hline \multicolumn{5}{|c|}{$\begin{array}{l}\mathrm{HF}=-648.6694088 \text { hartrees }(-407046.540716088 \mathrm{kcal} / \\
\text { Imaginary Frequencies: none found } \\
\text { Zero-point correction }=0.156807 \text { (Hartree/Particle) }\end{array}$} \\
\hline \multicolumn{4}{|c|}{ Thermal correction to Energy $=\quad 0$} & 0.166279 \\
\hline \multicolumn{4}{|c|}{ Thermal correction to Enthalpy= } & 0.167223 \\
\hline \multirow{2}{*}{\multicolumn{5}{|c|}{$\begin{array}{lr}\text { Thermal correction to Gibbs Free Energy }= & 0.121 \\
\text { Sum of electronic and zero-point Energies }= & -64\end{array}$}} \\
\hline & & & & \\
\hline \multicolumn{5}{|c|}{$\begin{array}{l}\text { Sum of electronic and zero-point Energies= } \\
\text { Sum of electronic and thermal Energies= }\end{array}$} \\
\hline \multicolumn{5}{|c|}{ Sum of electronic and thermal Enthalpies= } \\
\hline \multirow{2}{*}{\multicolumn{5}{|c|}{$\begin{array}{l}\text { Sum of electronic and thermal Free Energies= } \\
\text { Coordinates (from last standard orientation): }\end{array}$}} \\
\hline & & & & \\
\hline \multirow{2}{*}{$\begin{array}{l}\text { Center } \\
\text { Number }\end{array}$} & \multirow{2}{*}{$\begin{array}{l}\text { Atomic } \\
\text { Numbel }\end{array}$} & & \multicolumn{2}{|c|}{ Coordinates (Angstroms) } \\
\hline & & & $X \quad Y$ & Z \\
\hline 1 & 8 & 0.481540 & -0.474511 & \\
\hline 2 & 8 & 2.440418 & 1.982606 & -0.187436 \\
\hline 3 & 6 & 0.532233 & 0.718437 & 0.584897 \\
\hline 4 & 6 & 1.938482 & 0.878873 & -0.005176 \\
\hline 5 & 6 & 2.582204 & -0.387325 & 00763 \\
\hline 6 & 6 & 1.894786 & -1.5 & 8380 \\
\hline 7 & 1 & 2.283412 & -2.4 & 833 \\
\hline 8 & 6 & 0.531932 & -1.5 & \\
\hline 9 & 6 & -0.560892 & -1.10 & 470 \\
\hline 10 & 6 & -0.495861 & 0.439532 & -0.562066 \\
\hline 11 & 1 & 0.273805 & 1.606766 & 1.225116 \\
\hline 12 & 1 & 0.313681 & -2.481068 & 0.899670 \\
\hline 13 & 1 & 3.573731 & -0.340005 & -0.847812 \\
\hline 14 & 6 & -1.992740 & -1.440740 & -0.157954 \\
\hline 15 & 6 & -1.892432 & 0.880077 & 56813 \\
\hline 16 & 8 & -2.694661 & -0.185623 & 0.042239 \\
\hline 17 & 8 & -2.291877 & 2.014913 & -0.006501 \\
\hline 18 & 1 & -2.008576 & -1.973912 & 0.798097 \\
\hline 19 & 1 & -0.365769 & -1.512505 & -1.653904 \\
\hline 20 & 1 & -0.221311 & 0.945506 & -1.498026 \\
\hline 21 & 1 & -2.558600 & -2.011422 & -0.898879 \\
\hline
\end{tabular}


in $\mathrm{H}_{2}$ O MP2/6-31G(d)

$\mathrm{HF}=-644.9409158$ hartrees $(-404706.874073658 \mathrm{kcal} / \mathrm{mol})$

Imaginary Frequencies: none found

Zero-point correction $=0.158667$ (Hartree/Particle)

Thermal correction to Energy $=\quad 0.167922$

Thermal correction to Enthalpy= $\quad 0.168866$

Thermal correction to Gibbs Free Energy $=0.123706$

Sum of electronic and zero-point Energies $=\quad-646.626310$

Sum of electronic and thermal Energies $=\quad-646.617056$

Sum of electronic and thermal Enthalpies $=\quad-646.616112$

Sum of electronic and thermal Free Energies $=\quad-646.661271$

Coordinates (from last standard orientation):

\begin{tabular}{ccrrr} 
Center & Atomic & \multicolumn{3}{c}{ Coordinates } \\
Number & Number & \multicolumn{2}{c}{ X } & Yngstroms) \\
\hline 1 & 8 & 0.468667 & -0.512867 & 1.367031 \\
2 & 8 & 2.409873 & 1.999969 & -0.123616 \\
3 & 6 & 0.512804 & 0.694914 & 0.592055 \\
4 & 6 & 1.910809 & 0.880704 & 0.022503 \\
5 & 6 & 2.561505 & -0.363609 & -0.419252 \\
6 & 6 & 1.884386 & -1.524061 & -0.297727 \\
7 & 1 & 2.281026 & -2.458069 & -0.697032 \\
8 & 6 & 0.532187 & -1.542405 & 0.356439 \\
9 & 6 & -0.563411 & -1.109925 & -0.637730 \\
10 & 6 & -0.481451 & 0.423262 & -0.571855 \\
11 & 1 & 0.235114 & 1.574265 & 1.247220 \\
12 & 1 & 0.325557 & -2.511130 & 0.850028 \\
13 & 1 & 3.550228 & -0.299261 & -0.872189 \\
14 & 6 & -1.971968 & -1.431747 & -0.108366 \\
15 & 6 & -1.865524 & 0.883713 & -0.177538 \\
16 & 8 & -2.676452 & -0.171931 & 0.069508 \\
17 & 8 & -2.254314 & 2.032782 & -0.058520 \\
18 & 1 & -1.946373 & -1.926525 & 0.867629 \\
19 & 1 & -0.400826 & -1.524136 & -1.670891 \\
20 & 1 & -0.181919 & 0.926920 & -1.503966 \\
21 & 1 & -2.561020 & -2.030763 & -0.807197 \\
----------------------------------------------
\end{tabular}

in $\mathrm{CH}_{3} \mathrm{CN}$ B3LYP/6-31G(d)

$\mathrm{HF}=-648.6504935 \mathrm{hartrees}(-407034.671176185 \mathrm{kcal} / \mathrm{mol})$

Imaginary Frequencies: none found

Zero-point correction $=0.161434$ (Hartree/Particle)

Thermal correction to Energy $=\quad 0.170868$

Thermal correction to Enthalpy $=\quad 0.171812$

Thermal correction to Gibbs Free Energy $=\quad 0.125973$

Sum of electronic and zero-point Energies $=\quad-648.489060$

Sum of electronic and thermal Energies $=\quad-648.479626$

Sum of electronic and thermal Enthalpies $=\quad-648.478682$

Sum of electronic and thermal Free Energies $=\quad-648.524520$

Coordinates (from last standard orientation):

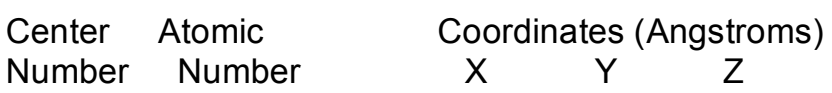




$\begin{array}{rrrrr}1 & 8 & 0.478757 & -0.475657 & 1.369467 \\ 2 & 8 & 2.442500 & 1.982779 & -0.181069 \\ 3 & 6 & 0.532233 & 0.715225 & 0.581131 \\ 4 & 6 & 1.941891 & 0.881295 & -0.005910 \\ 5 & 6 & 2.584279 & -0.387574 & -0.402819 \\ 6 & 6 & 1.900356 & -1.535756 & -0.257704 \\ 7 & 1 & 2.292232 & -2.484171 & -0.619469 \\ 8 & 6 & 0.534596 & -1.522877 & 0.389001 \\ 9 & 6 & -0.562183 & -1.101631 & -0.632031 \\ 10 & 6 & -0.498186 & 0.440799 & -0.563300 \\ 11 & 1 & 0.278623 & 1.577328 & 1.206471 \\ 12 & 1 & 0.318685 & -2.469405 & 0.894267 \\ 13 & 1 & 3.575590 & -0.338857 & -0.845466 \\ 14 & 6 & -1.995377 & -1.438172 & -0.155625 \\ 15 & 6 & -1.897946 & 0.882100 & -0.155607 \\ 16 & 8 & -2.697305 & -0.189455 & 0.041349 \\ 17 & 8 & -2.291425 & 2.014686 & -0.004097 \\ 18 & 1 & -2.005896 & -1.971448 & 0.799460 \\ 19 & 1 & -0.374479 & -1.500335 & -1.633753 \\ 20 & 1 & -0.225631 & 0.942793 & -1.495992 \\ 21 & 1 & -2.557315 & -2.015194 & -0.893545 \\ ------------------------------------------------------\end{array}$

\section{in DMSO B3LYP/6-31G(d)}

$\mathrm{HF}=-648.6504936$ hartrees $(-407034.671238936 \mathrm{kcal} / \mathrm{mol})$ Imaginary Frequencies: none found Zero-point correction $=0.161451$ (Hartree/Particle) Thermal correction to Energy $=\quad 0.170882$ Thermal correction to Enthalpy $=\quad 0.171826$ Thermal correction to Gibbs Free Energy $=\quad 0.126000$ Sum of electronic and zero-point Energies $=\quad-648.489042$ Sum of electronic and thermal Energies $=\quad-648.479612$ Sum of electronic and thermal Enthalpies $=\quad-648.478667$ Sum of electronic and thermal Free Energies $=\quad-648.524494$ Coordinates (from last standard orientation):

\begin{tabular}{|c|c|c|c|c|}
\hline \multirow{2}{*}{$\begin{array}{l}\text { Center } \\
\text { Number }\end{array}$} & \multirow{2}{*}{$\begin{array}{l}\text { Atomic } \\
\text { Number }\end{array}$} & & \multicolumn{2}{|c|}{ Coordinates (Angstroms) } \\
\hline & & & $X \quad Y$ & Z \\
\hline 1 & 8 & 0.478964 & -0.474456 & 1.371185 \\
\hline 2 & 8 & 2.441799 & 1.982515 & -0.185201 \\
\hline 3 & 6 & 0.532764 & 0.716375 & 0.581989 \\
\hline 4 & 6 & 1.942068 & 0.881135 & -0.006660 \\
\hline 5 & 6 & 2.584773 & -0.387932 & -0.401892 \\
\hline 6 & 6 & 1.900451 & -1.535894 & -0.256625 \\
\hline 7 & 1 & 2.292098 & -2.484728 & -0.617602 \\
\hline 8 & 6 & 0.534837 & -1.522009 & 0.390613 \\
\hline 9 & 6 & -0.560780 & -1.100503 & -0.632050 \\
\hline 10 & 6 & -0.498464 & $4 \quad 0.442073$ & -0.561927 \\
\hline 11 & 1 & 0.280269 & 1.578816 & 1.207114 \\
\hline 12 & 1 & 0.318123 & $3-2.468223$ & 0.896124 \\
\hline 13 & 1 & 3.576100 & -0.339964 & -0.844531 \\
\hline 14 & 6 & -1.994375 & $5-1.439084$ & -0.158460 \\
\hline 15 & 6 & -1.898907 & $7 \quad 0.881239$ & -0.154612 \\
\hline 16 & 8 & -2.697756 & $6 \quad-0.191043$ & 0.038746 \\
\hline 17 & 8 & -2.293111 & 2.013345 & -0.001349 \\
\hline
\end{tabular}




$\begin{array}{rrrrr}18 & 1 & -2.005779 & -1.973440 & 0.796012 \\ 19 & 1 & -0.372945 & -1.497337 & -1.634242 \\ 20 & 1 & -0.226503 & 0.945064 & -1.494195 \\ 21 & 1 & -2.554735 & -2.015474 & -0.897984\end{array}$

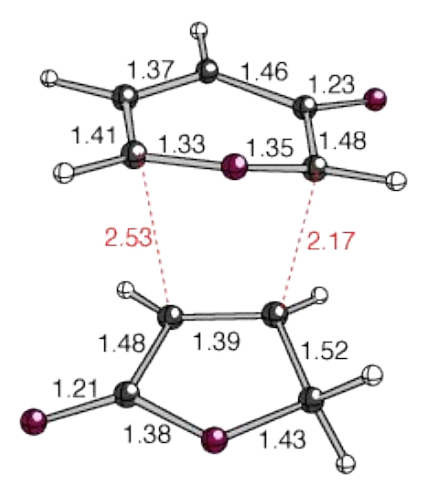

\section{B3LYP/6-31G(d)}

$\mathrm{HF}=-648.5639057$ hartrees $(-406980.336465807 \mathrm{kcal} / \mathrm{mol})$

Imaginary Frequencies: $1(-393.25491 / \mathrm{cm})$

Zero-point correction $=0.156801$ (Hartree/Particle)

Thermal correction to Energy $=\quad 0.167049$

Thermal correction to Enthalpy= $\quad 0.167993$

Thermal correction to Gibbs Free Energy $=\quad 0.120314$

Sum of electronic and zero-point Energies $=\quad-648.407105$

Sum of electronic and thermal Energies $=\quad-648.396857$

Sum of electronic and thermal Enthalpies $=\quad-648.395912$

Sum of electronic and thermal Free Energies $=\quad-648.443592$

Coordinates (from last standard orientation):

\begin{tabular}{|c|c|c|c|c|}
\hline \multirow{2}{*}{$\begin{array}{l}\text { Center } \\
\text { Number }\end{array}$} & \multirow{2}{*}{$\begin{array}{l}\text { Atomic } \\
\text { Number }\end{array}$} & \multicolumn{3}{|c|}{ Coordinates (Angstroms) } \\
\hline & & & $\begin{array}{ll}X & Y\end{array}$ & Z \\
\hline 1 & 8 & 0.455582 & 0.286684 & 1.423350 \\
\hline 2 & 6 & 0.334618 & 1.446428 & 0.776019 \\
\hline 3 & 6 & 1.358198 & 1.854604 & -0.106721 \\
\hline 4 & 6 & 2.344911 & 0.982230 & -0.486405 \\
\hline 5 & 6 & 2.260896 & -0.417956 & -0.099229 \\
\hline 6 & 6 & 1.124325 & -0.701504 & 0.796679 \\
\hline 7 & 6 & -0.415924 & -1.087820 & -0.678333 \\
\hline 8 & 6 & -0.913170 & 0.161976 & -1.008449 \\
\hline 9 & 1 & -0.380971 & 2.116866 & 1.233618 \\
\hline 10 & 1 & 1.140695 & -1.616832 & 1.378890 \\
\hline 11 & 1 & 3.175589 & 1.291067 & -1.113254 \\
\hline 12 & 6 & -1.501918 & -1.773875 & 0.137815 \\
\hline 13 & 6 & -2.218736 & 0.361806 & -0.348821 \\
\hline 14 & 8 & -2.486942 & -0.768019 & 0.397274 \\
\hline 15 & 8 & -2.971069 & 1.305519 & -0.380457 \\
\hline 16 & 1 & -1.176047 & -2.184202 & 1.097683 \\
\hline 17 & 1 & 0.267313 & -1.668190 & -1.290046 \\
\hline 18 & 1 & -0.574544 & 0.816733 & -1.797196 \\
\hline 19 & 1 & -1.962985 & -2.584374 & -0.442276 \\
\hline 20 & 1 & 1.333525 & 2.879088 & -0.467337 \\
\hline 21 & 8 & 2.994706 & -1.324869 & -0.502095 \\
\hline
\end{tabular}


B3LYP/6-31+G(d,p)

$\mathrm{HF}=-648.6046912$ hartrees $(-407005.929774912 \mathrm{kcal} / \mathrm{mol})$

Imaginary Frequencies: 1 (-399.1759 1/cm)

Zero-point correction $=0.156144$ (Hartree/Particle)

Thermal correction to Energy=

0.166389

Thermal correction to Enthalpy=

0.167333

Thermal correction to Gibbs Free Energy=

Sum of electronic and zero-point Energies=

0.119700

Sum of electronic and thermal Energies=

$-648.448547$

Sum of electronic and thermal Enthalpies= $-648.438302$

Sum of electronic and thermal Free Energies=

$-648.437358$

Coordinates (from last standard orientation):

\begin{tabular}{|c|c|c|c|c|}
\hline Center & Atomic & & ordinates & Angstroms) \\
\hline Number & Number & \rangle & $\begin{array}{ll}X & Y\end{array}$ & Z \\
\hline 1 & 8 & 0.468925 & 0.290374 & 1.426559 \\
\hline 2 & 6 & 0.350422 & 1.452546 & 0.782724 \\
\hline 3 & 6 & 1.368920 & 1.853304 & -0.109744 \\
\hline 4 & 6 & 2.351879 & 0.975868 & -0.493277 \\
\hline 5 & 6 & 2.260709 & -0.420287 & -0.100804 \\
\hline 6 & 6 & 1.121489 & -0.706190 & 0.788773 \\
\hline 7 & 6 & -0.415220 & -1.079072 & -0.668540 \\
\hline 8 & 6 & -0.917170 & 0.173878 & -0.995114 \\
\hline 9 & 1 & -0.357829 & 2.128185 & 1.244040 \\
\hline 10 & 1 & 1.137061 & -1.622229 & 1.369631 \\
\hline 11 & 1 & 3.180191 & 1.279094 & -1.125294 \\
\hline 12 & 6 & -1.501853 & -1.775207 & 0.138336 \\
\hline 13 & 6 & -2.228153 & 0.361650 & -0.345720 \\
\hline 14 & 8 & -2.496647 & -0.770920 & 0.393635 \\
\hline 15 & 8 & -2.990044 & 1.301241 & -0.385055 \\
\hline 16 & 1 & -1.183891 & -2.184107 & 1.100527 \\
\hline 17 & 1 & 0.257983 & -1.655779 & -1.294676 \\
\hline 18 & 1 & -0.584381 & 0.829628 & -1.785016 \\
\hline 19 & 1 & -1.959101 & -2.581504 & -0.448749 \\
\hline 20 & 1 & 1.345052 & 2.876089 & -0.474060 \\
\hline 21 & 8 & 2.995113 & -1.331734 & -0.505916 \\
\hline
\end{tabular}

\section{MP2/6-31G(d)}

$\mathrm{HF}=-644.7806363$ hartrees $(-404606.297084613 \mathrm{kcal} / \mathrm{mol})$

Imaginary Frequencies: $1(-341.30011 / \mathrm{cm})$

Zero-point correction $=0.158472$ (Hartree/Particle)

Thermal correction to Energy $=\quad 0.168698$

Thermal correction to Enthalpy= $\quad 0.169642$

Thermal correction to Gibbs Free Energy $=\quad 0.122057$

Sum of electronic and zero-point Energies $=\quad-646.514971$

Sum of electronic and thermal Energies $=\quad-646.504745$

Sum of electronic and thermal Enthalpies $=\quad-646.503801$

Sum of electronic and thermal Free Energies $=\quad-646.551386$

Coordinates (from last standard orientation):

\begin{tabular}{|c|c|}
\hline enter & Atomic \\
\hline Jumber & Number \\
\hline
\end{tabular}




$\begin{array}{rrrrr}1 & 8 & 0.390855 & 0.240582 & 1.425771 \\ 2 & 6 & 0.220487 & 1.404319 & 0.770040 \\ 3 & 6 & 1.252825 & 1.865207 & -0.073656 \\ 4 & 6 & 2.282984 & 1.039138 & -0.451986 \\ 5 & 6 & 2.244459 & -0.364312 & -0.089755 \\ 6 & 6 & 1.129201 & -0.700778 & 0.811697 \\ 7 & 6 & -0.367975 & -1.100070 & -0.711954 \\ 8 & 6 & -0.863655 & 0.146259 & -1.026058 \\ 9 & 1 & -0.520457 & 2.040655 & 1.240937 \\ 10 & 1 & 1.183515 & -1.620655 & 1.389029 \\ 11 & 1 & 3.113423 & 1.395391 & -1.055848 \\ 12 & 6 & -1.433793 & -1.793010 & 0.113099 \\ 13 & 6 & -2.165294 & 0.341139 & -0.356102 \\ 14 & 8 & -2.416732 & -0.785229 & 0.402266 \\ 15 & 8 & -2.924620 & 1.287937 & -0.385688 \\ 16 & 1 & -1.095958 & -2.206029 & 1.066762 \\ 17 & 1 & 0.343699 & -1.662129 & -1.312087 \\ 18 & 1 & -0.527654 & 0.808010 & -1.813580 \\ 19 & 1 & -1.910070 & -2.592924 & -0.466830 \\ 20 & 1 & 1.196700 & 2.894393 & -0.421160 \\ 21 & 8 & 3.003168 & -1.253798 & -0.509745 \\ -------------------------------------------------------\end{array}$

\section{in $\mathrm{H}_{2} \mathrm{O}$ B3LYP/6-31G(d)}

$\mathrm{HF}=-648.5924354$ hartrees $(-406998.239137854 \mathrm{kcal} / \mathrm{mol})$ Imaginary Frequencies: $1(-406.73711 / \mathrm{cm})$

Zero-point correction $=0.155016$ (Hartree/Particle)

Thermal correction to Energy $=\quad 0.165208$

Thermal correction to Enthalpy $=\quad 0.166152$

Thermal correction to Gibbs Free Energy $=\quad 0.118682$

Sum of electronic and zero-point Energies $=\quad-648.437420$

Sum of electronic and thermal Energies $=\quad-648.427228$

Sum of electronic and thermal Enthalpies $=\quad-648.426284$

Sum of electronic and thermal Free Energies $=\quad-648.473753$

Coordinates (from last standard orientation):

\begin{tabular}{llrrr} 
Center & Atomic & \multicolumn{3}{c}{ Coordinates (Angstroms) } \\
Number & Number & X & $Y$ & $Z$ \\
-1 & 8 & 0.466725 & 0.264604 & 1.431403 \\
- & 6 & 0.350728 & 1.442091 & 0.819702 \\
2 & 6 & 1.349567 & 1.859204 & -0.083287 \\
3 & 6 & 2.331249 & 0.988905 & -0.488281 \\
5 & 6 & 2.250268 & -0.407299 & -0.115049 \\
5 & 6 & 1.116034 & -0.719618 & 0.766321 \\
7 & 6 & -0.404683 & -1.079809 & -0.657530 \\
8 & 6 & -0.911006 & 0.169507 & -1.003867 \\
9 & 1 & -0.347757 & 2.114156 & 1.308938 \\
10 & 1 & 1.131642 & -1.654757 & 1.334616 \\
11 & 1 & 3.151872 & 1.312504 & -1.125284 \\
12 & 6 & -1.484108 & -1.768129 & 0.161080 \\
13 & 6 & -2.214420 & 0.355492 & -0.360290 \\
14 & 8 & -2.479959 & -0.751281 & 0.406073 \\
15 & 8 & -3.002960 & 1.282058 & -0.421753 \\
16 & 1 & -1.162240 & -2.166903 & 1.131381 \\
17 & 1 & 0.250644 & -1.670356 & -1.292575
\end{tabular}




$\begin{array}{rrrrr}18 & 1 & -0.569703 & 0.821447 & -1.797204 \\ 19 & 1 & -1.949910 & -2.579761 & -0.420903 \\ 20 & 1 & 1.319766 & 2.890192 & -0.431489 \\ 21 & 8 & 3.000432 & -1.308954 & -0.533258\end{array}$

\begin{tabular}{|c|c|c|c|c|}
\hline \multicolumn{5}{|c|}{$\begin{array}{l}\mathrm{HF}=-644.8161543 \text { hartrees }(-404628.584984793 \mathrm{kcal} / \mathrm{r} \\
\text { Imaginary Frequencies: } 1(-343.87161 / \mathrm{cm}) \\
\text { Zero-point correction }=0.156803(\text { Hartree/Particle })\end{array}$} \\
\hline Thermal c & correction & to Energy= & & $\begin{array}{l}\text { Particle) } \\
0.166856\end{array}$ \\
\hline \multicolumn{4}{|c|}{ Thermal correction to Enthalpy= } & \\
\hline \multicolumn{4}{|c|}{$\begin{array}{l}\text { Thermal correction to Enthalpy= } \\
\text { Thermal correction to Gibbs Free Energy= }\end{array}$} & \\
\hline \multicolumn{5}{|c|}{ Sum of electronic and zero-point Energies= } \\
\hline \multicolumn{5}{|c|}{ Sum of electronic and thermal Energies= } \\
\hline \multicolumn{5}{|c|}{ Sum of electronic and thermal Enthalpies= } \\
\hline \multirow{2}{*}{\multicolumn{5}{|c|}{$\begin{array}{l}\text { Sum of electronic and thermal Free Energies= } \\
\text { Coordinates (from last standard orientation): }\end{array}$}} \\
\hline & & & & \\
\hline \multirow{2}{*}{$\begin{array}{l}\text { Center } \\
\text { Number }\end{array}$} & \multirow{2}{*}{$\begin{array}{l}\text { Atomic } \\
\text { Number }\end{array}$} & \multicolumn{3}{|c|}{ Coordinates (Angstroms) } \\
\hline & & & & \\
\hline 1 & 8 & 0.398014 & 0.211544 & \\
\hline 2 & 6 & 0.22 & & 279 \\
\hline 3 & 6 & 1.229603 & 6916 & 1967 \\
\hline 4 & 6 & 2.2 & & 1237 \\
\hline 5 & 6 & 2.2 & -0. & \\
\hline 6 & & & & \\
\hline 7 & & & & \\
\hline 8 & & & & \\
\hline 9 & 1 & -0.506889 & 2.0 & 8600 \\
\hline 10 & 1 & 1.173097 & -1.666280 & 33341 \\
\hline 11 & 1 & 1712 & & -1.064782 \\
\hline 12 & 6 & -1.41 & & 0.119207 \\
\hline 13 & 6 & & & \\
\hline 14 & 8 & -2.4 & & \\
\hline 15 & 8 & -2.9 & & \\
\hline 16 & 1 & -1.0 & & 1 \\
\hline 17 & 1 & 0.33 & -1.6 & -1.329789 \\
\hline 18 & 1 & -0.525682 & 0.824191 & -1.810489 \\
\hline 19 & 1 & -1.896050 & -2.588656 & -0.470391 \\
\hline 20 & 1 & 1.159395 & 2.900473 & \\
\hline 21 & 8 & 3.025888 & -1.223776 & -0.52365 \\
\hline
\end{tabular}

in $\mathrm{CH}_{3}$ CN B3LYP/6-31G(d)

$\mathrm{HF}=-648.5786013$ hartrees $(-406989.558101763 \mathrm{kcal} / \mathrm{mol})$ Imaginary Frequencies: $1(-401.01681 / \mathrm{cm})$

Zero-point correction $=0.156560($ Hartree/Particle $)$

Thermal correction to Energy $=\quad 0.166762$

Thermal correction to Enthalpy= $\quad 0.167706$

Thermal correction to Gibbs Free Energy $=\quad 0.120178$

Sum of electronic and zero-point Energies $=\quad-648.422041$

Sum of electronic and thermal Energies $=\quad-648.411839$

Sum of electronic and thermal Enthalpies $=\quad-648.410895$

Sum of electronic and thermal Free Energies $=\quad-648.458423$ 
Coordinates (from last standard orientation):

\begin{tabular}{|c|c|c|c|c|}
\hline \multirow{2}{*}{$\begin{array}{l}\text { Center } \\
\text { Number }\end{array}$} & \multirow{2}{*}{$\begin{array}{l}\text { Atomic } \\
\text { Number }\end{array}$} & \multicolumn{3}{|c|}{ Coordinates (Angstroms) } \\
\hline & & & $\begin{array}{ll}X \\
X\end{array}$ & Z \\
\hline 1 & 8 & 0.467579 & 0.275485 & 1.429262 \\
\hline 2 & 6 & 0.351894 & 1.446062 & 0.804252 \\
\hline 3 & 6 & 1.358464 & 1.856861 & -0.094685 \\
\hline 4 & 6 & 2.338073 & 0.982884 & -0.492387 \\
\hline 5 & 6 & 2.256016 & -0.414376 & -0.110562 \\
\hline 6 & 6 & 1.120913 & -0.711804 & 0.778434 \\
\hline 7 & 6 & -0.410245 & -1.077705 & -0.664236 \\
\hline 8 & 6 & -0.914724 & 0.171903 & -1.000865 \\
\hline 9 & 1 & -0.345254 & 2.120853 & 1.285584 \\
\hline 10 & 1 & 1.133218 & -1.635831 & 1.350255 \\
\hline 11 & 1 & 3.159830 & 1.299094 & -1.128171 \\
\hline 12 & 6 & -1.491331 & -1.769155 & 0.151745 \\
\hline 13 & 6 & -2.221401 & 0.357784 & -0.354179 \\
\hline 14 & 8 & -2.486229 & -0.760219 & 0.401998 \\
\hline 15 & 8 & -3.000645 & 1.286925 & -0.406075 \\
\hline 16 & 1 & -1.167887 & -2.171345 & 1.115681 \\
\hline 17 & 1 & 0.255330 & -1.661819 & -1.292067 \\
\hline 18 & 1 & -0.575798 & 0.825957 & -1.790859 \\
\hline 19 & 1 & -1.950589 & -2.580167 & -0.428439 \\
\hline 20 & 1 & 1.333038 & 2.884225 & -0.447599 \\
\hline 21 & 8 & 2.998314 & -1.319153 & -0.521373 \\
\hline
\end{tabular}

\section{in DMSO B3LYP/6-31G(d)}

$\mathrm{HF}=-648.5786863$ hartrees $(-406989.611440113 \mathrm{kcal} / \mathrm{mol})$ Imaginary Frequencies: $1(-401.01891 / \mathrm{cm})$

Zero-point correction $=0.156559$ (Hartree/Particle)

Thermal correction to Energy $=\quad 0.166761$

Thermal correction to Enthalpy $=\quad 0.167705$

Thermal correction to Gibbs Free Energy $=\quad 0.120180$

Sum of electronic and zero-point Energies $=\quad-648.422127$

Sum of electronic and thermal Energies $=\quad-648.411926$

Sum of electronic and thermal Enthalpies $=\quad-648.410981$

Sum of electronic and thermal Free Energies $=\quad-648.458507$

Coordinates (from last standard orientation):

\begin{tabular}{|c|c|c|c|c|}
\hline \multirow{2}{*}{$\begin{array}{l}\text { Center } \\
\text { Number }\end{array}$} & \multirow{2}{*}{$\begin{array}{l}\text { Atomic } \\
\text { Number }\end{array}$} & \multicolumn{3}{|c|}{ Coordinates (Angstroms) } \\
\hline & & & $X \quad Y$ & Z \\
\hline 1 & 8 & 0.467673 & 0.275365 & 1.429294 \\
\hline 2 & 6 & 0.352025 & 1.446021 & 0.804464 \\
\hline 3 & 6 & 1.358439 & 1.856874 & -0.094605 \\
\hline 4 & 6 & 2.337979 & 0.982888 & -0.492471 \\
\hline 5 & 6 & 2.255994 & -0.414353 & -0.110643 \\
\hline 6 & 6 & 1.120946 & -0.711902 & 0.778351 \\
\hline 7 & 6 & -0.410287 & -1.077762 & -0.664109 \\
\hline 8 & 6 & -0.914724 & 0.171845 & -1.000856 \\
\hline 9 & 1 & -0.344922 & 2.120849 & 1.286033 \\
\hline 10 & 1 & 1.133233 & -1.635991 & 1.350095 \\
\hline 11 & 1 & 3.159653 & 1.299159 & -1.128338 \\
\hline 12 & 6 & -1.491429 & -1.769143 & 0.151830 \\
\hline
\end{tabular}




$\begin{array}{rrrrr}13 & 6 & -2.221379 & 0.357822 & -0.354229 \\ 14 & 8 & -2.486324 & -0.760115 & 0.401966 \\ 15 & 8 & -3.000628 & 1.287008 & -0.406207 \\ 16 & 1 & -1.168064 & -2.171282 & 1.115816 \\ 17 & 1 & 0.255207 & -1.661959 & -1.291934 \\ 18 & 1 & -0.575686 & 0.825893 & -1.790812 \\ 19 & 1 & -1.950702 & -2.580156 & -0.428342 \\ 20 & 1 & 1.332993 & 2.884266 & -0.447444 \\ 21 & 8 & 2.998393 & -1.319072 & -0.521486\end{array}$

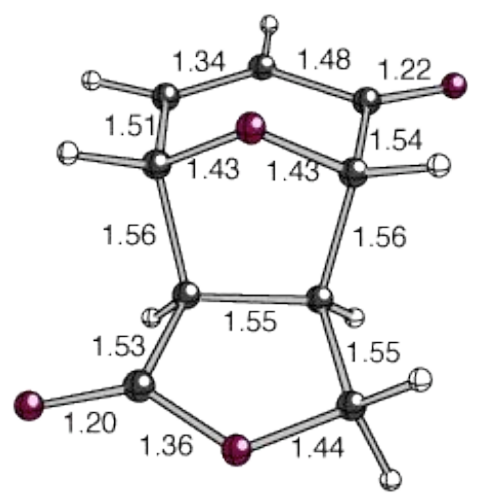

B3LYP/6-31G(d)

$\mathrm{HF}=-648.6366717$ hartrees $(-407025.997858467 \mathrm{kcal} / \mathrm{mol})$ Imaginary Frequencies: none found Zero-point correction $=0.161953$ (Hartree/Particle)

Thermal correction to Energy= 0.171431

Thermal correction to Enthalpy= 0.172375

Thermal correction to Gibbs Free Energy $=0.126228$

Sum of electronic and zero-point Energies= $-648.474718$ Sum of electronic and thermal Energies= $-648.465241$

Sum of electronic and thermal Enthalpies= Sum of electronic and thermal Free Energies= $-648.464297$ Coordinates (from last standard orientation):

\begin{tabular}{llrrr} 
Center & Atomic & \multicolumn{3}{c}{ Coordinates (Angstroms) } \\
Number & Number & $X$ & $Y$ & $Z$ \\
\hdashline 1 & 8 & 0.386066 & 0.326216 & 1.365992 \\
2 & 6 & 0.063812 & 1.302380 & 0.372135 \\
3 & 6 & 1.336805 & 1.823135 & -0.257028 \\
4 & 6 & 2.394741 & 1.005606 & -0.389156 \\
5 & 6 & 2.255031 & -0.415046 & 0.002520 \\
6 & 6 & 0.882887 & -0.771161 & 0.595940 \\
7 & 6 & -0.170326 & -0.941039 & -0.541324 \\
8 & 6 & -0.772200 & 0.476860 & -0.653381 \\
9 & 1 & -0.521933 & 2.094379 & 0.843073 \\
10 & 1 & 0.979691 & -1.643885 & 1.245206 \\
11 & 1 & 3.342853 & 1.319678 & -0.816375 \\
12 & 6 & -1.382506 & -1.824265 & -0.153200 \\
13 & 6 & -2.217406 & 0.340076 & -0.176769 \\
14 & 8 & -2.507302 & -0.959088 & 0.080386 \\
15 & 8 & -3.011033 & 1.231595 & -0.023535 \\
16 & 1 & -1.212035 & -2.396740 & 0.763001 \\
17 & 1 & 0.291127 & -1.303287 & -1.462565
\end{tabular}




$\begin{array}{rrrrr}18 & 1 & -0.761967 & 0.913485 & -1.654793 \\ 19 & 1 & -1.655441 & -2.517234 & -0.954588 \\ 20 & 1 & 1.358301 & 2.851387 & -0.612229 \\ 21 & 8 & 3.111566 & -1.260856 & -0.191488\end{array}$

\section{B3LYP/6-31+G(d,p)}

$\mathrm{HF}=-648.6751135$ hartrees $(-407050.120472385 \mathrm{kcal} / \mathrm{mol})$ Imaginary Frequencies: none found

Zero-point correction $=0.161199$ (Hartree/Particle)

Thermal correction to Energy $=\quad 0.170676$

Thermal correction to Enthalpy= $\quad 0.171620$

Thermal correction to Gibbs Free Energy $=\quad 0.125593$

Sum of electronic and zero-point Energies $=\quad-648.513915$

Sum of electronic and thermal Energies $=\quad-648.504438$

Sum of electronic and thermal Enthalpies $=\quad-648.503493$

Sum of electronic and thermal Free Energies $=\quad-648.549520$

Coordinates (from last standard orientation):

\begin{tabular}{|c|c|c|c|c|}
\hline \multirow{2}{*}{$\begin{array}{l}\text { Center } \\
\text { Number }\end{array}$} & \multirow{2}{*}{$\begin{array}{l}\text { Atomic } \\
\text { Number }\end{array}$} & \multicolumn{3}{|c|}{ Coordinates (Angstroms) } \\
\hline & & & $X \quad Y$ & Z \\
\hline 1 & 8 & 0.407270 & 0.302305 & 1.380102 \\
\hline 2 & 6 & 0.065639 & 1.293405 & 0.404732 \\
\hline 3 & 6 & 1.330187 & 1.829120 & -0.228883 \\
\hline 4 & 6 & 2.391939 & 1.018128 & -0.387364 \\
\hline 5 & 6 & 2.258281 & -0.408533 & -0.019786 \\
\hline 6 & 6 & 0.899317 & -0.784621 & 0.588575 \\
\hline 7 & 6 & -0.174012 & -0.943518 & -0.530073 \\
\hline 8 & 6 & -0.770959 & 0.478921 & -0.632266 \\
\hline 9 & 1 & -0.516361 & 2.075496 & 0.895450 \\
\hline 10 & 1 & 1.014141 & -1.663746 & 1.225409 \\
\hline 11 & 1 & 3.333753 & 1.344015 & -0.818325 \\
\hline 12 & 6 & -1.381861 & -1.823494 & -0.121070 \\
\hline 13 & 6 & -2.225073 & 0.342498 & -0.189036 \\
\hline 14 & 8 & -2.517157 & -0.953729 & 0.074591 \\
\hline 15 & 8 & -3.029593 & 1.233112 & -0.072123 \\
\hline 16 & 1 & -1.216889 & -2.358220 & 0.817873 \\
\hline 17 & 1 & 0.268309 & -1.305127 & -1.460513 \\
\hline 18 & 1 & -0.745924 & 0.925425 & -1.628548 \\
\hline 19 & 1 & -1.650690 & -2.542184 & -0.899377 \\
\hline 20 & 1 & 1.344730 & 2.863711 & -0.564463 \\
\hline 21 & 8 & 3.115504 & -1.250539 & -0.242129 \\
\hline
\end{tabular}

MP2/6-31G(d)

$\mathrm{HF}=-644.9025004$ hartrees $(-404682.768026004 \mathrm{kcal} / \mathrm{mol})$ Imaginary Frequencies: none found

Zero-point correction $=0.163897$ (Hartree/Particle)

Thermal correction to Energy $=\quad 0.173275$

Thermal correction to Enthalpy= $\quad 0.174219$

Thermal correction to Gibbs Free Energy $=0.128373$

Sum of electronic and zero-point Energies $=\quad-646.588199$

Sum of electronic and thermal Energies $=\quad-646.578822$

Sum of electronic and thermal Enthalpies $=\quad-646.577877$

Sum of electronic and thermal Free Energies $=\quad-646.623724$ 
Coordinates (from last standard orientation):

\begin{tabular}{|c|c|c|c|c|}
\hline Center & Atomic & & oordinates ( & Angstroms) \\
\hline Number & Number & & $\mathrm{Y}$ & Z \\
\hline 1 & 8 & -0.316392 & -0.426166 & 1.317532 \\
\hline 2 & 6 & -0.044755 & -1.351352 & 0.254618 \\
\hline 3 & 6 & -1.333418 & -1.796112 & -0.379394 \\
\hline 4 & 6 & -2.385539 & -0.953050 & -0.415251 \\
\hline 5 & 6 & -2.216162 & 0.422885 & 0.094464 \\
\hline 6 & 6 & -0.815512 & 0.720610 & 0.614802 \\
\hline 7 & 6 & 0.142497 & 0.903789 & -0.593176 \\
\hline 8 & 6 & 0.788907 & -0.483588 & -0.709498 \\
\hline 9 & 1 & 0.531467 & -2.181877 & 0.671181 \\
\hline 10 & 1 & -0.841477 & 1.568954 & 1.304237 \\
\hline 11 & 1 & -3.349200 & -1.224812 & -0.838109 \\
\hline 12 & 6 & 1.336291 & 1.831744 & -0.327464 \\
\hline 13 & 6 & 2.177869 & -0.307948 & -0.109258 \\
\hline 14 & 8 & 2.423977 & 1.008372 & 0.140818 \\
\hline 15 & 8 & 2.965350 & -1.189473 & 0.156426 \\
\hline 16 & 1 & 1.139552 & 2.580614 & 0.443244 \\
\hline 17 & 1 & -0.403237 & 1.221741 & -1.486919 \\
\hline 18 & 1 & 0.858453 & -0.890439 & -1.722945 \\
\hline 19 & 1 & 1.662362 & 2.338088 & -1.241685 \\
\hline 20 & 1 & -1.384799 & -2.790576 & -0.819598 \\
\hline 21 & 8 & -3.087208 & 1.289324 & 0.024166 \\
\hline
\end{tabular}

\section{in $\mathrm{H}_{2} \mathrm{O}$ B3LYP/6-31G(d)}

$\mathrm{HF}=-648.6693622$ hartrees $(-407046.511474122 \mathrm{kcal} / \mathrm{mol})$ Imaginary Frequencies: none found Zero-point correction $=0.156730$ (Hartree/Particle)

Thermal correction to Energy $=\quad 0.166210$ Thermal correction to Enthalpy= $\quad 0.167154$ Thermal correction to Gibbs Free Energy $=\quad 0.121161$ Sum of electronic and zero-point Energies $=\quad-648.512632$ Sum of electronic and thermal Energies $=\quad-648.503153$ Sum of electronic and thermal Enthalpies $=\quad-648.502208$ Sum of electronic and thermal Free Energies $=\quad-648.548201$ Coordinates (from last standard orientation):

\begin{tabular}{llrrr} 
Center & Atomic & \multicolumn{3}{c}{ Coordinates (Angstroms) } \\
Number & Number & X & $Y$ & $Z$ \\
\hdashline 1 & 8 & 0.377300 & 0.355555 & 1.354205 \\
2 & 6 & 0.059574 & 1.325681 & 0.345286 \\
3 & 6 & 1.333466 & 1.812060 & -0.303113 \\
4 & 6 & 2.393066 & 0.988951 & -0.403960 \\
5 & 6 & 2.246628 & -0.413903 & 0.029091 \\
6 & 6 & 0.865852 & -0.761471 & 0.595316 \\
7 & 6 & -0.162615 & -0.922591 & -0.567053 \\
8 & 6 & -0.786067 & 0.489899 & -0.662786 \\
9 & 1 & -0.515896 & 2.164974 & 0.821034 \\
10 & 1 & 0.937053 & -1.642153 & 1.256871 \\
11 & 1 & 3.342229 & 1.293352 & -0.841900 \\
12 & 6 & -1.359316 & -1.832744 & -0.221323
\end{tabular}




$\begin{array}{rrrrr}13 & 6 & -2.209578 & 0.324432 & -0.151475 \\ 14 & 8 & -2.477343 & -0.970347 & 0.115853 \\ 15 & 8 & -3.032895 & 1.195366 & 0.030982 \\ 16 & 1 & -1.178428 & -2.479395 & 0.642225 \\ 17 & 1 & 0.335366 & -1.264076 & -1.511973 \\ 18 & 1 & -0.808714 & 0.929677 & -1.669348 \\ 19 & 1 & -1.667812 & -2.446163 & -1.073373 \\ 20 & 1 & 1.356220 & 2.826049 & -0.700965 \\ 21 & 8 & 3.122177 & -1.261092 & -0.111348\end{array}$

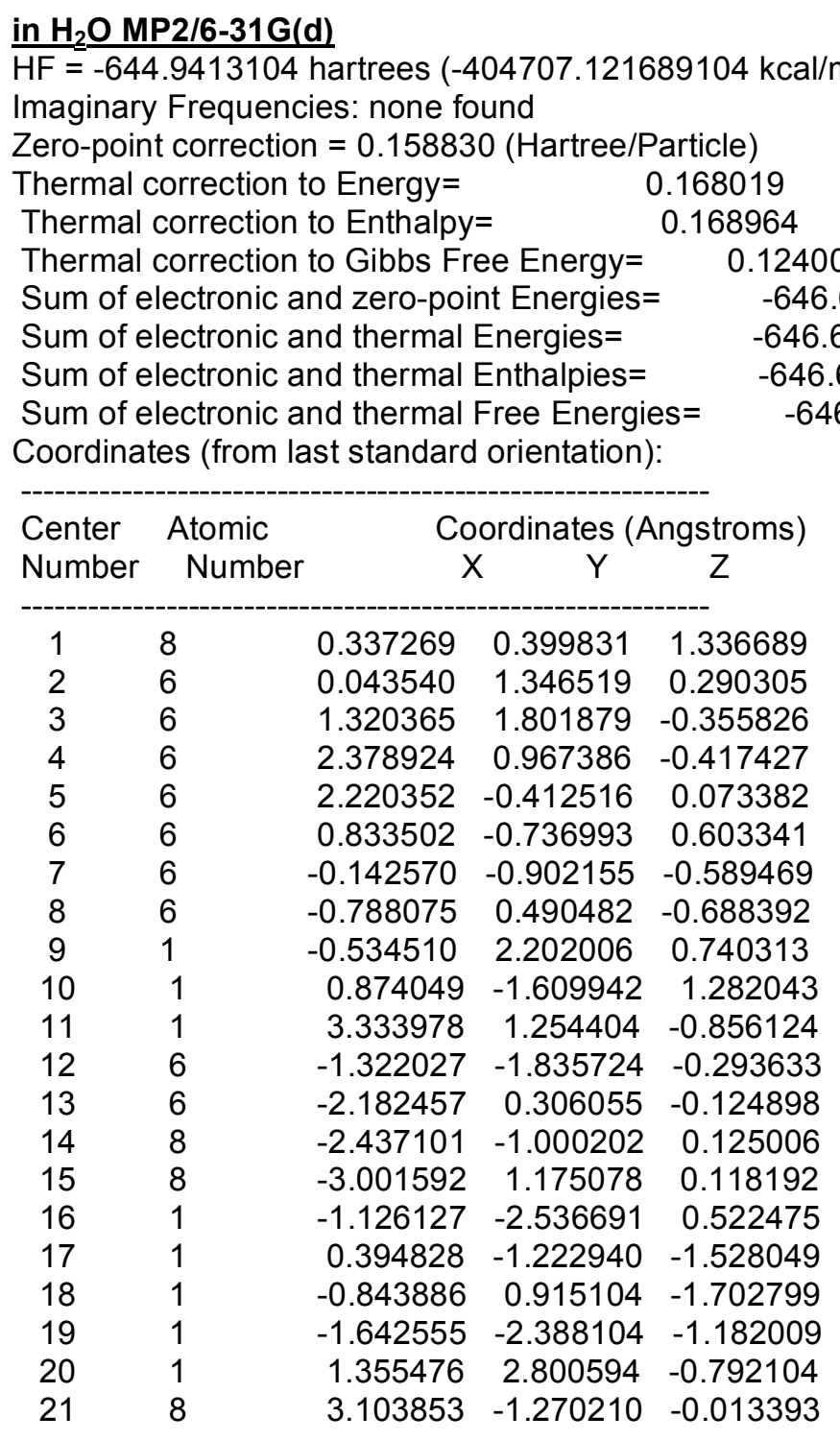

in $\mathrm{CH}_{3} \mathrm{CN}$ B3LYP/6-31G(d)

$\mathrm{HF}=-648.6504546$ hartrees $(-407034.646766046 \mathrm{kcal} / \mathrm{mol})$ Imaginary Frequencies: none found

Zero-point correction $=0.161369$ (Hartree/Particle)

Thermal correction to Energy $=\quad 0.170813$

Thermal correction to Enthalpy $=\quad 0.171757$ 


\begin{tabular}{|c|c|c|c|c|}
\hline \\
\hline \\
\hline \multicolumn{5}{|c|}{$\begin{array}{ll}\text { Sum of electronic and zero-point Energies }= & -648 \\
\text { Sum } & -648 \text { electronic }\end{array}$} \\
\hline \multicolumn{5}{|c|}{ Sum of electronic and thermal Enthalpies $=\quad-648$} \\
\hline \\
\hline & & & & \\
\hline \multirow{2}{*}{$\begin{array}{l}\text { Center } \\
\text { Number }\end{array}$} & \multirow{2}{*}{$\begin{array}{l}\text { Atomic } \\
\text { Number }\end{array}$} & \multicolumn{3}{|c|}{ Coordinates (Angstroms) } \\
\hline & & Coordinates (Angstroms) & $X \quad Y$ & Z \\
\hline 1 & 8 & 0.378580 & 0.345913 & 1.353003 \\
\hline \multirow{2}{*}{$\begin{array}{l}2 \\
3\end{array}$} & 6 & 0.062685 & & 0.349051 \\
\hline & 6 & 1.336745 & 13351 & -0.295923 \\
\hline \multirow{2}{*}{$\begin{array}{l}4 \\
5\end{array}$} & 6 & 2.394374 & 0.990902 & -0.404855 \\
\hline & 6 & 2.252261 & -0.415915 & 0.022865 \\
\hline 6 & 6 & 0.869476 & -0.764321 & 0.590735 \\
\hline & 6 & 3032 & & -0.563942 \\
\hline & & & & -0.661 \\
\hline 9 & 1 & -0.5 & & 0.817 \\
\hline 10 & 1 & & -1.637173 & 1.245503 \\
\hline 11 & 1 & 3.341871 & 1.297432 & -0.840004 \\
\hline 12 & 6 & -1.370637 & -1.829804 & -0.208261 \\
\hline & 6 & -2.213709 & 0.330776 & -0.153802 \\
\hline & 8 & -2.489938 & -0.967500 & 0.099335 \\
\hline 15 & 8 & -3.022014 & 1.209575 & 0.033443 \\
\hline 16 & 1 & -1.1 & -2.45 & 0.669242 \\
\hline \multirow{2}{*}{$\begin{array}{l}17 \\
18\end{array}$} & 1 & 0.30 & -1.266182 & -1.490983 \\
\hline & 1 & -0.799295 & 0.923512 & -1.664626 \\
\hline \multirow{2}{*}{$\begin{array}{l}19 \\
20\end{array}$} & 1 & -1.665065 & -2.466090 & -1.047415 \\
\hline & 1 & 1.359368 & 2.830531 & -0.681626 \\
\hline 21 & 8 & 3.125806 & -1.260146 & -0.117338 \\
\hline
\end{tabular}

in DMSO B3LYP/6-31G(d)

$\mathrm{HF}=-648.6505162$ hartrees $(-407034.685420662 \mathrm{kcal} / \mathrm{mol})$ Imaginary Frequencies: none found

Zero-point correction $=0.161351$ (Hartree/Particle)

Thermal correction to Energy $=\quad 0.170800$

Thermal correction to Enthalpy= $\quad 0.171744$

Thermal correction to Gibbs Free Energy $=0.125768$

Sum of electronic and zero-point Energies $=\quad-648.489165$

Sum of electronic and thermal Energies $=\quad-648.479716$

Sum of electronic and thermal Enthalpies $=\quad-648.478772$

Sum of electronic and thermal Free Energies $=\quad-648.524748$

Coordinates (from last standard orientation):

\begin{tabular}{|c|c|c|c|c|}
\hline \multirow{2}{*}{$\begin{array}{l}\text { Center } \\
\text { Number }\end{array}$} & \multirow{2}{*}{$\begin{array}{l}\text { Atomic } \\
\text { Number }\end{array}$} & \multicolumn{3}{|c|}{ Coordinates (Angstroms) } \\
\hline & & & $\begin{array}{ll}X & Y\end{array}$ & Z \\
\hline 1 & 8 & 0.373577 & 0.355666 & 1.348366 \\
\hline 2 & 6 & 0.062370 & 322913 & 0.338425 \\
\hline 3 & 6 & 707 & 1.810428 & -0.308024 \\
\hline 4 & 6 & 2.395312 & 0.985412 & -0.407783 \\
\hline 5 & 6 & 2.250303 & -0.417800 & 0.031072 \\
\hline 6 & 6 & 0.863746 & -0.759842 & 0.593170 \\
\hline 7 & 6 & -0.166510 & -0.923410 & -0.568717 \\
\hline
\end{tabular}




$\begin{array}{ccrrr}8 & 6 & -0.787126 & 0.488985 & -0.666711 \\ 9 & 1 & -0.502409 & 2.138874 & 0.801268 \\ 10 & 1 & 0.932679 & -1.630367 & 1.251683 \\ 11 & 1 & 3.344336 & 1.287284 & -0.842868 \\ 12 & 6 & -1.368825 & -1.830658 & -0.225136 \\ 13 & 6 & -2.211818 & 0.328575 & -0.147154 \\ 14 & 8 & -2.483253 & -0.970715 & 0.106441 \\ 15 & 8 & -3.019747 & 1.205593 & 0.049807 \\ 16 & 1 & -1.189094 & -2.477546 & 0.637443 \\ 17 & 1 & 0.313960 & -1.260750 & -1.492982 \\ 18 & 1 & -0.809444 & 0.922467 & -1.670584 \\ 19 & 1 & -1.669372 & -2.447469 & -1.076730 \\ 20 & 1 & 1.364059 & 2.824426 & -0.701863 \\ 21 & 8 & 3.124214 & -1.263611 & -0.097141 \\ ----------------------------------------------------\end{array}$

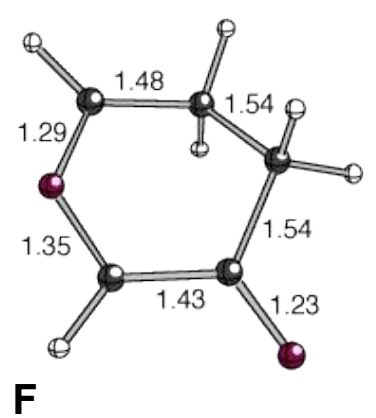

$\mathrm{HF}=-344.4980834$ hartrees $(-216175.992314334 \mathrm{kcal} / \mathrm{mol})$

Imaginary Frequencies: none found

Zero-point correction $=0.102023($ Hartree/Particle $)$

Thermal correction to Energy $=\quad 0.107963$

Thermal correction to Enthalpy= $\quad 0.108907$

Thermal correction to Gibbs Free Energy $=\quad 0.072421$

Sum of electronic and zero-point Energies $=\quad-344.396060$

Sum of electronic and thermal Energies $=\quad-344.390121$

Sum of electronic and thermal Enthalpies $=\quad-344.389176$

Sum of electronic and thermal Free Energies $=\quad-344.425662$

Coordinates (from last standard orientation):

\begin{tabular}{|c|c|c|c|c|}
\hline \multirow{2}{*}{$\begin{array}{l}\text { Center } \\
\text { Number }\end{array}$} & \multirow{2}{*}{$\begin{array}{l}\text { Atomic } \\
\text { Number }\end{array}$} & \multicolumn{3}{|c|}{ Coordinates (Angstroms) } \\
\hline & & & $X \quad Y$ & Z \\
\hline 1 & 8 & 0.999683 & -1.219005 & -0.098863 \\
\hline 2 & 8 & -2.335478 & 0.001216 & 0.088657 \\
\hline 3 & 6 & -0.340188 & -1.228176 & 0.091849 \\
\hline 4 & 6 & -1.105208 & -0.024330 & 0.006305 \\
\hline 5 & 6 & -0.286696 & 1.242674 & -0.295312 \\
\hline 6 & 6 & 1.130322 & 1.130657 & 0.291928 \\
\hline 7 & 1 & 1.044435 & 1.145917 & 1.397838 \\
\hline 8 & 6 & 1.748969 & -0.163852 & -0.079284 \\
\hline 9 & 1 & -0.755772 & -2.224655 & 0.085732 \\
\hline 10 & 1 & 2.801548 & $3 \quad-0.365478$ & -0.231245 \\
\hline 11 & 1 & -0.814881 & $1 \quad 2.108137$ & 0.112173 \\
\hline 12 & 1 & -0.248467 & 1.368370 & -1.387045 \\
\hline 13 & 1 & 1.776301 & 1.968184 & 0.011273 \\
\hline
\end{tabular}




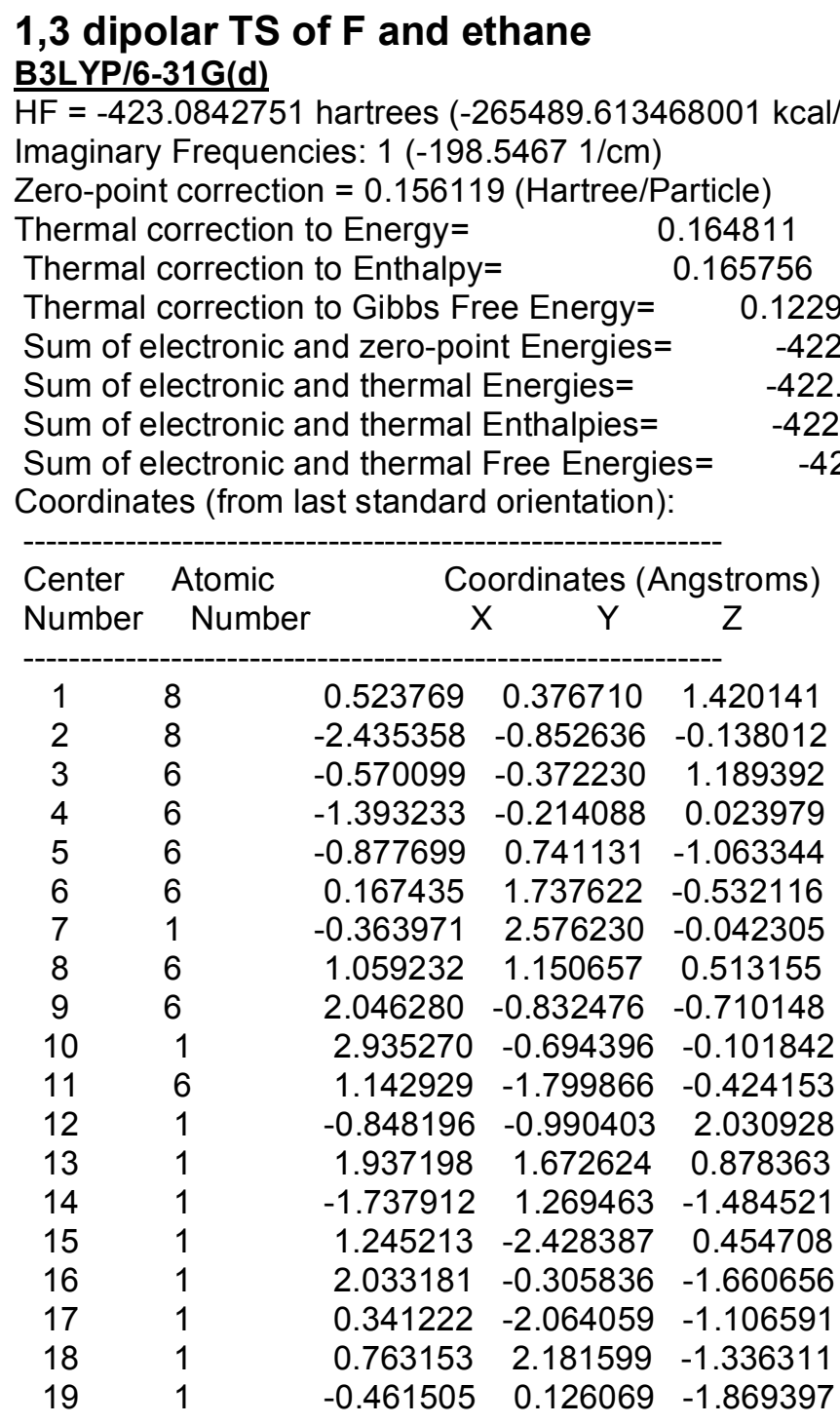

\section{in $\mathrm{H}_{2} \mathrm{O}$ B3LYP/6-31G(d)}

$\mathrm{HF}=-423.1017457 \mathrm{hartrees}(-265500.576444207 \mathrm{kcal} / \mathrm{mol})$

Imaginary Frequencies: $1(-240.31121 / \mathrm{cm})$

Zero-point correction $=0.154904$ (Hartree/Particle)

Thermal correction to Energy $=\quad 0.163469$

Thermal correction to Enthalpy= $\quad 0.164413$

Thermal correction to Gibbs Free Energy $=\quad 0.121969$

Sum of electronic and zero-point Energies $=\quad-422.946842$

Sum of electronic and thermal Energies $=\quad-422.938277$

Sum of electronic and thermal Enthalpies $=\quad-422.937333$

Sum of electronic and thermal Free Energies $=\quad-422.979776$

Coordinates (from last standard orientation):

\begin{tabular}{|c|c|}
\hline Center & Atomic \\
\hline & Number \\
\hline
\end{tabular}




$\begin{array}{rrrrr}1 & 8 & 0.537450 & 0.314310 & 1.436511 \\ 2 & 8 & -2.480478 & -0.760216 & -0.160675 \\ 3 & 6 & -0.610273 & -0.369232 & 1.188502 \\ 4 & 6 & -1.401340 & -0.159839 & 0.027644 \\ 5 & 6 & -0.861239 & 0.800007 & -1.037181 \\ 6 & 6 & 0.272726 & 1.697488 & -0.513861 \\ 7 & 1 & -0.172523 & 2.574505 & -0.001862 \\ 8 & 6 & 1.120964 & 1.044790 & 0.525652 \\ 9 & 6 & 2.011467 & -0.840223 & -0.694513 \\ 10 & 1 & 2.891959 & -0.783300 & -0.060344 \\ 11 & 6 & 1.063944 & -1.791489 & -0.489777 \\ 12 & 1 & -0.916491 & -0.990343 & 2.028039 \\ 13 & 1 & 2.046589 & 1.502741 & 0.878699 \\ 14 & 1 & -1.695632 & 1.415993 & -1.393748 \\ 15 & 1 & 1.111891 & -2.465197 & 0.360361 \\ 16 & 1 & 2.055164 & -0.278695 & -1.624148 \\ 17 & 1 & 0.281802 & -1.991820 & -1.215782 \\ 18 & 1 & 0.897178 & 2.096528 & -1.321491 \\ 19 & 1 & -0.533198 & 0.197826 & -1.895202 \\ ------------------------------------------\end{array}$

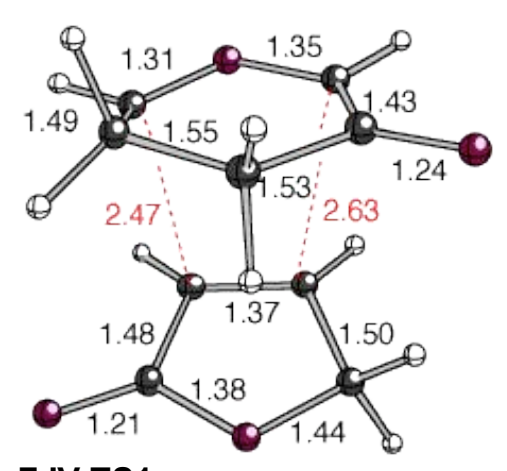

\section{F.IV.TS1}

$\mathrm{HF}=-649.7621721$ hartrees $(-407732.260614471 \mathrm{kcal} / \mathrm{mol})$ Imaginary Frequencies: $1(-247.87431 / \mathrm{cm})$

Zero-point correction $=0.179568$ (Hartree/Particle $)$

Thermal correction to Energy $=\quad 0.107963$

Thermal correction to Enthalpy $=\quad 0.108907$

Thermal correction to Gibbs Free Energy $=\quad 0.072421$

Sum of electronic and zero-point Energies $=\quad-344.396060$

Sum of electronic and thermal Energies $=\quad-344.390121$

Sum of electronic and thermal Enthalpies $=\quad-344.389176$

Sum of electronic and thermal Free Energies $=\quad-344.425662$

Coordinates (from last standard orientation):

\begin{tabular}{lrrrr} 
Center & \multicolumn{2}{c}{ Atomic } & \multicolumn{3}{c}{ Coordinates (Angstroms) } \\
Number & Number & X & $Y$ & \multicolumn{1}{c}{ Z } \\
\hline 1 & 8 & 1.372590 & -1.148693 & 1.233433 \\
2 & 8 & 2.235229 & 1.708689 & -0.703108 \\
3 & 6 & 1.834821 & 0.079467 & 0.916430 \\
4 & 8 & -1.838402 & 1.104341 & -0.637524 \\
5 & 6 & 1.787759 & 0.593075 & -0.420273 \\
6 & 6 & 1.062833 & -0.239636 & -1.483095 \\
7 & 6 & 0.636041 & -1.653600 & -1.024964
\end{tabular}




$\begin{array}{ccrrr}8 & 1 & 1.383386 & -2.394860 & -1.358981 \\ 9 & 6 & 0.550773 & -1.808391 & 0.458259 \\ 10 & 6 & -1.265650 & -0.280836 & 1.125028 \\ 11 & 1 & -1.467586 & -1.064224 & 1.841055 \\ 12 & 6 & -0.625749 & 0.907778 & 1.347640 \\ 13 & 6 & -1.008440 & 1.867230 & 0.255720 \\ 14 & 1 & 2.436523 & 0.520862 & 1.698439 \\ 15 & 1 & 0.228882 & -2.745043 & 0.901898 \\ 16 & 1 & 1.723636 & -0.301795 & -2.353236 \\ 17 & 1 & -0.165167 & 1.236946 & 2.268923 \\ 18 & 6 & -2.049520 & -0.158969 & -0.125262 \\ 19 & 8 & -2.744494 & -0.971950 & -0.686840 \\ 20 & 1 & 0.195982 & 0.345045 & -1.805925 \\ 21 & 1 & -0.314232 & -1.956757 & -1.476015 \\ 22 & 1 & -0.161857 & 2.276556 & -0.309517 \\ 23 & 1 & -1.596149 & 2.707470 & 0.648762 \\ ----------------------------------------------\end{array}$

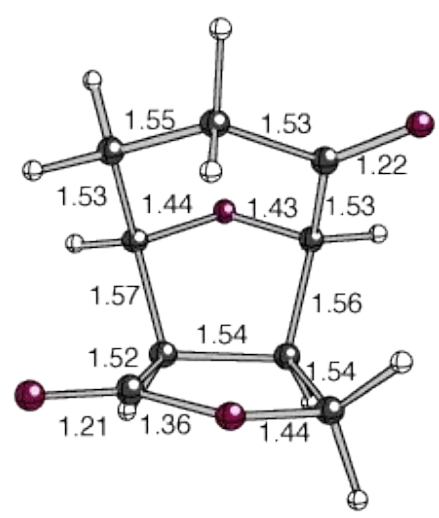

F.IV.TS1 product

$\mathrm{HF}=-649.8606353$ hartrees $(-407794.047257103 \mathrm{kcal} / \mathrm{mol})$

Imaginary Frequencies: none found

Zero-point correction $=0.186001$ (Hartree/Particle)

Thermal correction to Energy $=\quad 0.195766$

Thermal correction to Enthalpy $=\quad 0.196710$

Thermal correction to Gibbs Free Energy $=\quad 0.150132$

Sum of electronic and zero-point Energies $=\quad-649.674635$

Sum of electronic and thermal Energies $=\quad-649.664870$

Sum of electronic and thermal Enthalpies $=\quad-649.663925$

Sum of electronic and thermal Free Energies $=\quad-649.710504$

Coordinates (from last standard orientation):

\begin{tabular}{|c|c|c|c|c|}
\hline \multirow{2}{*}{$\begin{array}{l}\text { Center } \\
\text { Number }\end{array}$} & \multirow{2}{*}{$\begin{array}{l}\text { Atomic } \\
\text { Number }\end{array}$} & \multicolumn{3}{|c|}{ Coordinates (Angstroms) } \\
\hline & & & $\begin{array}{l}X \\
Y \quad Y\end{array}$ & Z \\
\hline 1 & 8 & 1.213338 & -0.985301 & 1.392377 \\
\hline 2 & 8 & 2.457183 & 1.203406 & -0.988835 \\
\hline 3 & 6 & 1.292866 & 0.365341 & 0.933110 \\
\hline 4 & 8 & -1.622197 & 1.271149 & -0.655622 \\
\hline 5 & 6 & 1.684283 & 0.374347 & -0.549966 \\
\hline 6 & 6 & 1.007870 & -0.667927 & -1.437889 \\
\hline 7 & 6 & 0.371339 & -1.859382 & -0.681712 \\
\hline 8 & 1 & 1.088726 & -2.685440 & -0.632732 \\
\hline 9 & 6 & 0.024572 & -1.499956 & 0.767488 \\
\hline
\end{tabular}




$\begin{array}{rrrrr}10 & 6 & -1.011082 & -0.341119 & 0.967714 \\ 11 & 1 & -1.667248 & -0.574972 & 1.810344 \\ 12 & 6 & -0.141537 & 0.910317 & 1.199307 \\ 13 & 6 & -0.725494 & 1.965065 & 0.238367 \\ 14 & 1 & 2.065329 & 0.884930 & 1.503420 \\ 15 & 1 & -0.266320 & -2.394681 & 1.321945 \\ 16 & 1 & 1.738510 & -1.000437 & -2.182226 \\ 17 & 1 & -0.183908 & 1.259073 & 2.233504 \\ 18 & 6 & -1.878343 & -0.002144 & -0.238999 \\ 19 & 8 & -2.669690 & -0.714801 & -0.801125 \\ 20 & 1 & 0.242960 & -0.118123 & -2.003038 \\ 21 & 1 & -0.515071 & -2.224667 & -1.209231 \\ 22 & 1 & 0.027324 & 2.468606 & -0.373654 \\ 23 & 1 & -1.306226 & 2.722837 & 0.772786 \\ ------------------------------------------------\end{array}$

\section{F.IV.TS2}

$\mathrm{HF}=-649.7541037$ hartrees $(-407727.197612787 \mathrm{kcal} / \mathrm{mol})$ Imaginary Frequencies: 1 (-252.0329 1/cm)

Zero-point correction $=0.179065$ (Hartree/Particle)

Thermal correction to Energy $=\quad 0.190062$

Thermal correction to Enthalpy $=\quad 0.191006$

Thermal correction to Gibbs Free Energy $=\quad 0.141742$

Sum of electronic and zero-point Energies $=\quad-649.575039$

Sum of electronic and thermal Energies $=\quad-649.564042$

Sum of electronic and thermal Enthalpies $=\quad-649.563098$

Sum of electronic and thermal Free Energies $=\quad-649.612362$

Coordinates (from last standard orientation):

\begin{tabular}{|c|c|c|c|c|}
\hline \multirow{2}{*}{$\begin{array}{l}\text { Center } \\
\text { Number }\end{array}$} & \multirow{2}{*}{$\begin{array}{l}\text { Atomic } \\
\text { Number }\end{array}$} & \multicolumn{3}{|c|}{ Coordinates (Angstroms) } \\
\hline & & & $X \quad Y$ & Z \\
\hline 1 & 8 & 1.599783 & -0.833987 & -1.223757 \\
\hline 2 & 6 & 1.266563 & -1.660248 & -0.252531 \\
\hline 3 & 8 & -2.160474 & -0.029047 & 0.886618 \\
\hline 4 & 6 & 1.510139 & -1.215100 & 1.156152 \\
\hline 5 & 6 & 1.161341 & 0.266798 & 1.401331 \\
\hline 6 & 6 & 1.458773 & 1.154255 & 0.189010 \\
\hline 7 & 6 & 1.546362 & 0.495021 & -1.095660 \\
\hline 8 & 6 & -1.146388 & -0.076222 & -1.191557 \\
\hline 9 & 1 & -0.924373 & 0.284037 & -2.184401 \\
\hline 10 & 6 & -1.105487 & $7 \quad-1.359677$ & -0.725559 \\
\hline 11 & 6 & -1.855814 & $4 \quad-1.394109$ & 0.586552 \\
\hline 12 & 1 & 1.415523 & $3-2.696166$ & -0.540785 \\
\hline 13 & 1 & 1.788882 & $2 \quad 1.026210$ & -2.005877 \\
\hline 14 & 1 & 1.723374 & 0.660291 & 2.252980 \\
\hline 15 & 1 & -0.973047 & $7-2.249155$ & -1.328252 \\
\hline 16 & 1 & -2.795687 & $7-1.954846$ & 0.487460 \\
\hline 17 & 6 & -1.827202 & 20.777781 & -0.197604 \\
\hline 18 & 8 & -2.098001 & 1.949815 & -0.224666 \\
\hline 19 & 1 & -1.299788 & $8-1.819468$ & 1.428547 \\
\hline 20 & 1 & 2.590136 & -1.364985 & 1.340167 \\
\hline 21 & 8 & 1.574854 & 2.371817 & 0.289918 \\
\hline 22 & 1 & 0.100557 & 0.392248 & 1.644290 \\
\hline 23 & 1 & 0.995411 & $1-1.877939$ & 1.860167 \\
\hline
\end{tabular}




\begin{tabular}{|c|c|c|c|c|}
\hline $\begin{array}{l}\text { F.IV.TS2 } \\
\text { HF = -64 } \\
\text { Imaginar } \\
\text { Zero-poir }\end{array}$ & $\begin{array}{l}\text { Frequenc } \\
\text { F } \\
\text { t correctior }\end{array}$ & $\begin{array}{l}\text { hartrees }(-4 \\
\text { ies: none fo } \\
n=0.18590\end{array}$ & $\begin{array}{l}407791.4182 \\
\text { ound } \\
05 \text { (Hartree/P }\end{array}$ & $\begin{array}{l}241207 \text { kca } \\
\text { Particle) }\end{array}$ \\
\hline Thermal & correction $t$ & to Energy= & & 0.195683 \\
\hline Thermal & correction & to Enthalpy & & 0.196627 \\
\hline Thermal & correction & to Gibbs Fr & ree Energy= & 0.150 \\
\hline Sum of $\epsilon$ & lectronic a & ind zero-poil & int Energies= & \\
\hline Sum of & lectronic & ind thermal & Energies= & -649 \\
\hline Sum of & lectronic & Ind thermal & Enthalpies= & \\
\hline Sum of & ectronic & ind thermal & Free Energie & \\
\hline Coordir & tes (from & ast standard & d orientation) & \\
\hline Center & Atomic & & oordinates (A & Angstroms) \\
\hline Number & Number & & $\begin{array}{l}X \\
\end{array}$ & Z \\
\hline 1 & 8 & 1.347749 & -0.988964 & -1.233218 \\
\hline 2 & 6 & 0.551812 & -1.732661 & -0.294113 \\
\hline 3 & 8 & -2.080827 & 0.257919 & 0.803033 \\
\hline 4 & 6 & 1.090694 & -1.448412 & 1.115530 \\
\hline 5 & 6 & 189184 & 0.065090 & 1.428132 \\
\hline 6 & 6 & 1.464393 & 0.917524 & 0.190538 \\
\hline 7 & 6 & 0.870864 & 0.359897 & -1.111817 \\
\hline 8 & 6 & -0.682629 & 0.192668 & -1.106485 \\
\hline 9 & 1 & -1.069977 & 0.389709 & -2.109348 \\
\hline 10 & 6 & -0.892430 & -1.260781 & -0.635436 \\
\hline 11 & 6 & -1.947314 & -1.141370 & 0.483269 \\
\hline 12 & 1 & 0.691549 & -2.790286 & -0.534923 \\
\hline 13 & 1 & 1.243569 & 0.940172 & -1.95617 \\
\hline 14 & 1 & 1.967234 & 0.276507 & 2.167140 \\
\hline 15 & 1 & -1.269160 & -1.892153 & -1.443433 \\
\hline 16 & 1 & -2.926407 & -1.502646 & 0.153412 \\
\hline 17 & 6 & -1.452227 & 1.055246 & -0.112154 \\
\hline 18 & 8 & -1.516259 & 2.254824 & -0.071113 \\
\hline 19 & 1 & -1.681688 & -1.664382 & 1.405378 \\
\hline 20 & 1 & 2.095672 & -1.883584 & 1.148362 \\
\hline 21 & 8 & 2.130245 & 1.929231 & 0.211849 \\
\hline 22 & 1 & 0.246517 & 0.427084 & 1.859472 \\
\hline 23 & 1 & 0.501351 & -1.967716 & 1.880912 \\
\hline
\end{tabular}

F.IV.TS3

$\mathrm{HF}=-649.7603709$ hartrees $(-407731.130343459 \mathrm{kcal} / \mathrm{mol})$

Imaginary Frequencies: $1(-256.97511 / \mathrm{cm})$

Zero-point correction $=0.179036($ Hartree/Particle $)$

Thermal correction to Energy $=\quad 0.190093$

Thermal correction to Enthalpy= $\quad 0.191037$

Thermal correction to Gibbs Free Energy $=0.141573$

Sum of electronic and zero-point Energies $=\quad-649.581334$

Sum of electronic and thermal Energies $=\quad-649.570278$

Sum of electronic and thermal Enthalpies $=\quad-649.569334$

Sum of electronic and thermal Free Energies $=\quad-649.618797$

Coordinates (from last standard orientation):

Center Atomic Coordinates (Angstroms) 


$\begin{array}{crrrr}\text { Number } & \text { Number } & \text { X } & Y & Z \\ -1 & 8 & 0.439541 & 0.327533 & 1.421741 \\ 2 & 6 & 0.284049 & 1.416587 & 0.716425 \\ 3 & 6 & 1.428560 & 1.903200 & -0.112154 \\ 4 & 6 & 2.199535 & 0.771990 & -0.818615 \\ 5 & 6 & 2.232036 & -0.531129 & -0.010074 \\ 6 & 6 & 1.233313 & -0.686362 & 1.014924 \\ 7 & 6 & -0.569535 & -1.104002 & -0.816149 \\ 8 & 6 & -0.997231 & 0.180561 & -0.997202 \\ 9 & 1 & -0.455299 & 2.089859 & 1.135931 \\ 10 & 1 & 1.222091 & -1.528409 & 1.692806 \\ 11 & 1 & 3.231956 & 1.071750 & -1.019651 \\ 12 & 6 & -1.600335 & -1.815753 & 0.027069 \\ 13 & 6 & -2.275513 & 0.366667 & -0.269798 \\ 14 & 8 & -2.567264 & -0.814788 & 0.373215 \\ 15 & 8 & -2.983788 & 1.340376 & -0.184481 \\ 16 & 1 & -1.215105 & -2.256845 & 0.950348 \\ 17 & 1 & 0.183747 & -1.635668 & -1.383149 \\ 18 & 1 & -0.671569 & 0.892752 & -1.742317 \\ 19 & 1 & -2.101817 & -2.608176 & -0.546189 \\ 20 & 1 & 2.111484 & 2.413416 & 0.592142 \\ 21 & 8 & 3.017174 & -1.435117 & -0.295801 \\ 22 & 1 & 1.086478 & 2.672499 & -0.811719 \\ 23 & 1 & 1.753461 & 0.534220 & -1.792152 \\ ----------------------------------------------\end{array}$

\section{F.IV.TS3product}

$\mathrm{HF}=-649.865355$ hartrees $(-407797.00891605 \mathrm{kcal} / \mathrm{mol})$

Imaginary Frequencies: none found

Zero-point correction $=0.185616$ (Hartree/Particle)

Thermal correction to Energy $=\quad 0.195573$

Thermal correction to Enthalpy $=\quad 0.196517$

Thermal correction to Gibbs Free Energy $=0.149209$

Sum of electronic and zero-point Energies $=\quad-649.679739$

Sum of electronic and thermal Energies $=\quad-649.669782$

Sum of electronic and thermal Enthalpies $=\quad-649.668838$

Sum of electronic and thermal Free Energies $=\quad-649.716146$

Coordinates (from last standard orientation):

\begin{tabular}{|c|c|c|c|c|}
\hline Center & Atomic & & ordinates ( & Angstroms) \\
\hline Number & Number & & $\mathrm{Y}$ & Z \\
\hline 1 & 8 & 0.442794 & 0.126072 & 1.416485 \\
\hline 2 & 6 & 0.034653 & 1.196953 & 0.547327 \\
\hline 3 & 6 & 1.290433 & 1.903647 & 0.026550 \\
\hline 4 & 6 & 2.229889 & 0.917306 & -0.703461 \\
\hline 5 & 6 & 2.217417 & -0.485527 & -0.101002 \\
\hline 6 & 6 & 0.880164 & -0.905053 & 0.522125 \\
\hline 7 & 6 & -0.245914 & -0.975197 & -0.537771 \\
\hline 8 & 6 & -0.778481 & 0.474312 & -0.562749 \\
\hline 9 & 1 & -0.586399 & 1.877759 & 1.134385 \\
\hline 10 & 1 & 1.020158 & -1.826087 & 1.092797 \\
\hline 11 & 1 & 3.263407 & 1.273714 & -0.739746 \\
\hline 12 & 6 & -1.468955 & -1.811047 & -0.077725 \\
\hline 13 & 6 & -2.257995 & 0.367882 & -0.204574 \\
\hline
\end{tabular}




$\begin{array}{rrrrr}14 & 8 & -2.600195 & -0.928840 & 0.006257 \\ 15 & 8 & -3.043806 & 1.274062 & -0.107345 \\ 16 & 1 & -1.317849 & -2.252334 & 0.912320 \\ 17 & 1 & 0.120534 & -1.322574 & -1.507699 \\ 18 & 1 & -0.693428 & 0.982377 & -1.527113 \\ 19 & 1 & -1.720997 & -2.606467 & -0.783895 \\ 20 & 1 & 1.807630 & 2.327983 & 0.894280 \\ 21 & 8 & 3.173008 & -1.232811 & -0.133895 \\ 22 & 1 & 1.017926 & 2.738741 & -0.630113 \\ 23 & 1 & 1.907343 & 0.799373 & -1.749547\end{array}$

\begin{tabular}{|c|c|c|c|c|}
\hline \multicolumn{5}{|c|}{$\begin{array}{l}\text { F.IV.TS4 } \\
\text { HF = -649.7568041 hartrees }(-407728.892140791 \mathrm{kcal} / \mathrm{n} \\
\text { Imaginary Frequencies: } 1(-230.66881 / \mathrm{cm}) \\
\text { Zero-point correction }=0.178855(\text { Hartree/Particle })\end{array}$} \\
\hline \multicolumn{3}{|c|}{ Thermal correction to Enerav $=$} & \multicolumn{2}{|c|}{ Thermal correction to Energy $=\quad 0.189951$} \\
\hline \multicolumn{4}{|c|}{ Thermal correction to Enthalpy= } & 0.190895 \\
\hline \multicolumn{4}{|c|}{$\begin{array}{lr}\text { Thermal correction to Enthalpy= } & 0.190895 \\
\text { Thermal correction to Gibbs Free Energy }= & 0.141\end{array}$} & \\
\hline \\
\hline \\
\hline \multicolumn{5}{|c|}{ Sum of electronic and thermal Enthalpies= } \\
\hline \multirow{2}{*}{\multicolumn{5}{|c|}{$\begin{array}{l}\text { Sum of electronic and thermal Free Energies= } \\
\text { Coordinates (from last standard orientation): }\end{array}$}} \\
\hline & & & & \\
\hline \multirow{2}{*}{$\begin{array}{l}\text { Center } \\
\text { Number }\end{array}$} & \multirow{2}{*}{$\begin{array}{l}\text { Atomic } \\
\text { Number }\end{array}$} & \multicolumn{3}{|c|}{ Coordinates (Angstroms) } \\
\hline & & & $X \quad Y$ & \\
\hline 1 & 8 & & & \\
\hline 2 & 8 & 2.304390 & 8457 & \\
\hline 3 & 6 & 0.900834 & 0.735047 & 1.030684 \\
\hline 4 & 6 & 1.890896 & 7773 & 528 \\
\hline 5 & 6 & & 149 & 200 \\
\hline 6 & 6 & & 543 & 050 \\
\hline 7 & 1 & & & \\
\hline 8 & & & & \\
\hline 9 & 6 & & & \\
\hline 10 & 6 & 93142 & 1382 & -0.979523 \\
\hline 11 & 1 & 0.509639 & 12105 & 1.671799 \\
\hline 12 & 1 & .418691 & -2.472172 & 1.136533 \\
\hline 13 & 1 & 3728 & -0.113799 & -1.048699 \\
\hline 14 & 6 & & & -0.221938 \\
\hline 15 & 6 & & & \\
\hline 16 & 8 & & -0.2 & \\
\hline 17 & 8 & & & \\
\hline 18 & 1 & -1.989454 & -2.140733 & 0.584903 \\
\hline 19 & 1 & -0.443863 & -1.659348 & -1.693474 \\
\hline 20 & 1 & -0.294697 & 1.029060 & -1.582428 \\
\hline 21 & 1 & -2.930670 & -1.855746 & -0.891647 \\
\hline 22 & 1 & & -2.417143 & \\
\hline 23 & 1 & 1.810478 & -0.184170 & -1.781424 \\
\hline
\end{tabular}

\section{F.IV.TS4product}

$\mathrm{HF}=-649.8640086$ hartrees $(-407796.164036586 \mathrm{kcal} / \mathrm{mol})$ Imaginary Frequencies: none found 


\begin{tabular}{|c|c|c|c|c|}
\hline \multicolumn{5}{|c|}{ Zero-point correction $=0.185519($ Hartree/Particle $)$} \\
\hline \multicolumn{4}{|c|}{ Thermal correction to Energy= } & 0.195499 \\
\hline \multicolumn{4}{|c|}{ Thermal correction to Enthalpy= } & 0.196443 \\
\hline \multirow{2}{*}{\multicolumn{5}{|c|}{$\begin{array}{lr}\text { Thermal correction to Gibbs Free Energy }= & 0.1488 \\
\text { Sum of electronic and zero-point Energies }= & -649\end{array}$}} \\
\hline & & & & \\
\hline \multicolumn{5}{|c|}{$\begin{array}{l}\text { Sum of electronic and zero-point Energles= } \\
\text { Sum of electronic and thermal Energies= }\end{array}$} \\
\hline \\
\hline \multirow{2}{*}{\multicolumn{5}{|c|}{$\begin{array}{l}\text { Sum of electronic and thermal Free Energies= } \\
\text { Coordinates (from last standard orientation): }\end{array}$}} \\
\hline & & & & \\
\hline \multirow{2}{*}{$\begin{array}{l}\text { Center } \\
\text { Number }\end{array}$} & \multirow{2}{*}{$\begin{array}{l}\text { Atomic } \\
\text { Number }\end{array}$} & & \multicolumn{2}{|c|}{ Coordinates (Angstroms) } \\
\hline & & & $X \quad Y$ & Z \\
\hline 1 & 8 & 0.444842 & -0.283819 & 1.400936 \\
\hline 2 & 8 & 2.509119 & 1.984186 & -0.088137 \\
\hline 3 & 6 & 0.475335 & 0.834829 & 05018 \\
\hline 4 & 6 & 1.885797 & 0.944082 & -0.086798 \\
\hline 5 & 6 & 2.419402 & -0.351750 & -0.695682 \\
\hline 6 & 6 & 010 & 9139 & 22033 \\
\hline 7 & 1 & 020 & -1.8 & 93532 \\
\hline 8 & 6 & 9116 & -1.4 & 0.534503 \\
\hline 9 & 6 & -0.557589 & -1.088505 & -0.576082 \\
\hline 10 & 6 & -0.566076 & 0.456671 & -0.579687 \\
\hline 11 & 1 & 0.223190 & 1.743368 & 1.054211 \\
\hline 12 & 1 & 0.163723 & -2.290575 & 1.139568 \\
\hline 13 & 1 & 511890 & -0.303800 & -0.717294 \\
\hline 14 & 6 & -2.021723 & -1.463804 & -0.233099 \\
\hline 15 & 6 & 6323 & 5845 & -0.143837 \\
\hline 16 & 8 & -2.748174 & -0.249659 & 0.013559 \\
\hline 17 & 8 & -2.384621 & 1.970020 & 0.049477 \\
\hline 18 & 1 & -2.095869 & -2.080557 & 0.667982 \\
\hline 19 & 1 & -0.267203 & -1.522191 & -1.536615 \\
\hline 20 & 1 & -0.354274 & 0.924712 & -1.545627 \\
\hline 21 & 1 & -2.518334 & -1.986472 & -1.056089 \\
\hline 22 & 1 & 1.965595 & -2.492783 & -0.638888 \\
\hline 23 & 1 & 2.089249 & -0.353860 & -1.745684 \\
\hline
\end{tabular}

G

\section{B3LYP/6-31G(d)}

$\mathrm{HF}=-307.375544$ hartrees $(-192881.22761544 \mathrm{kcal} / \mathrm{mol})$ Imaginary Frequencies: none found

Zero-point correction $=0.100848$ (Hartree/Particle)

Thermal correction to Energy $=\quad 0.106990$

Thermal correction to Enthalpy= $\quad 0.107934$

Thermal correction to Gibbs Free Energy $=\quad 0.070793$

Sum of electronic and zero-point Energies $=\quad-307.274696$

Sum of electronic and thermal Energies $=\quad-307.268554$

Sum of electronic and thermal Enthalpies $=\quad-307.267610$

Sum of electronic and thermal Free Energies $=\quad-307.304751$

Coordinates (from last standard orientation):

\begin{tabular}{lllll} 
Center & Atomic & & \multicolumn{2}{c}{ Coordinates (Angstroms) } \\
Number & Number & X & Y & Z \\
- \hdashline & 8 & -2.389576 & -0.006578 & 0.000106
\end{tabular}




\begin{tabular}{|c|c|c|c|c|}
\hline 2 & 6 & -0.329337 & -1.196030 & -0.000047 \\
\hline 3 & 6 & -1.146242 & -0.013589 & 0.000001 \\
\hline 4 & 6 & -0.334110 & 1.207010 & -0.000115 \\
\hline 5 & 6 & 1.070497 & 1.267169 & 0.000034 \\
\hline 6 & 1 & 1.553633 & 2.241892 & 0.000054 \\
\hline 7 & 6 & 1.827835 & 0.121421 & 0.000050 \\
\hline 8 & 1 & -0.829809 & -2.161244 & -0.000370 \\
\hline 9 & 1 & 2.912712 & 0.144526 & 0.000131 \\
\hline 10 & 1 & -0.899914 & 2.136799 & -0.000294 \\
\hline 11 & 6 & 1.139343 & -1.178952 & -0.000021 \\
\hline 12 & 1 & 1.506204 & -1.775834 & -0.858655 \\
\hline 13 & 1 & 1.505866 & -1.775689 & 0.858874 \\
\hline
\end{tabular}

in $\mathrm{H}_{2} \mathrm{O}$ B3LYP/6-31G(d)

$\mathrm{HF}=-307.4008572$ hartrees $(-192897.111901572 \mathrm{kcal} / \mathrm{mol})$

Imaginary Frequencies: none found

Zero-point correction $=0.099274($ Hartree/Particle $)$

Thermal correction to Energy $=\quad 0.105292$

Thermal correction to Enthalpy= $\quad 0.106237$

Thermal correction to Gibbs Free Energy $=0.069521$

Sum of electronic and zero-point Energies $=\quad-307.301583$

Sum of electronic and thermal Energies $=\quad-307.295565$

Sum of electronic and thermal Enthalpies $=\quad-307.294621$

Sum of electronic and thermal Free Energies $=\quad-307.331336$

Coordinates (from last standard orientation):

\begin{tabular}{|c|c|c|c|c|}
\hline \multirow{2}{*}{$\begin{array}{l}\text { Center } \\
\text { Number }\end{array}$} & \multirow{2}{*}{$\begin{array}{l}\text { Atomic } \\
\text { Number }\end{array}$} & \multicolumn{3}{|c|}{ Coordinates (Angstroms) } \\
\hline & & & $X \quad Y$ & Z \\
\hline 1 & 8 & 2.395979 & -0.010654 & 0.000603 \\
\hline 2 & 6 & 0.324393 & -1.193347 & -0.001291 \\
\hline 3 & 6 & 1.129936 & -0.016854 & -0.000020 \\
\hline 4 & 6 & 0.338318 & 1.202700 & -0.000260 \\
\hline 5 & 6 & -1.066464 & 1.272159 & -0.000169 \\
\hline 6 & 6 & -1.820908 & 0.123198 & -0.000132 \\
\hline 7 & 1 & 0.818565 & -2.167966 & -0.002103 \\
\hline 8 & 1 & -2.907588 & 0.147161 & 0.000321 \\
\hline 9 & 1 & -1.545470 & 2.250265 & -0.000161 \\
\hline 10 & 1 & 0.904803 & 2.135103 & -0.000343 \\
\hline 11 & 6 & -1.139014 & -1.172285 & 0.000100 \\
\hline 12 & 1 & -1.515066 & $6-1.783039$ & 0.860806 \\
\hline 13 & 1 & -1.520635 & $5-1.789722$ & -0.852712 \\
\hline
\end{tabular}

\section{1,3 dipolar TS of $G$ and ethene}

\section{B3LYP/6-31G(d)}

$\mathrm{HF}=-385.9616831$ hartrees $(-242194.815762081 \mathrm{kcal} / \mathrm{mol})$

Imaginary Frequencies: $1(-169.57231 / \mathrm{cm})$

Zero-point correction $=0.155213$ (Hartree/Particle)

Thermal correction to Energy $=\quad 0.163996$

Thermal correction to Enthalpy= $\quad 0.164940$

Thermal correction to Gibbs Free Energy $=\quad 0.121616$

Sum of electronic and zero-point Energies $=\quad-385.806471$

Sum of electronic and thermal Energies $=\quad-385.797687$ 
Sum of electronic and thermal Enthalpies $=\quad-385.796743$

Sum of electronic and thermal Free Energies $=\quad-385.840067$

Coordinates (from last standard orientation):

\begin{tabular}{ccccc} 
Center & Atomic & \multicolumn{3}{c}{ Coordinates (Angstroms) } \\
Number & Number & X & $Y$ & $Z$ \\
\hline 1 & 8 & -2.406020 & -0.960425 & -0.174662 \\
2 & 6 & -0.470371 & -0.427724 & 1.098334 \\
3 & 6 & -1.428010 & -0.219982 & 0.030007 \\
4 & 6 & -1.102344 & 0.935264 & -0.805303 \\
5 & 6 & 0.077042 & 1.668276 & -0.712172 \\
6 & 6 & 1.006062 & 1.422986 & 0.292981 \\
7 & 6 & 2.055249 & -0.885494 & -0.835819 \\
8 & 1 & 2.986164 & -0.671121 & -0.317580 \\
9 & 6 & 1.172009 & -1.795187 & -0.366526 \\
10 & 1 & -0.720364 & -1.177443 & 1.845298 \\
11 & 1 & 1.885371 & 2.048591 & 0.409623 \\
12 & 1 & 1.375369 & -2.363506 & 0.537420 \\
13 & 1 & 1.922300 & -0.409076 & -1.801304 \\
14 & 1 & 0.306890 & -2.110242 & -0.940411 \\
15 & 1 & 0.275303 & 2.446343 & -1.446980 \\
16 & 1 & -1.827090 & 1.175394 & -1.580045 \\
17 & 6 & 0.601832 & 0.537764 & 1.400729 \\
18 & 1 & 1.443628 & 0.095502 & 1.941597 \\
19 & 1 & 0.131789 & 1.233536 & 2.136291 \\
---1 & 1 &
\end{tabular}

\section{in $\mathrm{H}_{2} \mathrm{O}$ B3LYP/6-31G(d)}

$\mathrm{HF}=-385.9835357 \mathrm{hartrees}(-242208.528487107 \mathrm{kcal} / \mathrm{mol})$

Imaginary Frequencies: $1(-230.31551 / \mathrm{cm})$

Zero-point correction $=0.154011$ (Hartree/Particle)

Thermal correction to Energy $=\quad 0.162587$

Thermal correction to Enthalpy= $\quad 0.163532$

Thermal correction to Gibbs Free Energy= $\quad 0.120812$

Sum of electronic and zero-point Energies $=\quad-385.829525$

Sum of electronic and thermal Energies $=\quad-385.820948$

Sum of electronic and thermal Enthalpies $=\quad-385.820004$

Sum of electronic and thermal Free Energies $=\quad-385.862723$

Coordinates (from last standard orientation):

\begin{tabular}{|c|c|c|c|c|}
\hline \multirow{2}{*}{$\begin{array}{l}\text { Center } \\
\text { Number }\end{array}$} & \multirow{2}{*}{$\begin{array}{l}\text { Atomic } \\
\text { Number }\end{array}$} & & \multicolumn{2}{|c|}{ Coordinates (Angstroms) } \\
\hline & & & $X \quad Y$ & Z \\
\hline 1 & 8 & -2.493467 & -0.796052 & -0.188372 \\
\hline 2 & 6 & -0.478276 & -0.496653 & 1.049533 \\
\hline 3 & 6 & -1.432034 & -0.146661 & 0.024675 \\
\hline 4 & 6 & -1.040014 & 1.005522 & -0.765688 \\
\hline 5 & 6 & 0.186975 & 1.649361 & -0.647552 \\
\hline 6 & 6 & 1.094679 & 1.294483 & 0.349666 \\
\hline 7 & 6 & 1.990594 & -0.854692 & -0.852598 \\
\hline 8 & 1 & 2.910703 & -0.696415 & -0.296868 \\
\hline 9 & 6 & 1.067487 & -1.768913 & -0.453057 \\
\hline 10 & 1 & -0.763690 & $0 \quad-1.283419$ & 1.752229 \\
\hline 11 & 1 & 2.010187 & 1.862756 & 0.493285 \\
\hline 12 & 1 & 1.237242 & $2-2.392428$ & 0.420606 \\
\hline
\end{tabular}




$\begin{array}{rrrrr}13 & 1 & 1.916744 & -0.356084 & -1.813455 \\ 14 & 1 & 0.236890 & -2.056409 & -1.089744 \\ 15 & 1 & 0.443351 & 2.438305 & -1.354076 \\ 16 & 1 & -1.743798 & 1.329219 & -1.532514 \\ 17 & 6 & 0.626638 & 0.403488 & 1.424180 \\ 18 & 1 & 1.434391 & -0.097820 & 1.973976 \\ 19 & 1 & 0.169411 & 1.105100 & 2.178587\end{array}$

\begin{tabular}{|c|c|c|c|c|}
\hline $\begin{array}{l}\text { G.IV.TS1 } \\
\text { HF = -61 } \\
\text { Imaginar } \\
\text { Zero-poir }\end{array}$ & $\begin{array}{l}2.6397853 \\
\text { / Frequenc } \\
\text { it correctio }\end{array}$ & $\begin{array}{l}\text { hartrees }(-3 \\
\text { cies: } 1(-245 \\
\text { n }=0.17895\end{array}$ & $\begin{array}{l}384437.5916 \\
.23781 / \mathrm{cm}) \\
6 \text { (Hartree/P }\end{array}$ & $\begin{array}{l}573603 \text { kcal } \\
\text { Particle) }\end{array}$ \\
\hline Thermal & correction & to Energy= & & 0.189640 \\
\hline Thermal & correction & to Enthalpy & & 0.190584 \\
\hline Thermal & correction & to Gibbs Fr & ee Energy= & 0.1422 \\
\hline Sum of $\epsilon$ & lectronic a & and zero-poi & int Energies= & \\
\hline Sum of & lectronic a & and thermal & Energies= & -612 \\
\hline Sum o & & & Entholnina- & -612 \\
\hline Sum 0 & & & Free Energie & \\
\hline Coordi & & & & \\
\hline Center & Atomic & & ordinates (A & Angstroms) \\
\hline Number & Number & & $\mathrm{Y}$ & Z \\
\hline 1 & 8 & -2.171577 & -1.772728 & -0.674131 \\
\hline 2 & 6 & -1.760549 & 0547 & 2432 \\
\hline 3 & 8 & 1.713465 & 0184 & -0.572353 \\
\hline 4 & 6 & -1.782531 & -0.611534 & -0.440598 \\
\hline 5 & 6 & -1.198839 & 0.250943 & -1.467841 \\
\hline 6 & 6 & -0.555030 & 1.451514 & -1.202155 \\
\hline 7 & 1 & -0.035970 & 1.967981 & -2.005183 \\
\hline 8 & 6 & -0.520730 & 1.978343 & 0.088750 \\
\hline 9 & 6 & 1.294009 & 0.353551 & 1.113510 \\
\hline 10 & 1 & 497965 & 1.211937 & 1.737472 \\
\hline 11 & 6 & 0.555782 & -0.758586 & 1.411502 \\
\hline 12 & 6 & 0.879946 & -1.820873 & 0.393456 \\
\hline 13 & 1 & -2.307860 & -0.560997 & 1.658175 \\
\hline 14 & 1 & -0.068168 & 2.946896 & 0.276883 \\
\hline 15 & 1 & -1.232650 & -0.134195 & -2.483870 \\
\hline 16 & 1 & 0.106330 & -0.997016 & 2.367523 \\
\hline 17 & 1 & 452712 & -2.638445 & 0.852303 \\
\hline 18 & 6 & 2.045489 & 0.102212 & -0.136674 \\
\hline 19 & 8 & 2.809773 & 0.816975 & -0.738483 \\
\hline 20 & 1 & 0.009162 & -2.246372 & -0.117331 \\
\hline 21 & 6 & -1.415793 & 1.390276 & 1.104948 \\
\hline 22 & 1 & -1.109316 & 1.605683 & 2.133172 \\
\hline 23 & 1 & -2.376028 & 1.940227 & 0.976604 \\
\hline
\end{tabular}

pyrylium

$\mathrm{HF}=-268.4764625$ hartrees $(-168471.664983375 \mathrm{kcal} / \mathrm{mol})$

Imaginary Frequencies: none found

Zero-point correction $=0.089468$ (Hartree/Particle)

Thermal correction to Energy $=\quad 0.093814$

Thermal correction to Enthalpy $=\quad 0.094758$ 
Thermal correction to Gibbs Free Energy=

Sum of electronic and zero-point Energies=

Sum of electronic and thermal Energies=

Sum of electronic and thermal Enthalpies=

Sum of electronic and thermal Free Energies=

Coordinates (from last standard orientation):
0.061964

$-268.386995$

$-268.382648$

$-268.381704$

$-268.414498$

\begin{tabular}{ccrcc} 
Center & Atomic & \multicolumn{3}{c}{ Coordinates (Angstroms) } \\
Number & Number & X & $Y$ & $Z$ \\
- \hdashline 1 & 8 & 0.006055 & -1.330286 & 0.000001 \\
2 & 6 & -1.161066 & -0.688633 & -0.000023 \\
3 & 6 & -1.214720 & 0.686314 & 0.000015 \\
4 & 6 & -0.006959 & 1.396877 & -0.000018 \\
5 & 6 & 1.208139 & 0.697338 & -0.000001 \\
6 & 1 & 2.166133 & 1.205251 & 0.000010 \\
7 & 6 & 1.168296 & -0.677388 & 0.000006 \\
8 & 1 & 2.018004 & -1.349853 & 0.000032 \\
9 & 1 & -0.011561 & 2.482955 & 0.000000 \\
10 & 1 & -2.005387 & -1.367944 & 0.000012 \\
11 & 1 & -2.177774 & 1.184827 & 0.000067
\end{tabular}

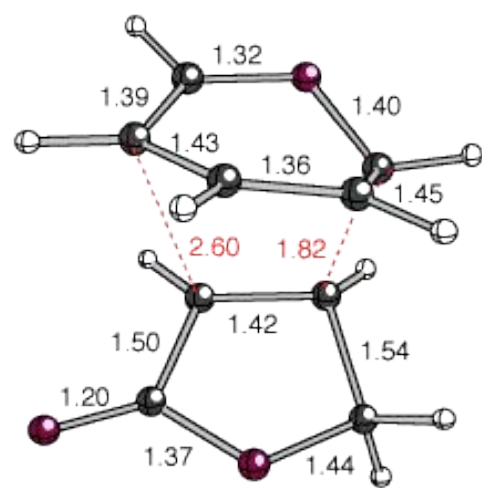

Pyrylium.4.TS1 (2+4)

$\mathrm{HF}=-573.6989954$ hartrees $(-360001.856603454 \mathrm{kcal} / \mathrm{mol})$

Imaginary Frequencies: $1(-310.68571 / \mathrm{cm})$

Zero-point correction $=0.165930($ Hartree/Particle $)$

Thermal correction to Energy=

0.174955

Thermal correction to Enthalpy=

0.175900

Thermal correction to Gibbs Free Energy $=\quad 0.131432$

Sum of electronic and zero-point Energies=

$-573.533066$

Sum of electronic and thermal Energies=

$-573.524040$

Sum of electronic and thermal Enthalpies $=\quad-573.523096$

Sum of electronic and thermal Free Energies $=\quad-573.567563$

Coordinates (from last standard orientation):

\begin{tabular}{llrrr} 
Center & Atomic & \multicolumn{3}{c}{ Coordinates (Angstroms) } \\
Number & Number & X & Y & Z \\
- \hdashline 1 & 8 & 2.100205 & -0.317742 & -0.772321 \\
2 & 6 & 1.332297 & -1.132853 & 0.069024 \\
3 & 8 & -1.968107 & -0.470364 & 0.597771 \\
4 & 6 & 1.107864 & -0.591461 & 1.397393 \\
5 & 6 & 0.936811 & 0.758339 & 1.496964
\end{tabular}




$\begin{array}{ccrrr}6 & 6 & 1.060941 & 1.517707 & 0.295563 \\ 7 & 1 & 0.775973 & 2.563234 & 0.245304 \\ 8 & 6 & 1.766856 & 0.959888 & -0.762298 \\ 9 & 6 & -0.618090 & 0.159170 & -1.158299 \\ 10 & 1 & -0.439995 & 0.684982 & -2.087841 \\ 11 & 6 & -0.242489 & -1.172658 & -0.843368 \\ 12 & 6 & -1.357562 & -1.665612 & 0.092348 \\ 13 & 1 & 1.705869 & -2.150091 & -0.005410 \\ 14 & 1 & 2.174940 & 1.523171 & -1.596074 \\ 15 & 1 & 0.684936 & 1.249620 & 2.430782 \\ 16 & 1 & 0.054053 & -1.821423 & -1.667001 \\ 17 & 1 & -2.098108 & -2.240130 & -0.473527 \\ 18 & 6 & -1.692021 & 0.599778 & -0.211804 \\ 19 & 8 & -2.206388 & 1.678253 & -0.110941 \\ 20 & 1 & -1.023078 & -2.260819 & 0.943767 \\ 21 & 1 & 0.992071 & -1.263505 & 2.240792 \\ ---------------------------------------\end{array}$

Pyrylium.4.TS1 product

$\mathrm{HF}=-573.7319161$ hartrees $(-360022.514671911 \mathrm{kcal} / \mathrm{mol})$

Imaginary Frequencies: none found

Zero-point correction $=0.169313($ Hartree/Particle $)$

Thermal correction to Energy $=\quad 0.177882$

Thermal correction to Enthalpy= $\quad 0.178826$

Thermal correction to Gibbs Free Energy $=\quad 0.135552$

Sum of electronic and zero-point Energies $=\quad-573.562603$

Sum of electronic and thermal Energies $=\quad-573.554035$

Sum of electronic and thermal Enthalpies $=\quad-573.553090$

Sum of electronic and thermal Free Energies $=\quad-573.596364$

Coordinates (from last standard orientation):

\begin{tabular}{|c|c|c|c|c|}
\hline \multirow{2}{*}{$\begin{array}{l}\text { Center } \\
\text { Number }\end{array}$} & \multirow{2}{*}{$\begin{array}{l}\text { Atomic } \\
\text { Number }\end{array}$} & \multicolumn{3}{|c|}{ Coordinates (Angstroms) } \\
\hline & & & $\begin{array}{ll}X \quad Y\end{array}$ & Z \\
\hline 1 & 8 & -2.309688 & 0.349098 & -0.489603 \\
\hline 2 & 6 & -1.238559 & 1.116708 & 0.298575 \\
\hline 3 & 8 & 2.137622 & 0.673170 & 0.260033 \\
\hline 4 & 6 & -0.964779 & 0.318953 & 1.537338 \\
\hline 5 & 6 & -0.690868 & -0.966071 & 1.297484 \\
\hline 6 & 6 & -0.689455 & -1.330646 & -0.186038 \\
\hline 7 & 1 & -0.488595 & -2.378638 & -0.400792 \\
\hline 8 & 6 & -1.989640 & -0.854660 & -0.702990 \\
\hline 9 & 6 & 0.361783 & -0.368815 & -0.900406 \\
\hline 10 & 1 & 0.462178 & -0.648363 & -1.951989 \\
\hline 11 & 6 & -0.031003 & 1.097551 & -0.684726 \\
\hline 12 & 6 & 1.259213 & 1.745040 & -0.115919 \\
\hline 13 & 1 & -1.688370 & 2.095980 & 0.442384 \\
\hline 14 & 1 & -2.706385 & -1.434723 & -1.286751 \\
\hline 15 & 1 & -0.483828 & $3-1.727610$ & 2.040907 \\
\hline 16 & 1 & -0.338304 & 1.596056 & -1.607161 \\
\hline 17 & 1 & 1.757903 & 2.350449 & -0.876787 \\
\hline 18 & 6 & 1.718700 & -0.532970 & -0.180979 \\
\hline 19 & 8 & 2.294430 & -1.565999 & 0.002002 \\
\hline 20 & 1 & 1.092150 & 2.362666 & 0.770069 \\
\hline 21 & 1 & -0.998009 & 0.783508 & 2.516637 \\
\hline
\end{tabular}


Pyrylium.4.TS1[1,2-alky]

$\mathrm{HF}=-573.6983276$ hartrees $(-360001.437552276 \mathrm{kcal} / \mathrm{mol})$

Imaginary Frequencies: $1(-211.19861 / \mathrm{cm})$

Zero-point correction $=0.166643$ (Hartree/Particle)

Thermal correction to Energy=

0.175252

Thermal correction to Enthalpy=

0.176196

Thermal correction to Gibbs Free Energy $=0.132799$

Sum of electronic and zero-point Energies=

$-573.531685$

Sum of electronic and thermal Energies=

$-573.523076$

Sum of electronic and thermal Enthalpies=

$-573.522131$

Sum of electronic and thermal Free Energies=

$-573.565529$

Coordinates (from last standard orientation):

\begin{tabular}{|c|c|c|c|c|}
\hline \multirow{2}{*}{$\begin{array}{l}\text { Center } \\
\text { Number }\end{array}$} & \multirow{2}{*}{$\begin{array}{l}\text { Atomic } \\
\text { Number }\end{array}$} & \multicolumn{3}{|c|}{ Coordinates (Angstroms) } \\
\hline & & & $\begin{array}{l}X \\
Y\end{array}$ & Z \\
\hline 1 & 8 & -1.921898 & -0.306295 & -1.021787 \\
\hline 2 & 6 & -1.888765 & 0.795614 & -0.261198 \\
\hline 3 & 8 & 1.964555 & 0.657885 & 0.582998 \\
\hline 4 & 6 & -1.463788 & 0.719895 & 1.071805 \\
\hline 5 & 6 & -1.063424 & -0.538304 & 1.604603 \\
\hline 6 & 6 & -0.767320 & -1.515247 & 0.702753 \\
\hline 7 & 1 & -0.400741 & -2.493714 & 0.995578 \\
\hline 8 & 6 & -0.788312 & -1.131263 & -0.733286 \\
\hline 9 & 6 & 0.465624 & -0.146462 & -1.057845 \\
\hline 10 & 1 & 0.689705 & -0.298417 & -2.117649 \\
\hline 11 & 6 & 0.100413 & 1.247494 & -0.730264 \\
\hline 12 & 6 & 1.199709 & 1.791801 & 0.164861 \\
\hline 13 & 1 & -2.505036 & 1.610345 & -0.630453 \\
\hline 14 & 1 & -0.814005 & -1.971322 & -1.422475 \\
\hline 15 & 1 & -0.965749 & -0.675368 & 2.676177 \\
\hline 16 & 1 & -0.286441 & 1.911909 & -1.498784 \\
\hline 17 & 1 & 1.835347 & 2.458244 & -0.434718 \\
\hline 18 & 6 & 1.675487 & -0.447523 & -0.161262 \\
\hline 19 & 8 & 2.240153 & -1.496250 & -0.049257 \\
\hline 20 & 1 & 0.881794 & 2.344899 & 1.050743 \\
\hline 21 & 1 & -1.515105 & 1.614659 & 1.684935 \\
\hline
\end{tabular}

Pyrylium.4.TS1[1,2-alky]product

$\mathrm{HF}=-573.7319161$ hartrees $(-360022.514671911 \mathrm{kcal} / \mathrm{mol})$

Imaginary Frequencies: none found

Zero-point correction $=0.169313$ (Hartree/Particle)

Thermal correction to Energy $=\quad 0.177882$

Thermal correction to Enthalpy= $\quad 0.178826$

Thermal correction to Gibbs Free Energy $=\quad 0.135552$

Sum of electronic and zero-point Energies $=\quad-573.562603$

Sum of electronic and thermal Energies $=\quad-573.554035$

Sum of electronic and thermal Enthalpies $=\quad-573.553090$

Sum of electronic and thermal Free Energies $=\quad-573.596364$

Coordinates (from last standard orientation):

\begin{tabular}{|c|c|}
\hline enter & Atomic \\
\hline Jum & Number \\
\hline
\end{tabular}




$\begin{array}{rrrrr}1 & 8 & -2.309688 & 0.349098 & -0.489603 \\ 2 & 6 & -1.238559 & 1.116708 & 0.298575 \\ 3 & 8 & 2.137622 & 0.673170 & 0.260033 \\ 4 & 6 & -0.964779 & 0.318953 & 1.537338 \\ 5 & 6 & -0.690868 & -0.966071 & 1.297484 \\ 6 & 6 & -0.689455 & -1.330646 & -0.186038 \\ 7 & 1 & -0.488595 & -2.378638 & -0.400792 \\ 8 & 6 & -1.989640 & -0.854660 & -0.702990 \\ 9 & 6 & 0.361783 & -0.368815 & -0.900406 \\ 10 & 1 & 0.462178 & -0.648363 & -1.951989 \\ 11 & 6 & -0.031003 & 1.097551 & -0.684726 \\ 12 & 6 & 1.259213 & 1.745040 & -0.115919 \\ 13 & 1 & -1.688370 & 2.095980 & 0.442384 \\ 14 & 1 & -2.706385 & -1.434723 & -1.286751 \\ 15 & 1 & -0.483828 & -1.727610 & 2.040907 \\ 16 & 1 & -0.338304 & 1.596056 & -1.607161 \\ 17 & 1 & 1.757903 & 2.350449 & -0.876787 \\ 18 & 6 & 1.718700 & -0.532970 & -0.180979 \\ 19 & 8 & 2.294430 & -1.565999 & 0.002002 \\ 20 & 1 & 1.092150 & 2.362666 & 0.770069 \\ 21 & 1 & -0.998009 & 0.783508 & 2.516637 \\ ----------------------------------------------------\end{array}$

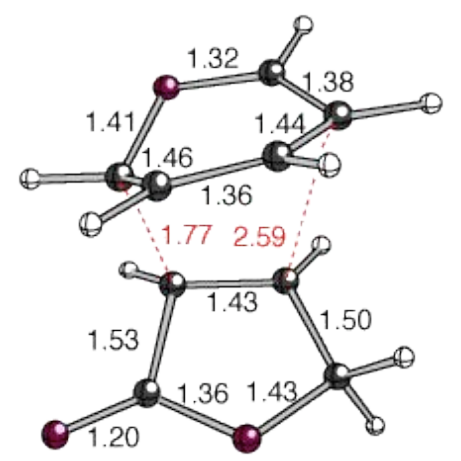

Pyrylium.4.TS2 (2+4)

$\mathrm{HF}=-573.6997949$ hartrees $(-360002.358297699 \mathrm{kcal} / \mathrm{mol})$

Imaginary Frequencies: $1(-255.94931 / \mathrm{cm})$

Zero-point correction $=0.165429$ (Hartree/Particle $)$

Thermal correction to Energy=

0.174489

Thermal correction to Enthalpy=

0.175433

Thermal correction to Gibbs Free Energy=

Sum of electronic and zero-point Energies=

0.130803

Sum of electronic and thermal Energies=

$-573.534366$

Sum of electronic and thermal Enthalpies=

$-573.525306$

Sum of electronic and thermal Free Energies=

$-573.524362$

Coordinates (from last standard orientation):

\begin{tabular}{llrrr}
$\begin{array}{l}\text { Center } \\
\text { Number }\end{array}$ & Atomic & \multicolumn{3}{c}{ Coordinates (Angstroms) } \\
-1 & 8 & -1.777723 & -1.003360 & -0.845412 \\
2 & 6 & -2.251932 & 0.212576 & -0.641694 \\
3 & 8 & 2.001682 & 0.821638 & 0.480511 \\
4 & 6 & -1.889617 & 0.948061 & 0.472598 \\
5 & 6 & -1.279695 & 0.249772 & 1.570418 \\
6 & 6 & -0.666712 & -0.928708 & 1.289243
\end{tabular}




$\begin{array}{ccrrr}7 & 6 & -0.613863 & -1.314543 & -0.119079 \\ 8 & 6 & 0.583960 & -0.342201 & -0.975946 \\ 9 & 1 & 0.627534 & -0.889165 & -1.917598 \\ 10 & 6 & 0.223946 & 1.035882 & -1.027567 \\ 11 & 6 & 1.128772 & 1.796913 & -0.099924 \\ 12 & 1 & -2.975571 & 0.526624 & -1.387866 \\ 13 & 1 & -0.329671 & -2.339271 & -0.341473 \\ 14 & 1 & -1.320724 & 0.666521 & 2.571407 \\ 15 & 1 & -0.267903 & 1.518341 & -1.863862 \\ 16 & 1 & 1.719174 & 2.524516 & -0.675058 \\ 17 & 6 & 1.837007 & -0.400404 & -0.100212 \\ 18 & 8 & 2.515748 & -1.357542 & 0.125618 \\ 19 & 1 & 0.630841 & 2.339754 & 0.710120 \\ 20 & 1 & -2.289757 & 1.950293 & 0.585367 \\ 21 & 1 & -0.142778 & -1.527600 & 2.026208 \\ ----------------------------------------------------\end{array}$

Pyrylium.4.TS2 (2+4)product

$\mathrm{HF}=-573.7302284$ hartrees $(-360021.455623284 \mathrm{kcal} / \mathrm{mol})$

Imaginary Frequencies: none found

Zero-point correction $=0.169299$ (Hartree/Particle)

Thermal correction to Energy $=\quad 0.177901$

Thermal correction to Enthalpy= $\quad 0.178845$

Thermal correction to Gibbs Free Energy $=\quad 0.135431$

Sum of electronic and zero-point Energies $=\quad-573.560930$

Sum of electronic and thermal Energies $=\quad-573.552328$

Sum of electronic and thermal Enthalpies $=\quad-573.551383$

Sum of electronic and thermal Free Energies $=\quad-573.594797$

Coordinates (from last standard orientation):

\begin{tabular}{|c|c|c|c|c|}
\hline \multirow{2}{*}{$\begin{array}{l}\text { Center } \\
\text { Number }\end{array}$} & \multirow{2}{*}{$\begin{array}{l}\text { Atomic } \\
\text { Number }\end{array}$} & \multicolumn{3}{|c|}{ Coordinates (Angstroms) } \\
\hline & & & $X \quad Y$ & Z \\
\hline 1 & 8 & -1.966655 & -0.971485 & -0.709734 \\
\hline 2 & 6 & -2.297309 & 0.232063 & -0.523022 \\
\hline 3 & 8 & 2.125913 & 0.739143 & 0.256297 \\
\hline 4 & 6 & -1.352499 & 1.110708 & 0.201481 \\
\hline 5 & 6 & -1.053302 & 0.380434 & 1.509116 \\
\hline 6 & 6 & -0.676758 & -0.890702 & 1.348968 \\
\hline 7 & 6 & -0.606246 & -1.315027 & -0.087428 \\
\hline 8 & 6 & 0.385399 & -0.405931 & -0.862604 \\
\hline 9 & 1 & 0.469009 & -0.747323 & -1.897723 \\
\hline 10 & 6 & -0.047075 & $5 \quad 1.058177$ & -0.725316 \\
\hline 11 & 6 & 1.186739 & 1.763332 & -0.095263 \\
\hline 12 & 1 & -3.254112 & 0.529121 & -0.955806 \\
\hline 13 & 1 & -0.484640 & o -2.376598 & -0.281632 \\
\hline 14 & 1 & -1.184877 & 0.888594 & 2.457988 \\
\hline 15 & 1 & -0.295901 & 1.509109 & -1.687976 \\
\hline 16 & 1 & 1.660800 & 2.432881 & -0.817554 \\
\hline 17 & 6 & 1.763163 & $3-0.489685$ & -0.175583 \\
\hline 18 & 8 & 2.398667 & $7-1.488911$ & -0.000639 \\
\hline 19 & 1 & 0.953597 & $7 \quad 2.328428$ & 0.810328 \\
\hline 20 & 1 & -1.735268 & 2.123794 & 0.320836 \\
\hline 21 & 1 & -0.404683 & $3-1.578207$ & 2.142044 \\
\hline
\end{tabular}


Pyrylium.4.TS3 (2+4)

$\mathrm{HF}=-573.6993191$ hartrees $(-360002.059728441 \mathrm{kcal} / \mathrm{mol})$

Imaginary Frequencies: $1(-317.00661 / \mathrm{cm})$

Zero-point correction $=0.165801$ (Hartree/Particle)

Thermal correction to Energy=

0.174903

Thermal correction to Enthalpy=

0.175847

$\begin{array}{lr}\text { Thermal correction to Gibbs Free Energy }= & 0.131214 \\ \text { Sum of electronic and zero-point Energies }= & -573.533\end{array}$

$-573.533518$

Sum of electronic and thermal Energies=

$-573.524416$

Sum of electronic and thermal Enthalpies=

Sum of electronic and thermal Free Energies=

$-573.523472$

Coordinates (from last standard orientation):

\begin{tabular}{|c|c|c|c|c|}
\hline \multirow{2}{*}{$\begin{array}{l}\text { Center } \\
\text { Number }\end{array}$} & \multirow{2}{*}{$\begin{array}{l}\text { Atomic } \\
\text { Number }\end{array}$} & \multicolumn{3}{|c|}{ Coordinates (Angstroms) } \\
\hline & & & $X \quad Y$ & Z \\
\hline 1 & 8 & 0.797561 & -0.362750 & 1.364580 \\
\hline 2 & 6 & 0.735377 & 0.932793 & 1.102142 \\
\hline 3 & 6 & 1.470845 & 1.494701 & 0.068114 \\
\hline 4 & 6 & 2.460317 & 0.694512 & -0.576272 \\
\hline 5 & 6 & 2.346006 & -0.658655 & -0.446695 \\
\hline 6 & 6 & 1.206117 & -1.160208 & 0.294803 \\
\hline 7 & 6 & -0.256715 & -1.085560 & -0.797912 \\
\hline 8 & 6 & -0.587009 & 0.272958 & -1.027902 \\
\hline 9 & 1 & 0.152949 & 1.496960 & 1.823709 \\
\hline 10 & 1 & 1.226059 & -2.196201 & 0.621973 \\
\hline 11 & 1 & 3.242589 & 1.159819 & -1.166788 \\
\hline 12 & 6 & -1.456242 & -1.638214 & 0.001047 \\
\hline 13 & 6 & -1.818722 & 0.628746 & -0.253477 \\
\hline 14 & 8 & -2.198791 & -0.491188 & 0.430323 \\
\hline 15 & 8 & -2.374245 & 1.690312 & -0.192194 \\
\hline 16 & 1 & -1.192105 & -2.215716 & 0.889104 \\
\hline 17 & 1 & 0.102510 & -1.680481 & -1.636292 \\
\hline 18 & 1 & -0.230268 & 0.908616 & -1.827361 \\
\hline 19 & 1 & -2.087127 & -2.252155 & -0.650247 \\
\hline 20 & 1 & 1.386556 & 2.562080 & -0.107918 \\
\hline 21 & 1 & 3.002803 & -1.360356 & -0.950958 \\
\hline
\end{tabular}

Pyrylium.4.TS3 (2+4)product

$\mathrm{HF}=-573.731086$ hartrees $(-360021.99377586 \mathrm{kcal} / \mathrm{mol})$

Imaginary Frequencies: none found

Zero-point correction $=0.169171$ (Hartree/Particle)

Thermal correction to Energy $=\quad 0.177819$

Thermal correction to Enthalpy= $\quad 0.178763$

Thermal correction to Gibbs Free Energy $=\quad 0.135043$

Sum of electronic and zero-point Energies $=\quad-573.561915$

Sum of electronic and thermal Energies $=\quad-573.553267$

Sum of electronic and thermal Enthalpies $=\quad-573.552323$

Sum of electronic and thermal Free Energies $=\quad-573.596043$

Coordinates (from last standard orientation):

\begin{tabular}{|c|c|}
\hline enter & Atomic \\
\hline Jum & Number \\
\hline
\end{tabular}




$\begin{array}{ccrrr}1 & 8 & -0.874569 & 0.321742 & 1.475044 \\ 2 & 6 & -0.624745 & -0.890785 & 1.225040 \\ 3 & 6 & -0.713373 & -1.332586 & -0.184607 \\ 4 & 6 & -2.103676 & -0.922708 & -0.657643 \\ 5 & 6 & -2.400947 & 0.357556 & -0.419721 \\ 6 & 6 & -1.286489 & 1.121110 & 0.232571 \\ 7 & 6 & -0.037035 & 1.091208 & -0.693573 \\ 8 & 6 & 0.350328 & -0.379037 & -0.897960 \\ 9 & 1 & -0.305618 & -1.494886 & 2.075961 \\ 10 & 1 & -1.536169 & 2.098367 & 0.638712 \\ 11 & 1 & -2.764976 & -1.656755 & -1.104725 \\ 12 & 6 & 1.253071 & 1.744436 & -0.129078 \\ 13 & 6 & 1.723091 & -0.529738 & -0.214899 \\ 14 & 8 & 2.148416 & 0.673992 & 0.225712 \\ 15 & 8 & 2.293108 & -1.566378 & -0.014345 \\ 16 & 1 & 1.087729 & 2.345248 & 0.768693 \\ 17 & 1 & -0.340764 & 1.578995 & -1.622141 \\ 18 & 1 & 0.408007 & -0.694303 & -1.941996 \\ 19 & 1 & 1.742904 & 2.365189 & -0.882908 \\ 20 & 1 & -0.453874 & -2.382116 & -0.311398 \\ 21 & 1 & -3.334234 & 0.848680 & -0.672257 \\ ------------------------------------------------------\end{array}$

Pyrylium.4.TS4 (2+4)

$\mathrm{HF}=-573.7003004$ hartrees $(-360002.675504004 \mathrm{kcal} / \mathrm{mol})$

Imaginary Frequencies: 1 (-266.9956 1/cm)

Zero-point correction $=0.165195$ (Hartree/Particle)

Thermal correction to Energy $=\quad 0.174326$

Thermal correction to Enthalpy= $\quad 0.175270$

Thermal correction to Gibbs Free Energy $=\quad 0.130582$

Sum of electronic and zero-point Energies $=\quad-573.535105$

Sum of electronic and thermal Energies $=\quad-573.525974$

Sum of electronic and thermal Enthalpies $=\quad-573.525030$

Sum of electronic and thermal Free Energies $=\quad-573.569718$

Coordinates (from last standard orientation):

\begin{tabular}{|c|c|c|c|c|}
\hline \multirow{2}{*}{$\begin{array}{l}\text { Center } \\
\text { Number }\end{array}$} & \multirow{2}{*}{$\begin{array}{l}\text { Atomic } \\
\text { Number }\end{array}$} & \multicolumn{3}{|c|}{ Coordinates (Angstroms) } \\
\hline & & & $\begin{array}{ll}X \quad Y\end{array}$ & Z \\
\hline 1 & 8 & 0.648800 & -0.426923 & 1.325259 \\
\hline 2 & 6 & 1.194855 & 0.773913 & 1.182829 \\
\hline 3 & 6 & 2.120071 & 1.034477 & 0.190077 \\
\hline 4 & 6 & 2.641277 & -0.068621 & -0.556209 \\
\hline 5 & 6 & 1.926444 & -1.227298 & -0.542497 \\
\hline 6 & 1 & 2.206555 & -2.105780 & -1.115056 \\
\hline 7 & 6 & 0.659854 & -1.218991 & 0.174783 \\
\hline 8 & 6 & -0.549717 & -0.420778 & -0.871357 \\
\hline 9 & 6 & -0.327900 & 0.976450 & -0.994163 \\
\hline 10 & 1 & 0.930381 & 1.461936 & 1.979368 \\
\hline 11 & 1 & 0.181782 & $2-2.169167$ & 0.403289 \\
\hline 12 & 1 & 3.563797 & $7 \quad 0.038691$ & -1.117247 \\
\hline 13 & 6 & -1.858987 & $7 \quad-0.550876$ & -0.081435 \\
\hline 14 & 6 & -1.455025 & $5 \quad 1.702504$ & -0.322368 \\
\hline 15 & 8 & -2.244749 & 0.697344 & 0.313442 \\
\hline 16 & 1 & -0.467526 & $6-1.046981$ & -1.759016 \\
\hline 17 & 1 & 0.297275 & $5 \quad 1.454251$ & -1.739161 \\
\hline
\end{tabular}




$\begin{array}{rrrrr}18 & 1 & 2.550814 & 2.027793 & 0.121924 \\ 19 & 1 & -1.175483 & 2.458571 & 0.417292 \\ 20 & 1 & -2.054244 & 2.211486 & -1.094570 \\ 21 & 8 & -2.421375 & -1.562355 & 0.214451 \\ - & 8 & -\end{array}$

\begin{tabular}{|c|c|c|c|c|}
\hline \multicolumn{5}{|c|}{$\begin{array}{l}\text { Pyrylium.4.TS4 }(2+4) \text { product } \\
\text { HF }=-573.7313845 \text { hartrees }(-360022.181087595 \mathrm{kcal} / \mathrm{n} \\
\text { Imaginary Frequencies: none found } \\
\text { Zero-point correction }=0.169231 \text { (Hartree } / \text { Particle) }\end{array}$} \\
\hline & \multirow{2}{*}{\multicolumn{2}{|c|}{ Thermal correction to Energy= }} & & 0.177711 \\
\hline \multicolumn{3}{|c|}{ Thermal correction to Enthalpy= } & & 0.178655 \\
\hline \multicolumn{4}{|c|}{ Thermal correction to Gibbs Free Energy= } & \\
\hline \multicolumn{4}{|c|}{ Sum of electronic and zero-point Energies= } & \\
\hline \multicolumn{3}{|c|}{$\begin{array}{l}\text { Sum of electronic and zero-point Energies= } \\
\text { Sum of electronic and thermal Energies= }\end{array}$} & Energies $=$ & \\
\hline \multicolumn{5}{|c|}{ Sum of electronic and thermal Enthalpies= } \\
\hline \multirow{2}{*}{\multicolumn{5}{|c|}{$\begin{array}{l}\text { Sum of electronic and thermal Free Energies= } \\
\text { Coordinates (from last standard orientation): }\end{array}$}} \\
\hline & & & & \\
\hline \multirow{2}{*}{$\begin{array}{l}\text { Center } \\
\text { Number }\end{array}$} & \multirow{2}{*}{$\begin{array}{l}\text { Atomic } \\
\text { Numbe }\end{array}$} & \multicolumn{3}{|c|}{ Coordinates (Angstroms) } \\
\hline & & & & \\
\hline 1 & 8 & 0.600932 & -0.935639 & 1.225924 \\
\hline 2 & 6 & 0.852526 & 0.279451 & 8980 \\
\hline 3 & 6 & 1.253866 & 1.137453 & 0.324049 \\
\hline 4 & 6 & 2.394327 & 0.382271 & -0.364277 \\
\hline 5 & 6 & 524 & $-0 . \varepsilon$ & 377748 \\
\hline 6 & 1 & 687 & -1. & 867 \\
\hline 7 & 6 & 478 & -1.2 & -0 \\
\hline 8 & 6 & 6732 & -0. & 868 \\
\hline 9 & 6 & 0.035060 & 1.095354 & -0.718717 \\
\hline 10 & 1 & 0.738624 & 0.590288 & 2.498495 \\
\hline 11 & 1 & 0.470534 & -2.348789 & -0.286166 \\
\hline 12 & 1 & 3.341028 & 0.880572 & -0.541462 \\
\hline 13 & 6 & 4597 & -0.5 & -0.181940 \\
\hline 14 & 6 & -1.2 & 1.73 & -0.237100 \\
\hline 15 & 8 & -2.065830 & 0.672973 & 0.324979 \\
\hline 16 & 1 & -0.447850 & -0.672488 & -1.967542 \\
\hline 17 & 1 & 0.412581 & 1.580101 & -1.620425 \\
\hline 18 & 1 & 1.499442 & 2.152935 & 0.632745 \\
\hline 19 & 1 & -1.159583 & 2.506123 & 0.530591 \\
\hline 20 & 1 & -1.822821 & 2.173189 & -1.081715 \\
\hline 21 & 8 & -2.284796 & -1.558022 & 0.007225 \\
\hline
\end{tabular}




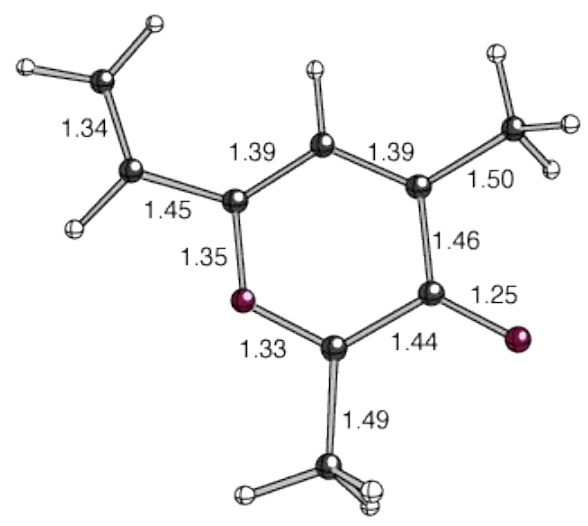

\section{A(s-cis)}

$\mathrm{HF}=-499.3717552$ hartrees $(-313360.770105552 \mathrm{kcal} / \mathrm{mol})$

Imaginary Frequencies: none found

Zero-point correction $=0.168212($ Hartree/Particle $)$

Thermal correction to Energy $=\quad 0.179396$

Thermal correction to Enthalpy= $\quad 0.180341$

Thermal correction to Gibbs Free Energy $=\quad 0.130611$

Sum of electronic and zero-point Energies $=\quad-499.203543$

Sum of electronic and thermal Energies $=\quad-499.192359$

Sum of electronic and thermal Enthalpies $=\quad-499.191415$

Sum of electronic and thermal Free Energies $=\quad-499.241144$

Coordinates (from last standard orientation):

\begin{tabular}{|c|c|c|c|c|}
\hline \multirow{2}{*}{$\begin{array}{l}\text { Center } \\
\text { Number }\end{array}$} & \multirow{2}{*}{$\begin{array}{l}\text { Atomic } \\
\text { Number }\end{array}$} & \multicolumn{3}{|c|}{ Coordinates (Angstroms) } \\
\hline & & & $X \quad Y$ & Z \\
\hline 1 & 8 & -0.486734 & -1.242476 & -0.000002 \\
\hline 2 & 8 & 2.778210 & 0.166687 & 0.000002 \\
\hline 3 & 6 & 0.841619 & -1.158563 & -0.000011 \\
\hline 4 & 6 & 1.530918 & 0.105945 & 0.000017 \\
\hline 5 & 6 & 0.651785 & 1.266264 & 0.000002 \\
\hline 6 & 6 & -0.722191 & 1.094860 & 0.000013 \\
\hline 7 & 1 & -1.378705 & 1.960174 & 0.000019 \\
\hline 8 & 6 & -1.306285 & -0.170792 & 0.000014 \\
\hline 9 & 6 & 1.568408 & -2.455033 & -0.000020 \\
\hline 10 & 1 & 2.227001 & $1-2.516386$ & -0.875250 \\
\hline 11 & 1 & 2.226141 & $1 \quad-2.516948$ & 0.875829 \\
\hline 12 & 1 & 0.874073 & $3 \quad-3.298506$ & -0.000592 \\
\hline 13 & 6 & 1.310175 & $5 \quad 2.608923$ & 0.000000 \\
\hline 14 & 1 & 1.967862 & 2.703765 & 0.872880 \\
\hline 15 & 1 & 1.967906 & 2.703737 & -0.872850 \\
\hline 16 & 1 & 0.585273 & 3.428105 & -0.000029 \\
\hline 17 & 6 & -2.707412 & $2-0.532887$ & 0.000018 \\
\hline 18 & 6 & -3.752059 & 90.311791 & -0.000007 \\
\hline 19 & 1 & -3.636476 & 1.391890 & -0.000363 \\
\hline 20 & 1 & -4.768722 & $2-0.066129$ & 0.000228 \\
\hline 21 & 1 & -2.885909 & $9-1.606441$ & -0.000031 \\
\hline
\end{tabular}




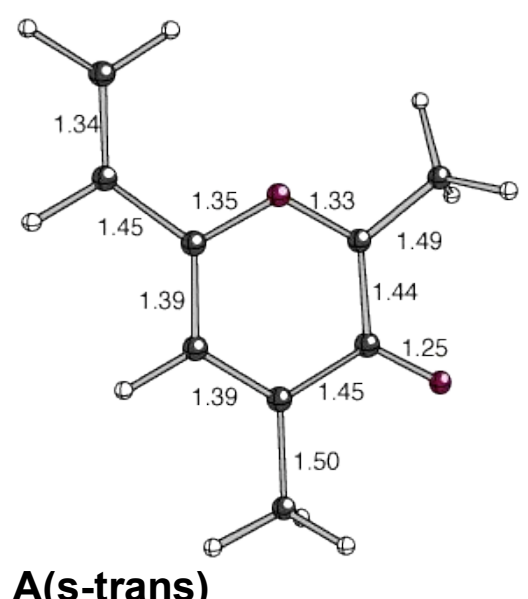

$\mathrm{HF}=-499.3727771$ hartrees $(-313361.411358021 \mathrm{kcal} / \mathrm{mol})$

Imaginary Frequencies: none found

Zero-point correction $=0.168364$ (Hartree/Particle)

Thermal correction to Energy $=\quad 0.179462$

Thermal correction to Enthalpy $=\quad 0.180406$

Thermal correction to Gibbs Free Energy $=0.131221$

Sum of electronic and zero-point Energies $=\quad-499.204413$

Sum of electronic and thermal Energies $=\quad-499.193315$

Sum of electronic and thermal Enthalpies $=\quad-499.192371$

Sum of electronic and thermal Free Energies $=\quad-499.241556$

Coordinates (from last standard orientation):

\begin{tabular}{|c|c|c|c|c|}
\hline \multirow{2}{*}{$\begin{array}{l}\text { Center } \\
\text { Number }\end{array}$} & \multirow{2}{*}{$\begin{array}{l}\text { Atomic } \\
\text { Number }\end{array}$} & \multicolumn{3}{|c|}{ Coordinates (Angstroms) } \\
\hline & & & $\begin{array}{ll}X \\
X\end{array}$ & Z \\
\hline 1 & 8 & 0.912735 & 0.750608 & 0.000021 \\
\hline 2 & 8 & -2.644930 & 0.779496 & -0.000171 \\
\hline 3 & 6 & -0.338446 & 1.206901 & 0.000064 \\
\hline 4 & 6 & -1.480279 & 0.327733 & 0.000258 \\
\hline 5 & 6 & -1.144479 & -1.086939 & 0.000037 \\
\hline 6 & 6 & 0.184773 & -1.481708 & -0.000005 \\
\hline 7 & 1 & 0.443756 & -2.537435 & -0.000059 \\
\hline 8 & 6 & 1.228118 & -0.558595 & 0.000030 \\
\hline 9 & 6 & -0.483440 & 2.685483 & -0.000031 \\
\hline 10 & 1 & -1.063296 & 3.004886 & -0.874908 \\
\hline 11 & 1 & -1.062160 & 3.005157 & 0.875510 \\
\hline 12 & 1 & 0.490451 & 3.180419 & -0.000703 \\
\hline 13 & 6 & -2.287508 & -2.050794 & -0.000024 \\
\hline 14 & 1 & -2.927763 & -1.872612 & 0.872871 \\
\hline 15 & 1 & -2.927849 & -1.872399 & -0.872813 \\
\hline 16 & 1 & -1.954188 & -3.092641 & -0.000168 \\
\hline 17 & 6 & 2.640679 & -0.869206 & 0.000004 \\
\hline 18 & 6 & 3.653240 & 0.013441 & -0.000084 \\
\hline 19 & 1 & 3.482310 & 1.085323 & 0.000144 \\
\hline 20 & 1 & 2.856387 & -1.935618 & -0.000072 \\
\hline 21 & 1 & 4.683955 & -0.323806 & -0.000096 \\
\hline
\end{tabular}




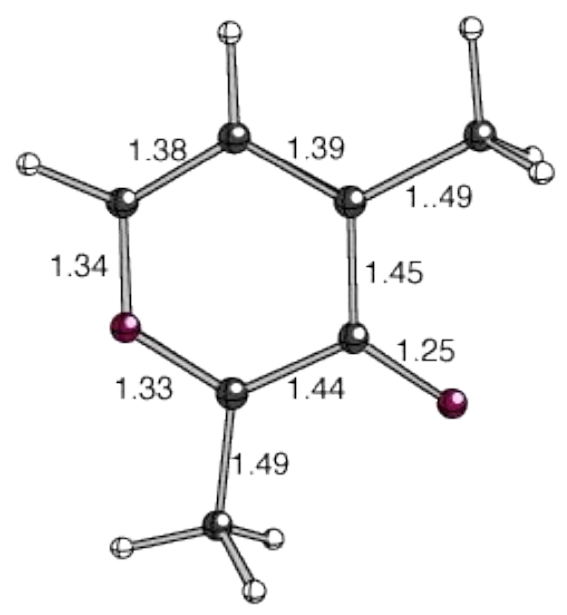

B

$\mathrm{HF}=-421.965054$ hartrees $(-264787.29103554 \mathrm{kcal} / \mathrm{mol})$ Imaginary Frequencies: none found

Zero-point correction $=0.135373$ (Hartree/Particle)

Thermal correction to Energy=

0.144112

Thermal correction to Enthalpy=

0.145056

Thermal correction to Gibbs Free Energy=

Sum of electronic and zero-point Energies=

0.101479

Sum of electronic and thermal Energies=

$-421.829681$

$-421.820942$

Sum of electronic and thermal Enthalpies $=\quad-421.819998$

Sum of electronic and thermal Free Energies $=\quad-421.863575$

Coordinates (from last standard orientation):

\begin{tabular}{|c|c|c|c|c|}
\hline \multirow{2}{*}{$\begin{array}{l}\text { Center } \\
\text { Number }\end{array}$} & \multirow{2}{*}{$\begin{array}{l}\text { Atomic } \\
\text { Number }\end{array}$} & \multicolumn{3}{|c|}{ Coordinates (Angstroms) } \\
\hline & & & $X \quad Y$ & Z \\
\hline 1 & 8 & 1.232094 & 1.245702 & -0.000034 \\
\hline 2 & 8 & -0.094723 & -2.056492 & -0.000124 \\
\hline 3 & 6 & 1.179533 & -0.085719 & 0.000053 \\
\hline 4 & 6 & -0.069868 & -0.807760 & -0.000065 \\
\hline 5 & 6 & -1.251260 & 0.038379 & -0.000017 \\
\hline 6 & 6 & -1.111502 & 1.424937 & -0.000031 \\
\hline 7 & 1 & -1.985551 & 2.070799 & -0.000100 \\
\hline 8 & 6 & 0.135646 & 2.011839 & -0.000017 \\
\hline 9 & 6 & 2.491920 & -0.782530 & 0.000091 \\
\hline 10 & 1 & 2.566772 & -1.439877 & 0.875200 \\
\hline 11 & 1 & 2.567042 & -1.439441 & -0.875331 \\
\hline 12 & 1 & 3.320202 & -0.070096 & 0.000369 \\
\hline 13 & 6 & -2.576118 & -0.653204 & 0.000083 \\
\hline 14 & 1 & -2.653746 & -1.314684 & -0.871993 \\
\hline 15 & 1 & -2.654078 & -1.313666 & 0.872939 \\
\hline 16 & 1 & -3.414261 & 0.049805 & -0.000410 \\
\hline 17 & 1 & 0.364546 & 3.067828 & 0.000012 \\
\hline
\end{tabular}




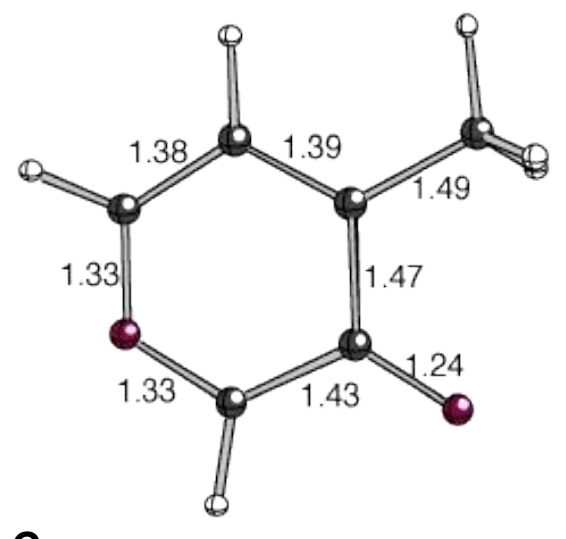

C

$\mathrm{HF}=-382.6386335$ hartrees $(-240109.568907585 \mathrm{kcal} / \mathrm{mol})$

Imaginary Frequencies: none found

Zero-point correction $=0.107892($ Hartree/Particle $)$

Thermal correction to Energy $=\quad 0.114768$

Thermal correction to Enthalpy= $\quad 0.115712$

Thermal correction to Gibbs Free Energy $=\quad 0.076829$

Sum of electronic and zero-point Energies $=\quad-382.530741$

Sum of electronic and thermal Energies $=\quad-382.523865$

Sum of electronic and thermal Enthalpies $=\quad-382.522921$

Sum of electronic and thermal Free Energies $=\quad-382.561805$

Coordinates (from last standard orientation):

\begin{tabular}{|c|c|c|c|c|}
\hline \multirow{2}{*}{$\begin{array}{l}\text { Center } \\
\text { Number }\end{array}$} & \multirow{2}{*}{$\begin{array}{l}\text { Atomic } \\
\text { Number }\end{array}$} & \multicolumn{3}{|c|}{ Coordinates (Angstroms) } \\
\hline & & & $\begin{array}{l}X \\
Y\end{array}$ & Z \\
\hline 1 & 8 & 1.917271 & 0.393770 & 0.000013 \\
\hline 2 & 8 & -1.413948 & 1.684001 & 0.000013 \\
\hline 3 & 6 & 0.903339 & 1.256471 & -0.000025 \\
\hline 4 & 6 & -0.477055 & 0.864995 & -0.000039 \\
\hline 5 & 6 & -0.663869 & -0.588185 & -0.000043 \\
\hline 6 & 6 & 0.440225 & -1.432167 & -0.000041 \\
\hline 7 & 1 & 0.314867 & -2.511682 & -0.000162 \\
\hline 8 & 6 & 1.726402 & -0.922360 & 0.000035 \\
\hline 9 & 6 & -2.073829 & -1.077824 & -0.000003 \\
\hline 10 & 1 & -2.605539 & -0.677856 & 0.872655 \\
\hline 11 & 1 & -2.606549 & -0.675247 & -0.870776 \\
\hline 12 & 1 & -2.139238 & -2.169505 & -0.001436 \\
\hline 13 & 1 & 2.650557 & -1.482828 & 0.000164 \\
\hline 14 & 1 & 1.228049 & 2.289372 & 0.000048 \\
\hline
\end{tabular}




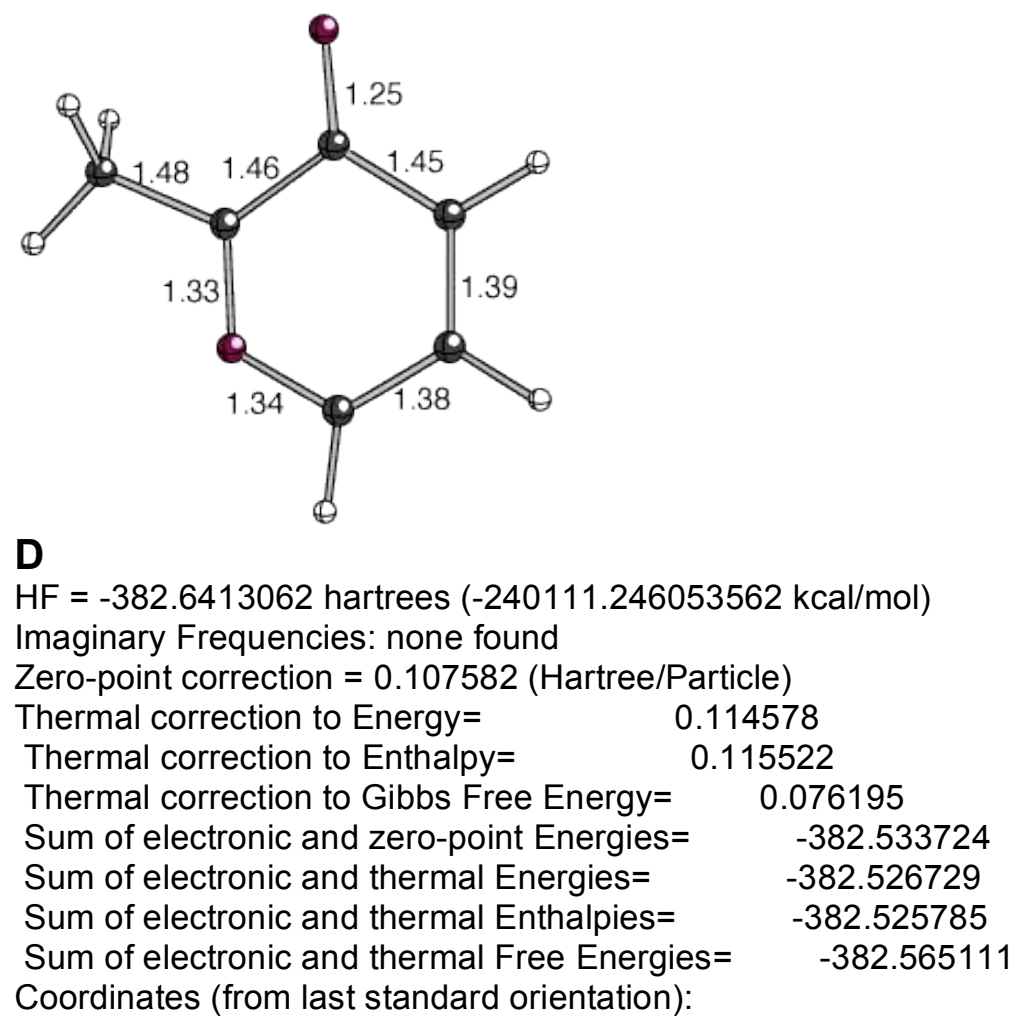

\begin{tabular}{|c|c|c|c|c|}
\hline \multirow{2}{*}{$\begin{array}{l}\text { Center } \\
\text { Number }\end{array}$} & \multirow{2}{*}{$\begin{array}{l}\text { Atomic } \\
\text { Number }\end{array}$} & \multicolumn{3}{|c|}{ Coordinates (Angstroms) } \\
\hline & & & $X \quad Y$ & Z \\
\hline 1 & 8 & 0.372548 & -1.368754 & -0.000049 \\
\hline 2 & 8 & -1.422183 & 1.698713 & 0.000039 \\
\hline 3 & 6 & -0.640870 & -0.512802 & -0.000081 \\
\hline 4 & 6 & -0.444173 & 0.929235 & -0.000011 \\
\hline 5 & 6 & 0.949989 & 1.307829 & -0.000041 \\
\hline 6 & 6 & 1.964307 & 0.358183 & 0.000039 \\
\hline 7 & 1 & 3.009857 & 0.651871 & -0.000033 \\
\hline 8 & 6 & 1.658332 & -0.986533 & 0.000042 \\
\hline 9 & 1 & 2.341088 & -1.823361 & 0.000230 \\
\hline 10 & 6 & -1.998154 & $4 \quad-1.113950$ & -0.000005 \\
\hline 11 & 1 & -2.558426 & $6-0.764209$ & 0.876047 \\
\hline 12 & 1 & -2.560293 & -0.759471 & -0.872898 \\
\hline 13 & 1 & -1.952490 & -2.205254 & -0.002868 \\
\hline 14 & 1 & 1.180760 & 2.368980 & -0.000058 \\
\hline
\end{tabular}




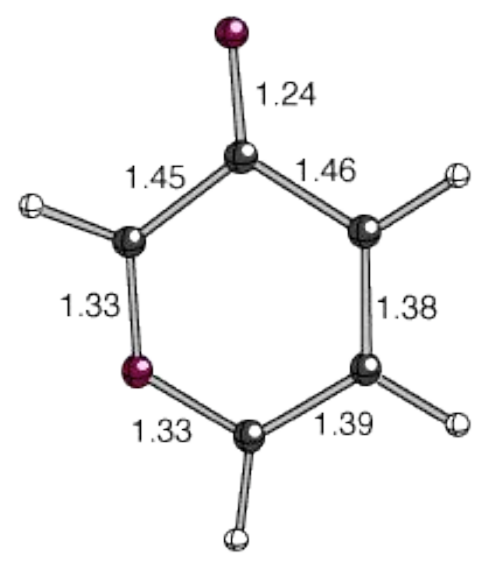

E

$\mathrm{HF}=-343.3138655$ hartrees $(-215432.883739905 \mathrm{kcal} / \mathrm{mol})$

Imaginary Frequencies: none found

Zero-point correction $=0.079960$ (Hartree/Particle $)$

Thermal correction to Energy $=\quad 0.085234$

Thermal correction to Enthalpy $=\quad 0.086178$

Thermal correction to Gibbs Free Energy $=\quad 0.050954$

Sum of electronic and zero-point Energies $=\quad-343.233906$

Sum of electronic and thermal Energies $=\quad-343.228632$

Sum of electronic and thermal Enthalpies $=\quad-343.227688$

Sum of electronic and thermal Free Energies $=\quad-343.262911$

Coordinates (from last standard orientation):

\begin{tabular}{|c|c|c|c|c|}
\hline Center & Atomic & & oordinates (A & Angstroms) \\
\hline Number & Numbe & & $\begin{array}{ll}X & Y\end{array}$ & Z \\
\hline 1 & 8 & 1.033576 & -1.169952 & 0.000012 \\
\hline 2 & 8 & -2.332575 & 0.009847 & -0.000201 \\
\hline 3 & 6 & -0.291833 & -1.164950 & 0.000178 \\
\hline 4 & 6 & -1.093209 & 0.039104 & -0.000025 \\
\hline 5 & 6 & -0.266718 & 1.238287 & 0.000126 \\
\hline 6 & 6 & 1.116420 & 1.174840 & 0.000012 \\
\hline 7 & 1 & 1.722383 & 2.076020 & 0.000340 \\
\hline 8 & 6 & 1.759464 & -0.052287 & -0.000115 \\
\hline 9 & 1 & 2.823291 & -0.240305 & -0.000804 \\
\hline 10 & 1 & -0.779666 & 2.195797 & 0.000324 \\
\hline 11 & 1 & -0.718755 & -2.160636 & 0.000598 \\
\hline
\end{tabular}

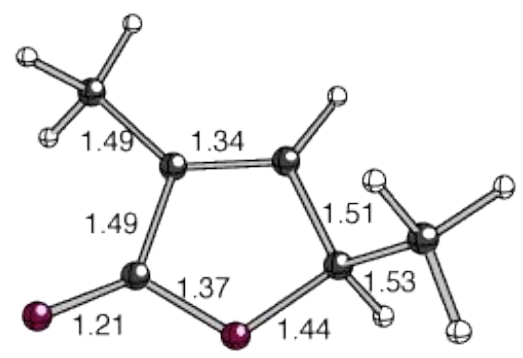

1

$\mathrm{HF}=-383.9074538$ hartrees $(-240905.766334038 \mathrm{kcal} / \mathrm{mol})$

Imaginary Frequencies: none found

Zero-point correction $=0.131040($ Hartree/Particle $)$ 


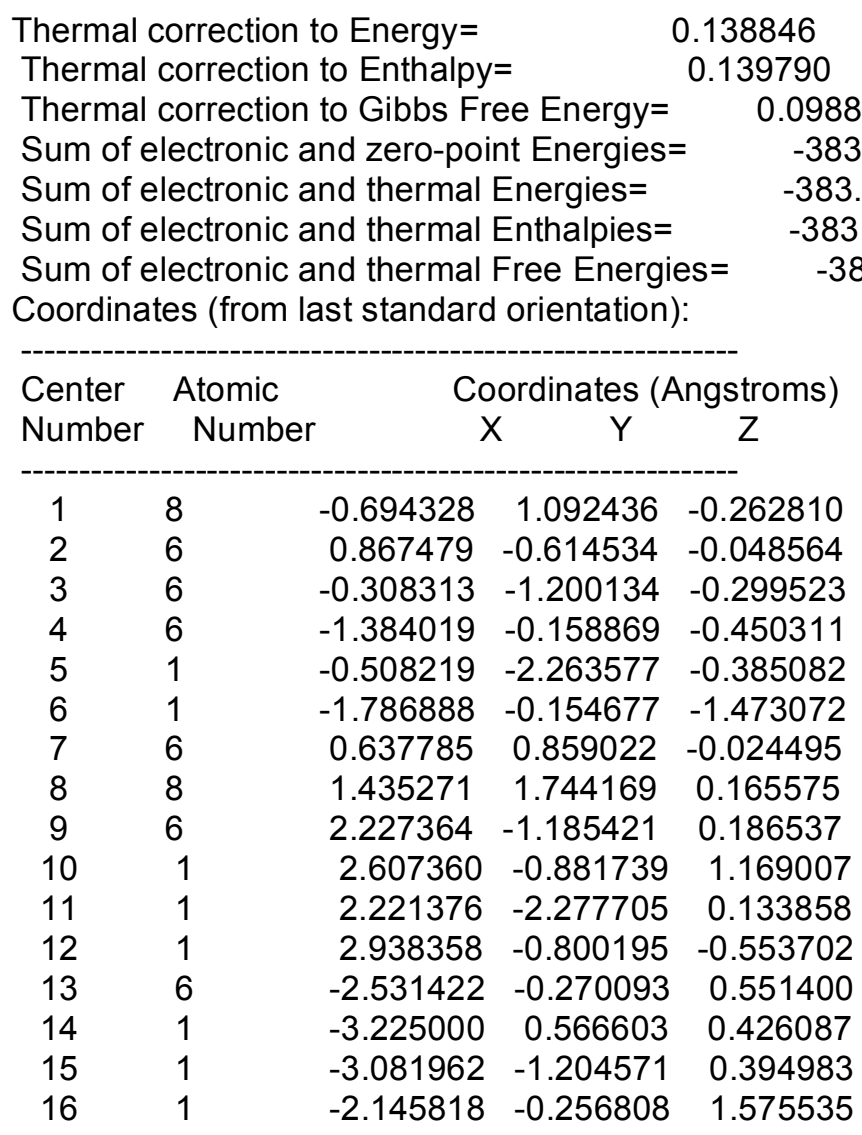

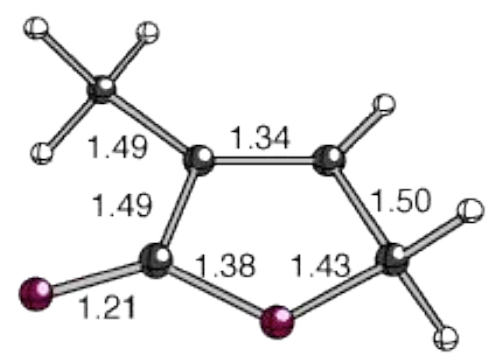

2

$\mathrm{HF}=-344.5872368$ hartrees $(-216231.936964368 \mathrm{kcal} / \mathrm{mol})$ Imaginary Frequencies: none found Zero-point correction $=0.102908$ (Hartree/Particle)

Thermal correction to Energy $=\quad 0.109273$

Thermal correction to Enthalpy $=\quad 0.110217$

Thermal correction to Gibbs Free Energy $=\quad 0.072560$

Sum of electronic and zero-point Energies $=\quad-344.484329$

Sum of electronic and thermal Energies $=\quad-344.477964$

Sum of electronic and thermal Enthalpies $=\quad-344.477020$

Sum of electronic and thermal Free Energies $=\quad-344.514677$

Coordinates (from last standard orientation):

$\begin{array}{llll}\text { Center } & \text { Atomic } & \text { Coordinates (Angstroms) } \\ \text { Number Number } & X & \text { Y }\end{array}$

$\begin{array}{lllll}1 & 8 & -1.358502 & 0.714327 & -0.000330\end{array}$




$\begin{array}{ccrcc}2 & 6 & 0.615787 & -0.514594 & 0.000062 \\ 3 & 6 & -0.385908 & -1.401026 & 0.000038 \\ 4 & 6 & -1.699590 & -0.675316 & 0.000107 \\ 5 & 1 & -0.308550 & -2.483204 & -0.000227 \\ 6 & 1 & -2.308897 & -0.890345 & -0.887391 \\ 7 & 6 & 0.010624 & 0.848844 & 0.000012 \\ 8 & 8 & 0.562246 & 1.921148 & 0.000151 \\ 9 & 6 & 2.097914 & -0.696985 & -0.000070 \\ 10 & 1 & 2.543957 & -0.215688 & 0.878081 \\ 11 & 1 & 2.374723 & -1.754931 & -0.000358 \\ 12 & 1 & 2.543679 & -0.215253 & -0.878137 \\ 13 & 1 & -2.307823 & -0.889919 & 0.888569 \\ - & 1 & \end{array}$

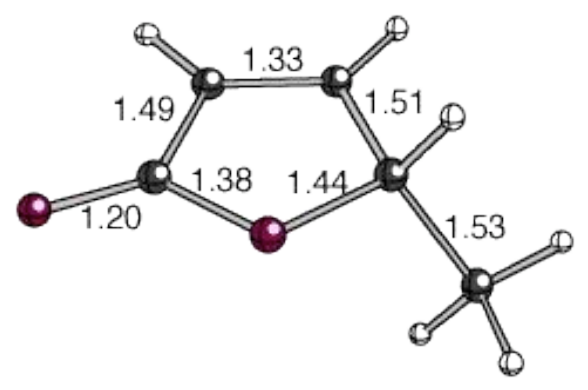

3

$\mathrm{HF}=-344.5844788$ hartrees $(-216230.206291788 \mathrm{kcal} / \mathrm{mol})$

Imaginary Frequencies: none found

Zero-point correction $=0.103094$ (Hartree/Particle)

Thermal correction to Energy $=\quad 0.109164$

Thermal correction to Enthalpy $=\quad 0.110108$

Thermal correction to Gibbs Free Energy $=\quad 0.073227$

Sum of electronic and zero-point Energies $=\quad-344.481385$

Sum of electronic and thermal Energies $=\quad-344.475315$

Sum of electronic and thermal Enthalpies $=\quad-344.474371$

Sum of electronic and thermal Free Energies $=\quad-344.511251$

Coordinates (from last standard orientation):

\begin{tabular}{|c|c|c|c|c|}
\hline \multirow{2}{*}{$\begin{array}{l}\text { Center } \\
\text { Number }\end{array}$} & \multirow{2}{*}{$\begin{array}{l}\text { Atomic } \\
\text { Number }\end{array}$} & \multicolumn{3}{|c|}{ Coordinates (Angstroms) } \\
\hline & & & $X \quad Y$ & Z \\
\hline 1 & 8 & -0.096819 & -0.912693 & 0.341565 \\
\hline 2 & 6 & -0.771605 & 1.237767 & -0.159326 \\
\hline 3 & 6 & 0.529918 & 1.315537 & 0.123281 \\
\hline 4 & 6 & 1.052958 & -0.056296 & 0.458854 \\
\hline 5 & 1 & 1.159498 & 2.199043 & 0.120782 \\
\hline 6 & 6 & -1.203611 & -0.176817 & -0.025648 \\
\hline 7 & 8 & -2.289721 & -0.671398 & -0.188095 \\
\hline 8 & 1 & 1.386724 & -0.099314 & 1.505409 \\
\hline 9 & 1 & -1.456719 & 2.024985 & -0.445256 \\
\hline 10 & 6 & 2.169238 & -0.555442 & -0.457194 \\
\hline 11 & 1 & 3.060800 & 0.072102 & -0.347154 \\
\hline 12 & 1 & 2.434698 & $3-1.584647$ & -0.199024 \\
\hline 13 & 1 & 1.845932 & -0.527934 & -1.502322 \\
\hline
\end{tabular}




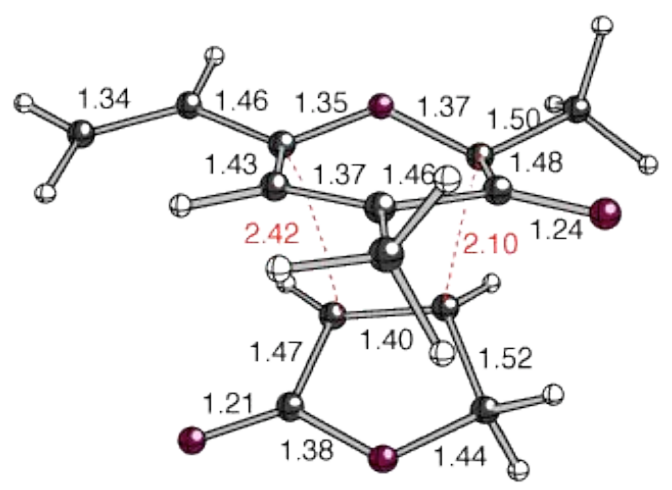

\begin{abstract}
A.IV.TS1 (s-cis)
$\mathrm{HF}=-804.6159533$ hartrees $(-504904.556855283 \mathrm{kcal} / \mathrm{mol})$

Imaginary Frequencies: $1(-406.44011 / \mathrm{cm})$

Zero-point correction $=0.245353$ (Hartree/Particle)

Thermal correction to Energy $=\quad 0.261199$

Thermal correction to Enthalpy= $\quad 0.262144$

Thermal correction to Gibbs Free Energy $=\quad 0.202665$

Sum of electronic and zero-point Energies $=\quad-804.370600$

Sum of electronic and thermal Energies $=\quad-804.354754$

Sum of electronic and thermal Enthalpies $=\quad-804.353810$

Sum of electronic and thermal Free Energies $=\quad-804.413289$

Coordinates (from last standard orientation):
\end{abstract}

\begin{tabular}{|c|c|c|c|c|}
\hline \multirow{2}{*}{$\begin{array}{l}\text { Center } \\
\text { Number }\end{array}$} & \multirow{2}{*}{$\begin{array}{l}\text { Atomic } \\
\text { Numbe }\end{array}$} & \multicolumn{3}{|c|}{ Coordinates (Angstroms) } \\
\hline & & & $\begin{array}{ll}X \quad Y\end{array}$ & Z \\
\hline 1 & 8 & 0.075216 & -1.757011 & -0.563988 \\
\hline 2 & 8 & -2.829484 & -0.202743 & 0.768304 \\
\hline 3 & 6 & -1.138082 & -1.129932 & -0.602709 \\
\hline 4 & 8 & -0.168750 & 2.428994 & -0.287920 \\
\hline 5 & 6 & -1.633851 & -0.501693 & 0.638113 \\
\hline 6 & 6 & -0.607240 & -0.173553 & 1.627272 \\
\hline 7 & 6 & 0.695031 & -0.467668 & 1.332980 \\
\hline 8 & 1 & 1.492816 & -0.162827 & 2.002395 \\
\hline 9 & 6 & 1.058731 & -1.173835 & 0.150367 \\
\hline 10 & 6 & 0.859391 & 0.686704 & -1.391255 \\
\hline 11 & 1 & 1.681503 & 0.226996 & -1.919200 \\
\hline 12 & 6 & -0.505019 & 0.562797 & -1.671994 \\
\hline 13 & 6 & -1.161302 & 1.821397 & -1.130167 \\
\hline 14 & 1 & -0.870462 & 0.141023 & -2.603520 \\
\hline 15 & 1 & -1.399662 & 2.512302 & -1.949780 \\
\hline 16 & 6 & 1.066371 & 1.854382 & -0.521546 \\
\hline 17 & 8 & 2.078743 & 2.283915 & -0.018002 \\
\hline 18 & 1 & -2.064795 & 1.657193 & -0.537444 \\
\hline 19 & 6 & -2.127663 & -1.842504 & -1.478270 \\
\hline 20 & 1 & -2.507040 & -2.742194 & -0.978252 \\
\hline 21 & 1 & -2.982875 & -1.188521 & -1.660345 \\
\hline 22 & 1 & -1.671681 & -2.137641 & -2.427582 \\
\hline 23 & 6 & -1.048099 & 0.496693 & 2.891548 \\
\hline 24 & 1 & -1.569087 & 1.431890 & 2.655998 \\
\hline 25 & 1 & -1.769495 & -0.130056 & 3.428745 \\
\hline 26 & 1 & -0.202922 & 0.713868 & 3.550253 \\
\hline 27 & 6 & 2.376078 & -1.739959 & -0.117418 \\
\hline
\end{tabular}




$\begin{array}{rrrrr}28 & 6 & 3.530606 & -1.315792 & 0.415098 \\ 29 & 1 & 4.470786 & -1.783088 & 0.141479 \\ 30 & 1 & 2.372383 & -2.548349 & -0.846007 \\ 31 & 1 & 3.585025 & -0.478060 & 1.103998 \\ -\end{array}$

\begin{abstract}
A.IV.TS1 product (s-cis)
$\mathrm{HF}=-804.6686651$ hartrees $(-504937.634036901 \mathrm{kcal} / \mathrm{mol})$

Imaginary Frequencies: none found

Zero-point correction $=0.249726$ (Hartree/Particle)

Thermal correction to Energy= $\quad 0.264847$

Thermal correction to Enthalpy= $\quad 0.265791$

Thermal correction to Gibbs Free Energy $=\quad 0.208112$

Sum of electronic and zero-point Energies $=\quad-804.418939$

Sum of electronic and thermal Energies $=\quad-804.403818$

Sum of electronic and thermal Enthalpies $=\quad-804.402874$

Sum of electronic and thermal Free Energies $=\quad-804.460554$

Coordinates (from last standard orientation):
\end{abstract}

\begin{tabular}{|c|c|c|c|c|}
\hline \multirow{2}{*}{$\begin{array}{l}\text { Center } \\
\text { Number }\end{array}$} & \multirow{2}{*}{$\begin{array}{l}\text { Atomic } \\
\text { Number }\end{array}$} & \multicolumn{3}{|c|}{ Coordinates (Angstroms) } \\
\hline & & & $X \quad Y$ & Z \\
\hline 1 & 8 & -0.140160 & -1.599825 & -0.819651 \\
\hline 2 & 8 & -2.933567 & 0.054593 & 0.533311 \\
\hline 3 & 6 & -1.152710 & -0.594765 & -0.967685 \\
\hline 4 & 8 & 0.312433 & 2.471349 & -0.042884 \\
\hline 5 & 6 & -1.774546 & -0.319008 & 0.417413 \\
\hline 6 & 6 & -0.860645 & -0.450733 & 1.581494 \\
\hline 7 & 6 & 0.436380 & -0.706148 & 1.326834 \\
\hline 8 & 1 & 1.180622 & -0.706106 & 2.119039 \\
\hline 9 & 6 & 0.920774 & -0.962379 & -0.094017 \\
\hline 10 & 6 & 1.078494 & 0.400722 & -0.880498 \\
\hline 11 & 1 & 1.864594 & 0.287964 & -1.629991 \\
\hline 12 & 6 & -0.317854 & 0.642139 & -1.471381 \\
\hline 13 & 6 & -0.696992 & 2.059476 & -0.990564 \\
\hline 14 & 1 & -0.305947 & 0.599312 & -2.563197 \\
\hline 15 & 1 & -0.691681 & 2.780558 & -1.813205 \\
\hline 16 & 6 & 1.366302 & 1.615495 & -0.010343 \\
\hline 17 & 8 & 2.353423 & 1.827174 & 0.649938 \\
\hline 18 & 1 & -1.664676 & 2.111042 & -0.486467 \\
\hline 19 & 6 & -2.195043 & -1.084518 & -1.958576 \\
\hline 20 & 1 & -2.658859 & -2.007200 & -1.598232 \\
\hline 21 & 1 & -2.982278 & -0.336447 & -2.080943 \\
\hline 22 & 1 & -1.724485 & -1.283324 & -2.926295 \\
\hline 23 & 6 & -1.435745 & -0.211070 & 2.948599 \\
\hline 24 & 1 & -1.920069 & 0.770323 & 2.997160 \\
\hline 25 & 1 & -2.210200 & -0.952080 & 3.178939 \\
\hline 26 & 1 & -0.660688 & -0.263760 & 3.718275 \\
\hline 27 & 6 & 2.127721 & -1.866186 & -0.125837 \\
\hline 28 & 6 & 3.387180 & -1.448488 & -0.004773 \\
\hline 29 & 1 & 4.213274 & -2.153869 & -0.001904 \\
\hline 30 & 1 & 1.888690 & -2.923926 & -0.218281 \\
\hline 31 & 1 & 3.634780 & -0.396018 & 0.115388 \\
\hline
\end{tabular}




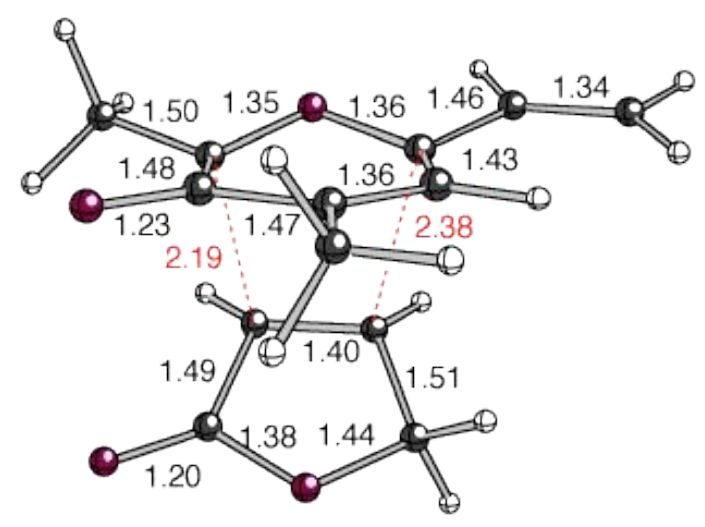

\begin{abstract}
A.IV.TS2 (s-cis)
$\mathrm{HF}=-804.6073028$ hartrees $(-504899.128580028 \mathrm{kcal} / \mathrm{mol})$

Imaginary Frequencies: $1(-433.67601 / \mathrm{cm})$

Zero-point correction $=0.245079$ (Hartree/Particle)

Thermal correction to Energy $=\quad 0.260994$

Thermal correction to Enthalpy= $\quad 0.261938$

Thermal correction to Gibbs Free Energy $=\quad 0.201987$

Sum of electronic and zero-point Energies $=\quad-804.362223$

Sum of electronic and thermal Energies $=\quad-804.346309$

Sum of electronic and thermal Enthalpies $=\quad-804.345365$

Sum of electronic and thermal Free Energies $=\quad-804.405316$

Coordinates (from last standard orientation):
\end{abstract}

\begin{tabular}{|c|c|c|c|c|}
\hline \multirow{2}{*}{$\begin{array}{l}\text { Center } \\
\text { Number }\end{array}$} & \multirow{2}{*}{$\begin{array}{l}\text { Atomic } \\
\text { Number }\end{array}$} & \multicolumn{3}{|c|}{ Coordinates (Angstroms) } \\
\hline & & & $X \quad Y$ & Z \\
\hline 1 & 8 & -0.927505 & -0.857641 & -1.307436 \\
\hline 2 & 6 & -1.653617 & -0.317520 & -0.289581 \\
\hline 3 & 8 & 1.205132 & 1.988544 & 1.033470 \\
\hline 4 & 6 & -1.244461 & -0.643375 & 1.042434 \\
\hline 5 & 6 & -0.021518 & -1.182300 & 1.314854 \\
\hline 6 & 6 & 0.910803 & -1.389184 & 0.200895 \\
\hline 7 & 6 & 0.406250 & -0.977410 & -1.129760 \\
\hline 8 & 6 & 0.655526 & 1.194660 & -1.073088 \\
\hline 9 & 1 & 0.876071 & 1.160814 & -2.132309 \\
\hline 10 & 6 & -0.531115 & 1.678425 & -0.521569 \\
\hline 11 & 6 & -0.184814 & 2.276790 & 0.824840 \\
\hline 12 & 1 & -1.363923 & 2.064466 & -1.095706 \\
\hline 13 & 1 & -0.320379 & 3.366940 & 0.818068 \\
\hline 14 & 6 & 1.761893 & 1.480320 & -0.121667 \\
\hline 15 & 8 & 2.947571 & 1.318289 & -0.252214 \\
\hline 16 & 1 & -0.744831 & 1.869609 & 1.672117 \\
\hline 17 & 1 & -1.942443 & -0.435685 & 1.849075 \\
\hline 18 & 8 & 2.042955 & -1.857668 & 0.334900 \\
\hline 19 & 6 & 1.157404 & -1.438014 & -2.338509 \\
\hline 20 & 1 & 2.199170 & -1.119907 & -2.248114 \\
\hline 21 & 1 & 1.157879 & -2.533742 & -2.396985 \\
\hline 22 & 1 & 0.715916 & -1.033283 & -3.253083 \\
\hline 23 & 6 & 0.443391 & -1.578074 & 2.682313 \\
\hline 24 & 1 & 0.682963 & -2.647326 & 2.712901 \\
\hline 25 & 1 & 1.372985 & -1.050279 & 2.925506 \\
\hline 26 & 1 & -0.308565 & -1.360274 & 3.446525 \\
\hline 27 & 6 & -3.030861 & -0.031830 & -0.690110 \\
\hline
\end{tabular}




$\begin{array}{rrrrr}28 & 6 & -4.051582 & 0.291741 & 0.115295 \\ 29 & 1 & -5.029191 & 0.521533 & -0.295243 \\ 30 & 1 & -3.185361 & -0.064253 & -1.767207 \\ 31 & 1 & -3.959306 & 0.343829 & 1.196601\end{array}$

\begin{abstract}
A.IV.TS2product (s-cis)
$\mathrm{HF}=-804.6645254$ hartrees $(-504935.036333754 \mathrm{kcal} / \mathrm{mol})$

Imaginary Frequencies: none found

Zero-point correction $=0.249522($ Hartree/Particle $)$

Thermal correction to Energy $=\quad 0.264670$

Thermal correction to Enthalpy $=\quad 0.265614$

Thermal correction to Gibbs Free Energy $=\quad 0.207670$

Sum of electronic and zero-point Energies $=\quad-804.415004$

Sum of electronic and thermal Energies $=\quad-804.399856$

Sum of electronic and thermal Enthalpies $=\quad-804.398911$

Sum of electronic and thermal Free Energies $=\quad-804.456856$

Coordinates (from last standard orientation):
\end{abstract}

\begin{tabular}{|c|c|c|c|c|}
\hline \multirow{2}{*}{$\begin{array}{l}\text { Center } \\
\text { Number }\end{array}$} & \multirow{2}{*}{$\begin{array}{l}\text { Atomic } \\
\text { Number }\end{array}$} & \multicolumn{3}{|c|}{ Coordinates (Angstroms) } \\
\hline & & & $\begin{array}{ll}X \quad Y\end{array}$ & Z \\
\hline 1 & 8 & 0.638561 & -0.909293 & 1.373894 \\
\hline 2 & 6 & 1.393867 & -0.258328 & 0.336643 \\
\hline 3 & 8 & -0.333418 & 2.269490 & -1.124743 \\
\hline 4 & 6 & 0.909065 & -0.752617 & -1.011391 \\
\hline 5 & 6 & -0.339167 & -1.225659 & -1.179138 \\
\hline 6 & 6 & -1.271270 & -1.179029 & -0.011602 \\
\hline 7 & 6 & -0.712574 & -0.431829 & 1.217016 \\
\hline 8 & 6 & -0.506610 & 1.106248 & 0.931424 \\
\hline 9 & 1 & -0.805730 & 1.678238 & 1.814963 \\
\hline 10 & 6 & 0.981031 & 1.241453 & 0.587397 \\
\hline 11 & 6 & 1.007861 & 2.210605 & -0.603932 \\
\hline 12 & 1 & 1.576333 & 1.617432 & 1.422902 \\
\hline 13 & 1 & 1.297599 & 3.221068 & -0.297388 \\
\hline 14 & 6 & -1.233296 & 1.690248 & -0.279080 \\
\hline 15 & 8 & -2.414280 & 1.673761 & -0.507876 \\
\hline 16 & 1 & 1.668221 & 1.892741 & -1.415479 \\
\hline 17 & 1 & 1.592946 & -0.713805 & -1.855549 \\
\hline 18 & 8 & -2.373258 & -1.694264 & -0.030339 \\
\hline 19 & 6 & -1.505676 & -0.726469 & 2.474509 \\
\hline 20 & 1 & -2.533600 & -0.374720 & 2.353415 \\
\hline 21 & 1 & -1.534727 & -1.802737 & 2.663270 \\
\hline 22 & 1 & -1.046961 & -0.226650 & 3.333460 \\
\hline 23 & 6 & -0.879722 & -1.783535 & -2.464006 \\
\hline 24 & 1 & -1.217425 & -2.815941 & -2.320327 \\
\hline 25 & 1 & -1.755666 & -1.212215 & -2.791673 \\
\hline 26 & 1 & -0.125608 & -1.764955 & -3.256196 \\
\hline 27 & 6 & 2.852891 & -0.497686 & 0.623156 \\
\hline 28 & 6 & 3.827333 & -0.742494 & -0.251779 \\
\hline 29 & 1 & 4.854303 & -0.859601 & 0.081540 \\
\hline 30 & 1 & 3.087247 & -0.418749 & 1.684371 \\
\hline 31 & 1 & 3.659830 & -0.843100 & -1.320106 \\
\hline
\end{tabular}




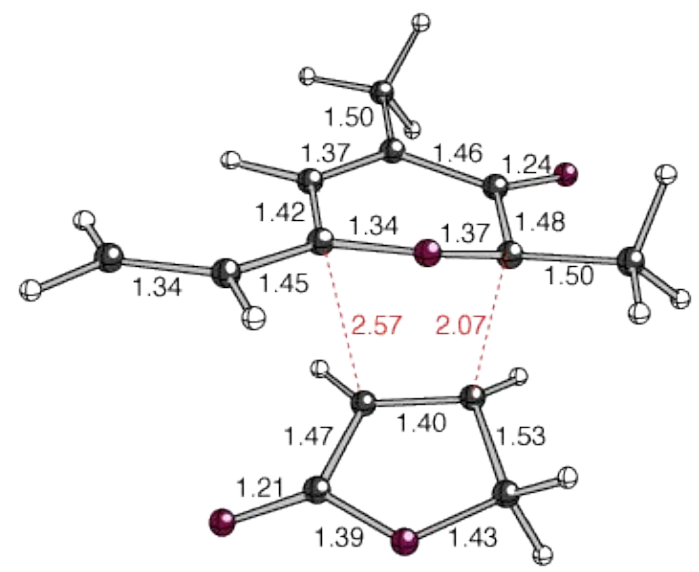
A.IV.TS3 (s-cis)
$\mathrm{HF}=-804.6133993$ hartrees $(-504902.954194743 \mathrm{kcal} / \mathrm{mol})$
Imaginary Frequencies: $1(-402.48981 / \mathrm{cm})$
Zero-point correction $=0.245022$ (Hartree/Particle)
Thermal correction to Energy $=\quad 0.261105$
Thermal correction to Enthalpy= $\quad 0.262049$
Thermal correction to Gibbs Free Energy $=\quad 0.201529$
Sum of electronic and zero-point Energies $=\quad-804.368378$
Sum of electronic and thermal Energies $=\quad-804.352295$
Sum of electronic and thermal Enthalpies $=\quad-804.351350$
Sum of electronic and thermal Free Energies $=\quad-804.411870$
Coordinates (from last standard orientation):

\begin{tabular}{ccrrr} 
Center & Atomic & \multicolumn{3}{c}{ Coordinates (Angstroms) } \\
Number & Number & X & $Y$ & $Z$ \\
- \hdashline 1 & 8 & 0.123901 & -0.008430 & 1.343402 \\
2 & 6 & -0.148450 & 1.143204 & 0.705157 \\
3 & 6 & 0.871928 & 1.712315 & -0.106837 \\
4 & 6 & 2.019186 & 1.034531 & -0.420450 \\
5 & 6 & 2.112961 & -0.370847 & -0.038863 \\
6 & 6 & 0.972058 & -0.897877 & 0.746847 \\
7 & 6 & -0.260338 & -1.460080 & -0.813694 \\
8 & 6 & -0.908071 & -0.312247 & -1.276558 \\
9 & 6 & -1.367512 & -2.323717 & -0.214865 \\
10 & 6 & -2.320138 & -0.338756 & -0.885084 \\
11 & 8 & -2.530657 & -1.493320 & -0.148922 \\
12 & 8 & -3.206427 & 0.455910 & -1.096290 \\
13 & 1 & -1.171720 & -2.710074 & 0.787527 \\
14 & 1 & 0.552187 & -1.944293 & -1.350273 \\
15 & 1 & -0.543893 & 0.396129 & -2.004921 \\
16 & 1 & -1.574780 & -3.177384 & -0.874244 \\
17 & 1 & 0.719510 & 2.727064 & -0.463583 \\
18 & 8 & 3.036950 & -1.119000 & -0.383207 \\
19 & 6 & 1.242793 & -2.103797 & 1.601933 \\
20 & 1 & 1.509943 & -2.956804 & 0.973277 \\
21 & 1 & 2.099556 & -1.914101 & 2.259552 \\
22 & 1 & 0.377628 & -2.351344 & 2.221037 \\
23 & 6 & -1.308506 & 1.833069 & 1.241261 \\
24 & 1 & -1.873826 & 1.248214 & 1.962587 \\
25 & 6 & -1.740551 & 3.050937 & 0.883966
\end{tabular}




$\begin{array}{rrrrr}26 & 1 & -1.232601 & 3.667767 & 0.148287 \\ 27 & 1 & -2.648514 & 3.461070 & 1.312110 \\ 28 & 6 & 3.163893 & 1.628668 & -1.182793 \\ 29 & 1 & 4.072809 & 1.637376 & -0.568801 \\ 30 & 1 & 3.397628 & 1.010657 & -2.057579 \\ 31 & 1 & 2.950410 & 2.652016 & -1.504950\end{array}$

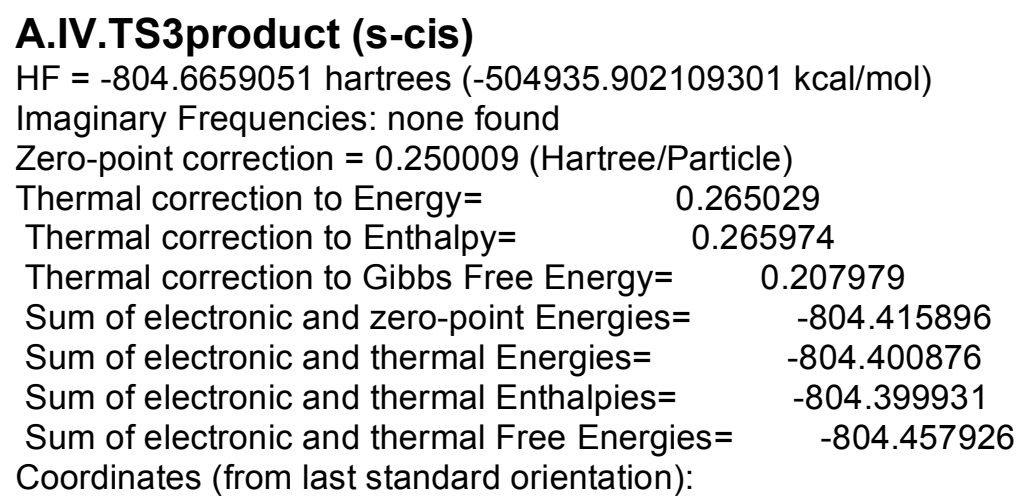

\begin{tabular}{|c|c|c|c|c|}
\hline \multirow{2}{*}{$\begin{array}{l}\text { Center } \\
\text { Number }\end{array}$} & \multirow{2}{*}{$\begin{array}{l}\text { Atomic } \\
\text { Number }\end{array}$} & \multicolumn{3}{|c|}{ Coordinates (Angstroms) } \\
\hline & & & $X \quad Y$ & Z \\
\hline 1 & 6 & -0.221013 & 1.061036 & 0.170951 \\
\hline 2 & 6 & 1.098235 & 1.477320 & -0.448186 \\
\hline 3 & 6 & 2.175300 & 0.671636 & -0.425654 \\
\hline 4 & 6 & 2.003430 & -0.702741 & 0.122757 \\
\hline 5 & 6 & 0.569505 & -1.049659 & 0.573017 \\
\hline 6 & 6 & -0.294264 & -1.210445 & -0.741954 \\
\hline 7 & 6 & -0.988362 & 0.161030 & -0.872497 \\
\hline 8 & 6 & -1.470863 & -2.200532 & -0.691685 \\
\hline 9 & 6 & -2.446536 & -0.117602 & -0.487078 \\
\hline 10 & 8 & -2.635668 & -1.451408 & -0.305527 \\
\hline 11 & 8 & -3.337812 & 0.680385 & -0.353720 \\
\hline 12 & 1 & -1.346842 & -3.013429 & 0.024790 \\
\hline 13 & 1 & 0.352742 & -1.454512 & -1.588128 \\
\hline 14 & 1 & -1.657833 & -2.635324 & -1.680180 \\
\hline 15 & 1 & 1.145631 & 2.442067 & -0.947205 \\
\hline 16 & 8 & 2.909201 & -1.520842 & 0.138466 \\
\hline 17 & 8 & 0.028761 & 0.111903 & 1.220285 \\
\hline 18 & 1 & -0.967652 & 0.599142 & -1.873956 \\
\hline 19 & 6 & 0.556927 & -2.201895 & 1.561133 \\
\hline 20 & 1 & 0.938021 & -3.114980 & 1.096237 \\
\hline 21 & 1 & 1.204062 & -1.965081 & 2.409933 \\
\hline 22 & 1 & -0.455478 & -2.371263 & 1.937827 \\
\hline 23 & 6 & 3.526960 & 1.032641 & -0.974539 \\
\hline 24 & 1 & 4.282993 & 1.007229 & -0.181183 \\
\hline 25 & 1 & 3.849491 & 0.307341 & -1.729955 \\
\hline 26 & 1 & 3.521946 & 2.032411 & -1.418598 \\
\hline 27 & 6 & -1.018073 & 2.192266 & 0.771046 \\
\hline 28 & 6 & -0.730678 & 3.491764 & 0.716950 \\
\hline 29 & 1 & -1.905319 & 1.863009 & 1.302356 \\
\hline 30 & 1 & 0.140434 & 3.896611 & 0.210882 \\
\hline 31 & 1 & -1.381459 & 4.217562 & 1.195572 \\
\hline
\end{tabular}




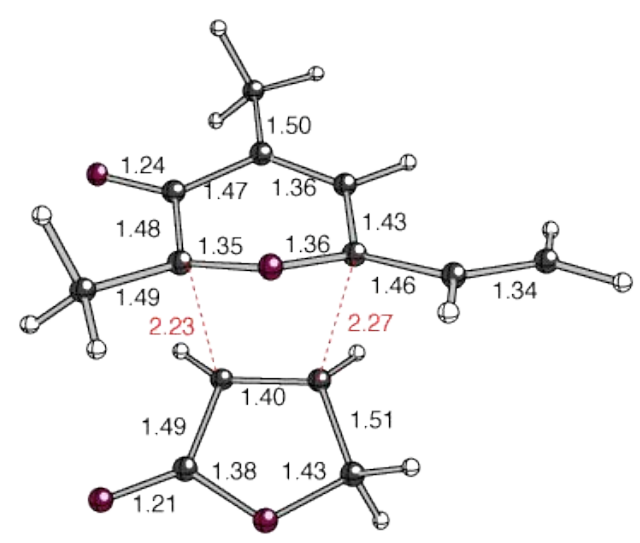

A.IV.TS4 (s-cis)

$\mathrm{HF}=-804.6113134$ hartrees $(-504901.645271634 \mathrm{kcal} / \mathrm{mol})$

Imaginary Frequencies: $1(-428.98421 / \mathrm{cm})$

Zero-point correction $=0.245195$ (Hartree/Particle)

Thermal correction to Energy $=\quad 0.261121$

Thermal correction to Enthalpy= $\quad 0.262065$

Thermal correction to Gibbs Free Energy $=\quad 0.202254$

Sum of electronic and zero-point Energies $=\quad-804.366118$

Sum of electronic and thermal Energies $=\quad-804.350193$

Sum of electronic and thermal Enthalpies $=\quad-804.349248$

Sum of electronic and thermal Free Energies $=\quad-804.409059$

Coordinates (from last standard orientation):

\begin{tabular}{ccrrr}
\hline Center & Atomic & \multicolumn{3}{c}{ Coordinates (Angstroms) } \\
Number & Number & X & $Y$ & $Z$ \\
- \hdashline 1 & 8 & -0.102056 & 0.290371 & 1.273786 \\
2 & 8 & -1.705842 & -2.586910 & -0.045584 \\
3 & 6 & -0.213825 & -0.983726 & 0.850112 \\
4 & 6 & -1.439817 & -1.393469 & 0.125822 \\
5 & 6 & -2.267823 & -0.296834 & -0.382587 \\
6 & 6 & -1.809652 & 0.977952 & -0.235002 \\
7 & 1 & -2.357308 & 1.813572 & -0.663446 \\
8 & 6 & -0.594990 & 1.273126 & 0.467991 \\
9 & 6 & 0.829159 & 0.748246 & -1.217480 \\
10 & 6 & 0.955528 & -0.629049 & -1.012697 \\
11 & 6 & 2.152022 & 1.381216 & -0.844489 \\
12 & 6 & 2.323254 & -0.885326 & -0.487425 \\
13 & 8 & 2.937938 & 0.326801 & -0.272797 \\
14 & 8 & 2.871857 & -1.933047 & -0.247323 \\
15 & 1 & 2.089416 & 2.199417 & -0.121059 \\
16 & 1 & 0.208939 & 1.197780 & -1.982075 \\
17 & 1 & 0.459560 & -1.410410 & -1.572989 \\
18 & 1 & 2.669335 & 1.760110 & -1.737120 \\
19 & 6 & 0.477652 & -1.986927 & 1.715289 \\
20 & 1 & -0.226530 & -2.393618 & 2.452164 \\
21 & 1 & 0.834403 & -2.819952 & 1.106263 \\
22 & 1 & 1.326580 & -1.534157 & 2.231262 \\
23 & 6 & -3.552862 & -0.659685 & -1.063792 \\
24 & 1 & -3.364140 & -1.373438 & -1.874234 \\
25 & 1 & -4.228627 & -1.168253 & -0.365917 \\
26 & 1 & -4.061788 & 0.221065 & -1.466562
\end{tabular}




$\begin{array}{rrrrr}27 & 6 & -0.234236 & 2.604788 & 0.956383 \\ 28 & 6 & -0.655026 & 3.778202 & 0.465289 \\ 29 & 1 & -0.303686 & 4.713941 & 0.887215 \\ 30 & 1 & 0.475298 & 2.589939 & 1.781569 \\ 31 & 1 & -1.352929 & 3.855201 & -0.364213\end{array}$

\section{A.IV.TS4product (s-cis)}

$\mathrm{HF}=-804.6676551$ hartrees $(-504937.000251801 \mathrm{kcal} / \mathrm{mol})$

Imaginary Frequencies: none found

Zero-point correction $=0.249359$ (Hartree/Particle)

Thermal correction to Energy $=\quad 0.264707$

Thermal correction to Enthalpy= $\quad 0.265652$

Thermal correction to Gibbs Free Energy $=\quad 0.206065$

Sum of electronic and zero-point Energies $=\quad-804.418296$

Sum of electronic and thermal Energies $=\quad-804.402948$

Sum of electronic and thermal Enthalpies $=\quad-804.402003$

Sum of electronic and thermal Free Energies $=\quad-804.461590$

Coordinates (from last standard orientation):

\begin{tabular}{|c|c|c|c|c|}
\hline \multirow{2}{*}{$\begin{array}{l}\text { Center } \\
\text { Number }\end{array}$} & \multirow{2}{*}{$\begin{array}{l}\text { Atomic } \\
\text { Number }\end{array}$} & \multicolumn{3}{|c|}{ Coordinates (Angstroms) } \\
\hline & & & $\begin{array}{l}X \\
Y\end{array}$ & Z \\
\hline 1 & 8 & -0.147599 & 0.283444 & 1.279110 \\
\hline 2 & 8 & -1.883746 & -2.502843 & 0.110158 \\
\hline 3 & 6 & -0.060192 & -0.981939 & 0.601651 \\
\hline 4 & 6 & -1.481381 & -1.351836 & 0.123894 \\
\hline 5 & 6 & -2.299957 & -0.214604 & -0.385111 \\
\hline 6 & 6 & -1.747988 & 1.011571 & -0.364821 \\
\hline 7 & 1 & -2.265609 & 1.869471 & -0.789230 \\
\hline 8 & 6 & -0.378442 & 1.253269 & 0.248713 \\
\hline 9 & 6 & 0.736377 & 0.881989 & -0.786045 \\
\hline 10 & 6 & 0.798001 & -0.651927 & -0.688965 \\
\hline 11 & 6 & 2.152902 & 1.323924 & -0.343434 \\
\hline 12 & 6 & 2.282387 & -0.981051 & -0.556887 \\
\hline 13 & 8 & 3.000227 & 0.161189 & -0.393284 \\
\hline 14 & 8 & 2.796364 & -2.068204 & -0.600714 \\
\hline 15 & 1 & 2.161762 & 1.703194 & 0.681475 \\
\hline 16 & 1 & 0.502485 & 1.243397 & -1.790010 \\
\hline 17 & 1 & 0.414497 & -1.189956 & -1.559032 \\
\hline 18 & 1 & 2.588979 & 2.078140 & -1.002177 \\
\hline 19 & 6 & 0.507704 & -2.021394 & 1.550003 \\
\hline 20 & 1 & -0.185559 & -2.182187 & 2.380010 \\
\hline 21 & 1 & 0.654403 & -2.969898 & 1.030450 \\
\hline 22 & 1 & 1.468120 & -1.685702 & 1.949399 \\
\hline 23 & 6 & -3.667148 & -0.532398 & -0.921468 \\
\hline 24 & 1 & -3.608874 & -1.282447 & -1.718262 \\
\hline 25 & 1 & -4.297197 & -0.966056 & -0.136403 \\
\hline 26 & 1 & -4.159743 & 0.363628 & -1.310195 \\
\hline 27 & 6 & -0.267394 & 2.627738 & 0.871413 \\
\hline 28 & 6 & -0.332210 & 3.779070 & 0.203059 \\
\hline 29 & 1 & -0.249388 & 4.733246 & 0.714711 \\
\hline 30 & 1 & -0.130961 & 2.620021 & 1.950807 \\
\hline 31 & 1 & -0.470832 & 3.821994 & -0.875727 \\
\hline
\end{tabular}




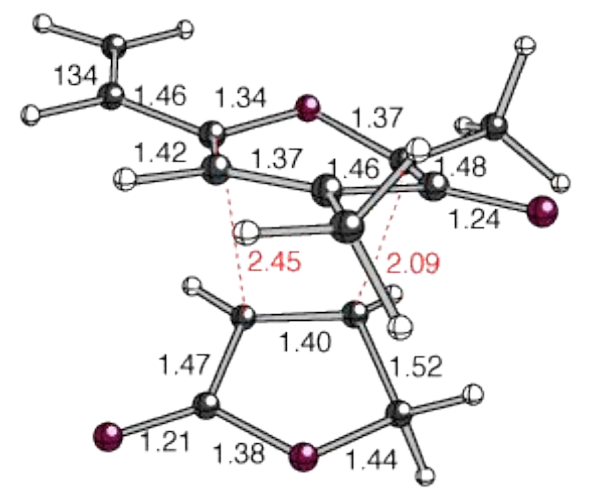

\section{A.IV.TS1 (s-trans)}

$\mathrm{HF}=-804.6171611$ hartrees $(-504905.314761861 \mathrm{kcal} / \mathrm{mol})$ Imaginary Frequencies: 1 (-402.2454 1/cm)

Zero-point correction $=0.245611$ (Hartree/Particle)

Thermal correction to Energy $=\quad 0.261361$

Thermal correction to Enthalpy= $\quad 0.262306$

Thermal correction to Gibbs Free Energy $=\quad 0.203213$

Sum of electronic and zero-point Energies $=\quad-804.371550$

Sum of electronic and thermal Energies $=\quad-804.355800$

Sum of electronic and thermal Enthalpies $=\quad-804.354855$

Sum of electronic and thermal Free Energies $=\quad-804.413948$

Coordinates (from last standard orientation):

\begin{tabular}{|c|c|c|c|c|}
\hline Center & Atomic & & oordinates ( & Angstroms) \\
\hline Number & Number & & $\begin{array}{ll}X & Y\end{array}$ & Z \\
\hline 1 & 8 & 1.095326 & -1.353811 & -0.202637 \\
\hline 2 & 8 & -2.422201 & -1.591217 & 0.253553 \\
\hline 3 & 6 & -0.213638 & -1.462981 & -0.585401 \\
\hline 4 & 8 & -1.359838 & 2.068836 & -0.602162 \\
\hline 5 & 6 & -1.257545 & -1.203974 & 0.427856 \\
\hline 6 & 6 & -0.839565 & -0.379167 & 1.559511 \\
\hline 7 & 6 & 0.454458 & 0.066858 & 1.589735 \\
\hline 8 & 1 & 0.782126 & 0.767820 & 2.352409 \\
\hline 9 & 6 & 1.419095 & -0.350746 & 0.630681 \\
\hline 10 & 6 & 6278 & 46860 & -1.163517 \\
\hline 11 & 1 & 1.725473 & 1.190356 & -1.416015 \\
\hline 12 & 6 & -0.290955 & 0.297573 & -1.711046 \\
\hline 13 & 6 & -1.624851 & 1.001357 & -1.524440 \\
\hline 14 & 1 & -0.117911 & -0.259141 & -2.627200 \\
\hline 15 & 1 & -1.967543 & 1.431996 & -2.474660 \\
\hline 16 & 6 & 0.003155 & 2.258771 & -0.473929 \\
\hline 17 & 8 & 0.464880 & 3.188829 & 0.144981 \\
\hline 18 & 1 & -2.423835 & 0.378987 & -1.113744 \\
\hline 19 & 6 & -0.434897 & -2.590612 & -1.551558 \\
\hline 20 & 1 & -0.410746 & -3.554345 & -1.028141 \\
\hline 21 & 1 & -1.424875 & -2.491788 & -2.001452 \\
\hline 22 & 1 & 0.332789 & -2.597236 & -2.330520 \\
\hline 23 & 6 & -1.867830 & -0.037432 & 2.592473 \\
\hline 24 & 1 & -2.720924 & 0.462794 & 2.119745 \\
\hline 25 & 1 & -2.264487 & & \\
\hline
\end{tabular}




$\begin{array}{rrrrr}26 & 1 & -1.458448 & 0.611341 & 3.371570 \\ 27 & 6 & 2.842776 & -0.079868 & 0.779949 \\ 28 & 1 & 3.076230 & 0.709739 & 1.490308 \\ 29 & 6 & 3.826588 & -0.709060 & 0.121610 \\ 30 & 1 & 3.622774 & -1.503656 & -0.589398 \\ 31 & 1 & 4.865635 & -0.445592 & 0.288535\end{array}$

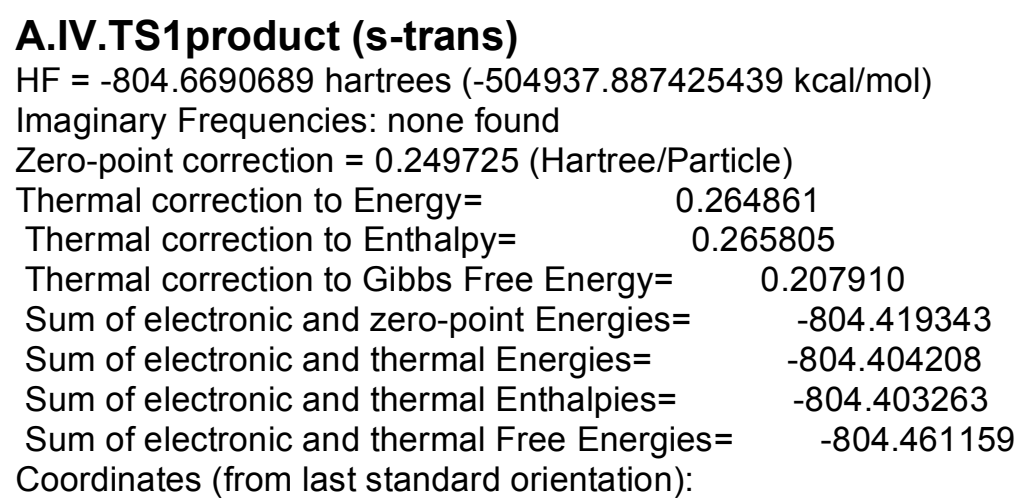

\begin{tabular}{|c|c|c|c|c|}
\hline \multirow{2}{*}{$\begin{array}{l}\text { Center } \\
\text { Number }\end{array}$} & \multirow{2}{*}{$\begin{array}{l}\text { Atomic } \\
\text { Number }\end{array}$} & \multicolumn{3}{|c|}{ Coordinates (Angstroms) } \\
\hline & & & $X \quad Y$ & Z \\
\hline 1 & 8 & 0.543966 & -1.556874 & -0.386041 \\
\hline 2 & 8 & -2.798357 & -0.740606 & 0.348763 \\
\hline 3 & 6 & -0.712194 & -1.028625 & -0.838019 \\
\hline 4 & 8 & -0.510606 & 2.463511 & -0.598187 \\
\hline 5 & 6 & -1.576792 & -0.702532 & 0.398320 \\
\hline 6 & 6 & -0.842149 & -0.264514 & 1.613274 \\
\hline 7 & 6 & 0.489043 & -0.095293 & 1.506212 \\
\hline 8 & 1 & .069083 & 0.327226 & 2.323096 \\
\hline 9 & 6 & 1.218194 & -0.454592 & 0.221341 \\
\hline 10 & 6 & 1.022853 & 30.688568 & -0.863322 \\
\hline 11 & 1 & 1.903054 & 0.723748 & -1.509279 \\
\hline 12 & 6 & -0.268680 & 0.284144 & -1.585342 \\
\hline 13 & 6 & -1.160505 & 1.541934 & -1.500447 \\
\hline 14 & 1 & -0.083669 & 0.014974 & -2.628053 \\
\hline 15 & 1 & -1.252421 & 2.036907 & -2.471867 \\
\hline 16 & 6 & 0.749921 & 2.065977 & -0.278456 \\
\hline 17 & 8 & 1.489513 & 2.729981 & 0.403058 \\
\hline 18 & 1 & -2.162161 & 1.347977 & -1.110184 \\
\hline 19 & 6 & -1.386590 & -2.054608 & -1.732585 \\
\hline 20 & 1 & -1.569860 & -2.980513 & -1.179932 \\
\hline 21 & 1 & -2.349438 & $8-1.674743$ & -2.083128 \\
\hline 22 & 1 & -0.746577 & $7 \quad-2.279020$ & -2.591378 \\
\hline 23 & 6 & -1.649414 & $4 \quad 0.030023$ & 2.845398 \\
\hline 24 & 1 & -2.426371 & 0.771467 & 2.629447 \\
\hline 25 & 1 & -2.167153 & $3-0.871069$ & 3.194452 \\
\hline 26 & 1 & -1.014985 & $5 \quad 0.404645$ & 3.653519 \\
\hline 27 & 6 & 2.665554 & $4-0.774515$ & 0.473114 \\
\hline 28 & 1 & 3.228110 & 0.041972 & 0.924350 \\
\hline 29 & 6 & 3.248108 & $3-1.933469$ & 0.175150 \\
\hline 30 & 1 & 2.689316 & $6-2.748025$ & -0.274266 \\
\hline 31 & 1 & 4.302858 & $\begin{array}{ll}3 & -2.098622\end{array}$ & 0.374650 \\
\hline
\end{tabular}




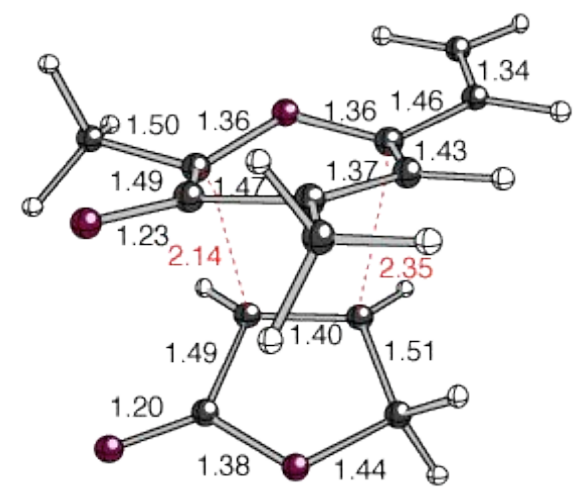

$\begin{array}{lc}\text { A.IV.TS2 (s-trans) } & \\ \text { HF = -804.6084591 hartrees }(-504899.854169841 \mathrm{kcal} / \mathrm{mol}) & \\ \text { Imaginary Frequencies: } 1(-431.4577 \text { 1/cm) } & \\ \text { Zero-point correction }=0.245195 \text { (Hartree/Particle) } \\ \text { Thermal correction to Energy= } & 0.261032 \\ \text { Thermal correction to Enthalpy= } & 0.261976 \\ \text { Thermal correction to Gibbs Free Energy= } & 0.202575 \\ \text { Sum of electronic and zero-point Energies }= & -804.363264 \\ \text { Sum of electronic and thermal Energies= } & -804.347427 \\ \text { Sum of electronic and thermal Enthalpies }= & -804.346483 \\ \text { Sum of electronic and thermal Free Energies }= & -804.405884 \\ \text { Coordinates (from last standard orientation): } & \end{array}$

\begin{tabular}{|c|c|c|c|c|}
\hline Center & Atomic & & Dordinates (A & roms) \\
\hline Number & Number & & $\begin{array}{ll}X & Y\end{array}$ & Z \\
\hline 1 & 8 & -1.350884 & 0.391685 & 1.024163 \\
\hline 2 & 6 & -1.707726 & 0.266910 & -0.277808 \\
\hline 3 & 8 & 1.733329 & -1.497826 & -1.251392 \\
\hline 4 & 6 & -0.959183 & 1.012707 & -1.238525 \\
\hline 5 & 6 & 0.241487 & 1.590842 & -0.937861 \\
\hline 6 & 6 & 0.795799 & 1.371179 & 0.400032 \\
\hline 7 & 6 & -0.028169 & 0.520737 & 1.297268 \\
\hline 8 & 6 & 0.394070 & -1.470922 & 0.639117 \\
\hline 9 & 1 & 0.222258 & -1.825313 & 1.647717 \\
\hline 10 & 6 & -0.462362 & -1.720562 & -0.435051 \\
\hline 11 & 6 & 0.393983 & -1.789650 & -1.679742 \\
\hline 12 & 1 & -1.399276 & -2.258406 & -0.365567 \\
\hline 13 & 1 & 0.376741 & -2.796270 & -2.119209 \\
\hline 14 & 6 & 1.790703 & -1.447235 & 0.123610 \\
\hline 15 & 8 & 2.828624 & -1.383040 & 0.729771 \\
\hline 16 & 1 & 0.131284 & -1.070867 & -2.462159 \\
\hline 17 & 1 & -1.379204 & 1.098304 & -2.238593 \\
\hline 18 & 8 & 1.876168 & 1.829102 & 0.778550 \\
\hline 19 & 6 & 0.303987 & 0.531577 & 2.756630 \\
\hline 20 & 1 & 1.343456 & 0.217521 & 2.882742 \\
\hline 21 & 1 & 0.220651 & 1.547651 & 3.161554 \\
\hline 22 & 1 & -0.361367 & -0.132004 & 3.315417 \\
\hline 23 & 6 & 1.040836 & 2.430103 & -1.886548 \\
\hline 24 & 1 & 1.178169 & 3.440759 & -1.484365 \\
\hline 25 & 1 & 2.046547 & 2.008899 & -2.000591 \\
\hline 26 & 1 & 0.562985 & 2.502138 & -2.868167 \\
\hline 27 & 6 & -3.095619 & -0.124488 & -0.504751 \\
\hline
\end{tabular}




\begin{tabular}{rrrrr}
28 & 1 & -3.402984 & -0.099600 & -1.548767 \\
29 & 6 & -3.967388 & -0.508559 & 0.437816 \\
30 & 1 & -3.697612 & -0.537209 & 1.488859 \\
31 & 1 & -4.982044 & -0.790804 & 0.177275 \\
\hline
\end{tabular}

\begin{abstract}
A.IV.TS2product (s-trans)
$\mathrm{HF}=-804.6655353$ hartrees $(-504935.670056103 \mathrm{kcal} / \mathrm{mol})$

Imaginary Frequencies: none found

Zero-point correction $=0.249480($ Hartree/Particle $)$

Thermal correction to Energy $=\quad 0.264617$

Thermal correction to Enthalpy $=\quad 0.265562$

Thermal correction to Gibbs Free Energy $=\quad 0.207714$

Sum of electronic and zero-point Energies $=\quad-804.416055$

Sum of electronic and thermal Energies $=\quad-804.400918$

Sum of electronic and thermal Enthalpies $=\quad-804.399974$

Sum of electronic and thermal Free Energies $=\quad-804.457821$

Coordinates (from last standard orientation):
\end{abstract}

\begin{tabular}{|c|c|c|c|c|}
\hline \multirow{2}{*}{$\begin{array}{l}\text { Center } \\
\text { Number }\end{array}$} & \multirow{2}{*}{$\begin{array}{l}\text { Atomic } \\
\text { Number }\end{array}$} & \multicolumn{3}{|c|}{ Coordinates (Angstroms) } \\
\hline & & & $X \quad Y$ & Z \\
\hline 1 & 8 & -1.148849 & -0.899495 & -0.830004 \\
\hline 2 & 6 & -1.447251 & 0.033666 & 0.211689 \\
\hline 3 & 8 & 1.045620 & 2.344271 & 0.592631 \\
\hline 4 & 6 & -0.668561 & -0.351863 & 1.460828 \\
\hline 5 & 6 & 0.486414 & -1.037316 & 1.383906 \\
\hline 6 & 6 & 1.029339 & -1.337122 & 0.024694 \\
\hline 7 & 6 & 0.243385 & -0.692419 & -1.138093 \\
\hline 8 & 6 & 0.364708 & 0.880752 & -1.138611 \\
\hline 9 & 1 & 0.438024 & 1.230890 & -2.172522 \\
\hline 10 & 6 & -0.897231 & 1.369892 & -0.416414 \\
\hline 11 & 6 & -0.386524 & 2.473591 & 0.526197 \\
\hline 12 & 1 & -1.657633 & 1.748824 & -1.102717 \\
\hline 13 & 1 & -0.618984 & 3.472016 & 0.141586 \\
\hline 14 & 6 & 1.524888 & 1.483370 & -0.350560 \\
\hline 15 & 8 & 2.700571 & 1.271809 & -0.492122 \\
\hline 16 & 1 & -0.770694 & 2.398355 & 1.547431 \\
\hline 17 & 1 & -1.080077 & -0.066741 & 2.427633 \\
\hline 18 & 8 & 2.008149 & -2.038810 & -0.150077 \\
\hline 19 & 6 & 0.572034 & -1.335707 & -2.470461 \\
\hline 20 & 1 & 1.627688 & -1.176495 & -2.705144 \\
\hline 21 & 1 & 0.391570 & -2.413065 & -2.429623 \\
\hline 22 & 1 & -0.048048 & -0.899689 & -3.259975 \\
\hline 23 & 6 & 1.284279 & -1.515203 & 2.562651 \\
\hline 24 & 1 & 1.394270 & -2.605072 & 2.534242 \\
\hline 25 & 1 & 2.297899 & -1.099632 & 2.534145 \\
\hline 26 & 1 & 0.809546 & -1.232460 & 3.506721 \\
\hline 27 & 6 & -2.928256 & 0.066911 & 0.477596 \\
\hline 28 & 1 & -3.231913 & 0.737725 & 1.281569 \\
\hline 29 & 6 & -3.836176 & -0.643183 & -0.188544 \\
\hline 30 & 1 & -3.548332 & -1.323216 & -0.983589 \\
\hline 31 & 1 & -4.893529 & -0.565842 & 0.047541 \\
\hline
\end{tabular}




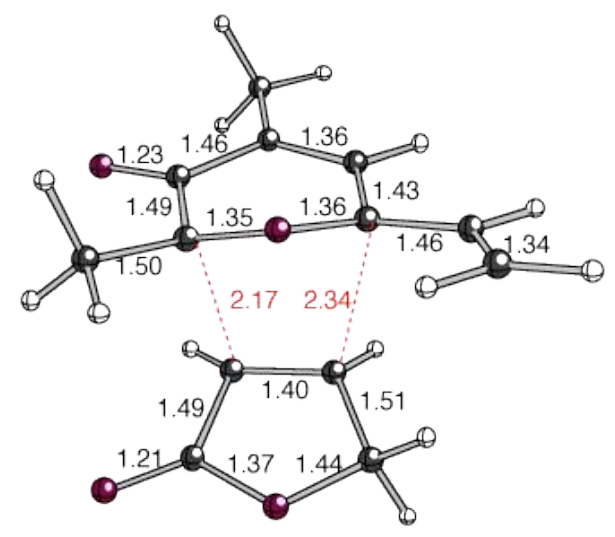

\begin{abstract}
A.IV.TS4 (s-trans)
$\mathrm{HF}=-804.6141068$ hartrees $(-504903.398158068 \mathrm{kcal} / \mathrm{mol})$

Imaginary Frequencies: $1(-413.96841 / \mathrm{cm})$

Zero-point correction $=0.245375$ (Hartree/Particle)

Thermal correction to Energy= $\quad 0.261114$

Thermal correction to Enthalpy $=\quad 0.262058$

Thermal correction to Gibbs Free Energy $=\quad 0.203079$

Sum of electronic and zero-point Energies $=\quad-804.368732$

Sum of electronic and thermal Energies $=\quad-804.352993$

Sum of electronic and thermal Enthalpies $=\quad-804.352049$

Sum of electronic and thermal Free Energies $=\quad-804.411028$

Coordinates (from last standard orientation):
\end{abstract}

\begin{tabular}{ccrrr}
\hline Center & Atomic & \multicolumn{3}{c}{ Coordinates (Angstroms) } \\
Number & Number & $X$ & $Y$ & $Z$ \\
\hline 1 & 8 & -0.016992 & 0.568459 & 1.120894 \\
2 & 8 & -2.353931 & -1.979337 & 0.307901 \\
3 & 6 & -0.450029 & -0.692075 & 0.880128 \\
4 & 6 & -1.794582 & -0.879102 & 0.277036 \\
5 & 6 & -2.356907 & 0.298639 & -0.384207 \\
6 & 6 & -1.591332 & 1.426177 & -0.444525 \\
7 & 1 & -1.929389 & 2.296993 & -1.002625 \\
8 & 6 & -0.318632 & 1.521661 & 0.203858 \\
9 & 6 & 0.883188 & 0.363020 & -1.435510 \\
10 & 6 & 0.630860 & -0.940101 & -0.989620 \\
11 & 6 & 2.325505 & 0.677136 & -1.136106 \\
12 & 6 & 1.917785 & -1.489627 & -0.473884 \\
13 & 8 & 2.844288 & -0.476386 & -0.457427 \\
14 & 8 & 2.180596 & -2.606575 & -0.101356 \\
15 & 1 & 2.477678 & 1.550347 & -0.488285 \\
16 & 1 & 0.367181 & 0.842637 & -2.256405 \\
17 & 1 & -0.077974 & -1.630668 & -1.428182 \\
18 & 1 & 2.902462 & 0.832712 & -2.057968 \\
19 & 6 & 0.017519 & -1.694827 & 1.886405 \\
20 & 1 & 1.003925 & -1.429386 & 2.271659 \\
21 & 1 & -0.697555 & -1.747535 & 2.717252 \\
22 & 1 & 0.066403 & -2.684342 & 1.428910 \\
23 & 6 & -3.721852 & 0.169409 & -0.989881 \\
24 & 1 & -3.757283 & -0.693343 & -1.665369 \\
25 & 1 & -4.471560 & -0.025049 & -0.213498 \\
26 & 1 & -4.010406 & 1.072057 & -1.536702
\end{tabular}




$\begin{array}{rrrrr}27 & 6 & 0.348296 & 2.801255 & 0.392312 \\ 28 & 1 & -0.005910 & 3.592512 & -0.265935 \\ 29 & 6 & 1.343161 & 3.047718 & 1.259175 \\ 30 & 1 & 1.710442 & 2.285017 & 1.938702 \\ 31 & 1 & 1.792424 & 4.033076 & 1.327259\end{array}$

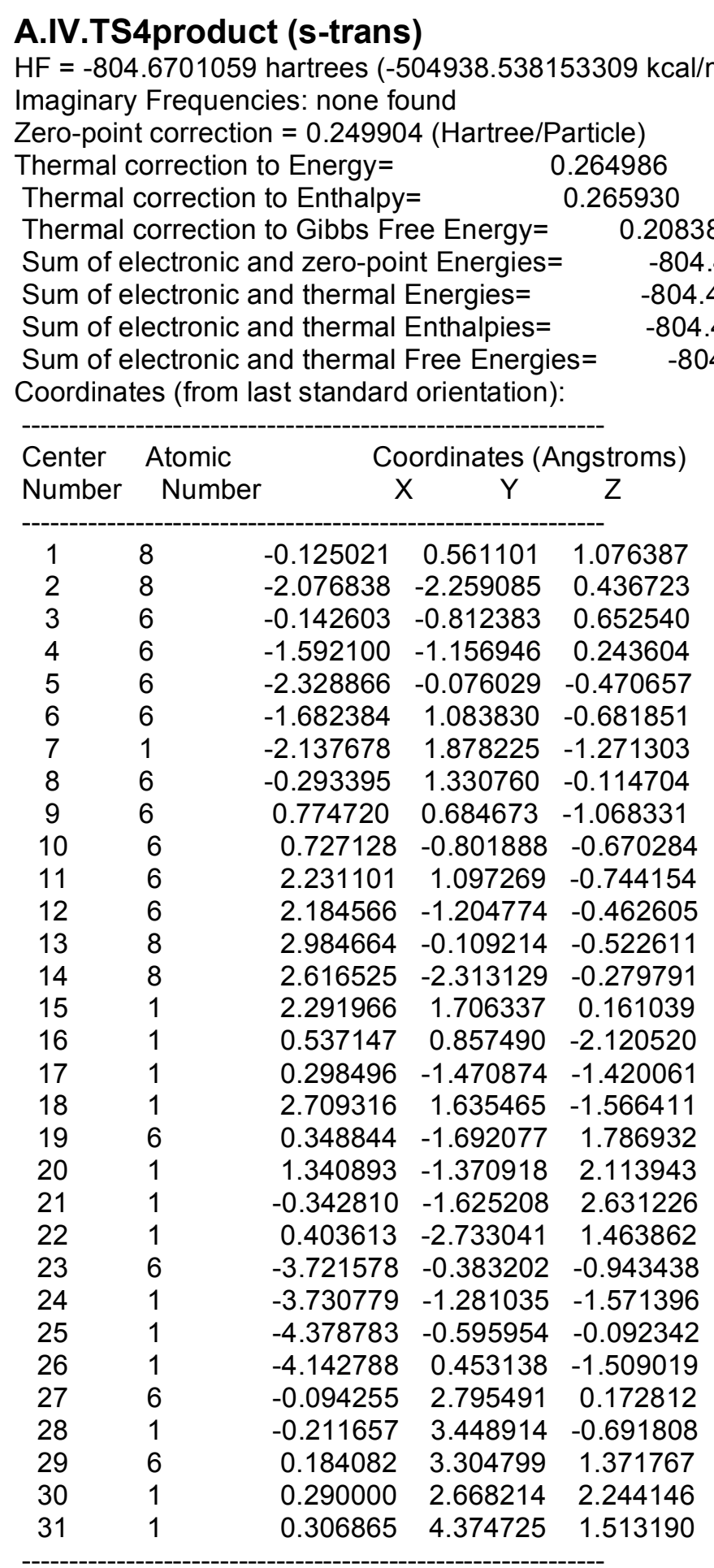




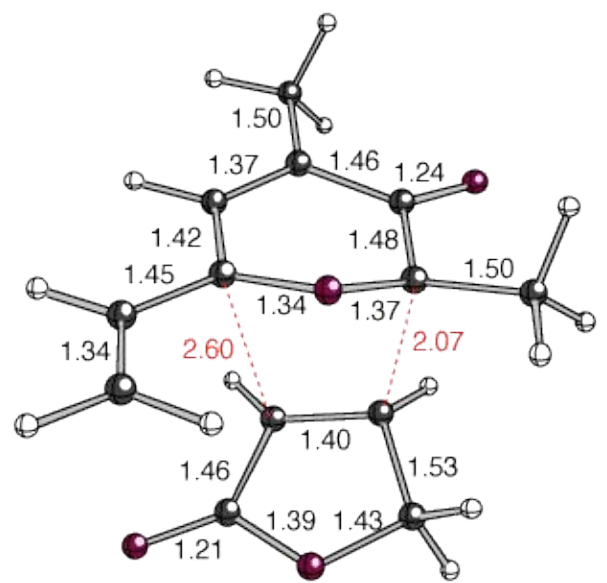

A.IV.TS3 (s-trans)

$\mathrm{HF}=-804.6150687$ hartrees $(-504904.001759937 \mathrm{kcal} / \mathrm{mol})$

Imaginary Frequencies: $1(-396.33601 / \mathrm{cm})$

Zero-point correction $=0.245169$ (Hartree/Particle)

Thermal correction to Energy=

0.261144

Thermal correction to Enthalpy=

0.262088

Thermal correction to Gibbs Free Energy=

Sum of electronic and zero-point Energies=

0.202266

Sum of electronic and thermal Energies=

$-804.369900$

Sum of electronic and thermal Enthalpies= $-804.353925$

Sum of electronic and thermal Free Energies= $-804.352981$

Coordinates (from last standard orientation):

\begin{tabular}{|c|c|c|c|c|}
\hline Center & Atomic & & Dordinates (A & Angstroms) \\
\hline Number & Number & & $\begin{array}{ll}X & Y\end{array}$ & Z \\
\hline 1 & 8 & -0.062186 & -0.133434 & 1.275249 \\
\hline 2 & 6 & 0.075644 & -1.275936 & 0.585786 \\
\hline 3 & 6 & -1.033505 & -1.738878 & -0.173639 \\
\hline 4 & 6 & -2.150312 & -0.973157 & -0.384247 \\
\hline 5 & 6 & -2.116023 & 0.417582 & 0.050405 \\
\hline 6 & 6 & -0.888790 & 0.833281 & 0.770833 \\
\hline 7 & 6 & 0.261379 & 1.385034 & -0.854478 \\
\hline 8 & 6 & 0.793915 & 0.222365 & -1.417002 \\
\hline 9 & 6 & 1.464776 & 2.138360 & -0.295438 \\
\hline 10 & 6 & 2.222352 & 0.125351 & -1.111392 \\
\hline 11 & 8 & 2.559645 & 1.218525 & -0.328987 \\
\hline 12 & 8 & 3.034661 & -0.718387 & -1.410761 \\
\hline 13 & 1 & 1.359560 & 2.500579 & 0.728961 \\
\hline 14 & 1 & -0.551206 & 1.949731 & -1.305929 \\
\hline 15 & 1 & 0.329869 & -0.423053 & -2.147262 \\
\hline 16 & 1 & 1.699272 & 2.997893 & -0.938106 \\
\hline 17 & 1 & -0.968526 & -2.747108 & -0.575928 \\
\hline 18 & 8 & -3.004452 & 1.243243 & -0.199762 \\
\hline 19 & 6 & -1.017113 & 2.017856 & 1.687000 \\
\hline 20 & 1 & -1.268111 & 2.912238 & 1.111501 \\
\hline 21 & 1 & -1.838736 & 1.856709 & 2.395242 \\
\hline 22 & 1 & -0.097748 & 2.182454 & 2.253418 \\
\hline 23 & 6 & 1.198516 & -2.118244 & 0.949176 \\
\hline 24 & 6 & -3.382068 & -1.457520 & -1.085659 \\
\hline
\end{tabular}




$\begin{array}{rrrrr}25 & 1 & -4.245008 & -1.437080 & -0.408674 \\ 26 & 1 & -3.634040 & -0.785733 & -1.914561 \\ 27 & 1 & -3.259943 & -2.476395 & -1.464611 \\ 28 & 6 & 2.202362 & -1.758673 & 1.763269 \\ 29 & 1 & 3.020572 & -2.439437 & 1.970604 \\ 30 & 1 & 1.205115 & -3.096087 & 0.474398 \\ 31 & 1 & 2.240789 & -0.778811 & 2.227350\end{array}$

\section{A.IV.TS3product (s-trans)}

$\mathrm{HF}=-804.6672473$ hartrees $(-504936.744353223 \mathrm{kcal} / \mathrm{mol})$

Imaginary Frequencies: none found

Zero-point correction $=0.249581$ (Hartree/Particle)

Thermal correction to Energy $=\quad 0.264897$

Thermal correction to Enthalpy= $\quad 0.265842$

Thermal correction to Gibbs Free Energy $=\quad 0.206904$

Sum of electronic and zero-point Energies $=\quad-804.417666$

Sum of electronic and thermal Energies $=\quad-804.402350$

Sum of electronic and thermal Enthalpies $=\quad-804.401406$

Sum of electronic and thermal Free Energies $=\quad-804.460343$

Coordinates (from last standard orientation):

\begin{tabular}{|c|c|c|c|c|}
\hline Center & Atomic & & ordinates & roms) \\
\hline Number & Number & $x$ & $\begin{array}{ll}X & Y\end{array}$ & Z \\
\hline 1 & 8 & 0.114515 & 0.114757 & 1.224492 \\
\hline 2 & 6 & -0.249719 & 1.022575 & 0.180761 \\
\hline 3 & 6 & 1.023618 & 1.642469 & -0.373453 \\
\hline 4 & 6 & 2.155746 & 0.921098 & -0.457985 \\
\hline 5 & 6 & 2.072534 & -0.514794 & -0.085263 \\
\hline 6 & 6 & 0.746624 & -0.988905 & 0.564509 \\
\hline 7 & 6 & -0.271678 & -1.312533 & -0.577211 \\
\hline 8 & 6 & -0.845095 & 0.069239 & -0.948274 \\
\hline 9 & 6 & -1.528480 & -2.096484 & -0.113700 \\
\hline 10 & 6 & -2.360413 & -0.091506 & -0.923167 \\
\hline 11 & 8 & -2.689304 & -1.333720 & -0.488328 \\
\hline 12 & 8 & -3.183937 & 0.720982 & -1.256902 \\
\hline 13 & 1 & -1.566425 & -2.229355 & 0.969250 \\
\hline 14 & 1 & 0.216182 & -1.821279 & -1.412439 \\
\hline 15 & 1 & -0.565062 & 0.432078 & -1.938640 \\
\hline 16 & 1 & -1.609009 & -3.075010 & -0.593054 \\
\hline 17 & 1 & 0.981582 & 2.672162 & -0.724408 \\
\hline 18 & 8 & 2.973502 & -1.305125 & -0.323286 \\
\hline 19 & 6 & 1.041040 & -2.082608 & 1.580966 \\
\hline 20 & 1 & 1.515310 & -2.934011 & 1.087754 \\
\hline 21 & 1 & 1.734246 & -1.702745 & 2.337146 \\
\hline 22 & 1 & 0.133201 & -2.410710 & 2.091900 \\
\hline 23 & 6 & -1.199536 & 2.068464 & 0.698486 \\
\hline 24 & 6 & 3.470852 & 1.432886 & -0.973985 \\
\hline 25 & 1 & 4.243306 & 1.371472 & -0.198251 \\
\hline 26 & 1 & 3.823004 & 0.819606 & -1.810584 \\
\hline 27 & 1 & 3.390165 & 2.473494 & -1.301447 \\
\hline 28 & 6 & -1.554979 & 2.195356 & 1.974798 \\
\hline 29 & 1 & -2.245134 & 2.973371 & 2.287249 \\
\hline 30 & 1 & -1.596900 & 2.738440 & -0.060940 \\
\hline 31 & 1 & -1.175754 & 1.525793 & \\
\hline
\end{tabular}




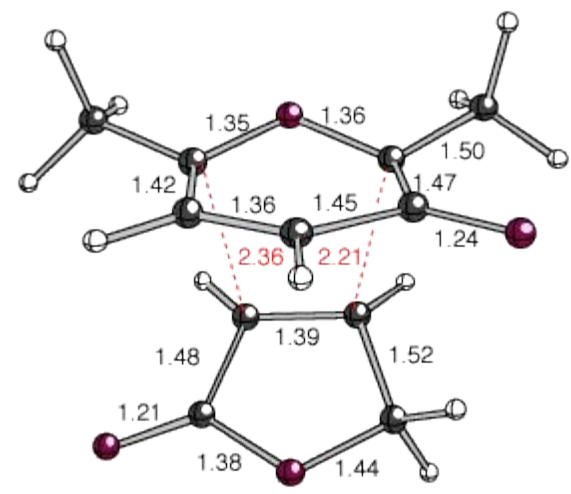

B.IV.TS1

$\mathrm{HF}=-727.2121384$ hartrees $(-456332.888967384 \mathrm{kcal} / \mathrm{mol})$

Imaginary Frequencies: 1 (-396.3048 1/cm)

Zero-point correction $=0.212549$ (Hartree/Particle)

Thermal correction to Energy $=\quad 0.225865$

Thermal correction to Enthalpy $=\quad 0.226809$

Thermal correction to Gibbs Free Energy $=\quad 0.173244$

Sum of electronic and zero-point Energies $=\quad-726.999590$

Sum of electronic and thermal Energies $=\quad-726.986274$

Sum of electronic and thermal Enthalpies $=\quad-726.985330$

Sum of electronic and thermal Free Energies $=\quad-727.038894$

Coordinates (from last standard orientation):

\begin{tabular}{|c|c|c|c|c|}
\hline \multirow{2}{*}{$\begin{array}{l}\text { Center } \\
\text { Number }\end{array}$} & \multirow{2}{*}{$\begin{array}{l}\text { Atomic } \\
\text { Number }\end{array}$} & \multicolumn{3}{|c|}{ Coordinates (Angstroms) } \\
\hline & & & $X \quad Y$ & Z \\
\hline 1 & 8 & 0.933446 & 1.472414 & -0.494716 \\
\hline 2 & 8 & 2.334738 & -1.346582 & 1.174976 \\
\hline 3 & 6 & 1.574800 & 0.281497 & -0.363172 \\
\hline 4 & 8 & -1.543093 & -1.895814 & 0.207960 \\
\hline 5 & 6 & 1.571915 & -0.397691 & 0.943348 \\
\hline 6 & 6 & 0.546098 & 0.081822 & 1.855075 \\
\hline 7 & 6 & -0.338245 & 1.041042 & 1.454647 \\
\hline 8 & 1 & -1.177406 & 1.332836 & 2.078863 \\
\hline 9 & 6 & -0.214736 & 1.661320 & 0.180219 \\
\hline 10 & 6 & -1.166772 & -0.057212 & -1.132350 \\
\hline 11 & 1 & -1.501625 & 0.723258 & -1.801437 \\
\hline 12 & 6 & -0.058424 & -0.881154 & -1.300911 \\
\hline 13 & 6 & -0.346990 & -2.162083 & -0.544554 \\
\hline 14 & 1 & 0.486756 & -0.380098 & 2.835457 \\
\hline 15 & 1 & 0.534869 & -0.914451 & -2.208041 \\
\hline 16 & 1 & -0.549459 & -2.985903 & -1.241706 \\
\hline 17 & 6 & -2.107375 & -0.708465 & -0.198643 \\
\hline 18 & 8 & -3.174972 & -0.321908 & 0.216909 \\
\hline 19 & 1 & 0.438318 & -2.478175 & 0.148429 \\
\hline 20 & 6 & 2.768585 & 0.146096 & -1.256544 \\
\hline 21 & 1 & 3.617496 & 0.715494 & -0.856221 \\
\hline 22 & 1 & 3.070878 & -0.902662 & -1.288852 \\
\hline 23 & 1 & 2.553414 & 0.509909 & -2.265265 \\
\hline 24 & 6 & -0.962182 & 2.903612 & -0.198091 \\
\hline 25 & 1 & -2.024570 & 2.772940 & 0.029655 \\
\hline
\end{tabular}




$\begin{array}{rrrrr}26 & 1 & -0.598641 & 3.777167 & 0.359268 \\ 27 & 1 & -0.851032 & 3.112101 & -1.265320\end{array}$

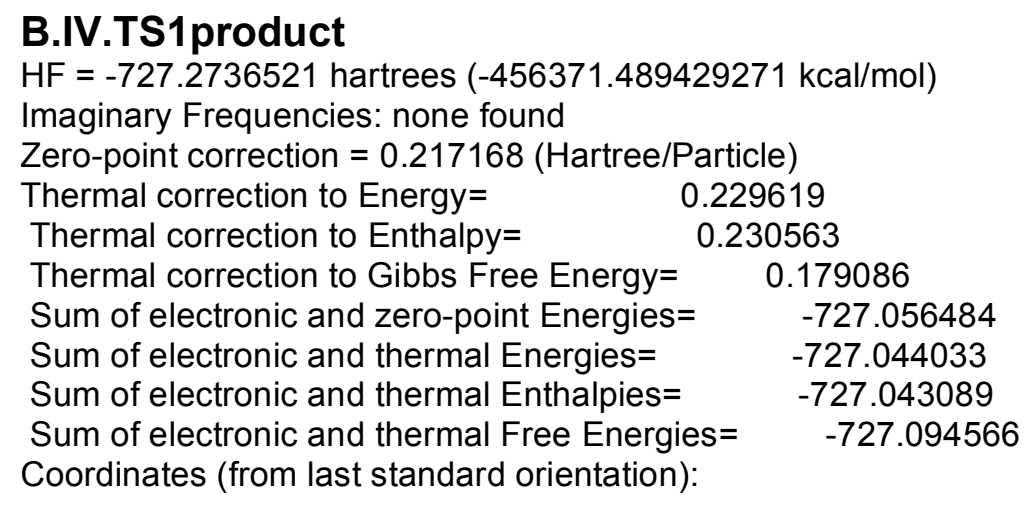

\begin{tabular}{|c|c|c|c|c|}
\hline \multirow{2}{*}{$\begin{array}{l}\text { Center } \\
\text { Number }\end{array}$} & \multirow{2}{*}{$\begin{array}{l}\text { Atomic } \\
\text { Number }\end{array}$} & \multicolumn{3}{|c|}{ Coordinates (Angstroms) } \\
\hline & & & $\begin{array}{l}X \\
Y\end{array}$ & Z \\
\hline 1 & 8 & 0.730165 & 1.536275 & -0.532963 \\
\hline 2 & 8 & 2.478415 & -0.868784 & 1.347293 \\
\hline 3 & 6 & 1.354866 & 0.242379 & -0.491092 \\
\hline 4 & 8 & -1.226436 & -2.097606 & 0.056591 \\
\hline 5 & 6 & 1.558000 & -0.148994 & 0.989478 \\
\hline 6 & 6 & 0.515308 & 0.343026 & 1.912640 \\
\hline 7 & 6 & -0.543855 & 1.004316 & 1.420287 \\
\hline 8 & 1 & -1.383553 & 1.288083 & 2.049864 \\
\hline 9 & 6 & -0.607488 & 1.331249 & -0.062368 \\
\hline 10 & 6 & -1.050354 & 0.059818 & -0.885766 \\
\hline 11 & 1 & -1.586700 & 0.380571 & -1.782851 \\
\hline 12 & 6 & 0.268510 & -0.670070 & -1.176219 \\
\hline 13 & 6 & 0.034774 & -2.102905 & -0.647335 \\
\hline 14 & 1 & 0.622311 & 0.089321 & 2.963367 \\
\hline 15 & 1 & 0.493127 & -0.690823 & -2.245429 \\
\hline 16 & 1 & -0.044756 & -2.827020 & -1.463452 \\
\hline 17 & 6 & -1.903247 & -0.931016 & -0.107091 \\
\hline 18 & 8 & -3.003389 & -0.745642 & 0.349179 \\
\hline 19 & 1 & 0.801414 & -2.446322 & 0.050920 \\
\hline 20 & 6 & 2.676510 & 0.303817 & -1.236776 \\
\hline 21 & 1 & 3.340384 & 1.039896 & -0.774478 \\
\hline 22 & 1 & 3.174547 & -0.668410 & -1.201807 \\
\hline 23 & 1 & 2.505807 & 0.590832 & -2.278849 \\
\hline 24 & 6 & -1.438797 & 2.571062 & -0.350260 \\
\hline 25 & 1 & -2.483259 & 2.390877 & -0.075668 \\
\hline 26 & 1 & -1.065101 & 3.427137 & 0.220198 \\
\hline 27 & 1 & -1.389628 & 2.815813 & -1.415612 \\
\hline
\end{tabular}




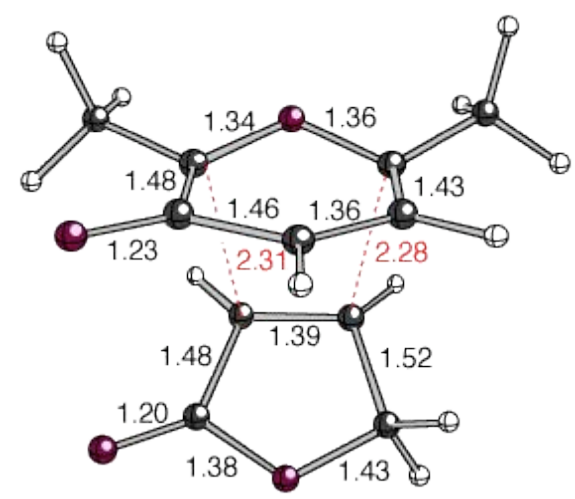

B.IV.TS2

$\mathrm{HF}=-727.2030727$ hartrees $(-456327.200149977 \mathrm{kcal} / \mathrm{mol})$ Imaginary Frequencies: $1(-401.54671 / \mathrm{cm})$

Zero-point correction $=0.212096$ (Hartree/Particle)

Thermal correction to Energy $=\quad 0.225582$

Thermal correction to Enthalpy= $\quad 0.226526$

Thermal correction to Gibbs Free Energy $=\quad 0.172146$

Sum of electronic and zero-point Energies $=\quad-726.990977$

Sum of electronic and thermal Energies $=\quad-726.977491$

Sum of electronic and thermal Enthalpies $=\quad-726.976547$

Sum of electronic and thermal Free Energies $=\quad-727.030927$

Coordinates (from last standard orientation):

\begin{tabular}{crrrr} 
Center & Atomic & \multicolumn{3}{c}{ Coordinates (Angstroms) } \\
Number & Number & X & $Y$ & $Z$ \\
-1 & 8 & 1.464176 & 0.844258 & -0.688017 \\
2 & 6 & 1.862317 & -0.225065 & 0.047395 \\
3 & 8 & -1.664716 & -1.823079 & 0.519073 \\
4 & 6 & 1.443982 & -0.257418 & 1.410462 \\
5 & 6 & 0.435435 & 0.542445 & 1.859791 \\
6 & 6 & -0.257266 & 1.437558 & 0.942958 \\
7 & 6 & 0.258822 & 1.387459 & -0.443142 \\
8 & 6 & -0.713885 & -0.575324 & -1.181225 \\
9 & 1 & -0.793783 & -0.063445 & -2.130323 \\
10 & 6 & 0.226675 & -1.542019 & -0.850888 \\
11 & 6 & -0.412663 & -2.437238 & 0.193213 \\
12 & 1 & 0.120048 & 0.544300 & 2.898357 \\
13 & 1 & 0.961824 & -1.941292 & -1.540640 \\
14 & 1 & -0.605455 & -3.438259 & -0.216721 \\
15 & 6 & -1.929013 & -0.791133 & -0.360481 \\
16 & 8 & -2.989143 & -0.221116 & -0.389166 \\
17 & 1 & 0.157867 & -2.552092 & 1.119271 \\
18 & 1 & 1.940211 & -0.961123 & 2.074092 \\
19 & 8 & -1.183374 & 2.191346 & 1.247990 \\
20 & 6 & -0.171506 & 2.439889 & -1.409753 \\
21 & 1 & -1.264037 & 2.475247 & -1.427228 \\
22 & 1 & 0.173322 & 3.428706 & -1.079835 \\
23 & 1 & 0.219731 & 2.242186 & -2.411049 \\
24 & 6 & 3.177024 & -0.788487 & -0.411668 \\
25 & 1 & 4.017398 & -0.149204 & -0.110348 \\
26 & 1 & 3.336471 & -1.778408 & 0.026755 \\
27 & 1 & 3.201326 & -0.881885 & -1.501348
\end{tabular}




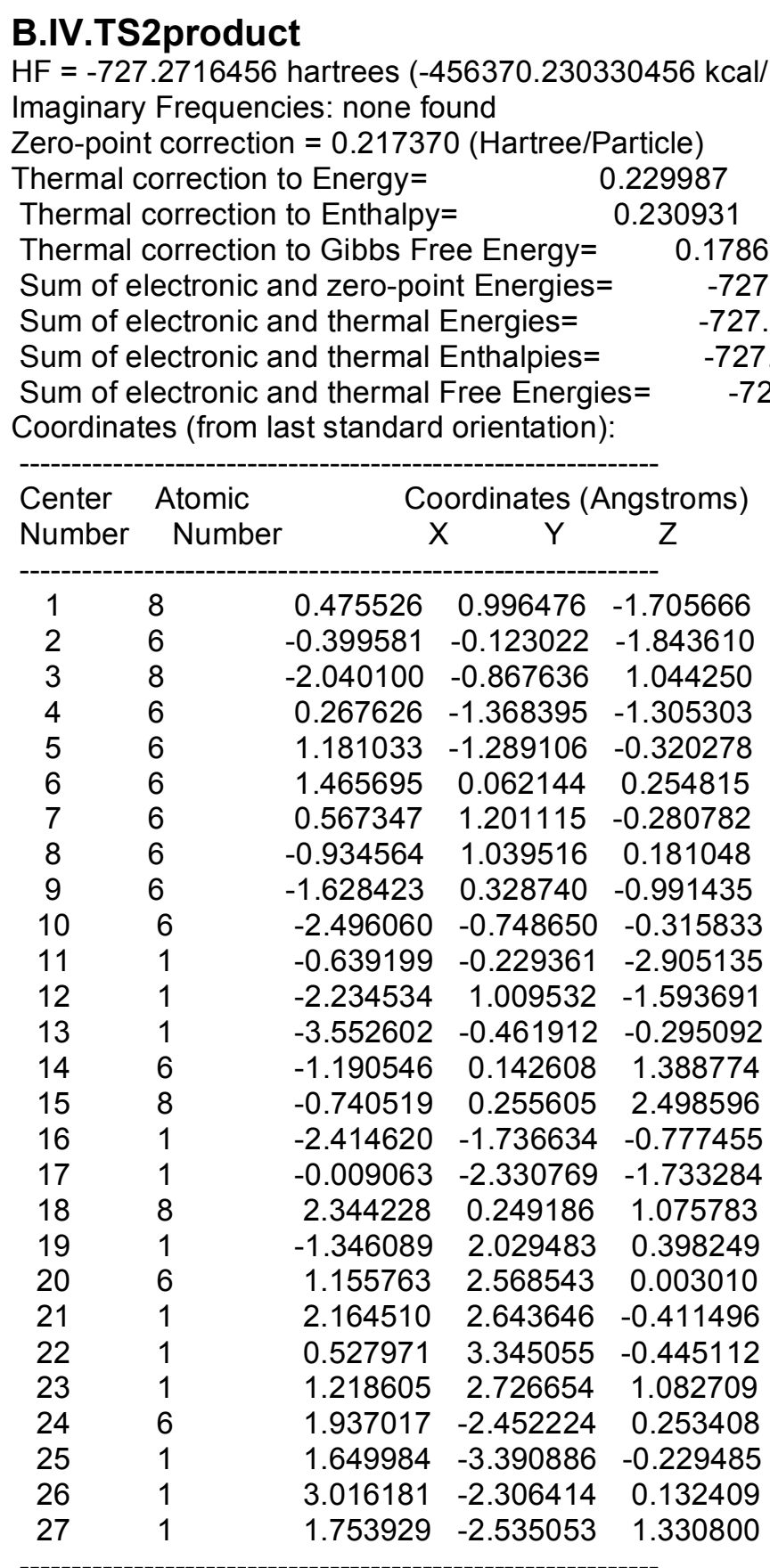




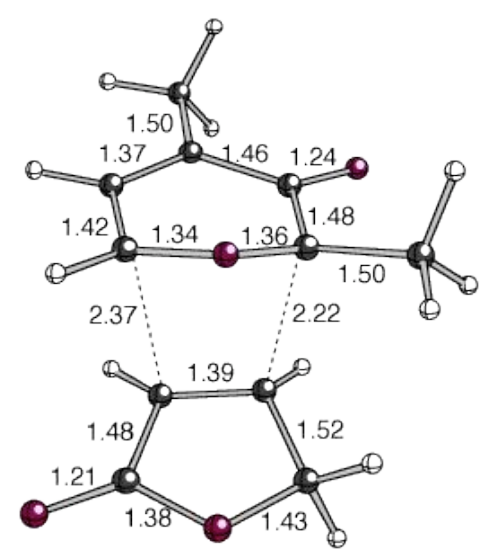

\section{B.IV.TS3}

$\mathrm{HF}=-727.2098311$ hartrees $(-456331.441113561 \mathrm{kcal} / \mathrm{mol})$

Imaginary Frequencies: $1(-414.59901 / \mathrm{cm})$

Zero-point correction $=0.212497($ Hartree/Particle $)$

Thermal correction to Energy $=\quad 0.226041$

Thermal correction to Enthalpy $=\quad 0.226985$

Thermal correction to Gibbs Free Energy $=\quad 0.172244$

Sum of electronic and zero-point Energies $=\quad-726.997334$

Sum of electronic and thermal Energies $=\quad-726.983790$

Sum of electronic and thermal Enthalpies $=\quad-726.982846$

Sum of electronic and thermal Free Energies $=\quad-727.037588$

Coordinates (from last standard orientation):

\begin{tabular}{ccrrr}
\hline Center & Atomic & \multicolumn{3}{c}{ Coordinates (Angstroms) } \\
Number & Number & X & $Y$ & $Z$ \\
\hline 1 & 8 & -0.021329 & 0.207409 & 1.441785 \\
2 & 6 & -0.019120 & -1.106396 & 1.187325 \\
3 & 6 & -1.159430 & -1.692708 & 0.588250 \\
4 & 6 & -2.109218 & -0.923192 & -0.031937 \\
5 & 6 & -1.857147 & 0.512310 & -0.151097 \\
6 & 6 & -0.655994 & 1.008571 & 0.550129 \\
7 & 6 & 0.786303 & 0.565027 & -1.071700 \\
8 & 6 & 1.149493 & -0.757953 & -0.841325 \\
9 & 1 & 0.670632 & -1.654812 & 1.816523 \\
10 & 6 & 2.007213 & 1.401896 & -0.727815 \\
11 & 6 & 2.506702 & -0.795046 & -0.254855 \\
12 & 8 & 2.935303 & 0.505865 & -0.104223 \\
13 & 8 & 3.179272 & -1.738353 & 0.086074 \\
14 & 1 & 1.836202 & 2.238061 & -0.045564 \\
15 & 1 & 0.104031 & 0.893460 & -1.848210 \\
16 & 1 & 0.729043 & -1.636725 & -1.306192 \\
17 & 1 & 2.461989 & 1.802520 & -1.644240 \\
18 & 1 & -1.238733 & -2.776973 & 0.611025 \\
19 & 8 & -2.567083 & 1.282788 & -0.811207 \\
20 & 6 & -0.594007 & 2.464590 & 0.892592 \\
21 & 1 & -0.665564 & 3.062665 & -0.019392 \\
22 & 1 & -1.450983 & 2.743692 & 1.518766 \\
23 & 1 & 0.324362 & 2.706130 & 1.432883 \\
24 & 6 & -3.367333 & -1.460453 & -0.640730 \\
25 & 1 & -4.248017 & -1.040577 & -0.139350 \\
26 & 1 & -3.441419 & -1.147507 & -1.688827
\end{tabular}




\section{B.IV.TS3product \\ $\mathrm{HF}=-727.2767571$ hartrees $(-456373.437847821 \mathrm{kcal} / \mathrm{mol})$ Imaginary Frequencies: none found \\ Zero-point correction $=0.217711$ (Hartree/Particle $)$ \\ Thermal correction to Energy $=\quad 0.230247$ \\ Thermal correction to Enthalpy= $\quad 0.231192$ \\ Thermal correction to Gibbs Free Energy= 0.179069 \\ Sum of electronic and zero-point Energies $=\quad-727.059046$ \\ Sum of electronic and thermal Energies $=\quad-727.046510$ \\ Sum of electronic and thermal Enthalpies $=\quad-727.045565$ \\ Sum of electronic and thermal Free Energies $=\quad-727.097688$ \\ Coordinates (from last standard orientation):}

\begin{tabular}{|c|c|c|c|c|}
\hline \multirow{2}{*}{$\begin{array}{l}\text { Center } \\
\text { Number }\end{array}$} & \multirow{2}{*}{$\begin{array}{l}\text { Atomic } \\
\text { Number }\end{array}$} & \multicolumn{3}{|c|}{ Coordinates (Angstroms) } \\
\hline & & & $X \quad Y$ & Z \\
\hline 1 & 6 & 0.133702 & -1.289409 & 0.632831 \\
\hline 2 & 6 & -1.235130 & -1.737145 & 0.183869 \\
\hline 3 & 6 & -2.191160 & -0.831617 & -0.095738 \\
\hline 4 & 6 & -1.810167 & 0.608761 & -0.038095 \\
\hline 5 & 6 & -0.354436 & 0.906194 & 0.396059 \\
\hline 6 & 6 & 0.567558 & 0.588459 & -0.849583 \\
\hline 7 & 6 & 1.015568 & -0.860611 & -0.571815 \\
\hline 8 & 1 & 0.630415 & -2.033244 & 1.259752 \\
\hline 9 & 6 & 1.904427 & 1.349720 & -0.951857 \\
\hline 10 & 6 & 2.475114 & -0.733132 & -0.130476 \\
\hline 11 & 8 & 2.909956 & 0.539947 & -0.314958 \\
\hline 12 & 8 & 3.166187 & -1.599271 & 0.338594 \\
\hline 13 & 1 & 1.906490 & 2.323462 & -0.460481 \\
\hline 14 & 1 & 0.003854 & 0.709644 & -1.777823 \\
\hline 15 & 1 & 2.193698 & 1.489305 & -1.999418 \\
\hline 16 & 1 & -1.420443 & -2.804829 & 0.075234 \\
\hline 17 & 8 & -2.573531 & 1.498974 & -0.377955 \\
\hline 18 & 8 & 0.027397 & -0.075220 & 1.373993 \\
\hline 19 & 1 & 0.946018 & -1.546228 & -1.420543 \\
\hline 20 & 6 & -0.235401 & 2.285950 & 1.017961 \\
\hline 21 & 1 & -0.483125 & 3.060864 & 0.288026 \\
\hline 22 & 1 & -0.936190 & 2.376106 & 1.852537 \\
\hline 23 & 1 & 0.775584 & 2.446073 & 1.402156 \\
\hline 24 & 6 & -3.596471 & -1.160299 & -0.512972 \\
\hline 25 & 1 & -4.318232 & -0.758534 & 0.207824 \\
\hline 26 & 1 & -3.834644 & -0.697792 & -1.477297 \\
\hline 27 & 1 & -3.745127 & -2.241493 & -0.588454 \\
\hline
\end{tabular}




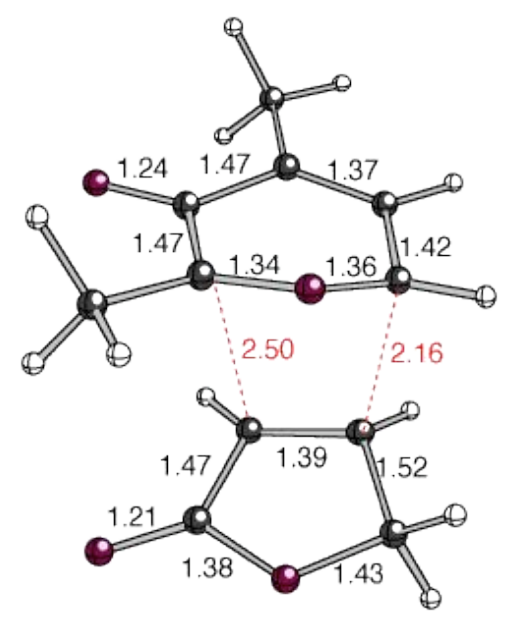

\section{B.IV.TS4}

$\mathrm{HF}=-727.2103862$ hartrees $(-456331.789444362 \mathrm{kcal} / \mathrm{mol})$ Imaginary Frequencies: 1 (-399.9694 1/cm)

Zero-point correction $=0.212419$ (Hartree/Particle)

Thermal correction to Energy $=\quad 0.225957$

Thermal correction to Enthalpy= $\quad 0.226901$

Thermal correction to Gibbs Free Energy $=\quad 0.172287$

Sum of electronic and zero-point Energies $=\quad-726.997967$

Sum of electronic and thermal Energies $=\quad-726.984429$

Sum of electronic and thermal Enthalpies $=\quad-726.983485$

Sum of electronic and thermal Free Energies $=\quad-727.038099$

Coordinates (from last standard orientation):

\begin{tabular}{|c|c|c|c|c|}
\hline \multirow{2}{*}{$\begin{array}{l}\text { Center } \\
\text { Number }\end{array}$} & \multirow{2}{*}{$\begin{array}{l}\text { Atomic } \\
\text { Number }\end{array}$} & \multicolumn{3}{|c|}{ Coordinates (Angstroms) } \\
\hline & & & $X \quad Y$ & Z \\
\hline 1 & 8 & 0.040977 & -0.297893 & 1.488763 \\
\hline 2 & 8 & 2.005072 & 1.878013 & -0.517745 \\
\hline 3 & 6 & 0.377903 & 0.800133 & 0.805487 \\
\hline 4 & 6 & 1.594744 & 0.821983 & -0.021627 \\
\hline 5 & 6 & 2.278809 & -0.466720 & -0.173098 \\
\hline 6 & 6 & 1.670153 & -1.584453 & 0.322723 \\
\hline 7 & 1 & 2.098505 & -2.573038 & 0.172562 \\
\hline 8 & 6 & 0.414321 & -1.501416 & 0.988374 \\
\hline 9 & 6 & -0.983949 & -1.475156 & -0.661758 \\
\hline 10 & 6 & -0.910407 & -0.161794 & -1.108881 \\
\hline 11 & 1 & 0.052864 & -2.312011 & 1.611442 \\
\hline 12 & 6 & -2.344733 & $3-1.631631$ & -0.001952 \\
\hline 13 & 6 & -2.132672 & 0.552091 & -0.701757 \\
\hline 14 & 8 & -2.906380 & -0.316228 & 0.045654 \\
\hline 15 & 8 & -2.482378 & 1.688565 & -0.918748 \\
\hline 16 & 1 & -2.321729 & -2.027018 & 1.018611 \\
\hline 17 & 1 & -0.527832 & -2.315536 & -1.171383 \\
\hline 18 & 1 & -0.266239 & 0.242131 & -1.876321 \\
\hline 19 & 1 & -2.999315 & -2.276769 & -0.603831 \\
\hline 20 & 6 & -0.224456 & 2.072099 & 1.288622 \\
\hline 21 & 1 & 0.551314 & 2.716103 & 1.719640 \\
\hline 22 & 1 & -0.664974 & 2.615523 & 0.445931 \\
\hline 23 & 1 & -1.004051 & 1.873196 & 2.027009 \\
\hline 24 & 6 & 3.595548 & -0.464582 & -0.886960 \\
\hline 25 & 1 & 3.486280 & -0.008182 & -1.877839 \\
\hline
\end{tabular}




\begin{tabular}{|c|c|c|c|c|}
\hline 26 & 1 & 4.322401 & 0.156791 & -0.350196 \\
\hline 27 & 1 & 4.002881 & -1.474165 & -0.994054 \\
\hline
\end{tabular}

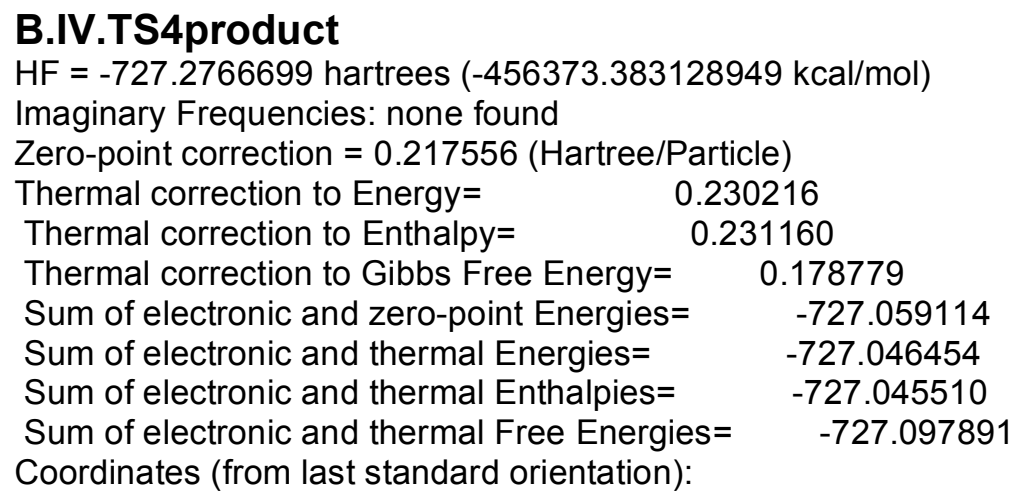

\begin{tabular}{|c|c|c|c|c|}
\hline \multirow{2}{*}{$\begin{array}{l}\text { Center } \\
\text { Number }\end{array}$} & \multirow{2}{*}{$\begin{array}{l}\text { Atomic } \\
\text { Number }\end{array}$} & \multicolumn{3}{|c|}{ Coordinates (Angstroms) } \\
\hline & & & $\begin{array}{l}X \\
Y\end{array}$ & Z \\
\hline 1 & 8 & 0.116917 & -0.399841 & 1.457125 \\
\hline 2 & 8 & 2.028318 & 1.930976 & -0.330246 \\
\hline 3 & 6 & 0.126647 & 0.690567 & 0.519414 \\
\hline 4 & 6 & 1.569836 & 0.844526 & -0.019409 \\
\hline 5 & 6 & 2.336993 & -0.422386 & -0.202609 \\
\hline 6 & 6 & 1.707940 & -1.575717 & 0.086691 \\
\hline 7 & 1 & 2.182067 & -2.538633 & -0.098409 \\
\hline 8 & 6 & 0.308925 & -1.561948 & 0.651952 \\
\hline 9 & 6 & -0.757255 & -1.379237 & -0.462570 \\
\hline 10 & 6 & -0.761308 & 0.147666 & -0.673884 \\
\hline 11 & 1 & 0.124324 & -2.437478 & 1.281912 \\
\hline 12 & 6 & -2.192675 & -1.679335 & 0.033288 \\
\hline 13 & 6 & -2.230243 & 0.554585 & -0.589411 \\
\hline 14 & 8 & -2.988379 & -0.504891 & -0.197944 \\
\hline 15 & 8 & -2.705217 & 1.632840 & -0.832147 \\
\hline 16 & 1 & -2.215260 & -1.893482 & 1.106636 \\
\hline 17 & 1 & -0.520374 & -1.941977 & -1.368469 \\
\hline 18 & 1 & -0.371601 & 0.487603 & -1.636051 \\
\hline 19 & 1 & -2.662660 & -2.506581 & -0.504882 \\
\hline 20 & 6 & -0.353445 & 1.953328 & 1.210385 \\
\hline 21 & 1 & 0.351206 & 2.232612 & 1.998575 \\
\hline 22 & 1 & -0.420446 & 2.775467 & 0.495858 \\
\hline 23 & 1 & -1.337731 & 1.790976 & 1.656082 \\
\hline 24 & 6 & 3.736969 & -0.311357 & -0.735603 \\
\hline 25 & 1 & 3.749805 & 0.236804 & -1.684239 \\
\hline 26 & 1 & 4.369859 & 0.256388 & -0.043736 \\
\hline 27 & 1 & 4.183411 & -1.298523 & -0.887049 \\
\hline
\end{tabular}




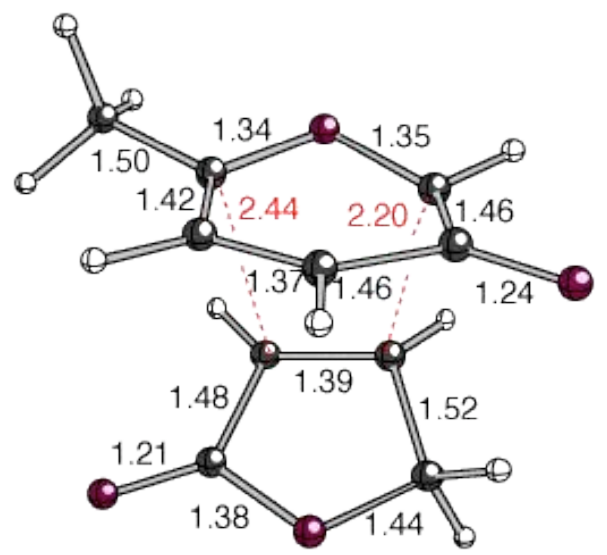

\section{C.IV.TS1}

$\mathrm{HF}=-687.8884024$ hartrees $(-431656.851390024 \mathrm{kcal} / \mathrm{mol})$

Imaginary Frequencies: $1(-389.12601 / \mathrm{cm})$

Zero-point correction $=0.184802($ Hartree/Particle $)$

Thermal correction to Energy=

0.196516

Thermal correction to Enthalpy= 0.197460

Thermal correction to Gibbs Free Energy=

Sum of electronic and zero-point Energies=

Sum of electronic and thermal Energies=

0.147001

$-687.703600$

$-687.691886$

Sum of electronic and thermal Enthalpies $=$ $-687.690942$

Sum of electronic and thermal Free Energies= $-687.741402$

Coordinates (from last standard orientation):

\begin{tabular}{|c|c|c|c|c|}
\hline \multirow{2}{*}{$\begin{array}{l}\text { Center } \\
\text { Number }\end{array}$} & \multirow{2}{*}{$\begin{array}{l}\text { Atomic } \\
\text { Number }\end{array}$} & & \multicolumn{2}{|c|}{ Coordinates (Angstroms) } \\
\hline & & & $X \quad Y$ & Z \\
\hline 1 & 8 & 0.802962 & 1.561832 & -0.902817 \\
\hline 2 & 8 & 2.849087 & -1.010447 & 0.483985 \\
\hline 3 & 6 & 1.558770 & 0.439603 & -0.861336 \\
\hline 4 & 8 & -0.986741 & -2.026672 & 0.441912 \\
\hline 5 & 6 & 1.960831 & -0.151601 & 0.416465 \\
\hline 6 & 6 & 1.128831 & 0.287498 & 1.529283 \\
\hline 7 & 6 & 0.081352 & 1.137084 & 1.310423 \\
\hline 8 & 1 & -0.616121 & 1.388046 & 2.103776 \\
\hline 9 & 6 & -0.149053 & 1.709065 & 0.029268 \\
\hline 10 & 6 & -1.209191 & -0.238003 & -0.994080 \\
\hline 11 & 1 & -1.784077 & $7 \quad 0.482472$ & -1.558737 \\
\hline 12 & 6 & -0.075242 & $2-0.921542$ & -1.414162 \\
\hline 13 & 6 & 0.003194 & -2.188606 & -0.587394 \\
\hline 14 & 1 & 1.340861 & $1-0.116456$ & 2.514173 \\
\hline 15 & 1 & 0.288768 & $8-0.917323$ & -2.435632 \\
\hline 16 & 1 & -0.262719 & -3.062501 & -1.196731 \\
\hline 17 & 6 & -1.787920 & $\begin{array}{ll}0 & -0.940627\end{array}$ & 0.166136 \\
\hline 18 & 8 & -2.757525 & $5-0.665252$ & 0.833745 \\
\hline
\end{tabular}




$\begin{array}{rrrrr}19 & 1 & 0.971715 & -2.374221 & -0.114704 \\ 20 & 6 & -1.086714 & 2.851959 & -0.206916 \\ 21 & 1 & -2.048532 & 2.641368 & 0.270480 \\ 22 & 1 & -0.694922 & 3.786382 & 0.216737 \\ 23 & 1 & -1.246896 & 3.005565 & -1.277082 \\ 24 & 1 & 2.240515 & 0.382004 & -1.703003\end{array}$

\section{C.IV.TS1product}

$\mathrm{HF}=-687.9531602$ hartrees $(-431697.487557102 \mathrm{kcal} / \mathrm{mol})$

Imaginary Frequencies: none found

Zero-point correction $=0.189561$ (Hartree/Particle)

Thermal correction to Energy $=\quad 0.200454$

Thermal correction to Enthalpy $=\quad 0.201398$

Thermal correction to Gibbs Free Energy $=\quad 0.152819$

Sum of electronic and zero-point Energies $=\quad-687.763599$

Sum of electronic and thermal Energies= $\quad-687.752706$

Sum of electronic and thermal Enthalpies= $\quad-687.751762$

Sum of electronic and thermal Free Energies $=\quad-687.800341$

Coordinates (from last standard orientation):

\begin{tabular}{|c|c|c|c|c|}
\hline \multirow{2}{*}{$\begin{array}{l}\text { Center } \\
\text { Number }\end{array}$} & \multirow{2}{*}{$\begin{array}{l}\text { Atomic } \\
\text { Number }\end{array}$} & & \multicolumn{2}{|c|}{ Coordinates (Angstroms) } \\
\hline & & & $X \quad Y$ & Z \\
\hline 1 & 8 & -0.479865 & -1.624809 & -0.940490 \\
\hline 2 & 8 & -3.021847 & 0.260113 & 0.588388 \\
\hline 3 & 6 & -1.304853 & -0.459795 & -0.943888 \\
\hline 4 & 8 & 0.584508 & 2.207741 & 0.305808 \\
\hline 5 & 6 & -1.927453 & -0.262109 & 0.443543 \\
\hline 6 & 6 & -1.053569 & -0.671117 & 1.561939 \\
\hline 7 & 6 & 0.194014 & -1.091369 & 1.294953 \\
\hline 8 & 1 & 0.906652 & -1.300155 & 2.088975 \\
\hline 9 & 6 & 0.657086 & -1.250198 & -0.145809 \\
\hline 10 & 6 & 1.032969 & 0.152047 & -0.766440 \\
\hline 11 & 1 & 810833 & 0.014708 & -1.522220 \\
\hline 12 & 6 & -0.297964 & 0.678768 & -1.328055 \\
\hline 13 & 6 & -0.456358 & 2.074742 & -0.686653 \\
\hline 14 & 1 & -1.438625 & $5-0.556916$ & 2.571121 \\
\hline 15 & 1 & -0.278020 & 0.753877 & -2.417280 \\
\hline 16 & 1 & -0.319623 & 2.874380 & -1.420438 \\
\hline 17 & 6 & 1.483492 & 1.190742 & 0.250736 \\
\hline 18 & 8 & 2.462091 & 1.145716 & 0.952771 \\
\hline 19 & 1 & -1.414368 & 2.221404 & -0.182855 \\
\hline 20 & 6 & 1.744109 & -2.301692 & -0.295091 \\
\hline 21 & 1 & 2.646729 & -1.980822 & 0.234960 \\
\hline 22 & 1 & 1.413047 & -3.260183 & 0.116600 \\
\hline
\end{tabular}




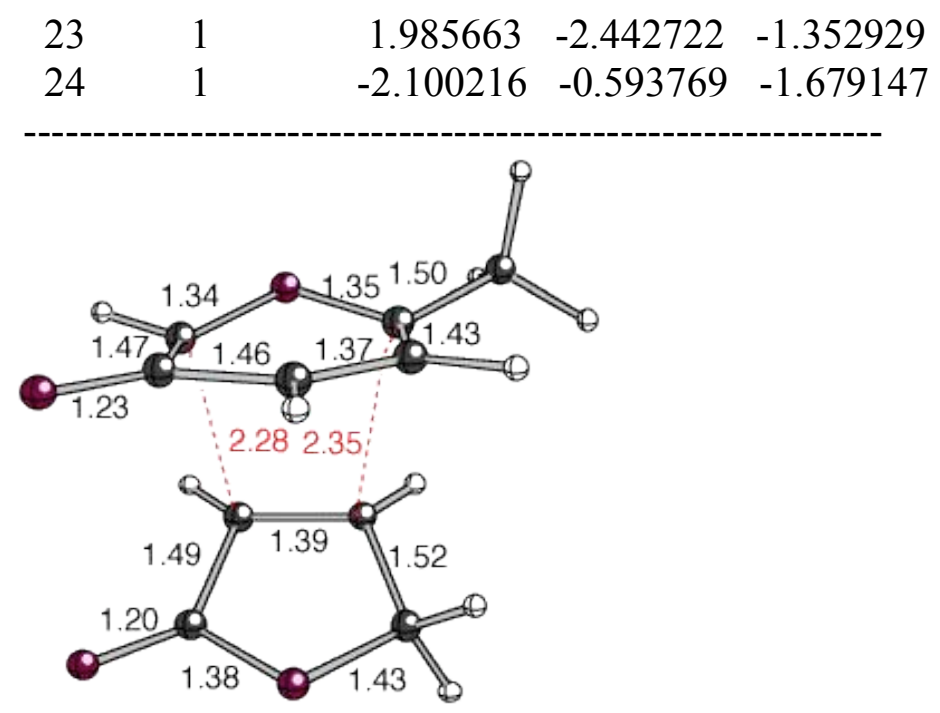

\section{C.IV.TS2}

$\mathrm{HF}=-687.8771542$ hartrees $(-431649.793032042 \mathrm{kcal} / \mathrm{mol})$ Imaginary Frequencies: 1 (-397.6700 1/cm)

Zero-point correction $=0.184240$ (Hartree/Particle $)$

Thermal correction to Energy $=\quad 0.196169$

Thermal correction to Enthalpy= $\quad 0.197113$

Thermal correction to Gibbs Free Energy $=0.145786$

Sum of electronic and zero-point Energies $=\quad-687.692914$

Sum of electronic and thermal Energies $=\quad-687.680985$

Sum of electronic and thermal Enthalpies $=\quad-687.680041$

Sum of electronic and thermal Free Energies $=\quad-687.731368$

Coordinates (from last standard orientation):

\begin{tabular}{|c|c|c|c|c|}
\hline \multirow{2}{*}{$\begin{array}{l}\text { Center } \\
\text { Number }\end{array}$} & \multirow{2}{*}{$\begin{array}{l}\text { Atomic } \\
\text { Number }\end{array}$} & \multicolumn{3}{|c|}{ Coordinates (Angstroms) } \\
\hline & & & $X \quad Y$ & Z \\
\hline 1 & 8 & 1.501480 & 0.623981 & -1.089362 \\
\hline 2 & 6 & 1.877101 & -0.128312 & -0.032360 \\
\hline 3 & 8 & -1.794921 & -1.285639 & 0.882141 \\
\hline 4 & 6 & 1.457086 & 0.312871 & 1.257248 \\
\hline 5 & 6 & 0.493410 & 1.266902 & 1.406561 \\
\hline 6 & 6 & 5976 & 1.829288 & 0.234883 \\
\hline 7 & 6 & 9703 & 1.255469 & -1.026494 \\
\hline 8 & 6 & -0.763981 & -0.744163 & -1.119690 \\
\hline 9 & 1 & -0.814988 & -0.584101 & -2.188283 \\
\hline 10 & 6 & 0.100347 & -1.609979 & -0.467095 \\
\hline 11 & 6 & -0.582169 & -2.043699 & 0.813521 \\
\hline 12 & 1 & 0.196302 & 1.634859 & 2.383667 \\
\hline 13 & 1 & 0.823841 & -2.249631 & -0.958557 \\
\hline 14 & 1 & -0.833959 & -3.112770 & 0.781816 \\
\hline 15 & 6 & -1.996833 & -0.601537 & -0.299712 \\
\hline 16 & 8 & -3.022669 & -0.024815 & -0.545958 \\
\hline 17 & 1 & -0.014599 & -1.854869 & 1.729619 \\
\hline 18 & 1 & 1.928096 & -0.147415 & 2.122190 \\
\hline 19 & 8 & -1.066997 & 2.676923 & 0.259372 \\
\hline 20 & 6 & 3.151374 & -0.885924 & -0.265186 \\
\hline 21 & 1 & 4.029961 & -0.232312 & -0.181865 \\
\hline
\end{tabular}




$\begin{array}{rrrrr}22 & 1 & 3.257070 & -1.682687 & 0.477309 \\ 23 & 1 & 3.160610 & -1.333860 & -1.262963 \\ 24 & 1 & 0.052148 & 1.733690 & -1.962540\end{array}$

\section{C.IV.TS2product}

$\mathrm{HF}=-687.9502495$ hartrees $(-431695.661063745 \mathrm{kcal} / \mathrm{mol})$

Imaginary Frequencies: none found

Zero-point correction $=0.189852$ (Hartree/Particle)

Thermal correction to Energy $=\quad 0.200897$

Thermal correction to Enthalpy= $\quad 0.201842$

Thermal correction to Gibbs Free Energy $=\quad 0.152570$

Sum of electronic and zero-point Energies $=\quad-687.760398$

Sum of electronic and thermal Energies= $\quad-687.749352$

Sum of electronic and thermal Enthalpies $=\quad-687.748408$

Sum of electronic and thermal Free Energies $=\quad-687.797679$

Coordinates (from last standard orientation):

\begin{tabular}{|c|c|c|c|c|}
\hline \multirow{2}{*}{$\begin{array}{l}\text { Center } \\
\text { Number }\end{array}$} & \multirow{2}{*}{$\begin{array}{l}\text { Atomic } \\
\text { Number }\end{array}$} & \multicolumn{3}{|c|}{ Coordinates (Angstroms) } \\
\hline & & & $X \quad Y$ & Z \\
\hline 1 & 8 & 0.279242 & -1.700351 & -1.311837 \\
\hline 2 & 6 & -0.294861 & -1.841943 & -0.009173 \\
\hline 3 & 8 & -1.749011 & 1.028250 & 1.098734 \\
\hline 4 & 6 & 0.653958 & -1.289337 & 1.032706 \\
\hline 5 & 6 & 1.518185 & -0.300841 & 0.733348 \\
\hline 6 & 6 & 1.451230 & 0.276314 & -0.644913 \\
\hline 7 & 6 & 0.305887 & -0.281117 & -1.500917 \\
\hline 8 & 6 & -1.120653 & 0.171982 & -1.018433 \\
\hline 9 & 6 & -1.607593 & -1.007954 & -0.156977 \\
\hline 10 & 6 & -2.200647 & $7 \quad-0.337713$ & 1.095025 \\
\hline 11 & 1 & -0.486642 & $2-2.905471$ & 0.155366 \\
\hline 12 & 1 & 0.494501 & $1-0.065863$ & -2.552708 \\
\hline 13 & 1 & -2.350131 & $1-1.622751$ & -0.670830 \\
\hline 14 & 1 & -3.295297 & $7-0.333073$ & 1.069980 \\
\hline 15 & 6 & -1.175643 & $3 \quad 1.382469$ & -0.087490 \\
\hline 16 & 8 & -0.786916 & 2.498849 & -0.306273 \\
\hline 17 & 1 & -1.877729 & $9-0.793424$ & 2.035198 \\
\hline 18 & 1 & 0.624800 & -1.718020 & 2.033286 \\
\hline 19 & 8 & 2.233459 & $9 \quad 1.112253$ & -1.055550 \\
\hline 20 & 1 & -1.753717 & 0.373408 & -1.886539 \\
\hline 21 & 6 & 2.537999 & 0.272185 & 1.673329 \\
\hline 22 & 1 & 2.494701 & $1 \quad-0.212181$ & 2.653184 \\
\hline 23 & 1 & 3.547001 & 10.151787 & 1.263100 \\
\hline 24 & 1 & 2.381143 & 1.349306 & 1.800339 \\
\hline
\end{tabular}




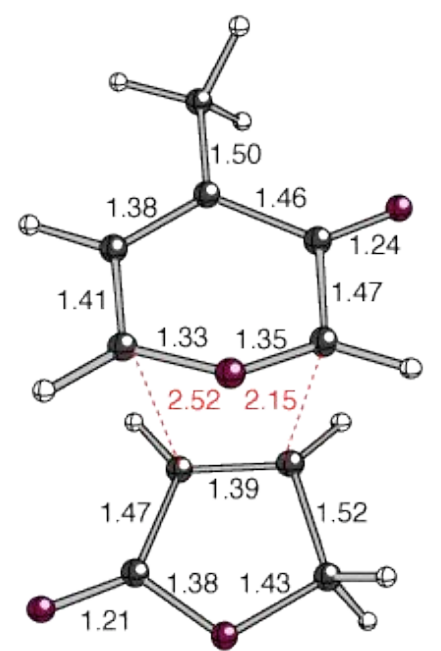

\section{C.IV.TS3}

$\mathrm{HF}=-687.8874122$ hartrees $(-431656.230029622 \mathrm{kcal} / \mathrm{mol})$ Imaginary Frequencies: $1(-401.52421 / \mathrm{cm})$

Zero-point correction $=0.184622($ Hartree/Particle $)$ Thermal correction to Energy= 0.196599 Thermal correction to Enthalpy= 0.197543

Thermal correction to Gibbs Free Energy= Sum of electronic and zero-point Energies= 0.145993 Sum of electronic and thermal Energies= $-687.702790$ Sum of electronic and thermal Enthalpies= $-687.690813$ $-687.689869$

Sum of electronic and thermal Free Energies= $-687.741419$ Coordinates (from last standard orientation):

\begin{tabular}{llrrr} 
Center & Atomic & \multicolumn{3}{c}{ Coordinates (Angstroms) } \\
Number & Number & X & $Y$ & $Z$ \\
-1 & 8 & -0.017793 & 0.027575 & 1.551244 \\
2 & 6 & -0.130564 & -1.200676 & 1.046465 \\
3 & 6 & -1.289892 & -1.554691 & 0.325116 \\
4 & 6 & -2.203569 & -0.609618 & -0.077155 \\
5 & 6 & -1.867869 & 0.800458 & 0.130061 \\
6 & 6 & -0.613003 & 1.022812 & 0.857507 \\
7 & 6 & 0.789290 & 1.005518 & -0.772891 \\
8 & 6 & 1.091825 & -0.332458 & -0.977423 \\
9 & 1 & 0.533973 & -1.915805 & 1.514030 \\
10 & 6 & 2.034262 & 1.628353 & -0.155965 \\
11 & 6 & 2.422164 & -0.628284 & -0.414481 \\
12 & 8 & 2.907167 & 0.535616 & 0.148812 \\
13 & 8 & 3.044239 & -1.663161 & -0.385157 \\
14 & 1 & 1.866016 & 2.193907 & 0.764837 \\
15 & 1 & 0.133972 & 1.597403 & -1.404047 \\
16 & 1 & 0.597520 & -1.023902 & -1.643070 \\
17 & 1 & 2.529445 & 2.290616 & -0.878771 \\
18 & 1 & -1.434049 & -2.607574 & 0.095893 \\
19 & 8 & -2.524285 & 1.745828 & -0.323032 \\
20 & 6 & -3.491736 & -0.923046 & -0.769909 \\
21 & 1 & -4.343979 & -0.595578 & -0.161425 \\
22 & 1 & -3.558926 & -0.357635 & -1.706921 \\
23 & 1 & -3.597080 & -1.991973 & -0.975686
\end{tabular}




\section{C.IV.TS3product \\ $\mathrm{HF}=-687.9583335$ hartrees $(-431700.733854585 \mathrm{kcal} / \mathrm{mol})$ \\ Imaginary Frequencies: none found \\ Zero-point correction $=0.189814$ (Hartree/Particle) \\ Thermal correction to Energy $=\quad 0.201014$ \\ Thermal correction to Enthalpy= $\quad 0.201958$ \\ Thermal correction to Gibbs Free Energy $=\quad 0.152000$ \\ Sum of electronic and zero-point Energies $=\quad-687.768520$ \\ Sum of electronic and thermal Energies $=\quad-687.757320$ \\ Sum of electronic and thermal Enthalpies $=\quad-687.756376$ \\ Sum of electronic and thermal Free Energies $=\quad-687.806333$ \\ Coordinates (from last standard orientation):}

\begin{tabular}{|c|c|c|c|c|}
\hline \multirow{2}{*}{$\begin{array}{l}\text { Center } \\
\text { Number }\end{array}$} & \multirow{2}{*}{$\begin{array}{l}\text { Atomic } \\
\text { Numbe }\end{array}$} & \multicolumn{3}{|c|}{ Coordinates (Angstroms) } \\
\hline & & & $X \quad Y$ & Z \\
\hline 1 & 6 & 0.108497 & -1.174617 & 0.519954 \\
\hline 2 & 6 & -1.267324 & -1.544781 & 0.017830 \\
\hline 3 & 6 & -2.229733 & -0.610662 & -0.108980 \\
\hline 4 & 6 & -1.840432 & 0.800311 & 0.166906 \\
\hline 5 & 6 & -0.393955 & 0.999405 & 0.640342 \\
\hline 6 & 6 & 0.585003 & 0.945457 & -0.573036 \\
\hline 7 & 6 & 0.978422 & -0.547189 & -0.613060 \\
\hline 8 & 1 & 0.609543 & -2.012614 & 1.008972 \\
\hline 9 & 6 & 1.930440 & 1.673370 & -0.331345 \\
\hline 10 & 6 & 2.457172 & -0.583364 & -0.232550 \\
\hline 11 & 8 & 2.937362 & 0.676510 & -0.086340 \\
\hline 12 & 8 & 3.132042 & -1.564851 & -0.060244 \\
\hline 13 & 1 & 1.903657 & 2.334693 & 0.539391 \\
\hline 14 & 1 & 0.109159 & 1.307563 & -1.487002 \\
\hline 15 & 1 & 2.241477 & 2.255455 & -1.204163 \\
\hline 16 & 1 & -1.450905 & -2.582795 & -0.255505 \\
\hline 17 & 8 & -2.580317 & 1.749281 & -0.037190 \\
\hline 18 & 8 & 0.006250 & -0.099995 & 1.458755 \\
\hline 19 & 1 & 0.839476 & -1.045642 & -1.575144 \\
\hline 20 & 6 & -3.638477 & -0.871919 & -0.558779 \\
\hline 21 & 1 & -4.352018 & -0.598205 & 0.227359 \\
\hline 22 & 1 & -3.888309 & -0.256330 & -1.430032 \\
\hline 23 & 1 & -3.786531 & -1.925990 & -0.810940 \\
\hline 24 & 1 & -0.325916 & 1.920244 & 1.223522 \\
\hline
\end{tabular}




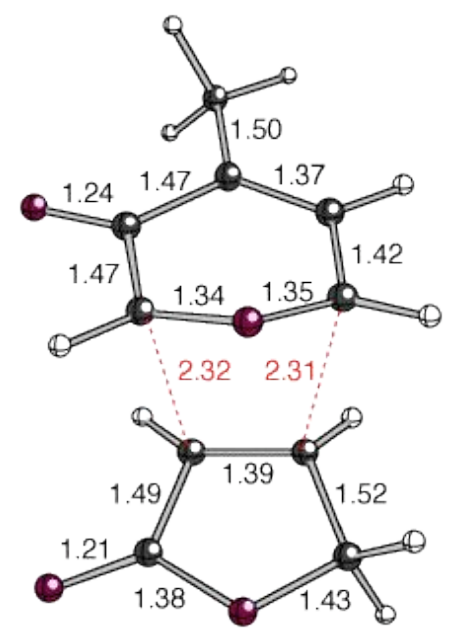

\section{C.IV.TS4}

$\mathrm{HF}=-687.8833914$ hartrees $(-431653.706937414 \mathrm{kcal} / \mathrm{mol})$ Imaginary Frequencies: $1(-410.52441 / \mathrm{cm})$

Zero-point correction $=0.184551$ (Hartree/Particle)

Thermal correction to Energy $=\quad 0.196492$

Thermal correction to Enthalpy $=\quad 0.197437$

Thermal correction to Gibbs Free Energy $=\quad 0.146010$

Sum of electronic and zero-point Energies $=\quad-687.698841$

Sum of electronic and thermal Energies $=\quad-687.686899$

Sum of electronic and thermal Enthalpies $=\quad-687.685955$

Sum of electronic and thermal Free Energies $=\quad-687.737381$

Coordinates (from last standard orientation):

\begin{tabular}{|c|c|c|c|c|}
\hline \multirow{2}{*}{$\begin{array}{l}\text { Center } \\
\text { Number }\end{array}$} & \multirow{2}{*}{$\begin{array}{l}\text { Atomic } \\
\text { Number }\end{array}$} & \multicolumn{3}{|c|}{ Coordinates (Angstroms) } \\
\hline & & & $X \quad Y$ & Z \\
\hline 1 & 8 & 0.017928 & -0.481620 & 1.485301 \\
\hline 2 & 8 & 2.007997 & 2.073989 & 0.005046 \\
\hline 3 & 6 & 0.342996 & 0.718003 & 0.990701 \\
\hline 4 & 6 & 1.601025 & 0.939282 & 0.272379 \\
\hline 5 & 6 & 2.292907 & -0.293295 & -0.129275 \\
\hline 6 & 6 & 1.667259 & -1.488979 & 0.100341 \\
\hline 7 & 1 & 2.093841 & -2.423433 & -0.257429 \\
\hline 8 & 6 & 0.422681 & -1.553545 & 0.779988 \\
\hline 9 & 6 & -1.018365 & -1.029975 & -0.943772 \\
\hline 10 & 6 & -0.952926 & $6 \quad 0.353306$ & -0.893823 \\
\hline 11 & 1 & 0.034353 & $3-2.479254$ & 1.188254 \\
\hline 12 & 6 & -2.365181 & $1-1.439478$ & -0.381677 \\
\hline 13 & 6 & -2.208428 & $8 \quad 0.858271$ & -0.274355 \\
\hline 14 & 8 & -2.961367 & $7-0.232703$ & 0.104829 \\
\hline 15 & 8 & -2.578173 & $\begin{array}{ll}3 & 1.988892\end{array}$ & -0.084119 \\
\hline 16 & 1 & -2.328095 & $5-2.155693$ & 0.445676 \\
\hline 17 & 1 & -0.499315 & $5-1.650141$ & -1.663124 \\
\hline 18 & 1 & -0.355515 & 1.007544 & -1.513439 \\
\hline 19 & 1 & -3.008003 & $3-1.861977$ & -1.166517 \\
\hline 20 & 6 & 3.620354 & $4-0.147913$ & -0.804516 \\
\hline 21 & 1 & 3.532607 & 0.532182 & -1.660035 \\
\hline 22 & 1 & 4.345559 & 0.318245 & -0.126379 \\
\hline 23 & 1 & 4.017288 & $3-1.110521$ & -1.139536 \\
\hline 24 & 1 & -0.137731 & 1.540532 & 1.508116 \\
\hline
\end{tabular}




\section{C.IV.TS4pro}

$\mathrm{HF}=-687.9571029$ hartrees $(-431699.961640779 \mathrm{kcal} / \mathrm{mol})$ Imaginary Frequencies: none found

Zero-point correction $=0.189717$ (Hartree/Particle)

Thermal correction to Energy $=\quad 0.200948$

Thermal correction to Enthalpy= $\quad 0.201892$

Thermal correction to Gibbs Free Energy $=0.151788$

Sum of electronic and zero-point Energies $=\quad-687.767386$

Sum of electronic and thermal Energies $=\quad-687.756155$

Sum of electronic and thermal Enthalpies= $\quad-687.755211$

Sum of electronic and thermal Free Energies $=\quad-687.805315$

Coordinates (from last standard orientation):

\begin{tabular}{|c|c|c|c|c|}
\hline \multirow{2}{*}{$\begin{array}{l}\text { Center } \\
\text { Number }\end{array}$} & \multirow{2}{*}{$\begin{array}{l}\text { Atomic } \\
\text { Number }\end{array}$} & \multicolumn{3}{|c|}{ Coordinates (Angstroms) } \\
\hline & & & $X \quad Y$ & Z \\
\hline 1 & 8 & 0.038661 & -0.424095 & 1.463508 \\
\hline 2 & 8 & 2.042613 & 2.064669 & 0.009610 \\
\hline 3 & 6 & 0.126494 & 0.754230 & 0.661258 \\
\hline 4 & 6 & 1.571666 & 0.953833 & 0.184465 \\
\hline 5 & 6 & 2.314810 & -0.301111 & -0.127019 \\
\hline 6 & 6 & 1.637096 & -1.458654 & -0.009812 \\
\hline 7 & 1 & 2.088295 & -2.405904 & -0.302108 \\
\hline 8 & 6 & 0.217974 & -1.475327 & 0.509022 \\
\hline 9 & 6 & -0.805431 & -1.107614 & -0.607370 \\
\hline 10 & 6 & -0.804499 & 0.435352 & -0.551567 \\
\hline 11 & 1 & -0.012663 & -2.420388 & 1.009125 \\
\hline 12 & 6 & -2.264006 & -1.490853 & -0.252926 \\
\hline 13 & 6 & -2.249668 & 0.830239 & -0.250318 \\
\hline 14 & 8 & -3.024593 & -0.278647 & -0.128251 \\
\hline 15 & 8 & -2.683385 & 1.944272 & -0.122464 \\
\hline 16 & 1 & -2.330368 & -2.022562 & 0.701733 \\
\hline 17 & 1 & -0.514193 & -1.508432 & -1.580421 \\
\hline 18 & 1 & -0.481753 & 0.944862 & -1.462542 \\
\hline 19 & 1 & -2.736242 & -2.099571 & -1.028900 \\
\hline 20 & 6 & 3.736113 & -0.171132 & -0.593483 \\
\hline 21 & 1 & 3.800298 & 0.495213 & -1.460883 \\
\hline 22 & 1 & 4.358884 & 0.279759 & 0.187976 \\
\hline 23 & 1 & 4.159122 & -1.144708 & -0.857941 \\
\hline 24 & 1 & -0.201048 & 1.618360 & 1.241225 \\
\hline
\end{tabular}




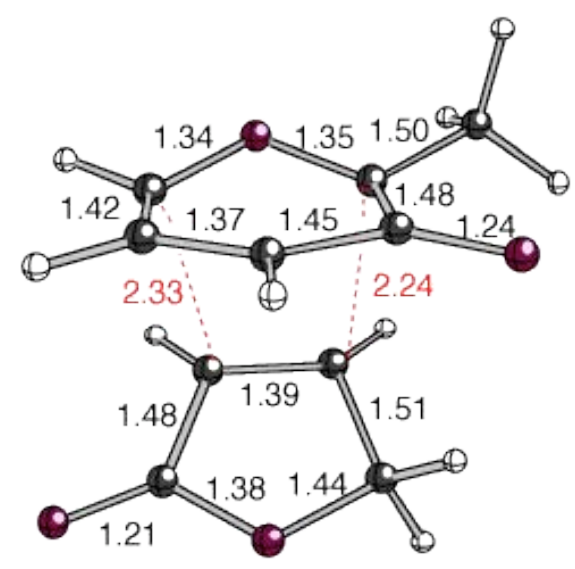

D.IV.TS1

$\mathrm{HF}=-687.8891257$ hartrees $(-431657.305268007 \mathrm{kcal} / \mathrm{mol})$

Imaginary Frequencies: $1(-404.22431 / \mathrm{cm})$

Zero-point correction $=0.184961$ (Hartree/Particle)

Thermal correction to Energy=

0.196608

Thermal correction to Enthalpy=

0.197552

Thermal correction to Gibbs Free Energy=

Sum of electronic and zero-point Energies=

0.147425

Sum of electronic and thermal Energies=

$-687.704164$

Sum of electronic and thermal Enthalpies= $-687.692518$

$-687.691573$

Sum of electronic and thermal Free Energies=

$-687.741701$

Coordinates (from last standard orientation):

\begin{tabular}{|c|c|c|c|c|}
\hline \multirow{2}{*}{$\begin{array}{l}\text { Center } \\
\text { Number }\end{array}$} & \multirow{2}{*}{$\begin{array}{l}\text { Atomic } \\
\text { Number }\end{array}$} & \multicolumn{3}{|c|}{ Coordinates (Angstroms) } \\
\hline & & & $\begin{array}{l}X \\
X\end{array}$ & Z \\
\hline 1 & 8 & 1.146797 & 1.340331 & -0.909348 \\
\hline 2 & 8 & 1.987913 & -1.121843 & 1.523034 \\
\hline 3 & 6 & 1.573040 & 0.150204 & -0.424712 \\
\hline 4 & 8 & -1.878933 & -1.266587 & 0.520763 \\
\hline 5 & 6 & 1.419169 & -0.146710 & 1.012962 \\
\hline 6 & 6 & 0.504597 & 0.740568 & 1.713451 \\
\hline 7 & 6 & -0.188026 & 1.695628 & 1.024410 \\
\hline 8 & 1 & -0.956537 & 2.299971 & 1.496025 \\
\hline 9 & 6 & 0.044368 & 1.874082 & -0.360980 \\
\hline 10 & 6 & -1.203175 & 0.097098 & -1.216287 \\
\hline 11 & 1 & -1.413264 & 0.718607 & -2.075847 \\
\hline 12 & 6 & -0.259932 & -0.922058 & -1.138851 \\
\hline 13 & 6 & -0.752459 & -1.907256 & -0.100267 \\
\hline 14 & 1 & -0.316535 & 2.733969 & -0.911239 \\
\hline 15 & 1 & 0.371666 & 0.587788 & 2.779580 \\
\hline 16 & 1 & 0.326107 & -1.268666 & -1.982611 \\
\hline 17 & 1 & -1.096559 & -2.834365 & -0.577672 \\
\hline 18 & 6 & -2.239992 & -0.142631 & -0.184916 \\
\hline 19 & 8 & -3.229243 & 0.497193 & 0.078724 \\
\hline 20 & 1 & -0.025829 & -2.164876 & 0.676120 \\
\hline 21 & 6 & 2.733869 & -0.427959 & -1.170723 \\
\hline 22 & 1 & 3.670459 & 0.051356 & -0.857228 \\
\hline 23 & 1 & 2.820202 & -1.488936 & -0.927247 \\
\hline 24 & 1 & 2.619265 & -0.293389 & -2.249785 \\
\hline
\end{tabular}




\section{D.IV.TS1product}

$\mathrm{HF}=-687.9528955$ hartrees $(-431697.321455205 \mathrm{kcal} / \mathrm{mol})$

Imaginary Frequencies: none found

Zero-point correction $=0.189651$ (Hartree/Particle)

Thermal correction to Energy $=\quad 0.200556$

Thermal correction to Enthalpy= $\quad 0.201500$

Thermal correction to Gibbs Free Energy $=\quad 0.152908$

Sum of electronic and zero-point Energies $=\quad-687.763244$

Sum of electronic and thermal Energies $=\quad-687.752340$

Sum of electronic and thermal Enthalpies $=\quad-687.751396$

Sum of electronic and thermal Free Energies $=\quad-687.799987$

Coordinates (from last standard orientation):

\begin{tabular}{|c|c|c|c|c|}
\hline \multirow{2}{*}{$\begin{array}{l}\text { Center } \\
\text { Number }\end{array}$} & \multirow{2}{*}{$\begin{array}{l}\text { Atomic } \\
\text { Number }\end{array}$} & \multicolumn{3}{|c|}{ Coordinates (Angstroms) } \\
\hline & & & $X \quad Y$ & Z \\
\hline 1 & 8 & 1.026885 & 1.079339 & -1.248410 \\
\hline 2 & 8 & 2.050266 & -0.379439 & 1.793064 \\
\hline 3 & 6 & 1.314655 & -0.116020 & -0.500662 \\
\hline 4 & 8 & -1.735537 & -1.326608 & 0.733744 \\
\hline 5 & 6 & 1.363178 & 0.249430 & 1.002277 \\
\hline 6 & 6 & 0.466748 & 1.350171 & 1.414665 \\
\hline 7 & 6 & -0.378751 & 1.881632 & 0.516942 \\
\hline 8 & 1 & -1.129388 & 2.616727 & 0.794631 \\
\hline 9 & 6 & -0.321616 & 1.394408 & -0.913478 \\
\hline 10 & 6 & -1.056026 & $6 \quad 0.013676$ & -1.088876 \\
\hline 11 & 1 & -1.494568 & $8-0.049834$ & -2.087638 \\
\hline 12 & 6 & 0.061724 & $4-1.010563$ & -0.830940 \\
\hline 13 & 6 & -0.514994 & $4-1.935621$ & 0.261847 \\
\hline 14 & 1 & -0.678939 & 2.150767 & -1.614287 \\
\hline 15 & 1 & 0.491191 & 1.644579 & 2.459777 \\
\hline 16 & 1 & 0.303668 & $3-1.582325$ & -1.730129 \\
\hline 17 & 1 & -0.768406 & $6-2.922185$ & -0.137454 \\
\hline 18 & 6 & -2.121762 & $2-0.277287$ & -0.039006 \\
\hline 19 & 8 & -3.150263 & 0.322292 & 0.143637 \\
\hline 20 & 1 & 0.144430 & -2.064127 & 1.123089 \\
\hline 21 & 6 & 2.636724 & $4-0.692906$ & -0.974946 \\
\hline 22 & 1 & 3.443911 & 0.028813 & -0.820560 \\
\hline 23 & 1 & 2.878770 & -1.596276 & -0.409498 \\
\hline 24 & 1 & 2.579243 & $3-0.932327$ & -2.041150 \\
\hline
\end{tabular}

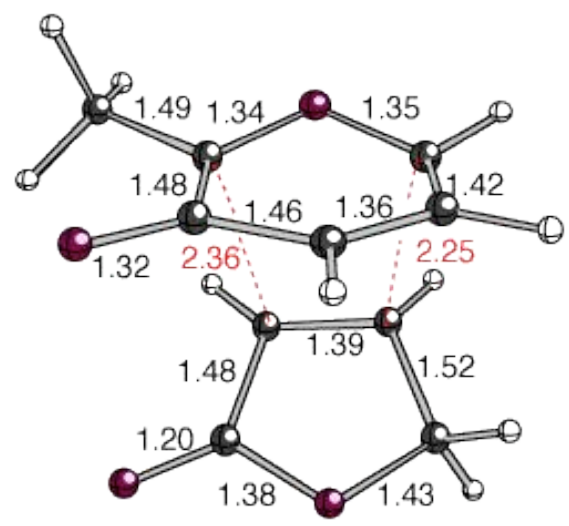




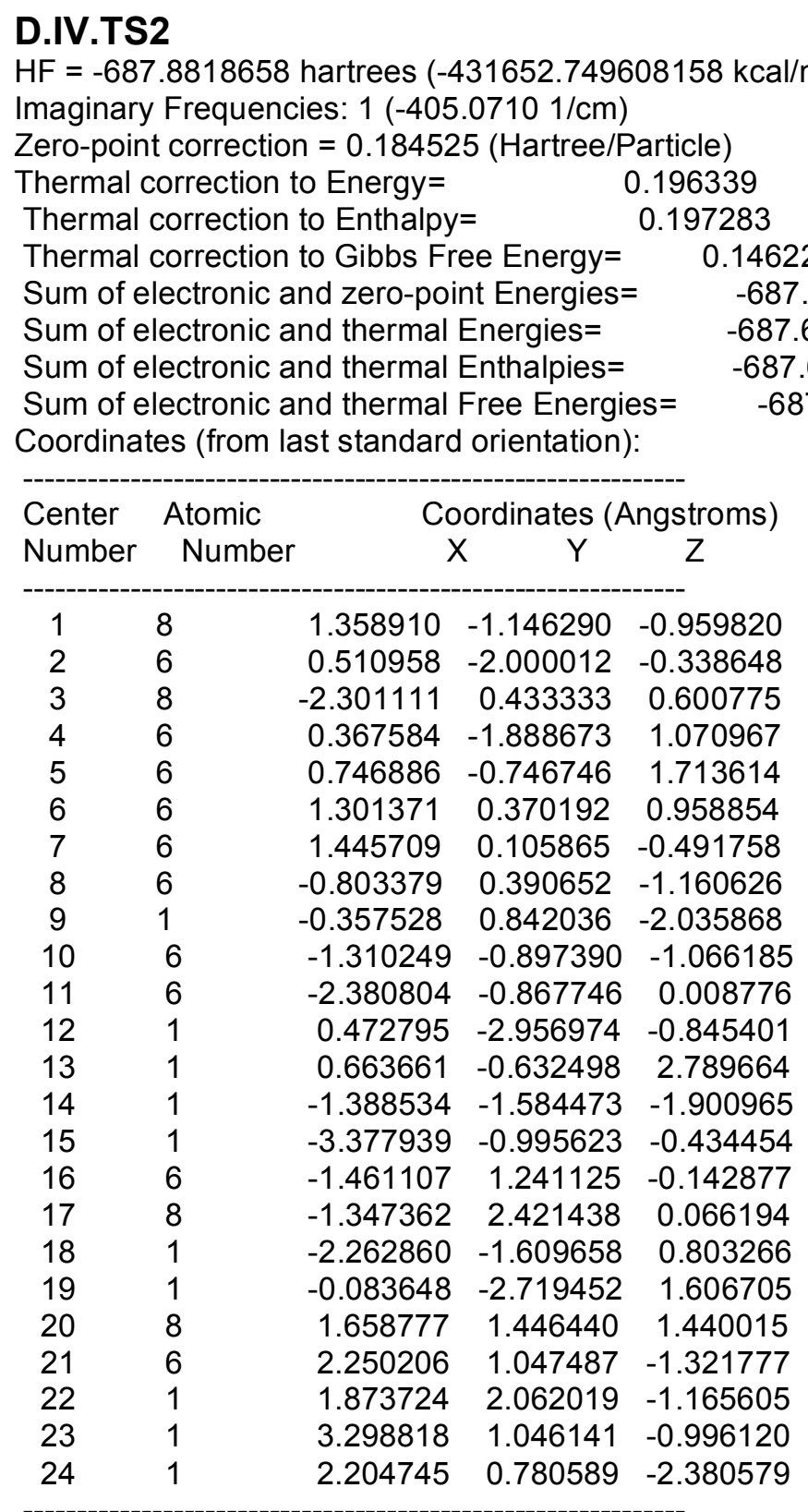

\section{D.IV.TS2product}

$\mathrm{HF}=-687.9495966$ hartrees $(-431695.251362466 \mathrm{kcal} / \mathrm{mol})$

Imaginary Frequencies: none found

Zero-point correction $=0.189377$ (Hartree/Particle)

Thermal correction to Energy $=\quad 0.200333$

Thermal correction to Enthalpy= $\quad 0.201277$

Thermal correction to Gibbs Free Energy $=\quad 0.152601$

Sum of electronic and zero-point Energies $=\quad-687.760220$

Sum of electronic and thermal Energies $=\quad-687.749264$

Sum of electronic and thermal Enthalpies $=\quad-687.748320$

Sum of electronic and thermal Free Energies $=\quad-687.796995$

Coordinates (from last standard orientation): 


\begin{tabular}{|c|c|c|c|c|}
\hline \multirow{2}{*}{$\begin{array}{l}\text { Center } \\
\text { Number }\end{array}$} & \multirow{2}{*}{$\begin{array}{l}\text { Atomic } \\
\text { Number }\end{array}$} & \multicolumn{3}{|c|}{ Coordinates (Angstroms) } \\
\hline & & & $\begin{array}{l}\mathrm{Y} \\
\mathrm{Y}\end{array}$ & Z \\
\hline 1 & 8 & 0.960893 & -1.509799 & -0.805112 \\
\hline 2 & 6 & -0.287715 & -1.838457 & -0.198139 \\
\hline 3 & 8 & -2.180356 & 0.935826 & 0.429854 \\
\hline 4 & 6 & -0.199534 & -1.625994 & 1.297899 \\
\hline 5 & 6 & 0.631394 & -0.691621 & 1.784381 \\
\hline 6 & 6 & 1.414429 & 0.155436 & 0.848463 \\
\hline 7 & 6 & 1.077112 & -0.079152 & -0.644637 \\
\hline 8 & 6 & -0.375981 & 0.416946 & -1.011993 \\
\hline 9 & 6 & -1.260045 & -0.835935 & -0.898505 \\
\hline 10 & 6 & -2.504347 & -0.348219 & -0.132138 \\
\hline 11 & 1 & -0.510879 & -2.881263 & -0.438859 \\
\hline 12 & 1 & -1.529291 & -1.245611 & -1.874698 \\
\hline 13 & 1 & -3.363131 & -0.219570 & -0.799027 \\
\hline 14 & 6 & -1.015155 & 1.436620 & -0.073507 \\
\hline 15 & 8 & -0.603192 & 2.528230 & 0.216903 \\
\hline 16 & 1 & -2.802734 & -1.002139 & 0.692144 \\
\hline 17 & 1 & -0.816262 & -2.236775 & 1.954021 \\
\hline 18 & 8 & 2.254779 & 0.951256 & 1.221437 \\
\hline 19 & 1 & -0.356109 & 0.845921 & -2.017952 \\
\hline 20 & 1 & 0.768962 & -0.511386 & 2.846623 \\
\hline 21 & 6 & 2.162314 & 0.452021 & -1.558891 \\
\hline 22 & 1 & 3.126775 & 0.003395 & -1.306740 \\
\hline 23 & 1 & 1.922626 & 0.218607 & -2.600926 \\
\hline 24 & 1 & 2.248230 & 1.534849 & -1.436848 \\
\hline
\end{tabular}

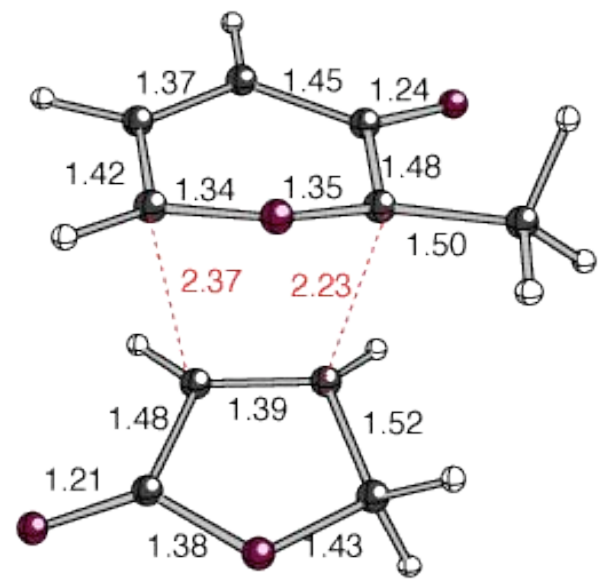

\section{D.IV.TS3}

$\mathrm{HF}=-687.8869517$ hartrees $(-431655.941061267 \mathrm{kcal} / \mathrm{mol})$

Imaginary Frequencies: $1(-413.77041 / \mathrm{cm})$

Zero-point correction $=0.184688($ Hartree/Particle $)$

Thermal correction to Energy $=\quad 0.196495$

Thermal correction to Enthalpy $=\quad 0.197440$

Thermal correction to Gibbs Free Energy $=\quad 0.146621$

Sum of electronic and zero-point Energies $=\quad-687.702264$

Sum of electronic and thermal Energies $=\quad-687.690456$

Sum of electronic and thermal Enthalpies $=\quad-687.689512$

Sum of electronic and thermal Free Energies $=\quad-687.740330$

Coordinates (from last standard orientation): 


\begin{tabular}{|c|c|c|c|c|}
\hline \multirow{2}{*}{$\begin{array}{l}\text { Center } \\
\text { Number }\end{array}$} & \multirow{2}{*}{$\begin{array}{l}\text { Atomic } \\
\text { Number }\end{array}$} & \multicolumn{3}{|c|}{ Coordinates (Angstroms) } \\
\hline & & & $\begin{array}{ll}X & Y\end{array}$ & Z \\
\hline 1 & 8 & 0.402517 & 0.172291 & 1.351290 \\
\hline 2 & 6 & 0.050021 & 1.401492 & 0.953088 \\
\hline 3 & 6 & 0.966143 & 2.156632 & 0.180744 \\
\hline 4 & 6 & 2.023927 & 1.542187 & -0.429352 \\
\hline 5 & 6 & 2.152918 & 0.095904 & -0.369975 \\
\hline 6 & 6 & 1.158024 & -0.569172 & 0.507480 \\
\hline 7 & 6 & -0.455139 & -0.704979 & -1.019143 \\
\hline 8 & 6 & -1.112070 & 0.515452 & -0.914739 \\
\hline 9 & 1 & -0.710774 & 1.844332 & 1.583106 \\
\hline 10 & 1 & 2.767744 & 2.094743 & -0.994711 \\
\hline 11 & 6 & -1.407057 & -1.747061 & -0.456934 \\
\hline 12 & 6 & -2.397941 & 0.316680 & -0.208569 \\
\hline 13 & 8 & -2.484833 & -1.013372 & 0.138013 \\
\hline 14 & 8 & -3.256346 & 1.115667 & 0.078151 \\
\hline 15 & 1 & -0.989570 & -2.409699 & 0.304605 \\
\hline 16 & 1 & 0.241293 & -0.968794 & -1.807502 \\
\hline 17 & 1 & -0.946442 & 1.395190 & -1.517971 \\
\hline 18 & 1 & -1.807934 & -2.372008 & -1.266531 \\
\hline 19 & 1 & 0.781540 & 3.221931 & 0.072091 \\
\hline 20 & 8 & 2.984713 & -0.572205 & -0.993373 \\
\hline 21 & 6 & 1.479663 & -1.936692 & 1.022189 \\
\hline 22 & 1 & 1.617019 & -2.625031 & 0.184313 \\
\hline 23 & 1 & 2.430277 & -1.919015 & 1.570329 \\
\hline 24 & 1 & 0.697509 & -2.303366 & 1.690889 \\
\hline
\end{tabular}

\section{D.IV.TS3product}

$\mathrm{HF}=-687.9553405$ hartrees $(-431698.855717155 \mathrm{kcal} / \mathrm{mol})$ Imaginary Frequencies: none found Zero-point correction $=0.189723$ (Hartree/Particle)

Thermal correction to Energy $=\quad 0.200575$ Thermal correction to Enthalpy $=\quad 0.201519$ Thermal correction to Gibbs Free Energy $=0.153073$ Sum of electronic and zero-point Energies $=\quad-687.765618$ Sum of electronic and thermal Energies $=\quad-687.754766$ Sum of electronic and thermal Enthalpies $=\quad-687.753822$ Sum of electronic and thermal Free Energies $=\quad-687.802267$ Coordinates (from last standard orientation):

\begin{tabular}{ccrrr} 
Center & Atomic & \multicolumn{3}{c}{ Coordinates (Angstroms) } \\
Number & Number & X & $Y$ & $Z$ \\
- \hdashline 1 & 6 & -0.110848 & 1.436022 & 0.476993 \\
2 & 6 & 1.098755 & 2.124577 & -0.109412 \\
3 & 6 & 2.194304 & 1.400699 & -0.388740 \\
4 & 6 & 2.166087 & -0.067989 & -0.196182 \\
5 & 6 & 0.830656 & -0.623925 & 0.358170 \\
6 & 6 & -0.203230 & -0.607839 & -0.838951 \\
7 & 6 & -0.942517 & 0.729369 & -0.627249 \\
8 & 1 & -0.720647 & 2.106209 & 1.086880 \\
9 & 6 & -1.345411 & -1.642405 & -0.799996 \\
10 & 6 & -2.310118 & 0.326366 & -0.069798
\end{tabular}




$\begin{array}{rrrrr}11 & 8 & -2.464473 & -1.020523 & -0.141005 \\ 12 & 8 & -3.144586 & 1.058223 & 0.394386 \\ 13 & 1 & -1.106878 & -2.555311 & -0.252824 \\ 14 & 1 & 0.323460 & -0.676630 & -1.793898 \\ 15 & 1 & -1.657294 & -1.916611 & -1.813891 \\ 16 & 1 & 1.046786 & 3.191919 & -0.315041 \\ 17 & 8 & 3.092812 & -0.795817 & -0.511308 \\ 18 & 8 & 0.304323 & 0.340295 & 1.288569 \\ 19 & 1 & -1.076619 & 1.339696 & -1.524474 \\ 20 & 6 & 1.037115 & -1.939440 & 1.085714 \\ 21 & 1 & 1.407112 & -2.704974 & 0.398825 \\ 22 & 1 & 1.781724 & -1.810767 & 1.876017 \\ 23 & 1 & 0.103198 & -2.272727 & 1.546010 \\ 24 & 1 & 3.105799 & 1.829170 & -0.796033 \\ ------------------------------------------\end{array}$

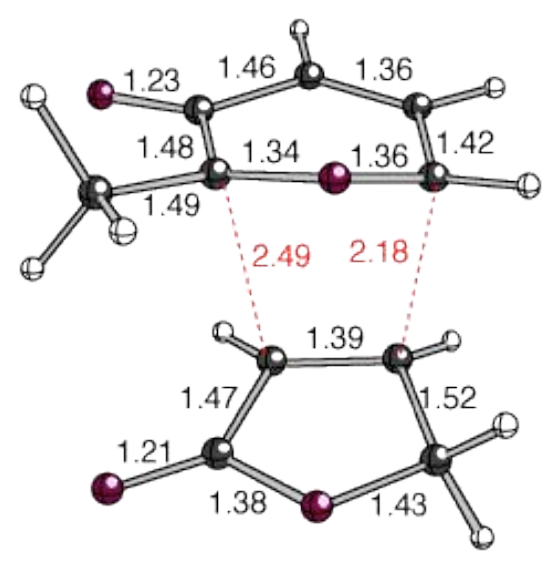

\section{D.IV.TS4}

$\mathrm{HF}=-687.8874812$ hartrees $(-431656.273327812 \mathrm{kcal} / \mathrm{mol})$ Imaginary Frequencies: $1(-399.00011 / \mathrm{cm})$

Zero-point correction $=0.184485$ (Hartree/Particle)

Thermal correction to Energy $=\quad 0.196341$

Thermal correction to Enthalpy $=\quad 0.197286$

Thermal correction to Gibbs Free Energy $=0.146271$

Sum of electronic and zero-point Energies $=\quad-687.702996$

Sum of electronic and thermal Energies $=\quad-687.691140$

Sum of electronic and thermal Enthalpies $=\quad-687.690195$

Sum of electronic and thermal Free Energies $=\quad-687.741211$

Coordinates (from last standard orientation):

\begin{tabular}{|c|c|c|c|c|}
\hline \multirow{2}{*}{$\begin{array}{l}\text { Center } \\
\text { Number }\end{array}$} & \multirow{2}{*}{$\begin{array}{l}\text { Atomic } \\
\text { Number }\end{array}$} & \multicolumn{3}{|c|}{ Coordinates (Angstroms) } \\
\hline & & & $\begin{array}{ll}X & Y\end{array}$ & Z \\
\hline 1 & 8 & 0.479867 & -0.448012 & 1.337369 \\
\hline 2 & 8 & 2.408261 & 1.606684 & -0.827810 \\
\hline 3 & 6 & 0.849850 & 0.641967 & 0.660442 \\
\hline 4 & 6 & 1.948296 & 0.576179 & -0.328740 \\
\hline 5 & 6 & 2.424456 & -0.773324 & -0.605671 \\
\hline 6 & 6 & 1.771394 & -1.850187 & -0.086140 \\
\hline 7 & 1 & 2.060492 & -2.868882 & -0.331526 \\
\hline 8 & 6 & 0.633641 & -1.660402 & 0.748847 \\
\hline 9 & 6 & -0.946970 & -1.367906 & -0.724300 \\
\hline 10 & 6 & -0.777667 & -0.039769 & -1.093608 \\
\hline
\end{tabular}




$\begin{array}{rrrrr}11 & 1 & 0.255691 & -2.453506 & 1.383849 \\ 12 & 1 & 3.296815 & -0.875544 & -1.243727 \\ 13 & 6 & -2.225082 & -1.431407 & 0.095734 \\ 14 & 6 & -1.852662 & 0.765124 & -0.485601 \\ 15 & 8 & -2.622068 & -0.071947 & 0.299836 \\ 16 & 8 & -2.093731 & 1.945303 & -0.582814 \\ 17 & 1 & -2.124114 & -1.905816 & 1.076967 \\ 18 & 1 & -0.649045 & -2.211228 & -1.335595 \\ 19 & 1 & -0.194160 & 0.349758 & -1.915108 \\ 20 & 1 & -3.017245 & -1.954932 & -0.456900 \\ 21 & 6 & 0.479042 & 1.943277 & 1.277676 \\ 22 & 1 & 1.381788 & 2.473028 & 1.604156 \\ 23 & 1 & -0.015412 & 2.576826 & 0.533267 \\ 24 & 1 & -0.199228 & 1.792752 & 2.120119 \\ ------------------------------------------------------\end{array}$

\section{D.IV.TS4product}

$\mathrm{HF}=-687.9551134$ hartrees $(-431698.713209634 \mathrm{kcal} / \mathrm{mol})$

Imaginary Frequencies: none found

Zero-point correction $=0.189568$ (Hartree/Particle)

Thermal correction to Energy $=\quad 0.200545$

Thermal correction to Enthalpy= $\quad 0.201489$

Thermal correction to Gibbs Free Energy $=\quad 0.152792$

Sum of electronic and zero-point Energies $=\quad-687.765545$

Sum of electronic and thermal Energies $=\quad-687.754568$

Sum of electronic and thermal Enthalpies $=\quad-687.753624$

Sum of electronic and thermal Free Energies $=\quad-687.802322$

Coordinates (from last standard orientation):

\begin{tabular}{|c|c|c|c|c|}
\hline \multirow{2}{*}{$\begin{array}{l}\text { Center } \\
\text { Number }\end{array}$} & \multirow{2}{*}{$\begin{array}{l}\text { Atomic } \\
\text { Number }\end{array}$} & \multicolumn{3}{|c|}{ Coordinates (Angstroms) } \\
\hline & & & $X \quad Y$ & Z \\
\hline 1 & 8 & 0.539347 & -0.497655 & 1.330789 \\
\hline 2 & 8 & 2.435946 & 1.745407 & -0.580939 \\
\hline 3 & 6 & 0.529777 & 0.621734 & 0.424270 \\
\hline 4 & 6 & 1.919219 & 0.690252 & -0.258432 \\
\hline 5 & 6 & 2.534862 & -0.626899 & -0.549291 \\
\hline 6 & 6 & 1.874845 & -1.750178 & -0.229156 \\
\hline 7 & 1 & 2.254882 & -2.735326 & -0.492645 \\
\hline 8 & 6 & 0.549066 & -1.647454 & 0.487508 \\
\hline 9 & 6 & -0.607462 & -1.349804 & -0.505021 \\
\hline 10 & 6 & -0.512492 & 0.179189 & -0.681747 \\
\hline 11 & 1 & 0.362856 & -2.523582 & 1.115917 \\
\hline 12 & 1 & 498471 & -0.621810 & -1.050955 \\
\hline 13 & 6 & -2.003310 & -1.554096 & 0.132276 \\
\hline 14 & 6 & -1.930408 & 0.692170 & -0.441553 \\
\hline 15 & 8 & -2.725848 & -0.318825 & 0.001632 \\
\hline 16 & 8 & -2.342268 & 1.809131 & -0.612867 \\
\hline 17 & 1 & -1.932748 & -1.790739 & 1.198883 \\
\hline 18 & 1 & -0.511756 & -1.900530 & -1.443700 \\
\hline 19 & 1 & -0.195461 & 0.514366 & -1.671875 \\
\hline 20 & 1 & -2.587092 & -2.331620 & -0.367104 \\
\hline 21 & 6 & 0.216585 & 1.890169 & 1.194749 \\
\hline 22 & 1 & 1.013833 & 2.088194 & 1.916502 \\
\hline 23 & 1 & 0.145226 & 2.739854 & 0.513529 \\
\hline 24 & 1 & -0.729728 & 1.786221 & 1.730903 \\
\hline
\end{tabular}




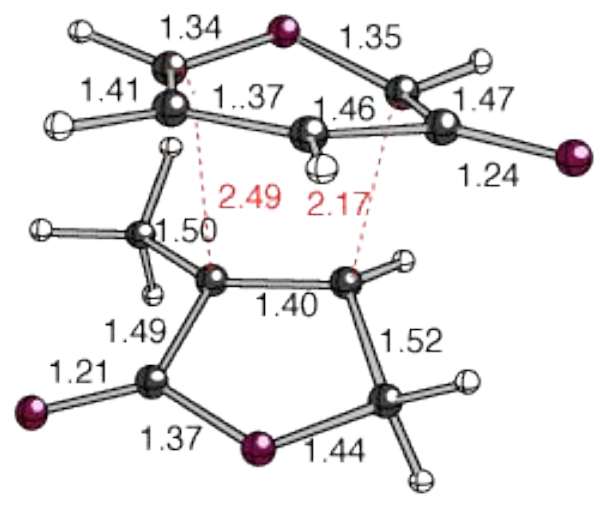

\section{E.II.TS1}

$\mathrm{HF}=-687.8831484$ hartrees $(-431653.554452484 \mathrm{kcal} / \mathrm{mol})$ Imaginary Frequencies: $1(-393.50791 / \mathrm{cm})$

Zero-point correction $=0.185224$ (Hartree/Particle)

Thermal correction to Energy $=\quad 0.196889$

Thermal correction to Enthalpy $=\quad 0.197834$

Thermal correction to Gibbs Free Energy $=\quad 0.147682$

Sum of electronic and zero-point Energies $=\quad-687.697924$

Sum of electronic and thermal Energies $=\quad-687.686259$

Sum of electronic and thermal Enthalpies $=\quad-687.685315$

Sum of electronic and thermal Free Energies $=\quad-687.735466$

Coordinates (from last standard orientation):

\begin{tabular}{ccccc}
\hline Center & Atomic & \multicolumn{3}{c}{ Coordinates } \\
Number & Number & X & $Y$ & $Z$ \\
- & & & \\
1 & 8 & 0.849547 & 1.841938 & -0.413465 \\
2 & 8 & 2.944765 & -1.037913 & -0.362026 \\
3 & 6 & 1.480839 & 0.741678 & -0.879654 \\
4 & 8 & -0.955478 & -1.862151 & 0.317976 \\
5 & 6 & 2.129949 & -0.201831 & 0.044956 \\
6 & 6 & 1.620569 & -0.109332 & 1.404521 \\
7 & 6 & 0.626704 & 0.786348 & 1.702707 \\
8 & 1 & 0.152903 & 0.806997 & 2.679207 \\
9 & 6 & 0.167653 & 1.696883 & 0.729692 \\
10 & 6 & -1.324486 & 0.228813 & -0.614691 \\
11 & 6 & -0.318933 & -0.370209 & -1.375178 \\
12 & 6 & -0.193051 & -1.802922 & -0.899504 \\
13 & 1 & 1.949966 & 0.922420 & -1.841181 \\
14 & 1 & -0.465811 & 2.545246 & 0.952838 \\
15 & 1 & 2.025352 & -0.786728 & 2.149532 \\
16 & 1 & -0.165764 & -0.142032 & -2.425601 \\
17 & 1 & -0.645212 & -2.488488 & -1.628508 \\
18 & 6 & -1.721631 & -0.729573 & 0.446264 \\
19 & 8 & -2.552175 & -0.600587 & 1.315551 \\
20 & 1 & 0.824517 & -2.141684 & -0.691320 \\
21 & 6 & -2.226693 & 1.362047 & -1.009783 \\
22 & 1 & -2.948115 & 1.030510 & -1.769730 \\
23 & 1 & -2.800875 & 1.712749 & -0.147593 \\
24 & 1 & -1.665757 & 2.199297 & -1.437930
\end{tabular}




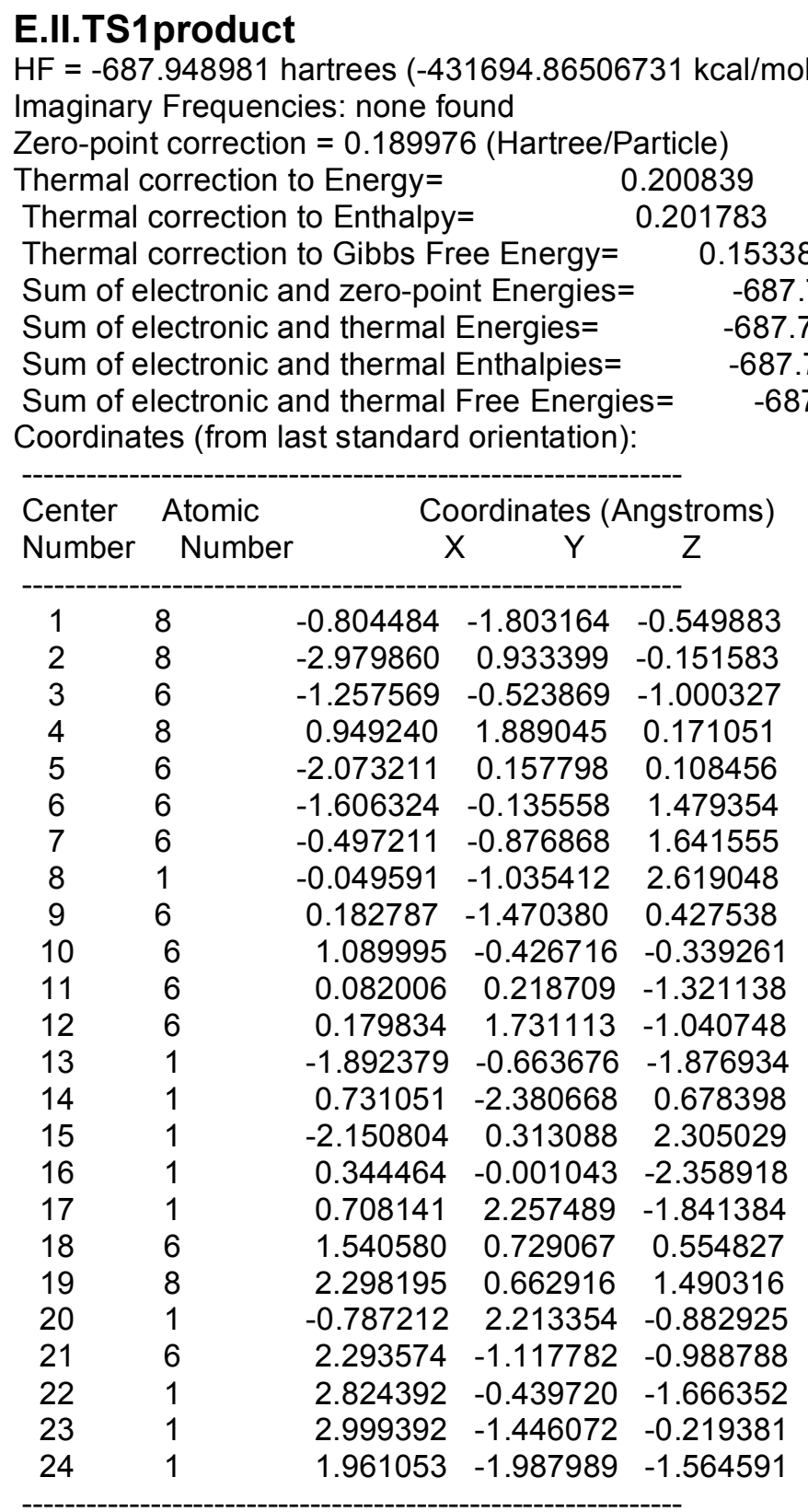

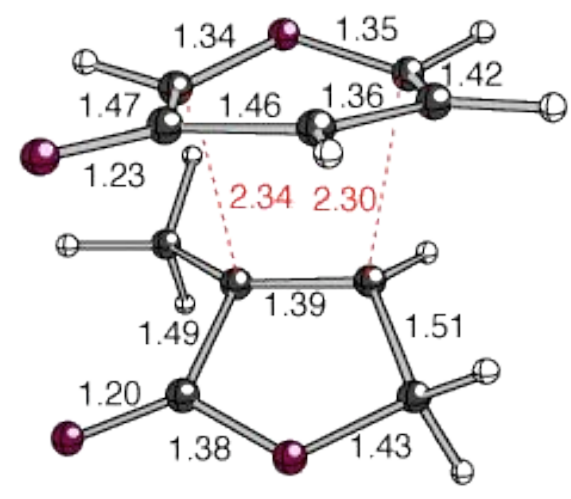




\begin{tabular}{|c|c|c|c|c|}
\hline \multicolumn{5}{|c|}{$\begin{array}{l}\text { E.II.TS2 } \\
\text { HF = -687.8726496 hartrees }(-431646.966350496 \mathrm{kcal} / \mathrm{n} \\
\text { Imaginary Frequencies: } 1(-414.57791 / \mathrm{cm}) \\
\text { Zero-point correction }=0.184784(\text { Hartree/Particle })\end{array}$} \\
\hline \multirow{2}{*}{\multicolumn{4}{|c|}{$\begin{array}{l}\text { Thermal correction to Energy= } \\
\text { Thermal correction to Enthalpy= }\end{array}$}} & 0.196575 \\
\hline & & & & 0.197519 \\
\hline \multicolumn{5}{|c|}{ Thermal correction to Gibbs Free Energy $=\quad 0.146$} \\
\hline \multicolumn{5}{|c|}{ Sum of electronic and zero-point Energies= } \\
\hline \multirow{2}{*}{\multicolumn{5}{|c|}{$\begin{array}{l}\text { Sum of electronic and thermal Energies= } \\
\text { Sum of electronic and thermal Enthalpies= }\end{array}$}} \\
\hline \multirow{3}{*}{\multicolumn{5}{|c|}{$\begin{array}{l}\text { Sum of electronic and thermal Enthalpies= } \\
\text { Sum of electronic and thermal Free Energies= } \\
\text { Coordinates (from last standard orientation): }\end{array}$}} \\
\hline & & & & \\
\hline & & & & \\
\hline \multirow{2}{*}{$\begin{array}{l}\text { Center } \\
\text { Number }\end{array}$} & \multirow{2}{*}{$\begin{array}{l}\text { Atomic } \\
\text { Number }\end{array}$} & \multicolumn{3}{|c|}{ Coordinates (Angstroms) } \\
\hline & & & $X \quad Y$ & Z \\
\hline 1 & 8 & & & \\
\hline 2 & 6 & -1.596162 & & \\
\hline 3 & 8 & 1.390494 & & -1. \\
\hline 4 & 6 & 33 & -0 . & \\
\hline 5 & 6 & -1.938309 & 0.554960 & -0.985719 \\
\hline 6 & 6 & -1.273199 & 1.342943 & 0.053085 \\
\hline 7 & 6 & -0.877921 & 0.539284 & 1.222031 \\
\hline 8 & 6 & & -0.4 & 0.583401 \\
\hline 9 & 6 & & & \\
\hline 10 & 6 & & & \\
\hline 11 & 1 & & & \\
\hline 12 & 1 & -0.606381 & 1.046001 & 2.141103 \\
\hline 13 & 1 & -2.313799 & 1.082455 & -1.856966 \\
\hline 14 & 1 & 0.662716 & -2.579546 & 0.250641 \\
\hline 15 & 1 & 1.664788 & -1.971320 & -1.998584 \\
\hline 16 & 6 & 1.66 & 0.515829 & -0.405131 \\
\hline 17 & 8 & & & \\
\hline 18 & 1 & & -1.3 & \\
\hline 19 & 1 & -2.5 & & 9606 \\
\hline 20 & 8 & -1.048101 & 2.550949 & -0.005504 \\
\hline 21 & 6 & 1.692906 & -0.483976 & 1.982282 \\
\hline 22 & 1 & 1.894531 & 0.534337 & 2.324924 \\
\hline 23 & 1 & 1.014622 & -0.976351 & 2.686045 \\
\hline 24 & 1 & 2.644768 & -1.031118 & 2.000345 \\
\hline
\end{tabular}

\section{E.II.TS2product}

$\mathrm{HF}=-687.944038$ hartrees $(-431691.76328538 \mathrm{kcal} / \mathrm{mol})$ Imaginary Frequencies: none found

Zero-point correction $=0.189687$ (Hartree/Particle)

Thermal correction to Energy $=\quad 0.200634$

Thermal correction to Enthalpy= $\quad 0.201578$

Thermal correction to Gibbs Free Energy $=\quad 0.152844$

Sum of electronic and zero-point Energies $=\quad-687.754351$

Sum of electronic and thermal Energies $=\quad-687.743404$

Sum of electronic and thermal Enthalpies $=\quad-687.742460$

Sum of electronic and thermal Free Energies $=\quad-687.791195$

Coordinates (from last standard orientation): 


\begin{tabular}{|c|c|c|c|c|}
\hline \multirow{2}{*}{$\begin{array}{l}\text { Center } \\
\text { Number }\end{array}$} & \multirow{2}{*}{$\begin{array}{l}\text { Atomic } \\
\text { Number }\end{array}$} & \multicolumn{3}{|c|}{ Coordinates (Angstroms) } \\
\hline & & & $\begin{array}{ll}X & Y\end{array}$ & Z \\
\hline 1 & 8 & -1.161803 & -0.863090 & 1.487668 \\
\hline 2 & 6 & -0.642967 & -1.719725 & 0.466630 \\
\hline 3 & 8 & 1.595361 & -0.074394 & -1.519150 \\
\hline 4 & 6 & -1.384984 & -1.471252 & -0.830942 \\
\hline 5 & 6 & -1.889002 & -0.254254 & -1.089511 \\
\hline 6 & 6 & -1.652975 & 0.847548 & -0.122343 \\
\hline 7 & 6 & -0.754529 & 0.441636 & 1.057092 \\
\hline 8 & 6 & 0.766806 & 0.237804 & 0.690171 \\
\hline 9 & 6 & 0.854507 & -1.283315 & 0.413856 \\
\hline 10 & 6 & 1.599825 & -1.386337 & -0.928773 \\
\hline 11 & 1 & -0.771397 & -2.751824 & 0.802829 \\
\hline 12 & 1 & -0.895676 & 1.144331 & 1.879418 \\
\hline 13 & 1 & -2.462284 & -0.024045 & -1.982873 \\
\hline 14 & 1 & 1.392993 & -1.807928 & 1.207363 \\
\hline 15 & 1 & 2.642221 & -1.691774 & -0.790748 \\
\hline 16 & 6 & 1.197460 & 0.881281 & -0.632439 \\
\hline 17 & 8 & 1.217473 & 2.054269 & -0.897801 \\
\hline 18 & 1 & 1.132980 & -2.069379 & -1.643731 \\
\hline 19 & 1 & -1.497323 & -2.293860 & -1.534193 \\
\hline 20 & 8 & -2.123206 & 1.962547 & -0.240904 \\
\hline 21 & 6 & 1.666777 & 0.745980 & 1.825657 \\
\hline 22 & 1 & 2.712984 & 0.463081 & 1.662773 \\
\hline 23 & 1 & 1.616561 & 1.837464 & 1.882411 \\
\hline 24 & 1 & 1.340829 & 0.323090 & 2.781864 \\
\hline
\end{tabular}

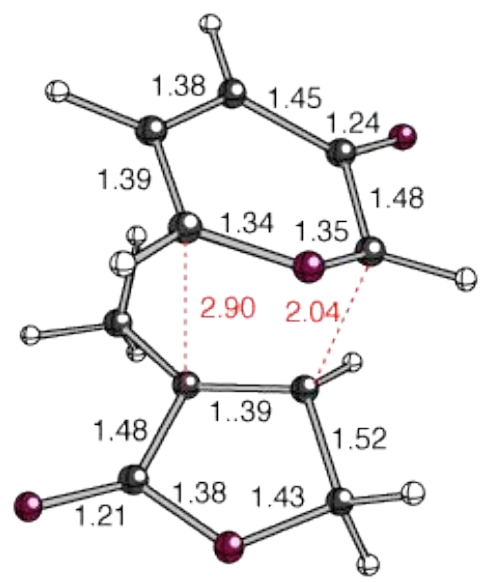

\section{E.II.TS3}

$\mathrm{HF}=-687.8840712$ hartrees $(-431654.133518712 \mathrm{kcal} / \mathrm{mol})$ Imaginary Frequencies: $1(-376.81861 / \mathrm{cm})$

Zero-point correction $=0.184664$ (Hartree/Particle)

Thermal correction to Energy $=\quad 0.196528$

Thermal correction to Enthalpy= $\quad 0.197472$

Thermal correction to Gibbs Free Energy $=\quad 0.146424$

Sum of electronic and zero-point Energies $=\quad-687.699408$

Sum of electronic and thermal Energies $=\quad-687.687543$

Sum of electronic and thermal Enthalpies $=\quad-687.686599$

Sum of electronic and thermal Free Energies $=\quad-687.737647$

Coordinates (from last standard orientation): 


\begin{tabular}{|c|c|c|c|c|}
\hline \multirow{2}{*}{$\begin{array}{l}\text { Center } \\
\text { Number }\end{array}$} & \multirow{2}{*}{$\begin{array}{l}\text { Atomic } \\
\text { Number }\end{array}$} & \multicolumn{3}{|c|}{ Coordinates (Angstroms) } \\
\hline & & & $\begin{array}{ll}X & Y\end{array}$ & Z \\
\hline 1 & 8 & 0.570456 & 0.454999 & -1.599660 \\
\hline 2 & 6 & 0.465530 & -0.877770 & -1.578054 \\
\hline 3 & 6 & 1.388190 & -1.640312 & -0.863041 \\
\hline 4 & 6 & 2.310169 & -1.043035 & -0.024084 \\
\hline 5 & 6 & 2.228131 & 0.379908 & 0.236151 \\
\hline 6 & 6 & 1.155622 & 1.054131 & -0.536039 \\
\hline 7 & 6 & -0.413177 & 1.062214 & 0.762944 \\
\hline 8 & 6 & -0.908469 & -0.233240 & 0.892478 \\
\hline 9 & 1 & -0.217049 & -1.261223 & -2.324359 \\
\hline 10 & 1 & 1.261630 & 2.122984 & -0.695539 \\
\hline 11 & 1 & 3.094218 & -1.612944 & 0.464473 \\
\hline 12 & 6 & -1.479640 & 1.849361 & 0.012563 \\
\hline 13 & 6 & -2.137105 & -0.353929 & 0.079970 \\
\hline 14 & 8 & -2.398547 & 0.873850 & -0.492751 \\
\hline 15 & 8 & -2.854836 & -1.311887 & -0.090916 \\
\hline 16 & 1 & -1.113495 & 2.435762 & -0.834347 \\
\hline 17 & 1 & 0.182866 & 1.541267 & 1.538385 \\
\hline 18 & 1 & -2.013742 & 2.522045 & 0.696341 \\
\hline 19 & 1 & 1.359708 & -2.718254 & -0.992078 \\
\hline 20 & 8 & 2.900106 & 1.019079 & 1.052302 \\
\hline 21 & 6 & -0.476837 & -1.297969 & 1.837106 \\
\hline 22 & 1 & -0.652858 & -0.993672 & 2.877533 \\
\hline 23 & 1 & -1.033847 & -2.219617 & 1.647201 \\
\hline 24 & 1 & 0.600651 & -1.500824 & 1.750626 \\
\hline
\end{tabular}

\section{E.II.TS3product}

$\mathrm{HF}=-687.9519247$ hartrees $(-431696.712268497 \mathrm{kcal} / \mathrm{mol})$ Imaginary Frequencies: none found Zero-point correction $=0.189814$ (Hartree/Particle)

Thermal correction to Energy $=\quad 0.200767$ Thermal correction to Enthalpy $=\quad 0.201712$ Thermal correction to Gibbs Free Energy $=0.153006$ Sum of electronic and zero-point Energies $=\quad-687.762111$ Sum of electronic and thermal Energies $=\quad-687.751157$ Sum of electronic and thermal Enthalpies $=\quad-687.750213$ Sum of electronic and thermal Free Energies $=\quad-687.798918$ Coordinates (from last standard orientation):

\begin{tabular}{|c|c|c|c|c|}
\hline \multirow{2}{*}{$\begin{array}{l}\text { Center } \\
\text { Number }\end{array}$} & \multirow{2}{*}{$\begin{array}{l}\text { Atomic } \\
\text { Number }\end{array}$} & & \multicolumn{2}{|c|}{ Coordinates (Angstroms) } \\
\hline & & & $\begin{array}{ll}X & Y\end{array}$ & Z \\
\hline 1 & 6 & 0.109694 & -1.107746 & -0.734926 \\
\hline 2 & 6 & 3013 & -1.779398 & -0.207642 \\
\hline 3 & 6 & 2.436476 & -1.036260 & 0.093933 \\
\hline 4 & 6 & 9098 & 0.437915 & -0.000569 \\
\hline 5 & 6 & 0.984717 & 0.942450 & -0.522105 \\
\hline 6 & 6 & -0.078283 & 0.902833 & 0.618390 \\
\hline 7 & 6 & -0.761194 & -0.473178 & 0.401177 \\
\hline 8 & 1 & -0.479001 & -1.774422 & -1.368934 \\
\hline 9 & 1 & 1.112288 & 1.926210 & -0.978761 \\
\hline 10 & 1 & 3.372725 & $5-1.458247$ & 0.448418 \\
\hline
\end{tabular}




$\begin{array}{rrrrr}11 & 6 & -1.227971 & 1.918945 & 0.449702 \\ 12 & 6 & -2.121970 & -0.108497 & -0.213250 \\ 13 & 8 & -2.329778 & 1.229700 & -0.167481 \\ 14 & 8 & -2.924323 & -0.879411 & -0.673825 \\ 15 & 1 & -0.965316 & 2.762163 & -0.194713 \\ 16 & 1 & 0.388820 & 1.011793 & 1.600681 \\ 17 & 1 & -1.570942 & 2.308694 & 1.413852 \\ 18 & 1 & 1.354806 & -2.859122 & -0.075157 \\ 19 & 8 & 3.215084 & 1.201500 & 0.368660 \\ 20 & 6 & -0.977902 & -1.349225 & 1.639424 \\ 21 & 1 & -1.575644 & -0.823474 & 2.391994 \\ 22 & 1 & -1.518124 & -2.261689 & 1.366965 \\ 23 & 1 & -0.022565 & -1.624359 & 2.096863 \\ 24 & 8 & 0.481378 & 0.036388 & -1.508106 \\ --------------------------------------------------\end{array}$

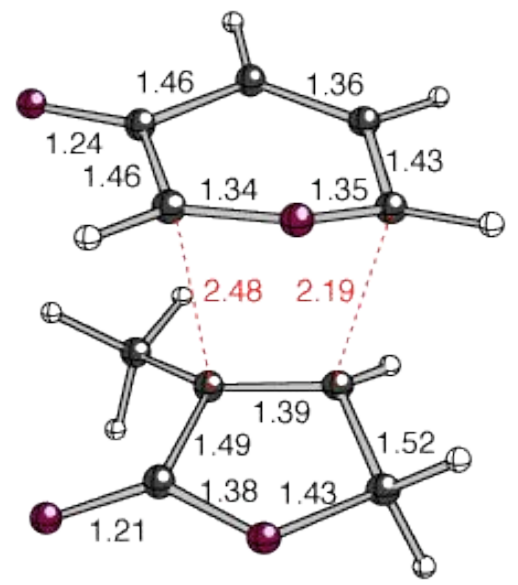

\section{E.II.TS4}

$\mathrm{HF}=-687.8781836$ hartrees $(-431650.438990836 \mathrm{kcal} / \mathrm{mol})$ Imaginary Frequencies: $1(-414.67081 / \mathrm{cm})$

Zero-point correction $=0.184671($ Hartree/Particle $)$

Thermal correction to Energy $=\quad 0.196572$

Thermal correction to Enthalpy= $\quad 0.197516$

Thermal correction to Gibbs Free Energy $=\quad 0.146607$

Sum of electronic and zero-point Energies $=\quad-687.693512$

Sum of electronic and thermal Energies $=\quad-687.681612$

Sum of electronic and thermal Enthalpies $=\quad-687.680668$

Sum of electronic and thermal Free Energies $=\quad-687.731577$

Coordinates (from last standard orientation):

\begin{tabular}{llrrr} 
Center & Atomic & \multicolumn{3}{c}{ Coordinates (Angstroms) } \\
Number & Number & X & $Y$ & Z \\
- \hdashline 1 & 8 & -0.492197 & -1.026687 & -1.290133 \\
2 & 8 & -2.288952 & 1.929342 & -0.448142 \\
3 & 6 & -0.777694 & 0.271756 & -1.174946 \\
4 & 6 & -1.927470 & 0.749153 & -0.409514 \\
5 & 6 & -2.550734 & -0.292216 & 0.408177 \\
6 & 6 & -1.965731 & -1.517443 & 0.511751 \\
7 & 1 & -2.355254 & -2.285286 & 1.175061 \\
8 & 6 & -0.782376 & -1.812679 & -0.229666 \\
9 & 6 & 0.834468 & -0.966271 & 0.987805
\end{tabular}




$\begin{array}{rrrrr}10 & 6 & 0.762661 & 0.407581 & 0.763152 \\ 11 & 1 & -0.352572 & 0.878193 & -1.966780 \\ 12 & 1 & -0.459617 & -2.834931 & -0.391892 \\ 13 & 1 & -3.461127 & -0.036880 & 0.942013 \\ 14 & 6 & 2.051949 & -1.482943 & 0.240506 \\ 15 & 6 & 1.853638 & 0.767049 & -0.186387 \\ 16 & 8 & 2.511435 & -0.385311 & -0.555658 \\ 17 & 8 & 2.179073 & 1.853872 & -0.596098 \\ 18 & 1 & 1.869929 & -2.328661 & -0.429495 \\ 19 & 1 & 0.522501 & -1.433081 & 1.915724 \\ 20 & 1 & 2.847248 & -1.769275 & 0.942587 \\ 21 & 6 & 0.171409 & 1.472588 & 1.641106 \\ 22 & 1 & 0.963429 & 1.923972 & 2.252887 \\ 23 & 1 & -0.283616 & 2.275782 & 1.054844 \\ 24 & 1 & -0.586506 & 1.060998 & 2.313391 \\ ------------------------------------------------------\end{array}$

\section{E.II.TS4product}

$\mathrm{HF}=-687.9506544$ hartrees $(-431695.915142544 \mathrm{kcal} / \mathrm{mol})$

Imaginary Frequencies: none found

Zero-point correction $=0.189762($ Hartree/Particle $)$

Thermal correction to Energy $=\quad 0.200773$

Thermal correction to Enthalpy $=\quad 0.201717$

Thermal correction to Gibbs Free Energy $=0.152793$

Sum of electronic and zero-point Energies $=\quad-687.760892$

Sum of electronic and thermal Energies $=\quad-687.749881$

Sum of electronic and thermal Enthalpies $=\quad-687.748937$

Sum of electronic and thermal Free Energies $=\quad-687.797862$

Coordinates (from last standard orientation):

\begin{tabular}{|c|c|c|c|c|}
\hline \multirow{2}{*}{$\begin{array}{l}\text { Center } \\
\text { Number }\end{array}$} & \multirow{2}{*}{$\begin{array}{l}\text { Atomic } \\
\text { Number }\end{array}$} & \multicolumn{3}{|c|}{ Coordinates (Angstroms) } \\
\hline & & & $X \quad Y$ & Z \\
\hline 1 & 8 & 0.458455 & 0.719477 & -1.412267 \\
\hline 2 & 8 & 2.516445 & -1.836985 & -0.168562 \\
\hline 3 & 6 & 0.559549 & -0.550849 & -0.762701 \\
\hline 4 & 6 & 1.986613 & -0.740778 & -0.227390 \\
\hline 5 & 6 & 2.619890 & 0.501674 & 0.272880 \\
\hline 6 & 6 & 1.907000 & 1.639317 & 0.270672 \\
\hline 7 & 1 & 2.292234 & 2.559126 & 0.706652 \\
\hline 8 & 6 & 0.515136 & 1.644653 & -0.322848 \\
\hline 9 & 6 & -0.527017 & 1.080205 & 0.692115 \\
\hline 10 & 6 & -0.498940 & $0 \quad-0.442740$ & 0.387917 \\
\hline 11 & 1 & 0.320325 & $5-1.342815$ & -1.474152 \\
\hline 12 & 1 & 0.249381 & $1 \quad 2.631967$ & -0.710888 \\
\hline 13 & 1 & 3.630322 & 0.428678 & 0.665169 \\
\hline 14 & 6 & -1.982895 & 1.506865 & 0.397278 \\
\hline 15 & 6 & -1.876914 & $4-0.719023$ & -0.234829 \\
\hline 16 & 8 & -2.660402 & $2 \quad 0.387122$ & -0.194108 \\
\hline 17 & 8 & -2.253625 & $5-1.764295$ & -0.696960 \\
\hline 18 & 1 & -2.045579 & $9 \quad 2.339497$ & -0.310008 \\
\hline 19 & 1 & -0.252257 & 1.314423 & 1.723288 \\
\hline 20 & 1 & -2.523169 & 1.782148 & 1.308533 \\
\hline 21 & 6 & -0.280358 & $8 \quad-1.393549$ & 1.570850 \\
\hline 22 & 1 & -1.073576 & $6-1.273330$ & 2.316648 \\
\hline 23 & 1 & -0.296266 & $6-2.430902$ & 1.224931 \\
\hline
\end{tabular}




\begin{tabular}{|c|c|c|c|c|}
\hline \multicolumn{5}{|c|}{$\begin{array}{l}\mathrm{HF}=-687.8848436 \text { hartrees }(-431654.618207436 \mathrm{kcal} / \\
\text { Imaginary Frequencies: } 1(-383.86621 / \mathrm{cm}) \\
\text { Zero-point correction }=0.185146(\text { Hartree/Particle })\end{array}$} \\
\hline \multirow{2}{*}{\multicolumn{3}{|c|}{$\begin{array}{l}\text { Thermal correction to Energy= } \\
\text { Thermal correction to Enthalpy= }\end{array}$}} & & $\begin{array}{l}\text { Particle) } \\
0.196705\end{array}$ \\
\hline & & & & 0.197649 \\
\hline \multirow{2}{*}{\multicolumn{5}{|c|}{$\begin{array}{l}\text { Thermal correction to Gibbs Free Energy= } \\
\text { Sum of electronic and zero-point Energies= }\end{array}$}} \\
\hline & & & & \\
\hline \multicolumn{5}{|c|}{ Sum of electronic and thermal Energies= } \\
\hline \multicolumn{5}{|c|}{ Sum of electronic and thermal Enthalpies= } \\
\hline \multirow{2}{*}{\multicolumn{5}{|c|}{$\begin{array}{l}\text { Sum of electronic and thermal Free Energies= } \\
\text { Coordinates (from last standard orientation): }\end{array}$}} \\
\hline & & & & \\
\hline \multirow{2}{*}{$\begin{array}{l}\text { Center } \\
\text { Number }\end{array}$} & Atomic & \multicolumn{3}{|c|}{ Coordinates (Angstroms) } \\
\hline & Number & & $X \quad Y$ & Z \\
\hline 1 & 8 & -1.860553 & & \\
\hline 2 & 8 & -1.435804 & 43 & \\
\hline 3 & 6 & -1.483429 & $-0 . \varepsilon$ & \\
\hline 4 & 8 & 1.689457 & & \\
\hline 5 & 6 & -1.546740 & -1.136967 & -0.5 \\
\hline 6 & 6 & -1.645168 & 0.066484 & -1.359322 \\
\hline 7 & 6 & -1.611148 & 9195 & -0.766950 \\
\hline 8 & 1 & 1330 & 257 & 0992 \\
\hline 9 & 6 & -1.5 & & \\
\hline 10 & 6 & 59 & & \\
\hline 11 & 1 & 3789 & & $1 . \varepsilon$ \\
\hline 12 & 6 & 0.685308 & 5139 & 1.123228 \\
\hline 13 & 6 & 1.442329 & -1.022313 & -0.060087 \\
\hline 14 & 1 & -1.669138 & -1.666037 & 1.594192 \\
\hline 15 & 1 & -1.764421 & 2.344440 & 1.166767 \\
\hline 16 & 1 & -1.705218 & -0.052006 & -2.436422 \\
\hline 17 & 1 & & & \\
\hline 18 & 6 & $1.3 \varsigma$ & 972 & -0.2 \\
\hline 19 & 8 & 1.633464 & 8671 & -0.725081 \\
\hline 20 & 6 & 2.771375 & -1.663000 & 0.337792 \\
\hline 21 & 1 & 2.592926 & -2.556756 & 0.946455 \\
\hline 22 & 1 & 3.323041 & -1.961184 & -0.558611 \\
\hline 23 & 1 & 3.387807 & -0.963245 & 0.911754 \\
\hline 24 & 1 & 0.851515 & -1.733173 & -0.645664 \\
\hline
\end{tabular}

\section{E.III.TS1 product (Me down)}

$\mathrm{HF}=-687.9524545$ hartrees $(-431697.044723295 \mathrm{kcal} / \mathrm{mol})$

Imaginary Frequencies: none found

Zero-point correction $=0.190090($ Hartree/Particle $)$

Thermal correction to Energy $=\quad 0.200871$

Thermal correction to Enthalpy= $\quad 0.201815$

Thermal correction to Gibbs Free Energy $=0.153209$

Sum of electronic and zero-point Energies $=\quad-687.762364$

Sum of electronic and thermal Energies $=\quad-687.751584$

Sum of electronic and thermal Enthalpies $=\quad-687.750639$ 
Sum of electronic and thermal Free Energies=

$-687.799245$

Coordinates (from last standard orientation):

\begin{tabular}{|c|c|c|c|c|}
\hline \multirow{2}{*}{$\begin{array}{l}\text { Center } \\
\text { Number }\end{array}$} & \multirow{2}{*}{$\begin{array}{l}\text { Atomic } \\
\text { Number }\end{array}$} & \multicolumn{3}{|c|}{ Coordinates (Angstroms) } \\
\hline & & & $\begin{array}{ll}X \quad Y \\
\end{array}$ & Z \\
\hline 1 & 8 & -1.649173 & 0.216978 & 1.530205 \\
\hline 2 & 8 & -1.714084 & -2.089640 & -1.124680 \\
\hline 3 & 6 & -1.073830 & -0.922031 & 0.885117 \\
\hline 4 & 8 & 1.699596 & 0.123707 & -0.946072 \\
\hline 5 & 6 & -1.592896 & -1.015023 & -0.557543 \\
\hline 6 & 6 & -1.840330 & 0.290504 & -1.205380 \\
\hline 7 & 6 & -1.524170 & 1.416230 & -0.543287 \\
\hline 8 & 1 & -1.585912 & 2.395616 & -1.010037 \\
\hline 9 & 6 & -1.013925 & 1.317948 & 0.878429 \\
\hline 10 & 6 & 0.506799 & 0.916193 & 0.930617 \\
\hline 11 & 1 & 0.979370 & 1.370803 & 1.804479 \\
\hline 12 & 6 & 0.462052 & -0.622591 & 0.969717 \\
\hline 13 & 6 & 1.384312 & -1.077313 & -0.188580 \\
\hline 14 & 1 & -1.367413 & -1.820537 & 1.430422 \\
\hline 15 & 1 & -1.230010 & 2.221933 & 1.449804 \\
\hline 16 & 1 & -2.219561 & 0.283548 & -2.223021 \\
\hline 17 & 1 & 0.824163 & -1.013468 & 1.923611 \\
\hline 18 & 6 & 1.299235 & 1.261366 & -0.323620 \\
\hline 19 & 8 & 1.532982 & 2.360987 & -0.757327 \\
\hline 20 & 6 & 2.684031 & -1.720792 & 0.274564 \\
\hline 21 & 1 & 2.474152 & -2.671325 & 0.778143 \\
\hline 22 & 1 & 3.337256 & -1.918761 & -0.580183 \\
\hline 23 & 1 & 3.219066 & -1.067999 & 0.973782 \\
\hline 24 & 1 & 0.866654 & -1.743025 & -0.884208 \\
\hline
\end{tabular}

\section{E.III.TS2 (Me down)}

$\mathrm{HF}=-687.8701209$ hartrees $(-431645.379565959 \mathrm{kcal} / \mathrm{mol})$

Imaginary Frequencies: $1(-399.99971 / \mathrm{cm})$

Zero-point correction $=0.184720$ (Hartree/Particle)

Thermal correction to Energy $=\quad 0.196419$

Thermal correction to Enthalpy $=\quad 0.197364$

Thermal correction to Gibbs Free Energy $=\quad 0.146447$

Sum of electronic and zero-point Energies $=\quad-687.685400$

Sum of electronic and thermal Energies $=\quad-687.673702$

Sum of electronic and thermal Enthalpies $=\quad-687.672757$

Sum of electronic and thermal Free Energies $=\quad-687.723673$

Coordinates (from last standard orientation):

\begin{tabular}{|c|c|c|c|c|}
\hline \multirow{2}{*}{$\begin{array}{l}\text { Center } \\
\text { Number }\end{array}$} & \multirow{2}{*}{$\begin{array}{l}\text { Atomic } \\
\text { Number }\end{array}$} & & \multicolumn{2}{|c|}{ Coordinates (Angstroms) } \\
\hline & & & $\begin{array}{ll}X \quad Y\end{array}$ & Z \\
\hline 1 & 8 & 1.363779 & -1.288810 & 1.276078 \\
\hline 2 & 8 & 2.624986 & 1.273450 & -0.839077 \\
\hline 3 & 6 & 1.758233 & -0.067377 & 0.901774 \\
\hline 4 & 8 & -1.793227 & 1.061207 & -0.548276 \\
\hline 5 & 6 & 1.974508 & 0.282580 & -0.513653 \\
\hline 6 & 6 & 1.357447 & -0.674723 & -1.427855 \\
\hline 7 & 6 & 0.632482 & -1.722226 & -0.937386 \\
\hline 8 & 1 & 0.142834 & -2.429429 & -1.600080 \\
\hline
\end{tabular}




$\begin{array}{ccccc}9 & 6 & 0.500098 & -1.929330 & 0.462945 \\ 10 & 6 & -1.165856 & -0.427454 & 1.145479 \\ 11 & 1 & -1.376729 & -1.104663 & 1.965784 \\ 12 & 6 & -0.383235 & 0.712890 & 1.258772 \\ 13 & 6 & -0.790603 & 1.662298 & 0.188226 \\ 14 & 1 & 2.353092 & 0.430877 & 1.660015 \\ 15 & 1 & 0.174881 & -2.874348 & 0.880345 \\ 16 & 1 & 1.506719 & -0.526839 & -2.492760 \\ 17 & 1 & 0.070435 & 1.092992 & 2.163784 \\ 18 & 6 & -2.242008 & -0.136284 & 0.111490 \\ 19 & 6 & -2.638790 & -1.182386 & -0.919024 \\ 20 & 1 & -3.492573 & -0.823078 & -1.501418 \\ 21 & 1 & -1.819767 & -1.389704 & -1.608080 \\ 22 & 1 & -2.932633 & -2.112300 & -0.418673 \\ 23 & 8 & -0.382012 & 2.766664 & -0.058110 \\ 24 & 1 & -3.148139 & 0.128477 & 0.681563 \\ ------------------------------------------\end{array}$

\section{E.III.TS2product (Me down)}

$\mathrm{HF}=-687.9447879$ hartrees $(-431692.233855129 \mathrm{kcal} / \mathrm{mol})$

Imaginary Frequencies: none found

Zero-point correction $=0.189922$ (Hartree/Particle)

Thermal correction to Energy $=\quad 0.200697$

Thermal correction to Enthalpy= $\quad 0.201641$

Thermal correction to Gibbs Free Energy $=\quad 0.153323$

Sum of electronic and zero-point Energies $=\quad-687.754866$

Sum of electronic and thermal Energies $=\quad-687.744091$

Sum of electronic and thermal Enthalpies $=\quad-687.743147$

Sum of electronic and thermal Free Energies $=\quad-687.791465$

Coordinates (from last standard orientation):

\begin{tabular}{|c|c|c|c|c|}
\hline \multirow{2}{*}{$\begin{array}{l}\text { Center } \\
\text { Number }\end{array}$} & \multirow{2}{*}{$\begin{array}{l}\text { Atomic } \\
\text { Number }\end{array}$} & \multicolumn{3}{|c|}{ Coordinates (Angstroms) } \\
\hline & & & $X \quad Y$ & Z \\
\hline 1 & 8 & -0.977352 & -1.638122 & -1.053492 \\
\hline 2 & 8 & -3.067924 & 0.570436 & 0.528764 \\
\hline 3 & 6 & -1.377180 & -0.259274 & -1.001558 \\
\hline 4 & 8 & 1.521121 & 1.350841 & 0.383510 \\
\hline 5 & 6 & -2.023641 & -0.030765 & 0.373538 \\
\hline 6 & 6 & -1.244423 & -0.644849 & 1.478834 \\
\hline 7 & 6 & -0.162730 & -1.386769 & 1.190029 \\
\hline 8 & 1 & 0.444866 & -1.825135 & 1.978078 \\
\hline 9 & 6 & 0.209439 & -1.668738 & -0.252142 \\
\hline 10 & 6 & 1.053522 & $2-0.557505$ & -0.961860 \\
\hline 11 & 1 & 1.427772 & -0.992828 & -1.892046 \\
\hline 12 & 6 & -0.025925 & $5 \quad 0.513692$ & -1.202669 \\
\hline 13 & 6 & 0.307047 & 1.606868 & -0.183932 \\
\hline 14 & 1 & -2.105783 & -0.075230 & -1.791081 \\
\hline 15 & 1 & 0.653720 & -2.661930 & -0.355344 \\
\hline 16 & 1 & -1.583246 & $6-0.462221$ & 2.494379 \\
\hline 17 & 1 & 0.008192 & 20.952932 & -2.203940 \\
\hline 18 & 6 & 2.173840 & 0.219601 & -0.244865 \\
\hline 19 & 6 & 3.027495 & $5-0.504985$ & 0.782889 \\
\hline 20 & 1 & 3.790720 & 0.171099 & 1.179540 \\
\hline 21 & 1 & 2.429202 & $2-0.866804$ & 1.622305 \\
\hline 22 & 1 & 3.535229 & 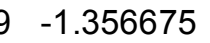 & 0.315994 \\
\hline
\end{tabular}




$\begin{array}{rrrrr}23 & 8 & -0.358499 & 2.562032 & 0.116336 \\ 24 & 1 & 2.835899 & 0.631652 & -1.018414\end{array}$

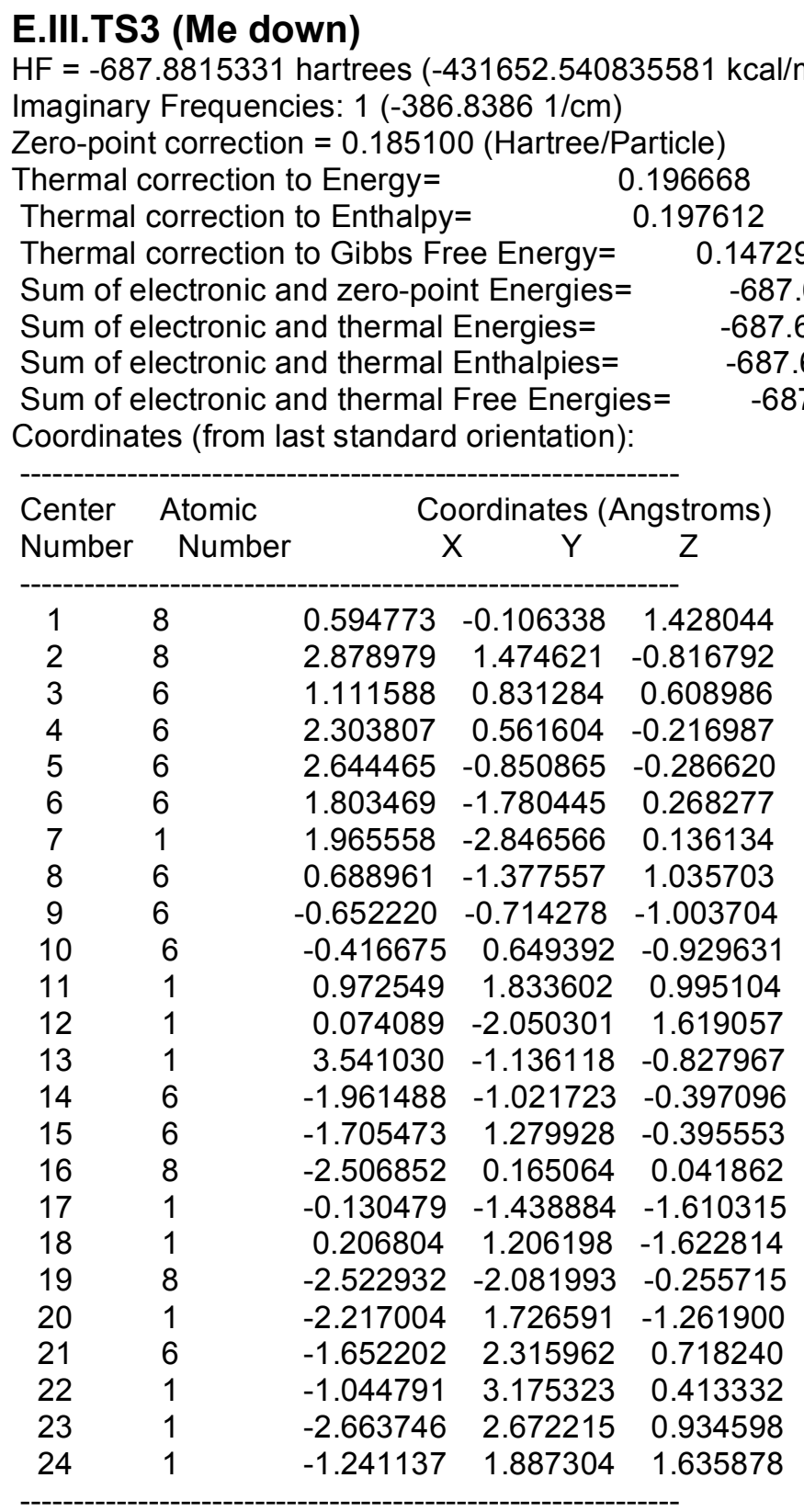

\section{E.III.TS3product (Me down)}

$\mathrm{HF}=-687.955253$ hartrees $(-431698.80081003 \mathrm{kcal} / \mathrm{mol})$

Imaginary Frequencies: none found

Zero-point correction $=0.190169$ (Hartree/Particle)

Thermal correction to Energy $=\quad 0.200958$

Thermal correction to Enthalpy $=\quad 0.201902$

Thermal correction to Gibbs Free Energy $=\quad 0.152988$

Sum of electronic and zero-point Energies $=\quad-687.765084$

Sum of electronic and thermal Energies $=\quad-687.754295$ 
Sum of electronic and thermal Enthalpies=

$-687.753351$

Sum of electronic and thermal Free Energies=

$-687.802265$

Coordinates (from last standard orientation):

\begin{tabular}{|c|c|c|c|c|}
\hline \multirow{2}{*}{$\begin{array}{l}\text { Center } \\
\text { Number }\end{array}$} & \multirow{2}{*}{$\begin{array}{l}\text { Atomic } \\
\text { Number }\end{array}$} & & \multicolumn{2}{|c|}{ Coordinates (Angstroms) } \\
\hline & & & $X \quad Y$ & Z \\
\hline 1 & 8 & 0.527844 & -0.345130 & 1.357842 \\
\hline 2 & 8 & 2.967475 & 1.570595 & -0.298712 \\
\hline 3 & 6 & 0.838711 & 0.748051 & 0.489412 \\
\hline 4 & 6 & 2.275800 & 0.601041 & -0.036431 \\
\hline 5 & 6 & 2.693122 & -0.798585 & -0.277959 \\
\hline 6 & 6 & 1.800800 & -1.784443 & -0.085872 \\
\hline 7 & 1 & 2.026577 & -2.819338 & -0.334982 \\
\hline 8 & 6 & 0.430486 & -1.455076 & 0.461889 \\
\hline 9 & 6 & -0.505316 & -0.902572 & -0.654235 \\
\hline 10 & 6 & -0.167474 & $4 \quad 0.604626$ & -0.694821 \\
\hline 11 & 1 & 0.756080 & 1.678326 & 1.050832 \\
\hline 12 & 1 & -0.016783 & $3-2.294391$ & 0.998160 \\
\hline 13 & 1 & 3.699152 & $2-0.967287$ & -0.651542 \\
\hline 14 & 6 & -1.961447 & $7 \quad-0.971711$ & -0.198450 \\
\hline 15 & 6 & -1.553670 & $0 \quad 1.299426$ & -0.535746 \\
\hline 16 & 8 & -2.488980 & 0.271744 & -0.120984 \\
\hline 17 & 1 & -0.394246 & $6-1.434420$ & -1.602467 \\
\hline 18 & 1 & 0.293205 & $5 \quad 0.929682$ & -1.630726 \\
\hline 19 & 8 & -2.578637 & $7-1.966886$ & 0.082399 \\
\hline 20 & 1 & -1.886749 & $9 \quad 1.632864$ & -1.525869 \\
\hline 21 & 6 & -1.656238 & 2.456512 & 0.443915 \\
\hline 22 & 1 & -0.970995 & 3.262669 & 0.159379 \\
\hline 23 & 1 & -2.675039 & 2.854937 & 0.441069 \\
\hline 24 & 1 & -1.421460 & 2.130747 & 1.461579 \\
\hline
\end{tabular}

\section{E.III.TS4 (Me down)}

$\mathrm{HF}=-687.8803609$ hartrees $(-431651.805268359 \mathrm{kcal} / \mathrm{mol})$

Imaginary Frequencies: $1(-398.65181 / \mathrm{cm})$

Zero-point correction $=0.184719$ (Hartree/Particle)

Thermal correction to Energy $=\quad 0.196463$

Thermal correction to Enthalpy= $\quad 0.197407$

Thermal correction to Gibbs Free Energy $=\quad 0.146433$

Sum of electronic and zero-point Energies $=\quad-687.695641$

Sum of electronic and thermal Energies $=\quad-687.683898$

Sum of electronic and thermal Enthalpies $=\quad-687.682954$

Sum of electronic and thermal Free Energies $=\quad-687.733928$

Coordinates (from last standard orientation):

\begin{tabular}{|c|c|c|c|c|}
\hline \multirow{2}{*}{$\begin{array}{l}\text { Center } \\
\text { Number }\end{array}$} & \multirow{2}{*}{$\begin{array}{l}\text { Atomic } \\
\text { Number }\end{array}$} & \multicolumn{3}{|c|}{ Coordinates (Angstroms) } \\
\hline & & & $\begin{array}{ll}X & Y\end{array}$ & Z \\
\hline 1 & 8 & 0.801861 & $1-0.343365$ & 1.448187 \\
\hline 2 & 8 & 2.989490 & $0 \quad 1.469359$ & -0.701022 \\
\hline 3 & 6 & 1.293538 & 0.682334 & 0.746904 \\
\hline 4 & 6 & 2.391759 & 0.504981 & -0.220566 \\
\hline 5 & 6 & 2.637608 & $8-0.894156$ & -0.555237 \\
\hline 6 & 6 & 1.815735 & $5-1.868265$ & -0.064300 \\
\hline 7 & 1 & 1.920168 & $3-2.907333$ & -0.365598 \\
\hline
\end{tabular}




$\begin{array}{ccrrr}8 & 6 & 0.772790 & -1.543658 & 0.841181 \\ 9 & 6 & -0.818538 & -0.761092 & -0.698181 \\ 10 & 6 & -0.404014 & 0.556317 & -0.793310 \\ 11 & 1 & 1.152870 & 1.640364 & 1.235316 \\ 12 & 1 & 0.258994 & -2.289213 & 1.435657 \\ 13 & 1 & 3.462759 & -1.118096 & -1.224183 \\ 14 & 6 & -2.100037 & -0.794810 & 0.117558 \\ 15 & 6 & -1.357601 & 1.397458 & -0.016282 \\ 16 & 8 & -2.270996 & 0.559507 & 0.580496 \\ 17 & 8 & -1.389506 & 2.593074 & 0.129310 \\ 18 & 1 & -2.028631 & -1.420433 & 1.014232 \\ 19 & 6 & -3.320733 & -1.217503 & -0.699814 \\ 20 & 1 & -3.223239 & -2.262104 & -1.017365 \\ 21 & 1 & -4.228263 & -1.120745 & -0.096351 \\ 22 & 1 & -3.424094 & -0.588816 & -1.589899 \\ 23 & 1 & -0.599547 & -1.534803 & -1.423274 \\ 24 & 1 & 0.199148 & 1.002928 & -1.572029 \\ -----------------------------------------\end{array}$

\section{E.III.TS4product (Me down)}

$\mathrm{HF}=-687.9558125$ hartrees $(-431699.151901875 \mathrm{kcal} / \mathrm{mol})$

Imaginary Frequencies: none found

Zero-point correction $=0.189800$ (Hartree/Particle)

Thermal correction to Energy $=\quad 0.200722$

Thermal correction to Enthalpy $=\quad 0.201666$

Thermal correction to Gibbs Free Energy $=\quad 0.152383$

Sum of electronic and zero-point Energies $=\quad-687.766012$

Sum of electronic and thermal Energies $=\quad-687.755091$

Sum of electronic and thermal Enthalpies $=\quad-687.754147$

Sum of electronic and thermal Free Energies $=\quad-687.803430$

Coordinates (from last standard orientation):

\begin{tabular}{|c|c|c|c|c|}
\hline \multirow{2}{*}{$\begin{array}{l}\text { Center } \\
\text { Number }\end{array}$} & \multirow{2}{*}{$\begin{array}{l}\text { Atomic } \\
\text { Number }\end{array}$} & \multicolumn{3}{|c|}{ Coordinates (Angstroms) } \\
\hline & & & $\begin{array}{l}X \\
Y\end{array}$ & Z \\
\hline 1 & 8 & 0.731764 & -0.330517 & 1.443918 \\
\hline 2 & 8 & 3.132690 & 1.348093 & -0.487396 \\
\hline 3 & 6 & 1.027289 & 0.727138 & 0.526880 \\
\hline 4 & 6 & 2.375931 & 0.452622 & -0.157435 \\
\hline 5 & 6 & 2.621980 & -0.979691 & -0.448808 \\
\hline 6 & 6 & 1.678451 & -1.881534 & -0.133698 \\
\hline 7 & 1 & 1.777525 & -2.931617 & -0.401922 \\
\hline 8 & 6 & 0.425840 & -1.433430 & 0.585710 \\
\hline 9 & 6 & -0.609646 & -0.824015 & -0.404522 \\
\hline 10 & 6 & -0.133683 & 0.639730 & -0.514062 \\
\hline 11 & 1 & 1.059813 & 1.676585 & 1.063238 \\
\hline 12 & 1 & 0.011367 & -2.232532 & 1.206952 \\
\hline 13 & 1 & 3.551188 & -1.237558 & -0.949013 \\
\hline 14 & 6 & -2.038689 & -0.723624 & 0.197084 \\
\hline 15 & 6 & -1.333404 & 1.483997 & -0.088530 \\
\hline 16 & 8 & -2.375630 & 0.682381 & 0.247636 \\
\hline 17 & 8 & -1.391190 & 2.684285 & -0.040751 \\
\hline 18 & 1 & -2.035027 & -1.070535 & 1.236885 \\
\hline 19 & 6 & -3.109295 & -1.455313 & -0.596482 \\
\hline 20 & 1 & -2.907287 & -2.532801 & -0.598408 \\
\hline 21 & 1 & -4.096820 & -1.290402 & -0.155632 \\
\hline
\end{tabular}




$\begin{array}{rrrrr}22 & 1 & -3.131016 & -1.103955 & -1.633962 \\ 23 & 1 & -0.625313 & -1.347605 & -1.363897 \\ 24 & 1 & 0.185861 & 0.961206 & -1.508336\end{array}$

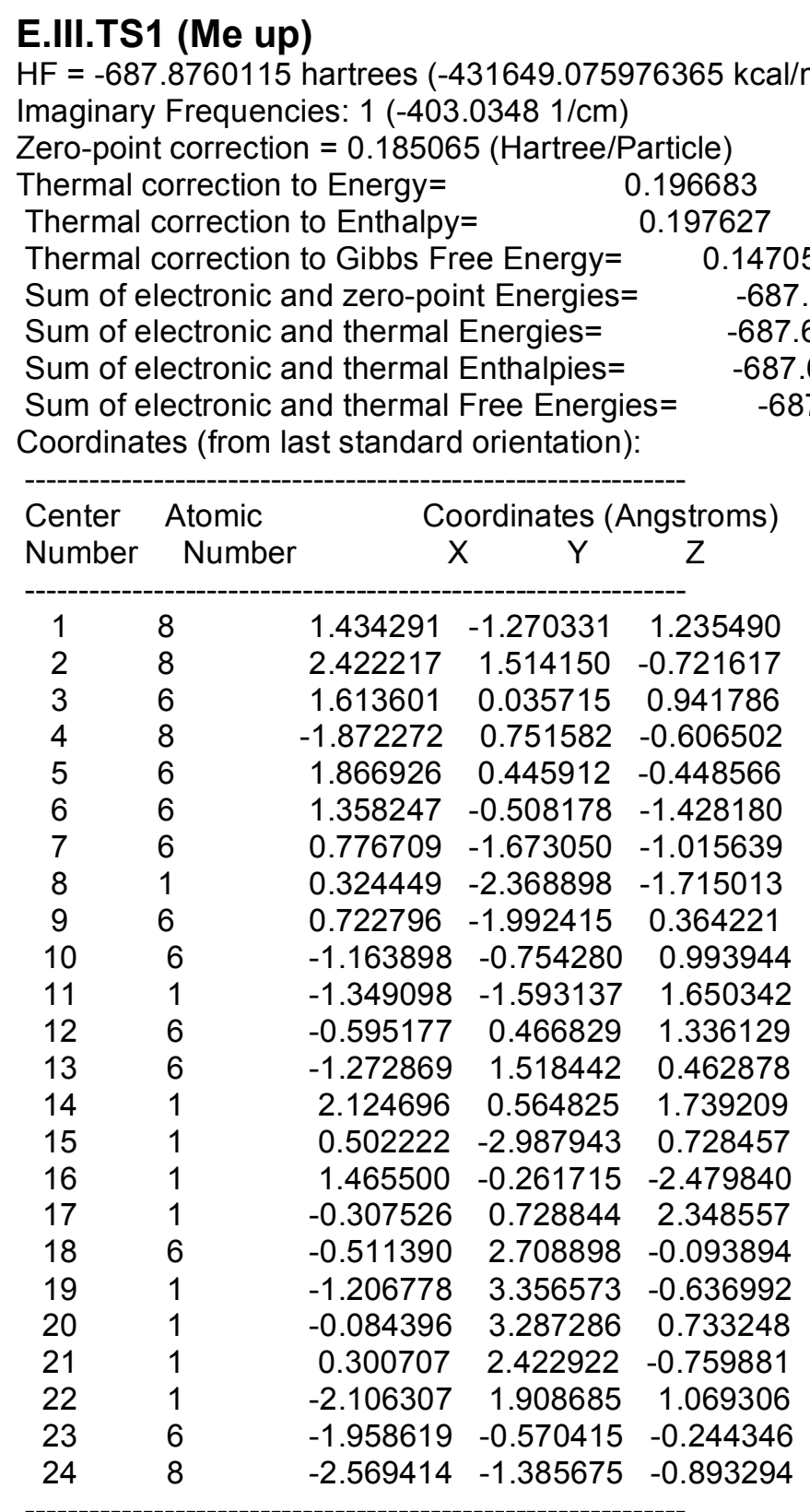

\section{E.III.TS1product (Me up)}

$\mathrm{HF}=-687.9475736$ hartrees $(-431693.981909736 \mathrm{kcal} / \mathrm{mol})$

Imaginary Frequencies: none found

Zero-point correction $=0.190164$ (Hartree/Particle)

Thermal correction to Energy $=\quad 0.200863$

Thermal correction to Enthalpy= $\quad 0.201807$

Thermal correction to Gibbs Free Energy $=\quad 0.153777$

Sum of electronic and zero-point Energies $=\quad-687.757410$ 


\begin{tabular}{|c|c|c|c|c|}
\hline \multirow{2}{*}{$\begin{array}{l}\text { Center } \\
\text { Number }\end{array}$} & \multirow{2}{*}{$\begin{array}{l}\text { Atomic } \\
\text { Number }\end{array}$} & \multicolumn{3}{|c|}{ Coordinates (Angstroms) } \\
\hline & & & X & Z \\
\hline 1 & 8 & -1.670432 & -0.468998 & 1.400380 \\
\hline 2 & 8 & -0.291273 & -2.537517 & -1.065636 \\
\hline 3 & 6 & -0.516153 & -1.164550 & 0.912342 \\
\hline 4 & 8 & 1.629652 & 0.894058 & -0.657654 \\
\hline 5 & 6 & -0.750494 & -1.520161 & -0.570456 \\
\hline 6 & 6 & -1.558670 & -0.543260 & -1.329061 \\
\hline 7 & 6 & -1.941982 & 0.595068 & -0.728882 \\
\hline 8 & 1 & -2.454058 & 1.388707 & -1.265384 \\
\hline 9 & 6 & -1.599375 & 0.794789 & 0.731685 \\
\hline 10 & 6 & -0.094754 & 1.213422 & 0.927534 \\
\hline 11 & 1 & -0.001121 & 1.958940 & 1.721964 \\
\hline 12 & 6 & 0.608613 & -0.123355 & 1.234342 \\
\hline 13 & 6 & 1.925208 & -0.016947 & 0.439077 \\
\hline 14 & 1 & -0.405214 & -2.093052 & 1.475254 \\
\hline 15 & 1 & -2.295342 & 1.474778 & 1.225065 \\
\hline 16 & 1 & -1.772485 & -0.773368 & -2.368474 \\
\hline 17 & 1 & 0.824516 & -0.236242 & 2.300077 \\
\hline 18 & 6 & 2.582628 & -1.277853 & -0.093774 \\
\hline 19 & 1 & 3.489022 & -1.014319 & -0.647380 \\
\hline 20 & 1 & 2.873855 & -1.916390 & 0.748296 \\
\hline 21 & 1 & 1.921416 & -1.849680 & -0.745573 \\
\hline 22 & 1 & 2.646351 & 0.508054 & 1.080105 \\
\hline 23 & 6 & 0.596872 & 1.719365 & -0.338572 \\
\hline 24 & 8 & 0.289766 & 2.674140 & -1.005760 \\
\hline
\end{tabular}

\section{E.III.TS2 (Me up)}

$\mathrm{HF}=-687.8756918$ hartrees $(-431648.875361418 \mathrm{kcal} / \mathrm{mol})$ Imaginary Frequencies: $1(-393.48111 / \mathrm{cm})$

Zero-point correction $=0.184761$ (Hartree/Particle)

Thermal correction to Energy $=\quad 0.196466$

Thermal correction to Enthalpy= $\quad 0.197410$

Thermal correction to Gibbs Free Energy $=0.146506$

Sum of electronic and zero-point Energies $=\quad-687.690931$

Sum of electronic and thermal Energies $=\quad-687.679226$

Sum of electronic and thermal Enthalpies $=\quad-687.678282$

Sum of electronic and thermal Free Energies $=\quad-687.729186$

Coordinates (from last standard orientation):

\begin{tabular}{llrrr} 
Center & Atomic & \multicolumn{3}{c}{ Coordinates (Angstroms) } \\
Number & Number & X & $Y$ & \multicolumn{1}{c}{ Z } \\
\hdashline 1 & 8 & -1.529469 & -1.291569 & -1.193316 \\
2 & 8 & -2.474545 & 1.651002 & 0.559141 \\
3 & 6 & -1.738791 & 0.010618 & -0.974586 \\
4 & 8 & 1.611580 & 0.859169 & 0.774172 \\
5 & 6 & -1.980693 & 0.539725 & 0.379362 \\
6 & 6 & -1.575816 & -0.391862 & 1.432062
\end{tabular}




$\begin{array}{ccrrr}7 & 6 & -1.014104 & -1.592512 & 1.106707 \\ 8 & 1 & -0.666267 & -2.281296 & 1.871829 \\ 9 & 6 & -0.843601 & -1.969495 & -0.252800 \\ 10 & 6 & 1.094500 & -0.810180 & -0.788629 \\ 11 & 1 & 1.369250 & -1.631040 & -1.441267 \\ 12 & 6 & 0.537457 & 0.382838 & -1.219473 \\ 13 & 6 & 0.918198 & 1.452388 & -0.260135 \\ 14 & 1 & -2.186118 & 0.514117 & -1.824876 \\ 15 & 1 & -0.649453 & -2.992355 & -0.551897 \\ 16 & 1 & -1.746021 & -0.094923 & 2.462098 \\ 17 & 1 & 0.287912 & 0.641179 & -2.239748 \\ 18 & 6 & 1.895117 & -0.520559 & 0.469865 \\ 19 & 8 & 0.710713 & 2.636363 & -0.306698 \\ 20 & 6 & 3.400180 & -0.706343 & 0.278010 \\ 21 & 1 & 3.934539 & -0.383456 & 1.176527 \\ 22 & 1 & 3.633987 & -1.761579 & 0.094389 \\ 23 & 1 & 3.758644 & -0.115588 & -0.571074 \\ 24 & 1 & 1.562610 & -1.102489 & 1.335330 \\ ---------------------------------------------------\end{array}$

\section{E.III.TS2product (Me up)}

$\mathrm{HF}=-687.9479697$ hartrees $(-431694.230466447 \mathrm{kcal} / \mathrm{mol})$

Imaginary Frequencies: none found

Zero-point correction $=0.189795$ (Hartree/Particle)

Thermal correction to Energy $=\quad 0.200630$

Thermal correction to Enthalpy= $\quad 0.201574$

Thermal correction to Gibbs Free Energy $=\quad 0.152775$

Sum of electronic and zero-point Energies $=\quad-687.758175$

Sum of electronic and thermal Energies $=\quad-687.747340$

Sum of electronic and thermal Enthalpies $=\quad-687.746395$

Sum of electronic and thermal Free Energies $=\quad-687.795194$

Coordinates (from last standard orientation):

\begin{tabular}{|c|c|c|c|c|}
\hline \multirow{2}{*}{$\begin{array}{l}\text { Center } \\
\text { Number }\end{array}$} & \multirow{2}{*}{$\begin{array}{l}\text { Atomic } \\
\text { Number }\end{array}$} & \multicolumn{3}{|c|}{ Coordinates (Angstroms) } \\
\hline & & & $X \quad Y$ & Z \\
\hline 1 & 8 & -1.162500 & -1.546935 & -1.094776 \\
\hline 2 & 8 & -2.932614 & 1.117113 & 0.189436 \\
\hline 3 & 6 & -1.281529 & -0.118391 & -1.085427 \\
\hline 4 & 8 & 1.526837 & 1.056626 & 0.752369 \\
\hline 5 & 6 & -2.054833 & 0.276397 & 0.183032 \\
\hline 6 & 6 & -1.621450 & -0.475586 & 1.388583 \\
\hline 7 & 6 & -0.699755 & -1.444046 & 1.263677 \\
\hline 8 & 1 & -0.326849 & -1.987181 & 2.129599 \\
\hline 9 & 6 & -0.160627 & -1.801481 & -0.106649 \\
\hline 10 & 6 & 0.997601 & $1-0.863615$ & -0.577666 \\
\hline 11 & 1 & 1.541719 & $9-1.370286$ & -1.378662 \\
\hline 12 & 6 & 0.215119 & 9.360309 & -1.089099 \\
\hline 13 & 6 & 0.582508 & 1.490031 & -0.130139 \\
\hline 14 & 1 & -1.837475 & 0.196096 & -1.968652 \\
\hline 15 & 1 & 0.101338 & $3-2.860963$ & -0.165226 \\
\hline 16 & 1 & -2.072693 & $3-0.202876$ & 2.338170 \\
\hline 17 & 1 & 0.491121 & 0.664245 & -2.102111 \\
\hline 18 & 6 & 1.971590 & -0.294170 & 0.479850 \\
\hline 19 & 8 & 0.142815 & $5 \quad 2.608659$ & -0.109049 \\
\hline 20 & 6 & 3.424112 & -0.255016 & 0.021965 \\
\hline
\end{tabular}




$\begin{array}{rrrrr}21 & 1 & 4.043139 & 0.262205 & 0.760786 \\ 22 & 1 & 3.809608 & -1.273332 & -0.104221 \\ 23 & 1 & 3.518578 & 0.271355 & -0.934522 \\ 24 & 1 & 1.898790 & -0.829560 & 1.432246\end{array}$

\begin{abstract}
E.III.TS3 (Me up)
$\mathrm{HF}=-687.8842021$ hartrees $(-431654.215659771 \mathrm{kcal} / \mathrm{mol})$

Imaginary Frequencies: $1(-385.16131 / \mathrm{cm})$

Zero-point correction $=0.184838$ (Hartree/Particle)

Thermal correction to Energy $=\quad 0.196580$

Thermal correction to Enthalpy $=\quad 0.197524$

Thermal correction to Gibbs Free Energy $=0.146581$

Sum of electronic and zero-point Energies $=\quad-687.699364$

Sum of electronic and thermal Energies $=\quad-687.687622$

Sum of electronic and thermal Enthalpies $=\quad-687.686678$

Sum of electronic and thermal Free Energies $=\quad-687.737621$

Coordinates (from last standard orientation):
\end{abstract}

\begin{tabular}{|c|c|c|c|c|}
\hline \multirow{2}{*}{$\begin{array}{l}\text { Center } \\
\text { Number }\end{array}$} & \multirow{2}{*}{$\begin{array}{l}\text { Atomic } \\
\text { Number }\end{array}$} & \multicolumn{3}{|c|}{ Coordinates (Angstroms) } \\
\hline & & & $X \quad Y$ & Z \\
\hline 1 & 8 & 0.816233 & -0.472006 & 1.411711 \\
\hline 2 & 8 & 2.751997 & 1.913881 & -0.407957 \\
\hline 3 & 6 & 1.173144 & 0.707792 & 0.867517 \\
\hline 4 & 6 & 2.300821 & 0.813642 & -0.076924 \\
\hline 5 & 6 & 2.730197 & -0.475374 & -0.597484 \\
\hline 6 & 6 & 2.024798 & -1.607313 & -0.280811 \\
\hline 7 & 1 & 2.252059 & -2.566745 & -0.736995 \\
\hline 8 & 6 & 0.972318 & -1.562214 & 0.659186 \\
\hline 9 & 6 & -0.646786 & -0.506479 & -0.967326 \\
\hline 10 & 6 & -0.481797 & $\begin{array}{ll}7 & 0.797001\end{array}$ & -0.531571 \\
\hline 11 & 1 & 0.976724 & 1.541332 & 1.533563 \\
\hline 12 & 1 & 0.480030 & -2.435198 & 1.067250 \\
\hline 13 & 1 & 3.582320 & -0.498122 & -1.269593 \\
\hline 14 & 6 & -1.828986 & $6-1.096706$ & -0.308540 \\
\hline 15 & 6 & -1.676646 & $6 \quad 1.116621$ & 0.363796 \\
\hline 16 & 8 & -2.356010 & o -0.143962 & 0.535495 \\
\hline 17 & 1 & -0.184281 & $1-0.978277$ & -1.821420 \\
\hline 18 & 1 & 0.002152 & $2 \quad 1.584845$ & -1.100703 \\
\hline 19 & 8 & -2.307938 & $8-2.200337$ & -0.416349 \\
\hline 20 & 6 & -2.627323 & $\begin{array}{l}3 \\
3\end{array}$ & -0.259065 \\
\hline 21 & 1 & -2.133528 & 3.111627 & -0.347494 \\
\hline 22 & 1 & -2.942827 & 1.812469 & -1.255678 \\
\hline 23 & 1 & -3.515961 & 2.252505 & 0.368640 \\
\hline 24 & 1 & -1.389382 & 1.449072 & 1.366572 \\
\hline
\end{tabular}

E.III.TS3product (Me up)

$\mathrm{HF}=-687.9570917$ hartrees $(-431699.954612667 \mathrm{kcal} / \mathrm{mol})$

Imaginary Frequencies: none found

Zero-point correction $=0.189876($ Hartree/Particle $)$

Thermal correction to Energy $=\quad 0.200796$

Thermal correction to Enthalpy= $\quad 0.201741$

Thermal correction to Gibbs Free Energy $=\quad 0.152351$

Sum of electronic and zero-point Energies $=\quad-687.767216$ 


\begin{tabular}{|c|c|c|c|c|}
\hline & & & & \\
\hline umber & Numbe & & $\mathrm{Y}$ & Z \\
\hline 1 & 8 & 0.743821 & -0.588171 & 1.365540 \\
\hline 2 & 8 & 2.920870 & 1.752289 & -0.114535 \\
\hline 3 & 6 & 0.930769 & 0.675625 & 0.723021 \\
\hline 4 & 6 & 2.296795 & 0.713081 & 0.019414 \\
\hline 5 & 6 & 2.726297 & -0.585470 & -0.545640 \\
\hline 6 & 6 & 1.891825 & -1.635343 & -0.466846 \\
\hline 7 & 1 & 2.119993 & -2.587184 & -0.942083 \\
\hline 8 & 6 & 0.577843 & -1.489586 & 0.267873 \\
\hline 9 & 6 & -0.476196 & -0.760080 & -0.623659 \\
\hline 10 & 6 & -0.226437 & 0.731479 & -0.317000 \\
\hline 11 & 1 & 0.888086 & 1.464906 & 1.476722 \\
\hline 12 & 1 & 0.209691 & -2.441744 & 0.655159 \\
\hline 13 & 1 & 3.689393 & -0.623433 & -1.046715 \\
\hline 14 & 6 & -1.897064 & -1.040892 & -0.143157 \\
\hline 15 & 6 & -1.571991 & 1.231900 & 0.283233 \\
\hline 16 & 8 & -2.473648 & 0.097637 & 0.308521 \\
\hline 17 & 1 & -0.394620 & -1.042116 & -1.675973 \\
\hline 18 & 1 & 0.046203 & 1.324017 & -1.194081 \\
\hline 19 & 8 & -2.457499 & -2.106632 & -0.144109 \\
\hline 20 & 6 & -2.223637 & 2.359339 & -0.501992 \\
\hline 21 & 1 & -1.589274 & 3.252471 & -0.473661 \\
\hline 22 & 1 & -2.369933 & 2.070377 & -1.548770 \\
\hline 23 & 1 & -3.198326 & 2.612294 & -0.074431 \\
\hline 24 & 1 & -1.438775 & 1.529109 & 1.329032 \\
\hline
\end{tabular}

\section{E.III.TS4 (Me up)}

$\mathrm{HF}=-687.8779676$ hartrees $(-431650.303448676 \mathrm{kcal} / \mathrm{mol})$ Imaginary Frequencies: $1(-397.77031 / \mathrm{cm})$

Zero-point correction $=0.184856($ Hartree/Particle $)$

Thermal correction to Energy $=\quad 0.196507$

Thermal correction to Enthalpy= $\quad 0.197451$

Thermal correction to Gibbs Free Energy $=\quad 0.146729$

Sum of electronic and zero-point Energies $=\quad-687.693111$

Sum of electronic and thermal Energies $=\quad-687.681461$

Sum of electronic and thermal Enthalpies $=\quad-687.680517$

Sum of electronic and thermal Free Energies $=\quad-687.731239$

Coordinates (from last standard orientation):

\begin{tabular}{llrrr} 
Center & Atomic & \multicolumn{3}{c}{ Coordinates (Angstroms) } \\
Number & Number & X & $Y$ & Z \\
- \hdashline 1 & 8 & 0.550568 & -0.576015 & 1.324686 \\
2 & 8 & 3.019529 & 1.513054 & -0.164298 \\
3 & 6 & 1.131316 & 0.542488 & 0.878591 \\
4 & 6 & 2.352379 & 0.500008 & 0.051357 \\
5 & 6 & 2.621749 & -0.822652 & -0.502202 \\
6 & 6 & 1.730721 & -1.839038 & -0.304460
\end{tabular}




$\begin{array}{ccrrr}7 & 1 & 1.859942 & -2.809595 & -0.776406 \\ 8 & 6 & 0.590740 & -1.651843 & 0.518070 \\ 9 & 6 & -0.783607 & -0.515770 & -1.079426 \\ 10 & 6 & -0.313600 & 0.768481 & -0.853406 \\ 11 & 1 & 0.937238 & 1.404492 & 1.507632 \\ 12 & 1 & 0.011355 & -2.478297 & 0.906322 \\ 13 & 1 & 3.522823 & -0.951335 & -1.093786 \\ 14 & 6 & -2.214408 & -0.585701 & -0.568539 \\ 15 & 6 & -1.368236 & 1.522281 & -0.116626 \\ 16 & 8 & -2.416901 & 0.664151 & 0.118459 \\ 17 & 8 & -1.377201 & 2.667766 & 0.257003 \\ 18 & 1 & -0.455423 & -1.162623 & -1.883002 \\ 19 & 1 & 0.421566 & 1.309916 & -1.433229 \\ 20 & 6 & -2.640846 & -1.727036 & 0.345952 \\ 21 & 1 & -3.709765 & -1.647623 & 0.565395 \\ 22 & 1 & -2.460444 & -2.693030 & -0.138959 \\ 23 & 1 & -2.096430 & -1.688925 & 1.292795 \\ 24 & 1 & -2.876069 & -0.581940 & -1.449434 \\ -------------------------------------------------------\end{array}$

\section{E.III.TS4product (Me up)}

$\mathrm{HF}=-687.9535575$ hartrees $(-431697.736866825 \mathrm{kcal} / \mathrm{mol})$

Imaginary Frequencies: none found

Zero-point correction $=0.190108$ (Hartree/Particle $)$

Thermal correction to Energy $=\quad 0.200909$

Thermal correction to Enthalpy= $\quad 0.201853$

Thermal correction to Gibbs Free Energy $=\quad 0.152782$

Sum of electronic and zero-point Energies $=\quad-687.763450$

Sum of electronic and thermal Energies $=\quad-687.752649$

Sum of electronic and thermal Enthalpies $=\quad-687.751705$

Sum of electronic and thermal Free Energies $=\quad-687.800776$

Coordinates (from last standard orientation):

\begin{tabular}{|c|c|c|c|c|}
\hline \multirow{2}{*}{$\begin{array}{l}\text { Center } \\
\text { Number }\end{array}$} & \multirow{2}{*}{$\begin{array}{l}\text { Atomic } \\
\text { Number }\end{array}$} & \multicolumn{3}{|c|}{ Coordinates (Angstroms) } \\
\hline & & & $X \quad Y$ & Z \\
\hline 1 & 8 & 0.493837 & -0.465791 & 1.315467 \\
\hline 2 & 8 & 3.138921 & 1.387062 & -0.075079 \\
\hline 3 & 6 & 0.923249 & 0.686447 & 0.585842 \\
\hline 4 & 6 & 2.349427 & 0.468529 & 0.056814 \\
\hline 5 & 6 & 2.636795 & -0.926194 & -0.350799 \\
\hline 6 & 6 & 1.654970 & -1.839253 & -0.279879 \\
\hline 7 & 1 & 1.789359 & -2.854795 & -0.647601 \\
\hline 8 & 6 & 0.309643 & -1.449563 & 0.293751 \\
\hline 9 & 6 & -0.544842 & -0.711153 & -0.785327 \\
\hline 10 & 6 & -0.095878 & $\begin{array}{ll}8 & 0.755746\end{array}$ & -0.591017 \\
\hline 11 & 1 & 0.889290 & 1.563503 & 1.233779 \\
\hline 12 & 1 & -0.188824 & $4-2.308153$ & 0.745944 \\
\hline 13 & 1 & 3.629145 & $5-1.143312$ & -0.735798 \\
\hline 14 & 6 & -2.089332 & $2-0.674883$ & -0.562382 \\
\hline 15 & 6 & -1.353047 & $\begin{array}{ll}7 & 1.505623\end{array}$ & -0.154266 \\
\hline 16 & 8 & -2.419124 & 0.669194 & -0.138437 \\
\hline 17 & 8 & -1.428219 & 2.664833 & 0.158398 \\
\hline 18 & 1 & -0.327102 & $2-1.097323$ & -1.783448 \\
\hline 19 & 1 & 0.323964 & $4 \quad 1.244944$ & -1.473583 \\
\hline 20 & 6 & -2.676549 & $9-1.644306$ & 0.452696 \\
\hline
\end{tabular}




$\begin{array}{llllr}21 & 1 & -3.754695 & -1.479481 & 0.536153 \\ 22 & 1 & -2.514558 & -2.681472 & 0.137629 \\ 23 & 1 & -2.229973 & -1.494521 & 1.440247 \\ 24 & 1 & -2.586555 & -0.817725 & -1.528717\end{array}$

\begin{abstract}
A.I.TS1 (s-cis) (Me down)
$\mathrm{HF}=-883.2529699$ hartrees $(-554250.071141949 \mathrm{kcal} / \mathrm{mol})$

Imaginary Frequencies: $1(-377.37871 / \mathrm{cm})$

Zero-point correction $=0.301354$ (Hartree/Particle)

Thermal correction to Energy $=\quad 0.320351$

Thermal correction to Enthalpy $=\quad 0.321295$

Thermal correction to Gibbs Free Energy $=0.255428$

Sum of electronic and zero-point Energies $=\quad-882.951616$

Sum of electronic and thermal Energies $=\quad-882.932619$

Sum of electronic and thermal Enthalpies $=\quad-882.931675$

Sum of electronic and thermal Free Energies $=\quad-882.997542$

Coordinates (from last standard orientation):
\end{abstract}

\begin{tabular}{|c|c|c|c|c|}
\hline \multirow{2}{*}{$\begin{array}{l}\text { Center } \\
\text { Number }\end{array}$} & \multirow{2}{*}{$\begin{array}{l}\text { Atomic } \\
\text { Number }\end{array}$} & \multicolumn{3}{|c|}{ Coordinates (Angstroms) } \\
\hline & & & $\begin{array}{ll}X & Y\end{array}$ & Z \\
\hline 1 & 8 & 0.687857 & -1.244796 & -1.324189 \\
\hline 2 & 8 & -2.503242 & -1.818931 & 0.107196 \\
\hline 3 & 6 & -0.667586 & -1.083938 & -1.200044 \\
\hline 4 & 8 & -0.864079 & 1.616701 & 1.287095 \\
\hline 5 & 6 & -1.292500 & -1.560249 & 0.056124 \\
\hline 6 & 6 & -0.411693 & -1.577154 & 1.220084 \\
\hline 7 & 6 & 0.901106 & -1.229104 & 1.043336 \\
\hline 8 & 1 & 1.568022 & -1.156180 & 1.896551 \\
\hline 9 & 6 & 1.450421 & -0.985117 & -0.242068 \\
\hline 10 & 6 & 0.530371 & 1.391143 & -0.545950 \\
\hline 11 & 6 & -0.739668 & 0.952748 & -0.962032 \\
\hline 12 & 6 & -1.713353 & 1.345778 & 0.146377 \\
\hline 13 & 1 & -1.062932 & 1.108099 & -1.989197 \\
\hline 14 & 6 & 0.439609 & 1.779393 & 0.873851 \\
\hline 15 & 8 & 1.312747 & 2.165044 & 1.620149 \\
\hline 16 & 6 & -1.387187 & -1.383178 & -2.485801 \\
\hline 17 & 1 & -1.407273 & -2.463757 & -2.671356 \\
\hline 18 & 1 & -2.423291 & -1.048161 & -2.406389 \\
\hline 19 & 1 & -0.898770 & -0.891533 & -3.331982 \\
\hline 20 & 6 & -1.003587 & -1.959671 & 2.541418 \\
\hline 21 & 1 & -1.851173 & -1.306438 & 2.778650 \\
\hline 22 & 1 & -1.403952 & -2.979786 & 2.503379 \\
\hline 23 & 1 & -0.267199 & -1.896929 & 3.347298 \\
\hline 24 & 6 & 2.870982 & -0.912323 & -0.543313 \\
\hline 25 & 6 & -2.525642 & 2.594610 & -0.194671 \\
\hline 26 & 1 & -3.209917 & 2.386868 & -1.025342 \\
\hline 27 & 1 & -3.118842 & 2.902115 & 0.671897 \\
\hline 28 & 1 & -1.869937 & 3.424324 & -0.479672 \\
\hline 29 & 1 & -2.391688 & 0.542653 & 0.441344 \\
\hline 30 & 6 & 1.662558 & 1.866304 & -1.402702 \\
\hline 31 & 1 & 2.607848 & 1.838153 & -0.854202 \\
\hline 32 & 1 & 1.759422 & 1.268612 & -2.314981 \\
\hline 33 & 1 & 1.496082 & 2.909645 & -1.709009 \\
\hline 34 & 6 & 3.846455 & -0.625909 & 0.332527 \\
\hline
\end{tabular}




$\begin{array}{rrrrr}35 & 1 & 4.881375 & -0.575014 & 0.010766 \\ 36 & 1 & 3.113266 & -1.078251 & -1.590839 \\ 37 & 1 & 3.650980 & -0.408555 & 1.378267\end{array}$

\begin{abstract}
A.I.TS1product (s-cis) (Me down)
$\mathrm{HF}=-883.3015141$ hartrees $(-554280.533112891 \mathrm{kcal} / \mathrm{mol})$

Imaginary Frequencies: none found

Zero-point correction $=0.305316$ (Hartree/Particle)

Thermal correction to Energy $=\quad 0.323476$

Thermal correction to Enthalpy $=\quad 0.324420$

Thermal correction to Gibbs Free Energy $=\quad 0.260640$

Sum of electronic and zero-point Energies $=\quad-882.996198$

Sum of electronic and thermal Energies $=\quad-882.978038$

Sum of electronic and thermal Enthalpies $=\quad-882.977094$

Sum of electronic and thermal Free Energies $=\quad-883.040874$

Coordinates (from last standard orientation):
\end{abstract}

\begin{tabular}{lrrrr} 
Center & Atomic & \multicolumn{3}{c}{ Coordinates (Angstroms) } \\
Number & Number & X & $Y$ & $Z$ \\
- \hdashline 1 & 8 & -0.093350 & -1.050233 & 1.571404 \\
2 & 8 & 2.954616 & -1.089356 & -0.147790 \\
3 & 6 & 1.085051 & -0.325375 & 1.186832 \\
4 & 8 & 0.120364 & 1.680367 & -1.561921 \\
5 & 6 & 1.741943 & -1.077141 & 0.010775 \\
6 & 6 & 0.804540 & -1.705479 & -0.953251 \\
7 & 6 & -0.512952 & -1.531986 & -0.740269 \\
8 & 1 & -1.249011 & -1.876762 & -1.461361 \\
9 & 6 & -1.031689 & -0.838686 & 0.509860 \\
10 & 6 & -0.963703 & 0.753659 & 0.334428 \\
11 & 6 & 0.496421 & 1.059844 & 0.729712 \\
12 & 6 & 1.111125 & 1.773936 & -0.500482 \\
13 & 1 & 0.530051 & 1.724542 & 1.597772 \\
14 & 6 & -1.059708 & 1.172273 & -1.130921 \\
15 & 8 & -2.024426 & 1.074094 & -1.851058 \\
16 & 6 & 2.019979 & -0.237600 & 2.381398 \\
17 & 1 & 2.305223 & -1.238889 & 2.716434 \\
18 & 1 & 2.932538 & 0.298864 & 2.109411 \\
19 & 1 & 1.522985 & 0.281215 & 3.206799 \\
20 & 6 & 1.394092 & -2.427937 & -2.131455 \\
21 & 1 & 2.074586 & -1.773766 & -2.687474 \\
22 & 1 & 1.989647 & -3.286646 & -1.799993 \\
23 & 1 & 0.612147 & -2.784450 & -2.807780 \\
24 & 6 & -2.359044 & -1.413318 & 0.947482 \\
25 & 6 & 1.437261 & 3.243162 & -0.267997 \\
26 & 1 & 2.239279 & 3.337006 & 0.473024 \\
27 & 1 & 1.768727 & 3.713557 & -1.198443 \\
28 & 1 & 0.559811 & 3.786977 & 0.099885 \\
29 & 1 & 1.998783 & 1.256713 & -0.874152 \\
30 & 6 & -2.007583 & 1.496620 & 1.176604 \\
31 & 1 & -3.013269 & 1.339578 & 0.781961 \\
32 & 1 & -1.977529 & 1.143937 & 2.212694 \\
33 & 1 & -1.802167 & 2.573371 & 1.173952 \\
34 & 6 & -3.473037 & -1.403092 & 0.215897 \\
35 & 1 & -4.392087 & -1.845369 & 0.589469
\end{tabular}




$\begin{array}{llllr}36 & 1 & -2.339628 & -1.867264 & 1.936035 \\ 37 & 1 & -3.513886 & -0.934866 & -0.764988\end{array}$

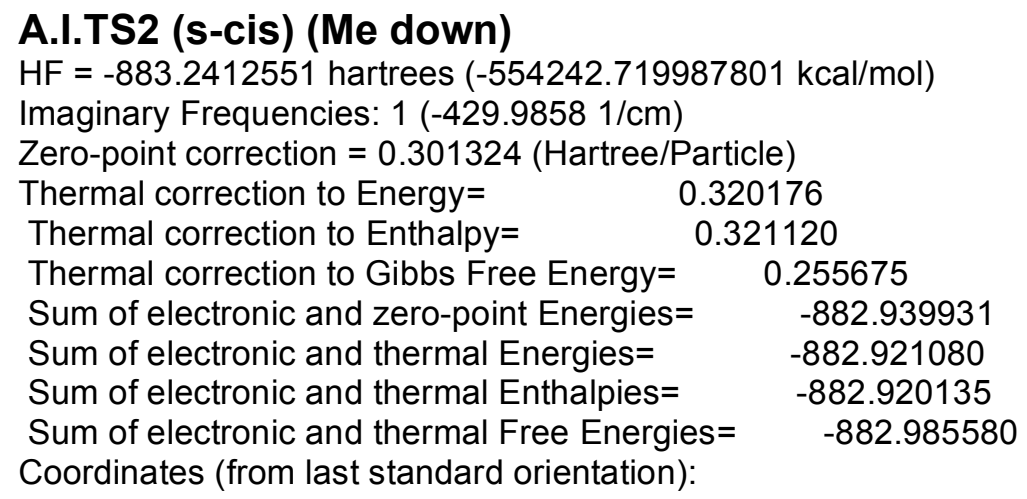

\begin{tabular}{|c|c|c|c|c|}
\hline \multirow{2}{*}{$\begin{array}{l}\text { Center } \\
\text { Number }\end{array}$} & \multirow{2}{*}{$\begin{array}{l}\text { Atomic } \\
\text { Number }\end{array}$} & \multicolumn{3}{|c|}{ Coordinates (Angstroms) } \\
\hline & & & $X \quad Y$ & Z \\
\hline 1 & 8 & -0.695270 & -1.158630 & -1.366400 \\
\hline 2 & 6 & -1.593544 & -0.601621 & -0.499506 \\
\hline 3 & 8 & 1.011017 & 1.557075 & 1.333207 \\
\hline 4 & 6 & -1.472558 & -0.997111 & 0.877358 \\
\hline 5 & 6 & -0.316079 & -1.511239 & 1.377649 \\
\hline 6 & 6 & 0.834727 & -1.632686 & 0.474472 \\
\hline 7 & 6 & 0.581239 & -1.272321 & -0.940344 \\
\hline 8 & 6 & 0.743630 & 1.003414 & -0.909984 \\
\hline 9 & 6 & -0.531458 & 1.344438 & -0.431208 \\
\hline 10 & 6 & -0.367127 & 1.840079 & 0.996346 \\
\hline 11 & 1 & -1.285256 & 1.805961 & -1.060828 \\
\hline 12 & 6 & 1.712684 & 1.217558 & 0.200827 \\
\hline 13 & 8 & 2.915292 & 1.131530 & 0.178730 \\
\hline 14 & 1 & -2.333090 & -0.845756 & 1.524031 \\
\hline 15 & 8 & 1.943860 & -2.030070 & 0.838234 \\
\hline 16 & 6 & 1.535245 & -1.814213 & -1.955907 \\
\hline 17 & 1 & 2.547832 & -1.492492 & -1.700983 \\
\hline 18 & 1 & 1.532708 & -2.911643 & -1.921920 \\
\hline 19 & 1 & 1.275106 & -1.492912 & -2.965809 \\
\hline 20 & 6 & -0.126280 & -1.956974 & 2.795033 \\
\hline 21 & 1 & 0.163060 & -3.013522 & 2.832494 \\
\hline 22 & 1 & 0.699708 & -1.398154 & 3.250168 \\
\hline 23 & 1 & -1.033112 & -1.815376 & 3.390555 \\
\hline 24 & 6 & -2.877206 & -0.333759 & -1.156801 \\
\hline 25 & 6 & 1.204576 & 1.210815 & -2.329941 \\
\hline 26 & 1 & 2.207739 & 0.806978 & -2.483845 \\
\hline 27 & 1 & 0.515847 & 0.756216 & -3.048964 \\
\hline 28 & 1 & 1.249414 & 2.285391 & -2.551178 \\
\hline 29 & 6 & -0.629597 & 3.338644 & 1.142500 \\
\hline 30 & 1 & -1.683830 & 3.559883 & 0.939015 \\
\hline 31 & 1 & -0.395616 & 3.664236 & 2.160814 \\
\hline 32 & 1 & -0.011722 & 3.913101 & 0.444453 \\
\hline 33 & 1 & -0.974594 & 1.290983 & 1.721566 \\
\hline 34 & 6 & -4.029957 & -0.003332 & -0.561253 \\
\hline 35 & 1 & -4.916664 & 0.209224 & -1.149336 \\
\hline 36 & 1 & -2.827722 & -0.383316 & -2.243161 \\
\hline
\end{tabular}




\begin{abstract}
A.I.TS2product (s-cis) (Me down)
$\mathrm{HF}=-883.2960561$ hartrees $(-554277.108163311 \mathrm{kcal} / \mathrm{mol})$

Imaginary Frequencies: none found

Zero-point correction $=0.305498$ (Hartree/Particle)

Thermal correction to Energy $=\quad 0.323560$

Thermal correction to Enthalpy= $\quad 0.324505$

Thermal correction to Gibbs Free Energy $=\quad 0.260674$

Sum of electronic and zero-point Energies $=\quad-882.990558$

Sum of electronic and thermal Energies $=\quad-882.972496$

Sum of electronic and thermal Enthalpies $=\quad-882.971552$

Sum of electronic and thermal Free Energies $=\quad-883.035383$

Coordinates (from last standard orientation):
\end{abstract}

\begin{tabular}{ccrrr} 
Center & Atomic & \multicolumn{3}{c}{ Coordinates (Angstroms) } \\
Number & Number & X & $Y$ & $Z$ \\
- \hdashline 1 & 8 & 0.004240 & -1.040452 & 1.555684 \\
2 & 6 & 1.031196 & -0.958936 & 0.551168 \\
3 & 8 & 0.346168 & 1.613514 & -1.624713 \\
4 & 6 & 0.529326 & -1.617773 & -0.717053 \\
5 & 6 & -0.778468 & -1.619402 & -1.028574 \\
6 & 6 & -1.717707 & -0.917990 & -0.106192 \\
7 & 6 & -1.057082 & -0.188709 & 1.089194 \\
8 & 6 & -0.267765 & 1.117365 & 0.619601 \\
9 & 6 & 1.165537 & 0.600606 & 0.380790 \\
10 & 6 & 1.568891 & 1.134278 & -1.010348 \\
11 & 1 & 1.854292 & 0.963677 & 1.149325 \\
12 & 6 & -0.695755 & 1.656303 & -0.749222 \\
13 & 8 & -1.775404 & 2.094451 & -1.053001 \\
14 & 1 & 1.248179 & -2.085120 & -1.385293 \\
15 & 8 & -2.923281 & -0.930568 & -0.278155 \\
16 & 6 & -2.067822 & -0.005002 & 2.207585 \\
17 & 1 & -2.881678 & 0.643714 & 1.875695 \\
18 & 1 & -2.498740 & -0.975009 & 2.469222 \\
19 & 1 & -1.597097 & 0.417210 & 3.097804 \\
20 & 6 & -1.371229 & -2.278123 & -2.240901 \\
21 & 1 & -2.101622 & -3.040052 & -1.946398 \\
22 & 1 & -1.914803 & -1.546453 & -2.849077 \\
23 & 1 & -0.598474 & -2.748803 & -2.855838 \\
24 & 6 & 2.278319 & -1.568240 & 1.137872 \\
25 & 6 & -0.345292 & 2.271249 & 1.633328 \\
26 & 1 & -1.368821 & 2.645065 & 1.719906 \\
27 & 1 & 0.003499 & 1.947667 & 2.618563 \\
28 & 1 & 0.287764 & 3.105076 & 1.309543 \\
29 & 6 & 2.588503 & 2.265897 & -0.980668 \\
30 & 1 & 3.546138 & 1.897403 & -0.595492 \\
31 & 1 & 2.747894 & 2.662561 & -1.987826 \\
32 & 1 & 2.250608 & 3.086642 & -0.338344 \\
33 & 1 & 1.933860 & 0.332162 & -1.659638 \\
34 & 6 & 3.186640 & -2.324347 & 0.522962 \\
35 & 1 & 4.068299 & -2.672534 & 1.053016 \\
36 & 1 & 2.419580 & -1.298448 & 2.184049 \\
37 & 1 & 3.103580 & -2.633367 & -0.514986
\end{tabular}




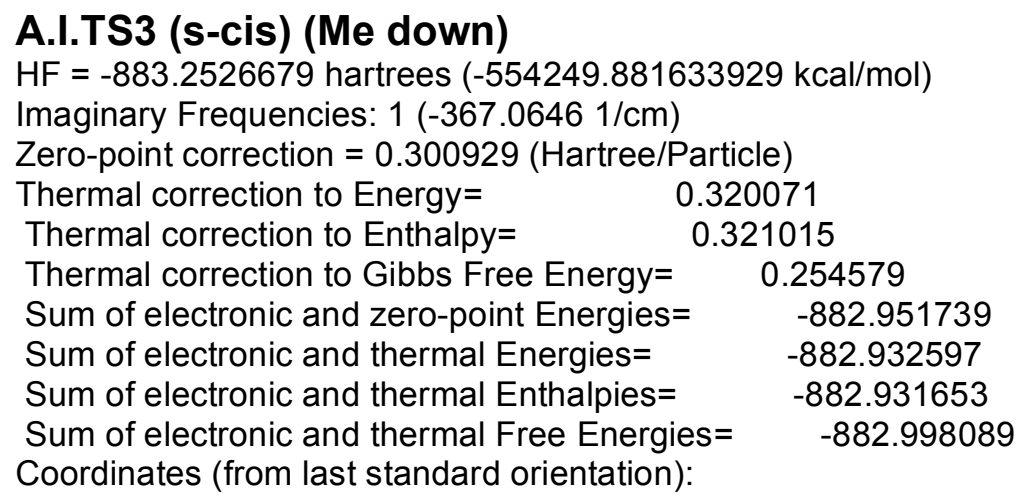

\begin{tabular}{|c|c|c|c|c|}
\hline \multirow{2}{*}{$\begin{array}{l}\text { Center } \\
\text { Number }\end{array}$} & \multirow{2}{*}{$\begin{array}{l}\text { Atomic } \\
\text { Number }\end{array}$} & \multicolumn{3}{|c|}{ Coordinates (Angstroms) } \\
\hline & & & $X \quad Y$ & Z \\
\hline 1 & 8 & -0.342648 & -0.012883 & -1.554514 \\
\hline 2 & 6 & -0.518974 & 1.205268 & -1.006624 \\
\hline 3 & 6 & -1.630956 & 1.404608 & -0.164289 \\
\hline 4 & 6 & -2.422746 & 0.364421 & 0.269000 \\
\hline 5 & 6 & -1.981186 & -0.995360 & -0.008569 \\
\hline 6 & 6 & -0.763304 & -1.116311 & -0.857524 \\
\hline 7 & 6 & 0.711921 & -1.215523 & 0.500736 \\
\hline 8 & 6 & 0.802634 & -0.023338 & 1.232739 \\
\hline 9 & 6 & 2.014763 & -1.303729 & -0.301964 \\
\hline 10 & 6 & 1.965749 & 0.741122 & 0.771084 \\
\hline 11 & 8 & 2.614730 & 0.004124 & -0.197438 \\
\hline 12 & 8 & 2.362544 & 1.829604 & 1.128796 \\
\hline 13 & 1 & 1.854477 & -1.471685 & -1.369417 \\
\hline 14 & 1 & 0.309785 & -2.121874 & 0.954428 \\
\hline 15 & 1 & -1.870970 & 2.427099 & 0.112946 \\
\hline 16 & 8 & -2.533042 & -2.010033 & 0.439034 \\
\hline 17 & 6 & -0.678253 & -2.374132 & -1.683092 \\
\hline 18 & 1 & -0.784297 & -3.242950 & -1.031140 \\
\hline 19 & 1 & -1.507109 & -2.402853 & -2.400563 \\
\hline 20 & 1 & 7959 & -2.434303 & -2.242486 \\
\hline 21 & 6 & 329521 & 2.225375 & -1.587111 \\
\hline 22 & 1 & 0.918395 & 1.889653 & -2.437264 \\
\hline 23 & 6 & 0.490116 & 3.468875 & -1.107868 \\
\hline 24 & 1 & -0.026628 & 3.827283 & -0.222690 \\
\hline 25 & 1 & 1.177625 & 4.160911 & -1.581485 \\
\hline 26 & 6 & -3.711821 & 0.547859 & 1.013107 \\
\hline 27 & 1 & -4.564147 & 0.260423 & 0.383619 \\
\hline 28 & 1 & -3.745646 & -0.110504 & 1.887755 \\
\hline 29 & 1 & 53168 & & 1.327788 \\
\hline 30 & 6 & 2.977018 & -2.357264 & 0.243943 \\
\hline 31 & 1 & 2.557032 & -3.361589 & 0.115403 \\
\hline 32 & 1 & 3.930390 & -2.307802 & -0.290887 \\
\hline 33 & 1 & 3.167463 & -2.192751 & 1.309534 \\
\hline 34 & 6 & 0.050015 & 0.365622 & 2.455536 \\
\hline 35 & 1 & 0.253162 & -0.329904 & 3.281488 \\
\hline 36 & 1 & 0.341734 & 1.371481 & 2.770787 \\
\hline 37 & 1 & -1.035712 & 0.341784 & 2.290556 \\
\hline
\end{tabular}




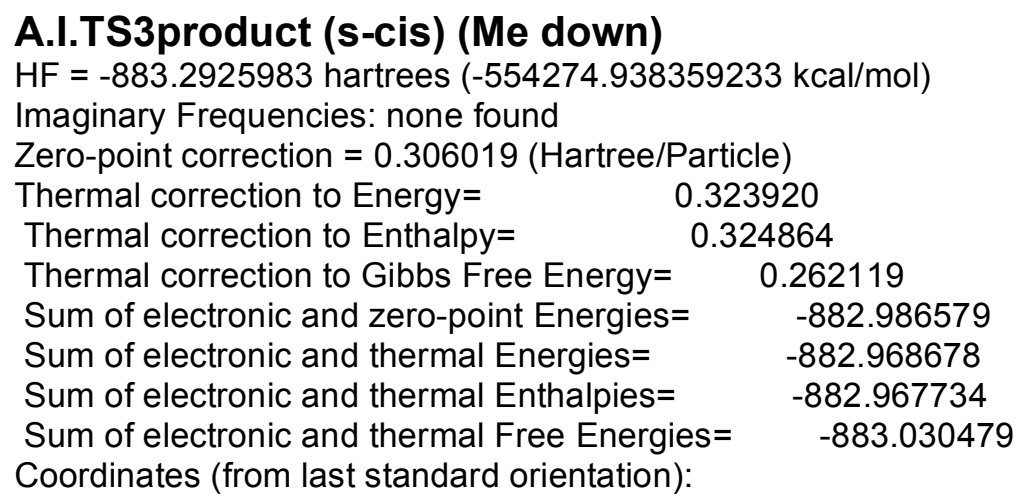

\begin{tabular}{|c|c|c|c|c|}
\hline \multirow{2}{*}{$\begin{array}{l}\text { Center } \\
\text { Number }\end{array}$} & \multirow{2}{*}{$\begin{array}{l}\text { Atomic } \\
\text { Number }\end{array}$} & \multicolumn{3}{|c|}{ Coordinates (Angstroms) } \\
\hline & & & $X \quad Y$ & Z \\
\hline 1 & 8 & 0.324894 & 0.126242 & -1.365736 \\
\hline 2 & 8 & 2.193147 & -2.524763 & -0.091853 \\
\hline 3 & 6 & 0.292194 & -1.124427 & -0.657050 \\
\hline 4 & 6 & 1.725712 & -1.398773 & -0.152071 \\
\hline 5 & 6 & 2.462636 & -0.199745 & 0.334374 \\
\hline 6 & 6 & 1.832242 & 0.988151 & 0.287212 \\
\hline 7 & 1 & 2.305267 & 1.869701 & 0.710989 \\
\hline 8 & 6 & 0.468999 & 1.141108 & -0.361897 \\
\hline 9 & 6 & -0.667382 & 0.713277 & 0.646795 \\
\hline 10 & 6 & -0.568060 & -0.836222 & 0.644667 \\
\hline 11 & 6 & -2.065226 & 0.943469 & 0.028407 \\
\hline 12 & 6 & -2.053903 & -1.238589 & 0.761489 \\
\hline 13 & 8 & -2.775425 & -0.216253 & 0.030124 \\
\hline 14 & 1 & -0.041305 & -1.225678 & 1.521524 \\
\hline 15 & 8 & -2.527675 & 1.977971 & -0.378826 \\
\hline 16 & 1 & -2.312180 & -1.103211 & 1.822585 \\
\hline 17 & 6 & -2.562073 & -2.611006 & 0.350149 \\
\hline 18 & 1 & -1.970708 & -3.392982 & 0.838868 \\
\hline 19 & 1 & -3.602428 & -2.720518 & 0.672184 \\
\hline 20 & 1 & -2.525105 & -2.764555 & -0.727621 \\
\hline 21 & 6 & -0.605410 & 1.408674 & 2.010737 \\
\hline 22 & 1 & -0.723402 & 2.489371 & 1.882723 \\
\hline 23 & 1 & -1.406442 & 1.063809 & 2.674168 \\
\hline 24 & 1 & 0.351176 & 1.211909 & 2.505629 \\
\hline 25 & 6 & -0.145056 & -2.188380 & -1.648920 \\
\hline 26 & 1 & -0.185390 & -3.173460 & -1.181626 \\
\hline 27 & 1 & -1.116203 & -1.932174 & -2.076916 \\
\hline 28 & 1 & 0.585841 & -2.233012 & -2.461718 \\
\hline 29 & 6 & 3.843645 & -0.414456 & 0.887384 \\
\hline 30 & 1 & 3.832786 & -1.164594 & 1.686067 \\
\hline 31 & 1 & 4.513425 & -0.801984 & 0.110876 \\
\hline 32 & 1 & 4.265315 & 0.516622 & 1.277464 \\
\hline 33 & 6 & 0.267475 & 2.477979 & -1.033257 \\
\hline 34 & 1 & -0.471442 & 2.450469 & -1.827852 \\
\hline 35 & 6 & 0.866869 & 3.628060 & -0.726815 \\
\hline 36 & 1 & 1.604802 & 3.733118 & 0.063421 \\
\hline 37 & 1 & 0.620495 & 4.536861 & -1.267665 \\
\hline
\end{tabular}




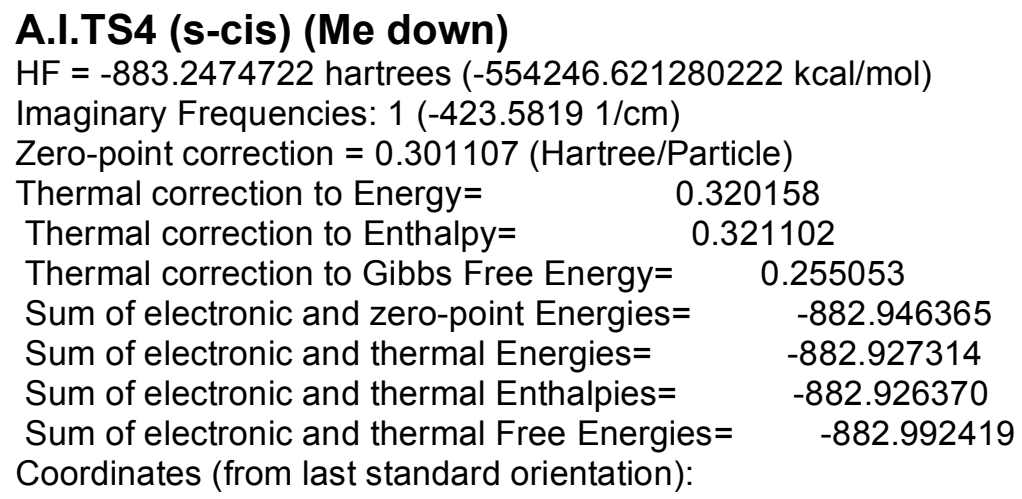

\begin{tabular}{|c|c|c|c|c|}
\hline \multirow{2}{*}{$\begin{array}{l}\text { Center } \\
\text { Number }\end{array}$} & \multirow{2}{*}{$\begin{array}{l}\text { Atomic } \\
\text { Number }\end{array}$} & \multicolumn{3}{|c|}{ Coordinates (Angstroms) } \\
\hline & & & $\begin{array}{l}X \quad Y \\
\end{array}$ & Z \\
\hline 1 & 8 & -0.257771 & 0.502262 & 1.429378 \\
\hline 2 & 8 & -2.804555 & -1.594244 & 0.132005 \\
\hline 3 & 6 & -0.879874 & -0.641357 & 1.103631 \\
\hline 4 & 6 & -2.079649 & -0.599684 & 0.260047 \\
\hline 5 & 6 & -2.339606 & 0.680149 & -0.413280 \\
\hline 6 & 6 & -1.402432 & 1.658536 & -0.315395 \\
\hline 7 & 1 & -1.524506 & 2.600170 & -0.844217 \\
\hline 8 & 6 & -0.205797 & 1.489739 & 0.476167 \\
\hline 9 & 6 & 1.049772 & 0.411793 & -0.817695 \\
\hline 10 & 6 & 0.536348 & -0.898080 & -0.856354 \\
\hline 11 & 6 & 2.393228 & 0.344406 & -0.096199 \\
\hline 12 & 6 & 1.464031 & -1.767815 & -0.093060 \\
\hline 13 & 8 & 2.458702 & -0.991991 & 0.449161 \\
\hline 14 & 8 & 1.422744 & -2.966740 & 0.063833 \\
\hline 15 & 1 & 2.465924 & 1.024547 & 0.755808 \\
\hline 16 & 1 & 0.952335 & 1.091338 & -1.658902 \\
\hline 17 & 6 & -0.691084 & -1.760374 & 2.071260 \\
\hline 18 & 1 & -1.539865 & -1.808813 & 2.765551 \\
\hline 19 & 1 & -0.654882 & -2.711275 & 1.533679 \\
\hline 20 & 1 & 0.235996 & -1.630138 & 2.633443 \\
\hline 21 & 6 & -3.617704 & 0.803830 & -1.186485 \\
\hline 22 & 1 & -3.694776 & -0.006516 & -1.920543 \\
\hline 23 & 1 & -4.482149 & 0.690847 & -0.521464 \\
\hline 24 & 1 & -3.690848 & 1.767580 & -1.699019 \\
\hline 25 & 6 & 0.556387 & 2.653370 & 0.963710 \\
\hline 26 & 1 & 1.037528 & 2.496638 & 1.927066 \\
\hline 27 & 6 & 0.721806 & 3.815067 & 0.320886 \\
\hline 28 & 1 & 0.273478 & 4.022772 & -0.647453 \\
\hline 29 & 1 & 1.323719 & 4.608066 & 0.752446 \\
\hline 30 & 6 & -0.309649 & -1.525761 & -1.930419 \\
\hline 31 & 1 & 0.335140 & -2.053367 & -2.645540 \\
\hline 32 & 1 & -1.008468 & -2.262150 & -1.522990 \\
\hline 33 & 1 & -0.880518 & -0.772741 & -2.480675 \\
\hline 34 & 6 & 3.581283 & 0.576042 & -1.028036 \\
\hline 35 & 1 & 3.576666 & 1.609407 & -1.393476 \\
\hline 36 & 1 & 4.519330 & 0.400001 & -0.492563 \\
\hline 37 & 1 & 3.540575 & -0.099835 & -1.888832 \\
\hline
\end{tabular}




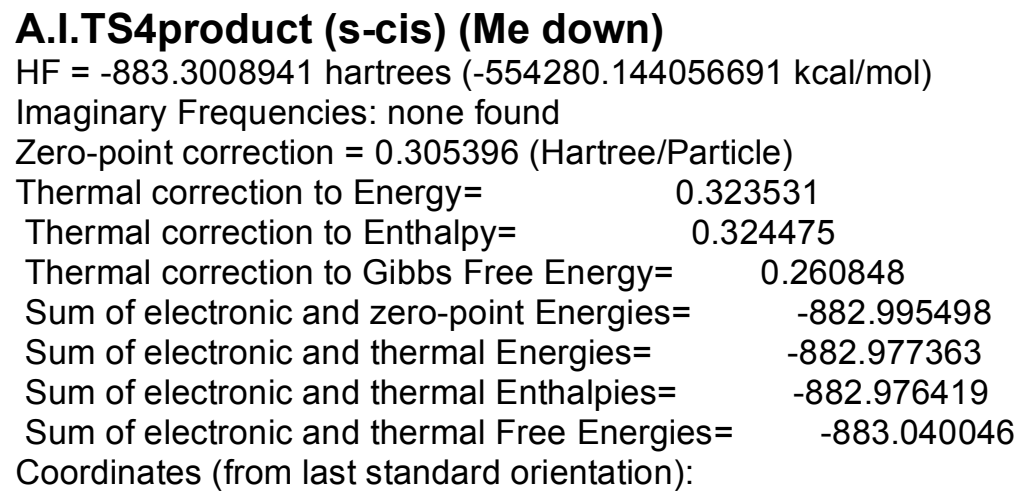

\begin{tabular}{|c|c|c|c|c|}
\hline \multirow{2}{*}{$\begin{array}{l}\text { Center } \\
\text { Number }\end{array}$} & \multirow{2}{*}{$\begin{array}{l}\text { Atomic } \\
\text { Number }\end{array}$} & \multicolumn{3}{|c|}{ Coordinates (Angstroms) } \\
\hline & & & $\begin{array}{ll}X & Y\end{array}$ & Z \\
\hline 1 & 8 & -0.317580 & 0.351854 & 1.417343 \\
\hline 2 & 8 & -2.962307 & -1.478169 & 0.085480 \\
\hline 3 & 6 & -0.709847 & -0.847227 & 0.730321 \\
\hline 4 & 6 & -2.120076 & -0.597856 & 0.148358 \\
\hline 5 & 6 & -2.364152 & 0.771986 & -0.383813 \\
\hline 6 & 6 & -1.350234 & 1.652025 & -0.323019 \\
\hline 7 & 1 & -1.437701 & 2.639183 & -0.771873 \\
\hline 8 & 6 & -0.051498 & 1.312405 & 0.386077 \\
\hline 9 & 6 & 0.889467 & 0.496642 & -0.568968 \\
\hline 10 & 6 & 0.341195 & -0.948417 & -0.468253 \\
\hline 11 & 6 & 2.353932 & 0.410265 & -0.059895 \\
\hline 12 & 6 & 1.563331 & -1.782178 & -0.060248 \\
\hline 13 & 8 & 2.642352 & -0.992899 & 0.148959 \\
\hline 14 & 8 & 1.615666 & -2.982813 & 0.033329 \\
\hline 15 & 1 & 2.451512 & 0.886330 & 0.919578 \\
\hline 16 & 1 & 0.860952 & 0.889853 & -1.589249 \\
\hline 17 & 6 & -0.726903 & -1.997170 & 1.723606 \\
\hline 18 & 1 & -1.503066 & -1.821244 & 2.473454 \\
\hline 19 & 1 & -0.943102 & -2.939408 & 1.217493 \\
\hline 20 & 1 & 0.239125 & -2.081806 & 2.225816 \\
\hline 21 & 6 & -3.708013 & 1.048349 & -0.996734 \\
\hline 22 & 1 & -3.933852 & 0.323706 & -1.786993 \\
\hline 23 & 1 & -4.501227 & 0.942944 & -0.247493 \\
\hline 24 & 1 & -3.754637 & 2.058667 & -1.413794 \\
\hline 25 & 6 & 0.567459 & 2.526251 & 1.045370 \\
\hline 26 & 1 & 0.794527 & 2.387209 & 2.100423 \\
\hline 27 & 6 & 0.837037 & 3.686201 & 0.446136 \\
\hline 28 & 1 & 0.621141 & 3.869744 & -0.604471 \\
\hline 29 & 1 & 1.290628 & 4.507906 & 0.992270 \\
\hline 30 & 6 & -0.222199 & -1.560935 & -1.761479 \\
\hline 31 & 1 & 0.563760 & -1.634303 & -2.520659 \\
\hline 32 & 1 & -0.595916 & -2.570639 & -1.571133 \\
\hline 33 & 1 & -1.033362 & -0.956621 & -2.175749 \\
\hline 34 & 6 & 3.386957 & 0.983368 & -1.017134 \\
\hline 35 & 1 & 3.232754 & 2.062456 & -1.127009 \\
\hline 36 & 1 & 4.399434 & 0.813794 & -0.638404 \\
\hline 37 & 1 & 3.305241 & 0.516193 & -2.005043 \\
\hline
\end{tabular}




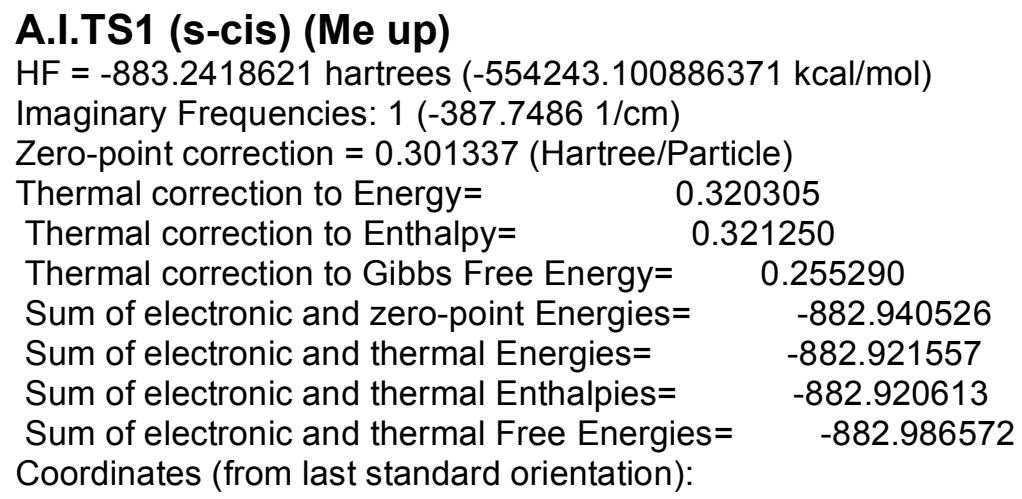

\begin{tabular}{|c|c|c|c|c|}
\hline \multirow{2}{*}{$\begin{array}{l}\text { Center } \\
\text { Number }\end{array}$} & \multirow{2}{*}{$\begin{array}{l}\text { Atomic } \\
\text { Number }\end{array}$} & \multicolumn{3}{|c|}{ Coordinates (Angstroms) } \\
\hline & & & $X \quad Y$ & Z \\
\hline 1 & 8 & 0.497453 & -1.415572 & -1.148937 \\
\hline 2 & 8 & -2.787657 & -1.400370 & 0.099476 \\
\hline 3 & 6 & -0.805300 & -0.991985 & -1.140355 \\
\hline 4 & 8 & -0.472674 & 2.134282 & 0.914864 \\
\hline 5 & 6 & -1.561335 & -1.234234 & 0.111387 \\
\hline 6 & 6 & -0.759886 & -1.197809 & 1.332506 \\
\hline 7 & 6 & 0.600418 & -1.116688 & 1.209615 \\
\hline 8 & 1 & 1.227909 & -1.044339 & 2.092008 \\
\hline 9 & 6 & 1.246445 & -1.174874 & -0.053901 \\
\hline 10 & 6 & 0.913644 & 1.237378 & -0.708922 \\
\hline 11 & 6 & -0.401637 & 1.079872 & -1.182392 \\
\hline 12 & 6 & -1.248062 & 2.006055 & -0.298896 \\
\hline 13 & 1 & -0.602871 & 1.112762 & -2.250877 \\
\hline 14 & 1 & -1.200774 & 2.986113 & -0.801309 \\
\hline 15 & 6 & 0.845471 & 1.853468 & 0.632921 \\
\hline 16 & 8 & 1.741024 & 2.086381 & 1.414692 \\
\hline 17 & 6 & -1.503827 & -1.294495 & -2.435584 \\
\hline 18 & 1 & -1.756475 & -2.360057 & -2.495508 \\
\hline 19 & 1 & -2.442391 & -0.738218 & -2.482008 \\
\hline 20 & 1 & -0.873999 & -1.032709 & -3.290578 \\
\hline 21 & 6 & -1.472216 & -1.292713 & 2.646813 \\
\hline 22 & 1 & -2.197627 & -0.476843 & 2.749410 \\
\hline 23 & 1 & -2.047993 & -2.223788 & 2.703536 \\
\hline 24 & 1 & -0.774165 & -1.252661 & 3.487584 \\
\hline 25 & 6 & 2.657794 & -1.469412 & -0.250448 \\
\hline 26 & 6 & -2.709917 & 1.751518 & 0.026032 \\
\hline 27 & 1 & -3.092768 & 2.598939 & 0.604107 \\
\hline 28 & 1 & -3.285870 & 1.690936 & -0.904437 \\
\hline 29 & 1 & -2.883793 & 0.835521 & 0.585782 \\
\hline 30 & 6 & 2.170073 & 1.310455 & -1.523175 \\
\hline 31 & 1 & 2.259769 & 2.305493 & -1.983874 \\
\hline 32 & 1 & 3.055680 & 1.160944 & -0.900847 \\
\hline 33 & 1 & 2.171647 & 0.574589 & -2.333807 \\
\hline 34 & 6 & 3.630020 & -1.313445 & 0.660727 \\
\hline 35 & 1 & 4.660940 & -1.544952 & 0.413971 \\
\hline 36 & 1 & 2.903797 & -1.817467 & -1.251328 \\
\hline 37 & 1 & 3.443707 & -0.930578 & 1.659446 \\
\hline
\end{tabular}




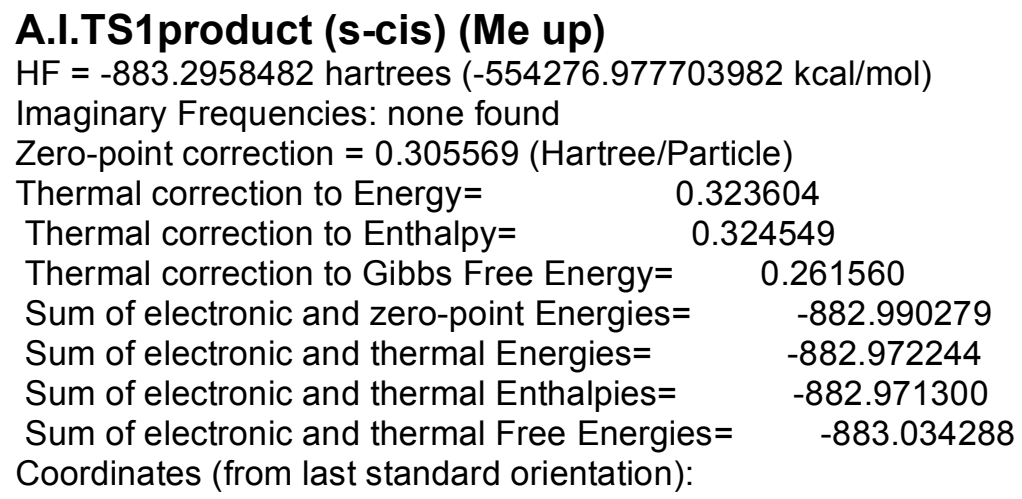

\begin{tabular}{|c|c|c|c|c|}
\hline \multirow{2}{*}{$\begin{array}{l}\text { Center } \\
\text { Number }\end{array}$} & \multirow{2}{*}{$\begin{array}{l}\text { Atomic } \\
\text { Number }\end{array}$} & \multicolumn{3}{|c|}{ Coordinates (Angstroms) } \\
\hline & & & $\begin{array}{ll}X & Y\end{array}$ & Z \\
\hline 1 & 8 & -0.327754 & -0.901413 & 1.605344 \\
\hline 2 & 8 & 2.726320 & -1.336827 & -0.020764 \\
\hline 3 & 6 & 0.930278 & -0.305162 & 1.238991 \\
\hline 4 & 8 & 0.458856 & 1.702612 & -1.488306 \\
\hline 5 & 6 & 1.521401 & -1.156867 & 0.090294 \\
\hline 6 & 6 & 0.548534 & -1.733310 & -0.868885 \\
\hline 7 & 6 & -0.752163 & -1.443176 & -0.689361 \\
\hline 8 & 1 & -1.504336 & -1.765073 & -1.403199 \\
\hline 9 & 6 & -1.213812 & -0.632330 & 0.509594 \\
\hline 10 & 6 & -0.958270 & 0.931093 & 0.253201 \\
\hline 11 & 6 & 0.459999 & 1.137758 & 0.830887 \\
\hline 12 & 6 & 1.152712 & 2.005582 & -0.243856 \\
\hline 13 & 1 & 0.416987 & 1.701906 & 1.767627 \\
\hline 14 & 1 & 0.906800 & 3.051049 & -0.012922 \\
\hline 15 & 6 & -0.793574 & 1.241245 & -1.237303 \\
\hline 16 & 8 & -1.623097 & 1.099122 & -2.102804 \\
\hline 17 & 6 & 1.845709 & -0.330983 & 2.453449 \\
\hline 18 & 1 & 2.018431 & -1.361448 & 2.776259 \\
\hline 19 & 1 & 2.814725 & 0.111381 & 2.210678 \\
\hline 20 & 1 & 1.386875 & 0.223000 & 3.278070 \\
\hline 21 & 6 & 1.092992 & -2.563289 & -1.996812 \\
\hline 22 & 1 & 1.820141 & -1.990624 & -2.583207 \\
\hline 23 & 1 & 1.625058 & -3.440615 & -1.611345 \\
\hline 24 & 1 & 0.291609 & -2.900957 & -2.659936 \\
\hline 25 & 6 & -2.607885 & -1.016032 & 0.945444 \\
\hline 26 & 6 & 2.653274 & 1.911329 & -0.460205 \\
\hline 27 & 1 & 2.950203 & 2.600049 & -1.257231 \\
\hline 28 & 1 & 3.173405 & 2.210590 & 0.456796 \\
\hline 29 & 1 & 2.976681 & 0.904760 & -0.725057 \\
\hline 30 & 6 & -2.020561 & 1.848083 & 0.870002 \\
\hline 31 & 1 & -1.700658 & 2.895611 & 0.814538 \\
\hline 32 & 1 & -2.972130 & 1.754232 & 0.342218 \\
\hline 33 & 1 & -2.178958 & 1.594154 & 1.923063 \\
\hline 34 & 6 & -3.680421 & -1.021670 & 0.154330 \\
\hline 35 & 1 & -4.656551 & -1.309213 & 0.533896 \\
\hline 36 & 1 & -2.682457 & -1.300623 & 1.992977 \\
\hline 37 & 1 & -3.629687 & -0.719764 & -0.889598 \\
\hline
\end{tabular}




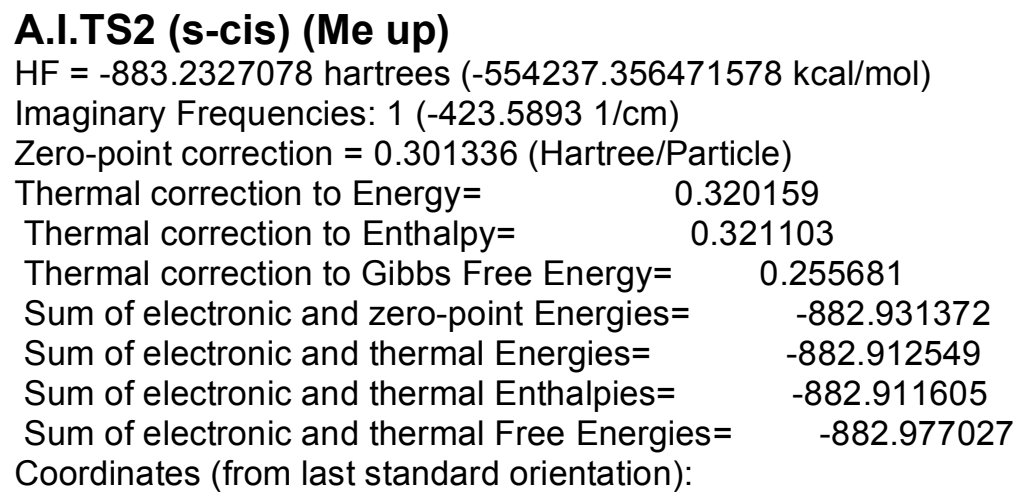

\begin{tabular}{|c|c|c|c|c|}
\hline \multirow{2}{*}{$\begin{array}{l}\text { Center } \\
\text { Number }\end{array}$} & \multirow{2}{*}{$\begin{array}{l}\text { Atomic } \\
\text { Number }\end{array}$} & \multicolumn{3}{|c|}{ Coordinates (Angstroms) } \\
\hline & & & $X \quad Y$ & Z \\
\hline 1 & 8 & -0.415331 & -1.210614 & -1.389516 \\
\hline 2 & 6 & -1.441248 & -0.657315 & -0.680337 \\
\hline 3 & 8 & 0.745205 & 1.958517 & 1.214178 \\
\hline 4 & 6 & -1.466378 & -0.956562 & 0.723944 \\
\hline 5 & 6 & -0.359763 & -1.381637 & 1.393058 \\
\hline 6 & 6 & 0.898164 & -1.489671 & 0.651621 \\
\hline 7 & 6 & 0.802578 & -1.235835 & -0.804576 \\
\hline 8 & 6 & 0.874201 & 1.039868 & -0.923647 \\
\hline 9 & 6 & -0.472652 & 1.380413 & -0.710975 \\
\hline 10 & 6 & -0.519557 & 2.211345 & 0.566177 \\
\hline 11 & 1 & -1.126662 & 1.672800 & -1.526373 \\
\hline 12 & 1 & -0.496284 & 3.260950 & 0.227149 \\
\hline 13 & 6 & 1.632351 & 1.441050 & 0.297114 \\
\hline 14 & 8 & 2.817106 & 1.366244 & 0.507744 \\
\hline 15 & 1 & -2.410522 & -0.830454 & 1.245562 \\
\hline 16 & 8 & 1.971156 & -1.806734 & 1.170952 \\
\hline 17 & 6 & 1.887567 & -1.814430 & -1.655849 \\
\hline 18 & 1 & 2.853774 & -1.450497 & -1.299344 \\
\hline 19 & 1 & 1.900257 & -2.906777 & -1.545024 \\
\hline 20 & 1 & 1.751059 & -1.570641 & -2.710554 \\
\hline 21 & 6 & -0.334311 & -1.738546 & 2.847668 \\
\hline 22 & 1 & 0.004695 & -2.771593 & 2.986781 \\
\hline 23 & 1 & 0.392302 & -1.106836 & 3.372440 \\
\hline 24 & 1 & -1.317977 & -1.622817 & 3.312939 \\
\hline 25 & 6 & -2.643925 & -0.505880 & -1.505259 \\
\hline 26 & 6 & 1.568103 & 1.137648 & -2.261113 \\
\hline 27 & 1 & 2.586904 & 0.748134 & -2.213156 \\
\hline 28 & 1 & 1.014205 & 0.611614 & -3.044650 \\
\hline 29 & 1 & 1.638158 & 2.192248 & -2.559811 \\
\hline 30 & 6 & -1.651601 & 2.101942 & 1.577412 \\
\hline 31 & 1 & -1.547548 & 2.892983 & 2.326588 \\
\hline 32 & 1 & -2.616695 & 2.231592 & 1.074875 \\
\hline 33 & 1 & -1.642510 & 1.143252 & 2.094641 \\
\hline 34 & 6 & -3.881394 & -0.222765 & -1.079048 \\
\hline 35 & 1 & -4.696377 & -0.102016 & -1.785065 \\
\hline 36 & 1 & -2.453762 & -0.611093 & -2.572045 \\
\hline 37 & 1 & -4.130907 & -0.097901 & -0.028950 \\
\hline
\end{tabular}




\section{A.I.TS2product (s-cis) (Me up)}

$\mathrm{HF}=-883.2923795$ hartrees $(-554274.801060045 \mathrm{kcal} / \mathrm{mol})$

Imaginary Frequencies: none found

Zero-point correction $=0.305703$ (Hartree/Particle)

Thermal correction to Energy $=\quad 0.323674$

Thermal correction to Enthalpy= $\quad 0.324618$

Thermal correction to Gibbs Free Energy $=\quad 0.261637$

Sum of electronic and zero-point Energies $=\quad-882.986677$

Sum of electronic and thermal Energies $=\quad-882.968705$

Sum of electronic and thermal Enthalpies $=\quad-882.967761$

Sum of electronic and thermal Free Energies $=\quad-883.030742$

Coordinates (from last standard orientation):

\begin{tabular}{|c|c|c|c|c|}
\hline \multirow{2}{*}{$\begin{array}{l}\text { Center } \\
\text { Number }\end{array}$} & \multirow{2}{*}{$\begin{array}{l}\text { Atomic } \\
\text { Number }\end{array}$} & \multicolumn{3}{|c|}{ Coordinates (Angstroms) } \\
\hline & & & $\begin{array}{l}X \quad Y \\
\end{array}$ & Z \\
\hline 1 & 8 & -0.002177 & -0.913523 & -1.601386 \\
\hline 2 & 6 & -1.088198 & -0.607685 & -0.703292 \\
\hline 3 & 8 & -0.344893 & 1.777791 & 1.523372 \\
\hline 4 & 6 & -0.829079 & -1.298220 & 0.620043 \\
\hline 5 & 6 & 0.416683 & -1.600784 & 1.026235 \\
\hline 6 & 6 & 1.560212 & -1.171670 & 0.167521 \\
\hline 7 & 6 & 1.162078 & -0.280144 & -1.031307 \\
\hline 8 & 6 & 0.601204 & 1.134522 & -0.562615 \\
\hline 9 & 6 & -0.930249 & 0.956139 & -0.630302 \\
\hline 10 & 6 & -1.438465 & 1.777212 & 0.568241 \\
\hline 11 & 1 & -1.344765 & 1.356298 & -1.560966 \\
\hline 12 & 1 & -1.552228 & 2.815586 & 0.227654 \\
\hline 13 & 6 & 0.833839 & 1.457230 & 0.922193 \\
\hline 14 & 8 & 1.883984 & 1.470486 & 1.512337 \\
\hline 15 & 1 & -1.678299 & -1.555852 & 1.246670 \\
\hline 16 & 8 & 2.705902 & -1.521300 & 0.381187 \\
\hline 17 & 6 & 2.263493 & -0.268203 & -2.075720 \\
\hline 18 & 1 & 3.168749 & 0.189303 & -1.669582 \\
\hline 19 & 1 & 2.507041 & -1.296451 & -2.354828 \\
\hline 20 & 1 & 1.948711 & 0.270297 & -2.972357 \\
\hline 21 & 6 & 0.750561 & -2.326053 & 2.297630 \\
\hline 22 & 1 & 1.324861 & -3.233787 & 2.081935 \\
\hline 23 & 1 & 1.383493 & -1.700826 & 2.937813 \\
\hline 24 & 1 & -0.152994 & -2.600616 & 2.849994 \\
\hline 25 & 6 & -2.356082 & -1.020429 & -1.408404 \\
\hline 26 & 6 & 1.121401 & 2.329479 & -1.380511 \\
\hline 27 & 1 & 2.198223 & 2.456880 & -1.238761 \\
\hline 28 & 1 & 0.916283 & 2.196520 & -2.446616 \\
\hline 29 & 1 & 0.633380 & 3.257016 & -1.058787 \\
\hline 30 & 6 & -2.720223 & 1.367115 & 1.274980 \\
\hline 31 & 1 & -2.955399 & 2.088909 & 2.063045 \\
\hline 32 & 1 & -3.552043 & 1.346326 & 0.563418 \\
\hline 33 & 1 & -2.630607 & 0.381271 & 1.735797 \\
\hline 34 & 6 & -3.369913 & -1.735844 & -0.924068 \\
\hline 35 & 1 & -4.237256 & -1.954976 & -1.540038 \\
\hline 36 & 1 & -2.394955 & -0.662498 & -2.437000 \\
\hline 37 & 1 & -3.388291 & -2.137016 & 0.084774 \\
\hline
\end{tabular}




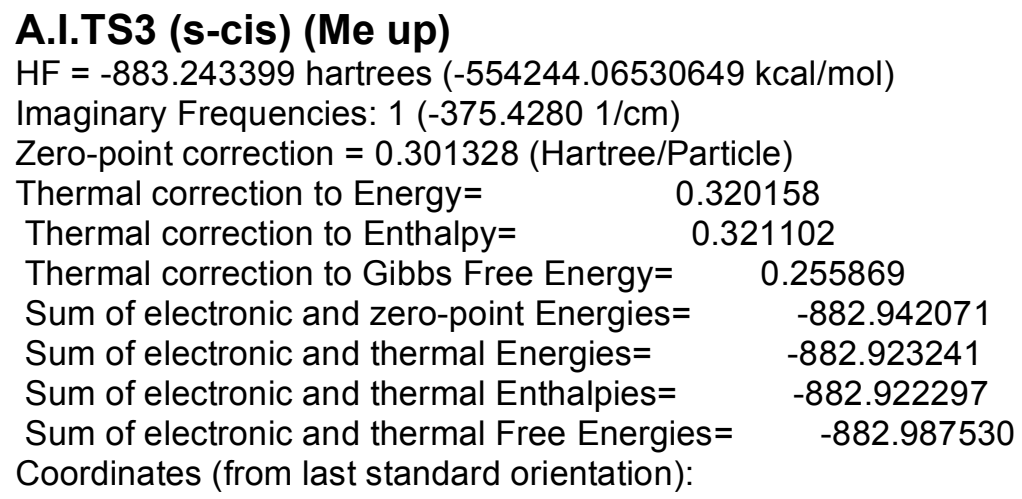

\begin{tabular}{|c|c|c|c|c|}
\hline Center & Atomic & & ordinates & Angstroms) \\
\hline Number & Numbe & & Y & Z \\
\hline 1 & 8 & 0.143904 & 0.365122 & -1.455354 \\
\hline 2 & 6 & 0.173014 & -0.960153 & -1.216487 \\
\hline 3 & 6 & 1.322740 & -1.508003 & -0.613768 \\
\hline 4 & 6 & 2.314276 & -0.720748 & -0.071109 \\
\hline 5 & 6 & 2.058190 & 0.706632 & 0.049391 \\
\hline 6 & 6 & 0.800795 & 1.203636 & -0.585486 \\
\hline 7 & 6 & -0.494010 & 1.180379 & 0.944430 \\
\hline 8 & 6 & -0.629437 & -0.133071 & 1.430633 \\
\hline 9 & 6 & -1.927996 & 1.576288 & 0.524268 \\
\hline 10 & 6 & -1.934559 & -0.669752 & 1.041329 \\
\hline 11 & 8 & -2.639335 & 0.327914 & 0.403004 \\
\hline 12 & 8 & -2.396872 & -1.778192 & 1.214899 \\
\hline 13 & 1 & 0.087254 & 1.913543 & 1.505220 \\
\hline 14 & 1 & 1.422177 & -2.589703 & -0.620171 \\
\hline 15 & 8 & 2.800588 & 1.500470 & 0.642289 \\
\hline 16 & 6 & 0.910246 & 2.616725 & -1.114475 \\
\hline 17 & 1 & 0.851726 & 3.339980 & -0.297449 \\
\hline 18 & 1 & 1.890227 & 2.755374 & -1.584284 \\
\hline 19 & 1 & 0.131277 & 2.829385 & -1.845221 \\
\hline 20 & 6 & -0.893780 & -1.689544 & -1.870797 \\
\hline 21 & 1 & -1.481984 & -1.101704 & -2.571499 \\
\hline 22 & 6 & -1.235323 & -2.960259 & -1.605092 \\
\hline 23 & 1 & -0.721928 & -3.559515 & -0.859579 \\
\hline 24 & 1 & -2.072024 & -3.427963 & -2.112326 \\
\hline 25 & 6 & 3.637565 & -1.245351 & 0.402522 \\
\hline 26 & 1 & 4.442602 & -0.928515 & -0.273499 \\
\hline 27 & 1 & 3.884413 & -0.830156 & 1.385190 \\
\hline 28 & 1 & 3.643742 & -2.338310 & 0.452457 \\
\hline 29 & 6 & -2.242893 & 2.408244 & -0.709312 \\
\hline 30 & 1 & -3.329101 & 2.525148 & -0.776637 \\
\hline 31 & 1 & -1.799278 & 3.405263 & -0.634358 \\
\hline 32 & 1 & -1.896058 & 1.921506 & -1.622405 \\
\hline 33 & 1 & -2.346431 & 2.101172 & 1.397505 \\
\hline 34 & 6 & 0.242242 & -0.846939 & 2.400088 \\
\hline 35 & 1 & 0.296105 & -0.303756 & 3.353493 \\
\hline 36 & 1 & -0.151509 & -1.849475 & 2.589877 \\
\hline 37 & 1 & 1.276087 & -0.933287 & 2.038166 \\
\hline
\end{tabular}




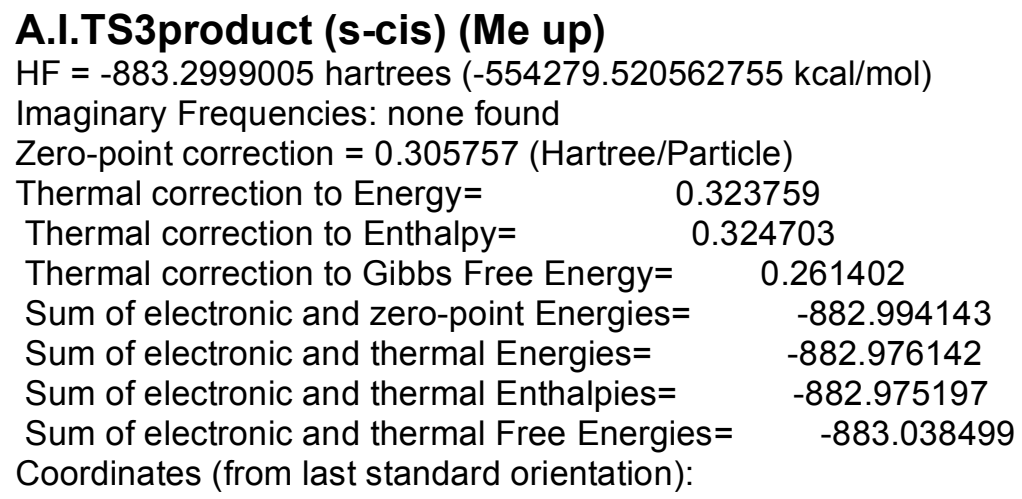

\begin{tabular}{|c|c|c|c|c|}
\hline Center & Atomic & & ordinates & Angstroms) \\
\hline Number & Numbe & & $\mathrm{Y}$ & Z \\
\hline 1 & 8 & 0.439835 & 0.233229 & -1.417274 \\
\hline 2 & 8 & 2.554435 & -2.225271 & -0.099219 \\
\hline 3 & 6 & 0.549363 & -1.060623 & -0.805634 \\
\hline 4 & 6 & 1.946573 & -1.167878 & -0.161214 \\
\hline 5 & 6 & 2.456935 & 0.085110 & 0.455910 \\
\hline 6 & 6 & 1.658487 & 1.167556 & 0.423248 \\
\hline 7 & 1 & 1.953842 & 2.074359 & 0.943525 \\
\hline 8 & 6 & 0.341984 & 1.164479 & -0.330031 \\
\hline 9 & 6 & -0.789223 & 0.485393 & 0.557539 \\
\hline 10 & 6 & -0.497381 & -1.026434 & 0.379415 \\
\hline 11 & 6 & -2.173853 & 0.645153 & -0.097475 \\
\hline 12 & 6 & -1.878127 & -1.653070 & 0.085922 \\
\hline 13 & 8 & -2.708771 & -0.567017 & -0.390100 \\
\hline 14 & 1 & -0.068219 & -1.487238 & 1.273919 \\
\hline 15 & 8 & -2.765577 & 1.673225 & -0.308902 \\
\hline 16 & 6 & -2.538632 & -2.303906 & 1.297006 \\
\hline 17 & 1 & -1.970033 & -3.188912 & 1.604521 \\
\hline 18 & 1 & -2.589008 & -1.614994 & 2.147038 \\
\hline 19 & 1 & -3.557408 & -2.616219 & 1.048406 \\
\hline 20 & 1 & -1.838896 & -2.375991 & -0.730608 \\
\hline 21 & 6 & -0.858667 & 1.032070 & 1.988578 \\
\hline 22 & 1 & -1.060430 & 2.107667 & 1.962601 \\
\hline 23 & 1 & -1.663332 & 0.558755 & 2.560553 \\
\hline 24 & 1 & 0.082254 & 0.858032 & 2.519673 \\
\hline 25 & 6 & 0.365677 & -2.113476 & -1.885516 \\
\hline 26 & 1 & 1.177742 & -2.033847 & -2.613532 \\
\hline 27 & 1 & 0.398910 & -3.118919 & -1.458249 \\
\hline 28 & 1 & -0.580504 & -1.963105 & -2.411787 \\
\hline 29 & 6 & 3.804032 & 0.033131 & 1.119994 \\
\hline 30 & 1 & 3.840713 & -0.768986 & 1.865510 \\
\hline 31 & 1 & 4.587051 & -0.192390 & 0.386592 \\
\hline 32 & 1 & 4.045237 & 0.983640 & 1.604946 \\
\hline 33 & 6 & -0.023838 & 2.505186 & -0.917721 \\
\hline 34 & 6 & 0.411278 & 3.702814 & -0.528076 \\
\hline 35 & 1 & 1.115226 & 3.855744 & 0.284797 \\
\hline 36 & 1 & 0.055417 & 4.602514 & -1.021021 \\
\hline 37 & 1 & -0.735582 & 2.433517 & -1.734602 \\
\hline
\end{tabular}




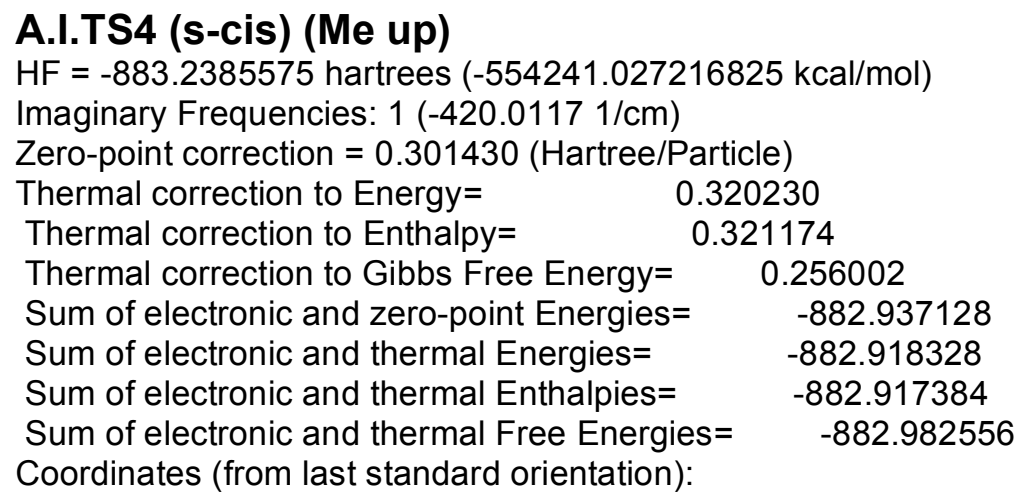

\begin{tabular}{|c|c|c|c|c|}
\hline \multirow{2}{*}{$\begin{array}{l}\text { Center } \\
\text { Number }\end{array}$} & \multirow{2}{*}{$\begin{array}{l}\text { Atomic } \\
\text { Number }\end{array}$} & \multicolumn{3}{|c|}{ Coordinates (Angstroms) } \\
\hline & & & $X \quad Y$ & Z \\
\hline 1 & 8 & 0.077715 & 0.585234 & 1.317727 \\
\hline 2 & 8 & -2.834814 & -1.310541 & 0.622991 \\
\hline 3 & 6 & -0.688915 & -0.507232 & 1.178744 \\
\hline 4 & 6 & -2.006790 & -0.394525 & 0.548034 \\
\hline 5 & 6 & -2.254929 & 0.856834 & -0.182092 \\
\hline 6 & 6 & -1.225757 & 1.728953 & -0.323670 \\
\hline 7 & 1 & -1.348024 & 2.641749 & -0.899994 \\
\hline 8 & 6 & 0.067232 & 1.501458 & 0.290398 \\
\hline 9 & 6 & 1.028345 & 0.230421 & -1.075509 \\
\hline 10 & 6 & 0.295526 & -0.975543 & -1.025988 \\
\hline 11 & 6 & 2.459736 & -0.133003 & -0.640549 \\
\hline 12 & 6 & 1.154964 & -2.018838 & -0.424150 \\
\hline 13 & 8 & 2.355892 & -1.455718 & -0.072716 \\
\hline 14 & 8 & 0.916005 & -3.192046 & -0.248008 \\
\hline 15 & 1 & 0.930455 & 0.915262 & -1.913055 \\
\hline 16 & 6 & -0.425208 & $8-1.595956$ & 2.162440 \\
\hline 17 & 1 & -1.093057 & $7-1.497185$ & 3.028345 \\
\hline 18 & 1 & -0.632658 & $8-2.563550$ & 1.698903 \\
\hline 19 & 1 & 0.613379 & $9-1.570162$ & 2.499345 \\
\hline 20 & 6 & -3.628026 & $6 \quad 1.065254$ & -0.745011 \\
\hline 21 & 1 & -4.372341 & 1.096804 & 0.059486 \\
\hline 22 & 1 & -3.693833 & 1.991731 & -1.323029 \\
\hline 23 & 1 & -3.907235 & 0.217199 & -1.380667 \\
\hline 24 & 6 & 0.916867 & 2.672364 & 0.591962 \\
\hline 25 & 1 & 1.461987 & 2.604968 & 1.529591 \\
\hline 26 & 6 & 1.060980 & 3.754589 & -0.181184 \\
\hline 27 & 1 & 0.554688 & 3.870049 & -1.136051 \\
\hline 28 & 1 & 1.710357 & 4.570009 & 0.120425 \\
\hline 29 & 6 & -0.804378 & -1.423519 & -1.950063 \\
\hline 30 & 1 & -0.377370 & - -2.046806 & -2.747121 \\
\hline 31 & 1 & -1.549433 & $\begin{array}{ll}3 & -2.035504\end{array}$ & -1.432485 \\
\hline 32 & 1 & -1.308583 & $\begin{array}{ll}3 & -0.574778\end{array}$ & -2.418984 \\
\hline 33 & 6 & 3.279422 & 0.714610 & 0.319490 \\
\hline 34 & 1 & 4.261059 & 0.247438 & 0.446969 \\
\hline 35 & 1 & 3.422182 & 1.724429 & -0.072984 \\
\hline 36 & 1 & 2.801846 & 0.771153 & 1.299710 \\
\hline 37 & 1 & 3.033793 & -0.233425 & -1.575471 \\
\hline
\end{tabular}




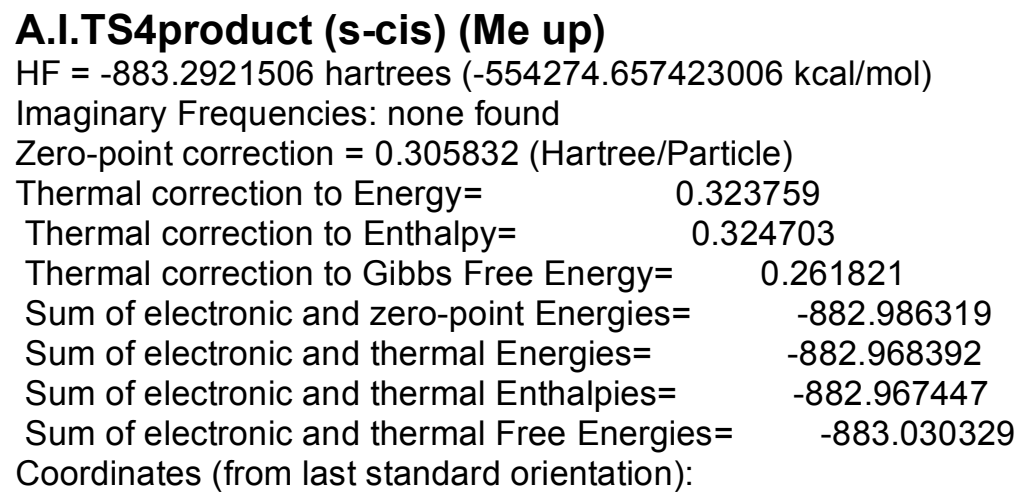

\begin{tabular}{|c|c|c|c|c|}
\hline \multirow{2}{*}{$\begin{array}{l}\text { Center } \\
\text { Number }\end{array}$} & \multirow{2}{*}{$\begin{array}{l}\text { Atomic } \\
\text { Number }\end{array}$} & \multicolumn{3}{|c|}{ Coordinates (Angstroms) } \\
\hline & & & $\begin{array}{ll}X & Y\end{array}$ & Z \\
\hline 1 & 8 & -0.123785 & 0.475561 & 1.294116 \\
\hline 2 & 8 & -2.762996 & -1.655777 & 0.608434 \\
\hline 3 & 6 & -0.491107 & -0.813411 & 0.768097 \\
\hline 4 & 6 & -1.993601 & -0.728310 & 0.421219 \\
\hline 5 & 6 & -2.421946 & 0.545185 & -0.220855 \\
\hline 6 & 6 & -1.480692 & 1.482728 & -0.420344 \\
\hline 7 & 1 & -1.703325 & 2.390617 & -0.976250 \\
\hline 8 & 6 & -0.075123 & 1.347662 & 0.155997 \\
\hline 9 & 6 & 0.827347 & 0.527222 & -0.830428 \\
\hline 10 & 6 & 0.357271 & -0.932281 & -0.580567 \\
\hline 11 & 6 & 2.376550 & 0.493921 & -0.572508 \\
\hline 12 & 6 & 1.653039 & -1.722415 & -0.378284 \\
\hline 13 & 8 & 2.728484 & -0.909331 & -0.449359 \\
\hline 14 & 8 & 1.755050 & -2.915533 & -0.235817 \\
\hline 15 & 1 & 0.646629 & 0.841400 & -1.862045 \\
\hline 16 & 6 & -0.233511 & -1.865498 & 1.833285 \\
\hline 17 & 1 & -0.883700 & -1.681383 & 2.692847 \\
\hline 18 & 1 & -0.444287 & -2.863906 & 1.447085 \\
\hline 19 & 1 & 0.807694 & -1.829192 & 2.161946 \\
\hline 20 & 6 & -3.853847 & 0.655244 & -0.662846 \\
\hline 21 & 1 & -4.526809 & 0.597162 & 0.200502 \\
\hline 22 & 1 & -4.037518 & 1.598666 & -1.185320 \\
\hline 23 & 1 & -4.125881 & -0.177643 & -1.320962 \\
\hline 24 & 6 & 0.402286 & 2.709715 & 0.614650 \\
\hline 25 & 1 & 0.623945 & 2.770390 & 1.677437 \\
\hline 26 & 6 & 0.519331 & 3.786678 & -0.162128 \\
\hline 27 & 1 & 0.302038 & 3.767702 & -1.228350 \\
\hline 28 & 1 & 0.849291 & 4.738661 & 0.242878 \\
\hline 29 & 6 & -0.383143 & -1.619007 & -1.744183 \\
\hline 30 & 1 & 0.278333 & -1.706185 & -2.612830 \\
\hline 31 & 1 & -0.682975 & -2.629410 & -1.453424 \\
\hline 32 & 1 & -1.269287 & -1.059905 & -2.053094 \\
\hline 33 & 6 & 2.976990 & 1.193650 & 0.642459 \\
\hline 34 & 1 & 4.015679 & 0.864067 & 0.744828 \\
\hline 35 & 1 & 2.968196 & 2.278220 & 0.521156 \\
\hline 36 & 1 & 2.438320 & 0.931238 & 1.556759 \\
\hline 37 & 1 & 2.890562 & 0.843652 & -1.473526 \\
\hline
\end{tabular}




\begin{tabular}{|c|c|c|c|c|}
\hline \multicolumn{5}{|c|}{$\begin{array}{l}\text { A.I.TS1 (s-trans) (Me down) } \\
\text { HF = -883.254915 hartrees }(-554251.29171165 \mathrm{kcal} / \mathrm{mc} \\
\text { Imaginary Frequencies: } 1(-364.88041 / \mathrm{cm}) \\
\text { Zero-point correction }=0.301398(\text { Hartree } / \text { Particle })\end{array}$} \\
\hline & \multicolumn{3}{|c|}{ Thermal correction to Energy= } & 0.320331 \\
\hline \multicolumn{4}{|c|}{ Thermal correction to Enthalpy= } & 0.321276 \\
\hline \multirow{2}{*}{\multicolumn{4}{|c|}{$\begin{array}{lr}\text { Thermal correction to Gibbs Free Energy }= & 0.2557 \\
\text { Sum of electronic and zero-point Energies }= & -882\end{array}$}} & \\
\hline & & & int Energies= & \\
\hline \multicolumn{5}{|c|}{ Sum of electronic and thermal Energies $=$} \\
\hline \multicolumn{5}{|c|}{ Sum of electronic and thermal Enthalpies= } \\
\hline \\
\hline \multicolumn{5}{|c|}{ Coordinates (from last standard orientation): } \\
\hline \multirow{2}{*}{$\begin{array}{l}\text { Center } \\
\text { Number }\end{array}$} & \multirow{2}{*}{\multicolumn{2}{|c|}{$\begin{array}{l}\text { Atomic } \\
\text { Number }\end{array}$}} & \multicolumn{2}{|c|}{ Coordinates (Angstroms) } \\
\hline & & & $X \quad Y$ & Z \\
\hline 1 & 8 & 1.314202 & -1.016531 & -0.847404 \\
\hline 2 & 8 & -1.946239 & -2.3 & 2882 \\
\hline 3 & 6 & -0.016500 & -1.179357 & -1.141547 \\
\hline 4 & 8 & -1.590931 & 1.396229 & 4834 \\
\hline 5 & 6 & -0.870422 & -1.795293 & -0.096697 \\
\hline 6 & 6 & -0.412774 & -1.598177 & 1.273726 \\
\hline 7 & 6 & 0.782129 & -0.9 & 68360 \\
\hline 8 & 1 & & & \\
\hline 9 & 6 & 1.6 & -0.6 & \\
\hline 10 & 6 & 0.307317 & 1.52 & 1677 \\
\hline 11 & 6 & -0.630096 & 0.774631 & 4192 \\
\hline 12 & 6 & -1.960509 & 0.917371 & -0.369950 \\
\hline 13 & 1 & -0.650016 & 0.838088 & -2.190151 \\
\hline 14 & 6 & -0.301507 & 1.879002 & 0.925444 \\
\hline 15 & 8 & 26 & & 1525 \\
\hline 16 & 6 & -0.2 & & 7347 \\
\hline 17 & 1 & 579 & -2.6 & -2 . \\
\hline 18 & 1 & -1.277316 & -1.5 & 14560 \\
\hline 19 & 1 & 0.377942 & -1.048870 & -3.254587 \\
\hline 20 & 6 & -1.284689 & -2.095617 & 2.384776 \\
\hline 21 & 1 & -2.290526 & -1.669357 & 2.295936 \\
\hline 22 & 1 & -1.406243 & $-3.1 \varepsilon$ & 2.321266 \\
\hline 23 & 1 & 30 & & \\
\hline 24 & 6 & & -0.1 & \\
\hline 25 & 1 & 3.242230 & & 1.604941 \\
\hline 26 & 6 & 3.963646 & -0.1 & -0.355187 \\
\hline 27 & 1 & 3.768404 & -0.506161 & -1.369799 \\
\hline 28 & 1 & 4.971009 & 0.157784 & -0.126132 \\
\hline 29 & 6 & -2.899223 & 1.923569 & -1.035042 \\
\hline 30 & 1 & -3.225693 & 1.549909 & -2.012399 \\
\hline 31 & 1 & -3.785066 & 2.075716 & -0.410998 \\
\hline 32 & 1 & -2.403202 & 2.889752 & -1.176553 \\
\hline 33 & 1 & -2.486835 & -0.028126 & -0.225685 \\
\hline 34 & 6 & 1.512667 & 2.240825 & -0.885481 \\
\hline 35 & 1 & 2.076764 & 2.675374 & -0.056020 \\
\hline 36 & 1 & 2.181611 & 1.583607 & -1.453795 \\
\hline 37 & 1 & 1.216769 & 3.059788 & -1.557222 \\
\hline
\end{tabular}




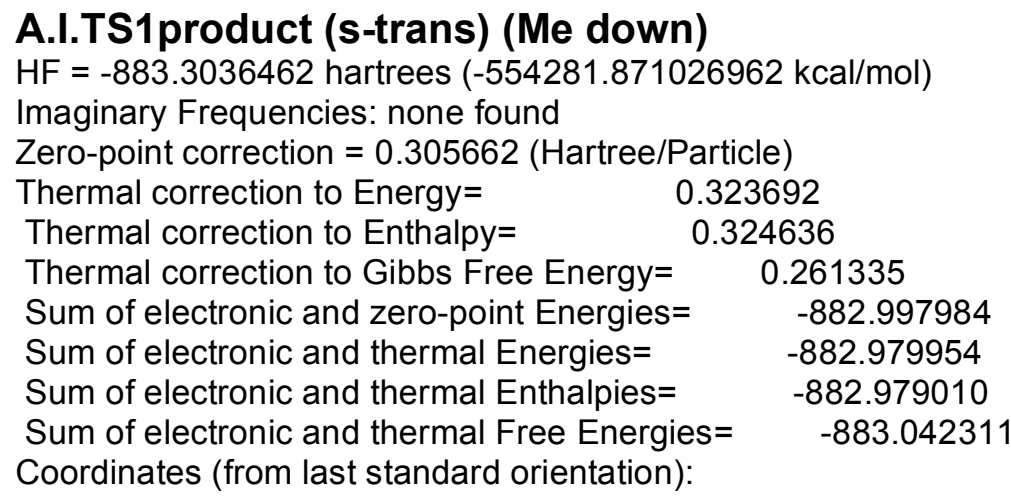

\begin{tabular}{|c|c|c|c|c|}
\hline \multirow{2}{*}{$\begin{array}{l}\text { Center } \\
\text { Number }\end{array}$} & \multirow{2}{*}{$\begin{array}{l}\text { Atomic } \\
\text { Number }\end{array}$} & \multicolumn{3}{|c|}{ Coordinates (Angstroms) } \\
\hline & & & $X \quad Y$ & Z \\
\hline 1 & 8 & 0.795354 & -1.152004 & -1.115225 \\
\hline 2 & 8 & -2.495805 & -1.874835 & -0.210388 \\
\hline 3 & 6 & -0.586165 & -0.759519 & -1.198400 \\
\hline 4 & 8 & -0.917888 & 1.897332 & 1.160913 \\
\hline 5 & 6 & -1.342690 & -1.492821 & -0.071757 \\
\hline 6 & 6 & -0.605925 & -1.625177 & 1.211096 \\
\hline 7 & 6 & 0.625895 & -1.086866 & 1.276061 \\
\hline 8 & 1 & 1.170748 & -1.050224 & 2.216534 \\
\hline 9 & 6 & 1.300167 & -0.496689 & 0.047338 \\
\hline 10 & 6 & 0.810776 & 1.007444 & -0.195721 \\
\hline 11 & 6 & -0.522546 & 0.792052 & -0.940339 \\
\hline 12 & 6 & -1.600828 & 1.458395 & -0.046049 \\
\hline 13 & 1 & -0.502923 & 1.283180 & -1.917679 \\
\hline 14 & 6 & 0.422374 & 1.700561 & 1.105447 \\
\hline 15 & 8 & 1.167604 & 2.035506 & 1.994035 \\
\hline 16 & 6 & -1.121526 & $6-1.139343$ & -2.568185 \\
\hline 17 & 1 & -1.033719 & $9-2.217991$ & -2.725990 \\
\hline 18 & 1 & -2.178499 & $9-0.872722$ & -2.648009 \\
\hline 19 & 1 & -0.554272 & $2-0.621722$ & -3.347769 \\
\hline 20 & 6 & -1.315504 & $4-2.285719$ & 2.358847 \\
\hline 21 & 1 & -2.269755 & $5-1.788503$ & 2.565253 \\
\hline 22 & 1 & -1.554558 & $8-3.327675$ & 2.115995 \\
\hline 23 & 1 & -0.703132 & $2-2.268969$ & 3.264817 \\
\hline 24 & 6 & 2.791295 & $5-0.660795$ & 0.151133 \\
\hline 25 & 1 & 3.236091 & $1-0.161763$ & 1.010938 \\
\hline 26 & 6 & 3.537843 & $\begin{array}{ll}3 & -1.361599\end{array}$ & -0.699716 \\
\hline 27 & 1 & 3.098816 & -1.867082 & -1.553802 \\
\hline 28 & 1 & 4.611498 & $3-1.451104$ & -0.561559 \\
\hline 29 & 6 & -2.281306 & $6 \quad 2.661799$ & -0.684196 \\
\hline 30 & 1 & -2.866378 & 2.343540 & -1.554579 \\
\hline 31 & 1 & -2.956277 & 3.143699 & 0.029285 \\
\hline 32 & 1 & -1.542497 & 3.400971 & -1.013902 \\
\hline 33 & 1 & -2.359645 & 0.743615 & 0.283699 \\
\hline 34 & 6 & 1.841506 & 1.840276 & -0.965863 \\
\hline 35 & 1 & 2.710388 & 2.057599 & -0.339246 \\
\hline 36 & 1 & 2.176191 & 1.300591 & -1.856586 \\
\hline 37 & 1 & 1.403618 & 2.794575 & -1.280266 \\
\hline
\end{tabular}




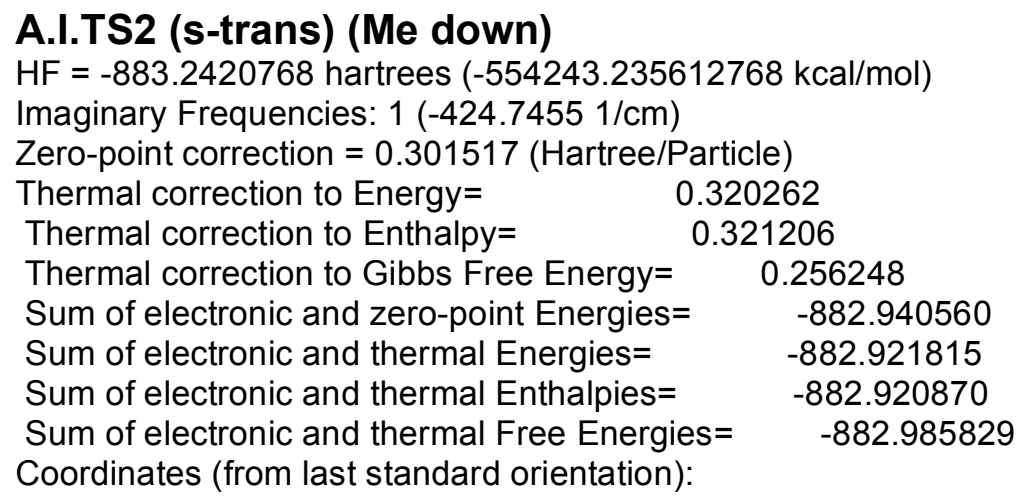

\begin{tabular}{|c|c|c|c|c|}
\hline \multirow{2}{*}{$\begin{array}{l}\text { Center } \\
\text { Number }\end{array}$} & \multirow{2}{*}{$\begin{array}{l}\text { Atomic } \\
\text { Number }\end{array}$} & \multicolumn{3}{|c|}{ Coordinates (Angstroms) } \\
\hline & & & $X \quad Y$ & Z \\
\hline 1 & 8 & 1.599180 & -0.591613 & 0.750319 \\
\hline 2 & 6 & 1.750619 & 0.038132 & -0.445725 \\
\hline 3 & 8 & -2.048898 & 0.892127 & -0.753534 \\
\hline 4 & 6 & 1.167227 & -0.604722 & -1.588492 \\
\hline 5 & 6 & 0.185122 & -1.541579 & -1.466836 \\
\hline 6 & 6 & -0.307253 & -1.847778 & -0.120045 \\
\hline 7 & 6 & 0.393213 & -1.160258 & 0.994025 \\
\hline 8 & 6 & -0.562678 & 0.868818 & 1.037168 \\
\hline 9 & 6 & 0.099886 & 1.513247 & -0.020822 \\
\hline 10 & 6 & -0.883267 & $\begin{array}{ll}7 & 1.641512\end{array}$ & -1.170802 \\
\hline 11 & 1 & 0.834019 & 2.294966 & 0.144135 \\
\hline 12 & 6 & -1.947662 & 0.561648 & 0.575217 \\
\hline 13 & 8 & -2.867670 & 0.108341 & 1.209104 \\
\hline 14 & 1 & 1.518548 & $8-0.292469$ & -2.569916 \\
\hline 15 & 8 & -1.231597 & -2.632913 & 0.103879 \\
\hline 16 & 6 & 0.265686 & -1.784581 & 2.347283 \\
\hline 17 & 1 & -0.792603 & $3-1.851891$ & 2.610506 \\
\hline 18 & 1 & 0.652976 & $6-2.811486$ & 2.320394 \\
\hline 19 & 1 & 0.812999 & $9-1.221256$ & 3.104915 \\
\hline 20 & 6 & -0.444370 & $0-2.271484$ & -2.613423 \\
\hline 21 & 1 & -0.288644 & $4-3.352336$ & -2.515678 \\
\hline 22 & 1 & -1.529536 & $6-2.117677$ & -2.599964 \\
\hline 23 & 1 & -0.041019 & $9-1.941969$ & -3.575691 \\
\hline 24 & 6 & 2.974126 & $6 \quad 0.833987$ & -0.564517 \\
\hline 25 & 1 & 3.160301 & 1.219504 & -1.565622 \\
\hline 26 & 6 & 3.823563 & 1.123409 & 0.429054 \\
\hline 27 & 1 & 3.672660 & 0.751232 & 1.437546 \\
\hline 28 & 1 & 4.703807 & 1.732359 & 0.251061 \\
\hline 29 & 6 & -0.346975 & 1.201369 & 2.493386 \\
\hline 30 & 1 & -0.931463 & 0.543579 & 3.140054 \\
\hline 31 & 1 & 0.709802 & 1.139039 & 2.770234 \\
\hline 32 & 1 & -0.679140 & 2.229168 & 2.689167 \\
\hline 33 & 6 & -1.286153 & 3.087877 & -1.457083 \\
\hline 34 & 1 & -0.424043 & 3.656388 & -1.825003 \\
\hline 35 & 1 & -2.071796 & 3.115482 & -2.218360 \\
\hline 36 & 1 & -1.663583 & 3.573928 & -0.551419 \\
\hline 37 & 1 & -0.537915 & 1.168321 & -2.094850 \\
\hline
\end{tabular}




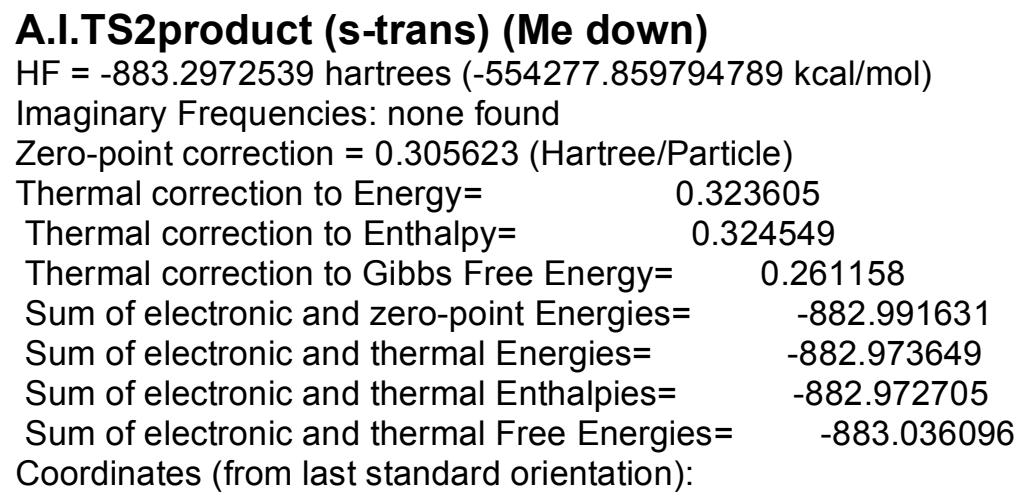

\begin{tabular}{|c|c|c|c|c|}
\hline \multirow{2}{*}{$\begin{array}{l}\text { Center } \\
\text { Number }\end{array}$} & \multirow{2}{*}{$\begin{array}{l}\text { Atomic } \\
\text { Number }\end{array}$} & \multicolumn{3}{|c|}{ Coordinates (Angstroms) } \\
\hline & & & $\begin{array}{ll}X & Y\end{array}$ & Z \\
\hline 1 & 8 & 0.145867 & 1.467883 & 1.028287 \\
\hline 2 & 6 & -0.696523 & 1.339282 & -0.120327 \\
\hline 3 & 8 & -0.706846 & -1.938367 & -1.200321 \\
\hline 4 & 6 & 0.171467 & 1.318976 & -1.368749 \\
\hline 5 & 6 & 1.420497 & 0.823037 & -1.337983 \\
\hline 6 & 6 & 1.904979 & 0.245901 & -0.050831 \\
\hline 7 & 6 & 0.875259 & 0.230584 & 1.108046 \\
\hline 8 & 6 & -0.269537 & -0.855824 & 0.871252 \\
\hline 9 & 6 & -1.368201 & -0.061759 & 0.133143 \\
\hline 10 & 6 & -1.737429 & -0.924650 & -1.094937 \\
\hline 11 & 1 & -2.241045 & 0.101903 & 0.771122 \\
\hline 12 & 6 & 0.111283 & -1.966019 & -0.112397 \\
\hline 13 & 8 & 0.980069 & -2.789066 & 0.020192 \\
\hline 14 & 1 & -0.244233 & 1.721790 & -2.290884 \\
\hline 15 & 8 & 3.040406 & -0.174237 & 0.082721 \\
\hline 16 & 6 & 1.600979 & 0.172159 & 2.440592 \\
\hline 17 & 1 & 2.156770 & -0.764548 & 2.524158 \\
\hline 18 & 1 & 2.319848 & 0.993772 & 2.498297 \\
\hline 19 & 1 & 0.901092 & 0.263311 & 3.273597 \\
\hline 20 & 6 & 2.369171 & 0.803181 & -2.501857 \\
\hline 21 & 1 & 3.280588 & 1.363740 & -2.265487 \\
\hline 22 & 1 & 2.684681 & -0.222519 & -2.723367 \\
\hline 23 & 1 & 1.910716 & 1.235954 & -3.395923 \\
\hline 24 & 6 & -1.689483 & 2.470385 & -0.166490 \\
\hline 25 & 1 & -2.348146 & 2.462806 & -1.035461 \\
\hline 26 & 6 & -1.797626 & 3.425953 & 0.754009 \\
\hline 27 & 1 & -1.142359 & 3.452443 & 1.618424 \\
\hline 28 & 1 & -2.543002 & 4.210943 & 0.664618 \\
\hline 29 & 6 & -0.757112 & -1.515722 & 2.172148 \\
\hline 30 & 1 & 0.035332 & -2.119672 & 2.621881 \\
\hline 31 & 1 & -1.086198 & -0.760195 & 2.891780 \\
\hline 32 & 1 & -1.606002 & -2.177977 & 1.968114 \\
\hline 33 & 6 & -3.096145 & -1.607052 & -0.996304 \\
\hline 34 & 1 & -3.895742 & -0.857307 & -0.999386 \\
\hline 35 & 1 & -3.247890 & -2.281101 & -1.844629 \\
\hline 36 & 1 & -3.177263 & -2.192303 & -0.073759 \\
\hline 37 & 1 & -1.682603 & -0.351320 & -2.026016 \\
\hline
\end{tabular}




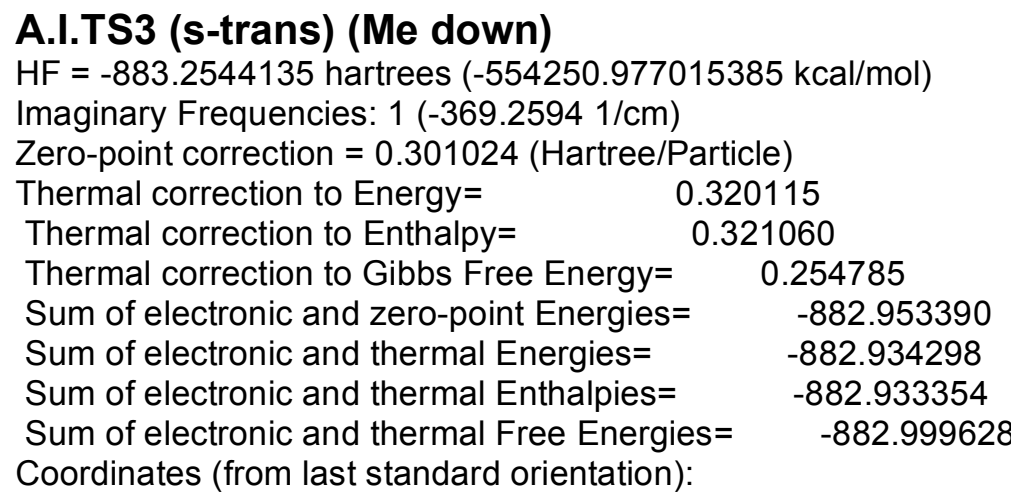

\begin{tabular}{|c|c|c|c|c|}
\hline \multirow{2}{*}{$\begin{array}{l}\text { Center } \\
\text { Number }\end{array}$} & \multirow{2}{*}{$\begin{array}{l}\text { Atomic } \\
\text { Number }\end{array}$} & \multicolumn{3}{|c|}{ Coordinates (Angstroms) } \\
\hline & & & $\begin{array}{ll}X & Y\end{array}$ & Z \\
\hline 1 & 8 & -0.300892 & 0.289168 & -1.473578 \\
\hline 2 & 6 & -0.506120 & 1.430079 & -0.791433 \\
\hline 3 & 6 & -1.662814 & 1.538833 & 0.004691 \\
\hline 4 & 6 & -2.488936 & 0.466557 & 0.263612 \\
\hline 5 & 6 & -2.048481 & -0.851756 & -0.166587 \\
\hline 6 & 6 & -0.785634 & -0.885869 & -0.955821 \\
\hline 7 & 6 & 0.589224 & -1.221661 & 0.466839 \\
\hline 8 & 6 & 0.707026 & -0.118776 & 1.324204 \\
\hline 9 & 6 & 1.930036 & -1.317384 & -0.267586 \\
\hline 10 & 6 & 1.938822 & 0.612733 & 1.001920 \\
\hline 11 & 8 & 2.591489 & -0.062320 & -0.009133 \\
\hline 12 & 8 & 2.384878 & 1.628023 & 1.488696 \\
\hline 13 & 1 & 1.827940 & -1.381183 & -1.352926 \\
\hline 14 & 1 & 0.104065 & -2.140540 & 0.797875 \\
\hline 15 & 1 & -1.893233 & 2.520657 & 0.410802 \\
\hline 16 & 8 & -2.634320 & -1.907500 & 0.111919 \\
\hline 17 & 6 & -0.679382 & -2.021168 & -1.941859 \\
\hline 18 & 1 & -0.752243 & -2.975057 & -1.416084 \\
\hline 19 & 1 & -1.518424 & -1.977008 & -2.646448 \\
\hline 20 & 1 & 0.249450 & -1.976448 & -2.514190 \\
\hline 21 & 6 & 0.347365 & 2.547763 & -1.130578 \\
\hline 22 & 6 & -3.808260 & 0.574342 & 0.967792 \\
\hline 23 & 1 & -4.634246 & 0.382496 & 0.270490 \\
\hline 24 & 1 & -3.887982 & -0.189710 & 1.748590 \\
\hline 25 & 1 & -3.953245 & 1.566102 & 1.406446 \\
\hline 26 & 6 & 2.797970 & -2.472116 & 0.230552 \\
\hline 27 & 1 & 2.331892 & -3.433257 & -0.015050 \\
\hline 28 & 1 & 3.783263 & -2.432190 & -0.243848 \\
\hline 29 & 1 & 2.932073 & -2.416620 & 1.315753 \\
\hline 30 & 6 & -0.082918 & 0.184983 & 2.548131 \\
\hline 31 & 1 & 0.067932 & -0.585642 & 3.317013 \\
\hline 32 & 1 & 0.223760 & 1.149520 & 2.962551 \\
\hline 33 & 1 & -1.161041 & 0.208296 & 2.339734 \\
\hline 34 & 6 & 1.433554 & 2.482596 & -1.917517 \\
\hline 35 & 1 & 2.036420 & 3.364284 & -2.105468 \\
\hline 36 & 1 & 0.071778 & 3.488334 & -0.660072 \\
\hline 37 & 1 & 1.753890 & 1.554070 & -2.376556 \\
\hline
\end{tabular}




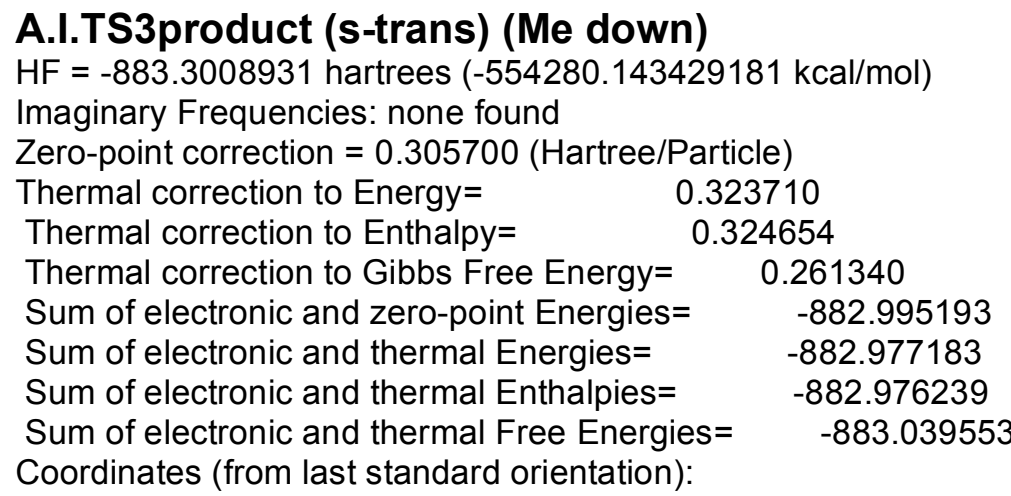

\begin{tabular}{|c|c|c|c|c|}
\hline \multirow{2}{*}{$\begin{array}{l}\text { Center } \\
\text { Number }\end{array}$} & \multirow{2}{*}{$\begin{array}{l}\text { Atomic } \\
\text { Number }\end{array}$} & \multicolumn{3}{|c|}{ Coordinates (Angstroms) } \\
\hline & & & $\begin{array}{l}X \\
Y\end{array}$ & Z \\
\hline 1 & 8 & 0.430830 & 0.484525 & 1.279078 \\
\hline 2 & 6 & 0.264357 & 1.235033 & 0.077512 \\
\hline 3 & 6 & 1.593994 & 1.262345 & -0.663492 \\
\hline 4 & 6 & 2.473616 & 0.249437 & -0.560768 \\
\hline 5 & 6 & 2.037322 & -0.958132 & 0.187752 \\
\hline 6 & 6 & 0.646612 & -0.868279 & 0.857703 \\
\hline 7 & 6 & -0.431871 & -1.090364 & -0.270407 \\
\hline 8 & 6 & -0.782294 & 0.349103 & -0.730953 \\
\hline 9 & 6 & -1.787147 & -1.686389 & 0.184506 \\
\hline 10 & 6 & -2.201633 & 0.568990 & -0.186176 \\
\hline 11 & 8 & -2.677562 & -0.563538 & 0.388467 \\
\hline 12 & 8 & -2.855958 & 1.576921 & -0.268933 \\
\hline 13 & 1 & -1.715863 & -2.187782 & 1.150944 \\
\hline 14 & 1 & -0.015228 & -1.708365 & -1.071226 \\
\hline 15 & 1 & 1.817197 & 2.134064 & -1.275705 \\
\hline 16 & 8 & 2.700126 & -1.983859 & 0.220581 \\
\hline 17 & 6 & 0.587957 & -1.766217 & 2.083307 \\
\hline 18 & 1 & 0.673445 & -2.817705 & 1.797915 \\
\hline 19 & 1 & 1.423732 & -1.532325 & 2.748626 \\
\hline 20 & 1 & -0.341229 & -1.603779 & 2.635023 \\
\hline 21 & 6 & -0.174350 & 2.639501 & 0.401027 \\
\hline 22 & 6 & 3.834254 & 0.221049 & -1.197875 \\
\hline 23 & 1 & 4.617457 & 0.143420 & -0.434562 \\
\hline 24 & 1 & 3.943450 & -0.658368 & -1.842083 \\
\hline 25 & 1 & 4.014573 & 1.123394 & -1.789509 \\
\hline 26 & 6 & -2.410363 & -2.638415 & -0.831137 \\
\hline 27 & 1 & -1.797359 & -3.541805 & -0.927376 \\
\hline 28 & 1 & -3.413798 & -2.932209 & -0.508762 \\
\hline 29 & 1 & -2.490799 & -2.169802 & -1.818298 \\
\hline 30 & 6 & -0.799197 & 0.607070 & -2.243905 \\
\hline 31 & 1 & -1.518263 & -0.049151 & -2.745916 \\
\hline 32 & 1 & -1.103101 & 1.638496 & -2.449803 \\
\hline 33 & 1 & 0.185882 & 0.430427 & -2.685325 \\
\hline 34 & 6 & -0.209030 & 3.148884 & 1.629969 \\
\hline 35 & 1 & -0.520307 & 4.175199 & 1.800413 \\
\hline 36 & 1 & -0.448119 & 3.246829 & -0.458971 \\
\hline 37 & 1 & 0.055469 & 2.555383 & 2.498701 \\
\hline
\end{tabular}




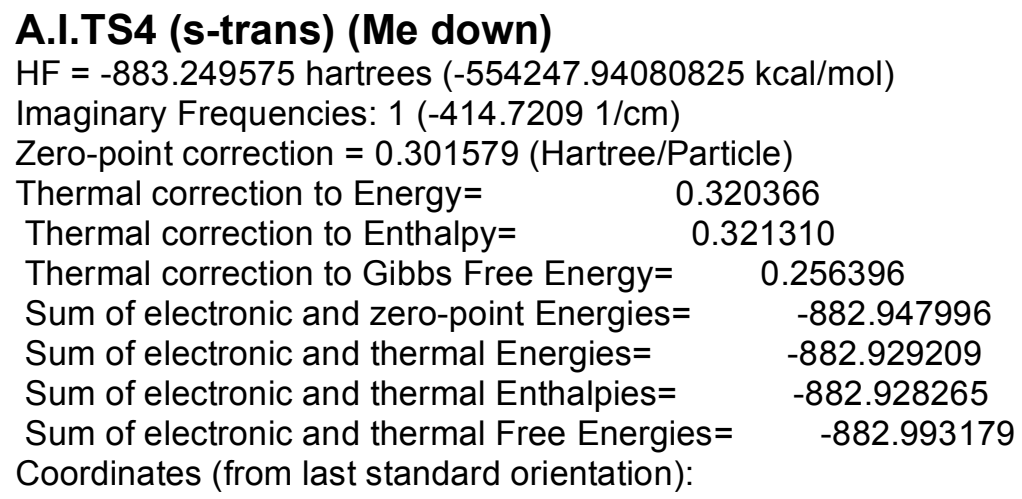

\begin{tabular}{|c|c|c|c|c|}
\hline \multirow{2}{*}{$\begin{array}{l}\text { Center } \\
\text { Number }\end{array}$} & \multirow{2}{*}{$\begin{array}{l}\text { Atomic } \\
\text { Number }\end{array}$} & \multicolumn{3}{|c|}{ Coordinates (Angstroms) } \\
\hline & & & $X \quad Y$ & Z \\
\hline 1 & 8 & -0.190306 & 0.822734 & 1.204119 \\
\hline 2 & 8 & -2.884780 & -1.369012 & 0.497324 \\
\hline 3 & 6 & -0.844393 & -0.353794 & 1.112392 \\
\hline 4 & 6 & -2.116428 & -0.407970 & 0.374015 \\
\hline 5 & 6 & -2.374200 & 0.719359 & -0.530455 \\
\hline 6 & 6 & -1.399139 & 1.654789 & -0.677319 \\
\hline 7 & 1 & -1.507337 & 2.466000 & -1.394337 \\
\hline 8 & 6 & -0.179294 & 1.616488 & 0.092891 \\
\hline 9 & 6 & 0.996603 & 0.214423 & -1.044608 \\
\hline 10 & 6 & 0.416256 & $6-1.037872$ & -0.756882 \\
\hline 11 & 6 & 2.364342 & 0.247913 & -0.382615 \\
\hline 12 & 6 & 1.381253 & $3-1.787164$ & 0.090543 \\
\hline 13 & 8 & 2.436907 & $7-0.968613$ & 0.396175 \\
\hline 14 & 8 & 1.315695 & $5-2.931015$ & 0.477875 \\
\hline 15 & 1 & 2.476211 & 11.073670 & 0.327514 \\
\hline 16 & 1 & 0.867113 & 0.700714 & -2.006040 \\
\hline 17 & 6 & -0.632895 & $5-1.271185$ & 2.270604 \\
\hline 18 & 1 & -1.411587 & $7 \quad-1.109032$ & 3.027201 \\
\hline 19 & 1 & -0.708942 & $2-2.309149$ & 1.939396 \\
\hline 20 & 1 & 0.350030 & -1.107758 & 2.717524 \\
\hline 21 & 6 & -3.683917 & 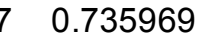 & -1.259004 \\
\hline 22 & 1 & -3.831824 & $4-0.209155$ & -1.794075 \\
\hline 23 & 1 & -4.517008 & $8 \quad 0.814122$ & -0.550246 \\
\hline 24 & 1 & -3.744716 & 1.568669 & -1.965945 \\
\hline 25 & 6 & 0.610109 & 2.843846 & 0.235637 \\
\hline 26 & 6 & -0.506237 & $7 \quad-1.835092$ & -1.641885 \\
\hline 27 & 1 & 0.083890 & -2.502563 & -2.283150 \\
\hline 28 & 1 & -1.185712 & $2-2.463277$ & -1.059425 \\
\hline 29 & 1 & -1.102426 & -1.184875 & -2.287821 \\
\hline 30 & 6 & 3.518875 & 0.282051 & -1.382611 \\
\hline 31 & 1 & 3.510706 & 1.228091 & -1.936371 \\
\hline 32 & 1 & 4.474429 & 0.197199 & -0.856086 \\
\hline 33 & 1 & 3.442552 & $2-0.542798$ & -2.098919 \\
\hline 34 & 6 & 1.362763 & 3.176202 & 1.292471 \\
\hline 35 & 1 & 1.925783 & 4.103929 & 1.307357 \\
\hline 36 & 1 & 0.559546 & 3.508353 & -0.625225 \\
\hline 37 & 1 & 1.416963 & 2.547334 & 2.175664 \\
\hline
\end{tabular}




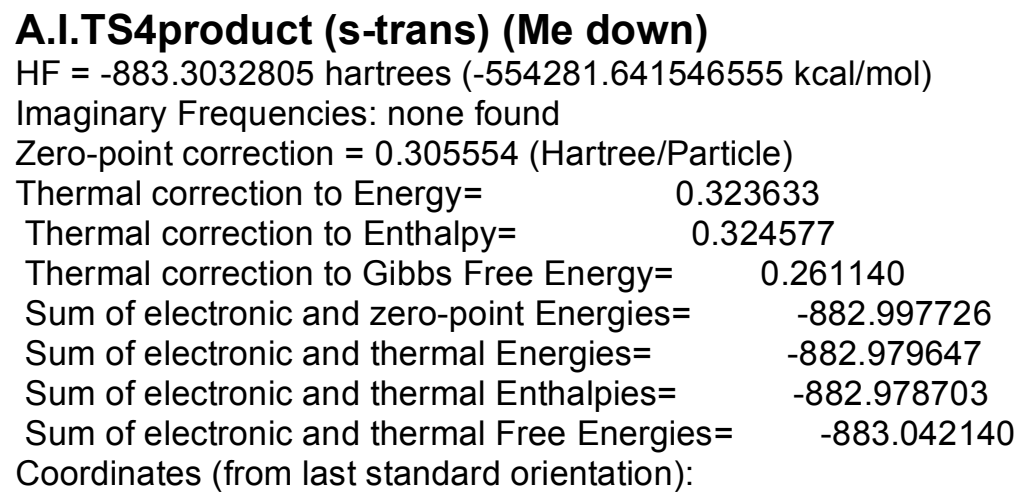

\begin{tabular}{|c|c|c|c|c|}
\hline \multirow{2}{*}{$\begin{array}{l}\text { Center } \\
\text { Number }\end{array}$} & \multirow{2}{*}{$\begin{array}{l}\text { Atomic } \\
\text { Number }\end{array}$} & \multicolumn{3}{|c|}{ Coordinates (Angstroms) } \\
\hline & & & $\begin{array}{ll}X & Y\end{array}$ & Z \\
\hline 1 & 8 & -0.294678 & 0.622727 & 1.232089 \\
\hline 2 & 8 & -2.895670 & -1.510269 & 0.346691 \\
\hline 3 & 6 & -0.651992 & -0.695147 & 0.781132 \\
\hline 4 & 6 & -2.088455 & -0.604809 & 0.217327 \\
\hline 5 & 6 & -2.402630 & 0.634028 & -0.548107 \\
\hline 6 & 6 & -1.421435 & 1.542381 & -0.683797 \\
\hline 7 & 1 & -1.554121 & 2.419728 & -1.315151 \\
\hline 8 & 6 & -0.094912 & 1.388360 & 0.043783 \\
\hline 9 & 6 & 0.861199 & 0.453875 & -0.781683 \\
\hline 10 & 6 & 0.369012 & -0.969505 & -0.415302 \\
\hline 11 & 6 & 2.334575 & 0.506811 & -0.300436 \\
\hline 12 & 6 & 1.632284 & -1.680888 & 0.088902 \\
\hline 13 & 8 & 2.684791 & -0.832444 & 0.126707 \\
\hline 14 & 8 & 1.731222 & -2.845753 & 0.383344 \\
\hline 15 & 1 & 2.423953 & 1.140255 & 0.585999 \\
\hline 16 & 1 & 0.796516 & 0.657807 & -1.854602 \\
\hline 17 & 6 & -0.592131 & -1.647094 & . 1.964282 \\
\hline 18 & 1 & -1.355217 & -1.368778 & 2.696273 \\
\hline 19 & 1 & -0.779948 & -2.672828 & 1.642752 \\
\hline 20 & 1 & 0.389849 & -1.602235 & 2.440073 \\
\hline 21 & 6 & -3.768951 & 0.741434 & -1.163827 \\
\hline 22 & 1 & -3.980351 & -0.125727 & -1.799473 \\
\hline 23 & 1 & -4.541441 & 0.747685 & -0.386115 \\
\hline 24 & 1 & -3.866359 & 1.653911 & -1.759421 \\
\hline 25 & 6 & 0.457231 & 2.748547 & 0.383807 \\
\hline 26 & 6 & -0.206769 & -1.815656 & -1.562623 \\
\hline 27 & 1 & 0.559862 & -1.995792 & -2.323816 \\
\hline 28 & 1 & -0.535677 & -2.788032 & -1.186218 \\
\hline 29 & 1 & -1.051922 & -1.320813 & -2.048375 \\
\hline 30 & 6 & 3.328089 & 0.952237 & -1.362326 \\
\hline 31 & 1 & 3.125303 & 1.989086 & -1.654594 \\
\hline 32 & 1 & 4.351956 & 0.896378 & -0.980528 \\
\hline 33 & 1 & 3.256872 & 0.319710 & -2.254323 \\
\hline 34 & 6 & 0.699009 & 3.184981 & 1.619011 \\
\hline 35 & 1 & 1.082856 & 4.185426 & 1.797028 \\
\hline 36 & 1 & 0.633065 & 3.396007 & -0.475422 \\
\hline 37 & 1 & 0.514739 & 2.556801 & 2.484413 \\
\hline
\end{tabular}




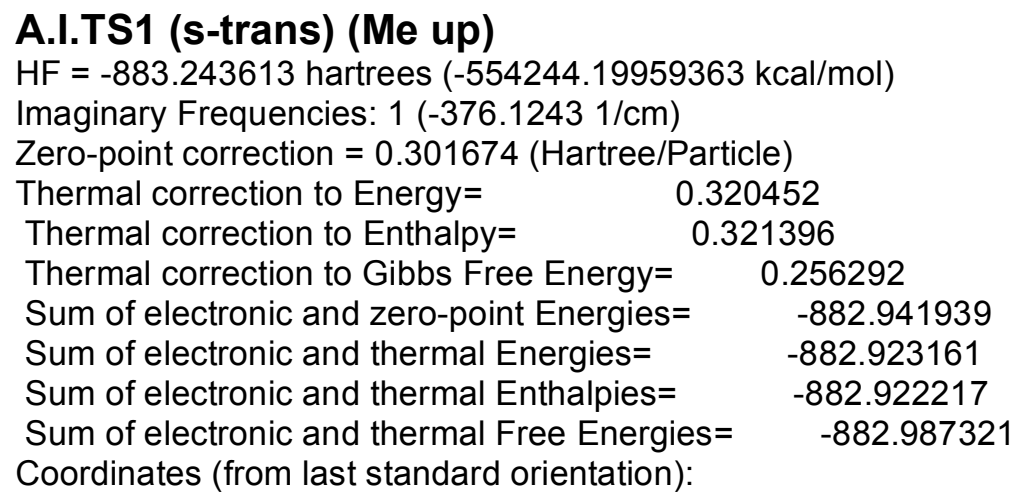

\begin{tabular}{|c|c|c|c|c|}
\hline \multirow{2}{*}{$\begin{array}{l}\text { Center } \\
\text { Number }\end{array}$} & \multirow{2}{*}{$\begin{array}{l}\text { Atomic } \\
\text { Number }\end{array}$} & \multicolumn{3}{|c|}{ Coordinates (Angstroms) } \\
\hline & & & $\begin{array}{ll}X & Y\end{array}$ & Z \\
\hline 1 & 8 & 0.985066 & -1.392213 & -0.610379 \\
\hline 2 & 8 & -2.466845 & -1.850381 & -0.122338 \\
\hline 3 & 6 & -0.332485 & -1.239713 & -0.961837 \\
\hline 4 & 8 & -1.048043 & 2.161885 & 0.440415 \\
\hline 5 & 6 & -1.325472 & -1.438344 & 0.122356 \\
\hline 6 & 6 & -0.870276 & -1.048276 & 1.453156 \\
\hline 7 & 6 & 0.450978 & -0.721363 & 1.609431 \\
\hline 8 & 1 & 0.827984 & -0.373779 & 2.567091 \\
\hline 9 & 6 & 1.391320 & -0.864977 & 0.557387 \\
\hline 10 & 6 & 0.828506 & 1.341855 & -0.639534 \\
\hline 11 & 6 & -0.281832 & 0.833074 & -1.336916 \\
\hline 12 & 6 & -1.465010 & 1.686242 & -0.859588 \\
\hline 13 & 1 & -0.215112 & 0.653774 & -2.407815 \\
\hline 14 & 1 & -1.458022 & 2.563475 & -1.527209 \\
\hline 15 & 6 & 0.326827 & 2.127852 & 0.508407 \\
\hline 16 & 8 & 0.946013 & 2.669873 & 1.396751 \\
\hline 17 & 6 & -0.631804 & -1.874142 & -2.290443 \\
\hline 18 & 1 & -0.683246 & -2.965007 & -2.189189 \\
\hline 19 & 1 & -1.608465 & -1.538009 & -2.644760 \\
\hline 20 & 1 & 0.137198 & -1.624249 & -3.026920 \\
\hline 21 & 6 & -1.862583 & -1.066550 & 2.574805 \\
\hline 22 & 1 & -2.705582 & -0.401478 & 2.352177 \\
\hline 23 & 1 & -2.286908 & -2.070298 & 2.693621 \\
\hline 24 & 1 & -1.408563 & -0.756497 & 3.520041 \\
\hline 25 & 6 & 2.821515 & -0.868000 & 0.797954 \\
\hline 26 & 1 & 3.118260 & -0.425424 & 1.745855 \\
\hline 27 & 6 & 3.750958 & -1.359209 & -0.037044 \\
\hline 28 & 1 & 3.483770 & -1.825457 & -0.980197 \\
\hline 29 & 1 & 4.804933 & -1.322178 & 0.217693 \\
\hline 30 & 6 & -2.898561 & 1.186759 & -0.810131 \\
\hline 31 & 1 & -3.548139 & 2.018077 & -0.517189 \\
\hline 32 & 1 & -3.204901 & 0.855483 & -1.808812 \\
\hline 33 & 1 & -3.053108 & 0.359048 & -0.122014 \\
\hline 34 & 6 & 2.218971 & 1.531098 & -1.156665 \\
\hline 35 & 1 & 2.228798 & 2.306703 & -1.936682 \\
\hline 36 & 1 & 2.887734 & 1.856802 & -0.356027 \\
\hline 37 & 1 & 2.627525 & 0.617868 & -1.603289 \\
\hline
\end{tabular}




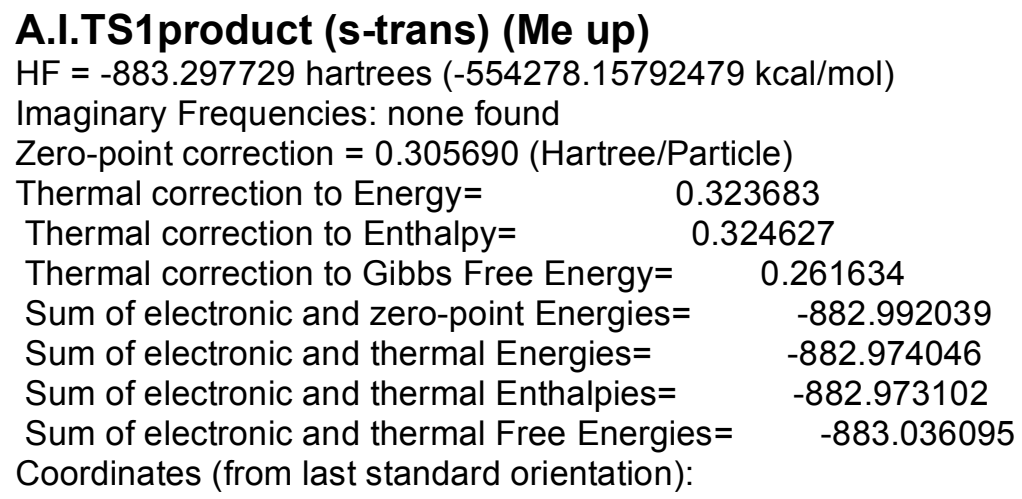

\begin{tabular}{|c|c|c|c|c|}
\hline \multirow{2}{*}{$\begin{array}{l}\text { Center } \\
\text { Number }\end{array}$} & \multirow{2}{*}{$\begin{array}{l}\text { Atomic } \\
\text { Number }\end{array}$} & \multicolumn{3}{|c|}{ Coordinates (Angstroms) } \\
\hline & & & $\begin{array}{ll}X \quad Y\end{array}$ & Z \\
\hline 1 & 8 & 0.825027 & -1.143265 & -1.079303 \\
\hline 2 & 8 & -2.488201 & -1.657008 & -0.119803 \\
\hline 3 & 6 & -0.537177 & -0.686434 & -1.182875 \\
\hline 4 & 8 & -0.990396 & 1.966952 & 0.915269 \\
\hline 5 & 6 & -1.318039 & -1.319633 & -0.005107 \\
\hline 6 & 6 & -0.573382 & -1.485228 & 1.267037 \\
\hline 7 & 6 & 0.692792 & -1.033981 & 1.309387 \\
\hline 8 & 1 & 1.258679 & -1.033414 & 2.237257 \\
\hline 9 & 6 & 1.362861 & -0.476292 & 0.064319 \\
\hline 10 & 6 & 0.900921 & $1 \quad 1.039674$ & -0.179578 \\
\hline 11 & 6 & -0.329935 & 0.869003 & -1.096964 \\
\hline 12 & 6 & -1.358430 & 1.848185 & -0.488868 \\
\hline 13 & 1 & -0.098637 & 1.186164 & -2.118644 \\
\hline 14 & 1 & -1.158481 & 2.832740 & -0.933365 \\
\hline 15 & 6 & 0.324473 & 1.671966 & 1.090213 \\
\hline 16 & 8 & 0.911688 & 1.884397 & 2.122643 \\
\hline 17 & 6 & -1.101372 & $2-1.156803$ & -2.514559 \\
\hline 18 & 1 & -1.088860 & - -2.248995 & -2.567960 \\
\hline 19 & 1 & -2.136864 & $4-0.827090$ & -2.627452 \\
\hline 20 & 1 & -0.498976 & $6-0.757670$ & -3.336249 \\
\hline 21 & 6 & -1.307613 & $3-2.092569$ & 2.428475 \\
\hline 22 & 1 & -2.214199 & $9-1.520691$ & 2.655630 \\
\hline 23 & 1 & -1.633240 & o & 2.191135 \\
\hline 24 & 1 & -0.676322 & $2-2.123770$ & 3.320970 \\
\hline 25 & 6 & 2.851072 & $2-0.679983$ & 0.140207 \\
\hline 26 & 1 & 3.323441 & $1 \quad-0.215921$ & 1.004940 \\
\hline 27 & 6 & 3.567625 & $5-1.380692$ & -0.736287 \\
\hline 28 & 1 & 3.103564 & $4-1.855829$ & -1.594440 \\
\hline 29 & 1 & 4.640011 & $1 \quad-1.502776$ & -0.614111 \\
\hline 30 & 6 & -2.847552 & $2 \quad 1.571851$ & -0.606622 \\
\hline 31 & 1 & -3.406696 & 2.372785 & -0.113038 \\
\hline 32 & 1 & -3.133918 & 1.562081 & -1.664488 \\
\hline 33 & 1 & -3.133973 & 0.617450 & -0.164974 \\
\hline 34 & 6 & 2.001048 & 1.933366 & -0.764228 \\
\hline 35 & 1 & 1.585433 & 2.896446 & -1.084272 \\
\hline 36 & 1 & 2.775437 & 2.135534 & -0.018830 \\
\hline 37 & 1 & 2.464908 & 1.451684 & -1.629860 \\
\hline
\end{tabular}




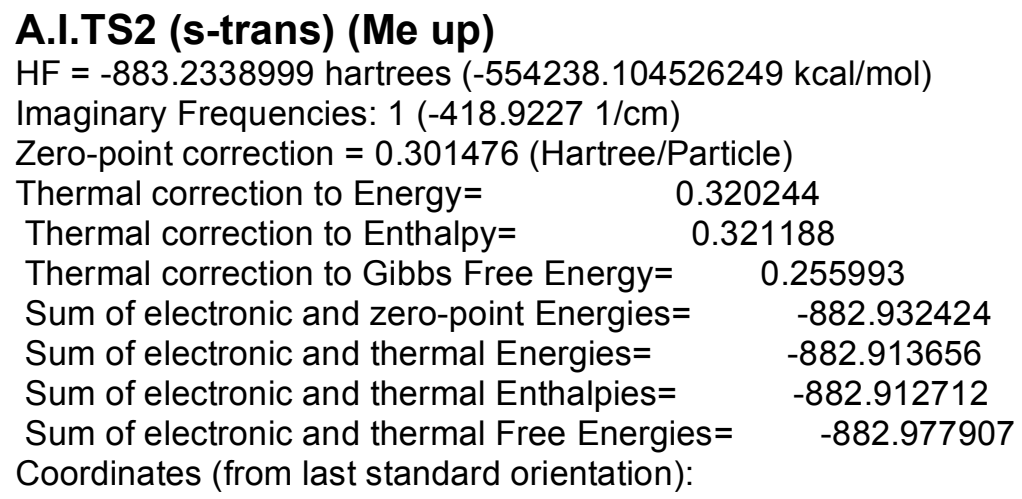

\begin{tabular}{|c|c|c|c|c|}
\hline \multirow{2}{*}{$\begin{array}{l}\text { Center } \\
\text { Number }\end{array}$} & \multirow{2}{*}{$\begin{array}{l}\text { Atomic } \\
\text { Number }\end{array}$} & \multicolumn{3}{|c|}{ Coordinates (Angstroms) } \\
\hline & & & K $\quad Y$ & Z \\
\hline 1 & 8 & -1.282110 & -1.200044 & 0.510080 \\
\hline 2 & 6 & -1.692243 & 0.091483 & 0.416847 \\
\hline 3 & 8 & 1.626165 & 1.405673 & -1.226034 \\
\hline 4 & 6 & -1.057233 & 1.026454 & 1.300708 \\
\hline 5 & 6 & 0.134504 & 0.761277 & 1.905179 \\
\hline 6 & 6 & 0.803186 & -0.498280 & 1.580692 \\
\hline 7 & 6 & 0.042919 & -1.415954 & 0.697288 \\
\hline 8 & 6 & 0.462529 & -0.609642 & -1.367680 \\
\hline 9 & 6 & -0.489452 & 0.417990 & -1.492267 \\
\hline 10 & 6 & 0.283811 & 1.719917 & -1.655512 \\
\hline 11 & 1 & -1.404458 & 0.287379 & -2.061286 \\
\hline 12 & 1 & 0.333139 & 1.893785 & -2.744015 \\
\hline 13 & 6 & 1.800812 & 0.040464 & -1.238030 \\
\hline 14 & 8 & 2.886300 & -0.478386 & -1.163892 \\
\hline 15 & 1 & -1.567790 & 1.971371 & 1.469380 \\
\hline 16 & 8 & 1.910821 & -0.817388 & 2.020593 \\
\hline 17 & 6 & 0.427281 & -2.860198 & 0.757463 \\
\hline 18 & 1 & 1.490982 & -2.956247 & 0.528883 \\
\hline 19 & 1 & 0.288228 & -3.234312 & 1.780321 \\
\hline 20 & 1 & -0.168904 & -3.469848 & 0.076821 \\
\hline 21 & 6 & 0.822697 & 1.681931 & 2.865757 \\
\hline 22 & 1 & 0.971242 & 1.190667 & 3.834466 \\
\hline 23 & 1 & 1.824133 & 1.927884 & 2.492929 \\
\hline 24 & 1 & 0.256254 & 2.605156 & 3.021550 \\
\hline 25 & 6 & -3.085982 & 0.262671 & 0.003480 \\
\hline 26 & 1 & -3.443424 & 1.289639 & 0.056611 \\
\hline 27 & 6 & -3.901743 & -0.705595 & -0.432635 \\
\hline 28 & 1 & -3.581189 & -1.741084 & -0.491030 \\
\hline 29 & 1 & -4.921790 & -0.484291 & -0.728983 \\
\hline 30 & 6 & 0.379497 & -1.921183 & -2.113788 \\
\hline 31 & 1 & 1.186585 & -2.597696 & -1.825733 \\
\hline 32 & 1 & -0.583711 & -2.416177 & -1.958131 \\
\hline 33 & 1 & 0.482222 & -1.734288 & -3.190998 \\
\hline 34 & 6 & -0.171014 & 3.026433 & -1.021138 \\
\hline 35 & 1 & 0.455357 & 3.846418 & -1.385897 \\
\hline 36 & 1 & -1.208547 & 3.237897 & -1.304232 \\
\hline 37 & 1 & -0.095151 & 2.998313 & 0.065190 \\
\hline
\end{tabular}




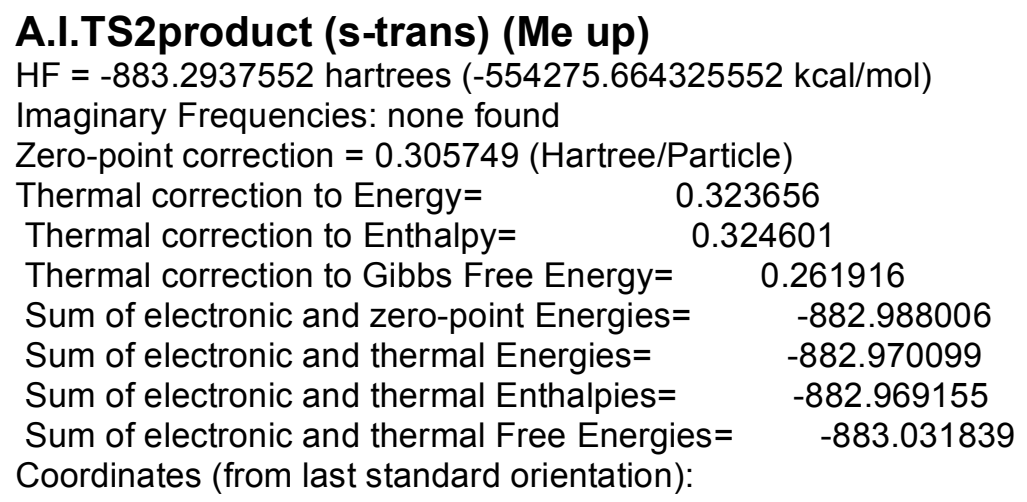

\begin{tabular}{|c|c|c|c|c|}
\hline \multirow{2}{*}{$\begin{array}{l}\text { Center } \\
\text { Number }\end{array}$} & \multirow{2}{*}{$\begin{array}{l}\text { Atomic } \\
\text { Number }\end{array}$} & \multicolumn{3}{|c|}{ Coordinates (Angstroms) } \\
\hline & & $r$ & K $\quad Y$ & Z \\
\hline 1 & 8 & -0.209583 & -1.545787 & -0.840151 \\
\hline 2 & 6 & -1.129647 & -0.870880 & 0.025374 \\
\hline 3 & 8 & -0.325084 & 2.320576 & 0.623530 \\
\hline 4 & 6 & -0.528114 & -0.812934 & 1.421787 \\
\hline 5 & 6 & 0.801445 & -0.848121 & 1.620256 \\
\hline 6 & 6 & 1.685351 & -0.857039 & 0.417502 \\
\hline 7 & 6 & 0.942565 & -0.681688 & -0.928388 \\
\hline 8 & 6 & 0.290007 & 0.764704 & -1.067606 \\
\hline 9 & 6 & -1.187653 & 0.532544 & -0.687310 \\
\hline 10 & 6 & -1.566812 & 1.823046 & 0.060992 \\
\hline 11 & 1 & -1.824196 & 0.401508 & -1.566939 \\
\hline 12 & 1 & -1.882523 & 2.557201 & -0.692852 \\
\hline 13 & 6 & 0.752428 & 1.779212 & -0.009658 \\
\hline 14 & 8 & 1.882595 & 2.109864 & 0.243925 \\
\hline 15 & 1 & -1.209481 & -0.775466 & 2.269229 \\
\hline 16 & 8 & 2.888745 & -1.022376 & 0.490459 \\
\hline 17 & 6 & 1.811536 & -1.153733 & -2.079654 \\
\hline 18 & 1 & 2.703494 & -0.528411 & -2.162961 \\
\hline 19 & 1 & 2.140729 & -2.178032 & -1.887201 \\
\hline 20 & 1 & 1.259204 & -1.136205 & -3.021832 \\
\hline 21 & 6 & 1.470443 & -0.863355 & 2.964186 \\
\hline 22 & 1 & 2.113764 & -1.744595 & 3.063188 \\
\hline 23 & 1 & 2.120430 & 0.012149 & 3.075638 \\
\hline 24 & 1 & 0.736663 & -0.866915 & 3.775629 \\
\hline 25 & 6 & -2.451705 & -1.593746 & 0.032445 \\
\hline 26 & 1 & -3.190848 & -1.190990 & 0.723719 \\
\hline 27 & 6 & -2.742111 & -2.647972 & -0.727267 \\
\hline 28 & 1 & -2.012342 & -3.069189 & -1.410760 \\
\hline 29 & 1 & -3.718334 & -3.121980 & -0.680322 \\
\hline 30 & 6 & 0.477482 & 1.420677 & -2.446858 \\
\hline 31 & 1 & 1.533764 & 1.631454 & -2.636393 \\
\hline 32 & 1 & 0.094048 & 0.777800 & -3.244225 \\
\hline 33 & 1 & -0.064219 & 2.372713 & -2.494906 \\
\hline 34 & 6 & -2.625560 & 1.779791 & 1.150883 \\
\hline 35 & 1 & -2.779919 & 2.783757 & 1.557814 \\
\hline 36 & 1 & -3.577239 & 1.429701 & 0.736118 \\
\hline 37 & 1 & -2.334301 & 1.124249 & 1.974855 \\
\hline
\end{tabular}




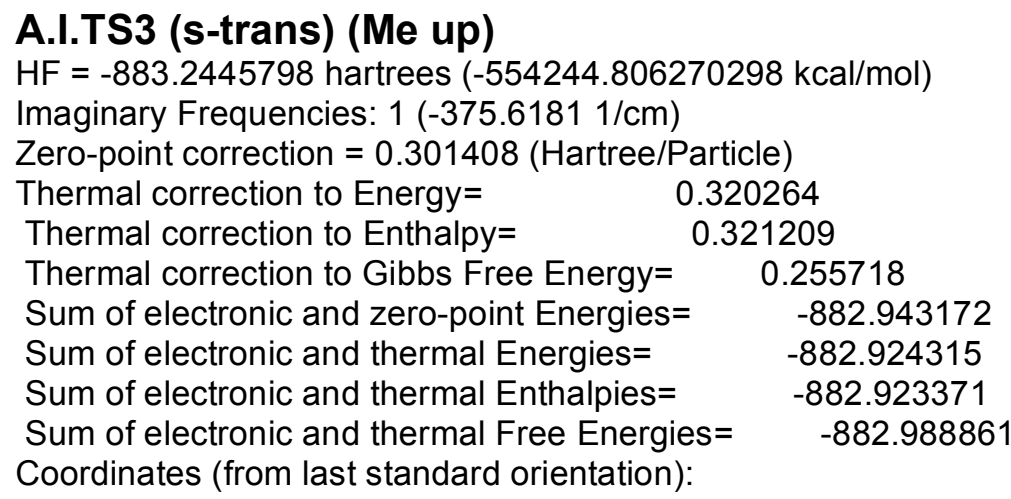

\begin{tabular}{|c|c|c|c|c|}
\hline \multirow{2}{*}{$\begin{array}{l}\text { Center } \\
\text { Number }\end{array}$} & \multirow{2}{*}{$\begin{array}{l}\text { Atomic } \\
\text { Number }\end{array}$} & \multicolumn{3}{|c|}{ Coordinates (Angstroms) } \\
\hline & & & $\begin{array}{l}X \quad Y \\
\end{array}$ & Z \\
\hline 1 & 8 & -0.076109 & 0.338352 & 1.398952 \\
\hline 2 & 6 & -0.238538 & -0.989603 & 1.261938 \\
\hline 3 & 6 & -1.460640 & -1.472016 & 0.757064 \\
\hline 4 & 6 & -2.415248 & -0.642552 & 0.208113 \\
\hline 5 & 6 & -2.051724 & 0.746707 & -0.016657 \\
\hline 6 & 6 & -0.726849 & 1.175605 & 0.523079 \\
\hline 7 & 6 & 0.456293 & 0.984788 & -1.090781 \\
\hline 8 & 6 & 0.506890 & -0.364533 & -1.483367 \\
\hline 9 & 6 & 1.928689 & 1.351374 & -0.805152 \\
\hline 10 & 6 & 1.818325 & -0.925871 & -1.140342 \\
\hline 11 & 8 & 2.595517 & 0.087375 & -0.619943 \\
\hline 12 & 8 & 2.235149 & -2.056165 & -1.268691 \\
\hline 13 & 1 & -0.141444 & 1.698750 & -1.659302 \\
\hline 14 & 1 & -1.636002 & -2.542844 & 0.824771 \\
\hline 15 & 8 & -2.756677 & 1.563827 & -0.624655 \\
\hline 16 & 6 & -0.705527 & 2.617878 & 0.979076 \\
\hline 17 & 1 & -0.649680 & 3.294271 & 0.123000 \\
\hline 18 & 1 & -1.643514 & 2.847567 & 1.496710 \\
\hline 19 & 1 & 0.127972 & 2.811032 & 1.652565 \\
\hline 20 & 6 & 0.762171 & -1.833413 & 1.878169 \\
\hline 21 & 6 & -3.796635 & -1.084788 & -0.173545 \\
\hline 22 & 1 & -4.541123 & -0.654565 & 0.509219 \\
\hline 23 & 1 & -4.053505 & -0.718373 & -1.173010 \\
\hline 24 & 1 & -3.895227 & -2.174063 & -0.143796 \\
\hline 25 & 6 & 2.369443 & 2.277745 & 0.317082 \\
\hline 26 & 1 & 3.461355 & 2.349723 & 0.293696 \\
\hline 27 & 1 & 1.960642 & 3.282970 & 0.180119 \\
\hline 28 & 1 & 2.075462 & 1.897263 & 1.296643 \\
\hline 29 & 1 & 2.302078 & 1.776356 & -1.750334 \\
\hline 30 & 6 & -0.451756 & -1.108727 & -2.341375 \\
\hline 31 & 1 & -0.534997 & -0.644010 & -3.333521 \\
\hline 32 & 1 & -0.117628 & -2.142607 & -2.465773 \\
\hline 33 & 1 & -1.465840 & -1.110164 & -1.918015 \\
\hline 34 & 6 & 1.910410 & -1.409890 & 2.430567 \\
\hline 35 & 1 & 2.616545 & -2.117324 & 2.851481 \\
\hline 36 & 1 & 2.179135 & -0.360222 & 2.468792 \\
\hline 37 & 1 & 0.540917 & -2.897087 & 1.838228 \\
\hline
\end{tabular}




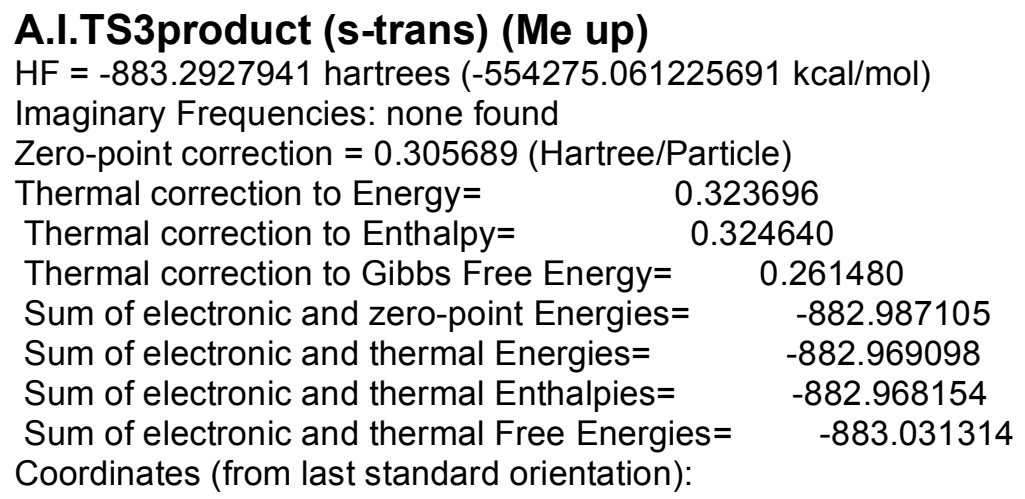

\begin{tabular}{|c|c|c|c|c|}
\hline Center & Atomic & & ordinates & Angstroms) \\
\hline Number & Numbe & & Y & Z \\
\hline 1 & 8 & 0.312396 & 0.343013 & 1.253031 \\
\hline 2 & 6 & 0.289598 & 1.253872 & 0.156005 \\
\hline 3 & 6 & 1.671274 & 1.278608 & -0.485589 \\
\hline 4 & 6 & 2.491932 & 0.213638 & -0.426510 \\
\hline 5 & 6 & 1.948552 & -1.041970 & 0.159567 \\
\hline 6 & 6 & 0.492457 & -0.959564 & 0.675600 \\
\hline 7 & 6 & -0.423725 & -0.957572 & -0.616491 \\
\hline 8 & 6 & -0.739510 & 0.552513 & -0.812158 \\
\hline 9 & 6 & -1.843298 & -1.571375 & -0.633631 \\
\hline 10 & 6 & -2.171069 & 0.672802 & -0.251692 \\
\hline 11 & 8 & -2.703372 & -0.562391 & -0.049086 \\
\hline 12 & 8 & -2.786662 & 1.685886 & -0.044978 \\
\hline 13 & 1 & 0.135939 & -1.383330 & -1.455431 \\
\hline 14 & 1 & 1.985810 & 2.195851 & -0.979861 \\
\hline 15 & 8 & 2.582746 & -2.085278 & 0.174374 \\
\hline 16 & 6 & 0.263540 & -1.975615 & 1.782629 \\
\hline 17 & 1 & 0.354461 & -2.996812 & 1.409503 \\
\hline 18 & 1 & 1.023992 & -1.833081 & 2.556070 \\
\hline 19 & 1 & -0.717511 & -1.825246 & 2.236924 \\
\hline 20 & 6 & -0.073956 & 2.635452 & 0.635186 \\
\hline 21 & 6 & 3.897821 & 0.179917 & -0.956631 \\
\hline 22 & 1 & 4.610261 & -0.021787 & -0.148155 \\
\hline 23 & 1 & 4.020746 & -0.630073 & -1.684220 \\
\hline 24 & 1 & 4.167475 & 1.129150 & -1.428859 \\
\hline 25 & 6 & -2.156420 & -2.921718 & -0.009958 \\
\hline 26 & 1 & -3.170078 & -3.221627 & -0.293763 \\
\hline 27 & 1 & -1.459990 & -3.681014 & -0.381622 \\
\hline 28 & 1 & -2.105033 & -2.898812 & 1.077911 \\
\hline 29 & 1 & -2.119553 & -1.635253 & -1.697065 \\
\hline 30 & 6 & -0.730596 & 1.063105 & -2.258400 \\
\hline 31 & 1 & -1.418564 & 0.484833 & -2.885661 \\
\hline 32 & 1 & -1.056200 & 2.107703 & -2.296672 \\
\hline 33 & 1 & 0.269833 & 0.985738 & -2.695276 \\
\hline 34 & 6 & -0.116858 & 2.996674 & 1.915199 \\
\hline 35 & 1 & -0.364383 & 4.014832 & 2.200412 \\
\hline 36 & 1 & 0.074005 & 2.286123 & 2.712462 \\
\hline 37 & 1 & -0.270508 & 3.360353 & -0.152185 \\
\hline
\end{tabular}




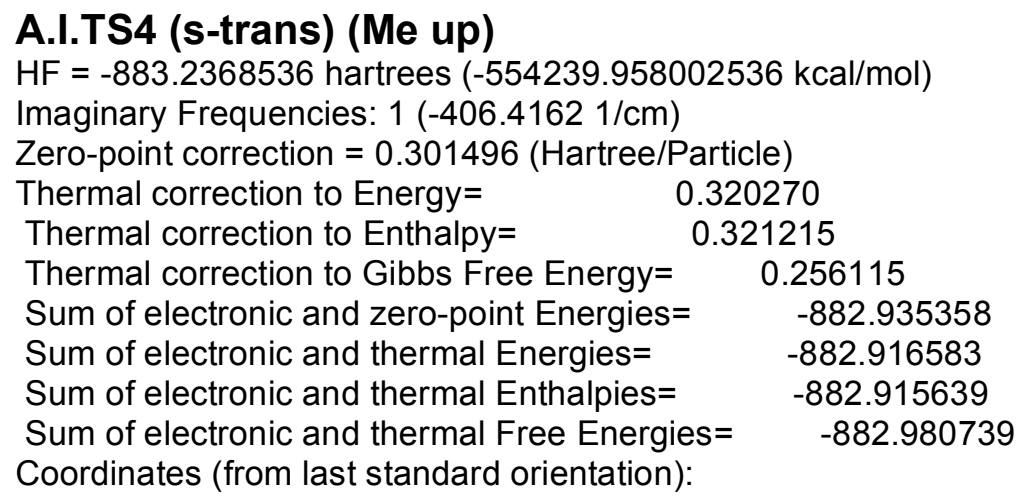

\begin{tabular}{|c|c|c|c|c|}
\hline \multirow{2}{*}{$\begin{array}{l}\text { Center } \\
\text { Number }\end{array}$} & \multirow{2}{*}{$\begin{array}{l}\text { Atomic } \\
\text { Number }\end{array}$} & \multicolumn{3}{|c|}{ Coordinates (Angstroms) } \\
\hline & & & $\begin{array}{ll}X & Y\end{array}$ & Z \\
\hline 1 & 8 & 0.119728 & 0.852237 & 1.065744 \\
\hline 2 & 8 & -2.883758 & -1.019764 & 0.894428 \\
\hline 3 & 6 & -0.683179 & -0.220921 & 1.174300 \\
\hline 4 & 6 & -2.028345 & -0.178506 & 0.592566 \\
\hline 5 & 6 & -2.260808 & 0.889012 & -0.390005 \\
\hline 6 & 6 & -1.205942 & 1.664987 & -0.747512 \\
\hline 7 & 1 & -1.305869 & 2.418855 & -1.525457 \\
\hline 8 & 6 & 0.090464 & 1.544310 & -0.113291 \\
\hline 9 & 6 & 0.954953 & -0.035311 & -1.264302 \\
\hline 10 & 6 & 0.208511 & -1.172775 & -0.886623 \\
\hline 11 & 6 & 2.411358 & -0.341304 & -0.877478 \\
\hline 12 & 6 & 1.100347 & -2.074674 & -0.120911 \\
\hline 13 & 8 & 2.331623 & -1.483353 & 0.001917 \\
\hline 14 & 8 & 0.859845 & -3.167852 & 0.339122 \\
\hline 15 & 1 & 0.792973 & 0.445404 & -2.224764 \\
\hline 16 & 6 & -0.407024 & -1.095481 & 2.349640 \\
\hline 17 & 1 & 0.645896 & -1.037473 & 2.634106 \\
\hline 18 & 1 & -1.033436 & -0.797958 & 3.200955 \\
\hline 19 & 1 & -0.658835 & -2.129795 & 2.103844 \\
\hline 20 & 6 & -3.640691 & 1.014077 & -0.960774 \\
\hline 21 & 1 & -3.691056 & 1.782747 & -1.737568 \\
\hline 22 & 1 & -3.966896 & 0.053261 & -1.375134 \\
\hline 23 & 1 & -4.360981 & 1.261854 & -0.172014 \\
\hline 24 & 6 & 0.920117 & 2.767148 & -0.115604 \\
\hline 25 & 6 & -0.946815 & -1.798028 & -1.622076 \\
\hline 26 & 1 & -0.569723 & -2.586984 & -2.286541 \\
\hline 27 & 1 & -1.660203 & -2.269256 & -0.939684 \\
\hline 28 & 1 & -1.477561 & -1.065966 & -2.236091 \\
\hline 29 & 6 & 3.329904 & 0.681678 & -0.232311 \\
\hline 30 & 1 & 4.316190 & 0.227422 & -0.092938 \\
\hline 31 & 1 & 3.443098 & 1.563343 & -0.869887 \\
\hline 32 & 1 & 2.953144 & 0.998906 & 0.740000 \\
\hline 33 & 1 & 2.891825 & -0.691092 & -1.805776 \\
\hline 34 & 6 & 1.464413 & 3.348149 & 0.957033 \\
\hline 35 & 1 & 2.039621 & 4.264112 & 0.864835 \\
\hline 36 & 1 & 1.019967 & 3.231299 & -1.095653 \\
\hline 37 & 1 & 1.358776 & 2.927020 & 1.952151 \\
\hline
\end{tabular}




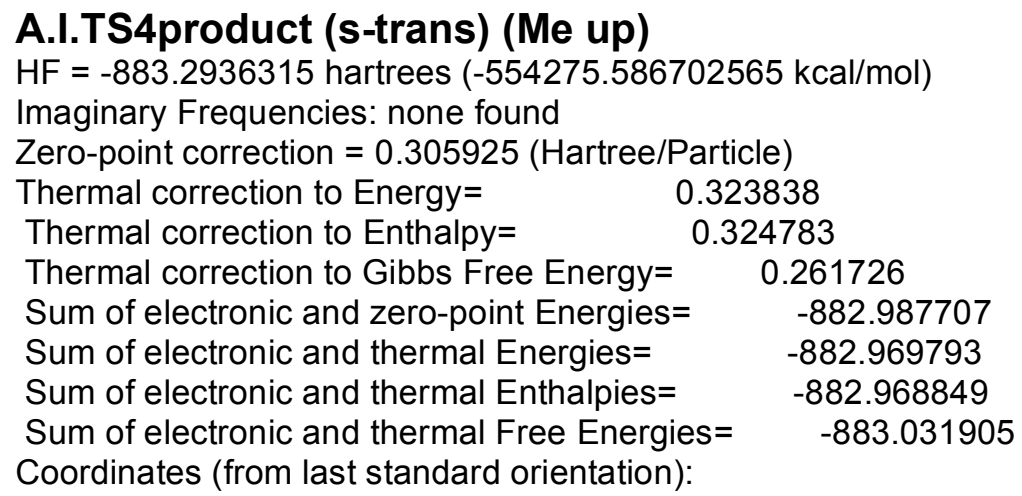

\begin{tabular}{|c|c|c|c|c|}
\hline \multirow{2}{*}{$\begin{array}{l}\text { Center } \\
\text { Number }\end{array}$} & \multirow{2}{*}{$\begin{array}{l}\text { Atomic } \\
\text { Number }\end{array}$} & \multicolumn{3}{|c|}{ Coordinates (Angstroms) } \\
\hline & & & $X \quad Y$ & Z \\
\hline 1 & 8 & -0.191209 & 0.488029 & 1.229020 \\
\hline 2 & 8 & -3.013185 & -1.297309 & 0.104100 \\
\hline 3 & 6 & -0.737866 & -0.736019 & 0.723952 \\
\hline 4 & 6 & -2.140312 & -0.443398 & 0.132411 \\
\hline 5 & 6 & -2.346594 & 0.910799 & -0.445415 \\
\hline 6 & 6 & -1.275299 & 1.718960 & -0.514655 \\
\hline 7 & 1 & -1.330632 & 2.684035 & -1.016602 \\
\hline 8 & 6 & 0.064172 & 1.301363 & 0.084236 \\
\hline 9 & 6 & 0.731877 & 0.310033 & -0.965086 \\
\hline 10 & 6 & 0.266597 & -1.085098 & -0.444958 \\
\hline 11 & 6 & 2.266099 & $9 \quad 0.132821$ & -1.092853 \\
\hline 12 & 6 & 1.563806 & -1.686342 & 0.135542 \\
\hline 13 & 8 & 2.627531 & $1-0.893918$ & -0.139927 \\
\hline 14 & 8 & 1.676601 & -2.730727 & 0.725813 \\
\hline 15 & 1 & 0.335223 & $\begin{array}{ll}3 & 0.533923\end{array}$ & -1.958665 \\
\hline 16 & 6 & -0.863773 & $3-1.724359$ & 1.874698 \\
\hline 17 & 1 & 0.096140 & -1.872663 & 2.367982 \\
\hline 18 & 1 & -1.586061 & $1-1.335935$ & 2.598912 \\
\hline 19 & 1 & -1.230083 & $3 \quad-2.686918$ & 1.513674 \\
\hline 20 & 6 & -3.715573 & $3 \quad 1.243942$ & -0.967837 \\
\hline 21 & 1 & -3.731694 & 2.231478 & -1.438449 \\
\hline 22 & 1 & -4.047365 & 0.494555 & -1.694876 \\
\hline 23 & 1 & -4.452487 & 1.233022 & -0.156193 \\
\hline 24 & 6 & 0.811934 & 2.538780 & 0.508326 \\
\hline 25 & 6 & -0.277321 & $1 \quad-2.057637$ & -1.502698 \\
\hline 26 & 1 & 0.467973 & $3-2.236616$ & -2.286101 \\
\hline 27 & 1 & -0.513550 & -3.021035 & -1.041927 \\
\hline 28 & 1 & -1.182920 & D -1.671084 & -1.977886 \\
\hline 29 & 6 & 3.224261 & $1 \quad 1.299785$ & -0.927503 \\
\hline 30 & 1 & 4.243573 & 0.956663 & -1.131255 \\
\hline 31 & 1 & 2.985009 & 2.095049 & -1.642241 \\
\hline 32 & 1 & 3.188624 & 1.709163 & 0.082088 \\
\hline 33 & 1 & 2.434980 & -0.302321 & -2.088898 \\
\hline 34 & 6 & 1.098180 & 2.853187 & 1.770653 \\
\hline 35 & 1 & 1.600271 & 3.784760 & 2.015819 \\
\hline 36 & 1 & 1.059599 & 3.225314 & -0.300059 \\
\hline 37 & 1 & 0.844364 & 2.189091 & 2.589748 \\
\hline
\end{tabular}




\begin{tabular}{|c|c|c|c|c|}
\hline \multicolumn{5}{|c|}{$\begin{array}{l}\mathrm{HF}=-727.2028938 \text { hartrees }(-456327.087888438 \mathrm{kcal} / \\
\text { Imaginary Frequencies: } 1(-386.65951 / \mathrm{cm}) \\
\text { Zero-point correction }=0.213110(\text { Hartree } / \text { Particle })\end{array}$} \\
\hline & \multicolumn{4}{|c|}{ Thermal correction to Energy $=\quad 0.226337$} \\
\hline \multicolumn{4}{|c|}{ Thermal correction to Enthalpy= } & 0.227281 \\
\hline \multicolumn{5}{|c|}{ Thermal correction to Gibbs Free Energy $=0.1738$} \\
\hline \multicolumn{5}{|c|}{ Sum of electronic and zero-point Energies= } \\
\hline \multirow{2}{*}{\multicolumn{5}{|c|}{ Sum of electronic and thermal Energies $=$}} \\
\hline & & & & \\
\hline \multicolumn{5}{|c|}{ Sum of electronic and thermal Free Energies= } \\
\hline \multicolumn{5}{|c|}{ Coordinates (from last standard orientation): } \\
\hline \multirow{2}{*}{$\begin{array}{l}\text { Center } \\
\text { Number }\end{array}$} & \multirow{2}{*}{\multicolumn{2}{|c|}{$\begin{array}{l}\text { Atomic } \\
\text { Number }\end{array}$}} & \multicolumn{2}{|c|}{ Coordinates (Angstroms) } \\
\hline & & & $X \quad Y$ & Z \\
\hline 1 & 8 & -1.450213 & -1.343050 & -1.10035 \\
\hline 2 & 8 & -2.537504 & 00 & \\
\hline 3 & 6 & -1.629009 & -0.003850 & 2380 \\
\hline 4 & 8 & 1.295392 & 0879 & 7291 \\
\hline 5 & 6 & -2.097353 & 0.685422 & 20195 \\
\hline 6 & 6 & -1.872263 & -0.082136 & 35495 \\
\hline 7 & 6 & .257126 & & 6693 \\
\hline 8 & 1 & & & \\
\hline 9 & 6 & 51 & & \\
\hline 10 & 6 & 1.114740 & -0 & 5377 \\
\hline 11 & 6 & 0.491464 & 5081 & 3605 \\
\hline 12 & 6 & 0.753892 & 1.611725 & 5170 \\
\hline 13 & 1 & -1.966883 & 0.364670 & -2.055347 \\
\hline 14 & 1 & -0.649175 & -2.913505 & -0.107971 \\
\hline 15 & 1 & -2.168617 & 9524 & 2.281488 \\
\hline 16 & 1 & 0.445435 & & -2.099688 \\
\hline 17 & 6 & & & \\
\hline 18 & 8 & 2.220297 & 02979 & 1.544298 \\
\hline 19 & 6 & 1.766083 & 2.642651 & -0.533273 \\
\hline 20 & 1 & 1.348575 & & 376835 \\
\hline 21 & 1 & 2.002309 & 3.349366 & 0.267850 \\
\hline 22 & 1 & 2.693985 & 2.159108 & -0.856698 \\
\hline 23 & 1 & -0.151865 & 2.124818 & 0.297810 \\
\hline 24 & 6 & 1.669546 & -1.790905 & -1.382308 \\
\hline 25 & 1 & 1.945130 & -2.624228 & -0.729800 \\
\hline 26 & 1 & 0.957969 & -2.140367 & -2.137612 \\
\hline 27 & 1 & 2.579525 & -1.476496 & -1.912310 \\
\hline
\end{tabular}

\section{E.I.TS1product (Me down)}

$\mathrm{HF}=-727.268803$ hartrees $(-456368.44657053 \mathrm{kcal} / \mathrm{mol})$ Imaginary Frequencies: none found Zero-point correction $=0.217745$ (Hartree/Particle)

Thermal correction to Energy $=\quad 0.230111$ Thermal correction to Enthalpy= $\quad 0.231055$ Thermal correction to Gibbs Free Energy $=0.179368$ Sum of electronic and zero-point Energies $=\quad-727.051058$ Sum of electronic and thermal Energies $=\quad-727.038692$ Sum of electronic and thermal Enthalpies $=\quad-727.037748$ 
Sum of electronic and thermal Free Energies=

$-727.089435$

Coordinates (from last standard orientation):

\begin{tabular}{|c|c|c|c|c|}
\hline \multirow{2}{*}{$\begin{array}{l}\text { Center } \\
\text { Number }\end{array}$} & \multirow{2}{*}{$\begin{array}{l}\text { Atomic } \\
\text { Number }\end{array}$} & \multicolumn{3}{|c|}{ Coordinates (Angstroms) } \\
\hline & & & $\begin{array}{l}X \\
Y\end{array}$ & Z \\
\hline 1 & 8 & 1.300869 & -1.082179 & 1.413545 \\
\hline 2 & 8 & 2.694832 & 1.618286 & -0.344278 \\
\hline 3 & 6 & 1.275808 & 0.315176 & 1.107463 \\
\hline 4 & 8 & -1.271538 & 0.850446 & -1.222099 \\
\hline 5 & 6 & 2.088624 & 0.572669 & -0.170593 \\
\hline 6 & 6 & 1.993889 & -0.503013 & -1.179613 \\
\hline 7 & 6 & 1.186716 & -1.549789 & -0.937926 \\
\hline 8 & 1 & 0.997978 & -2.314964 & -1.686274 \\
\hline 9 & 6 & 0.477671 & -1.650435 & 0.394227 \\
\hline 10 & 6 & -0.822756 & -0.755588 & 0.465515 \\
\hline 11 & 6 & -0.254177 & 0.603867 & 0.937996 \\
\hline 12 & 6 & -0.709949 & 1.628854 & -0.128608 \\
\hline 13 & 1 & 1.728220 & 0.864152 & 1.935046 \\
\hline 14 & 1 & 0.273973 & -2.688999 & 0.662848 \\
\hline 15 & 1 & 2.543070 & -0.374238 & -2.107720 \\
\hline 16 & 1 & -0.651633 & 0.874870 & 1.919785 \\
\hline 17 & 6 & -1.415434 & -0.460908 & -0.911307 \\
\hline 18 & 8 & -1.933953 & -1.255604 & -1.656104 \\
\hline 19 & 6 & -1.764500 & 2.609807 & 0.366042 \\
\hline 20 & 1 & -1.338219 & 3.262334 & 1.136495 \\
\hline 21 & 1 & -2.121549 & 3.234420 & -0.457946 \\
\hline 22 & 1 & -2.623401 & 2.081571 & 0.795591 \\
\hline 23 & 1 & 0.133477 & 2.177848 & -0.555321 \\
\hline 24 & 6 & -1.868375 & -1.393867 & 1.386765 \\
\hline 25 & 1 & -2.281762 & -2.293113 & 0.919294 \\
\hline 26 & 1 & -1.409368 & -1.665914 & 2.343140 \\
\hline 27 & 1 & -2.697568 & -0.706190 & 1.586791 \\
\hline
\end{tabular}

\section{E.I.TS2 (Me down)}

$\mathrm{HF}=-727.1853374$ hartrees $(-456316.071071874 \mathrm{kcal} / \mathrm{mol})$

Imaginary Frequencies: $1(-406.18861 / \mathrm{cm})$

Zero-point correction $=0.212814$ (Hartree/Particle)

Thermal correction to Energy $=\quad 0.226084$

Thermal correction to Enthalpy $=\quad 0.227028$

Thermal correction to Gibbs Free Energy $=0.173196$

Sum of electronic and zero-point Energies $=\quad-726.972524$

Sum of electronic and thermal Energies $=\quad-726.959254$

Sum of electronic and thermal Enthalpies $=\quad-726.958310$

Sum of electronic and thermal Free Energies $=\quad-727.012142$

Coordinates (from last standard orientation):

\begin{tabular}{|c|c|c|c|c|}
\hline Center & Atomic & & oordinates $(A$ & ngstroms) \\
\hline Number & Number & & $\begin{array}{ll}X & Y\end{array}$ & Z \\
\hline 1 & 8 & -1.524005 & 0.417090 & 1.538150 \\
\hline 2 & 8 & -2.292665 & -0.867281 & -1.688629 \\
\hline 3 & 6 & -1.676059 & -0.485484 & 0.559466 \\
\hline 4 & 8 & 1.797869 & -0.246086 & -1.093745 \\
\hline 5 & 6 & -1.870420 & -0.079855 & -0.842794 \\
\hline
\end{tabular}




$\begin{array}{ccrrr}6 & 6 & -1.529010 & 1.320478 & -1.079374 \\ 7 & 6 & -0.997616 & 2.072847 & -0.072437 \\ 8 & 1 & -0.689989 & 3.101862 & -0.233462 \\ 9 & 6 & -0.829610 & 1.529510 & 1.231911 \\ 10 & 6 & 1.125003 & 0.251123 & 1.094625 \\ 11 & 1 & 1.337144 & 0.541245 & 2.118982 \\ 12 & 6 & 0.584431 & -0.988464 & 0.753755 \\ 13 & 6 & 1.039084 & -1.299600 & -0.635447 \\ 14 & 1 & -2.141279 & -1.401519 & 0.904405 \\ 15 & 1 & -0.665909 & 2.157371 & 2.099423 \\ 16 & 1 & -1.709525 & 1.726360 & -2.069860 \\ 17 & 6 & 2.104083 & 0.642395 & -0.001628 \\ 18 & 6 & 2.207804 & 2.072596 & -0.509672 \\ 19 & 1 & 3.056258 & 2.158556 & -1.195364 \\ 20 & 1 & 1.306905 & 2.371653 & -1.044599 \\ 21 & 1 & 2.374714 & 2.758368 & 0.328934 \\ 22 & 8 & 0.834576 & -2.288711 & -1.291389 \\ 23 & 1 & 3.101410 & 0.356057 & 0.372907 \\ 24 & 6 & 0.302836 & -2.133736 & 1.698635 \\ 25 & 1 & -0.275469 & -1.810520 & 2.569733 \\ 26 & 1 & -0.232392 & -2.936742 & 1.185237 \\ 27 & 1 & 1.248777 & -2.553649 & 2.066325 \\ ----------------------------------------------------\end{array}$

\section{E.I.TS2product (Me down)}

$\mathrm{HF}=-727.2605466$ hartrees $(-456363.265596966 \mathrm{kcal} / \mathrm{mol})$

Imaginary Frequencies: none found

Zero-point correction $=0.217747$ (Hartree/Particle)

Thermal correction to Energy $=\quad 0.230093$

Thermal correction to Enthalpy= $\quad 0.231037$

Thermal correction to Gibbs Free Energy $=0.179733$

Sum of electronic and zero-point Energies $=\quad-727.042799$

Sum of electronic and thermal Energies $=\quad-727.030454$

Sum of electronic and thermal Enthalpies $=\quad-727.029510$

Sum of electronic and thermal Free Energies $=\quad-727.080813$

Coordinates (from last standard orientation):

\begin{tabular}{ccrrr}
\hline & Center & Atomic & \multicolumn{3}{c}{ Coordinates (Angstroms) } \\
Number & Number & X & $Y$ & $Z$ \\
- \hdashline & 8 & 0.970944 & -1.003135 & 1.645068 \\
2 & 8 & 3.049796 & 0.008160 & -0.891530 \\
3 & 6 & 1.372093 & 0.127109 & 0.855538 \\
4 & 8 & -1.497877 & 0.747679 & -1.182910 \\
5 & 6 & 2.010557 & -0.420807 & -0.429597 \\
6 & 6 & 1.236518 & -1.539388 & -1.024309 \\
7 & 6 & 0.155599 & -2.005484 & -0.378140 \\
8 & 1 & -0.450325 & -2.804379 & -0.798844 \\
9 & 6 & -0.216761 & -1.456303 & 0.985067 \\
10 & 6 & -1.054867 & -0.136949 & 0.981027 \\
11 & 1 & -1.442576 & -0.008287 & 1.995811 \\
12 & 6 & 0.025295 & 0.916522 & 0.638638 \\
13 & 6 & -0.293427 & 1.274145 & -0.820731 \\
14 & 1 & 2.111505 & 0.700369 & 1.416475 \\
15 & 1 & -0.665844 & -2.234167 & 1.608064
\end{tabular}




$\begin{array}{rrrrr}16 & 1 & 1.576959 & -1.938312 & -1.975372 \\ 17 & 6 & -2.163330 & 0.131804 & -0.051663 \\ 18 & 6 & -3.013697 & -1.030167 & -0.538329 \\ 19 & 1 & -3.776026 & -0.670541 & -1.235847 \\ 20 & 1 & -2.411950 & -1.781080 & -1.055375 \\ 21 & 1 & -3.521943 & -1.504095 & 0.309098 \\ 22 & 8 & 0.375907 & 1.933099 & -1.572859 \\ 23 & 1 & -2.830639 & 0.895508 & 0.370548 \\ 24 & 6 & -0.011929 & 2.192853 & 1.493089 \\ 25 & 1 & 0.231508 & 1.956238 & 2.534489 \\ 26 & 1 & 0.714628 & 2.918753 & 1.114998 \\ 27 & 1 & -1.001759 & 2.663552 & 1.470267 \\ --------------------------------------------------\end{array}$

\section{E.I.TS3 (Me down)}

$\mathrm{HF}=-727.2008459$ hartrees $(-456325.802810709 \mathrm{kcal} / \mathrm{mol})$

Imaginary Frequencies: $1(-367.36351 / \mathrm{cm})$

Zero-point correction $=0.212799$ (Hartree/Particle)

Thermal correction to Energy $=\quad 0.226044$

Thermal correction to Enthalpy $=\quad 0.226988$

Thermal correction to Gibbs Free Energy $=\quad 0.173154$

Sum of electronic and zero-point Energies $=\quad-726.988047$

Sum of electronic and thermal Energies $=\quad-726.974802$

Sum of electronic and thermal Enthalpies $=\quad-726.973858$

Sum of electronic and thermal Free Energies $=\quad-727.027692$

Coordinates (from last standard orientation):

\begin{tabular}{|c|c|c|c|c|}
\hline \multirow{2}{*}{$\begin{array}{l}\text { Center } \\
\text { Number }\end{array}$} & \multirow{2}{*}{$\begin{array}{l}\text { Atomic } \\
\text { Number }\end{array}$} & \multicolumn{3}{|c|}{ Coordinates (Angstroms) } \\
\hline & & & $\begin{array}{ll}X & Y\end{array}$ & Z \\
\hline 1 & 8 & 0.539462 & 0.638968 & 1.484788 \\
\hline 2 & 8 & 2.733543 & 1.253308 & -1.269646 \\
\hline$\overline{3}$ & 6 & 0.995376 & 1.162167 & 0.323128 \\
\hline 4 & 6 & 2.219148 & 0.636016 & -0.330463 \\
\hline 5 & 6 & 2.646259 & -0.642930 & 0.197853 \\
\hline 6 & 6 & 1.875070 & -1.284106 & 1.150607 \\
\hline 7 & 1 & 2.112620 & -2.290367 & 1.482930 \\
\hline 8 & 6 & 0.768831 & -0.657554 & 1.719277 \\
\hline 9 & 6 & -0.637970 & -0.800417 & -0.848867 \\
\hline 10 & 6 & -0.472285 & 0.577211 & -0.976190 \\
\hline 11 & 1 & 0.845169 & 2.235407 & 0.288897 \\
\hline 12 & 1 & 0.174782 & -1.052560 & 2.532214 \\
\hline 13 & 1 & 3.563991 & -1.078765 & -0.184316 \\
\hline 14 & 6 & -1.857563 & -1.056541 & -0.055561 \\
\hline 15 & 6 & -1.787912 & 1.207948 & -0.505124 \\
\hline 16 & 8 & -2.453417 & 0.155171 & 0.220198 \\
\hline 17 & 1 & 0.064471 & 1.022487 & -1.813670 \\
\hline 18 & 8 & -2.330023 & -2.108487 & 0.310470 \\
\hline 19 & 1 & -2.378959 & 1.397273 & -1.414493 \\
\hline 20 & 6 & -1.794620 & 2.476287 & 0.335389 \\
\hline 21 & 1 & -1.312138 & 3.296719 & -0.208027 \\
\hline 22 & 1 & -2.829146 & 2.768916 & 0.538371 \\
\hline 23 & 1 & -1.290683 & 2.327379 & 1.292790 \\
\hline 24 & 6 & 0.092421 & -1.889328 & -1.551243 \\
\hline 25 & 1 & -0.234905 & -2.864770 & -1.180890 \\
\hline 26 & 1 & -0.092718 & -1.848251 & -2.633155 \\
\hline
\end{tabular}




\section{E.I.TS3product (Me down) \\ $\mathrm{HF}=-727.2704762$ hartrees $(-456369.496520262 \mathrm{kcal} / \mathrm{mol})$ \\ Imaginary Frequencies: none found \\ Zero-point correction $=0.218002$ (Hartree/Particle) \\ Thermal correction to Energy $=\quad 0.230320$ \\ Thermal correction to Enthalpy= $\quad 0.231264$ \\ Thermal correction to Gibbs Free Energy= 0.179773 \\ Sum of electronic and zero-point Energies $=\quad-727.052474$ \\ Sum of electronic and thermal Energies $=\quad-727.040156$ \\ Sum of electronic and thermal Enthalpies $=\quad-727.039212$ \\ Sum of electronic and thermal Free Energies $=\quad-727.090703$ \\ Coordinates (from last standard orientation):}

\begin{tabular}{|c|c|c|c|c|}
\hline \multirow{2}{*}{$\begin{array}{l}\text { Center } \\
\text { Number }\end{array}$} & \multirow{2}{*}{$\begin{array}{l}\text { Atomic } \\
\text { Number }\end{array}$} & \multicolumn{3}{|c|}{ Coordinates (Angstroms) } \\
\hline & & & $\begin{array}{ll}X \quad Y\end{array}$ & Z \\
\hline 1 & 8 & -0.569954 & -0.064812 & -1.504255 \\
\hline 2 & 8 & -3.032768 & 1.563386 & 0.406390 \\
\hline 3 & 6 & -0.893973 & 0.898533 & -0.497387 \\
\hline 4 & 6 & -2.322716 & 0.653210 & 0.012895 \\
\hline 5 & 6 & -2.709014 & -0.774635 & 0.064702 \\
\hline 6 & 6 & -1.794612 & -1.706305 & -0.254746 \\
\hline 7 & 1 & -2.004211 & -2.768895 & -0.152643 \\
\hline 8 & 6 & -0.434538 & -1.277349 & -0.758232 \\
\hline 9 & 6 & 0.531231 & -0.850657 & 0.396438 \\
\hline 10 & 6 & 0.125127 & 0.625585 & 0.651917 \\
\hline 11 & 1 & -0.838079 & 1.895669 & -0.933693 \\
\hline 12 & 1 & 0.020888 & -2.030270 & -1.405081 \\
\hline 13 & 1 & -3.712631 & -1.013902 & 0.405051 \\
\hline 14 & 6 & 1.939022 & -0.735052 & -0.207711 \\
\hline 15 & 6 & 1.472218 & 1.396066 & 0.592170 \\
\hline 16 & 8 & 2.405495 & 0.530381 & -0.103247 \\
\hline 17 & 1 & -0.341977 & 0.787309 & 1.627441 \\
\hline 18 & 8 & 2.572221 & -1.628763 & -0.710116 \\
\hline 19 & 1 & 1.852570 & 1.500084 & 1.616247 \\
\hline 20 & 6 & 1.485537 & 2.755637 & -0.085819 \\
\hline 21 & 1 & 0.776056 & 3.433097 & 0.402186 \\
\hline 22 & 1 & 2.484638 & 3.196178 & -0.018336 \\
\hline 23 & 1 & 1.224427 & 2.669589 & -1.144550 \\
\hline 24 & 6 & 0.567129 & -1.785539 & 1.609852 \\
\hline 25 & 1 & 0.927086 & -2.776140 & 1.313341 \\
\hline 26 & 1 & 1.246331 & -1.404174 & 2.380295 \\
\hline 27 & 1 & -0.427503 & -1.887049 & 2.055104 \\
\hline
\end{tabular}

\section{E.I.TS4 (Me down)}

$\mathrm{HF}=-727.1984516$ hartrees $(-456324.300363516 \mathrm{kcal} / \mathrm{mol})$

Imaginary Frequencies: $1(-403.33071 / \mathrm{cm})$

Zero-point correction $=0.212882($ Hartree/Particle $)$

Thermal correction to Energy $=\quad 0.226164$

Thermal correction to Enthalpy= $\quad 0.227108$

Thermal correction to Gibbs Free Energy $=\quad 0.173399$ 


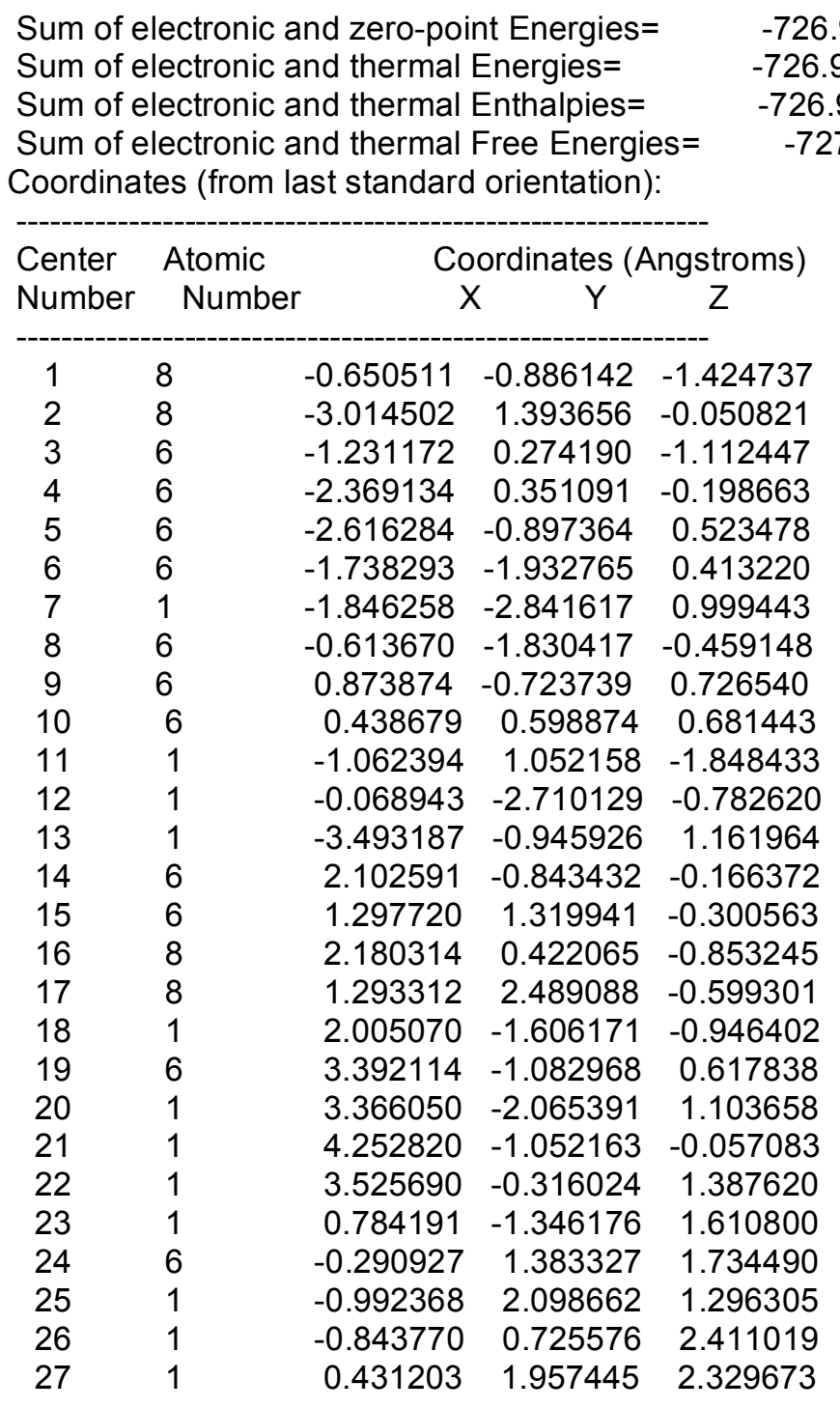

\section{E.I.TS4product (Me down)}

$\mathrm{HF}=-727.2708302$ hartrees $(-456369.718658802 \mathrm{kcal} / \mathrm{mol})$

Imaginary Frequencies: none found

Zero-point correction $=0.217635($ Hartree/Particle $)$

Thermal correction to Energy $=\quad 0.230100$

Thermal correction to Enthalpy= $\quad 0.231044$

Thermal correction to Gibbs Free Energy $=\quad 0.178928$

Sum of electronic and zero-point Energies $=\quad-727.053195$

Sum of electronic and thermal Energies $=\quad-727.040730$

Sum of electronic and thermal Enthalpies $=\quad-727.039786$

Sum of electronic and thermal Free Energies $=\quad-727.091902$

Coordinates (from last standard orientation):

\begin{tabular}{llrrr} 
Center & Atomic & & \multicolumn{3}{c}{ Coordinates (Angstroms) } \\
Number & Number & X & Y & Z \\
\hline 1 & 8 & -0.701238 & -0.527799 & -1.537163 \\
2 & 8 & -3.145632 & 1.248310 & 0.235170
\end{tabular}




\begin{tabular}{ccrrr}
3 & 6 & -1.015407 & 0.584994 & -0.694784 \\
4 & 6 & -2.372780 & 0.340761 & -0.018795 \\
5 & 6 & -2.616379 & -1.071494 & 0.357877 \\
6 & 6 & -1.654702 & -1.979256 & 0.127085 \\
7 & 1 & -1.745867 & -3.009955 & 0.464638 \\
8 & 6 & -0.394412 & -1.564646 & -0.599936 \\
9 & 6 & 0.621388 & -0.883099 & 0.364096 \\
10 & 6 & 0.167837 & 0.601059 & 0.336401 \\
11 & 1 & -1.058169 & 1.494848 & -1.295664 \\
12 & 1 & 0.035792 & -2.400639 & -1.158898 \\
13 & 1 & -3.554936 & -1.305841 & 0.852265 \\
14 & 6 & 2.073706 & -0.874690 & -0.183790 \\
15 & 6 & 1.346456 & 1.336484 & -0.317343 \\
16 & 8 & 2.377411 & 0.490315 & -0.552415 \\
17 & 8 & 1.393187 & 2.509314 & -0.585553 \\
18 & 1 & 2.140213 & -1.455199 & -1.110979 \\
19 & 6 & 3.120871 & -1.358921 & 0.808559 \\
20 & 1 & 2.958991 & -2.417362 & 1.043384 \\
21 & 1 & 4.126216 & -1.247146 & 0.391804 \\
22 & 1 & 3.068464 & -0.785334 & 1.740772 \\
23 & 1 & 0.589598 & -1.319844 & 1.366050 \\
24 & 6 & -0.153245 & 1.264781 & 1.681766 \\
25 & 1 & -0.455254 & 2.303953 & 1.524340 \\
26 & 1 & -0.961663 & 0.742200 & 2.201292 \\
27 & 1 & 0.726801 & 1.263369 & 2.333870 \\
-------------------------------------------------- \\
\hline
\end{tabular}

\section{E.I.TS1 (Me up)}

$\mathrm{HF}=-727.1928552$ hartrees $(-456320.788566552 \mathrm{kcal} / \mathrm{mol})$ Imaginary Frequencies: $1(-403.16301 / \mathrm{cm})$

Zero-point correction $=0.213034$ (Hartree/Particle)

Thermal correction to Energy $=\quad 0.226277$

Thermal correction to Enthalpy= $\quad 0.227221$

Thermal correction to Gibbs Free Energy $=0.173555$

Sum of electronic and zero-point Energies $=\quad-726.979821$

Sum of electronic and thermal Energies $=\quad-726.966579$

Sum of electronic and thermal Enthalpies $=\quad-726.965634$

Sum of electronic and thermal Free Energies $=\quad-727.019300$

Coordinates (from last standard orientation):

\begin{tabular}{|c|c|c|c|c|}
\hline \multirow{2}{*}{$\begin{array}{l}\text { Center } \\
\text { Number }\end{array}$} & \multirow{2}{*}{$\begin{array}{l}\text { Atomic } \\
\text { Number }\end{array}$} & & \multicolumn{2}{|c|}{ Coordinates (Angstroms) } \\
\hline & & & $X \quad Y$ & Z \\
\hline 1 & 8 & -0.548237 & -1.962727 & -0.771059 \\
\hline 2 & 8 & -3.198900 & 0.266274 & -0.048012 \\
\hline 3 & 6 & -1.367025 & -0.909050 & -0.979108 \\
\hline 4 & 8 & 0.993246 & 1.721413 & 0.685308 \\
\hline 5 & 6 & -2.157357 & -0.367945 & 0.139953 \\
\hline 6 & 6 & -1.541985 & -0.616780 & 1.438834 \\
\hline 7 & 6 & -0.396436 & -1.358987 & 1.523474 \\
\hline 8 & 1 & 0.124576 & -1.502590 & 2.464918 \\
\hline 9 & 6 & 0.158116 & -1.953111 & 0.366867 \\
\hline 10 & 6 & 1.400641 & $1-0.158811$ & -0.611402 \\
\hline 11 & 6 & 0.352011 & 10.526461 & -1.229377 \\
\hline 12 & 6 & 0.269437 & 1.894919 & -0.555244 \\
\hline 13 & 1 & -1.826924 & $4-0.942038$ & -1.961523 \\
\hline
\end{tabular}




$\begin{array}{rrrrr}14 & 1 & 0.904902 & -2.735152 & 0.404060 \\ 15 & 1 & -2.023127 & -0.203047 & 2.319622 \\ 16 & 1 & 0.179197 & 0.473413 & -2.300229 \\ 17 & 6 & -1.045642 & 2.611793 & -0.300367 \\ 18 & 1 & -0.833562 & 3.610432 & 0.094386 \\ 19 & 1 & -1.594817 & 2.724206 & -1.241254 \\ 20 & 1 & -1.690476 & 2.088622 & 0.402312 \\ 21 & 1 & 0.886409 & 2.559244 & -1.182394 \\ 22 & 6 & 1.781097 & 0.603427 & 0.607782 \\ 23 & 8 & 2.619000 & 0.334360 & 1.437447 \\ 24 & 6 & 2.382566 & -1.098426 & -1.257754 \\ 25 & 1 & 3.060007 & -0.540608 & -1.919173 \\ 26 & 1 & 3.000346 & -1.593279 & -0.502963 \\ 27 & 1 & 1.880063 & -1.854703 & -1.869182 \\ --------------------------------------------------\end{array}$

\section{E.I.TS1product (Me up)}

$\mathrm{HF}=-727.26389$ hartrees $(-456365.3636139 \mathrm{kcal} / \mathrm{mol})$ Imaginary Frequencies: none found

Zero-point correction $=0.217898$ (Hartree/Particle)

Thermal correction to Energy $=\quad 0.230207$

Thermal correction to Enthalpy= $\quad 0.231151$

Thermal correction to Gibbs Free Energy $=\quad 0.180020$

Sum of electronic and zero-point Energies $=\quad-727.045992$

Sum of electronic and thermal Energies $=\quad-727.033683$

Sum of electronic and thermal Enthalpies $=\quad-727.032739$

Sum of electronic and thermal Free Energies $=\quad-727.083870$

Coordinates (from last standard orientation):

\begin{tabular}{|c|c|c|c|c|}
\hline \multirow{2}{*}{$\begin{array}{l}\text { Center } \\
\text { Number }\end{array}$} & \multirow{2}{*}{$\begin{array}{l}\text { Atomic } \\
\text { Number }\end{array}$} & \multicolumn{3}{|c|}{ Coordinates (Angstroms) } \\
\hline & & & $X \quad Y$ & Z \\
\hline 1 & 8 & -0.311280 & -1.684248 & -1.340825 \\
\hline 2 & 8 & -2.997896 & -0.198817 & 0.327423 \\
\hline 3 & 6 & -1.077492 & -0.493829 & -1.111199 \\
\hline 4 & 8 & 0.522853 & 1.607478 & 0.983297 \\
\hline 5 & 6 & -1.883298 & -0.678509 & 0.190046 \\
\hline 6 & 6 & -1.203474 & -1.465848 & 1.238094 \\
\hline 7 & 6 & 0.050323 & -1.894531 & 1.020575 \\
\hline 8 & 1 & 0.631404 & -2.395648 & 1.789544 \\
\hline 9 & 6 & 0.691557 & -1.625721 & -0.322463 \\
\hline 10 & 6 & 1.239030 & -0.148339 & -0.447565 \\
\hline 11 & 6 & 0.051712 & 0.588897 & -1.115448 \\
\hline 12 & 6 & -0.034139 & 1.911006 & -0.327863 \\
\hline 13 & 1 & -1.782540 & -0.369909 & -1.935215 \\
\hline 14 & 1 & 1.455800 & -2.368023 & -0.561661 \\
\hline 15 & 1 & -1.740120 & -1.630701 & 2.167589 \\
\hline 16 & 1 & 0.259006 & 0.800835 & -2.168621 \\
\hline 17 & 6 & -1.372294 & 2.612413 & -0.171749 \\
\hline 18 & 1 & -1.240353 & 3.527754 & 0.413275 \\
\hline 19 & 1 & -1.751632 & 2.893592 & -1.160907 \\
\hline 20 & 1 & -2.118944 & 1.985106 & 0.315535 \\
\hline 21 & 1 & 0.669652 & 2.611497 & -0.797800 \\
\hline 22 & 6 & 1.373567 & 0.551229 & 0.910754 \\
\hline 23 & 8 & 2.102863 & 0.232722 & 1.816716 \\
\hline 24 & 6 & & -0.098500 & -1.217248 \\
\hline
\end{tabular}




$\begin{array}{lllll}25 & 1 & 3.365635 & -0.542328 & -0.618866 \\ 26 & 1 & 2.473464 & -0.651292 & -2.158602 \\ 27 & 1 & 2.852080 & 0.932418 & -1.452764\end{array}$

\begin{tabular}{|c|c|c|c|c|}
\hline \multicolumn{5}{|c|}{$\begin{array}{l}\mathrm{HF}=-727.1927004 \text { hartrees }(-456320.691428004 \mathrm{kcal} / \mathrm{r} \\
\text { Imaginary Frequencies: } 1(-408.69751 / \mathrm{cm}) \\
\text { Zero-point correction }=0.212720(\text { Hartree } / \text { Particle })\end{array}$} \\
\hline \multirow{2}{*}{\multicolumn{3}{|c|}{ Thermal correction to Enthalpy= }} & \\
\hline & & & \multicolumn{2}{|r|}{$\begin{array}{l}0.226036 \\
0.226980\end{array}$} \\
\hline \multicolumn{5}{|c|}{ Thermal correction to Gibbs Free Energy= } \\
\hline \multicolumn{5}{|c|}{ Sum of electronic and zero-point Energies= } \\
\hline \multirow{2}{*}{\multicolumn{5}{|c|}{$\begin{array}{l}\text { Sum of electronic and thermal Energies= } \\
\text { Sum of electronic and thermal Enthalpies= }\end{array}$}} \\
\hline \multirow{3}{*}{\multicolumn{5}{|c|}{$\begin{array}{l}\text { Sum of electronic and thermal Enthalpies= } \\
\text { Sum of electronic and thermal Free Energies= } \\
\text { Coordinates (from last standard orientation): }\end{array}$}} \\
\hline & & & & \\
\hline & & & & \\
\hline \multirow{2}{*}{$\begin{array}{l}\text { Center } \\
\text { Number }\end{array}$} & \multirow{2}{*}{$\begin{array}{l}\text { Atomic } \\
\text { Number }\end{array}$} & \multicolumn{3}{|c|}{ Coordinates (Angstroms) } \\
\hline & & & $X \quad Y$ & Z \\
\hline 1 & 8 & & & \\
\hline 2 & 8 & 2.214656 & & -1. \\
\hline 3 & 6 & & & \\
\hline 4 & 8 & -1.475505 & 0.206635 & -1.27 \\
\hline 5 & 6 & 1.906581 & 0.372005 & -0.769375 \\
\hline 6 & 6 & 1.753325 & -1.008389 & -1.229976 \\
\hline 7 & 6 & 1.334273 & -1.9 & -0.362237 \\
\hline 8 & 1 & 968 & $-2 . c$ & 0611 \\
\hline 9 & 6 & & & \\
\hline 10 & 6 & & & 547 \\
\hline 11 & 1 & 430 & & 245 \\
\hline 12 & 6 & 4611 & & 55276 \\
\hline 13 & 6 & 38459 & 1.230956 & -0.472941 \\
\hline 14 & 1 & 1.976179 & 1.440170 & 1.165288 \\
\hline 15 & 1 & & -2.427455 & 1.762161 \\
\hline 16 & 1 & & -1.222444 & -2.269602 \\
\hline 17 & 6 & & & -0.5 \\
\hline 18 & 8 & & & $-0 . \varepsilon$ \\
\hline 19 & 6 & & -1.354319 & -0.403659 \\
\hline 20 & 1 & -3.544914 & -1.468844 & -1.404611 \\
\hline 21 & 1 & -3.249498 & -2.296929 & 0.140580 \\
\hline 22 & 1 & -3.669853 & -0.567877 & 0.121325 \\
\hline 23 & 1 & -1.110678 & -1.787369 & -1.059467 \\
\hline 24 & 6 & -0.627860 & 1.566137 & 2.061171 \\
\hline 25 & 1 & & 1.141396 & \\
\hline 26 & 1 & 475 & & \\
\hline 27 & 1 & -1.643279 & 1.716785 & 2.450935 \\
\hline
\end{tabular}

\section{E.I.TS2product (Me up)}

$\mathrm{HF}=-727.2638019$ hartrees $(-456365.308330269 \mathrm{kcal} / \mathrm{mol})$

Imaginary Frequencies: none found

Zero-point correction $=0.217664($ Hartree/Particle $)$

Thermal correction to Energy=

0.230034 


\begin{tabular}{|c|c|c|c|c|}
\hline \multicolumn{5}{|c|}{ Thermal correction to Enthalpy= } \\
\hline \multicolumn{5}{|c|}{ Thermal correction to Gibbs Free Energy= } \\
\hline \multirow{2}{*}{\multicolumn{5}{|c|}{$\begin{array}{l}\text { Sum of electronic and zero-point Energies= } \\
\text { Sum of electronic and thermal Energies= }\end{array}$}} \\
\hline & & & & \\
\hline \multicolumn{5}{|c|}{ Sum of electronic and thermal Enthalpies $=\quad-727$} \\
\hline \multirow{2}{*}{\multicolumn{5}{|c|}{ Sum of electronic and thermal Free Energies $=\quad-72$}} \\
\hline & & & & \\
\hline \multirow{2}{*}{$\begin{array}{l}\text { Center } \\
\text { Number }\end{array}$} & Atomic & \multicolumn{3}{|c|}{ Coordinates (Angstroms) } \\
\hline & Numbe & & $X \quad Y$ & Z \\
\hline 1 & 8 & 1.219721 & -1.047443 & 1.509610 \\
\hline 2 & 8 & 2.889662 & 950 & -0.689658 \\
\hline 3 & 6 & 1.268515 & 4076 & 0.935892 \\
\hline 4 & 8 & -1.406933 & 0.475744 & -1.332661 \\
\hline 5 & 6 & 2.081820 & 0.185197 & -0.366222 \\
\hline 6 & 6 & 1.785964 & -1.021445 & -1.178220 \\
\hline 7 & 6 & 792 & 3733 & -0.707589 \\
\hline 8 & 1 & & -2 & -1.288405 \\
\hline 9 & 6 & 185 & 3635 & 0.662384 \\
\hline 10 & 6 & -0.928915 & -0.770560 & 0.656256 \\
\hline 11 & 1 & -1.535380 & -0.978008 & 1.542411 \\
\hline 12 & 6 & -0.256267 & 0.621498 & 0.742338 \\
\hline 13 & 6 & -0.596242 & 1.283690 & -0.595008 \\
\hline 14 & 1 & 1.769390 & 0.936368 & 1.633719 \\
\hline 15 & 1 & 4318 & 0558 & 1.131078 \\
\hline 16 & 1 & & 3404 & -2.135381 \\
\hline 17 & 6 & -1.80 & -0.7 & -0.614401 \\
\hline 18 & 8 & -0.240601 & 2.366700 & -0.980289 \\
\hline 19 & 6 & -3.304959 & -0.645505 & -0.334826 \\
\hline 20 & 1 & -3.860655 & -0.500470 & -1.265925 \\
\hline 21 & 1 & -3.646786 & -1.575808 & 0.133428 \\
\hline 22 & 1 & -3.540291 & 0.186272 & 0.338458 \\
\hline 23 & 1 & -1.601255 & -1.552531 & -1.289425 \\
\hline 24 & 6 & -0.739326 & 1.511093 & 1.897469 \\
\hline 25 & 1 & -0.4634 & 1.062408 & 2.857747 \\
\hline 26 & 1 & -0.282190 & 2.502295 & 1.821254 \\
\hline 27 & 1 & -1.827597 & 1.637558 & 1.876593 \\
\hline
\end{tabular}

\section{E.I.TS3 (Me up)}

$\mathrm{HF}=-727.2041841$ hartrees $(-456327.897564591 \mathrm{kcal} / \mathrm{mol})$ Imaginary Frequencies: $1(-369.36471 / \mathrm{cm})$

Zero-point correction $=0.212565$ (Hartree/Particle)

Thermal correction to Energy $=\quad 0.225983$

Thermal correction to Enthalpy= $\quad 0.226927$

Thermal correction to Gibbs Free Energy $=\quad 0.172437$

Sum of electronic and zero-point Energies $=\quad-726.991619$

Sum of electronic and thermal Energies $=\quad-726.978201$

Sum of electronic and thermal Enthalpies $=\quad-726.977257$

Sum of electronic and thermal Free Energies $=\quad-727.031748$

Coordinates (from last standard orientation):

$\begin{array}{llll}\text { Center } & \text { Atomic } & \text { Coordinates (Angstroms) } \\ \text { Number } & \text { Number } & X & Y\end{array}$




\begin{tabular}{rrrrr}
1 & 8 & -0.791658 & 0.107764 & -1.671590 \\
2 & 8 & -2.618924 & 1.840777 & 0.864338 \\
3 & 6 & -1.085020 & 1.048443 & -0.744114 \\
4 & 6 & -2.230460 & 0.884873 & 0.184566 \\
5 & 6 & -2.734732 & -0.472218 & 0.235367 \\
6 & 6 & -2.098008 & -1.467225 & -0.482700 \\
7 & 1 & -2.387517 & -2.508916 & -0.380180 \\
8 & 6 & -1.064951 & -1.166093 & -1.369126 \\
9 & 6 & 0.649857 & -0.484970 & 0.866180 \\
10 & 6 & 0.529128 & 0.848403 & 0.485456 \\
11 & 1 & -0.897956 & 2.048216 & -1.124210 \\
12 & 1 & -0.588865 & -1.871334 & -2.036888 \\
13 & 1 & -3.601067 & -0.678149 & 0.856062 \\
14 & 6 & 1.729558 & -1.106100 & 0.070380 \\
15 & 6 & 1.713377 & 1.152272 & -0.431656 \\
16 & 8 & 2.279356 & -0.138342 & -0.740560 \\
17 & 1 & 0.151315 & 1.615852 & 1.160368 \\
18 & 8 & 2.134804 & -2.245788 & 0.085769 \\
19 & 6 & 2.768252 & 2.035381 & 0.231739 \\
20 & 1 & 2.361338 & 3.034381 & 0.425581 \\
21 & 1 & 3.094783 & 1.600695 & 1.181980 \\
22 & 1 & 3.638814 & 2.135303 & -0.423356 \\
23 & 1 & 1.415076 & 1.590386 & -1.389279 \\
24 & 6 & 0.023562 & -1.177337 & 2.024488 \\
25 & 1 & 0.270645 & -2.242428 & 2.006755 \\
26 & 1 & 0.380792 & -0.754608 & 2.973211 \\
27 & 1 & -1.069353 & -1.057248 & 2.022799 \\
--------------------------------------------------- \\
\hline
\end{tabular}

\section{E.I.TS3product (Me up)}

$\mathrm{HF}=-727.2719852$ hartrees $(-456370.443432852 \mathrm{kcal} / \mathrm{mol})$

Imaginary Frequencies: none found

Zero-point correction $=0.217788($ Hartree/Particle $)$

Thermal correction to Energy $=\quad 0.230178$

Thermal correction to Enthalpy= $\quad 0.231122$

Thermal correction to Gibbs Free Energy $=\quad 0.179319$

Sum of electronic and zero-point Energies $=\quad-727.054197$

Sum of electronic and thermal Energies $=\quad-727.041807$

Sum of electronic and thermal Enthalpies $=\quad-727.040863$

Sum of electronic and thermal Free Energies $=\quad-727.092666$

Coordinates (from last standard orientation):

\begin{tabular}{|c|c|c|c|c|}
\hline \multirow{2}{*}{$\begin{array}{l}\text { Center } \\
\text { Number }\end{array}$} & \multirow{2}{*}{$\begin{array}{l}\text { Atomic } \\
\text { Number }\end{array}$} & & \multicolumn{2}{|c|}{ Coordinates (Angstroms) } \\
\hline & & & $\begin{array}{ll}X & Y\end{array}$ & Z \\
\hline 1 & 8 & -0.796105 & -0.406373 & -1.518916 \\
\hline 2 & 8 & -3.004155 & 1.771999 & 0.145330 \\
\hline 3 & 6 & -0.996298 & 0.801689 & -0.779307 \\
\hline 4 & 6 & -2.358553 & 0.759882 & -0.069805 \\
\hline 5 & 6 & -2.757551 & -0.587008 & 0.395460 \\
\hline 6 & 6 & -1.896562 & -1.607214 & 0.241001 \\
\hline 7 & 1 & -2.108172 & -2.596937 & 0.639780 \\
\hline 8 & 6 & -0.592961 & -1.380878 & -0.492163 \\
\hline 9 & 6 & 0.497285 & -0.703257 & 0.408094 \\
\hline 10 & 6 & 0.163462 & $2 \quad 0.804477$ & 0.261388 \\
\hline
\end{tabular}




$\begin{array}{rrrrr}11 & 1 & -0.973922 & 1.646510 & -1.471179 \\ 12 & 1 & -0.219501 & -2.296604 & -0.955664 \\ 13 & 1 & -3.721470 & -0.686653 & 0.886619 \\ 14 & 6 & 1.846965 & -0.850798 & -0.307540 \\ 15 & 6 & 1.473318 & 1.448205 & -0.267341 \\ 16 & 8 & 2.349645 & 0.358349 & -0.645664 \\ 17 & 1 & -0.141643 & 1.278224 & 1.198507 \\ 18 & 8 & 2.416735 & -1.886536 & -0.541812 \\ 19 & 6 & 2.190138 & 2.328147 & 0.747292 \\ 20 & 1 & 1.579171 & 3.206559 & 0.983841 \\ 21 & 1 & 2.381139 & 1.779104 & 1.676310 \\ 22 & 1 & 3.149322 & 2.669572 & 0.346812 \\ 23 & 1 & 1.289796 & 2.013023 & -1.187244 \\ 24 & 6 & 0.612666 & -1.262653 & 1.830343 \\ 25 & 1 & 0.894278 & -2.320194 & 1.797671 \\ 26 & 1 & 1.384707 & -0.733110 & 2.398799 \\ 27 & 1 & -0.334125 & -1.162557 & 2.369721\end{array}$

\section{E.I.TS4 (Me up)}

$\mathrm{HF}=-727.1950652$ hartrees $(-456322.175363652 \mathrm{kcal} / \mathrm{mol})$

Imaginary Frequencies: $1(-399.90711 / \mathrm{cm})$

Zero-point correction $=0.212958$ (Hartree/Particle)

Thermal correction to Energy= $\quad 0.226142$

Thermal correction to Enthalpy $=\quad 0.227086$

Thermal correction to Gibbs Free Energy $=0.173766$

Sum of electronic and zero-point Energies $=\quad-726.982107$

Sum of electronic and thermal Energies $=\quad-726.968923$

Sum of electronic and thermal Enthalpies $=\quad-726.967979$

Sum of electronic and thermal Free Energies $=\quad-727.021299$

Coordinates (from last standard orientation):

\begin{tabular}{|c|c|c|c|c|}
\hline \multirow{2}{*}{$\begin{array}{l}\text { Center } \\
\text { Number }\end{array}$} & \multirow{2}{*}{$\begin{array}{l}\text { Atomic } \\
\text { Number }\end{array}$} & \multicolumn{3}{|c|}{ Coordinates (Angstroms) } \\
\hline & & & $X \quad Y$ & Z \\
\hline 1 & 8 & -0.311996 & -1.068336 & -1.224828 \\
\hline 2 & 8 & -3.038161 & 1.126215 & -0.584329 \\
\hline 3 & 6 & -1.020512 & 0.061690 & -1.183452 \\
\hline 4 & 6 & -2.288761 & 0.152410 & -0.462757 \\
\hline 5 & 6 & -2.533037 & -0.987083 & 0.422244 \\
\hline 6 & 6 & -1.564935 & -1.927162 & 0.604968 \\
\hline 7 & 1 & -1.681942 & -2.740192 & 1.316791 \\
\hline 8 & 6 & -0.341131 & -1.851820 & -0.124858 \\
\hline 9 & 6 & 0.869387 & -0.424529 & 1.072548 \\
\hline 10 & 6 & 0759 & 0.816546 & 0.751356 \\
\hline 11 & 1 & -0.804394 & 0.736099 & -2.004423 \\
\hline 12 & 1 & 0.300111 & -2.716874 & -0.231509 \\
\hline 13 & 1 & -3.487196 & -1.028884 & 0.938822 \\
\hline 14 & 6 & 2.274123 & -0.472057 & 0.473679 \\
\hline 15 & 6 & 1.263014 & 1.497126 & -0.181066 \\
\hline 16 & 8 & 2.328218 & 0.659132 & -0.416397 \\
\hline 17 & 8 & 1.186388 & 2.597026 & -0.671797 \\
\hline 18 & 1 & 0.678834 & -0.924935 & 2.016230 \\
\hline 19 & 6 & 2.771815 & -1.704940 & -0.268562 \\
\hline 20 & 1 & 3.815021 & -1.556769 & -0.563369 \\
\hline 21 & 1 & 2.724438 & -2.588027 & 0.378992 \\
\hline
\end{tabular}




$\begin{array}{rrrrr}22 & 1 & 2.188943 & -1.884255 & -1.175212 \\ 23 & 1 & 2.973913 & -0.266582 & 1.299587 \\ 24 & 6 & -0.645591 & 1.647176 & 1.548668 \\ 25 & 1 & -1.307884 & 2.233023 & 0.905898 \\ 26 & 1 & -1.259723 & 1.025436 & 2.205971 \\ 27 & 1 & -0.086504 & 2.355537 & 2.174422 \\ - & & \end{array}$

\section{E.I.TS4product (Me up) \\ $\mathrm{HF}=-727.2688897$ hartrees $(-456368.500975647 \mathrm{kcal} / \mathrm{mol})$ \\ Imaginary Frequencies: none found \\ Zero-point correction $=0.217983$ (Hartree/Particle) \\ Thermal correction to Energy $=\quad 0.230327$ \\ Thermal correction to Enthalpy $=\quad 0.231271$ \\ Thermal correction to Gibbs Free Energy $=0.179563$ \\ Sum of electronic and zero-point Energies $=\quad-727.050907$ \\ Sum of electronic and thermal Energies $=\quad-727.038563$ \\ Sum of electronic and thermal Enthalpies $=\quad-727.037618$ \\ Sum of electronic and thermal Free Energies $=\quad-727.089327$ \\ Coordinates (from last standard orientation):}

\begin{tabular}{|c|c|c|c|c|}
\hline \multirow{2}{*}{$\begin{array}{l}\text { Center } \\
\text { Number }\end{array}$} & \multirow{2}{*}{$\begin{array}{l}\text { Atomic } \\
\text { Number }\end{array}$} & \multicolumn{3}{|c|}{ Coordinates (Angstroms) } \\
\hline & & & $X \quad Y$ & Z \\
\hline 1 & 8 & -0.403510 & -0.713379 & -1.385774 \\
\hline 2 & 8 & -3.209779 & 1.050518 & -0.229828 \\
\hline 3 & 6 & -0.929446 & 0.466454 & -0.771299 \\
\hline 4 & 6 & -2.348478 & 0.187953 & -0.256034 \\
\hline 5 & 6 & -2.546767 & -1.182409 & 0.270204 \\
\hline 6 & 6 & -1.497392 & -2.018961 & 0.308975 \\
\hline 7 & 1 & -1.565230 & -3.003521 & 0.768186 \\
\hline 8 & 6 & -0.169824 & -1.580113 & -0.271790 \\
\hline 9 & 6 & 0.598547 & -0.684463 & 0.750758 \\
\hline 10 & 6 & 0.085152 & 0.737236 & 0.389619 \\
\hline 11 & 1 & -0.949961 & 1.277871 & -1.500439 \\
\hline 12 & 1 & 0.401473 & $\begin{array}{ll}3 & -2.437005\end{array}$ & -0.632176 \\
\hline 13 & 1 & -3.532003 & $3-1.438752$ & 0.649477 \\
\hline 14 & 6 & 2.142751 & $1-0.594460$ & 0.586787 \\
\hline 15 & 6 & 1.311593 & 31.417493 & -0.238878 \\
\hline 16 & 8 & 2.412162 & 0.638092 & -0.122796 \\
\hline 17 & 8 & 1.332496 & 2.498981 & -0.767812 \\
\hline 18 & 1 & 0.357023 & $3-0.974998$ & 1.776356 \\
\hline 19 & 6 & 2.846073 & $3-1.724411$ & -0.149140 \\
\hline 20 & 1 & 3.922257 & $7-1.530992$ & -0.182143 \\
\hline 21 & 1 & 2.686399 & 9 & 0.364824 \\
\hline 22 & 1 & 2.484549 & $9-1.806016$ & -1.178479 \\
\hline 23 & 1 & 2.590787 & -0.479615 & 1.581535 \\
\hline 24 & 6 & -0.445394 & $4 \quad 1.606688$ & 1.536027 \\
\hline 25 & 1 & 0.328841 & 1.763474 & 2.295107 \\
\hline 26 & 1 & -0.747083 & 2.586154 & 1.154196 \\
\hline 27 & 1 & -1.308883 & 1.142877 & 2.021861 \\
\hline
\end{tabular}




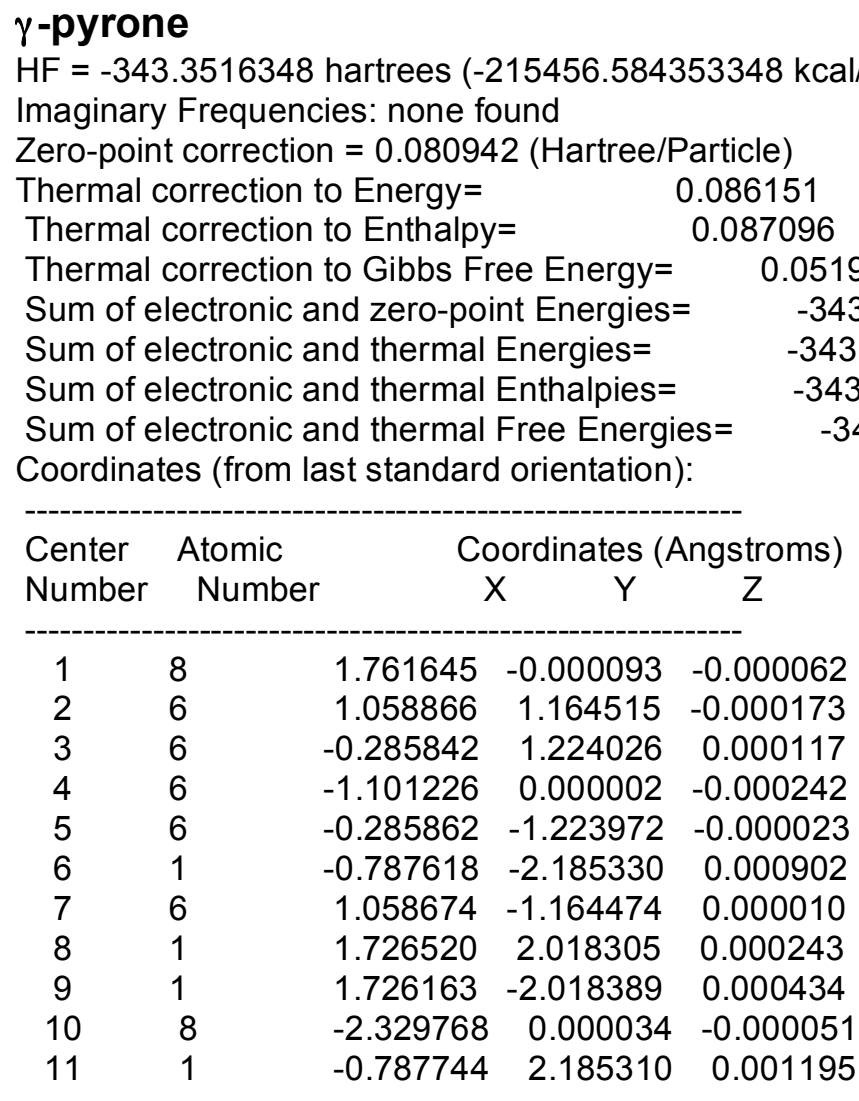

\section{1,3 dipolar TS of $\gamma$-pyrone and ethene}

$\mathrm{HF}=-421.8661787$ hartrees $(-264725.245796037 \mathrm{kcal} / \mathrm{mol})$

Imaginary Frequencies: 1 (-595.4717 1/cm)

Zero-point correction $=0.134283($ Hartree/Particle $)$

Thermal correction to Energy $=\quad 0.141810$

Thermal correction to Enthalpy= $\quad 0.142755$

Thermal correction to Gibbs Free Energy $=\quad 0.102253$

Sum of electronic and zero-point Energies $=\quad-421.731896$

Sum of electronic and thermal Energies $=\quad-421.724368$

Sum of electronic and thermal Enthalpies $=\quad-421.723424$

Sum of electronic and thermal Free Energies $=\quad-421.763926$

Coordinates (from last standard orientation):

\begin{tabular}{|c|c|c|c|c|}
\hline \multirow{2}{*}{$\begin{array}{l}\text { Center } \\
\text { Number }\end{array}$} & \multirow{2}{*}{$\begin{array}{l}\text { Atomic } \\
\text { Number }\end{array}$} & \multicolumn{3}{|c|}{ Coordinates (Angstroms) } \\
\hline & & & $\begin{array}{ll}X & Y\end{array}$ & Z \\
\hline 1 & 8 & 0.857812 & 0.000341 & 1.342972 \\
\hline 2 & 6 & 535748 & -1.117386 & 0.595524 \\
\hline 3 & 6 & -0.767810 & -1.181961 & 0.058908 \\
\hline 4 & 6 & -1.595624 & 0.000011 & -0.105094 \\
\hline 5 & 6 & -0.767953 & 1.182090 & 0.058528 \\
\hline 6 & 1 & -1.114535 & 2.121728 & -0.364727 \\
\hline 7 & 6 & 0.535739 & 1.117787 & 0.594982 \\
\hline 8 & 6 & 1.699339 & 0.713416 & -0.927671 \\
\hline 9 & 1 & 2.588284 & 1.204558 & -0.538067 \\
\hline 10 & 6 & 1.698984 & -0.713965 & -0.92759 \\
\hline
\end{tabular}




$\begin{array}{rrrrr}11 & 1 & 0.986234 & -2.016348 & 1.009937 \\ 12 & 1 & 0.986085 & 2.016765 & 1.008791 \\ 13 & 1 & 2.587633 & -1.205597 & -0.537934 \\ 14 & 1 & 1.249036 & 1.236696 & -1.765321 \\ 15 & 1 & 1.248275 & -1.237195 & -1.765023 \\ 16 & 8 & -2.788725 & -0.000192 & -0.439097 \\ 17 & 1 & -1.114250 & -2.121747 & -0.364140\end{array}$

\section{1,3 dipolar TS of $\boldsymbol{\gamma}$-pyrone and alkene IV (unstable) $\mathrm{HF}=-648.5314989$ hartrees $(-406960.000874739 \mathrm{kcal} / \mathrm{mol})$ Imaginary Frequencies: $1(-527.47871 / \mathrm{cm})$ \\ Zero-point correction $=0.156781$ (Hartree/Particle) \\ Thermal correction to Energy $=\quad 0.166626$ \\ Thermal correction to Enthalpy= $\quad 0.167570$ \\ Thermal correction to Gibbs Free Energy $=\quad 0.120954$ \\ Sum of electronic and zero-point Energies $=\quad-648.374718$ \\ Sum of electronic and thermal Energies $=\quad-648.364873$ \\ Sum of electronic and thermal Enthalpies $=\quad-648.363929$ \\ Sum of electronic and thermal Free Energies $=\quad-648.410544$ \\ Coordinates (from last standard orientation):}

\begin{tabular}{|c|c|c|c|c|}
\hline \multirow{2}{*}{$\begin{array}{l}\text { Center } \\
\text { Number }\end{array}$} & \multirow{2}{*}{$\begin{array}{l}\text { Atomic } \\
\text { Number }\end{array}$} & \multicolumn{3}{|c|}{ Coordinates (Angstroms) } \\
\hline & & & $\begin{array}{ll}X & Y\end{array}$ & Z \\
\hline 1 & 8 & 1.072982 & 1.406511 & 1.220811 \\
\hline 2 & 6 & 0.915249 & 1.533997 & -0.165565 \\
\hline 3 & 8 & -1.715369 & -0.611897 & -1.149657 \\
\hline 4 & 6 & 1.528890 & 0.496553 & -0.937268 \\
\hline 5 & 6 & 1.863690 & -0.792495 & -0.365770 \\
\hline 6 & 6 & 1.108369 & -0.949369 & 0.871767 \\
\hline 7 & 1 & 0.840183 & -1.947256 & 1.207422 \\
\hline 8 & 6 & 0.607896 & 0.163548 & 1.574876 \\
\hline 9 & 6 & -1.234862 & 0.501246 & 0.826154 \\
\hline 10 & 1 & -1.655659 & 0.828246 & 1.769875 \\
\hline 11 & 6 & -0.898855 & 1.413428 & -0.237126 \\
\hline 12 & 6 & -1.439056 & 0.752759 & -1.505810 \\
\hline 13 & 1 & 1.125302 & 2.557618 & -0.473039 \\
\hline 14 & 1 & 0.388464 & 0.083720 & 2.635988 \\
\hline 15 & 1 & -1.161980 & 2.460387 & -0.099328 \\
\hline 16 & 1 & -2.375092 & 1.227615 & -1.823062 \\
\hline 17 & 6 & -1.783122 & -0.743584 & 0.214414 \\
\hline 18 & 8 & -2.185868 & -1.741716 & 0.755486 \\
\hline 19 & 1 & -0.745872 & 0.741922 & -2.349778 \\
\hline 20 & 8 & 2.568269 & -1.658419 & -0.890898 \\
\hline 21 & 1 & 1.655340 & 0.635419 & -2.008046 \\
\hline
\end{tabular}

\section{1,3 dipolar product of $\boldsymbol{\gamma}$-pyrone and alkene IV}

$\mathrm{HF}=-648.5833679$ hartrees $(-406992.549190929 \mathrm{kcal} / \mathrm{mol})$

Imaginary Frequencies: none found

Zero-point correction $=0.161237$ (Hartree/Particle)

Thermal correction to Energy $=\quad 0.170377$

Thermal correction to Enthalpy $=\quad 0.171321$

Thermal correction to Gibbs Free Energy $=\quad 0.126350$ 


\begin{tabular}{|c|c|c|c|c|}
\hline \multirow{3}{*}{\begin{tabular}{l} 
Center \\
Number \\
\hdashline 1
\end{tabular}} & \multirow{2}{*}{$\begin{array}{l}\text { Atomic } \\
\text { Number }\end{array}$} & \multicolumn{3}{|c|}{ Coordinates (Angstroms) } \\
\hline & & & K $\quad Y$ & Z \\
\hline & 8 & -1.110079 & -0.816900 & 1.388653 \\
\hline 2 & 6 & -0.695537 & -1.408635 & 0.145160 \\
\hline 3 & 8 & 2.133042 & 0.113805 & -1.074541 \\
\hline 4 & 6 & -1.168704 & -0.390855 & -0.899202 \\
\hline 5 & 6 & -2.311404 & 0.458394 & -0.510433 \\
\hline 6 & 6 & -1.004452 & 0.982030 & -0.082000 \\
\hline 7 & 1 & -0.592820 & 1.892355 & -0.510642 \\
\hline 8 & 6 & -0.442612 & 0.445231 & 1.236304 \\
\hline 9 & 6 & 1.038983 & -0.008932 & 1.020202 \\
\hline 10 & 1 & 1.567562 & -0.066557 & 1.974965 \\
\hline 11 & 6 & 0.851237 & -1.373839 & 0.308858 \\
\hline 12 & 6 & 1.741057 & -1.266829 & -0.943632 \\
\hline 13 & 1 & -1.135249 & -2.403201 & 0.056357 \\
\hline 14 & 1 & -0.619446 & 1.064700 & 2.114845 \\
\hline 15 & 1 & 1.153933 & -2.216465 & 0.935086 \\
\hline 16 & 1 & 2.650971 & -1.867100 & -0.845340 \\
\hline 17 & 6 & 1.833604 & 0.842038 & 0.038652 \\
\hline 18 & 8 & 2.147527 & 1.998656 & 0.154347 \\
\hline 19 & 1 & 1.239099 & -1.555071 & -1.871623 \\
\hline 20 & 8 & -3.473478 & 0.702069 & -0.692433 \\
\hline 21 & 1 & -0.893167 & -0.501317 & -1.945308 \\
\hline
\end{tabular}

\footnotetext{
1,3 dipolar TS of $\gamma$-pyrone and alkene IV (diradical pathway) (stable) $\mathrm{HF}=-648.5315862$ hartrees $(-406960.055656362 \mathrm{kcal} / \mathrm{mol})$ Imaginary Frequencies: 1 (-688.2698 1/cm) Zero-point correction $=0.156067$ (Hartree/Particle) Thermal correction to Energy $=\quad 0.166139$ Thermal correction to Enthalpy $=\quad 0.167083$ Thermal correction to Gibbs Free Energy $=0.119809$ Sum of electronic and zero-point Energies $=\quad-648.375519$ Sum of electronic and thermal Energies $=\quad-648.365447$ Sum of electronic and thermal Enthalpies $=\quad-648.364503$ Sum of electronic and thermal Free Energies $=\quad-648.411777$ Coordinates (from last standard orientation):

\begin{tabular}{|c|c|c|c|c|}
\hline \multirow{2}{*}{$\begin{array}{l}\text { Center } \\
\text { Number }\end{array}$} & \multirow{2}{*}{$\begin{array}{l}\text { Atomic } \\
\text { Number }\end{array}$} & & \multicolumn{2}{|c|}{ Coordinates (Angstroms) } \\
\hline & & & $\begin{array}{ll}X & Y\end{array}$ & Z \\
\hline 1 & 8 & -1.093034 & -1.444136 & 1.176312 \\
\hline 2 & 6 & -0.913193 & -1.524470 & -0.214724 \\
\hline 3 & 8 & 1.698121 & 0.635269 & -1.128711 \\
\hline 4 & 6 & -1.531004 & -0.462023 & -0.959497 \\
\hline 5 & 6 & -1.820817 & 0.824681 & -0.357882 \\
\hline 6 & 6 & -1.118522 & 0.926653 & 0.919810 \\
\hline 7 & 1 & -0.870849 & 1.908517 & 1.312238 \\
\hline 8 & 6 & -0.651631 & -0.215616 & 1.589744 \\
\hline
\end{tabular}
}




\begin{tabular}{|c|c|c|c|c|}
\hline 9 & 6 & 1.235562 & -0.535245 & 0.814762 \\
\hline 10 & 1 & 1.638801 & -0.887754 & 1.756451 \\
\hline 11 & 6 & 0.874315 & -1.413345 & -0.272622 \\
\hline 12 & 6 & 1.423400 & -0.719185 & -1.521905 \\
\hline 13 & 1 & -1.139842 & -2.535269 & -0.552283 \\
\hline 14 & 1 & -0.432399 & -0.182507 & 2.653075 \\
\hline 15 & 1 & 1.142545 & -2.463315 & -0.165810 \\
\hline 16 & 1 & 2.360887 & -1.186740 & -1.845467 \\
\hline 17 & 6 & 1.782095 & 0.723778 & 0.239564 \\
\hline 18 & 8 & 2.197269 & 1.702582 & 0.806456 \\
\hline 19 & 1 & 0.734351 & -0.686265 & -2.368504 \\
\hline 20 & 8 & -2.481287 & 1.728828 & -0.877052 \\
\hline 21 & 1 & -1.683270 & -0.578379 & -2.029246 \\
\hline
\end{tabular}

\section{1,3 dipolar product of $\gamma$-pyrone and alkene IV (diradical pathway) \\ $\mathrm{HF}=-648.5833667$ hartrees $(-406992.548437917 \mathrm{kcal} / \mathrm{mol})$ \\ Imaginary Frequencies: none found \\ Zero-point correction $=0.161245($ Hartree/Particle $)$ \\ Thermal correction to Energy $=\quad 0.170385$ \\ Thermal correction to Enthalpy= $\quad 0.171329$ \\ Thermal correction to Gibbs Free Energy $=\quad 0.126352$ \\ Sum of electronic and zero-point Energies $=\quad-648.422122$ \\ Sum of electronic and thermal Energies $=\quad-648.412981$ \\ Sum of electronic and thermal Enthalpies $=\quad-648.412037$ \\ Sum of electronic and thermal Free Energies $=\quad-648.457015$ \\ Coordinates (from last standard orientation):}

\begin{tabular}{llrrr} 
Center & Atomic & \multicolumn{3}{c}{ Coordinates (Angstroms) } \\
Number & Number & X & $Y$ & $Z$ \\
-1 & 8 & -1.110299 & -0.813530 & 1.390039 \\
2 & 6 & -0.696569 & -1.408333 & 0.147769 \\
3 & 8 & 2.133285 & 0.111892 & -1.074828 \\
4 & 6 & -1.170441 & -0.392782 & -0.898400 \\
5 & 6 & -2.311788 & 0.458356 & -0.510577 \\
6 & 6 & -1.003681 & 0.981413 & -0.085207 \\
7 & 1 & -0.592585 & 1.891325 & -0.515196 \\
8 & 6 & -0.441983 & 0.447690 & 1.234437 \\
9 & 6 & 1.039134 & -0.008133 & 1.020007 \\
10 & 1 & 1.566824 & -0.065302 & 1.975279 \\
11 & 6 & 0.850377 & -1.373650 & 0.310107 \\
12 & 6 & 1.738969 & -1.268015 & -0.943468 \\
13 & 1 & -1.136512 & -2.403069 & 0.061717 \\
14 & 1 & -0.618459 & 1.069613 & 2.111356 \\
15 & 1 & 1.153824 & -2.215657 & 0.936786 \\
16 & 1 & 2.647942 & -1.869901 & -0.846342 \\
17 & 6 & 1.835217 & 0.841038 & 0.038151 \\
18 & 8 & 2.151155 & 1.997137 & 0.153451 \\
19 & 1 & 1.235433 & -1.555315 & -1.870885 \\
20 & 8 & -3.473808 & 0.702891 & -0.691834 \\
21 & 1 & -0.894540 & -0.504317 & -1.944259
\end{tabular}




\section{Investigations on Interconversion of 2 to its endo/exo atropisomer:}

Our efforts towards locating a pathway that interconverts 2 and its endo/exo atropisomer has lead to several intermediates and transition state structures that are close in energy to each other. Although we have not found a complete pathway for the interconversion, the calculations described below suggest that the associated energy surface is somewhat flat.

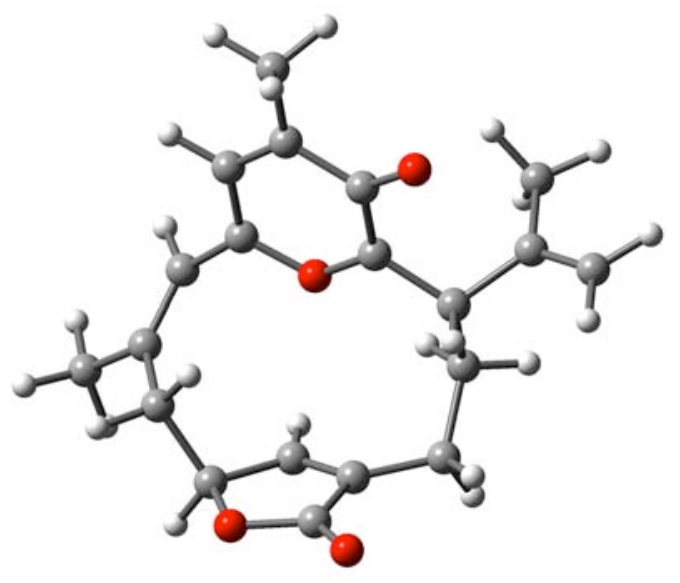

$\mathrm{HF}=-1076.1889022$ hartrees $(-675319.298019522 \mathrm{kcal} / \mathrm{mol})$ Imaginary Frequencies: $1(-32.61221 / \mathrm{cm})$

Zero-point correction $=0.379758$ (Hartree/Particle)

Thermal correction to Energy $=\quad 0.401404$

Thermal correction to Enthalpy= $\quad 0.402348$

Thermal correction to Gibbs Free Energy $=\quad 0.330126$

Sum of electronic and zero-point Energies $=\quad-1075.809144$

Sum of electronic and thermal Energies $=\quad-1075.787498$

Sum of electronic and thermal Enthalpies $=\quad-1075.786554$

Sum of electronic and thermal Free Energies $=\quad-1075.858776$

Coordinates (from last standard orientation):

\begin{tabular}{ccccc} 
Center & Atomic & \multicolumn{3}{c}{ Coordinates } \\
Number & Number & X & $Y$ & $Z$ \\
- \hdashline 1 & 8 & 0.218969 & 0.691991 & -0.519066 \\
2 & 6 & 1.646343 & -1.262434 & -0.240896 \\
3 & 1 & 1.517663 & -1.745819 & 0.739707 \\
4 & 8 & 3.270783 & 0.604087 & 1.315274 \\
5 & 6 & 1.361174 & 0.208330 & -0.017238 \\
6 & 8 & -3.167792 & -1.289617 & 1.364028 \\
7 & 6 & 2.249261 & 1.050016 & 0.755150 \\
8 & 8 & -1.823611 & -2.971173 & 2.081893 \\
9 & 6 & 1.877299 & 2.456319 & 0.782872 \\
10 & 6 & 0.692281 & 2.875243 & 0.197363 \\
11 & 1 & 0.391941 & 3.918855 & 0.243293 \\
12 & 6 & -0.166648 & 1.981301 & -0.426129 \\
13 & 6 & -1.467644 & 2.331948 & -1.021357 \\
14 & 1 & -1.425133 & 3.072266 & -1.821852 \\
15 & 6 & -2.681044 & 1.898861 & -0.626221 \\
16 & 6 & -2.873746 & 0.974508 & 0.555244 \\
17 & 1 & -3.655880 & 1.375905 & 1.212950
\end{tabular}




\begin{tabular}{rrrrr}
18 & 1 & -1.954152 & 0.911471 & 1.143856 \\
19 & 6 & -3.262252 & -0.477276 & 0.181750 \\
20 & 1 & -4.299554 & -0.525172 & -0.171911 \\
21 & 6 & -2.286783 & -1.090316 & -0.775278 \\
22 & 1 & -2.139049 & -0.708196 & -1.778191 \\
23 & 6 & -1.588609 & -2.050905 & -0.163107 \\
24 & 6 & -0.416460 & -2.842091 & -0.659913 \\
25 & 1 & -0.030773 & -3.452856 & 0.164418 \\
26 & 1 & -0.762076 & -3.548778 & -1.426676 \\
27 & 6 & 0.718219 & -1.988350 & -1.269934 \\
28 & 1 & 0.301082 & -1.275940 & -1.989876 \\
29 & 1 & 1.354525 & -2.668744 & -1.843677 \\
30 & 6 & 3.109147 & -1.512561 & -0.641939 \\
31 & 6 & 3.805130 & -2.495762 & -0.067345 \\
32 & 1 & 4.811629 & -2.743164 & -0.394324 \\
33 & 1 & 3.411031 & -3.069259 & 0.767672 \\
34 & 6 & 3.663349 & -0.694366 & -1.780537 \\
35 & 1 & 3.794938 & 0.351202 & -1.478138 \\
36 & 1 & 2.996187 & -0.701759 & -2.653854 \\
37 & 1 & 4.639024 & -1.074396 & -2.097618 \\
38 & 6 & -3.931478 & 2.359251 & -1.333003 \\
39 & 1 & -3.708307 & 3.070083 & -2.134109 \\
40 & 1 & -4.623539 & 2.838377 & -0.627792 \\
41 & 1 & -4.477147 & 1.515320 & -1.777602 \\
42 & 6 & -2.148425 & -2.205574 & 1.209142 \\
43 & 6 & 2.811023 & 3.381145 & 1.494888 \\
44 & 1 & 3.823432 & 3.277627 & 1.085493 \\
45 & 1 & 2.492166 & 4.425550 & 1.430199 \\
46 & 1 & 2.894385 & 3.091400 & 2.549922 \\
---------------------------------------------- \\
\hline
\end{tabular}

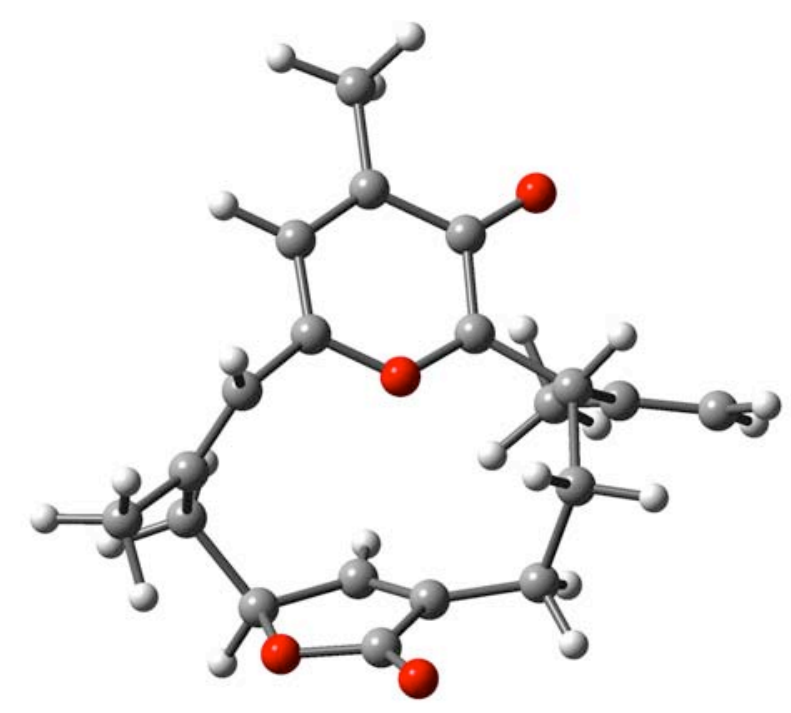

$\mathrm{HF}=-1076.1884698$ hartrees $(-675319.026684198 \mathrm{kcal} / \mathrm{mol})$ Imaginary Frequencies: $1(-15.25331 / \mathrm{cm})$

Zero-point correction $=0.380209$ (Hartree/Particle)

Thermal correction to Energy $=\quad 0.401733$

Thermal correction to Enthalpy= $\quad 0.402677$

Thermal correction to Gibbs Free Energy $=0.331232$

Sum of electronic and zero-point Energies $=\quad-1075.808261$ 

$\begin{array}{ll}\text { Sum of electronic and thermal Energies= } & -1075.786737 \\ \text { Sum of electronic and thermal Enthalpies= } & -1075.785793 \\ \text { Sum of electronic and thermal Free Energies }= & -1075.857238 \\ \text { Coordinates (from last standard orientation): } & \end{array}$

\begin{tabular}{|c|c|c|c|c|}
\hline \multirow{2}{*}{$\begin{array}{l}\text { Center } \\
\text { Number }\end{array}$} & \multirow{2}{*}{$\begin{array}{l}\text { Atomic } \\
\text { Number }\end{array}$} & \multicolumn{3}{|c|}{ Coordinates (Angstroms) } \\
\hline & & & $\begin{array}{ll}\mathrm{Y} \\
\mathrm{y}\end{array}$ & Z \\
\hline 1 & 8 & -0.284201 & 0.585284 & -0.605731 \\
\hline 2 & 6 & -1.852837 & -1.247146 & -0.788046 \\
\hline 3 & 1 & -2.535657 & -1.178161 & -1.649346 \\
\hline 4 & 6 & -1.554413 & 0.197286 & -0.477155 \\
\hline 5 & 8 & 3.444252 & -0.825690 & 0.349785 \\
\hline 6 & 8 & 3.034595 & -2.224102 & -1.387035 \\
\hline 7 & 6 & 0.137708 & 1.858559 & -0.478817 \\
\hline 8 & 6 & 1.562797 & 2.090599 & -0.781854 \\
\hline 9 & 1 & 1.751917 & 2.565322 & -1.746319 \\
\hline 10 & 6 & 2.627077 & 1.808147 & -0.010965 \\
\hline 11 & 6 & 2.537415 & 1.231763 & 1.389169 \\
\hline 12 & 1 & 1.582307 & 1.499509 & 1.854648 \\
\hline 13 & 1 & 3.326928 & 1.691920 & 1.994634 \\
\hline 14 & 6 & 2.701690 & -0.320171 & 1.471603 \\
\hline 15 & 1 & 3.270521 & -0.569303 & 2.376147 \\
\hline 16 & 6 & 1.402258 & -1.068625 & 1.422076 \\
\hline 17 & 1 & 0.632958 & -0.931847 & 2.174355 \\
\hline 18 & 6 & 1.350967 & -1.845237 & 0.335855 \\
\hline 19 & 6 & 0.281097 & 9707 & -0.210950 \\
\hline 20 & 1 & 0.785340 & -3.585804 & -0.691826 \\
\hline 21 & 1 & -0.328429 & -3.149305 & 0.600728 \\
\hline 22 & 6 & -0.646094 & -2.100833 & -1.277550 \\
\hline 23 & 1 & -1.085617 & -2.931524 & -1.839100 \\
\hline 24 & 1 & -0.044357 & -1.529033 & -1.992336 \\
\hline 25 & 6 & -2.667938 & -1.980967 & 0.295629 \\
\hline 26 & 6 & -3.421745 & -3.027067 & -0.056384 \\
\hline 27 & 1 & -3.979764 & -3.598372 & 0.680515 \\
\hline 28 & 1 & -3.529408 & -3.340671 & -1.092317 \\
\hline 29 & 6 & -2.581739 & -1.523687 & 1.729384 \\
\hline 30 & 1 & -3.081722 & -0.555429 & 1.838737 \\
\hline 31 & 1 & -1.544330 & -1.405152 & 2.067658 \\
\hline 32 & 1 & -3.073759 & -2.238621 & 2.396141 \\
\hline 33 & 6 & 4.021840 & 2.111793 & -0.497905 \\
\hline 34 & 1 & 4.016957 & 2.539734 & -1.504596 \\
\hline 35 & 1 & 4.625478 & 1.197898 & -0.512712 \\
\hline 36 & 1 & 4.521305 & 2.820982 & 0.176293 \\
\hline 37 & 6 & 2.654573 & -1.695726 & -0.372242 \\
\hline 38 & 6 & -0.812040 & 2.832855 & -0.211142 \\
\hline 39 & 6 & -2.607815 & 1.148113 & -0.216674 \\
\hline 40 & 6 & -2.159008 & 2.521191 & -0.069430 \\
\hline 41 & 8 & -3.806468 & 0.798176 & -0.134729 \\
\hline 42 & 6 & -3.211561 & 3.547064 & 0.203903 \\
\hline 43 & 1 & -3.954158 & 3.545507 & -0.603740 \\
\hline 44 & 1 & -3.769181 & 3.280400 & 1.110414 \\
\hline 45 & 1 & -2.794943 & 4.552625 & 0.312629 \\
\hline 46 & 1 & -0.471205 & 3.860750 & -0.119968 \\
\hline
\end{tabular}




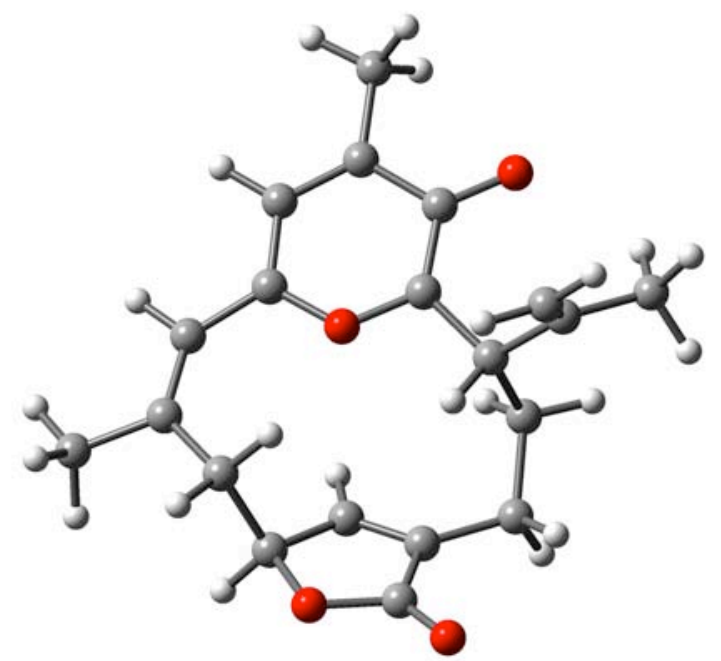

$\mathrm{HF}=-1076.1971749$ hartrees $(-675324.489221499 \mathrm{kcal} / \mathrm{mol})$ Imaginary Frequencies: none found Zero-point correction $=0.380896$ (Hartree/Particle)

Thermal correction to Energy $=\quad 0.403146$ Thermal correction to Enthalpy= $\quad 0.404090$ Thermal correction to Gibbs Free Energy $=\quad 0.330047$ Sum of electronic and zero-point Energies $=\quad-1075.816278$ Sum of electronic and thermal Energies $=\quad-1075.794029$ Sum of electronic and thermal Enthalpies $=\quad-1075.793085$ Sum of electronic and thermal Free Energies $=\quad-1075.867128$ Coordinates (from last standard orientation):

\begin{tabular}{|c|c|c|c|c|}
\hline \multirow{2}{*}{$\begin{array}{l}\text { Center } \\
\text { Number }\end{array}$} & \multirow{2}{*}{$\begin{array}{l}\text { Atomic } \\
\text { Number }\end{array}$} & \multicolumn{3}{|c|}{ Coordinates (Angstroms) } \\
\hline & & & $\begin{array}{l}X \\
Y\end{array}$ & Z \\
\hline 1 & 8 & 0.371149 & 0.877494 & 0.208685 \\
\hline 2 & 6 & 1.012101 & -1.390483 & 0.116305 \\
\hline 3 & 1 & 0.080642 & -1.386140 & 0.692196 \\
\hline 4 & 8 & 3.747578 & -0.139502 & -0.336354 \\
\hline 5 & 6 & 1.430099 & 0.060605 & 0.095776 \\
\hline 6 & 8 & -3.658076 & -0.898970 & 0.347710 \\
\hline 7 & 6 & 2.748247 & 0.586759 & -0.148140 \\
\hline 8 & 8 & -3.133435 & -3.102937 & 0.449957 \\
\hline 9 & 6 & 2.816092 & 2.043487 & -0.174735 \\
\hline 10 & 6 & 666434 & 2.805390 & -0.064695 \\
\hline 11 & 1 & .717775 & 3.889155 & -0.126503 \\
\hline 12 & 6 & 0.412698 & 2.217832 & 0.115871 \\
\hline 13 & 6 & -0.835481 & 2.947598 & 0.136753 \\
\hline 14 & 1 & -0.681163 & 3.996003 & -0.113306 \\
\hline 15 & 6 & -2.114541 & 2.570802 & 0.389425 \\
\hline 16 & 6 & -2.563698 & 1.200571 & 0.842527 \\
\hline 17 & 1 & -3.386828 & 1.320146 & 1.557947 \\
\hline 18 & 1 & -1.762323 & 0.662895 & 1.353995 \\
\hline 19 & 6 & -3.103749 & 0.269760 & -0.280001 \\
\hline 20 & 1 & -3.912512 & 0.766789 & -0.829782 \\
\hline 21 & 6 & -2.020099 & -0.263159 & -1.168050 \\
\hline 22 & 1 & -1.419745 & 0.384673 & -1.795384 \\
\hline 23 & 6 & -1.854315 & -1.574526 & -0.953813 \\
\hline 24 & 6 & -0.767204 & -2.510082 & -1.405972 \\
\hline
\end{tabular}




$\begin{array}{rrrrr}25 & 1 & -0.814490 & -3.406715 & -0.776655 \\ 26 & 1 & -0.953636 & -2.847808 & -2.434403 \\ 27 & 6 & 0.633727 & -1.865031 & -1.332748 \\ 28 & 1 & 0.664009 & -1.010257 & -2.018356 \\ 29 & 1 & 1.379478 & -2.569782 & -1.708567 \\ 30 & 6 & 1.974851 & -2.304525 & 0.891750 \\ 31 & 6 & 1.839655 & -2.382062 & 2.220198 \\ 32 & 1 & 2.499830 & -2.999468 & 2.823417 \\ 33 & 1 & 1.073661 & -1.827478 & 2.758228 \\ 34 & 6 & 3.019419 & -3.105016 & 0.156123 \\ 35 & 1 & 3.604525 & -2.455097 & -0.499568 \\ 36 & 1 & 2.566359 & -3.900533 & -0.451434 \\ 37 & 1 & 3.698577 & -3.586465 & 0.866300 \\ 38 & 6 & -3.220320 & 3.591389 & 0.265297 \\ 39 & 1 & -2.853319 & 4.559226 & -0.088671 \\ 40 & 1 & -3.720553 & 3.746436 & 1.231438 \\ 41 & 1 & -3.999618 & 3.252771 & -0.432175 \\ 42 & 6 & -2.911805 & -2.004553 & 0.004341 \\ 43 & 6 & 4.171128 & 2.641009 & -0.382095 \\ 44 & 1 & 4.595842 & 2.287239 & -1.329614 \\ 45 & 1 & 4.147423 & 3.734533 & -0.379213 \\ 46 & 1 & 4.858909 & 2.286612 & 0.395424 \\ --------------------------------------------------------\end{array}$

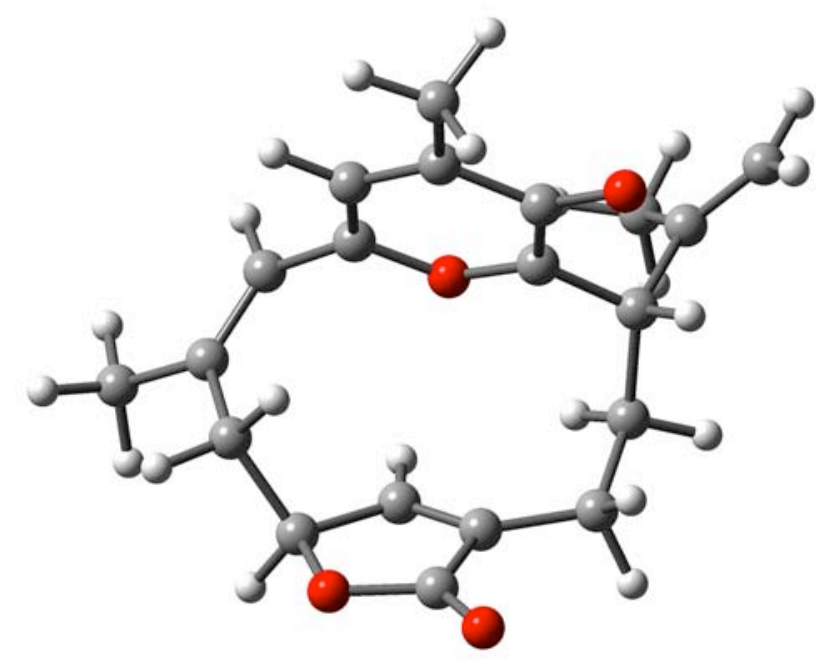

$\mathrm{HF}=-1076.1952919$ hartrees $(-675323.307620169 \mathrm{kcal} / \mathrm{mol})$ Imaginary Frequencies: none found

Zero-point correction $=0.380144$ (Hartree/Particle)

Thermal correction to Energy= $\quad 0.402682$

Thermal correction to Enthalpy $=\quad 0.403626$

Thermal correction to Gibbs Free Energy $=0.328666$

Sum of electronic and zero-point Energies $=\quad-1075.815148$

Sum of electronic and thermal Energies $=\quad-1075.792610$

Sum of electronic and thermal Enthalpies $=\quad-1075.791666$

Sum of electronic and thermal Free Energies $=\quad-1075.866625$

Coordinates (from last standard orientation):

$\begin{array}{llll}\text { Center } & \text { Atomic } & \text { Coordinates (Angstroms) } \\ \text { Number } & \text { Number } & X & Y\end{array}$




$\begin{array}{rrrrr}1 & 8 & 0.473558 & 0.622907 & -0.700548 \\ 2 & 6 & 2.083853 & -1.146864 & -0.315911 \\ 3 & 1 & 2.550580 & -1.487940 & 0.613966 \\ 4 & 8 & 2.896396 & 0.503084 & 1.912932 \\ 5 & 6 & 1.456487 & 0.161958 & 0.073841 \\ 6 & 8 & -3.207171 & -1.262770 & 1.169183 \\ 7 & 6 & 1.958037 & 0.918445 & 1.199562 \\ 8 & 8 & -1.963849 & -2.650808 & 2.463625 \\ 9 & 6 & 1.275766 & 2.182261 & 1.417492 \\ 10 & 6 & 0.234961 & 2.570668 & 0.578569 \\ 11 & 1 & -0.286946 & 3.508652 & 0.747754 \\ 12 & 6 & -0.181247 & 1.782084 & -0.480840 \\ 13 & 6 & -1.309855 & 2.038697 & -1.403416 \\ 14 & 1 & -1.085985 & 2.575697 & -2.326749 \\ 15 & 6 & -2.578381 & 1.653651 & -1.169341 \\ 16 & 6 & -2.971263 & 0.887188 & 0.078285 \\ 17 & 1 & -3.940971 & 1.238649 & 0.451448 \\ 18 & 1 & -2.236221 & 1.050025 & 0.871283 \\ 19 & 6 & -3.064119 & -0.648985 & -0.122494 \\ 20 & 1 & -3.952568 & -0.906569 & -0.713338 \\ 21 & 6 & -1.808539 & -1.246786 & -0.684668 \\ 22 & 1 & -1.443081 & -0.995025 & -1.671308 \\ 23 & 6 & -1.212286 & -2.015464 & 0.232366 \\ 24 & 6 & 0.089115 & -2.763997 & 0.200702 \\ 25 & 1 & 0.508468 & -2.766326 & 1.214800 \\ 26 & 1 & -0.134583 & -3.818894 & -0.013544 \\ 27 & 6 & 1.132277 & -2.258853 & -0.819912 \\ 28 & 1 & 0.632847 & -1.952465 & -1.745793 \\ 29 & 1 & 1.776352 & -3.102690 & -1.094213 \\ 30 & 6 & 3.229109 & -0.864062 & -1.303636 \\ 31 & 6 & 4.489379 & -0.799767 & -0.861987 \\ 32 & 1 & 5.313867 & -0.579598 & -1.536632 \\ 33 & 1 & 4.732101 & -0.936146 & 0.187326 \\ 34 & 6 & 2.869661 & -0.620582 & -2.749902 \\ 35 & 1 & 2.111021 & 0.166878 & -2.850051 \\ 36 & 1 & 2.454773 & -1.518625 & -3.225959 \\ 37 & 1 & 3.749681 & -0.316263 & -3.324580 \\ 38 & 6 & -3.679604 & 1.953644 & -2.154280 \\ 39 & 1 & -3.306071 & 2.482380 & -3.036127 \\ 40 & 1 & -4.460028 & 2.569586 & -1.688148 \\ 41 & 1 & -4.172995 & 1.032695 & -2.493488 \\ 42 & 6 & -2.106399 & -2.054746 & 1.425991 \\ 43 & 6 & 1.747212 & 3.011065 & 2.568372 \\ 44 & 1 & 2.807214 & 3.264007 & 2.438956 \\ 45 & 1 & 1.166521 & 3.930590 & 2.685705 \\ 46 & 1 & 1.699578 & 2.424751 & 3.494399 \\ ---------------------------------------------- \\ \end{array}$




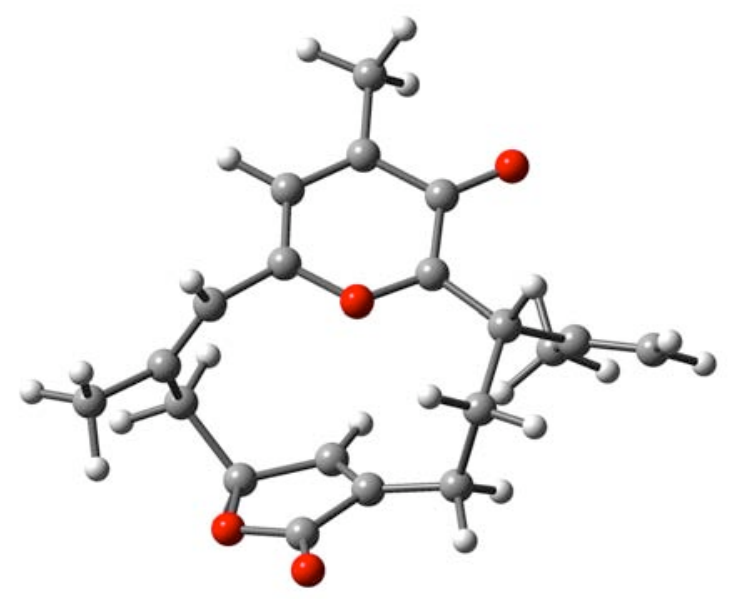

$\mathrm{HF}=-1076.1886298$ hartrees $(-675319.127085798 \mathrm{kcal} / \mathrm{mol})$ Imaginary Frequencies: none found

Zero-point correction $=0.380600$ (Hartree/Particle)

Thermal correction to Energy $=\quad 0.402826$

Thermal correction to Enthalpy= $\quad 0.403771$

Thermal correction to Gibbs Free Energy $=\quad 0.329991$

Sum of electronic and zero-point Energies $=\quad-1075.808030$

Sum of electronic and thermal Energies $=\quad-1075.785803$

Sum of electronic and thermal Enthalpies $=\quad-1075.784859$

Sum of electronic and thermal Free Energies $=\quad-1075.858639$

Coordinates (from last standard orientation):

\begin{tabular}{|c|c|c|c|c|}
\hline \multirow{2}{*}{$\begin{array}{l}\text { Center } \\
\text { Number }\end{array}$} & \multirow{2}{*}{$\begin{array}{l}\text { Atomic } \\
\text { Number }\end{array}$} & \multicolumn{3}{|c|}{ Coordinates (Angstroms) } \\
\hline & & & $X \quad Y$ & Z \\
\hline 1 & 8 & -0.288618 & 0.561293 & -0.784851 \\
\hline 2 & 6 & -1.922580 & -1.192329 & -0.867812 \\
\hline 3 & 1 & -2.528025 & -1.124627 & -1.785953 \\
\hline 4 & 6 & -1.562196 & 0.238724 & -0.555149 \\
\hline 5 & 8 & 3.397332 & -0.941249 & 0.579117 \\
\hline 6 & 8 & 3.049172 & -2.540082 & -0.989127 \\
\hline 7 & 6 & 0.238753 & 1.786237 & -0.617302 \\
\hline 8 & 6 & 1.660835 & 1.893892 & -1.001391 \\
\hline 9 & 1 & 1.847470 & 2.168239 & -2.041206 \\
\hline 10 & 6 & 2.723110 & 1.679721 & -0.207484 \\
\hline 11 & 6 & 2.625458 & 1.302590 & 1.259512 \\
\hline 12 & 1 & 1.733716 & 1.743274 & 1.719737 \\
\hline 13 & 1 & 3.493970 & 1.720046 & 1.781260 \\
\hline 14 & 6 & 2.591916 & -0.231059 & 1.533593 \\
\hline 15 & 1 & 3.023258 & -0.413418 & 2.526743 \\
\hline 16 & 6 & 1.234736 & -0.862694 & 1.416546 \\
\hline 17 & 1 & 0.403720 & -0.554193 & 2.041911 \\
\hline 18 & 6 & 1.229479 & -1.787250 & 0.450061 \\
\hline 19 & 6 & 0.155247 & -2.685164 & -0.092374 \\
\hline 20 & 1 & 0.662445 & -3.568789 & -0.495702 \\
\hline 21 & 1 & -0.494203 & $3-3.034970$ & 0.715870 \\
\hline 22 & 6 & -0.716806 & -2.108004 & -1.239428 \\
\hline 23 & 1 & -1.156342 & -2.968842 & -1.753267 \\
\hline 24 & 1 & -0.074580 & -1.604069 & -1.969374 \\
\hline 25 & 6 & -2.840440 & -1.867662 & 0.166895 \\
\hline
\end{tabular}




$\begin{array}{rrrrr}26 & 6 & -3.692960 & -2.810096 & -0.247419 \\ 27 & 1 & -4.322528 & -3.353778 & 0.451647 \\ 28 & 1 & -3.810749 & -3.061110 & -1.299244 \\ 29 & 6 & -2.734380 & -1.495153 & 1.625108 \\ 30 & 1 & -3.133533 & -0.487243 & 1.778065 \\ 31 & 1 & -1.699165 & -1.509657 & 1.988828 \\ 32 & 1 & -3.314481 & -2.187740 & 2.243073 \\ 33 & 6 & 4.123582 & 1.822774 & -0.748292 \\ 34 & 1 & 4.124223 & 2.082616 & -1.810826 \\ 35 & 1 & 4.678092 & 0.887109 & -0.616978 \\ 36 & 1 & 4.671103 & 2.603418 & -0.202598 \\ 37 & 6 & 2.612217 & -1.843130 & -0.107774 \\ 38 & 6 & -0.608201 & 2.802247 & -0.204764 \\ 39 & 6 & -2.526906 & 1.246034 & -0.185695 \\ 40 & 6 & -1.961385 & 2.570249 & 0.019508 \\ 41 & 8 & -3.742395 & 0.987678 & -0.042577 \\ 42 & 6 & -2.911908 & 3.643081 & 0.444372 \\ 43 & 1 & -3.688213 & 3.781127 & -0.318855 \\ 44 & 1 & -3.448496 & 3.330406 & 1.348730 \\ 45 & 1 & -2.409047 & 4.597593 & 0.625204 \\ 46 & 1 & -0.187989 & 3.795433 & -0.071833 \\ -----------------------------------------------------\end{array}$


Nucleus-Independent Chemical Shifts (NICS) Calculations: A ghost atom was placed in the center of the 6-membered ring. The following systems were examined: $\mathbf{E}$ (oxidopyrylium), $\boldsymbol{\gamma}$-pyrone, $\mathbf{F}$, and $\mathbf{G}$ on their own as well as in the 1,3-dipolar cycoladdition transition state structure with ethene (the structure for the $\mathbf{E}+$ ethane transition state is shown here as an example). NICS on E.IV.TS1-4 were also calculated.

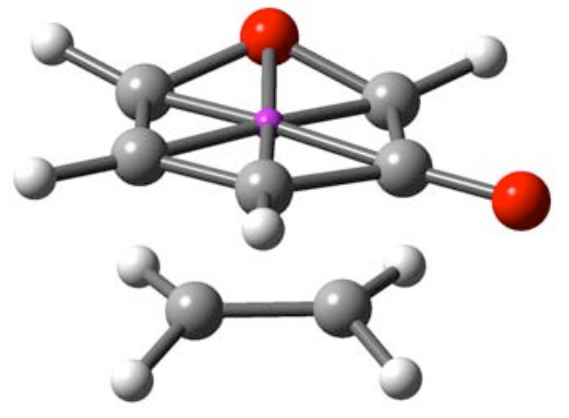

Table S1.

\begin{tabular}{|l|c|}
\hline & $\begin{array}{c}\text { NICS (B3LYP/6- } \\
\mathbf{3 1 1 + G ( d , p ) ~}\end{array}$ \\
\hline E & -1.9 \\
\hline E + ethene TS & -8.3 \\
\hline$\gamma$-pyrone & 0.1 \\
\hline$\gamma$-pyrone + ethene TS & -8.3 \\
\hline F & 2.9 \\
\hline F + ethene TS & -2.0 \\
\hline G & 0.8 \\
\hline G + ethene TS & -4.4 \\
\hline E.IV.TS1 & -7.5 \\
\hline E.IV.TS2 & -6.8 \\
\hline E.IV.TS3 & -7.1 \\
\hline E.IV.TS4 & -7.1 \\
\hline
\end{tabular}


NMR Calculations: Chemical shifts for hydrogens 12 and 13, shown in the pictures below (left: $\mathbf{E}+$ ethene; right: $\mathbf{F}+$ ethene), were calculated with B3LYP/6-311+G(d,p).
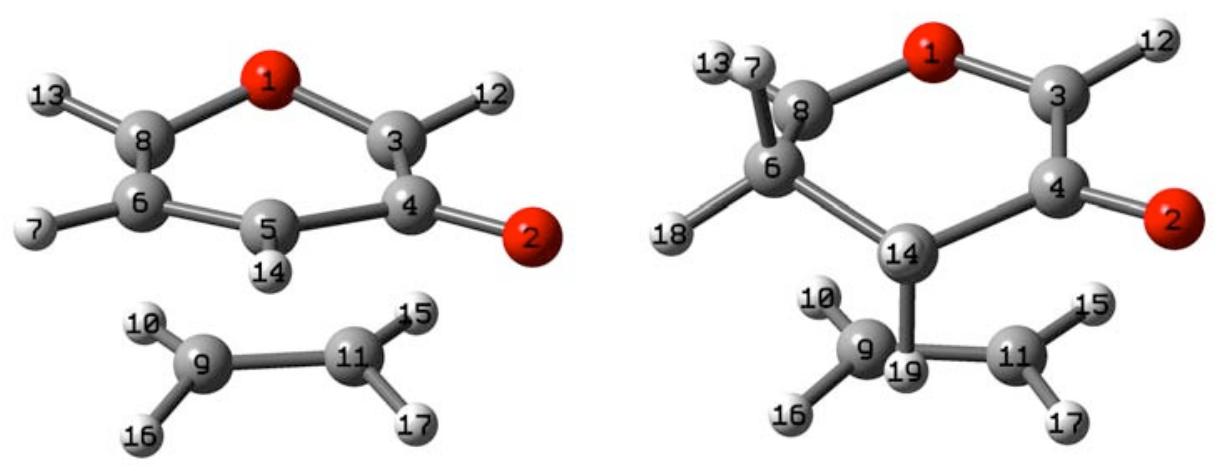

Table S2.

\begin{tabular}{|l|c|l|c|}
\hline E + ethene & $\begin{array}{c}\text { Chemical shift (ppm) } \\
\text { (B3LYP/6-311+G(2d,p) }\end{array}$ & F + ethene & $\begin{array}{c}\text { Chemical shift (ppm) } \\
\text { (B3LYP/6-311+G(d,p) }\end{array}$ \\
\hline Hydrogen 12 & 7.33 & Hydrogen 12 & 7.84 \\
\hline Hydrogen 13 & 7.32 & Hydrogen 13 & 7.54 \\
\hline
\end{tabular}


Table S3. Energies (overall exothermicity for reaction; barrier), Dipole moments, BSSE (for some) of the transition state structures.

\begin{tabular}{|c|c|c|c|c|c|c|c|c|c|}
\hline & $\Delta \mathbf{E}$ & $\Delta \mathrm{E} \ddagger$ & $\begin{array}{l}\Delta \mathbf{G} \text { at } \\
25^{\circ} \mathrm{C}\end{array}$ & $\begin{array}{l}\Delta \mathrm{G} \ddagger \text { at } \\
25^{\circ} \mathrm{C}\end{array}$ & $\begin{array}{l}\Delta \mathbf{G} \text { at } \\
150^{\circ} \mathrm{C}\end{array}$ & $\begin{array}{l}\Delta \mathrm{G} \ddagger \text { at } \\
150^{\circ} \mathrm{C}\end{array}$ & $\begin{array}{l}\text { Dipole } \\
\text { Moment }\end{array}$ & $\begin{array}{c}\text { BSSE } \\
\text { [B3LYP/6- } \\
\text { 31G(d)] }\end{array}$ & $\begin{array}{c}\text { BSSE } \\
\text { [B3LYP/6- } \\
311+G(d, p)]\end{array}$ \\
\hline $\begin{array}{l}\text { A.IV.TS1 } \\
\text { (s-cis) }\end{array}$ & -16.5 & 13.9 & -1.4 & 28.3 & 5.4 & 34.5 & 4.23 & & \\
\hline $\begin{array}{l}\text { A.IV.TS2 } \\
\text { (s-cis) }\end{array}$ & -14.0 & 19.2 & 1.0 & 33.3 & 7.6 & 39.4 & 5.81 & & \\
\hline $\begin{array}{l}\text { A.IV.TS3 } \\
\text { (s-cis) }\end{array}$ & -14.6 & 15.3 & 0.3 & 29.2 & 6.9 & 35.1 & 2.78 & & \\
\hline $\begin{array}{l}\text { A.IV.TS4 } \\
\text { (s-cis) }\end{array}$ & -16.1 & 16.7 & -2.0 & 31.0 & 4.2 & 37.1 & 6.91 & & \\
\hline $\begin{array}{l}\text { A.IVTS1 } \\
\text { (s-trans) }\end{array}$ & -16.2 & 13.9 & -1.5 & 28.1 & 5.1 & 34.3 & 4.50 & & \\
\hline $\begin{array}{l}\text { A.IVTS2 } \\
\text { (s-trans) }\end{array}$ & -14.1 & 19.1 & 0.6 & 33.2 & 7.2 & 39.3 & 7.25 & & \\
\hline $\begin{array}{l}\text { A.IVTS3 } \\
\text { (s-trans) }\end{array}$ & -15.1 & 14.9 & -1.0 & 28.9 & 5.3 & 34.9 & 2.62 & & \\
\hline $\begin{array}{l}\text { A.IVTS4 } \\
\text { (s-trans) }\end{array}$ & -16.7 & 15.6 & -1.8 & 30.0 & 4.8 & 36.2 & 6.92 & & \\
\hline B.IV.TS1 & -23.6 & 12.1 & -8.6 & 26.3 & -1.9 & 32.5 & 4.77 & & \\
\hline B.IV.TS2 & -22.3 & 17.5 & -7.6 & 31.3 & -1.1 & 37.3 & 8.38 & & \\
\hline B.IV.TS3 & -25.2 & 13.5 & -10.6 & 27.1 & -4.0 & 33.0 & 2.57 & & \\
\hline B.IV.TS4 & -25.3 & 13.1 & -10.7 & 26.8 & -4.2 & 32.7 & 7.13 & & \\
\hline C.IV.TS1 & -27.4 & 10.3 & -13.4 & 23.6 & -7.1 & 29.4 & 4.74 & & \\
\hline C.IV.TS2 & -25.4 & 17.0 & -11.7 & 29.9 & -5.6 & 35.4 & 8.87 & & \\
\hline C.IV.TS3 & -30.5 & 10.8 & -17.1 & 23.6 & -11.2 & 29.1 & 2.58 & & \\
\hline C.IV.TS4 & -29.8 & 13.3 & -16.5 & 26.1 & -10.6 & 31.6 & 7.71 & & \\
\hline D.IV.TS1 & -25.3 & 11.8 & -11.1 & 25.5 & -4.7 & 31.5 & 4.54 & & \\
\hline D.IV.TS2 & -23.4 & 16.1 & -9.2 & 29.3 & -2.8 & 35.0 & 7.83 & & \\
\hline D.IV.TS3 & -26.8 & 13.0 & -12.5 & 26.4 & -6.1 & 32.2 & 1.90 & & \\
\hline D.IV.TS4 & -26.8 & 12.5 & -12.5 & 25.8 & -6.2 & 31.5 & 7.16 & & \\
\hline E.IV.TS1 & -29.6 & 9.7 & -15.9 & 22.9 & -9.7 & 28.6 & 4.29 & $\begin{array}{l}3.9 \text { (5.9 in its } \\
\text { product) }\end{array}$ & $\begin{array}{l}1.3 \text { (2.6 in its } \\
\text { product) }\end{array}$ \\
\hline E.IV.TS2 & -27.0 & 15.3 & -13.4 & 28.0 & -7.3 & 33.5 & 8.24 & $\begin{array}{l}3.5 \text { (5.9 in its } \\
\text { product) }\end{array}$ & $\begin{array}{l}1.2 \text { (2.6 in its } \\
\text { product) }\end{array}$ \\
\hline E.IV.TS3 & -32.4 & 10.1 & -19.0 & 22.9 & -13.0 & 28.5 & 1.82 & $\begin{array}{l}3.3 \text { (5.7 in its } \\
\text { product) }\end{array}$ & $\begin{array}{l}1.3 \text { (2.6 in its } \\
\text { product) }\end{array}$ \\
\hline E.IV.TS4 & -31.6 & 12.5 & -18.2 & 25.3 & -12.2 & 30.8 & 7.71 & $\begin{array}{l}3.3 \text { (5.6 in its } \\
\text { product) }\end{array}$ & $\begin{array}{c}1.3 \text { (2.6 in its } \\
\text { product) }\end{array}$ \\
\hline F.IV.TS1 & -56.1 & 1.7 & -42.4 & 14.7 & -36.2 & 20.4 & 3.93 & & \\
\hline F.IV.TS2 & -53.6 & 6.4 & -39.8 & 19.2 & -33.6 & 24.6 & 8.18 & & \\
\hline F.IV.TS3 & -59.3 & 2.5 & -46.0 & 15.1 & -39.9 & 20.5 & 2.19 & & \\
\hline F.IV.TS4 & -58.6 & 4.6 & -45.3 & 17.2 & -39.4 & 22.5 & 8.01 & & \\
\hline $\begin{array}{l}\text { A.I.TS1 } \\
\text { (s-cis) } \\
\text { (Me } \\
\text { down) }\end{array}$ & -10.3 & 17.8 & 5.8 & 33.0 & 12.7 & 39.4 & 3.84 & & \\
\hline $\begin{array}{l}\text { A.I.TS2 } \\
\text { (s-cis) } \\
\text { (Me } \\
\text { down) }\end{array}$ & -6.7 & 25.1 & 9.3 & 40.5 & 16.2 & 47.0 & 7.08 & & \\
\hline $\begin{array}{l}\text { A.I.TS3 } \\
\text { (s-cis) } \\
\text { (Me } \\
\text { down) }\end{array}$ & -4.2 & 17.7 & 12.3 & 32.7 & 19.5 & 38.9 & 2.21 & & \\
\hline $\begin{array}{l}\text { A.I.TS4 } \\
\text { (s-cis) }\end{array}$ & -9.8 & 21.1 & 6.3 & 36.2 & 13.3 & 42.5 & 6.98 & & \\
\hline
\end{tabular}




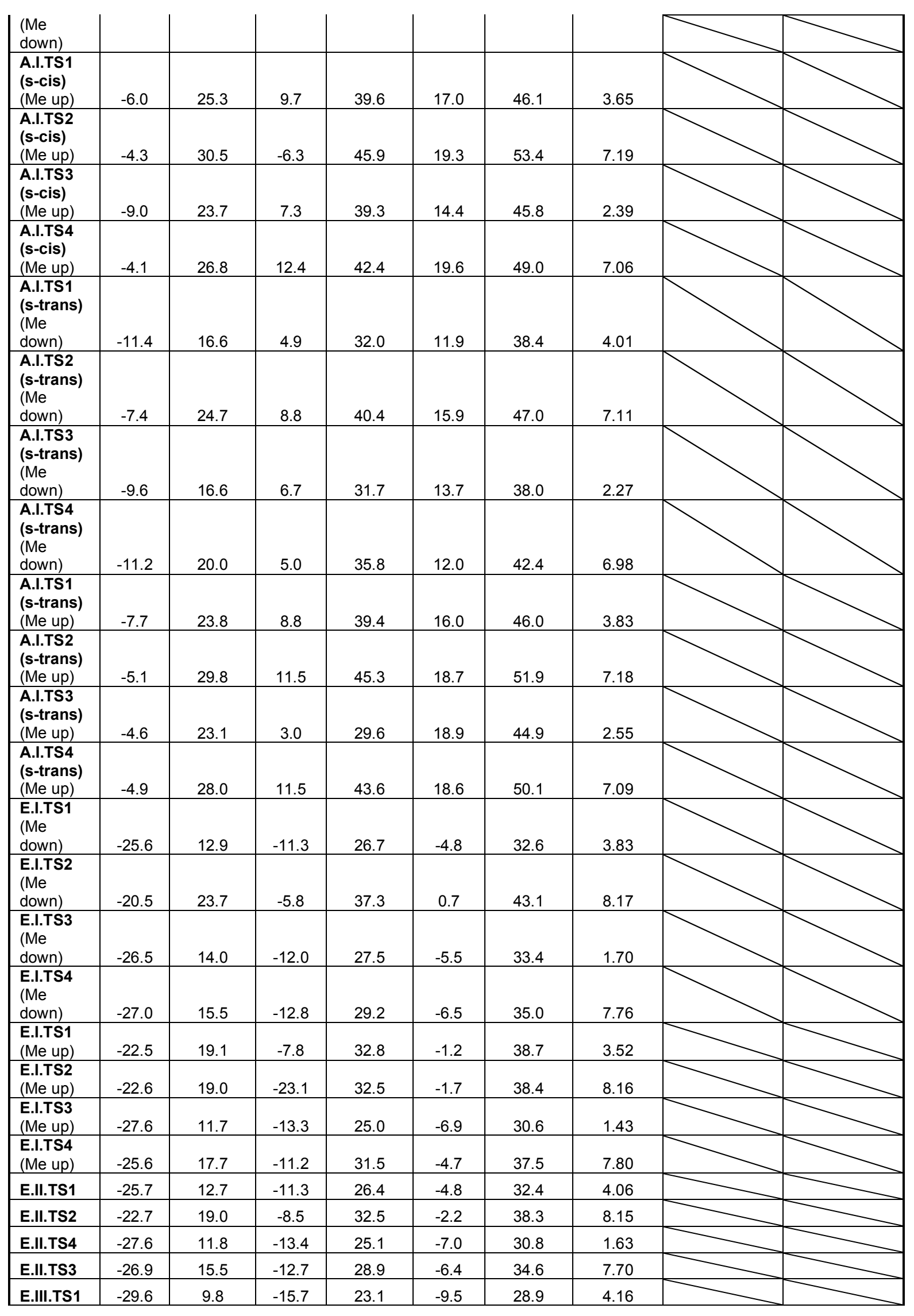




\begin{tabular}{|l|l|l|l|l|l|l|l|}
$\begin{array}{l}\text { (Me } \\
\text { down) }\end{array}$ & & & & & & & \\
\hline $\begin{array}{l}\text { E.III.TS2 } \\
\text { (Me } \\
\text { down) }\end{array}$ & -24.9 & 18.7 & -10.9 & 31.7 & -4.6 & 37.2 & 8.23 \\
\hline $\begin{array}{l}\text { E.III.TS3 } \\
\text { (Me } \\
\text { down) }\end{array}$ & -31.3 & 11.8 & -17.6 & 25.1 & -11.5 & 30.8 & 1.76 \\
\hline $\begin{array}{l}\text { E.III.TS4 } \\
\text { (Me } \\
\text { down) }\end{array}$ & -31.9 & 12.3 & -18.4 & 25.2 & -12.3 & 30.8 & 7.78 \\
\hline $\begin{array}{l}\text { E.III.TS1 } \\
\text { (Me up) }\end{array}$ & -26.5 & 15.3 & -12.3 & 28.4 & -6.0 & 34.0 & 3.97 \\
\hline $\begin{array}{l}\text { E.III.TS2 } \\
\text { (Me up) }\end{array}$ & -27.0 & 15.3 & -13.2 & 28.2 & -7.1 & 33.8 & 8.26 \\
\hline $\begin{array}{l}\text { E.III.TS3 } \\
\text { (Me up) }\end{array}$ & -32.7 & 10.0 & -19.2 & 22.9 & -13.2 & 28.5 & 1.52 \\
\hline $\begin{array}{l}\text { E.III.TS4 } \\
\text { (Me up) }\end{array}$ & -30.3 & 13.9 & -16.7 & 26.9 & -10.6 & 32.5 & 7.81 \\
\hline
\end{tabular}




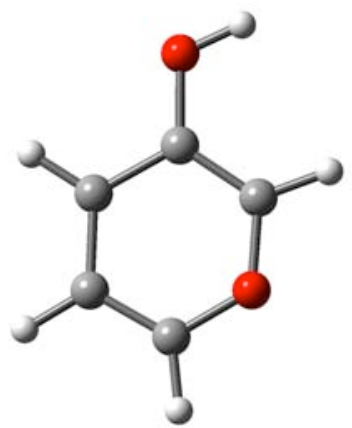

$\mathrm{HF}=-343.6913033$ hartrees $(-215669.729733783 \mathrm{kcal} / \mathrm{mol})$

Imaginary Frequencies: none found

Zero-point correction $=0.093405($ Hartree/Particle $)$

Thermal correction to Energy $=\quad 0.098818$

Thermal correction to Enthalpy= $\quad 0.099763$

Thermal correction to Gibbs Free Energy $=\quad 0.064420$

Sum of electronic and zero-point Energies $=\quad-343.597898$

Sum of electronic and thermal Energies $=\quad-343.592485$

Sum of electronic and thermal Enthalpies $=\quad-343.591541$

Sum of electronic and thermal Free Energies $=\quad-343.626883$

Temperature 298.150 Kelvin. Pressure $1.00000 \mathrm{Atm}$.

Coordinates (from last standard orientation):

\begin{tabular}{ccrrr}
\hline & Center & Atomic & \multicolumn{3}{c}{ Coordinates (Angstroms) } \\
Number & Number & $X$ & $Y$ & $Z$ \\
\hline 1 & 8 & 1.071977 & -1.172848 & -0.000121 \\
2 & 8 & -2.291650 & 0.133743 & 0.000641 \\
3 & 6 & -0.258715 & -1.165844 & -0.000165 \\
4 & 6 & -0.962348 & 0.032211 & -0.000483 \\
5 & 6 & -0.210427 & 1.227449 & -0.000427 \\
6 & 6 & 1.180227 & 1.172010 & 0.000272 \\
7 & 1 & 1.784219 & 2.072471 & 0.000799 \\
8 & 6 & 1.797748 & -0.063231 & 0.000052 \\
9 & 1 & -0.682972 & -2.163380 & 0.000793 \\
10 & 1 & 2.862666 & -0.259586 & 0.000787 \\
11 & 1 & -0.740589 & 2.175556 & -0.000979 \\
12 & 1 & -2.744851 & -0.727801 & -0.001044
\end{tabular}

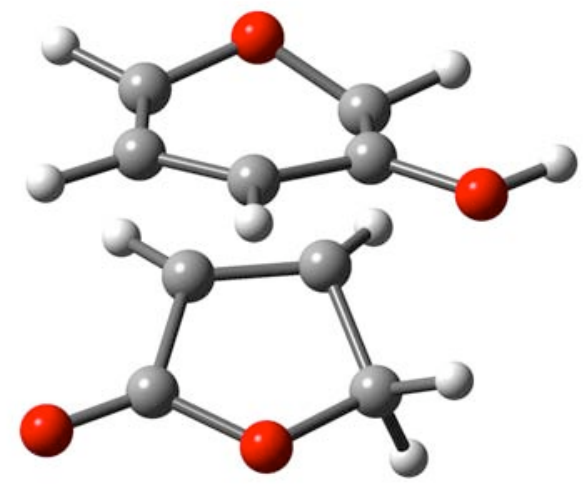

$\mathrm{HF}=-648.9208484$ hartrees $(-407204.321579484 \mathrm{kcal} / \mathrm{mol})$ 
Imaginary Frequencies: $1(-416.76411 / \mathrm{cm})$

Zero-point correction $=0.169954$ (Hartree/Particle)

Thermal correction to Energy $=\quad 0.180265$

Thermal correction to Enthalpy= $\quad 0.181209$

Thermal correction to Gibbs Free Energy $=\quad 0.133549$

Sum of electronic and zero-point Energies $=\quad-648.750895$

Sum of electronic and thermal Energies $=\quad-648.740583$

Sum of electronic and thermal Enthalpies $=\quad-648.739639$

Sum of electronic and thermal Free Energies $=\quad-648.787300$

Coordinates (from last standard orientation):

\begin{tabular}{|c|c|c|c|c|}
\hline \multirow{2}{*}{$\begin{array}{l}\text { Center } \\
\text { Number }\end{array}$} & \multirow{2}{*}{$\begin{array}{l}\text { Atomic } \\
\text { Number }\end{array}$} & \multicolumn{3}{|c|}{ Coordinates (Angstroms) } \\
\hline & & & $X \quad Y$ & Z \\
\hline 1 & 8 & -1.309671 & 1.351672 & 1.109098 \\
\hline 2 & 8 & -2.221246 & -1.520572 & -0.853075 \\
\hline 3 & 6 & -1.489442 & 0.017155 & 0.875083 \\
\hline 4 & 8 & 1.585597 & -1.313365 & -0.473912 \\
\hline 5 & 6 & 50788 & -0.374174 & -0.502558 \\
\hline 6 & 6 & 5909 & 0.412824 & -1.484268 \\
\hline 7 & 6 & -0.338399 & 1.529460 & -1.053268 \\
\hline 8 & 1 & 0.289350 & 2.098059 & -1.731723 \\
\hline 9 & 6 & -0.418711 & 1.932593 & 0.289128 \\
\hline 10 & 6 & 1.193435 & 0.434495 & 0.985906 \\
\hline 11 & 1 & 1.553179 & 1.240835 & 1.612988 \\
\hline 12 & 6 & 38554 & $4-0.6$ & 1.390 \\
\hline 13 & 6 & .774131 & $1-1.8$ & 0.585281 \\
\hline 14 & 1 & -2.139717 & $7 \quad-0.421713$ & 1.628033 \\
\hline 15 & 1 & -0.087787 & 2.903844 & 0.638392 \\
\hline 16 & 1 & -1.084810 & 0.116144 & -2.526731 \\
\hline 17 & 1 & 0.080643 & -0.734708 & 2.439690 \\
\hline 18 & 1 & 1.378467 & $7-2.496630$ & 1.215073 \\
\hline 19 & 6 & 1.984868 & -0.028620 & -0.198079 \\
\hline 20 & 8 & 2.780009 & 0.579874 & -0.857976 \\
\hline 21 & 1 & -0.026846 & -2.423790 & 0.134879 \\
\hline 22 & 1 & -2.726417 & -1.933635 & -0.129448 \\
\hline
\end{tabular}

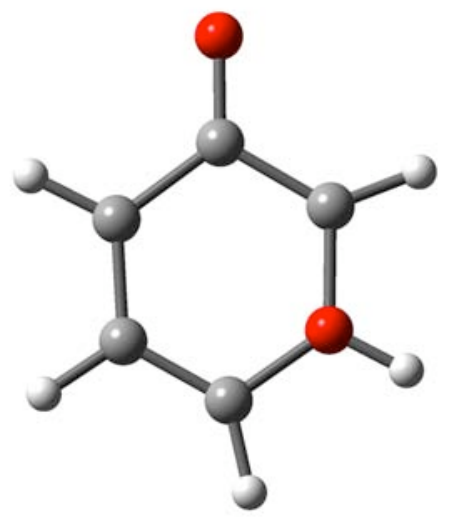

$\mathrm{HF}=-343.5430479$ hartrees $(-215576.697987729 \mathrm{kcal} / \mathrm{mol})$

Imaginary Frequencies: none found

Zero-point correction $=0.089553($ Hartree/Particle $)$ 


\begin{tabular}{|c|c|c|c|c|c|}
\hline \multirow{2}{*}{\multicolumn{6}{|c|}{ Thermal correction to Energy= }} \\
\hline & & & & & \\
\hline \multicolumn{6}{|c|}{ Thermal correction to Enthalpy= } \\
\hline \multicolumn{6}{|c|}{ Thermal correction to Gibbs Free Energy $=0.0593$} \\
\hline \multicolumn{6}{|c|}{ Sum of electronic and zero-point Energies= } \\
\hline \multirow{2}{*}{\multicolumn{6}{|c|}{ Sum of electronic and thermal Energies= }} \\
\hline & & & & & \\
\hline \multicolumn{6}{|c|}{ Sum of electronic and thermal Free Energies= } \\
\hline \multirow{2}{*}{$\begin{array}{l}\text { Center } \\
\text { Number }\end{array}$} & \multirow{2}{*}{$\begin{array}{l}\text { Atomic } \\
\text { Number }\end{array}$} & & \multicolumn{3}{|c|}{ Coordinates (Angstroms) } \\
\hline & & & $X \quad Y$ & Z & Z \\
\hline 1 & 8 & & & & \\
\hline 2 & 8 & & 012 & & 3220 \\
\hline 3 & 6 & 0.357536 & 9693 & & 3041 \\
\hline 4 & 6 & 1.149205 & 0.030523 & -0.00 & 00012 \\
\hline 5 & 6 & 0.33 & 3643 & & 2694 \\
\hline 6 & 6 & -1.060549 & 1.252612 & 0.00 & 00019 \\
\hline 7 & 1 & -1.618632 & 2.184550 & -0.00 & 00096 \\
\hline 8 & 6 & & 0.094118 & -0. & 02088 \\
\hline 9 & 1 & 0.701058 & -2.206191 & 0.00 & 05604 \\
\hline 10 & 1 & -2.860351 & -0.083723 & & 005717 \\
\hline 11 & 1 & & 2.196601 & & 05500 \\
\hline 12 & 1 & -1.552547 & -1.937771 & & 000244 \\
\hline
\end{tabular}

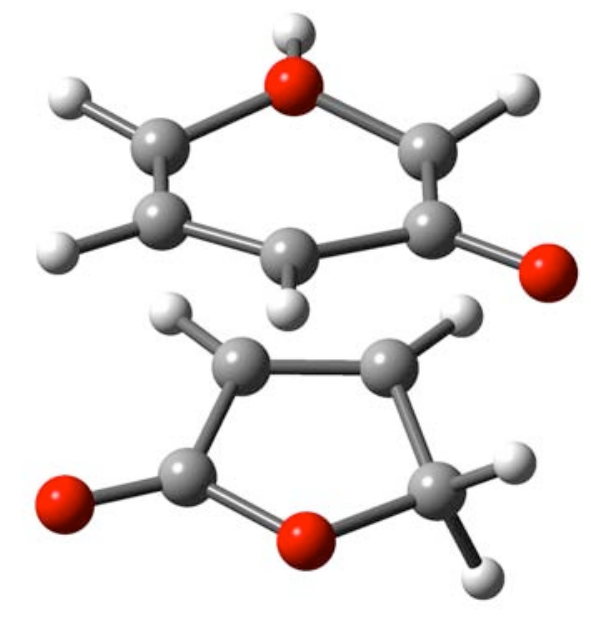

$\mathrm{HF}=-648.8087091$ hartrees $(-407133.953047341 \mathrm{kcal} / \mathrm{mol})$

Imaginary Frequencies: $1(-228.26251 / \mathrm{cm})$

Zero-point correction $=0.166210$ (Hartree/Particle)

Thermal correction to Energy $=\quad 0.177356$

Thermal correction to Enthalpy $=\quad 0.178300$

Thermal correction to Gibbs Free Energy $=\quad 0.128816$

Sum of electronic and zero-point Energies $=\quad-648.642499$

Sum of electronic and thermal Energies $=\quad-648.631353$

Sum of electronic and thermal Enthalpies $=\quad-648.630409$

Sum of electronic and thermal Free Energies $=\quad-648.679893$

Temperature 298.150 Kelvin. Pressure 1.00000 Atm.

$\begin{array}{llll}\text { Center } & \text { Atomic } & & \text { Coordinates (Angstroms) } \\ \text { Number } & \text { Number } & X & \text { Y }\end{array}$




\begin{tabular}{ccrrr}
\hline 1 & 8 & 1.430078 & -1.124181 & 1.156561 \\
2 & 8 & 2.039770 & 1.792476 & -0.818170 \\
3 & 6 & 1.654822 & 0.256672 & 0.877389 \\
4 & 8 & -1.670965 & 1.033213 & -0.714741 \\
5 & 6 & 1.690761 & 0.654960 & -0.520748 \\
6 & 6 & 1.198530 & -0.363986 & -1.454101 \\
7 & 6 & 0.660826 & -1.563690 & -1.026584 \\
8 & 1 & 0.196795 & -2.252643 & -1.725803 \\
9 & 6 & 0.631656 & -1.922326 & 0.311074 \\
10 & 6 & -1.369009 & -0.294909 & 1.145198 \\
11 & 1 & -1.594289 & -1.111415 & 1.820039 \\
12 & 6 & -0.778054 & 0.903139 & 1.429432 \\
13 & 6 & -1.072902 & 1.854105 & 0.297597 \\
14 & 1 & 2.232155 & 0.726086 & 1.664605 \\
15 & 1 & 0.391493 & -2.877988 & 0.754786 \\
16 & 1 & 1.253717 & -0.128508 & -2.512702 \\
17 & 1 & -0.434910 & 1.244936 & 2.400585 \\
18 & 1 & -1.795690 & 2.613527 & 0.623234 \\
19 & 6 & -1.944673 & -0.220805 & -0.229376 \\
20 & 8 & -2.485397 & -1.080268 & -0.871734 \\
21 & 1 & -0.204676 & 2.364503 & -0.129490 \\
22 & 1 & 1.415767 & -1.367375 & 2.110140 \\
---------------------------------
\end{tabular}

